\title{
NISTIR 6997
}

\section{User Manual for Finite Element and Finite Difference Programs: A Parallel Version of NIST IR 6269}

Robert B. Bohn

Edward J. Garboczi

U. S. DEPARTMENT OF COMMERCE Technology Administration National Institute of Standards and Technology

Information Technology Laboratory Building and Fire Research Laboratory Gaithersburg, MD 20899

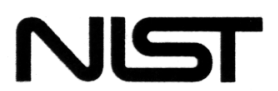

National Instifute of Standards and Technology 


\section{NISTIR 6997}

\section{User Manual for Finite Element and Finite Difference Programs: A Parallel Version of NIST IR 6269}

Robert B. Bohn

Edward J. Garboczi

U. S. DEPARTMENT OF COMMERCE Technology Administration National Institute of Standards and Technology Information Technology Laboratory Building and Fire Research Laboratory Gaithersburg, MD 20899

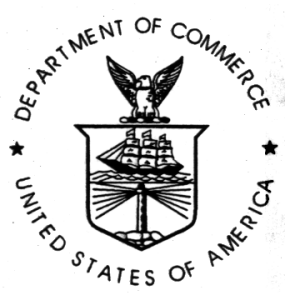

U.S. DEPARTMENT OF COMMERCE Donald L. Evans, Secretary TECHNOLOGY ADMINISTRATION Phillip J. Bond, Under Secretary for Technology NATIONAL INSTITUTE OF STANDARDS AND TECHNOLOGY

Arden L. Bement, Jr., Director 


\title{
User Manual for Finite Element and Finite Difference Programs: A Parallel Version of NISTIR-6269
}

\author{
Robert B. Bohn \\ Information Technology Laboratory \\ Edward J. Garboczi \\ Building and Fire Research Laboratory \\ NIST \\ Gaithersburg, MD 20899
}

March 4, 2003

\begin{abstract}
This document contains the descriptions, algorithms, user information and listings of the parallel Fortran90/MPI versions of the suite of programs found in NIST-IR 6269 [1], Finite Element and Finite Difference Programs for Computing the Linear Electric and Elastic Properties of Digital Images of Random Materials. These programs use 3-D digital image data on random materials as input and then calculate the effective properties of the random material when subjected to applied fields (for example, mechanical/thermal stresses and AC/DC electric fields).

The purpose behind this undertaking is to execute these programs in a parallel computing environment (for example, Linux clusters), so as to decrease real-time execution, increase the potential problem size, and increase digital resolution/problem accuracy.

Keywords: computer modeling, concrete, elastic moduli; electrical conductivity; finite difference; finite element; MPI; parallel computing; random materials; thermal elasticity
\end{abstract}




\section{Contents}

1 Introduction $\quad 4$

2 Program Theory 5

2.1 Data Model and Memory Management . . . . . . . . . . . . . . . . . 5

$2.1 .1 \quad$ Finite Element . . . . . . . . . . . . . . . . . . . 9

$2.1 .2 \quad$ Finite Difference . . . . . . . . . . . . . . . . . . . 9

2.2 Parallel Algorithms . . . . . . . . . . . . . . . . . . . . . . 12

$2.2 .1 \quad$ Finite Element . . . . . . . . . . . . . . . . . . . . 12

2.2 .2 Finite Difference . . . . . . . . . . . . . . . . . . . . 13

3 Program Operation and USER information $\quad 14$

3.1 Notable changes from the serial version . . . . . . . . . . . . . . . . . 14

3.2 Inputs . . . . . . . . . . . . . . . . . . . . . . . . 14

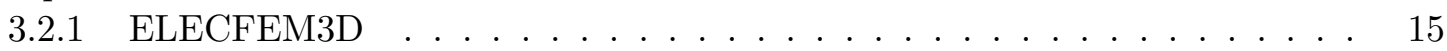

$3.2 .2 \quad$ ELAS3D . . . . . . . . . . . . . . . . . . . . . . 15

3.2 .3 THERMAL3D . . . . . . . . . . . . . . . . . . . 15

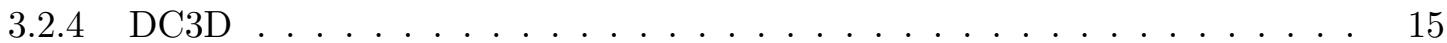

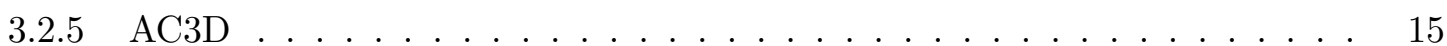

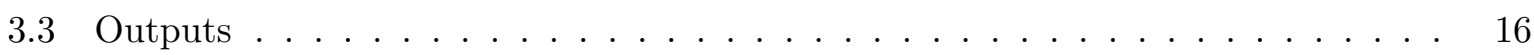

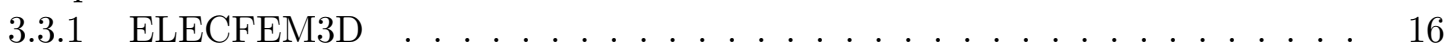

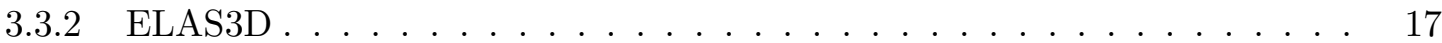

3.3 .3 THERMAL3D . . . . . . . . . . . . . . . . . . 17

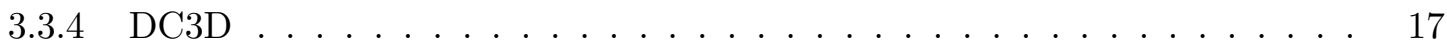

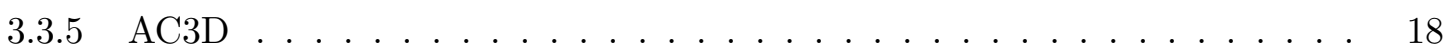

$3.4 \mathrm{MAIN} \ldots \ldots \ldots \ldots \ldots \ldots \ldots \ldots$

3.5 Finite Element Subroutines . . . . . . . . . . . . . . . . . . . 19

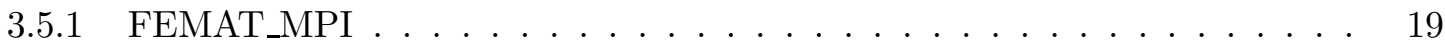

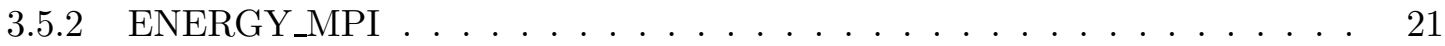

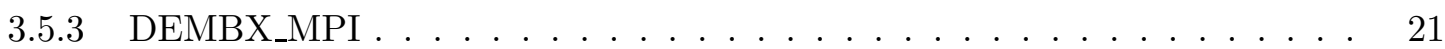

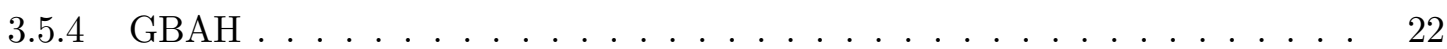

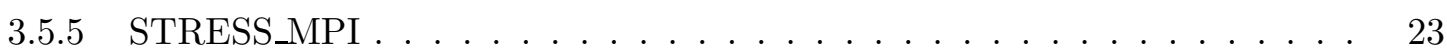

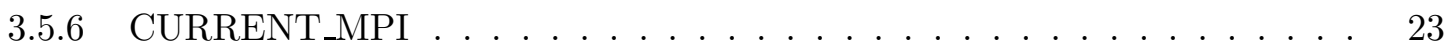

3.6 Finite Difference Subroutines . . . . . . . . . . . . . . . . . . . . . . 23

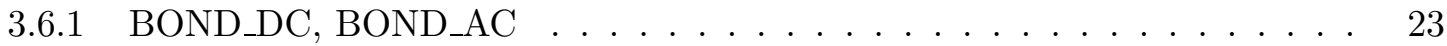

3.6 .2 DEMBX_DC, DEMBX_AC . . . . . . . . . . . . . . 24

3.6.3 CURRENT_DC, CURRENT_AC . . . . . . . . . . . . . . 24

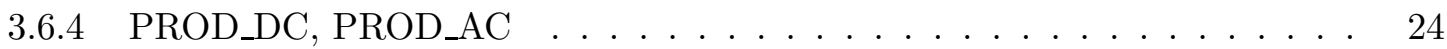

3.7 Other Subroutines . . . . . . . . . . . . . . . . . . . . 24

3.7 .1 z_ghost_int,z_ghost_dp,z_ghost_cmplx . . . . . . . . . . . . . 24

3.7.2 xy_ghost_dp,xy_ghost_cmplx . . . . . . . . . . . . . . . . 24

3.7 .3 t2b,b2t,t2b_dp,b2t_dp, t2b_cmplx,b2t_cmplx . . . . . . . . . . . 25

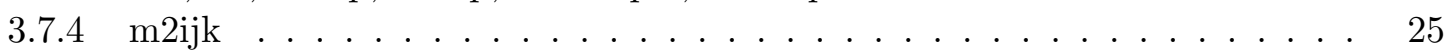

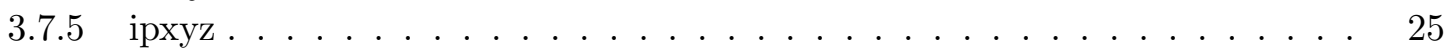

3.7 .6 phasemod_init . . . . . . . . . . . . . . . . 25

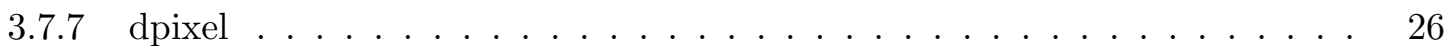

3.7 .8 dassig . . . . . . . . . . . . . . . . . . . 26

3.8 Makefiles and Execution scripts . . . . . . . . . . . . . . . 26

3.8.1 PC Linux Cluster using PBS . . . . . . . . . . . . . . 26 
3.8 .2 SGI Origin 2000 using NQS . . . . . . . . . . . . . . . 28

4 MPI Primer $\quad 29$

4.1 Initialization . . . . . . . . . . . . . . . . . . . . . . . . 29

4.2 Sending and Receiving Data . . . . . . . . . . . . . . . . . . 30

4.3 Built-in Mathematical Functions . . . . . . . . . . . . . . . . . . 31

4.4 Summary . . . . . . . . . . . . . . . . . . . . . 31

5 Disclaimer $\quad 31$

6 Program Listings $\quad 32$

6.1 Finite Element . . . . . . . . . . . . . . . . . . . . . . . . . 32

$6.1 .1 \quad$ ELECFEM3D_MPI.f . . . . . . . . . . . . . . . . . . . 32

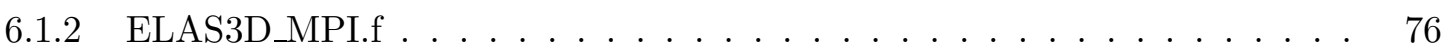

6.1 .3 THERMAL3D_MPI.f . . . . . . . . . . . . . . . . . . 129

6.2 Finite Difference . . . . . . . . . . . . . . . . . . . . . 210

6.2 .1 DC3D_MPI.f . . . . . . . . . . . . . . . . . . 210

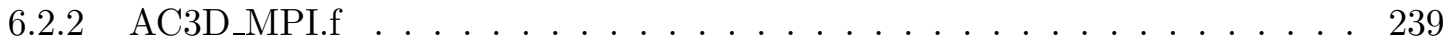

7 Tools $\quad \mathbf{2 7 2}$

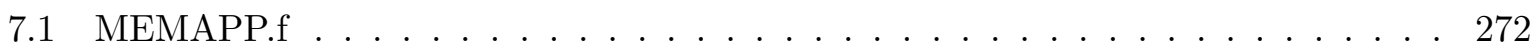

\section{List of Tables}

1 Array redimensioning from serial to parallel versions of ELECFEM3D, ELAS3D and THERMAL3D . . . . . . . . . . . . . . . . . . . . 10

\section{List of Figures}

1 Depiction of Data Set split across 8 processing nodes. . . . . . . . . . . . 6

2 Depiction of $\mathrm{d} 1$ and $\mathrm{d} 2$ values for Root and Processing Node $1 \ldots \ldots$. . . . . . 7

3 Depiction of Top and Bottom Ghost Layers from Root and Node 1. d2+1(Node1) $=\mathrm{d} 1($ Node 2$) ; \mathrm{d} 1-1($ Node 1$)=\mathrm{d} 2$ (Root) $; \mathrm{d} 2+1($ Root $)=\mathrm{d} 2$ (Node1) but, $\mathrm{d} 1-1$ (Root)

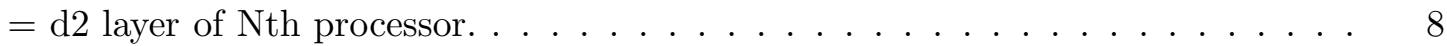

4 Depiction of original finite element data set in a serial based program showing the equivalency of the $\mathrm{Z}$ layers. The Real layers are real data. . . . . . . . . . . . . . 10

5 Depiction of ghost layers in the parallel finite element program showing the equivalency of the $\mathrm{Z}$ layers across 4 processors for the $n z=6, n z 2=8$ case. Note how the $d 1-1$ layer of the bottom node \& the $d 2+1$ layer of the top node are unused in the calculation. . . . . . . . . . . . . . . . . . 


\section{Introduction}

Calculating the effective properties of random materials is not a trivial procedure. Due to their random composition, random phase shape, and widely varying length scales, these properties cannot be determined analytically, but instead necessitate a numerical computation. But before any computing may begin, detailed microstructural information must be in hand. Some of this information may be obtained experimentally using x-ray tomography or similar techniques or developed in models. In any event, the input is converted to a 2 or 3 -D digital image that represents the overall structure of the composite.

One application is to the properties of cement and concrete. They are complex mixtures that can contain 20 to 30 distinct individual chemical phases in the same mixture. The nominal sizes of the data sets are $100^{3}$ to $300^{3}$ voxels, containing several thousand particles. Even though this seems to be a large number of voxels, one would like to increase the potential problem size and increase the digital resolution of the original data image. But the possible overall sizes of problems have been ultimately bound by the available computational resources of a serial based machine. In addition, the wall clock (real) time to perform these calculations routinely reach into the 100 hour regime, making even larger computations impractical.

The original programs from NISTIR-6269 calculate the overall effective properties of the above systems but they suffer from several limitations. Resolution problems (hence accuracy) are affected if the dataset is too large ( $\geq 512^{3}$ voxels) since lack of computer memory becomes a critical issue. At present, datasets this size must be split up into smaller files and processed individually, each producing a subset of results that must be approximately linked together.

Using parallel processing allows one to have the power and storage capacity of several processors. It is then possible to divide a large dataset exactly across multiple processors and, in essence, each processor operates on a dataset of reduced size. In addition, any large arrays calculated in the serial version only have their corresponding sections calculated on each processor as well. The overall functionality of the program is not compromised by operating on smaller sections, but one can gain theoretical speed-ups of execution time on the order of the number of processors used and so be able to handle large problems.

Parallel processing also supports data transfer, i.e. send and receive mechanisms, between the processors. This is important for calculations involving nearest neighbors. Splitting the data across $N$ processors sets up $N-1$ imposed boundaries on the data by direct consequence of the split. Of course, it is necessary to know which data is needed by which processor and when in order to have a successfully operating program.

To accommodate these large calculations and decrease the time to perform them, the suite of original programs from NISTIR-6269 have been converted to run in a parallel environment in FORTRAN90 with MPI (Message Passing Interface). This conversion allows datasets of size $400^{3}$ or greater to be routinely used without any compromise of numerical accuracy. 


\section{Program Theory}

This section will examine the basics behind the parallel versions by discussing their data models, memory management, and algorithms. Although one can probably comprehend the principles of the programming model, it is important to have the necessary mathematical background from the original NISTIR 6269 to provide a more complete understanding of the relationships between the serial and parallel versions.

Briefly, these programs operate by performing a series of matrix operations (additions and multiplications) on very large arrays, dimensioned as large as the original data set or greater, in order to minimize the overall energy of the system in question. The minimization technique used is a conjugate gradient solver [2]. This is an iterative procedure which is carried out until a certain minimum threshold (energy gradient) is reached. The serial case defines these arrays as $1-\mathrm{D}$ vectors and then operates or computes each element sequentially and therefore independently from each other. This is good behavior for a program to have if the parallel adaptation is to be made. In fact, it ensures that the operations can proceed in a parallel fashion. Therefore, it is necessary to give each processor a piece of the original data array and most of the calculations can proceed independently until special cases of communication are warranted. It is important to reduce the amount of time one spends communicating to gain the maximum time savings from parallel computations.

The theoretical core behind each program is similar, therefore many of the parallel algorithms were readily modified to adapt to the specific physical case (mechanical/thermal stress, electric fields). Differences between the codes is manifested by a few simple items, namely the dimensionality of the problem (arrays), how many arrays are included in the minimization, the specific applied field and if the program itself is either based on a finite element or finite difference method of solution of the original partial differential equations.

\subsection{Data Model and Memory Management}

In a parallel environment with a set of $N$ processors, the program is typically set up such that one processor is arbitrarily selected as the root node $(\mathrm{rank}=0)$ and the others as workers $(\mathrm{rank}=1 \ldots \mathrm{N}$ 1). For a given processor, $\mathrm{P}$, we call processors with rank equal to $P-1$ and $P+1, P$ 's south and north neighbor, respectively. Root is in charge of the I/O, assigning data to the workers (using MPI) and does its share of calculating. It should be noted that the user does not actually assign the root or worker identity to any specific processor in the cluster, but the program makes the requirement that the conditions exist and it is the operating system's duty to carry it out. The user can always specify a processor to carry out a certain function in the code. See Section 4, MPI Primer for details on how each processor is uniquely identified within a program.

As mentioned previously, each matrix element can be calculated individually and independently from each other. So these parallel programs take advantage of the 3 -D nature of the data (stored in array pix) by splitting it (along the $\mathrm{z}$-direction) across multiple processing nodes. Each matrix element is addressed by a unique triplet of $(x, y, z)$ coordinates and only portions (z-specific) of these large arrays exist on all the processors. This data is divided as evenly as possible over $N$ compute nodes in the $\mathrm{z}$ direction. The number of data layers, $\mathbf{N}_{k}$, each of the $N$ processors receives per array is approximately $\frac{n z}{N}$. Therefore, the data model is to take a large array and treat it as $n z 2$-D arrays with dimensions of $(n x, n y)$. Now each processing node

only has to dedicate $\frac{1}{N}$ (Figure 1 ) the amount of memory to data storage for an equivalent sized problem on a serial machine. Theoretically, this calculation should speed up by a factor of $\mathrm{N}$ the amount of time to execute the same problem on a serial based machine. Additionally, problems which are $\mathrm{N}$ times as large can be run as well. 


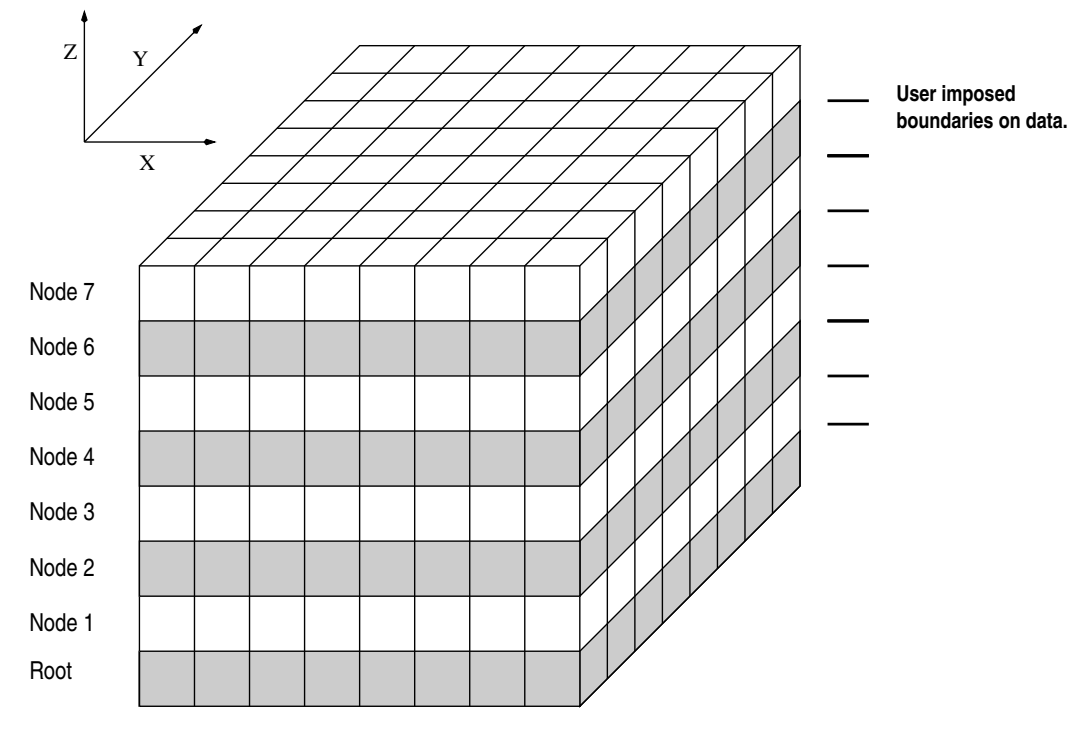

Figure 1: Depiction of Data Set split across 8 processing nodes.

Given the magnitude of $n z$ and the number of processors, root calculates the number of data layers of pix each processing node receives. The lower and upper limits of the $\mathrm{z}$ extents for each processor are $d 1$ and $d 2$. Each node gets its own copy of the layers of pix from root; root stores a master copy of the $d 1$ and $d 2$ values for all the nodes in arrays $d 1 s$ and $d 2 s$. Next, root passes the contiguous layers of the original data in which the value of pix's $k$ indices lie $(d 1 \leq k \leq d 2)$ on the proper processing node. The proper assignment of an array of this type using FORTRAN90 notation is: $p i x(n x, n y, d 1: d 2)$. The last index in the form $d 1: d 2$, is the range of the $k$ values used. For a given processing node, these values are unique.

The inherent question after splitting the original data across a number of processing nodes is: Does a node have all the data it needs to carry out its assigned tasks? We know for these problems, which need nearest neighbor information, that they cannot have the required data after the initial split due to the user imposed boundaries on the dataset. Therefore inter-node communication (data transfer) is necessary. This requires the processors to know which nodes have the data they need and a mechanism for the data transfer.

Since a voxel needs information from its nearest neighbors to perform a correct calculation, problems arise when a processor attempts to calculate using a voxel located in either its top layer $(z=d 2)$ or its bottom layer $(z=d 1)$. Since this problem arises for all voxels in their respective $d 1$ or $d 2$ layers, a given node will need an entire data slice (one 2-d array) from its north and south neighbors, respectively. To be exact, processor $P$ needs the south node $(P-1)$ to send its values of $p i x(i, j, d 2)$ and the north node $(P+1)$ to send its values of $p i x(i, j, d 1)$. In another notation, using the rank value as a subscript, processor $P$ needs $p i x(i, j, d 2)_{P-1}$ and $\operatorname{pix}(i, j, d 1)_{P+1}$.

The preferred way for handling this situation is to increase the z-size of the array on each node by 2 . The new layers occupy $k=d 1-1$ and $k=d 2+1$ per processor. They are referred to commonly as ghost layers (Figure 3). These layers are created before any of the calculations proceed since pix does not change during a calculation. This method allows the calculations to proceed uninterrupted unless global sums or other similar actions are called for. We define a new array called vox which is a copy of pix, but also containing the 2 extra data layers. It has no serial counterpart, but will function like pix from the serial code. It is dimensioned in 


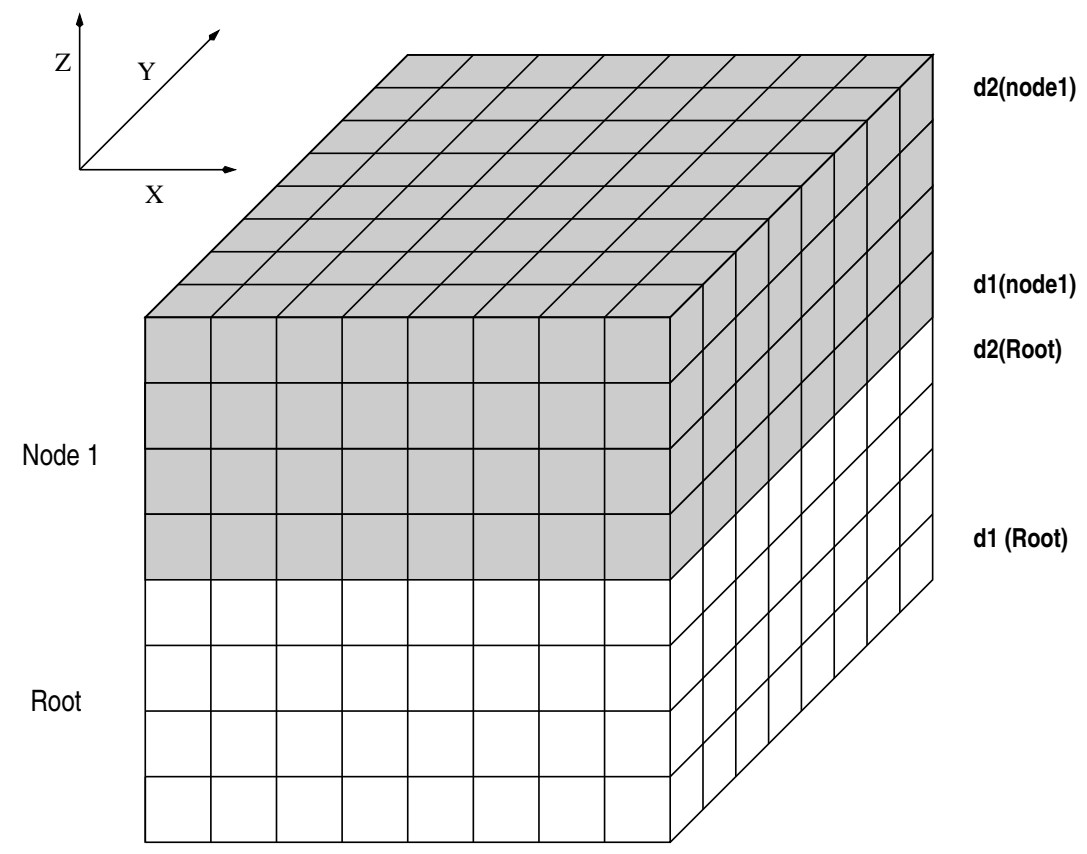

Figure 2: Depiction of $\mathrm{d} 1$ and $\mathrm{d} 2$ values for Root and Processing Node 1

FORTRAN90 as: $\operatorname{vox}(i, j, d 1-1: d 2+1)$. To emphasize its identity,

$$
\begin{gathered}
\operatorname{vox}(i, j, d 1: d 2)_{P}=p i x(i, j, d 1: d 2)_{P} \\
\operatorname{vox}(i, j, d 1-1)_{P}=p i x(i, j, d 2)_{P-1} \\
\operatorname{vox}(i, j, d 2+1)_{P}=p i x(i, j, d 1)_{P+1}
\end{gathered}
$$

This makes the total amount of memory usage per node increase slightly. However, it obviates the need for additional inter-node communication during a given calculation that would increase the overall run time of the job. Also remember that one is gaining substantial memory savings compared to the serial version, so this cost is acceptable.

This situation gives rise to two special cases, namely: What is considered south of processor 0 and north of processor $N-1$ ? The key to this is to know that the original data, pix, has periodic boundary conditions and behaves in a cyclic fashion. Therefore, south of processor 0 is processor $N-1$ and north of processor $N-1$ is processor 0 . This leads to the following assignments.

Processor 0:

$$
\begin{gathered}
\operatorname{vox}(i, j, d 1: d 2)_{0}=\operatorname{pix}(i, j, d 1: d 2)_{0} \\
\operatorname{vox}(i, j, d 1-1)_{0}=\operatorname{pix}(i, j, d 2)_{N-1} \\
\operatorname{vox}(i, j, d 2+1)_{0}=\operatorname{pix}(i, j, d 1)_{1}
\end{gathered}
$$

Processor N-1:

$$
\begin{gathered}
\operatorname{vox}(i, j, d 1: d 2)_{N-1}=\operatorname{pix}(i, j, d 1: d 2)_{N-1} \\
\operatorname{vox}(i, j, d 1-1)_{N-1}=\operatorname{pix}(i, j, d 2)_{N-2} \\
\operatorname{vox}(i, j, d 2+1)_{N-1}=\operatorname{pix}(i, j, d 1)_{0}
\end{gathered}
$$




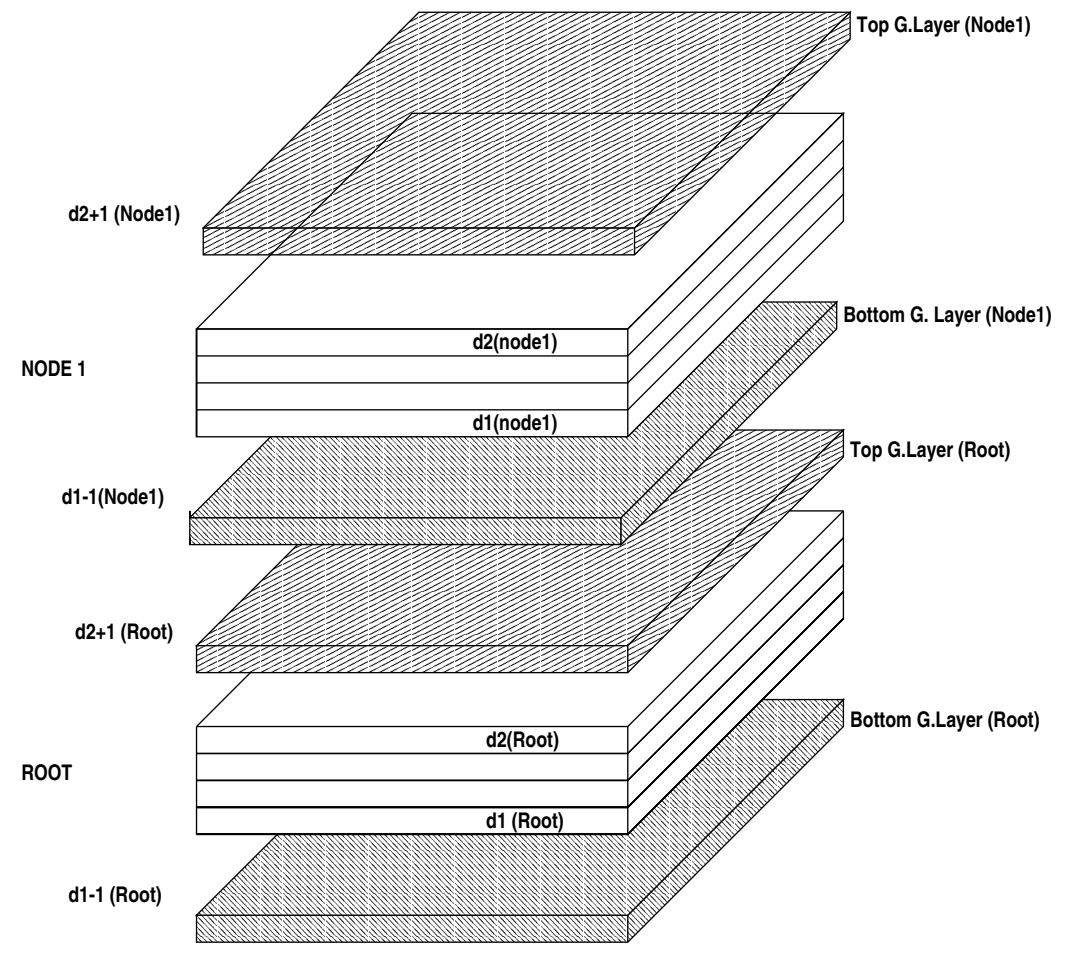

Figure 3: Depiction of Top and Bottom Ghost Layers from Root and Node 1. d2+1(Node1) = $\mathrm{d} 1($ Node2); d1-1(Node1) $=\mathrm{d} 2$ (Root); $\mathrm{d} 2+1$ (Root) $=\mathrm{d} 2$ (Node1) but, $\mathrm{d} 1-1$ (Root) $=\mathrm{d} 2$ layer of Nth processor.

In the serial code, memory allocation of the array pix and subsequent large arrays is handled in a static way by DIMENSION statements. In each statement, the user substitutes the numerical value of $n s$, where $n s=n x \times n y \times n z$, into the DIMENSION statement of each individual array. The parallel versions incorporate FORTRAN90's ALLOCATABLE and ALLOCATE statements. Dynamic memory allocation for the large arrays is based on the values of $n x, n y, n z, d 1$ and $d 2$. The user only needs to correctly enter the values for $n x, n y$ and $n z$ and the program handles the necessary allocation procedures. Assuming $n x, n y, n z, d 1$ and $d 2$ are already known, then examples of using ALLOCATE per node for arrays pix and vox are:

$$
\text { integer*2, allocatable : : pix }(:,:,:), \operatorname{vox}(:,:,:)
$$

C

C myrank on ROOT equals 0 ,

c so just ROOT does this:

if (myrank.eq.0) then

allocate (pix $(n x, n y, n z))$

end if

c But all nodes do this:

allocate (vox $(n x, n y, d 1-1: d 2+1))$

In this example, only root allocates memory for pix and all processors allocate memory space 
for its portion of vox. Root is the only processor which needs the entire pix array since it must pass out specific allotments to the workers. In conjunction with the DEALLOCATE statement, the memory used for pix is released after all passing of data is complete. Also vox is defined by its $d 1$ and $d 2$ limits and not the entire value, nz. This small range is at the heart of defining subsections of arrays per processor for parallel computations. Furthermore, this type of memory allocation used with the array vox is applied to all the large arrays found throughout all the programs. See Table 1 for a description of the array dimensions per program.

\subsubsection{Finite Element}

In the finite element programs, each voxel, of type INTEGER ${ }^{*} 2$, must know the positions of its 27 nearest neighbors in a cubic array since that is a mathematical requirement of the calculation. In the serial versions, this is accomplished by using an array, $i b$, which is dimensioned as $i b(n s, 27)$. This array is of type INTEGER $* 4$ and uses $54(27 \times 2)$ times the amount of memory as the original dataset. In fact, it serves no purpose as a calculating device, but is only used as a look-up (hash) table since it stores the 1-D positions of the 27 nearest neighbors for a given voxel.

In the parallel program, vox is dimensioned as a rank 3 array, vox $(n x, n y, d 1-1: d 2+1)$. With this arrangement, it is trivial to find the indices of 27 nearest neighbors for a given voxel, $\operatorname{vox}(i, j, k)$. The three nearest neighbors (including the voxel itself) in the z-direction have indices of $(i, j, k-1),(i, j, k)$ and $(i, j, k+1)$. Therefore the set of 27 nearest neighbors for this element is generated by adding \pm 1 or 0 to any or all of the indices of the $(i, j, k)$ triplet. The lowest neighbor has indices of $(i-1, j-1, k-1)$ and the highest has $(i+1, j+1, k+1)$. These values can be calculated on the fly or generated by using an adequately defined set of triply nested do-loops.

Special allowances have to be made when the current voxel is on the outside edges of the data cube (i.e. $i=1$ or $n x$ or $j=1$ or $n y$ ). At these extremes, the value of $i$ or $j$ is interrogated and the values of $i-1, i+1$ are compared to 0 and $n x$. For example, if $i-i$ (or $j-1$ ) equals 0 , a modification takes place and the $(i-1)^{t h}\left(\right.$ or $\left.(j-1)^{t h}\right)$ neighbor is replaced by the voxel with $i=n x$ (or $j=n y$ ). A similar modification takes place when the voxel having $i=n x(j=n y)$.

In this instance, the voxel with $i=1$ (or $j=1$ ) is used. This procedure is justified due to the periodic nature of the data.

Therefore, by switching over to a parallel implementation and this new indexing scheme, one has a dramatic improvement in memory savings since the additional storage of particle positions is no longer needed. This memory is now free to be put to better use.

Some small arrays $(d k, c m o d$, sigma, etc...) that appear throughout the calculations have dimensions that are determined by the number of phases nphase one has in the original dataset; this number is known a-priori, like $n x, n y$ and $n z$. Arrays which need this value are defined as allocatable as well. This increases the flexibility of the program and contributes to a saving of memory by implementing dynamic allocation of additional arrays. In the serial version of ELAS3D, $d k$ is dimensioned to $d k(100,8,3,8,3)$; the first index represents the phase number. Therefore, the new code allocates $d k$ as $d k$ (nphase, $8,3,8,3)$.

\subsubsection{Finite Difference}

In the finite difference programs, only the nearest neighbors are used to update a nodal voltage, so that the fact that the nearest neighbors are at $\Delta \mathrm{i}=\Delta \mathrm{j}=\Delta \mathrm{k}= \pm 1$ was used from the start. This made an array like $i b$ in the finite element programs, even in the serial version, unnecessary. For an $n x \times n y \times n z$ size microstructure in the serial code, the program actually stores an array that is $(n x+2) \times(n y+2) \times(n z+2)$ in size. The extra two layers of voxels in each direction 
are periodic continuations of the microstructure. The real microstructure lies from $2, n x+1$; $2, n y+1$; and $2, n z+1$, and is recorded in the array list in the serial version. Therefore, in a manner of speaking, the serial code has a set of ghost layers in the $\mathrm{x}, \mathrm{y}$ and $\mathrm{z}$ directions already built in. The parallel codes can determine (as described in the previous finite element section) if the position is real or belongs to a ghost layer and the array list becomes obsolete.

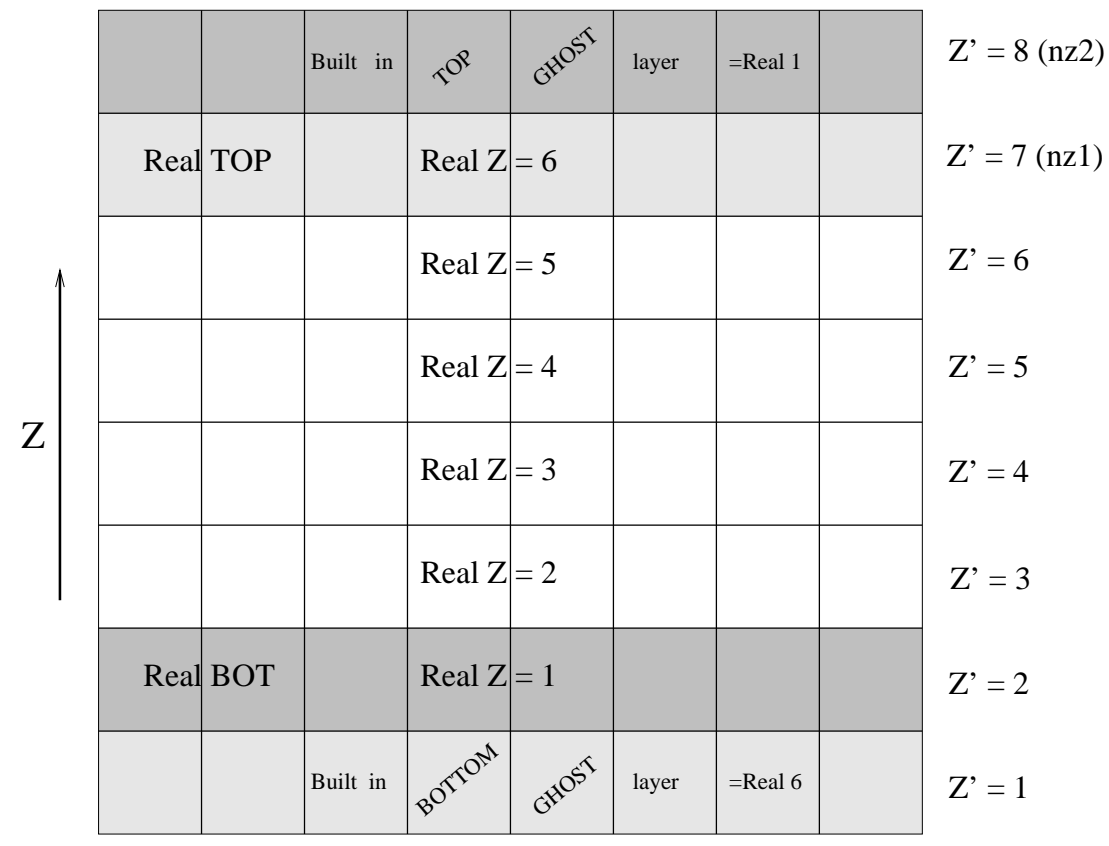

Figure 4: Depiction of original finite element data set in a serial based program showing the equivalency of the $\mathrm{Z}$ layers. The Real layers are real data.

With periodic updates of the $\mathrm{x}$ and $\mathrm{y}$ ghost sites, one can operate entirely on the real sites of the array, and freely take $\Delta \mathrm{i}=\Delta \mathrm{j}=\Delta \mathrm{k}= \pm 1$ to update each real site. The periodic boundary conditions are enforced via the regular updates of the boundary sites $(i=1$ and $n x+2, j=1$ and $n y+2$, and $k=1$ and $n z+2$ ).

However, in creating parallel implementations for the finite difference codes, there is a unique twist since the serial versions already have 2 Z-ghost layers built in. (Figure 4) In the serial

\begin{tabular}{|r||l|l|l|l|}
\hline Variable & Serial & ELECFEM3D & ELAS3D & THERMAL3D \\
\hline \hline pix/vox & ns & $($ nx,ny,d1-1:d2+1) & $($ nx,ny,d1-1:d2+1) & $(\mathrm{nx}, \mathrm{ny}, \mathrm{d} 1-1: \mathrm{d} 2+1)$ \\
\hline $\mathrm{X}$ & $\mathrm{ns}$ & $(\mathrm{nx}, \mathrm{ny}, \mathrm{d} 1-1: \mathrm{d} 2+1)$ & & \\
\hline $\mathrm{E}$ & $(\mathrm{ns}, 3)$ & & $(\mathrm{nx}, \mathrm{ny}, \mathrm{d} 1-1: \mathrm{d} 2+1,3)$ & \\
\hline $\mathrm{T}$ & $(\mathrm{ns}+2,3)$ & & & $(\mathrm{nx}, \mathrm{ny}, \mathrm{d} 1-1: \mathrm{d} 2+1,3),(2,3)$ \\
\hline $\mathrm{dk}$ & $(100,3,3),(100,8,3,8,3)$ & $($ nphase $, 3,3)$ & $($ nphase $, 8,3,8,3)$ & $(\mathrm{nph}), 8,3,8,3)$ \\
\hline phasemod & $(100,2)$ & $($ nphase, 2$)$ & $($ nphase, 2$)$ & $($ nphase, 2$)$ \\
\hline sigma & $(100,3,3)$ & $($ nphase $, 3,3)$ & & $($ nphase $, 3,3)$ \\
\hline eigen & $(100,3,3)$ & & & $($ nphase $, 3,3)$ \\
\hline
\end{tabular}

Table 1: Array redimensioning from serial to parallel versions of ELECFEM3D, ELAS3D and THERMAL3D 
codes, the real data lies in layers $Z^{\prime}=2$ through $n z+1$. The serial ghost layers contain the information from the first $(z=1)$ and last $(z=n z)$ real data in the $Z^{\prime}=1$ and $Z^{\prime}=n z 2$ layers, respectively. For the rest of the discussion, $n z 2=n z+2$.

\begin{tabular}{|c|c|}
\hline & $Z^{\prime}=1, d 2+1$ \\
\hline & built-in top ghost layer \\
\hline & $\mathrm{Z}^{\prime}=7, \mathrm{~d} 1$ (dlow, dhigh) \\
\hline 1 & $Z^{\prime}=6, d 1-1$ \\
\hline $\mathrm{Z}$ & $Z^{\prime}=7, d 2+1$ \\
\hline & $\mathrm{Z}^{\prime}=6, \mathrm{~d} 2$ (dhigh) \\
\hline & $Z^{\prime}=5, \mathrm{~d} 1$ (dlow) \\
\hline & $Z^{\prime}=4, \mathrm{~d} 1-1$ \\
\hline & $Z^{\prime}=5, \mathrm{~d} 2+1$ \\
\hline & $\mathrm{Z}^{\prime}=4, \mathrm{~d} 2$ (dhigh) \\
\hline & $\mathrm{Z}^{\prime}=3, \mathrm{~d} 1$ (dlow) \\
\hline & $Z^{\prime}=2, \mathrm{~d} 1-1$ \\
\hline & $Z^{\prime}=3, d 2+1$ \\
\hline & $\mathrm{Z}^{\prime}=2, \mathrm{~d} 2$ (dlow, dhigh) \\
\hline & built-in bottom ghost layer \\
\hline & $Z^{\prime}=8, d 1-1$ \\
\hline
\end{tabular}

Figure 5: Depiction of ghost layers in the parallel finite element program showing the equivalency of the $\mathrm{Z}$ layers across 4 processors for the $n z=6, n z 2=8$ case. Note how the $d 1-1$ layer of the bottom node \& the $d 2+1$ layer of the top node are unused in the calculation.

The parallel code is still set-up to split the layers dependent (i.e. calculate $d 1$ and $d 2$ per node) on the overall extent of z (Figure 5). Therefore, each node in the finite difference case will receive $\frac{n z 2}{N}$ layers and not $\frac{n z}{N}$ like a finite element problem since $n z 2$ is the overall extent of $z$. Therefore, before the parallelization and data split is made, it is known that the bottom node will already have the information it will need for its $d 1-1$ ghost layer and the top node will have the layer which it needs for its $d 2+1$ ghost layer. And interestingly enough, they are already stored in the bottom node's d1 and top node's d2 layers, respectively, before the split. Therefore, when the initial ghost layer production of vox (and subsequent arrays) in the parallel versions, the bottom node will only need to receive its $d 2+1$ (top ghost) layer and the top node will need it's $d 1-1$ (bottom ghost) layer through a message passing procedure.

Since it is known that before the splitting takes place, the bottom and top nodes already have 1 needed ghost layer, the code is designed with a new set of variables called dlow and dhigh which allow for the special cases of the lowest and topmost nodes and makes a one-to-one correspondence for $d 1$ with dlow and $d 2$ with dhigh for all other nodes. viz:

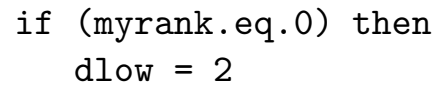




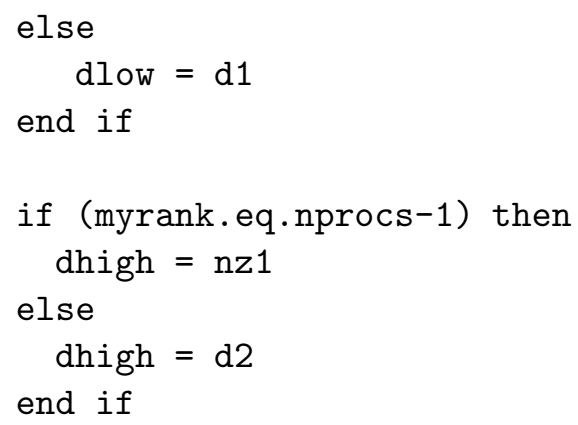

This type of code ensures that only the real data is used and the extra ghost layers (made by subroutine $z_{-}$ghost) for Node 0 and the the northernmost node, remain out of the central calculation.

Figures 4 and 5 depict the case of $n z=6$ for the serial and parallel cases. This increases the $\mathrm{Z}$ extent of this array to $n z+2$, or 8 . The $\mathrm{Z}=1$ and $\mathrm{Z}=8$ layers are the built in ghost layers, while layers with $2 \leq \mathrm{Z} \leq 6$ (nz) are the real data. The shading of the layers Real $=1$ and Real=6 corresponds to the ghost layer equvalency in the figure.

Figure 5 shows how the splitting of arrays is performed in the parallel case, with the number of processors arbitrarily set to 4 . In this example, each processing node receives, $\frac{n z 2}{N}$, or 2 layers per node. Notice how the values of dlow and dhigh are dependent on the rank values; namely dlow on root $($ myrank $=0)$ and dhigh on the northernmost $($ myrank $=$ nprocs -1$)$. Interestingly, in this example, dlow and dhigh are equal (dlow,dhigh $=2)$ on rank=0 and are also equal $($ dlow, dhigh $=7)$ on rank=nprocs -1 .

When a nodal calculation occurs, the extrema on the Z-type loops occur between dlow and dhigh and in addition, one needs to know the values at $\Delta \mathrm{k}= \pm 1$. Therefore, it can be seen from the figure, the lowest ghost layer on Node 0 will be unused as well as the top most ghost layer on Node 3. And by induction, this will occur for all instances. These type of calculations appear in subroutines PROD_nC where matrix calculations and updates are carried out.

\subsection{Parallel Algorithms}

This section states the problem to be solved, describes the serial version and then uses that information to make relationships that are useful for the development of parallel algorithms for these programs. The suite of programs provided in NISTIR-6269 can be broken down into two distinct kinds of problems. The first set calculates properties based on the finite element method and the other uses finite difference techniques.

\subsubsection{Finite Element}

As alluded to earlier, the main problem to be solved is the minimization of the system's energy in response to an applied field. Specifically, the total energy stored in the elastic case or the total energy dissipated in the electrical conductivity case is maximized, such that the gradient of the energy with respect to the variables of the problems is zero. To minimize the energy, $\mathrm{E}_{n}$, a function of many variables, $\mathrm{u}_{m}$, the various partial derivatives must equal zero,

$$
\frac{\partial E_{n}}{\partial u_{m}}=0
$$

In the electrical conductivity case, the energy, $\mathrm{E}_{n}$ is:

$$
E_{n}=\frac{1}{2} u_{r} D_{r s} u_{s}
$$


where the elements, $\mathrm{D}_{r s}$ are the elements of the stiffness matrix.

The energy in the elastic case, $\mathrm{E}_{n}$ is:

$$
E_{n}=\frac{1}{2} u_{r} D_{r s} u_{s}+b_{r} u_{r}+C
$$

The energy in the thermal case, $\mathrm{E}_{n}$ is:

$$
E_{n}=\frac{1}{2} u_{r} D_{r s} u_{s}+b_{r} u_{r}+C+T_{r} u_{r}+Y
$$

The energies are described using expressions that are quadratic in the $u$ array; for ELECFEM3D this means quadratic in the nodal voltages and in ELAS3D, it is quadratic in the nodal displacements. The array $D$, is called the stiffness matrix. It contains all the physical information of the material properties of the system, along with the geometrical properties of each finite element used in this case. All finite elements are just made from voxels (3-D) or pixels (2-D), so are cubes or square.

The basic problem being solved is a composite one: what are the effective properties of a mixture of material phases with different properties. The elastic case just has elastic moduli, the electrical case just has conductivities, while the thermal case has thermal expansion constants as well as elastic moduli. In each case, a system energy is minimized in order to solve the relevant partial differential equations. In the elastic and thermoelastic cases, the overall stored energy is being minimized. In the electrical case, where current flows, the dissipated energy is being minimized. The electrical conductivities could also be interpreted as thermal conductivities if so desired, expanding the range of problem application.

\subsubsection{Finite Difference}

The energy equations for the finite difference programs are a little different. For a real conduction problem, with conductivity (which could be variable in space, but not time), the dissipated energy is:

$$
U=\int d^{3} r E_{i} \sigma_{i j} E_{j}
$$

where $\mathrm{E}_{i}$ is the electric field at a certain point in space, and the integral is over all space. However, to derive the steady state conductivity problem, one starts rather from the charge conservation equation,

$$
\nabla \cdot \vec{j}+\frac{\partial \rho}{\partial t}=0
$$

where $\vec{j}$ is the current density and $\rho$ is the charge density. For steady state problems, one ends up with simply $\nabla \cdot \vec{j}=0$ or $\nabla \cdot(\sigma \vec{E})=0$. Using the definition of $\mathrm{E}$ in terms of the gradient of the voltage results in the Laplace equation, $\sigma \nabla^{2} V=0$, in regions of uniform values of the conductivity. The appropriate boundary conditions between regions of different conductivity are applied in the program in subroutine BOND, which gives the correct value for the conductance of bonds that pass from one region to another. The program then essentially assumes that the microstructure is made up of a finite number of uniform conductivity regions.

Upon translating into finite difference language, one ends up with the equation $\mathrm{Au}=0$, where $\mathrm{u}$ is the array of voltages, and $\mathrm{A}$ is a matrix made up of the conductivities and the voxel geometry. To use the conjugate gradient method requires that an energy in quadratic form be minimized, so this equation is squared, resulting in $\mathrm{u}^{\dagger} \mathrm{A}^{\dagger} \mathrm{Au}=0$. The quadratic form being minimized is then really $\mathrm{A}^{\dagger} \mathrm{A}$, or the square of a matrix similar to the stiffness matrix in the finite element problems. This is why the finite difference programs do not usually converge as rapidly as do the finite element programs, since the square of the stiffness matrix is used instead of the matrix itself. 


\section{Program Operation and USER information}

Users familiar with the serial version of the code will find it relatively easy to use this new parallel version. This section lists changes with respect to the serial code, describes the necessary user input, generated output, describes the program's operative behavior with specific regard to the MPI subroutines, and finally gives a description of the other supporting subroutines for the finite difference and finite element programs.

\subsection{Notable changes from the serial version}

Here is a list of the major changes that the user who is accustomed to the serial code may notice.

1. The parallel version defaults to a double precision calculation.

2. All of the principal arrays used in this code have been changed from a one-dimensional vector representation into a $3-\mathrm{D}$ vector representation, i.e. the vector pix $(m)$ from the serial version has its parallel counterpart, $p i x(i, j, k)$. This 3 -D representation describes the data in a more natural way and also allows easier manipulation in a multiple processing environment for communication between processing nodes.

3. The array $\operatorname{vox}(n x, n y, d 1-1: d 2+1)$ is used throughout this program in lieu of pix and is invoked to do the rest of the computations. Pix itself is dynamically allocated and is deallocated as soon as the array vox is created.

4. The principal data arrays in this program have been changed. The arrays $u, b, g b, h$, and $A h$ were originally dimensioned as $(n s, 3)$ in the serial problem, but in this new paradigm they are changed to reflect the 3 -dimensionality of the problem. They are now dimensioned on a per node basis (akin to the array vox) to $(n x, n y, d 1-1: d 2+1,3)$; each has their own set of top and bottom ghost layers as well.

5. Arrays that are dependent on $n x, n y, n z$ (like the aforementioned set) and nphase e.g., $d k$, cmod and prob are now dynamically allocated. This saves the user time editing the code for his problem since the dimensions do not have to be changed from problem to problem. One only has to change the respective values of $n x, n y, n z$ and nphase.

\section{$3.2 \quad$ Inputs}

This section will briefly describe each of the individual input items (and their data types) a user needs in order to use this code. The following list of variables are the same as in the serial code, except for the flag, pflag, which is used for timing information. To find these variables in the code, search for occurrences of USER - they are located nearby. The variables are listed in the order in which they are found in the program. The input that is generic to all the codes will be presented first and then in each subsection, a description of the specific input per program is presented.

1. $\mathbf{n x}, \mathbf{n y}, \mathbf{n z}$ (integer*4): The dimensionality of the data, i.e. $p i x(n x, n y, n z)$.

2. nphase (integer*4): The number of phases represented in your microstructure.

3. gtest (double precision): The stopping criteria which is compared to $g g(=g b \times g b)$.

4. pflag (integer*4): A flag used for printing timing information. 0 suppresses printing and 1 prints out all per processor timing information .

5. Input file name (Unit 9) (character string): The name of the file which contains the 3 -D image under investigation. The input file contains 2 -byte integers in which the value of each element represents the phase at that position in the microstructure $(1,2,3, \ldots$, nphase $)$. 
6. Output file name (Unit 7) (character string): The main set of output is generated and placed into this file as the job proceeds. Description of its contents is in the Output section.

7. npoints (integer*4): This integer tells the program how many distinct input files will be run. Typically this value equals 1 , but can be changed.

8. $\mathbf{k m a x}$ (integer*4): Maximum number of times subroutine DEMBX_MPI will be called.

9. ldemb (integer*4): Number of conjugate gradient steps performed during each call of DEMBX_MPI.

\subsubsection{ELECFEM3D}

1. sigma(nphase,3,3) (integer*4): The electrical conductivity tensor of each phase. The user can make the value of sigma to be different for each phase of the microstructure if so desired.

2. ex,ey,ez (double precision): Global electric field applied to microstructure.

\subsubsection{ELAS3D}

1. phasemod(nphase,2) (integer*4): An array which contains the Young's modulus, phasemod( $i, 1)$, and Poisson ratio, phasemod $(i, 2)$, of the $\mathrm{i}^{\text {th }}$ phase. This array is now initialized in its own subroutine called phasemod_init instead of within MAIN.

2. exx,eyy,ezz,exz,eyz,exy (double precision): Global strains applied to microstructure.

\subsubsection{THERMAL3D}

1. phasemod(nphase,2) (integer*4): An array which contains the Young's modulus, phasemod( $i, 1)$, and Poisson ratio, phasemod $(i, 2)$, of the $\mathrm{i}^{\text {th }}$ phase. This array is now initialized in its own subroutine called phasemod_init instead of within MAIN.

2. eigen(nphase,6) (double precision): Thermal strains of each phase: eigen(nphase, 1$)=$ $\mathrm{xx}$, eigen $($ nphase, 2$)=\mathrm{yy}$, eigen $($ nphase, 3$)=\mathrm{zz}$, eigen $($ nphase, 4$)=\mathrm{xz}$, eigen (nphase, 5$)$ $=\mathrm{yz}$, eigen $($ nphase, 6$)=\mathrm{xy}$.

\subsubsection{DC3D}

1. sigma(nphase,3) (double precision) Values of the diagonal elements of the conductivity tensor for each phase (conductivity tensor is diagonal only). 1,2,3 =x,y,z, respectively.

2. ex,ey,ez (double precision): Components of applied field, $E=(e x, e y, e z)$.

\subsubsection{AC3D}

1. sigma(nphase,3) (double complex) Values of the diagonal elements of the conductivity tensor for each phase (conductivity tensor is diagonal only). 1,2,3 =x,y,z, respectively.

2. ex,ey,ez (double precision): Components of applied field, $E=(e x, e y, e z)$.

3. nfreq (integer*4) : Indicates how many frequencies are to be used at which to compute the complex conductivity. The program was originally set up to simulate the experimental probe of impedance spectroscopy, which scans a sample over a number of frequencies of applied electrical signal. In the numerical code, one often desires to scan over a similar set of frequencies. When doing so, the program converges better if the complex voltages 
from the last computation are used for the initial voltage values for the next frequency computation. When the frequencies are close, like they usually are in a sweep, this works well and save an appreciable amount of computer time. If the frequencies are far apart, there is little benefit to this procedure, and one might as well use a uniform field initial condition at all frequencies.

4. ncheck (integer*4) : Subroutine DEMBX_AC will write out the total current and norm of the gradient squared every ncheck gradient steps.

\subsection{Outputs}

The main set of output data is generated and placed into the output file Unit7 which the user names within the program. The output that is generic to all the codes will be presented first and then in each subsection, a description of the specific output per program is presented.

All programs report the following information in the first few lines of the output file.

- Prints out "MICRO" (which microstructure is currently being calculated); the program can be adjusted to use multiple input files.

- Prints out the values for $n x, n y, n z, n s, n p r o c s$, where $n p r o c s=$ number of processors for this job.

- The volume fraction of each phase.

- The input values of the applied fields (electrical, mechanical, thermal)

- Values of conductivity tensors or bulk and shear elastic moduli per phase. This is dependent on the specific program, but always appears at this time.

- Final $\mathrm{C}=C$

- Initial Energy $=E_{0}$

- $\operatorname{gg}=g_{0}$

The programs will now print out the following information after each ldemb or ncheck conjugate gradient steps until convergence.

- Energy $=E_{1} g g=g g_{1}$

- Number of conjugate steps = up to that point

- Root took $S_{1}$ s for ldemb conjugate steps.

- Intermediate results

The last list entry, Intermediate results, refers to the specific observables, which the given program calculates.

If there is more than one microstructure under investigation, each node will print its execution time for that microstructure. Otherwise, they will print their overall execution time.

\subsubsection{ELECFEM3D}

The intermediate results appear as:

- Current in $\mathrm{x}$ direction $=\mathbf{c u x x p}$

- Current in y direction = cuyyp

- Current in z direction $=$ cuzzp 
The final results of this program are displayed as:

Average current in $\mathrm{x}$ direction $=\mathbf{c u x x p}$

Average current in y direction $=$ cuyyp

Average current in $\mathrm{z}$ direction $=$ cuzzp

ic number of conjugate gradient cycles needed

\subsubsection{ELAS3D}

- The phase number, its Young's modulus phasemod $(i, 1)$, and Poisson ratio phasemod( $(i, 2)$ which are defined in phasemod_init.

The intermediate results appear as:

- stresses: xx,yy,zz,xz,yz,xy strxxp,stryyp,strzzp,strxzp,stryzp,strxyp

- strains : xx,yy,zz,xz,yz,xy sxxp,syyp,szzp,sxzp,syzp,sxyp

where strxxp, stryyp, strzzp, strxyp, strxzp, stryzp are the six Voigt volume averaged total stresses and sxxp, syyp, szzp, sxyp, sxzp, syzp are the six Voigt volume averaged total strains.

When the program finishes, the overall bulk modulus, shear modulus, Young's modulus and Poisson's ratio are printed as well.

bulk modulus $=$ bulk

shear modulus $=$ shear

Youngs modulus = young

Poissons ratio $=$ pois

\subsubsection{THERMAL3D}

The intermediate results appear as:

- stresses: xx,yy,zz,xz,yz,xy strxxp,stryyp,strzzp,strxzp,stryzp,strxyp

- strains : xx,yy,zz,xz,yz,xy sxxp,syyp,szzp,sxzp,syzp,sxyp

- macrostrains in same order u2(1,1),u2(1,2),u2(1,3),u2(2,1),u2(2,2), u2(2,3)

- average of the macrostrains $(\mathrm{u} 2(\mathbf{1}, \mathbf{1})+\mathrm{u} 2(\mathbf{1 , 2})+\mathrm{u} \mathbf{2}(\mathbf{1 , 3})) / \mathbf{3})$

When the program finishes, the above values are once again outputted but with their final values.

\subsubsection{DC3D}

The intermediate results appear as:

- Current in $\mathrm{x}$ direction $=\mathbf{c u x x p}$

- Current in y direction = cuyyp

- Current in $\mathrm{z}$ direction $=$ cuzzp

The final results of this program are displayed as:

Average current in $\mathrm{x}$ direction $=\mathbf{c u x x p}$

Average current in y direction $=$ cuyyp

Average current in $\mathrm{z}$ direction $=$ cuzzp

ic number of conjugate gradient cycles needed 


\subsubsection{AC3D}

The intermediate results appear as:

- No. nf angular frequency $=\mathbf{w}$ radians

- icc : The number of the current iterations which is a multiple of ncheck until it reaches the final value.

- gg : A complex number that is the value of the gradient squared $(g b \times g b)$.

- $\operatorname{cuxxp}=\operatorname{cuxxp}$

- $\operatorname{cuyyp}=$ cuyyp

- $\operatorname{cuzzp}=$ cuzzp

At the end of the calculation for a given frequency, the program reports the following:

Average current in $\mathrm{x}$ direction $=$ cuxxp

Average current in y direction $=$ cuyyp

Average current in $\mathrm{z}$ direction $=$ cuzzp

ic number of conjugate gradient cycles needed

\subsection{MAIN}

The programs are essentially the same as the serial version until the initial data set is read from the input file. Throughout this program, when I/O has to be done, it is only done by the root node $($ rank $=0)$. Dynamic allocation of several arrays based on the problem size $(n x, n y, n z)$ as well as those based on nphase also occur here. There are some small difference in details between the finite element and finite difference programs, which will be obvious when looking more closely at the programs.

- Initialize variables.

- Initialize MPI.

- Root calculates z extents, $d 1$ and $d 2$.

- Root reads in original data set and passes appropriate sections to other processing nodes.

- Dynamically allocate large arrays per processor.

- Call FEMAT_MPI - this computes the local stiffness matrices and any other auxiliary arrays needed. The local stiffness matrices are used in ENERGY_MPI and DEMBX_MPI to construct the global stiffness matrix as the various arrays are updated in the conjugate gradient process.

- Calculate initial $u$ array per processor.

- Call ENERGY_MPI - this computes the initial energy of the system, based on the initial conditions, and also computes the initial value of $g b$, the gradient of the energy array. Both of these are needed as initial inputs to DEMBX_MPI.

- Enter loop which calls DEMBX_MPI and ENERGY_MPI. DEMBX_MPI is the conjugate gradient subroutine that actually performs the conjugate gradient update to the variables of the problem. It is performed ldemb number of times, and is then exited and ENERGY_MPI is then called again. This is done in order to see how the relaxation is going, and whether the energy has relaxed enough. It is usually not possible, a priori, to set the value of gtest so that good results are obtained in a reasonable amount of time. If gtest is set too small, many conjugate gradient cycles could be wasted to try to push the accuracy of the answer beyond what is necessary. If gtest is too large, then the final answer obtained might not be accurate enough. 
- After convergence, calculate final results, i.e. stress, strain, or current per pixel and then produce a global summation.

\subsection{Finite Element Subroutines}

In this section, a discussion of the functionality of the MPI based subroutines used in the parallel codes, ELECFEM3D_MPI.f, ELAS3D_MPI.f and THERMAL3D_MPI.f are presented. Since each program will have its own specific needs, the operations will be essentially identical except for the dimensionality of the operand in question. See Table 1 for the variations in dimensionalities.

\subsubsection{FEMAT_MPI}

A generic form of this subroutine is used to calculate

- Elements of the stiffness array, $d k$.

- Values needed by the minimization procedure: array $b$ and variable $C$.

The term, $C$, and array, $b$, are calculated in essentially the same manner as in the serial code; there are contributions to each from the $\mathrm{x}, \mathrm{y}, \mathrm{z}$ faces, the $\mathrm{xy}, \mathrm{xz}, \mathrm{yz}$ edges and the corners of the original microstructure dataset. But $C$ and $b$ are per-processor values in the parallel code. This means that each processor will calculate its own contribution, based on its chunk of data, to the overall value. Once a node determines its contribution, all partial results are sent to the master node who adds them together and passes back the result of this operation and broadcasts it to all nodes.

The nominal loops to calculate the positional contributions to $C$ and $b$ are closely related to the serial case. One usually loops over 2 of the 3 Cartesian coordinates, $(\mathrm{x}, \mathrm{y}, \mathrm{z})$. In the code, a loop in the $\mathrm{x}$ direction uses the variable $i$ as its index, viz: $i=1, n x$. Similar arrangements are made for the $\mathrm{y}$ and $\mathrm{z}$ directions using the variables $j$ and $k$, respectively as in $j=1, n y$ and $k=1, n z$. Some k-type loops in the subroutine (z-direction) use the limits $k=1, n z-1$, but it is important to be mindful that the data, as well as other large arrays, are split across the processing nodes in the z-direction. Additional accommodations and precautions have to be made for this fact, but at the same time the code must be generic enough so all processors can execute it. It is especially important for the $k=1, n z-1$ loops.

This is accomplished by introducing a new variable, $d n$, in concert with an if statement. $d n$ is initially assigned to $d 2$, but if $d 2$ equals $n z$, then it takes on the value $n z-1$. Note that this only occurs at the processor with the highest rank value. So during these calculations, all levels on the lower ranks are included and the $d 2$ is excluded only on the highest rank.

Another interesting artifact of this calculation occurs when calculating the contributions for $C$ and $b$ from the $\mathrm{Z}$-face, $\mathrm{x}=\mathrm{nx} \mathrm{z}=\mathrm{nz}$ edge, $\mathrm{y}=\mathrm{ny} \mathrm{z}=\mathrm{nz}$ edge and $\mathrm{x}=\mathrm{nx} \mathrm{y}=\mathrm{ny} \mathrm{z}=\mathrm{nz}$ corner. In these 4 cases, only the highest rank processor is needed since this is the one where $k$ has values of $n z$ and $n z-1$.

The accuracy of $C$ is increased in this algorithm. At each instance when the contribution of $C$ is calculated (called cterm in the code), it is compared to zero (0). A positive cterm is added to cpos. Likewise a negative cterm is added to cneg. At the end of the calculation, cpos and cneg are summed on a node, which yields the overall $C$ per processor. This prevents adding very large numbers of one sign with very small numbers of the opposite sign.

As mentioned, the elements of array $b$ are calculated with a per-processor methodology as well. In the original serial codes, ELECFEM3D.f and ELAS3D.f, the elements of $b$ are calculated as $b(i b(m, i s(m m)))$ and $b(i b(m, i s(m m)), n n)$, respectively. Notice the dependence on the hash table array, $i b$. In the parallel versions, the need for the hash table $i b$ is eliminated and $b$ is an array of rank 3 or 4 , respectively. 
But a calculation for an element of $b$ is more complicated than generating a constant term like $C$. One must notice that the corresponding loop variables $(i, j, k)$ are not the same as the indices of $b$, namely $(i p x, i p y, i p z)$ which is currently being calculated. These indices can be thought of as a set of influenced positional values. Therefore, a calculation using $\operatorname{vox}(i, j, k)$ will influence the values of 27 separate and distinct elements of $b$, which correspond to its 27 nearest neighbors. In other words, a contribution to an element of $b$ comes from 27 distinct elements of vox. The subroutine ipxyz is used to generate the values of an influenced triplet (ipx,ipy,ipz) using a given set of parameters $(i, j, k$ and $m m)$.

So one saves on the overall amount of memory for this calculation by eliminating $i b$, but the price to be paid is executing ipxyz multiple times.

After the distinct faces, edges and corner loops are finished, $b$ is essentially complete. However no one node has the final values of $b(i, j, d 1)$ and $b(l, m, d 2)$. Part of the results are found on contiguous nodes, i.e. processor $P-1$ and $P+1$, respectively. Therefore, it is necessary to pass the bottom ghost layer of processor $P+1$ to $P$ then add to the $d 2$ level of $P$ once it is at the requesting node. This addition completes the calculation of $b$. A similar set of calculations involving the top layer of $P-1$ and the $d 1$ level of $P$ are also required. In a succinct manner, it can be expressed as:

$$
\begin{aligned}
& B(i, j, d 1)_{P}=b(i, j, d 1)_{P}+b(i, j, d 2+1)_{P-1} \\
& B(i, j, d 2)_{P}=b(i, j, d 2)_{P}+b(i, j, d 1-1)_{P+1}
\end{aligned}
$$

where capital $B$ represents the final value of the element and lower-case $b$ 's represent the partial results.

At this point $b$ is completely determined and updating the top and bottom ghost layers is the final step. This is accomplished by another use of subroutines b2t_dp and t2b_dp.

THERMAL3D_MPI.f uses a larger and slightly more complex form of FEMAT_MPI. Remember that THERMAL3D_MPI.f incorporates 1 additional large array, $T$. $T$ is a linear term in displacements (like $b$ ) but arises from thermal strains and the constant term $Y$ is similar to $C$, but arising from the thermal strains, not the applied or macrostrains. Since the overall system strains (macrostrains) are dynamic variables in the thermoelastic problem, the value of $C$ will change throughout the operation of the program. However, the value of $Y$ is constant, since it is a function only of the elastic moduli and the thermal strains, which are system variables and thus do not change. In the serial case, the array $T$ was defined differently than $b$ was in ELAS3D. Instead of being defined as $T(n s, 3)$, it was defined as $T(n s+2,3)$. This additional expansion of dimensions is handled by making 2 arrays for the parallel counterpart. The first is to dimension $T$ as $T(n x, n y, d 1-1: d 2+1,3)$ (like $b$ in ELAS3D_MPI.f) but the 6 extra terms of $T(n s+1: n s+2,3)$ are put into a new array called $T 2$ and dimensioned $T 2(2,3)$. Therefore, the generic array in THERMAL3D_MPI.f, $X(n s+2,3)$ is converted to 2 arrays, one with the standard parallel dimensions of $X(n x, n y, d 1-1: d 2+1)$ and then also $X 2(2,3)$.

Calculations for $T$ elements are handled in a similar manner as $b$ from ELAS3D_MPI.f. Again, the $(i, j, k)$ indices of the loop do not correspond to the element in question. The program also uses subroutine ipxyz and calculates a set of influenced positional parameters as well. To complete the calculation for $T$, implement equations similar to (16) and (17) above and do the final updating of the ghost layers.

Although the $T 2$ array is small $(2,3)$, it has contributions from the faces, edges and corner like the array $b$ in ELAS3D_MPI.f. At the end of determining these per processor arrays, one must also form the element-by-element sum to end up with the final version of $T 2$. This is accomplished by using a call to MPI_ALLREDUCE:

do $\operatorname{ipp}=1,2$

do $\mathrm{jpp}=1,3$ 


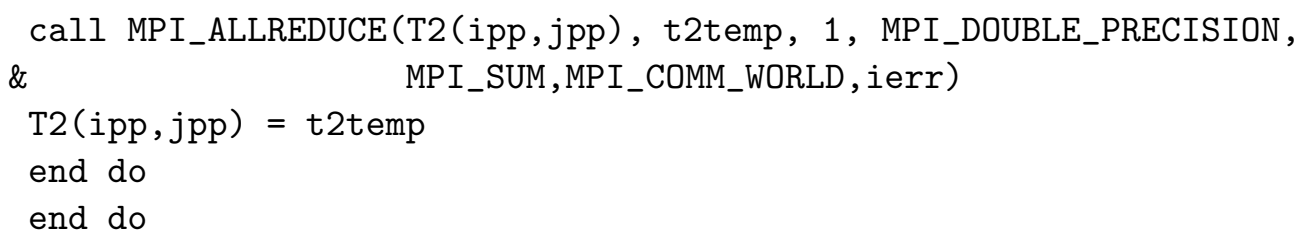

t2temp is a temporary variable used to store the global sum of the per-processor $T 2(i p p, j p p)$ values. Then it reassigns $T 2(i p p, j p p)$ with the global sum before it ends. After calculating $T 2$, this implementation also has to calculate an array $b$. This term is linear in displacements as well and is generated identically to $b$ in ELAS3D_MPI.f.

\subsubsection{ENERGY_MPI}

This subroutine calculates the gradient of the energy, $g b$, but calls the subroutine GBAH to do so. In the serial version, the majority of the subroutine ENERGY was dedicated to calculating the $g b$ array. It was much easier to create a subroutine for this calculation since the next subroutine, DEMBX_MPI, has the same functional form for creating its $A h$ array.

The differences here appear when calculating utot. One needs to get the per-processor value dutot and then make a global sum with a call to MPI_ALLREDUCE. The last two lines in the subroutine are simple assignments:

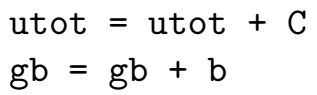

The first line does an update on a single variable, utot. But the second line does an update of the entire array $g b$ with array $b$ using FORTRAN90 syntax. Keep in mind, each processor is just updating its own local copy of $g b$ with its local copy of $b$.

In THERMAL3D_MPI.f, there is additional complexity for this subroutine. After it updates $g b$ (like above), it must calculate its value for the "constant" macrostrain energy term, $C$, using the $z$ con and $u 2$ arrays. This value $C$ is added to utot (also above), but to get the final value of utot, one must add the constant $Y$ from FEMAT_MPI.

\subsubsection{DEMBX_MPI}

This subroutine calculates the gradient relaxation process and the $h$ and $A h$ arrays, but calls the subroutine GBAH to generate them. It behaves similarly to the serial version, except in cases when FORTRAN90 array syntax was implemented for lines like:

$\mathrm{u}=\mathrm{u}-\mathrm{lambda} * \mathrm{~h}$

$\mathrm{gb}=\mathrm{gb}-\mathrm{lambda} * \mathrm{Ah}$

In the FORTRAN77 serial version, these lines looked like:

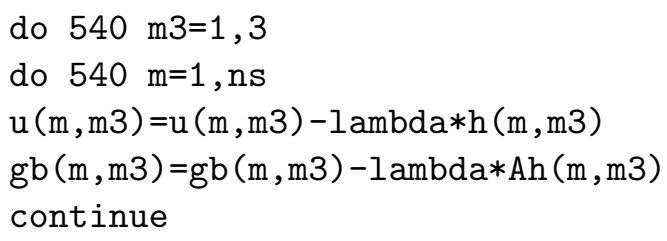




\subsubsection{GBAH}

When calculating the $g b$ or $A h$ arrays in the serial program, one can see that they are essentially the same calculation with a 1:1 correspondence between the arrays $u$ and $h$ as well as $g b$ and $A h$. Therefore, it is logical to create this subroutine and give input parameters which determine if one is calculating $g b$ or $A h$.

Regardless if one wants $g b$ or $A h$, the local array that is calculated in GBAH is the array om; the place from which GBAH is called and its parameters determine if $g b$ or $A h$ is going to be calculated.

In this subroutine, elements of the array om (output matrix) are being calculated on a per node basis. In addition to that, FORTRAN90 syntax to sum over all values of an array index to calculate the terms removes the extra loop $(n=1,3)$ that was found in the serial version. An example calculation from ELAS3D_MPI.f and ELAS3D.f should illustrate the point. Note that the last index of om, $j$, would not appear in ELECFEM3D_MPI.f (cf. Table 1).

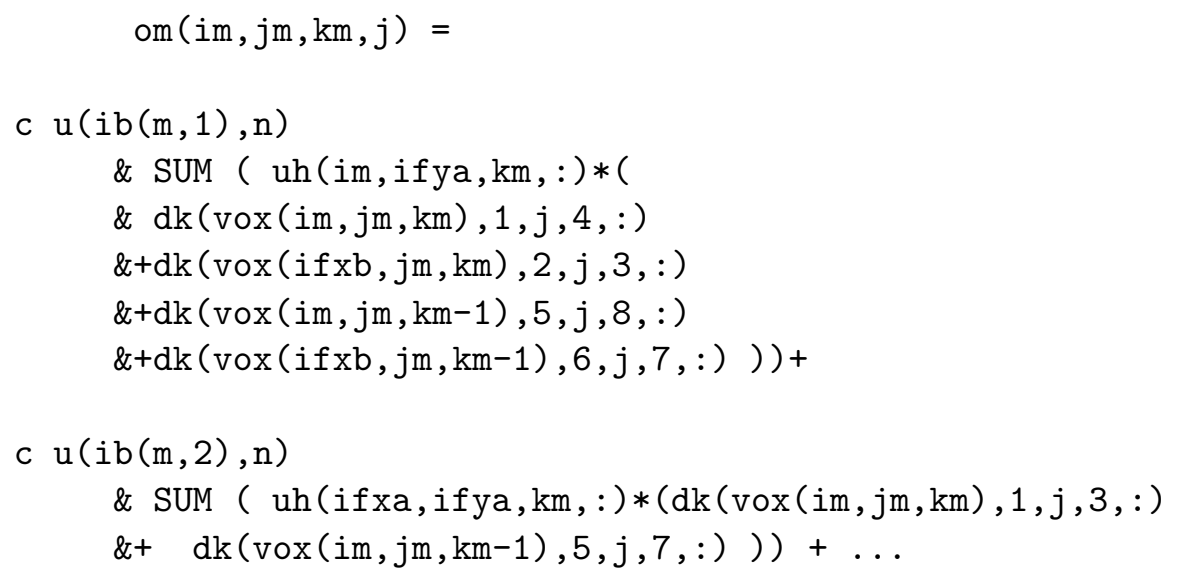

The $u(i b(m, 1), n)$ and $u(i b(m, 2), n)$ terms in the comments refer to the like terms found in the serial version, ie.

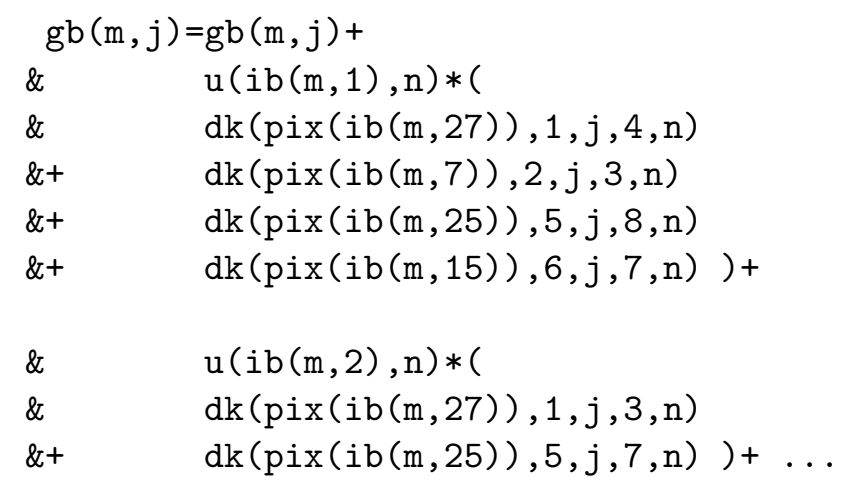

om exhibits the same behavior as $A h$ and $g b$, in other words, it must also have top and bottom ghost layers as well. Therefore, before GBAH returns om back to the calling routine, it has already created the required ghost layers by judicious use of the subroutines $\mathbf{t} \mathbf{2 b} \mathbf{b} \mathbf{d} \mathbf{p}$ and b2t_dp.

But the most intriguing part of the calculation involves the determination for the necessary elements of $u h$ and vox in the above calculation. Note that $u h$ is merely a parameter; $u h$ equals $u$ when called from subroutine ENERGY_MPI but equals $h$ when called from DEMBX_MPI. This parameter is named $u h$ to remind the user of its duality. 
Before the code was written, it was necessary to determine what the $(i, j, k)$ indices for the $u h$ and vox arrays for each of the 27 terms will be, keeping in mind $i b$ and Table 3 . After one has done this, their $(i, j, k)$ indices can be deduced. However, one must also keep in mind the periodic nature of $u h$ and $v o x$ in the $\mathrm{x}$ and y directions; periodicity in the $\mathrm{z}$ direction is assured due to the ghost layers. Therefore when looping over $i m$ and $j m$ (ie. $\mathrm{x}$ and y directions), special precautions (in the form of if-statements) are taken when $(i m+1)>n x$ or $(i m-1)<0$, when the value of that particular i-type coordinate is assigned to 1 or $n x$, respectively, with similar arrangements for $j m$.

For a given set of $(i m, j m, k m)$ loop variables, if the serial code says to invoke $i b(m, 1)$, this corresponds to the listing in Table 3 for $i b(m, 1)$, which says the required neighbor has a $(\Delta \mathrm{i}, \Delta \mathrm{j}, \Delta \mathrm{k})$ setting of $(0,1,0)$. This implies that the proper element to use in this calculation would be $(i m, j m+1, k m)$. There is a change in variable for $j m+1$ which is now assigned to if $y a$. This specialized nomenclature is summarized here:

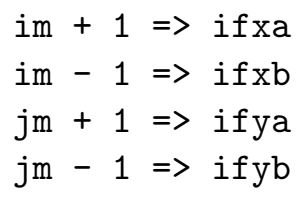

Therefore, when one encounters a if $x a$, if $x b$, ifya or ifyb term, it can immediately be identified as a term that needs to be fixed or adjusted according to the above specifications. This occurs not only for $u h$, but also for $\operatorname{vox}(i m, j m, k m)$ as it is a parameter for $d k$.

\subsubsection{STRESS_MPI}

This subroutine calculates the individual stresses and strains per pixel per processor. MPI code is needed to sum the individual contributions per processor onto the root (master) node (like the $C$ calculation). The 12 macroscopic stresses and strains are broadcast to each node, but root is the lone processor who uses these values for output purposes.

\subsubsection{CURRENT_MPI}

This subroutine behaves very like STRESS_MPI, except it calculates the current contribution from each pixel. A call to MPI_ALLREDUCE is needed to sum the individual contributions per processor onto the root (master) node. The 3 macroscopic current contributions $(\mathrm{x}, \mathrm{y}, \mathrm{z})$ are available to each node, but root is the lone processor who uses these values for output purposes.

\subsection{Finite Difference Subroutines}

In this section, a discussion of the functionality of the MPI based subroutines used in the parallel codes, DC3D_MPI.f and AC3D_MPI.f are presented. The dual labeling of the subroutines in this section reflects the fact that they are used in DC3D_MPI.f or AC3D_MPI.f.

\subsubsection{BOND_DC, BOND_AC}

The subroutine bond applies the correct boundary conditions between regions of different conductivity by choosing the correct value for the conductivity of a bond that extends across a boundary. Remember that in the finite difference programs, the node is thought of as being at the center of a pixel, with nearest neighbor bonds connecting nodes. A bond that connects nodes that are in regions of different conductivities gets assigned a conductance that is a function of the voxel geometry and is a series combination of the two different values of conductivities. When a 
bond connects two nodes of the same conductivity, then that value of the conductivity is simply used to assign the conductance of the bond.

\subsubsection{DEMBX_DC, DEMBX_AC}

This is structurally like DEMBX_MPI from the finite element codes.

\subsubsection{CURRENT_DC, CURRENT_AC}

This is structurally like CURRENT $\_$MPI from the finite element codes.

\subsubsection{PROD_DC, PROD_AC}

Analogously to the subroutine GBAH, in the finite element programs, these routines perform the large matrix multiply necessary for the conjugate gradient updating process. Generically, this subroutine does the matrix multiplication calculation: $y w=A \times x w$, with $x w$ as the input vector. In practice, this subroutine uses the parameter sets, $(u, g b)$ and $(h, A h)$, where $u$ and $h$ are passed as the input vectors $(x w)$ and used to calculate new $g b$ and $A h$ (yw) arrays. The large array $A$ in the above equation is substituted with the $g x, g y$ and $g z$ arrays which describe the bond conductance network of the microstructure.

The final calculation on vector $y w$ is to correct for terms at periodic boundaries. This is a straightforward per processor operation for the updates involving the $\mathrm{x}$ and $\mathrm{y}$ faces. But the $\mathrm{Z}$ face updates for the genuine ghost layers occur through a message passing procedure when using multiple processors. In this case, the bottom node gets the information for it's genuine bottom ghost layer $(n z=1)$ from the top most node, (level $n z=n z 1)$ called highrank in the code. The top node gets it genuine top ghost layer $(n z=n z 2)$ from Node 0, (level $n z=2)$, called lowrank. These allowances must take place in conjunction with the actual calling of $\mathbf{z}$ ghost_dp since z_ghost_dp by itself will not create the aformentioned genuine ghost layers needed for vector $y w$.

\subsection{Other Subroutines}

In this section, a discussion of the functionality of the MPI based subroutines used by all the parallel codes are presented.

\subsection{1 z_ghost_int,z_ghost_dp,z_ghost_cmplx}

Subroutines to create a ghost layer with INTEGER*2, DOUBLE PRECISION or DOUBLE COMPLEX elements in the Z-direction of the data cube. They internally call the $\mathbf{t} \mathbf{2} \mathbf{b} \mathbf{X X}$ and b2t_XX subroutines below.

call z_ghost_int (vox, nx, ny, nz, d1, d2)

call z_ghost_dp ( $u, n x, n y, n z, d 1, d 2)$

call z_ghost_cmplx ( u, nx, ny, nz, d1, d2)

\subsection{2 xy_ghost_dp,xy_ghost_cmplx}

Subroutine to create a ghost layer with DOUBLE PRECISION or DOUBLE COMPLEX elements in the $\mathrm{x}$ and $\mathrm{y}$ directions of the data cube. Only used in finite difference programs, DC3D_MPI.f and AC3D_MPI.f. 
call xy_ghost_dp (y, nx, ny, nz, d1, d2)

call xy_ghost_cmp $(\mathrm{y}, \mathrm{nx}, \mathrm{ny}, \mathrm{nz}, \mathrm{d} 1, \mathrm{~d} 2)$

\subsection{3 t2b,b2t,t2b_dp,b2t_dp, t2b_cmplx,b2t_cmplx}

These subroutines, called by z_ghost_(int,dp,cmplx), are used to create top and bottom ghost layers. t2 $\mathbf{b}$ and $\mathbf{b 2 t}$ are used with array datn and manipulate data that are 2 bytes long. Calls to $\mathbf{t} \mathbf{2} \mathbf{b}$ or $\mathbf{b} \mathbf{2 t}$ look like:

call t2b(bot, top, nx, ny)

call b2t (bot, top, nx, ny)

This sequence will create a new bottom ghost layer on node $N+1$ with the value in bot as the new bottom ghost layer and then make a new top ghost layer on node $N-1$ with top as the new top ghost layer.

The subroutines t2b_dp and $\mathbf{b 2 t}$ t_dp are used for all other arrays since they are used with double precision values, hence the "dp" suffix.

The calls to t2b_dp or b2t_dp are similar:

call t2b_dp(bot,top, nx,ny,i)

call b2t_dp(bot,top, nx, ny,i)

The last parameter, an integer, can in general be anything, but for our specific problem, $i$ equals 3 , which is a direct consequence of the dimensionality of $u, b, g b, h$ and $A h$ in the elastic and thermal programs.

\subsection{4 m2ijk}

Typically, one is accustomed to using this function to determine the 1-d ordinal count of array elements. The function to perform this is:

$$
m=n x \times n y \times(k-1)+n x \times(j-1)+i
$$

where $(i, j, k)$ is the set of indices in an array; $n x$ and $n y$ are the dimensions in the $\mathrm{x}$ and $\mathrm{y}$ directions, respectively; and $m$ is the ordinal count.

However, this subroutine is the inverse function of the equation above, i.e. it takes $m, n x$, $n y$ and $n z$ as the input and returns the $(i, j, k)$ triplet. It does this through a series of modular mathematical functions. The call to $\mathbf{m} 2 \mathbf{i j k}$ is:

call m2ijk(m,i,j,k,nx,ny,nx)

\subsection{5 ipxyz}

This subroutine is used to calculate which element of the array $b$ will be calculated when using an input triplet of $(i, j, k)$, returning it as the triplet $(i p x, i p y, i p z)$. The call to ipxyz is:

call ipxyz (mm,i,j,k, ipx, ipy,ipz,nx,ny,nz)

\subsection{6 phasemod_init}

This subroutine initializes the values in the phasemod array here instead of in the main program. It occurs here now as it is easier to modify. The call to phasemod init is:

call phasemod_init(phasemod,nphase) 


\subsection{7 dpixel}

This subroutine reads in the original data set. The call to dpixel is:

call dpixel (nx,ny,nz,ns, nphase,pix)

\subsection{8 dassig}

This subroutine calculates the volume fraction of phase $i$. The call to dassig is:

call dassig(nx, ny, nz, nphase,prob,pix)

\subsection{Makefiles and Execution scripts}

\subsubsection{PC Linux Cluster using PBS}

This is an example of the Makefile used to create the executable using the Portland Group FORTRAN90 compiler and the LAM libraries.

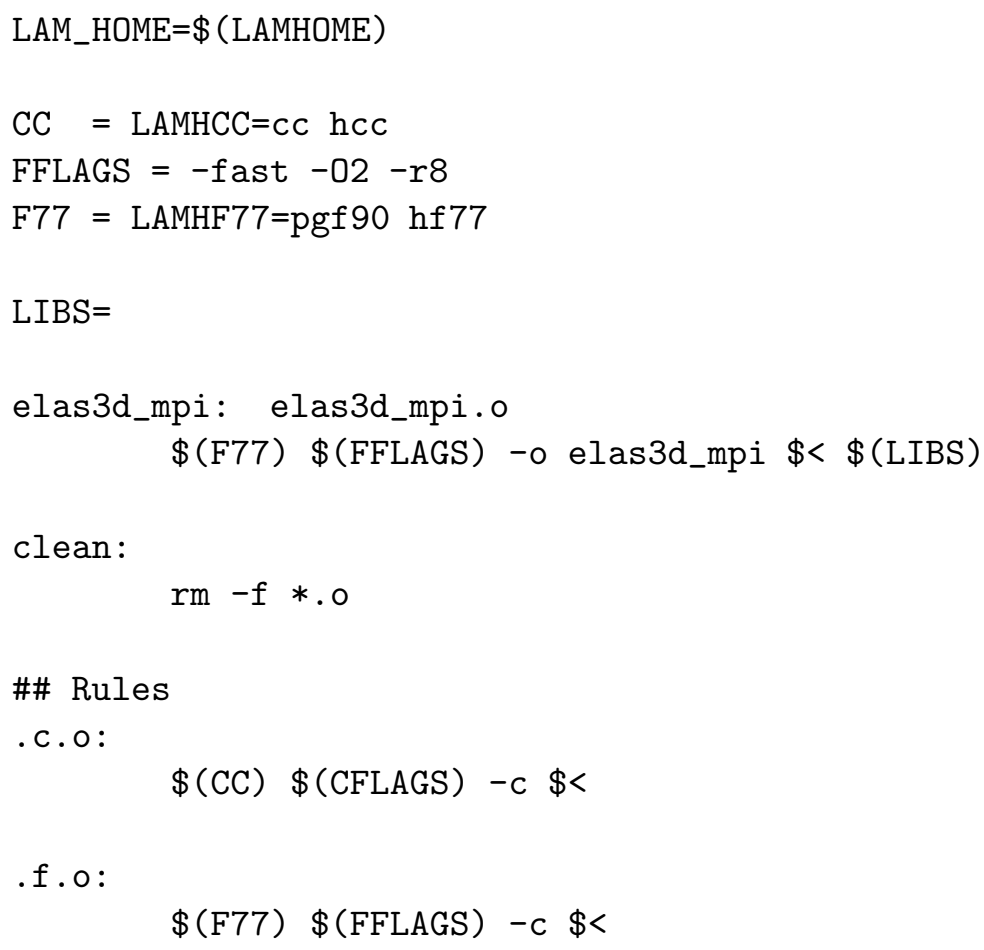

This is a PBS execution script to submit this job in an 8 cpu queue using LAM.

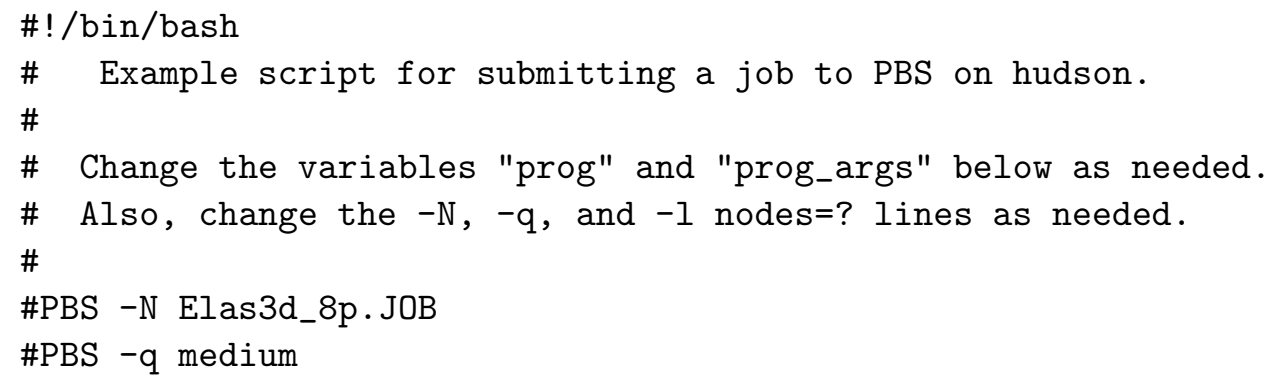




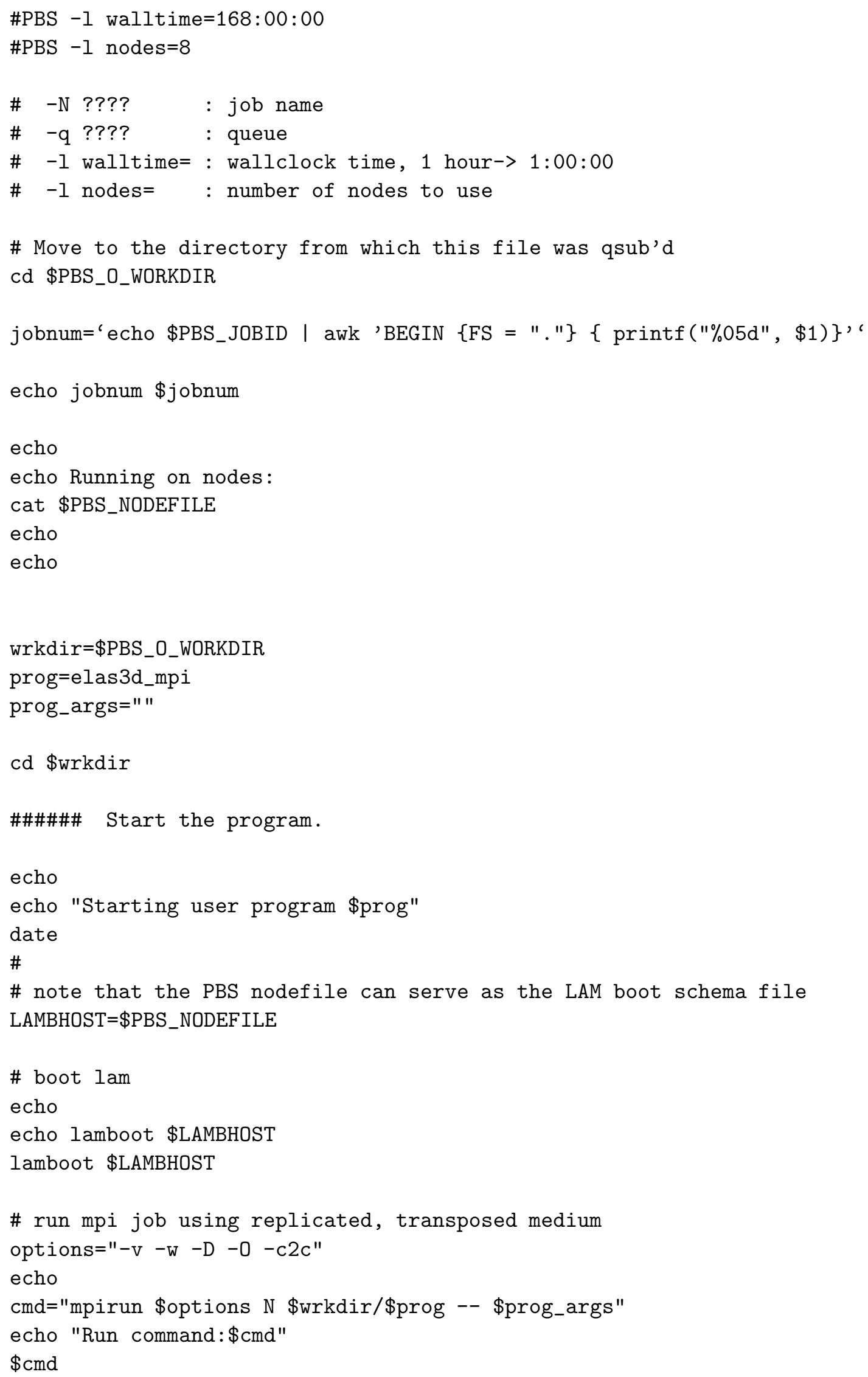


\#Kill lam daemons and clean up hostfile

echo

echo \$LAMHOME/bin/wipe -v \$LAMBHOST

\$LAMHOME/bin/wipe -v \$LAMBHOST

date

echo

exit

\subsubsection{SGI Origin 2000 using NQS}

To compile on the SGI Origin 2000 with the Fortran90 compiler:

f90 -o elas3d_mpi -02 elas3d_mpi.f -lmpi

To submit this job, this example using 8 processors can be modified to suit:

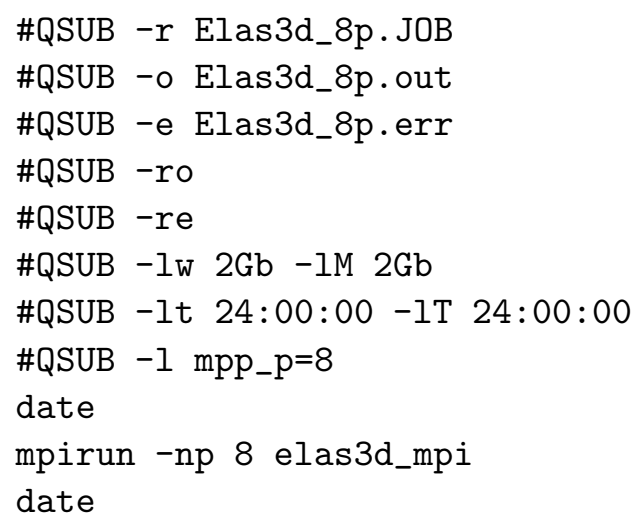




\section{MPI Primer}

MPI is a series of subroutines which are used to facilitate parallel processing. These routines allow data transfer between processing nodes and also support a variety of mathematical functions. They can be invoked using the $\mathrm{C}$ or FORTRAN languages; however, since the programs are written in FORTRAN90, only the FORTRAN bindings will be discussed. This section will focus on the bare essentials of MPI needed to understand the programs.

All MPI programs have a few things in common. They need to use a MPI include file, they must be initialized, finalized and the communicating processors must belong to the same communicating group. Here is a list of the generic MPI calls the user will encounter as they read the code. Any variables used in these calls will be defined in turn. Italicized font will be used when there are multiple options available.

- MPI_INIT(ierr)

- MPI_FINALIZE(ierr)

- MPI_COMM_SIZE( MPI_Comm, nprocs, ierr )

- MPI_COMM_RANK( MPI_Comm, myrank, ierr )

- MPI_SEND(X,count,MPI_Data_Type,dest,itag,MPI_Comm,ierr)

- MPI_RECV(Y,count,MPI_Data_Type,source,itag,MPI_Comm,status,ierr)

- MPI_IRECV(Y,count,MPI_Data_Type,source,itag,MPI_Comm,irequest,ierr)

- MPI_WAIT(irequest,status,ierr)

- MPI_BARRIER(MPI_Comm,ierr)

- MPI_ALLREDUCE(dA,A,count,MPI_Data_Type,MPI_Op,MPI_Comm,ierr)

\subsection{Initialization}

The include file always comes before any MPI calls; its location is system dependent. MPI INIT is the first genuine MPI call and does initialization. MPI_FINALIZE is called at the end when MPI is no longer needed, usually near the end of the program. The integer ierr is used as a return code for the subroutine call. The simplest MPI program in FORTRAN is:

include 'mpif.h'

integer ierr

call MPI_INIT(ierr)

call MPI_FINALIZE(ierr)

end

To do something useful with MPI, make calls to MPI_COMM_SIZE and MPI_COMM_RANK within the communicating group MPI_COMM_WORLD.

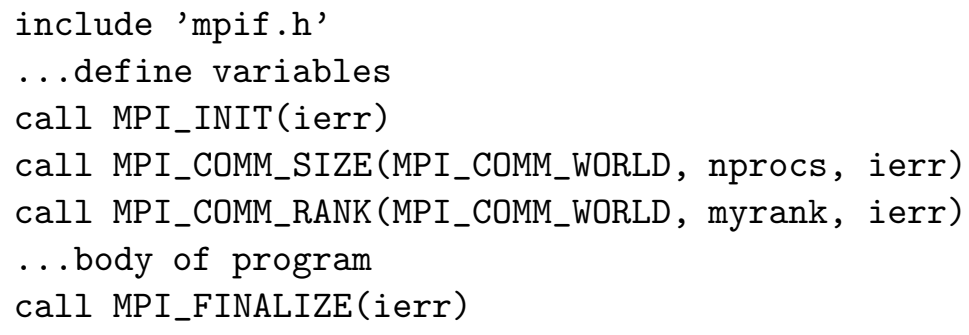


The call to MPI_COMM_SIZE returns the number of total processors that the job is running on and stores that value as an integer in the variable nprocs. The call to MPI_COMM_RANK returns the value of the rank, between 0 and nprocs -1 , and stores it into the local copy of the variable myrank. Here is also an example of the communicating group in action. All processors belong to MPI_COMM_WORLD by default, so the MPI_COMM_SIZE and MPI_COMM_RANK calls happen globally over all processors.

\subsection{Sending and Receiving Data}

The essence of doing distributed memory parallel processing involves sending and receiving data from one processing node to another. The core vehicles for data transfer are MPI_SEND and MPI_RECV. There are some particulars of which the user must be aware. The correct MPI_Data_Type, MPI_Comm and itag variables must be set. The programs in the manual use numerous data types: integer*2, integer*4, double precision, double complex and byte. For each one of these, there is a corresponding MPI data type.

Assume processor $P$ sends a $2-\mathrm{D}$ slice of array $p i x(n y, n y, k)$ of data type INTEGER ${ }^{*} 2$ to a 2-D array on processor $P+1$ named $Y(n x, n y)$. The number of elements count is simply $n x \times n y$. But since they are being sent "byte-wise", one is really sending $2 \times n x \times n y$ elements. Therefore, the necessary pair of matching SEND and RECV calls are:

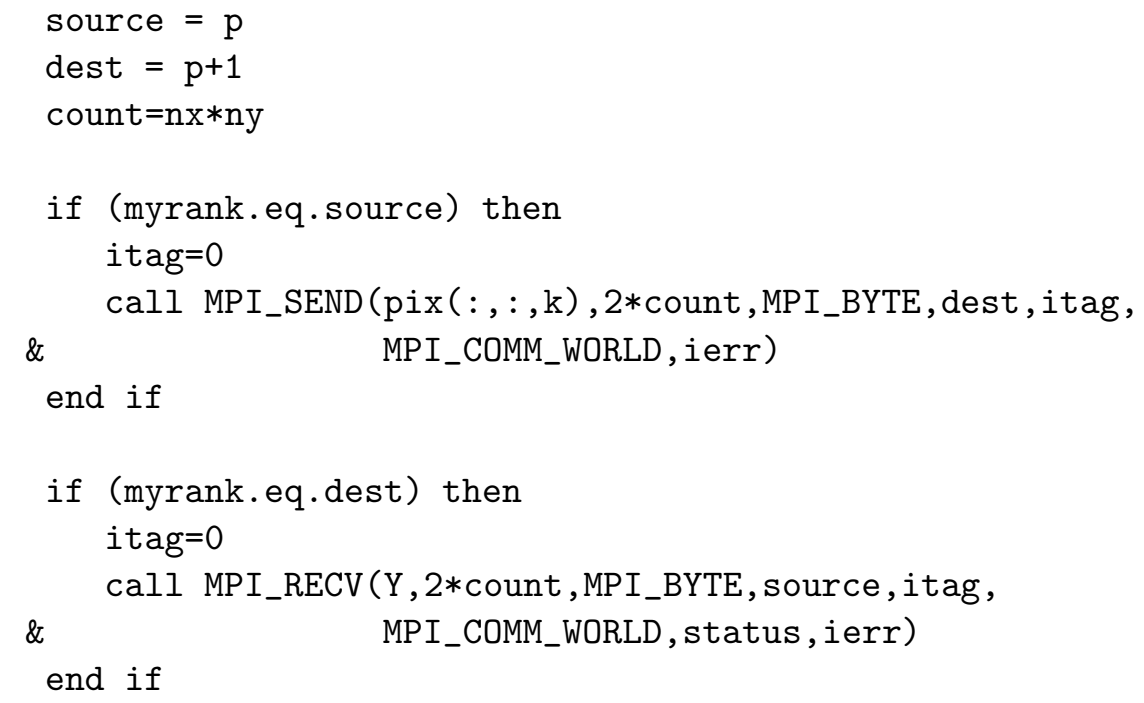

This MPI_SEND and MPI_RECV calls are technically called a blocking send and a blocking receive, respectively. In other words, the processor waits (or blocks) for the message to complete before it continues with the executable.

The timing of sending and receiving can be crucial at times. One processor may already know that it has to be sending data, but the receiving node may not be ready. In such cases, it is possible to turn a data receive into a two-stage process with calls to MPI_IRECV and MPI_WAIT. MPI_IRECV performs a non-blocking receive using the handle irequest. The MPI_WAIT keeps the MPI_IRECV from completing until it gets irequest and a legitimate status. This method is used in the subroutines $\mathbf{t} \mathbf{2} \mathbf{b}, \mathbf{b} \mathbf{2 t}$ and their variants. Of course there is a matching MPI_SEND call posted by another processor, but it is not shown.

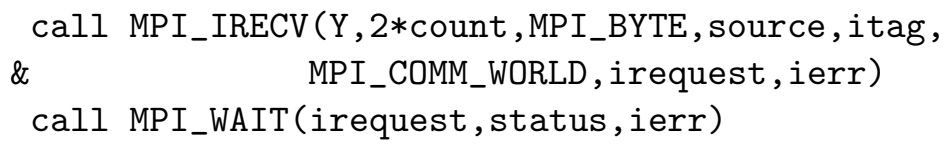


There are also cases in which one processor may finish its series of calculations before other processors do. Therefore a mechanism for pausing is built into MPI as MPI_BARRIER. No processor will pass this statement until all are ready to pass.

call MPI_BARRIER (MPI_COMM_WORLD, ierr)

There have been several integer variables used in this section that have never been explicitly defined in the code examples. It is good programming practice to define them at the beginning of the code, so they are listed here for completeness.

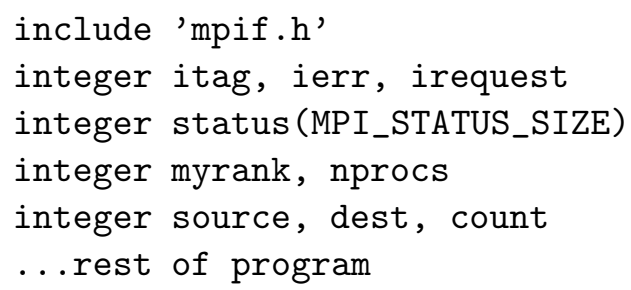

\subsection{Built-in Mathematical Functions}

There are multiple instances in the code of getting a sum of all the elements in one of the large principal arrays. Since the arrays per processor are only slices, summing up their elements would result in nprocs sums; the sum of these sums is the global sum of interest. After the operation, the result is sent to all processors. Fortunately, there is a call in MPI which will perform global mathematical procedures and return the result back to root $($ myrank $=0)$. To do this, MPI_Data_Type and which operation to perform must be defined.

Here is an example of using FORTRAN90 and MPI_SUM to perform the global sum of array $A$. Each processor has a portion of $A$, so the partial sum on a processor is made by using the FORTRAN90 operation, SUM on array $A$. Then the operation MPI_SUM performs a global summation of a single $($ count $=1)$ real variable $d X$ from each node and sums it into variable $X$ and all nodes have this information.

$\mathrm{dX}=\operatorname{SUM}(\mathrm{A})$

count $=1$

call MPI_ALLREDUCE (dX , X, count, MPI_REAL, MPI_SUM, MPI_COMM_WORLD, ierr)

\subsection{Summary}

This section was a brief summary to using MPI with FORTRAN, listing specifically which MPI statements were used in the parallel codes described in this manual. Further information on MPI can be found on the web at: http://www.mpi-forum.org/

\section{Disclaimer}

Certain commercial equipment is identified in this paper to specify the experimental procedure. In no case does such identification imply endorsement by the National Institute of Standards and Technology, nor does it indicate that the products are necessarily the best available for the purpose. 


\section{Program Listings}

\subsection{Finite Element}

\subsubsection{ELECFEM3D_MPI.f}

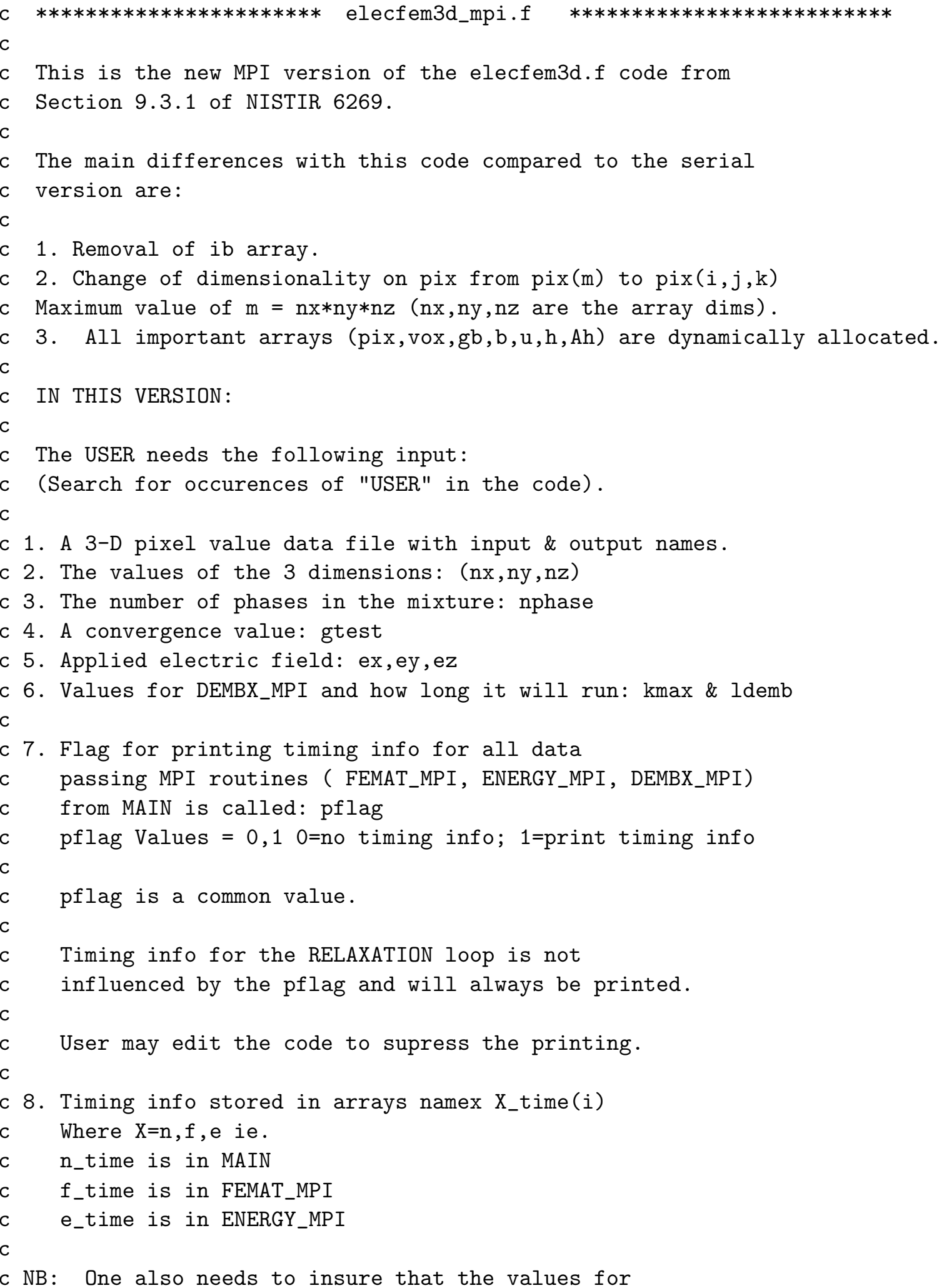




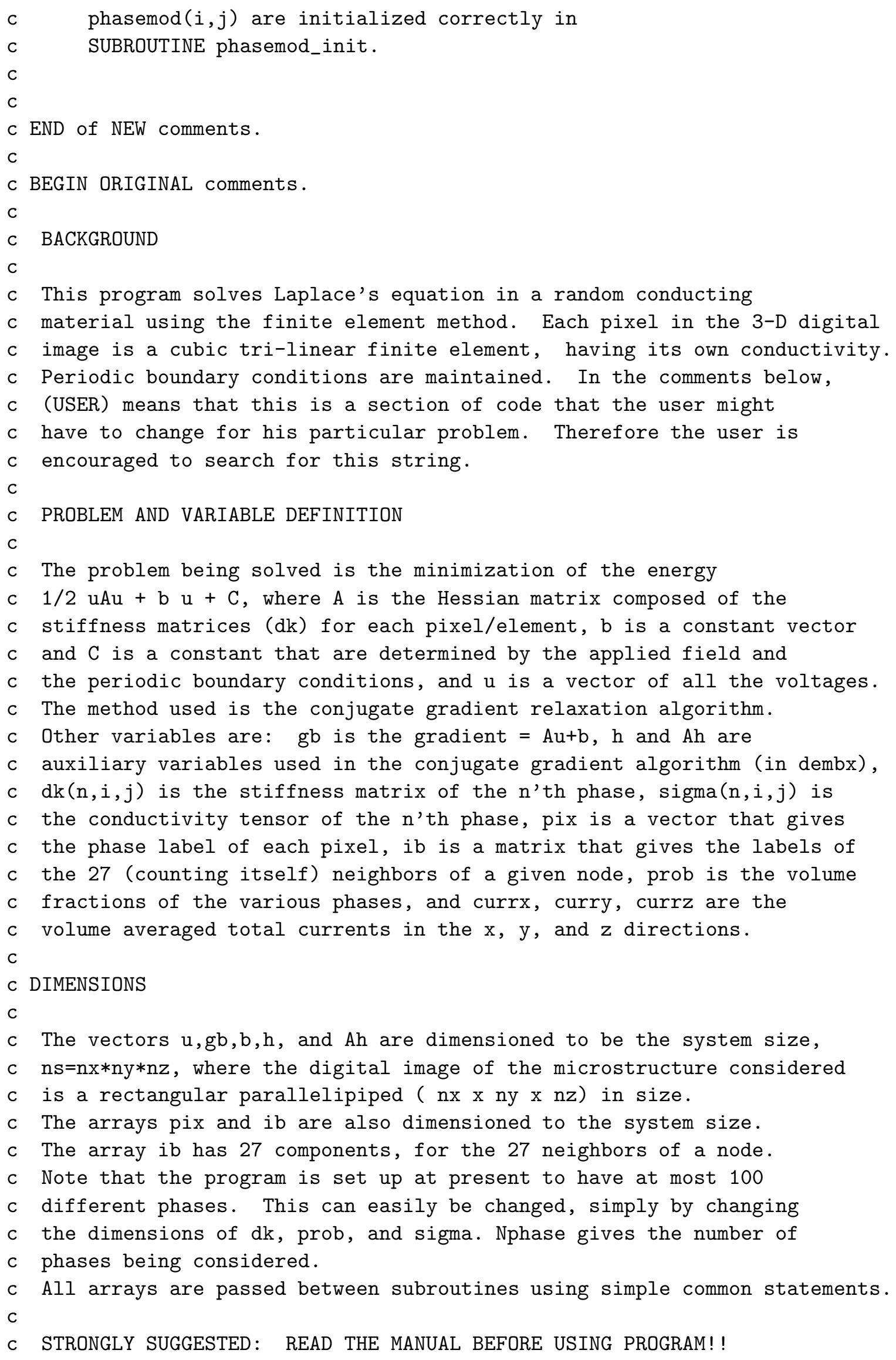




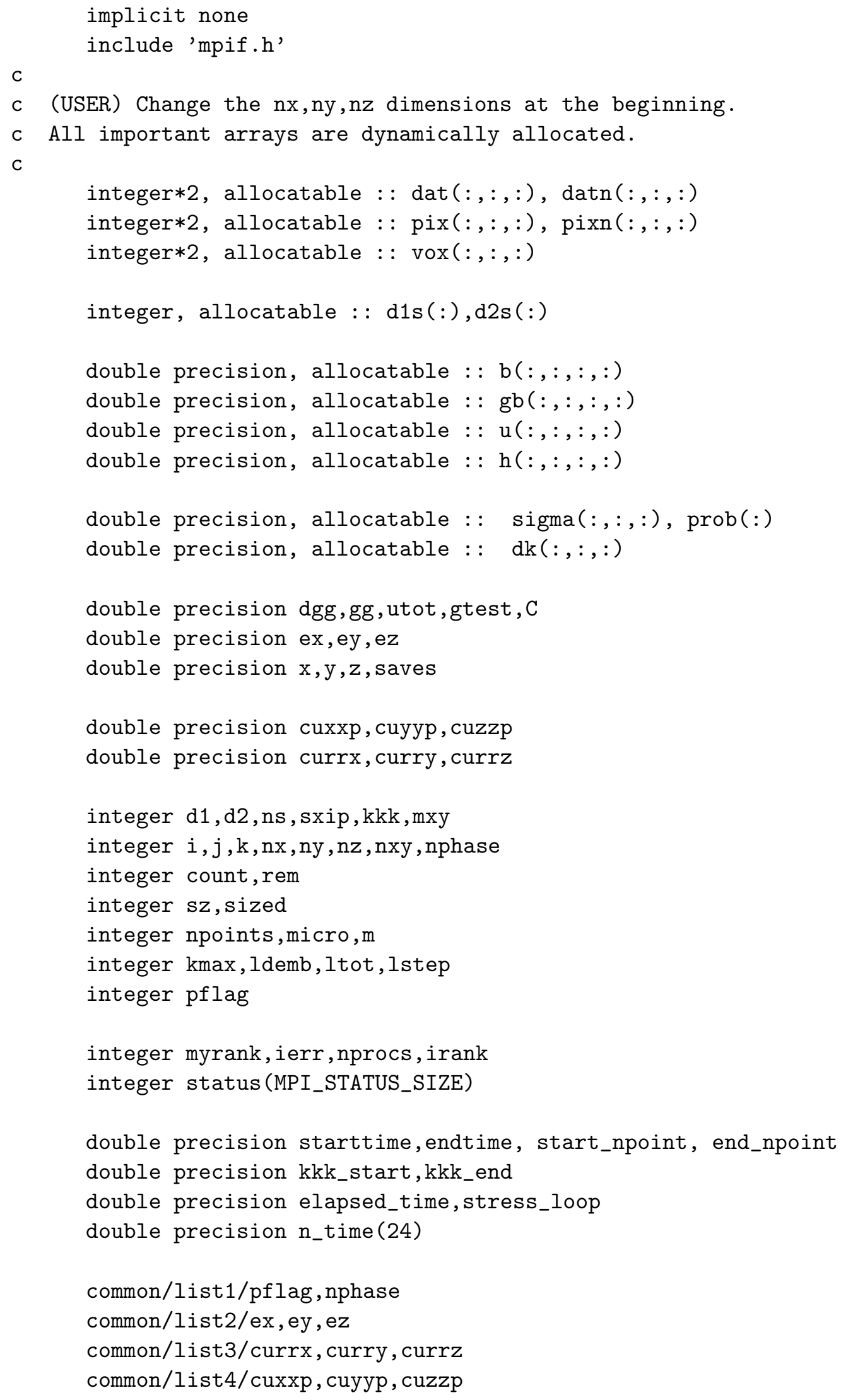




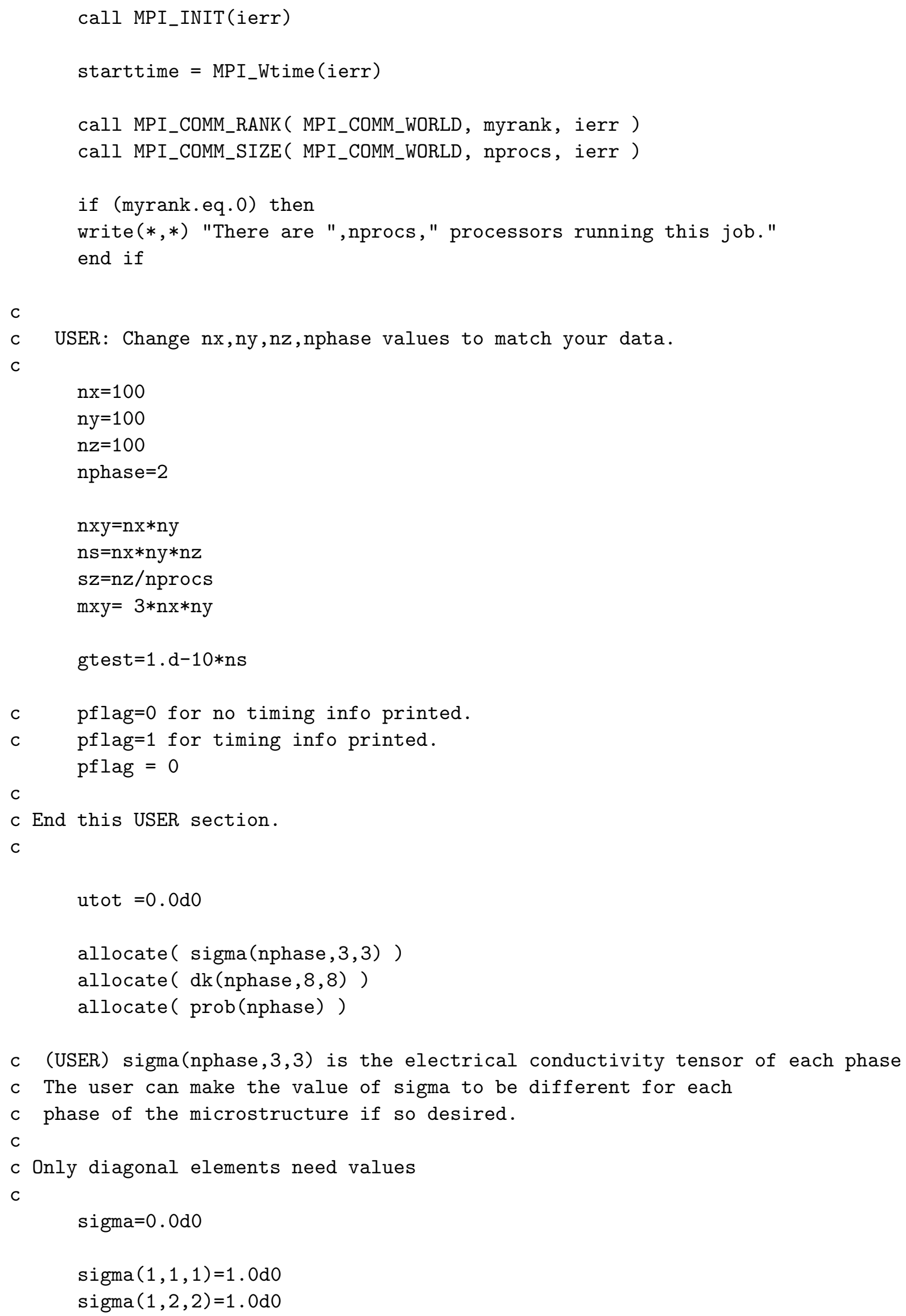




$$
\begin{aligned}
& \operatorname{sigma}(1,3,3)=1.0 \mathrm{~d} 0 \\
& \operatorname{sigma}(2,1,1)=200.0 \mathrm{~d} 0 \\
& \operatorname{sigma}(2,2,2)=200.0 \mathrm{~d} 0 \\
& \operatorname{sigma}(2,3,3)=200.0 \mathrm{~d} 0
\end{aligned}
$$

c (USER) Set applied electric field.

$\mathrm{ex}=1.0 \mathrm{~d} 0$

ey $=1.0 \mathrm{~d} 0$

ez $=1.0 \mathrm{~d} 0$

C

c Calculate d1s(n) \& d2s(n); These hold the d1 and d2

$c$ values for processor $n$.

c

if (myrank.eq.0) then

allocate (d1s (0:nprocs-1))

allocate (d2s (0:nprocs-1))

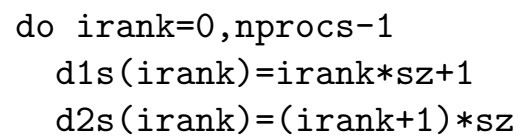

c Send all d1s(i) and d2s(i) from ROOT

$c$ to NODE $i$ \& store into $d 1$ \& $d 2$

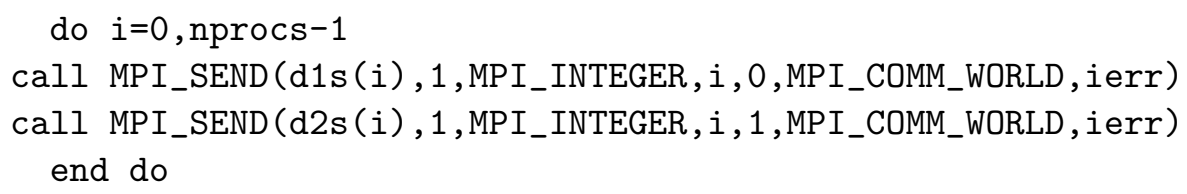


C

c Allocate other arrays which need d1\&d2 values.

C

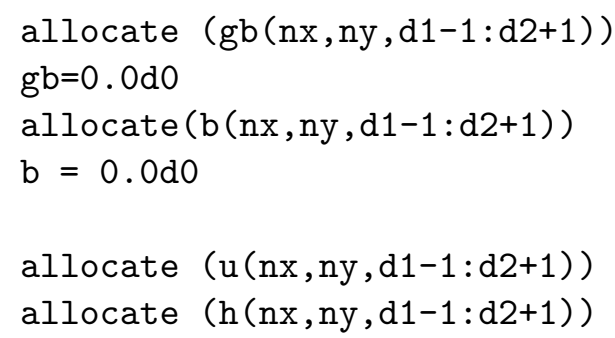

C

c Want the ability to calculate on a series

c of input files based on a value \& some if statements.

C

c Compute the average stress and strain in each microstructure.

c (USER) npoints is the number of microstructures to use.

npoints $=1$

c (USER) Unit 9 is the microstructure input file,

c unit 7 is the results output file.

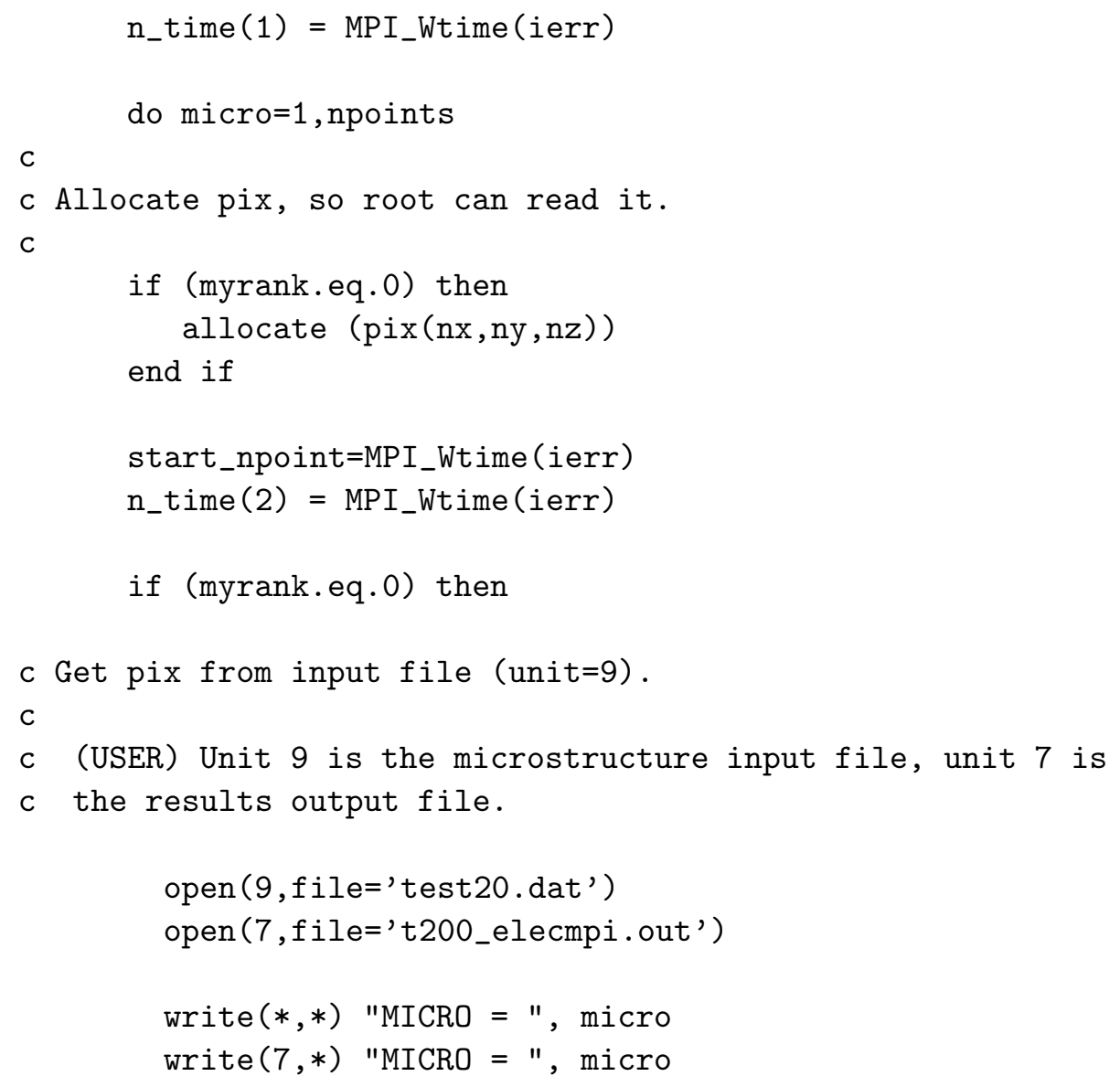


C

c Finally... read in pix

C

write $(*, *)$ "call dpixel"

call dpixel (nx, ny, nz,ns,pix)

write $(*, *)$ "back from dpixel"

c ns=total number of sites

write $(7,9010) \mathrm{nx}, \mathrm{ny}, \mathrm{nz}, \mathrm{ns}, \mathrm{nprocs}$

9010 format (' $n x=$ ', i4,' $n y=$ ', i4,' $n z=$ ', i4,' $n s=$ 'i8,' nprocs= ',i4)

end if

cal1 MPI_BARRIER(MPI_COMM_WORLD, ierr)

c Now that the nodes are set up correctly,

c one can pass the data from the root node (myrank=0)

c to all the rest.

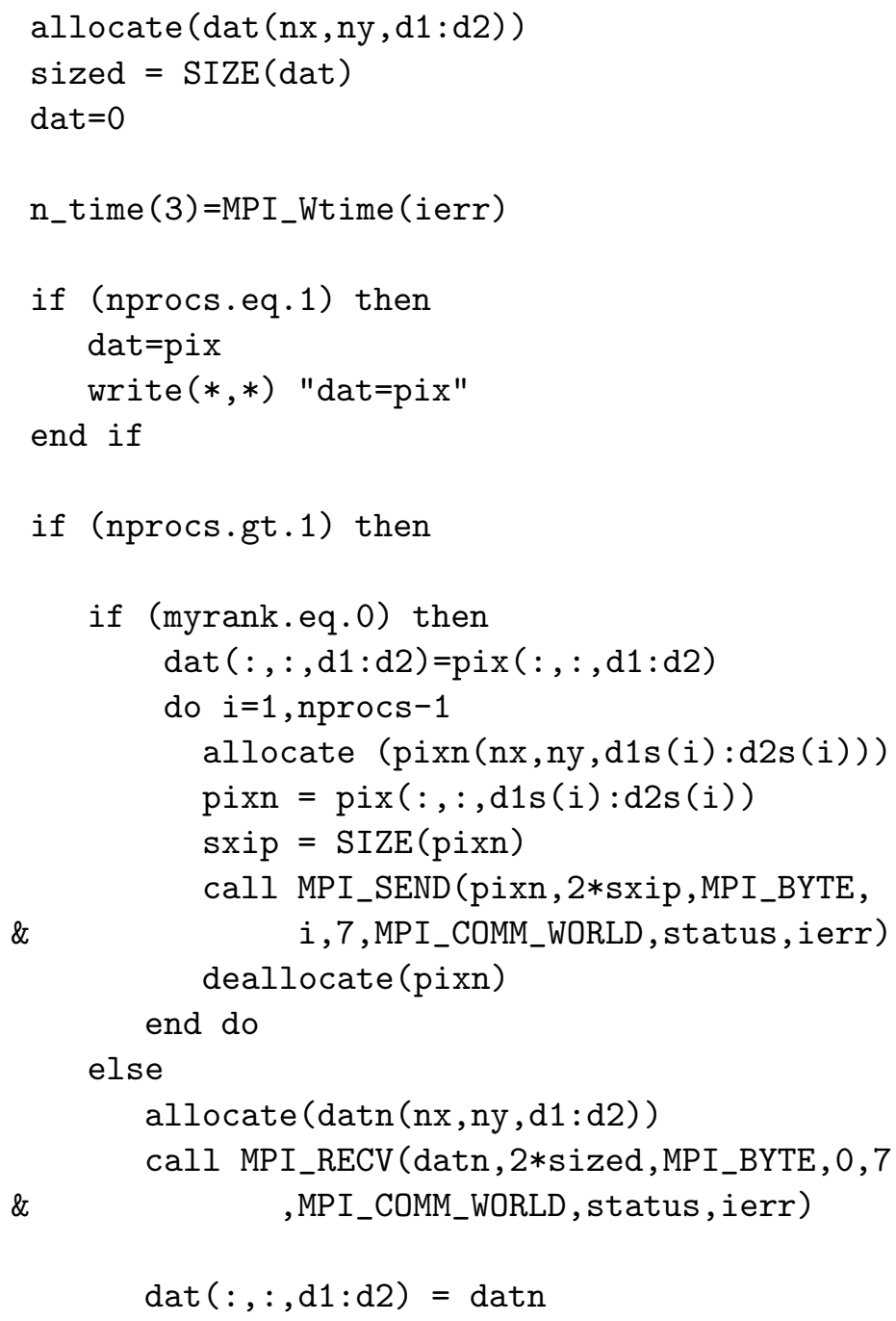




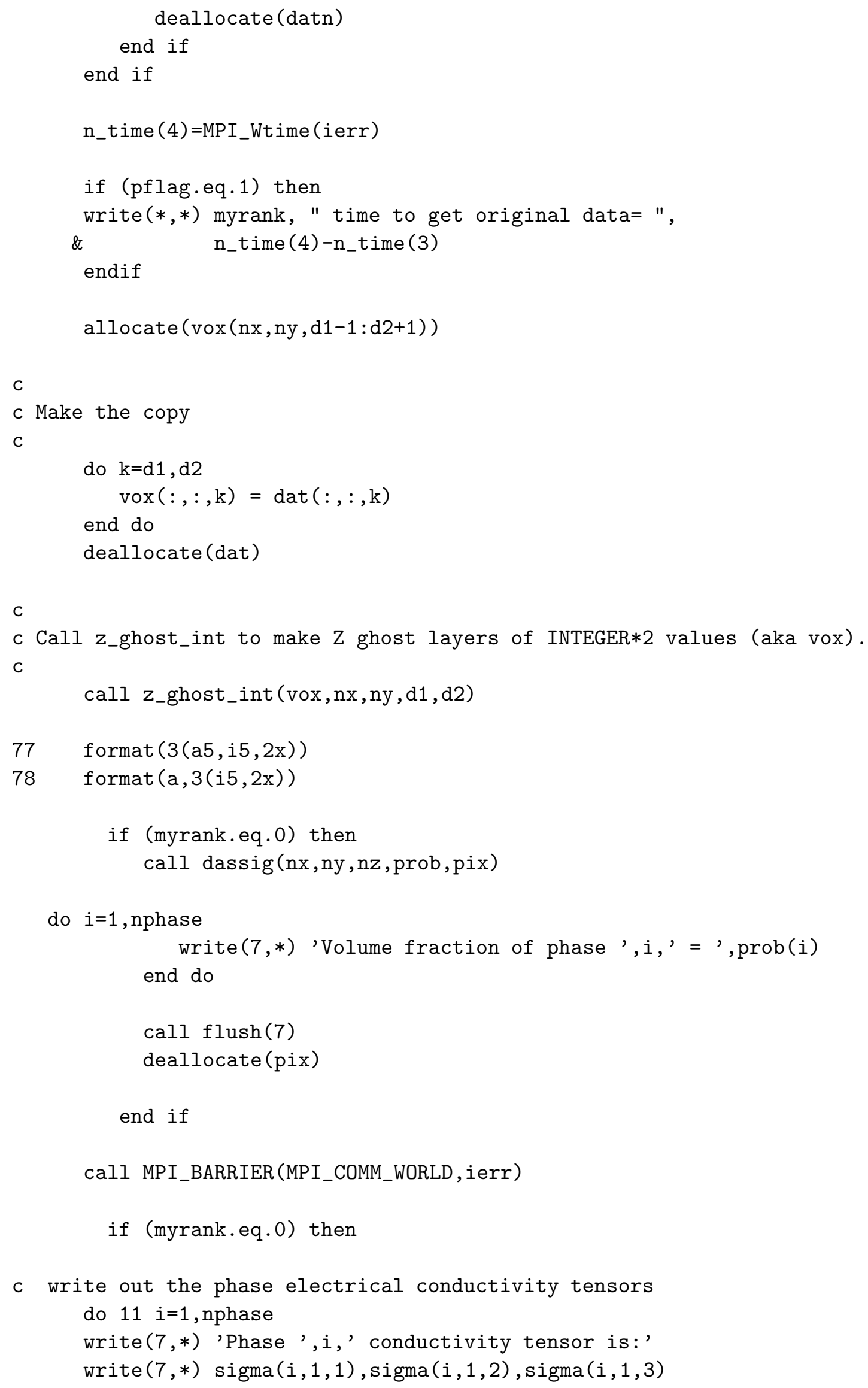




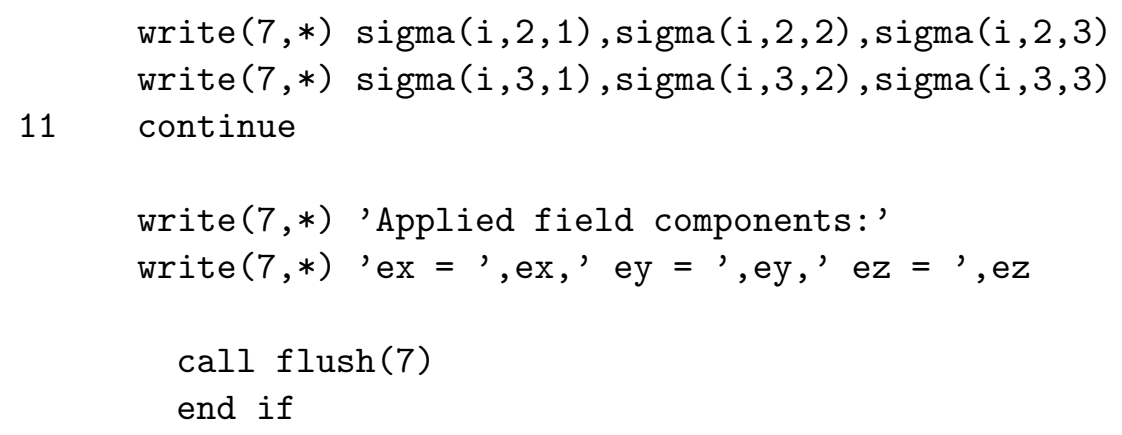

c Call energy to get initial energy and initial gradient

n_time (15)=MPI_Wtime (ierr) 


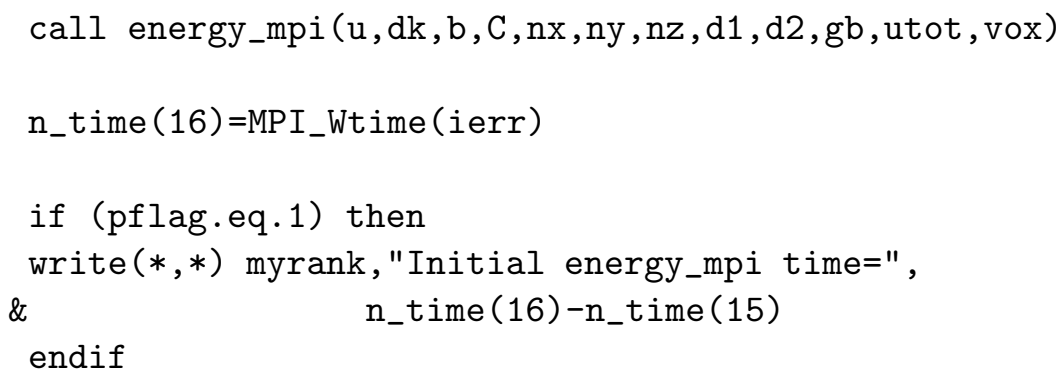

c $g g$ is the norm squared of the gradient ( $g g=g b * g b)$

$\mathrm{dgg}=0.0 \mathrm{~d} 0$

$\mathrm{gg}=0.0 \mathrm{~d} 0$

$\operatorname{dgg}=\operatorname{SUM}(\mathrm{gb}(:, \mathrm{:}, \mathrm{d} 1: \mathrm{d} 2) * \mathrm{gb}(:, \mathrm{:}, \mathrm{d} 1: \mathrm{d} 2))$

call MPI_ALLREDUCE (dgg,gg, 1,MPI_DOUBLE_PRECISION, $\&$

MPI_SUM, MPI_COMM_WORLD, ierr)

n_time (17)=MPI_Wtime (ierr)

if (myrank.eq.0) then

write $(*, *)$ " Initial Energy = ",utot, " gg = ",gg

write $(7, *)$ " Initial Energy = ",utot, " gg = ",gg call flush (7)

end if

elapsed_time $=0.0 \mathrm{~d} 0$

n_time (18) =MPI_Wtime (ierr)

$k \mathrm{kk}=0$

$\mathrm{kkk}=\mathrm{kkk}+1$

do $\mathrm{kkk}=1$, $\mathrm{kmax}$

kkk_start $=$ MPI_Wtime $($ ierr $)$

c call dembx_mpi to go into the conjugate gradient solver

call dembx_mpi (nx, ny, nz,d1,d2, Lstep,gb, u,vox,h,

\&

$\mathrm{gg}, \mathrm{dk}$, gtest , ldemb, kkk)

ltot=ltot+Lstep

call energy_mpi ( $\mathrm{u}, \mathrm{dk}, \mathrm{b}, \mathrm{C}, \mathrm{nx}, \mathrm{ny}, \mathrm{nz}, \mathrm{d} 1, \mathrm{~d} 2, \mathrm{gb}$, utot, vox)

kkk_end $=$ MPI_Wtime (ierr)

elapsed_time=elapsed_time+(kkk_end-kkk_start)

if (myrank.eq.0) then

write $(7, *)$ "Energy = ", utot," gg = ",gg

write $(7, *)$ "Number of conjugate steps = ", ltot 


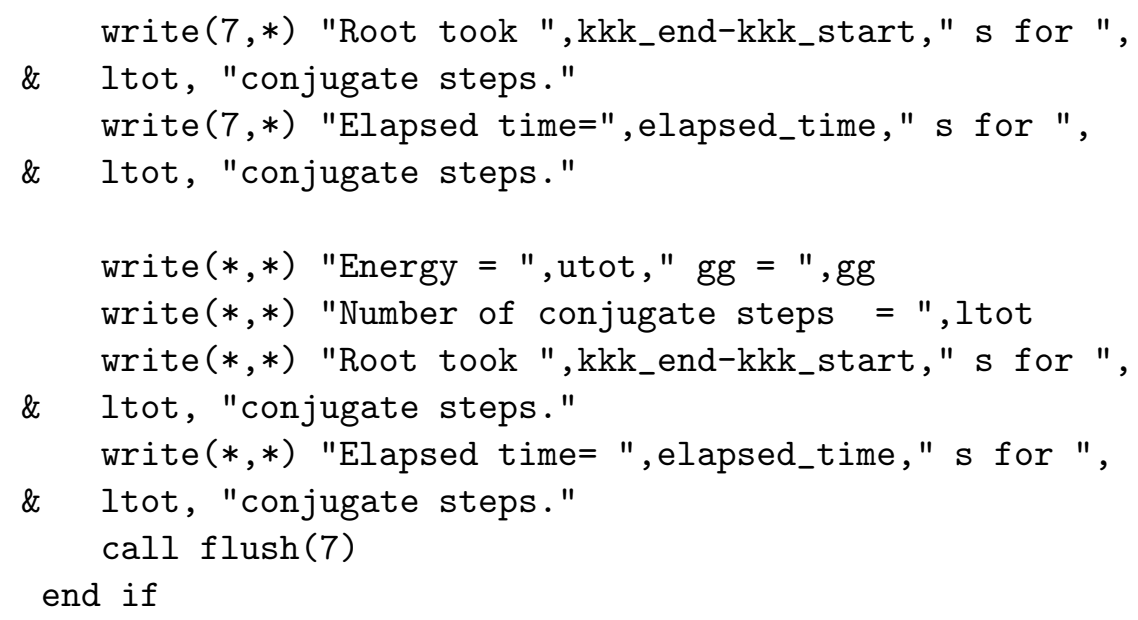




\section{call flush(7)}

c Output final currents

write $(*, *)$

write $(*, *)$ ' Current in $\mathrm{x}$ direction $=$ ', $\operatorname{cuxxp}$

write $(*, *)$ ' Current in y direction $=$ ', cuyyp

write $(*, *)$ ' Current in $\mathrm{z}$ direction $=$, , cuzzp

close (unit=9)

close (unit=7)

end if

deallocate(vox)

end do

C

c Do another calculation using loop var: npoints

C

deallocate (u)

deallocate (b)

deallocate (gb)

deallocate $(\mathrm{h})$

n_time (24) $=$ MPI_Wtime(ierr)

CALL MPI_FINALIZE(ierr)

end

C

$\mathrm{C} * * * * * * * * * * * * * * * * * * * * * * * * * * * * * * * * * * * * * * * * * * * * * * * * * * * * * * * * * * *$

$\mathrm{C}$

subroutine femat_mpi ( $n x, n y, n z, d 1, d 2$, vox, sigma,b,dk,C)

implicit none

include 'mpif.h'

integer i,ierr, nx, j, ny, nz

integer d1, d2, myrank, nprocs, mxy

integer ipx,ipy,ipz

integer $n x y, k, n m, i j k, m m, i i, j j, k k, l l$

integer $i 8, \mathrm{dn}, \mathrm{m}, \mathrm{m} 8$

integer pflag,nphase

integer status(MPI_STATUS_SIZE) 


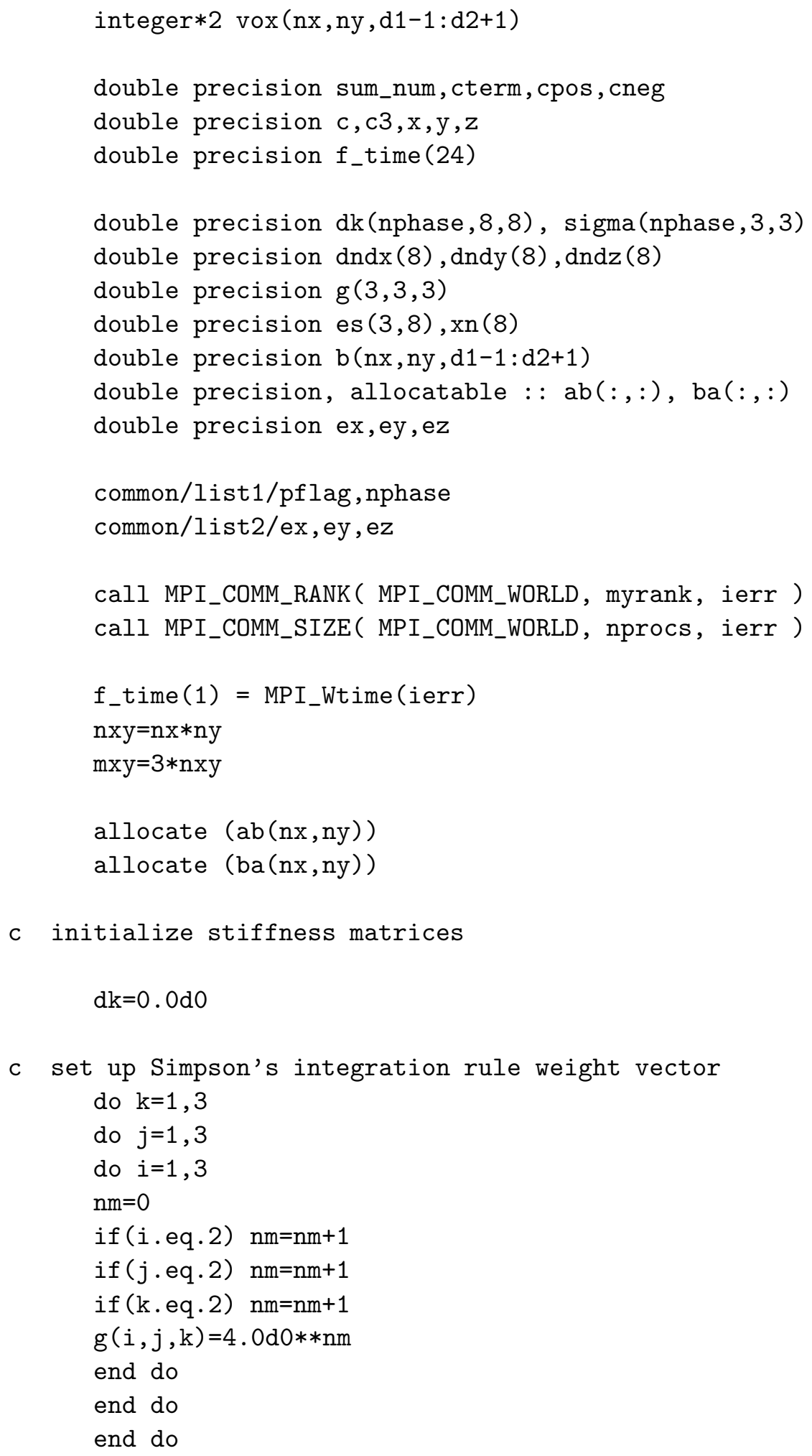
initialize stiffness matrices

$\mathrm{dk}=0.0 \mathrm{dO}$

c set up Simpson's integration rule weight vector

do $k=1,3$

do $j=1,3$

do $i=1,3$

$\mathrm{nm}=0$

if (i.eq.2) $n m=n m+1$

if (j.eq.2) $n m=n m+1$

if (k.eq.2) $\mathrm{nm}=\mathrm{nm}+1$

$\mathrm{g}(\mathrm{i}, \mathrm{j}, \mathrm{k})=4$. Od0**nm

end do

end do

end do

C

c loop over the nphase kinds of pixels and Simpson's rule quadrature

c points in order to compute the stiffness matrices. Stiffness matrices

$c$ of trilinear finite elements are quadratic in $\mathrm{x}, \mathrm{y}$, and $\mathrm{z}$, so that

c Simpson's rule quadrature gives exact results. 


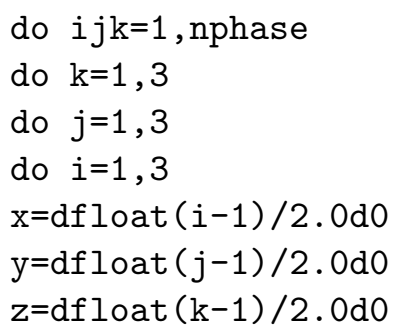

c now build electric field matrix

$$
\begin{aligned}
& \text { es }=0.0 \mathrm{~d} 0 \\
& \text { es }(1,:)=\operatorname{dndx} \\
& \text { es }(2,:)=d n d y \\
& \text { es }(3,:)=\operatorname{dnd} z
\end{aligned}
$$

c Matrix multiply to determine value at $(\mathrm{x}, \mathrm{y}, \mathrm{z})$, multiply by

c proper weight, and sum_num into dk, the stiffness matrix

f_time(2) $=$ MPI_Wtime(ierr)

do $i i=1,8$

do $j j=1,8$ 
c Define sum over strain matrices and elastic moduli matrix for

c stiffness matrix

sum_num $=0.0 \mathrm{~d} 0$

do $k \mathrm{k}=1,3$

do $11=1,3$

sum_num=sum_num+es $(k k, i i) * \operatorname{sigma}(i j k, k k, 1 l) * e s(l l, j j)$

end do; end do

$d k(i j k, i i, j j)=d k(i j k, i i, j j)+g(i, j, k) * s u m \_n u m / 216$.

end do; end do

end do; end do; end do; end do

f_time(3) $=$ MPI_Wtime(ierr)

if (pflag.eq.1) then

write $(*, *)$ myrank, "time to calculate dk = ",f_time(3)-f_time(2)

endif

c Initialize $\mathrm{b}$ and $\mathrm{C}$

$\mathrm{b}=0.0 \mathrm{~d} 0$

$\mathrm{C}=0.0 \mathrm{~d} 0$

$\mathrm{c} 3=0.0 \mathrm{~d} 0$

999 format $(4(i 4,1 x),, 3(f 9.6,1 x))$

C

c $\mathrm{x}=\mathrm{nx}$ face

c

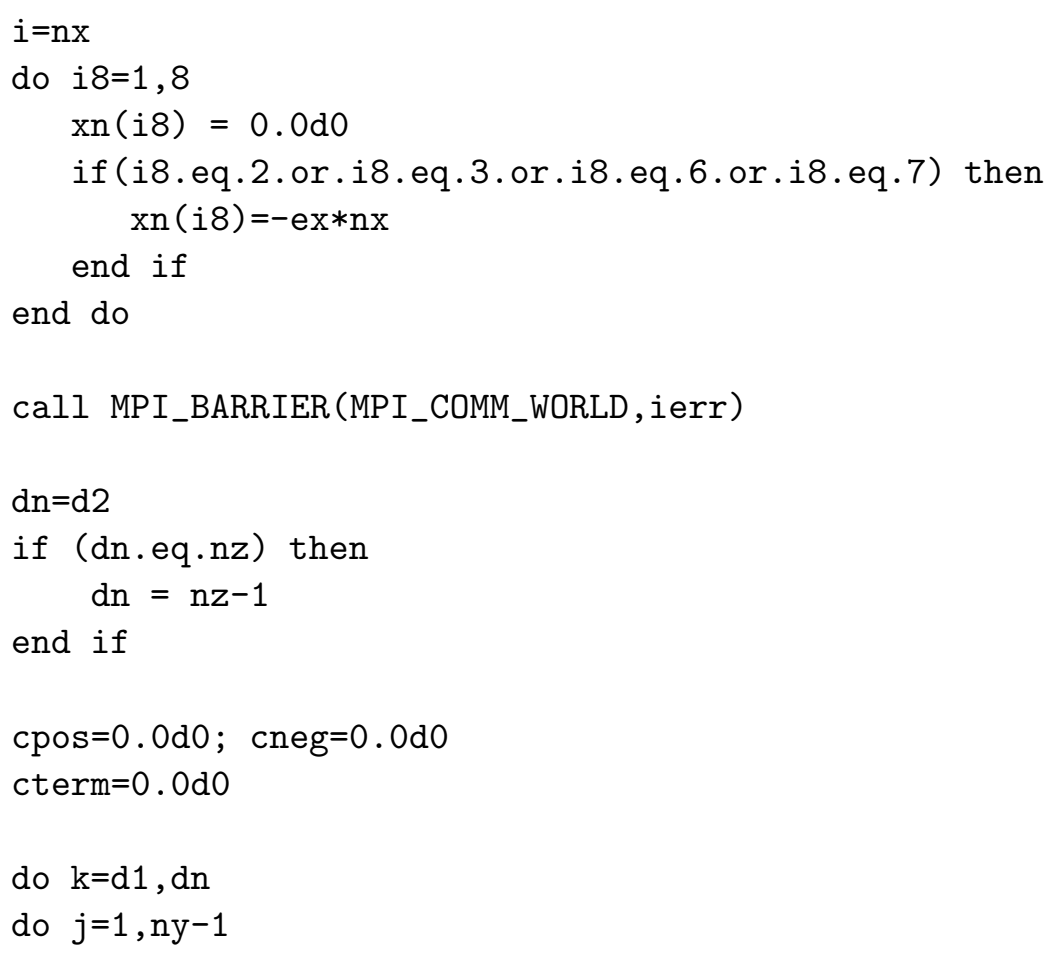


do $\mathrm{m} 8=1,8$

sum_num=sum_num+xn $(\mathrm{m} 8) * \mathrm{dk}(\operatorname{vox}(i \mathrm{i}, j \mathrm{j}, \mathrm{kk}), \mathrm{m} 8, \mathrm{~mm})$

cterm $=0.5 \mathrm{~d} 0 * \mathrm{xn}(\mathrm{m} 8) * \mathrm{dk}(\operatorname{vox}(\mathrm{i} i, j \mathrm{j}, \mathrm{kk}), \mathrm{m} 8, \mathrm{~mm}) * \mathrm{xn}(\mathrm{mm})$

if (cterm.ge.0.0d0) then

cpos $=$ cpos + cterm

else

cneg $=$ cneg + cterm

end if

end do

$b(i p x, i p y, i p z)=b(i p x, i p y, i p z)+$ sum_num

end do

end do; end do

C

C

C

$\mathrm{C}$

C

C

c

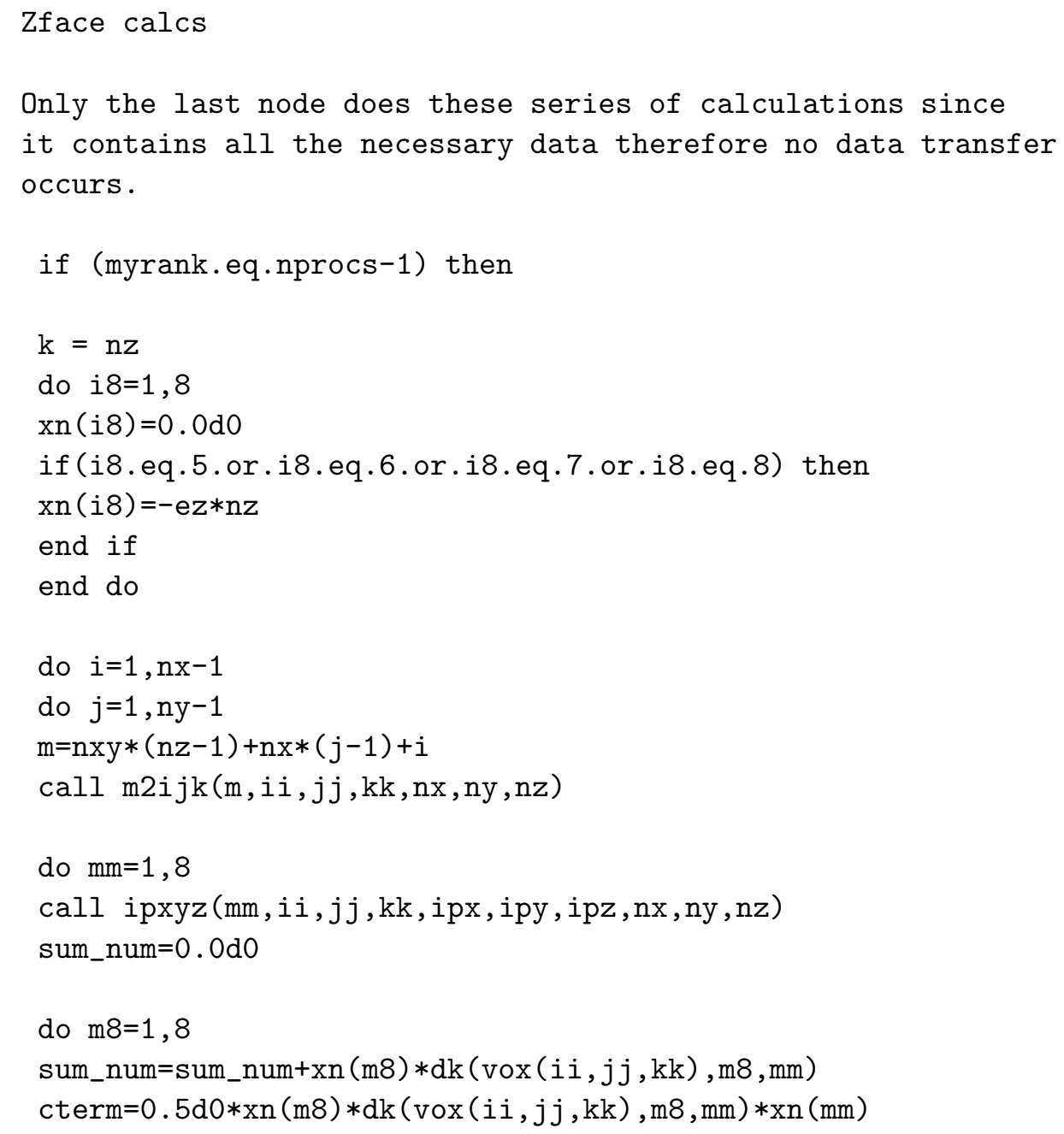




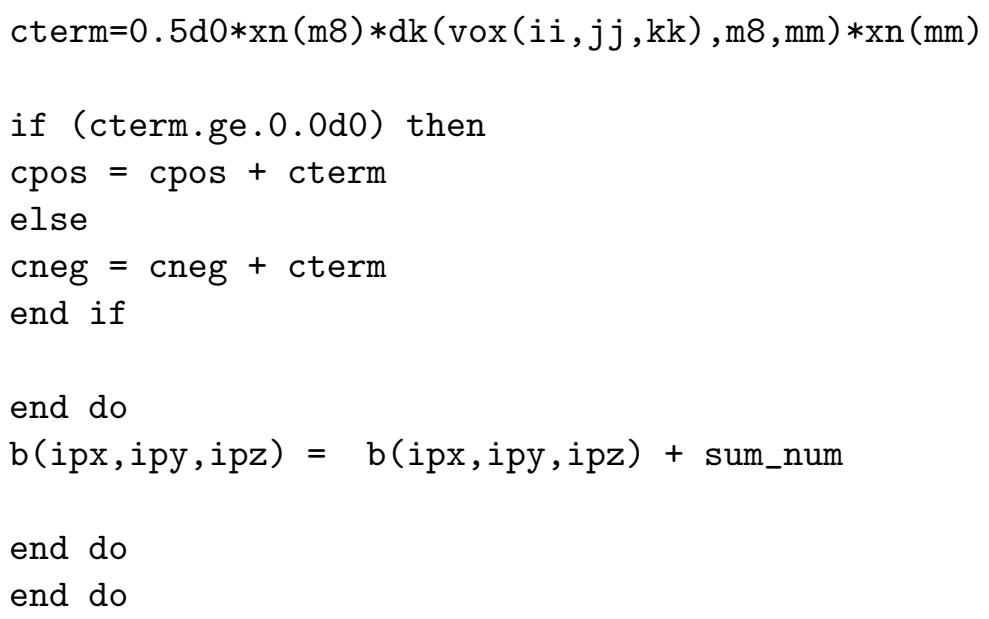




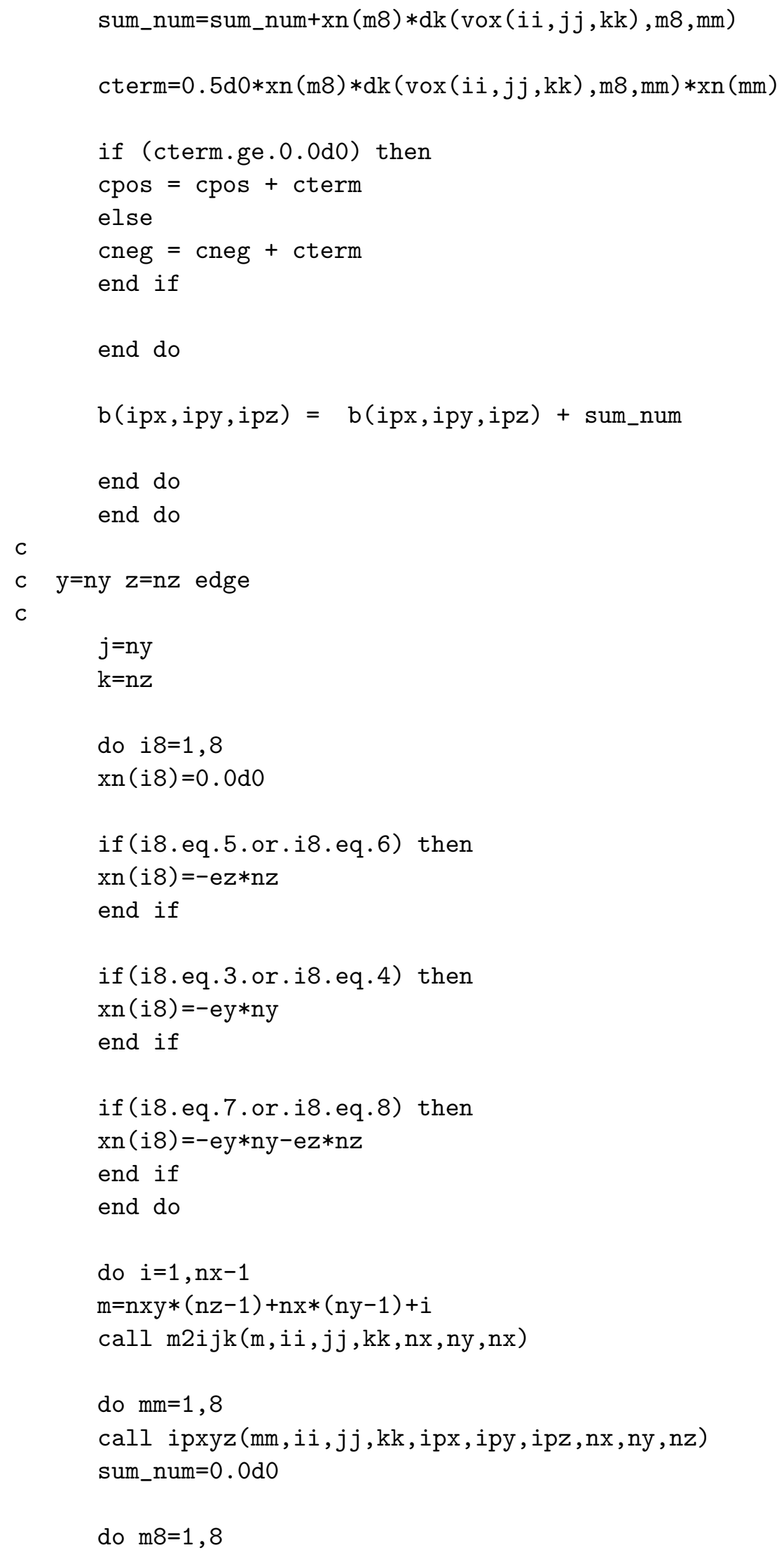




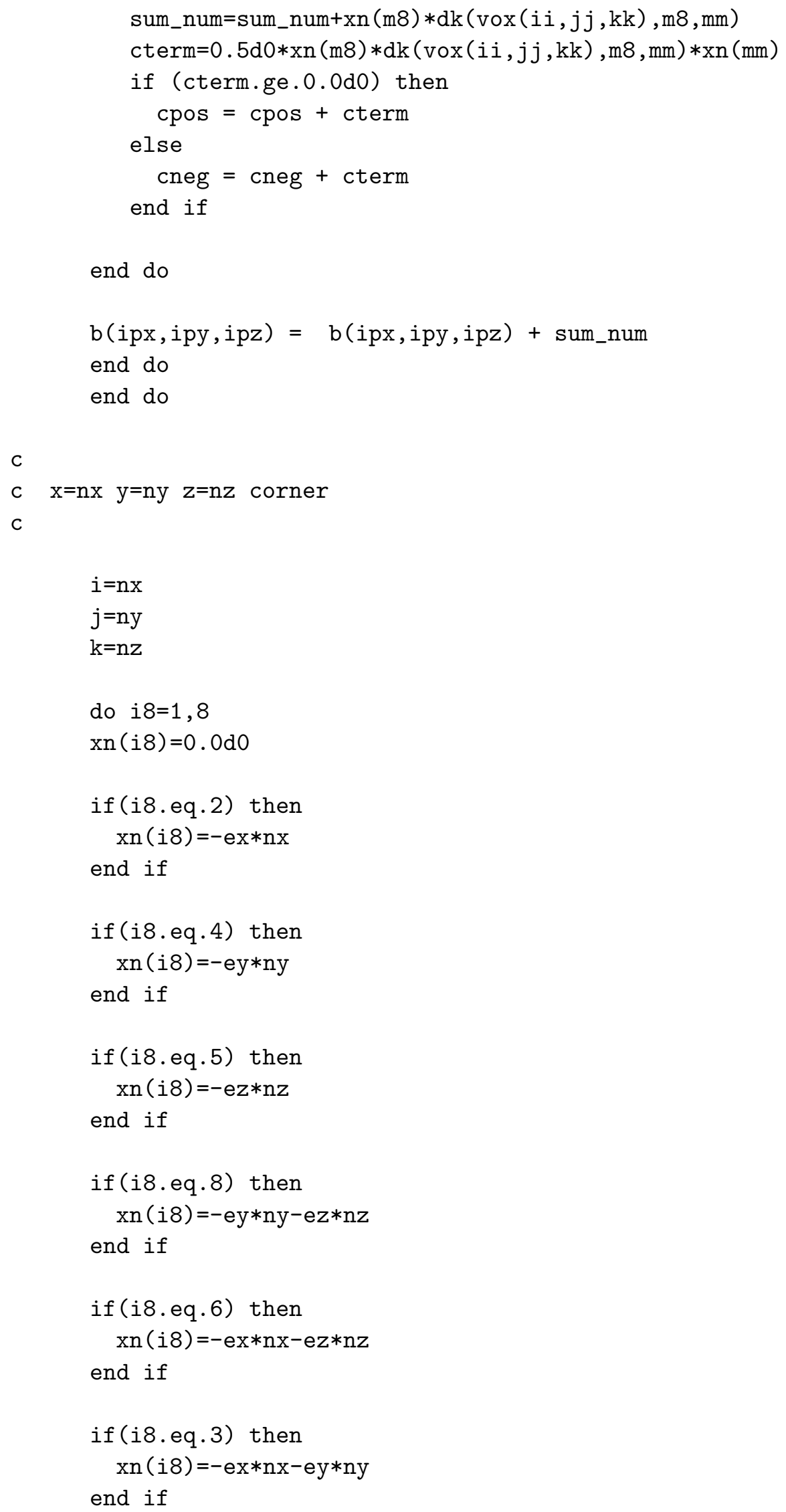




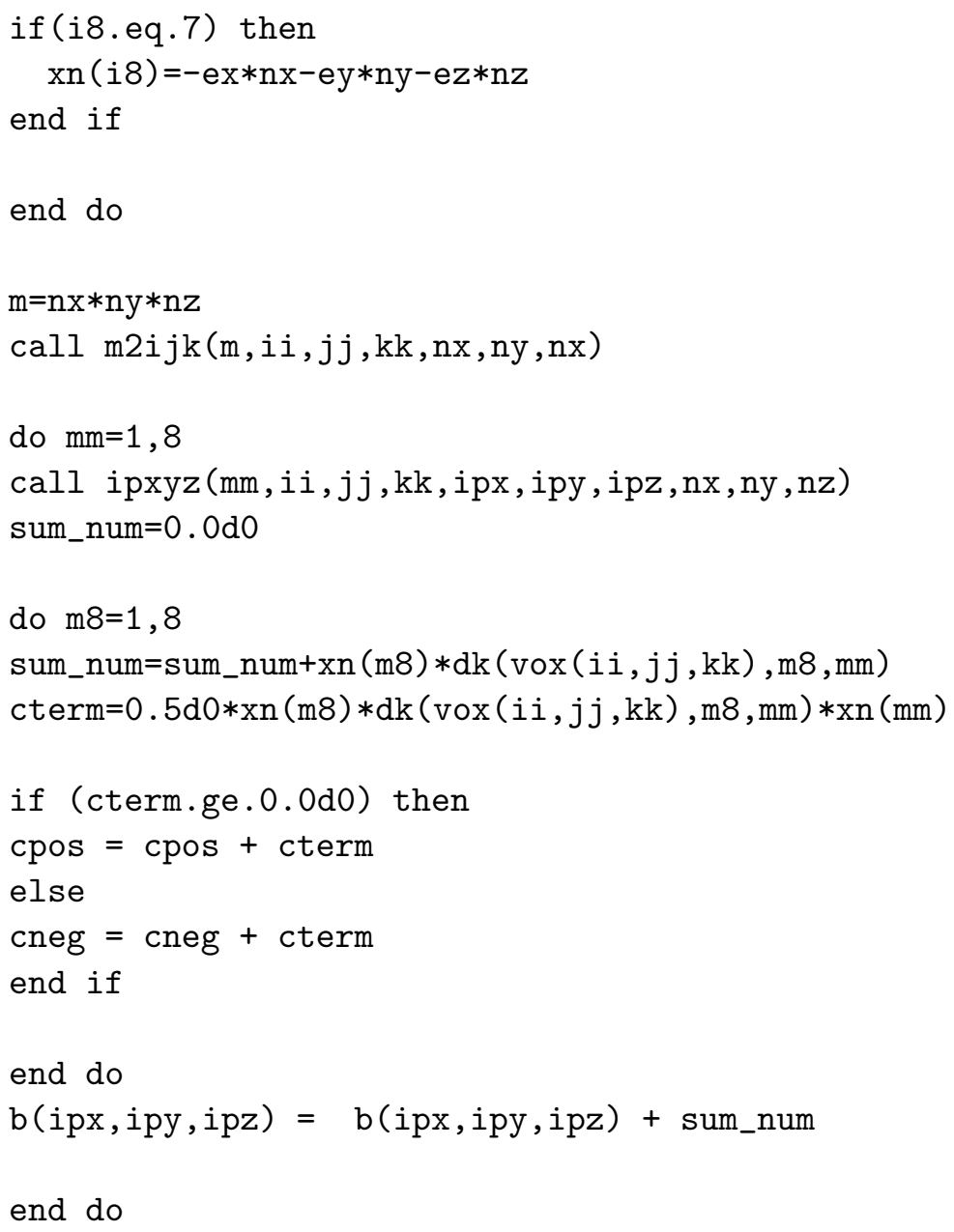




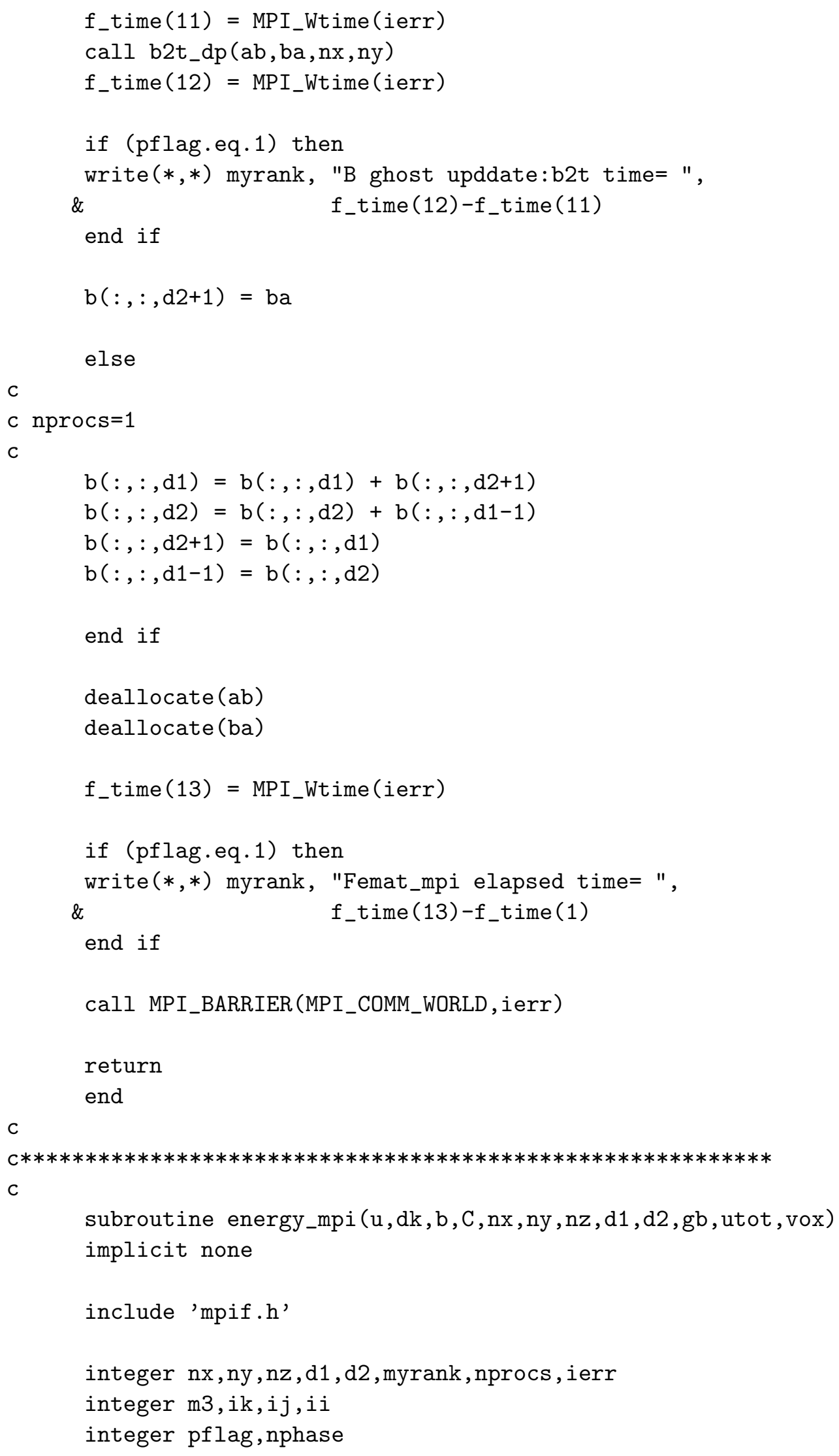




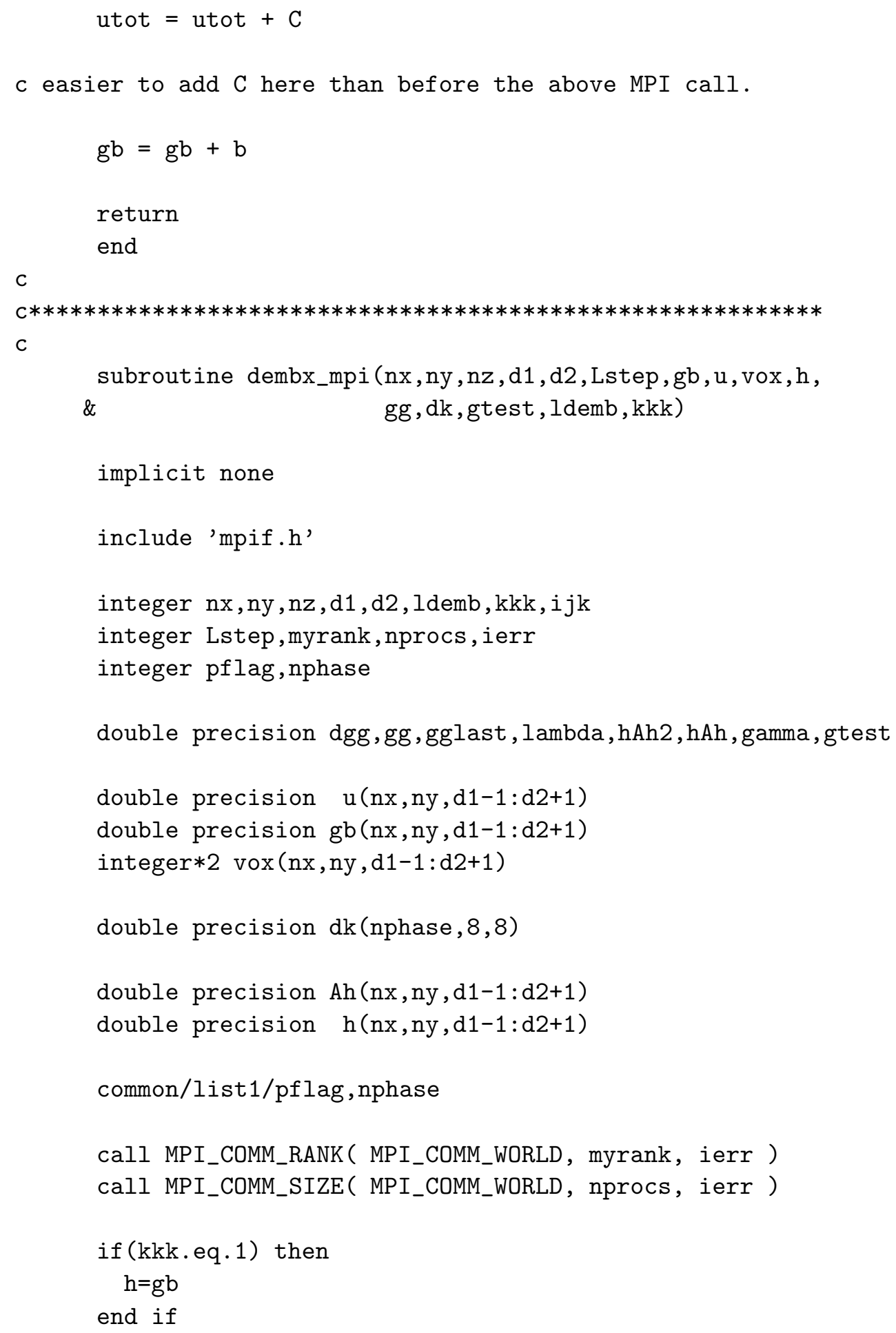

c Lstep counts the number of conjugate gradient steps taken in c each call to dembx

Lstep $=0$ 


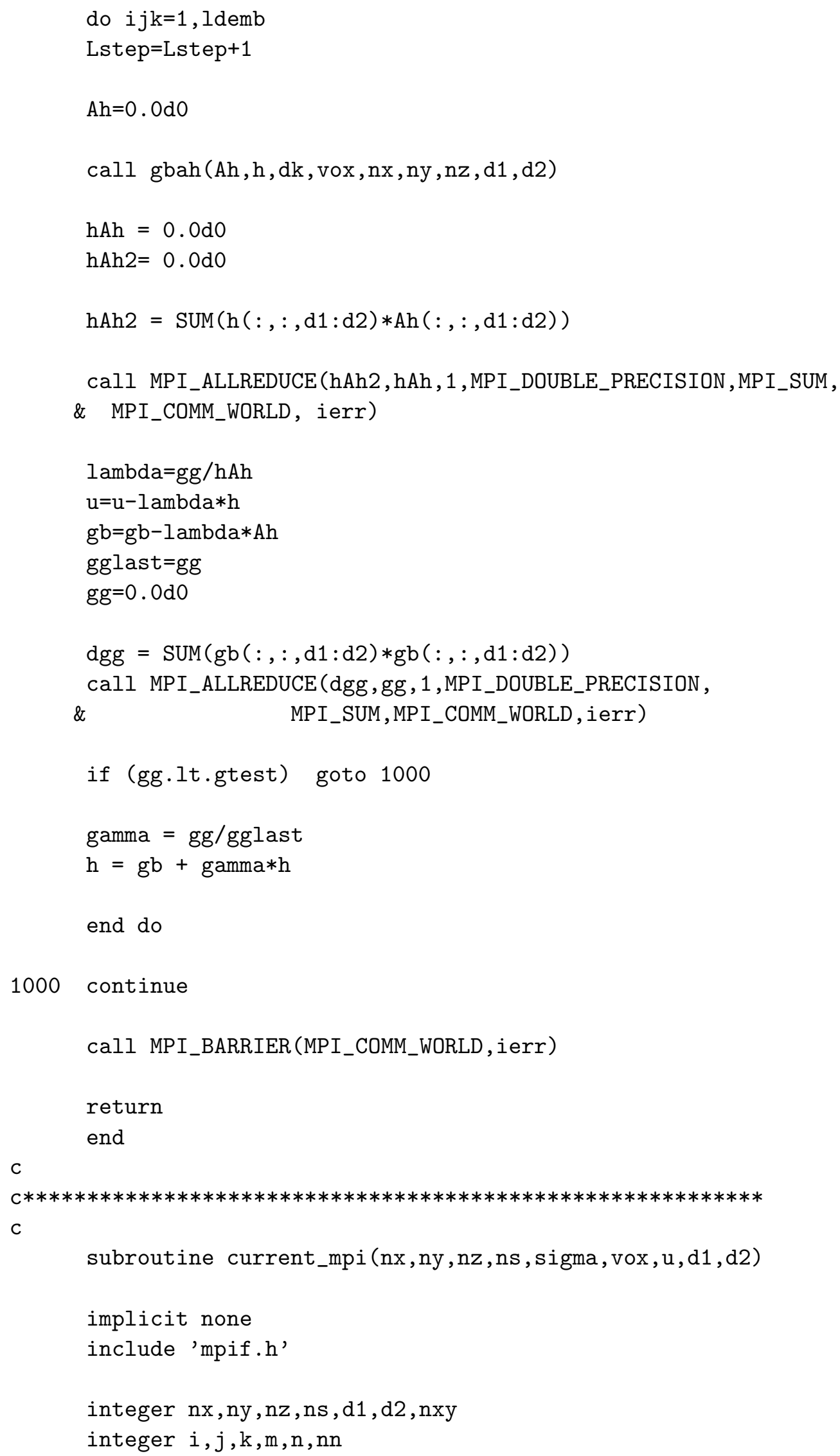




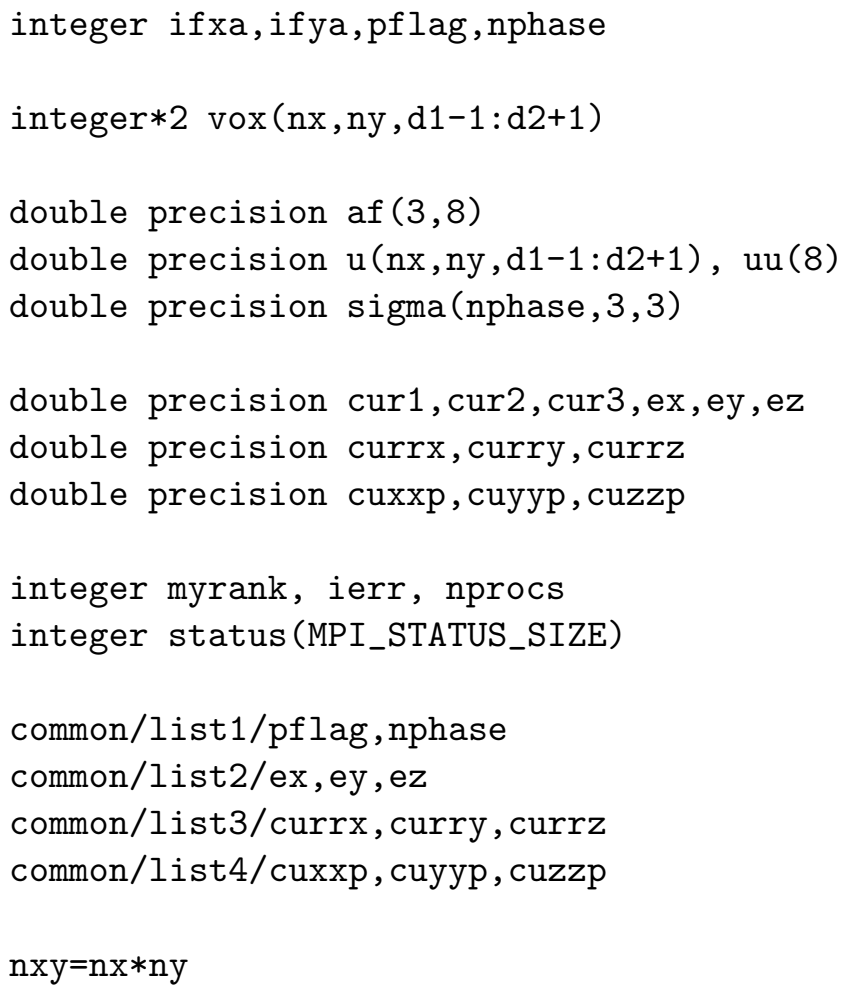

c af is the average field matrix, average field in a pixel is af*u(pixel).

$c$ The matrix af relates the nodal voltages to the average field in the pixel.

c Set up single element average field matrix

$$
\begin{aligned}
& \text { af }(1,1)=0.25 \mathrm{~d} 0 \\
& \text { af }(1,2)=-0.25 \mathrm{~d} 0 \\
& \text { af }(1,3)=-0.25 \mathrm{~d} 0 \\
& \text { af }(1,4)=0.25 \mathrm{~d} 0 \\
& \text { af }(1,5)=0.25 \mathrm{~d} 0 \\
& \text { af }(1,6)=-0.25 \mathrm{~d} 0 \\
& \text { af }(1,7)=-0.25 \mathrm{~d} 0 \\
& \text { af }(1,8)=0.25 \mathrm{~d} 0 \\
& \text { af }(2,1)=0.25 \mathrm{~d} 0 \\
& \text { af }(2,2)=0.25 \mathrm{~d} 0 \\
& \text { af }(2,3)=-0.25 \mathrm{~d} 0 \\
& \text { af }(2,4)=-0.25 \mathrm{~d} 0 \\
& \text { af }(2,5)=0.25 \mathrm{~d} 0 \\
& \text { af }(2,6)=0.25 \mathrm{~d} 0 \\
& \text { af }(2,7)=-0.25 \mathrm{~d} 0 \\
& \text { af }(2,8)=-0.25 \mathrm{~d} 0 \\
& \text { af }(3,1)=0.25 \mathrm{~d} 0 \\
& \text { af }(3,2)=0.25 \mathrm{~d} 0 \\
& \text { af }(3,3)=0.25 \mathrm{~d} 0 \\
& \text { af }(3,4)=0.25 \mathrm{~d} 0 \\
& \text { af }(3,5)=-0.25 \mathrm{~d} 0
\end{aligned}
$$


af $(3,6)=-0.25 \mathrm{~d} 0$

af $(3,7)=-0.25 \mathrm{~d} 0$

af $(3,8)=-0.25 \mathrm{~d} 0$

C

now compute current in each pixel

currx $=0.0 \mathrm{~d} 0$

curry $=0.0 \mathrm{~d} 0$

currz $=0.0 \mathrm{~d} 0$

c

compute average field in each pixel

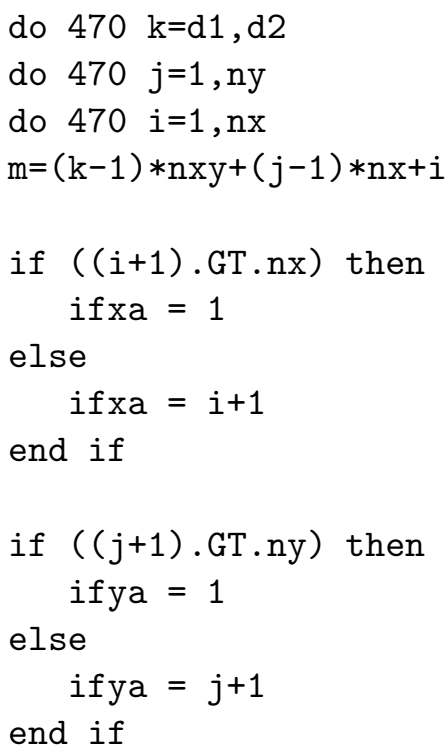

c load in elements of 8-vector using pd. bd. conds.

$$
\begin{aligned}
& u u(1)=u(i, j, k) \\
& u u(2)=u(\text { ifxa, } j, k) \\
& u u(3)=u(\text { ifxa, ifya, } k) \\
& u u(4)=u(i, \text { ifya, } k) \\
& u u(5)=u(i, j, k+1) \\
& u u(6)=u(\text { ifxa, } j, k+1) \\
& u u(7)=u(\text { ifxa, ifya, } k+1) \\
& u u(8)=u(i, \text { ifya }, k+1)
\end{aligned}
$$

c Correct for periodic boundary conditions, some voltages are wrong

c for a pixel on a periodic boundary. Since they come from an opposite

c face, need to put in applied fields to correct them.

if (i.eq. nx) then

$\mathrm{uu}(2)=\mathrm{uu}(2)-\mathrm{ex} * \mathrm{nx}$

$\mathrm{uu}(3)=\mathrm{uu}(3)-\mathrm{ex} * \mathrm{nx}$

$\mathrm{uu}(6)=\mathrm{uu}(6)-\mathrm{ex} * \mathrm{nx}$

$\mathrm{uu}(7)=\mathrm{uu}(7)-\mathrm{ex} * \mathrm{nx}$

end if

if (j.eq.ny) then 


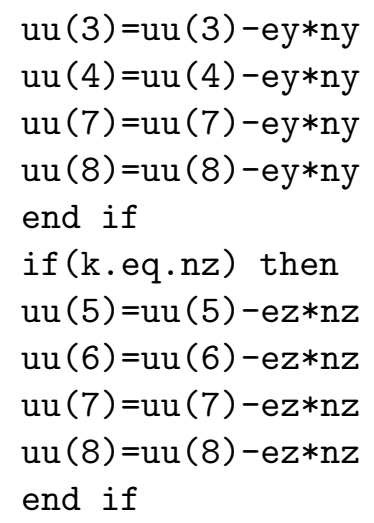

c Volume average currents cuxxp=cuxxp/dfloat (ns) cuyyp=cuyyp/dfloat (ns) cuzzp=cuzzp/dfloat (ns)

return 
end

$\mathrm{C}$

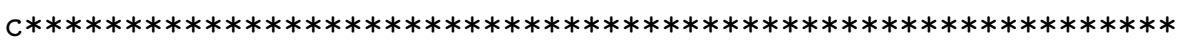

C

subroutine gbah (om, uh, dk, vox, nx, ny, nz,d1,d2)

implicit none

include 'mpif.h'

integer $\mathrm{nx}, \mathrm{ny}, \mathrm{nz}, \mathrm{d} 1, \mathrm{~d} 2, \mathrm{mxy}, \mathrm{pflag}$, nphase

integer im, jm, $\mathrm{km}$, if $x a$, if $x b$, ifya, ifyb

integer myrank, nprocs, ierr

double precision $\mathrm{uh}(\mathrm{nx}, \mathrm{ny}, \mathrm{d} 1-1: \mathrm{d} 2+1)$

double precision om ( $\mathrm{nx}, \mathrm{ny}, \mathrm{d} 1-1: \mathrm{d} 2+1)$

double precision gb_time(6)

integer*2 $\operatorname{vox}(\mathrm{nx}, \mathrm{ny}, \mathrm{d} 1-1: \mathrm{d} 2+1)$

double precision dk(nphase, 8,8)

common/list1/pflag, nphase

call MPI_COMM_RANK ( MPI_COMM_WORLD, myrank, ierr )

call MPI_COMM_SIZE( MPI_COMM_WORLD, nprocs, ierr )

gb_time(1) = MPI_Wtime (ierr)

$\mathrm{om}=0.0 \mathrm{~d} 0$

do $\mathrm{km}=\mathrm{d} 1, \mathrm{~d} 2$

do $j m=1$, ny

do $i m=1, n x$

if $((\mathrm{im}+1) . \mathrm{GT} . \mathrm{nx})$ then

if $x a=1$

else

ifxa $=i m+1$

end if

if ( $(\mathrm{im}-1)$.LE.0) then if $x b=n x$

else

if $x b=i m-1$

end if

if $((j m+1)$.GT.ny) then ifya $=1$

else 


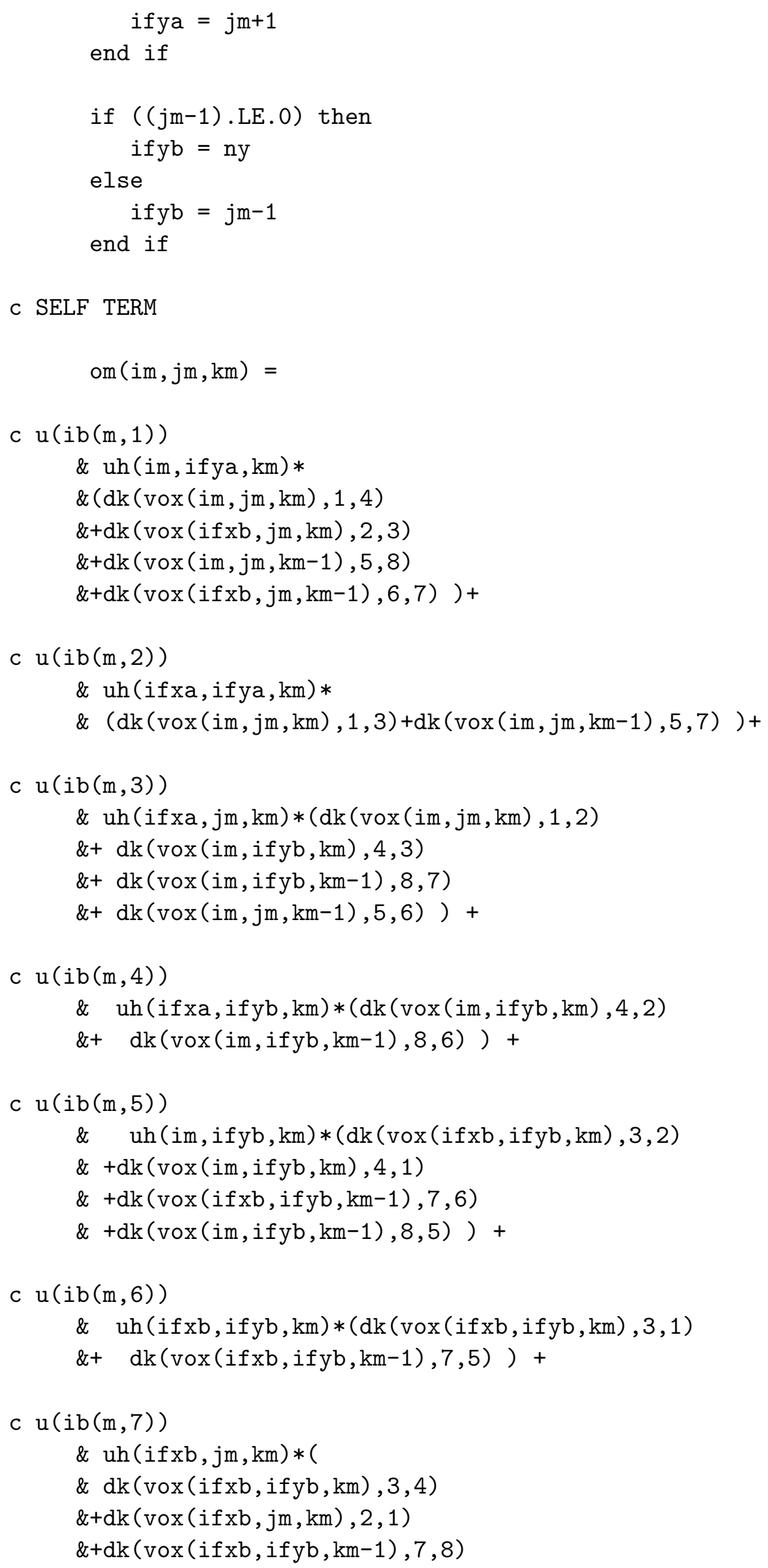




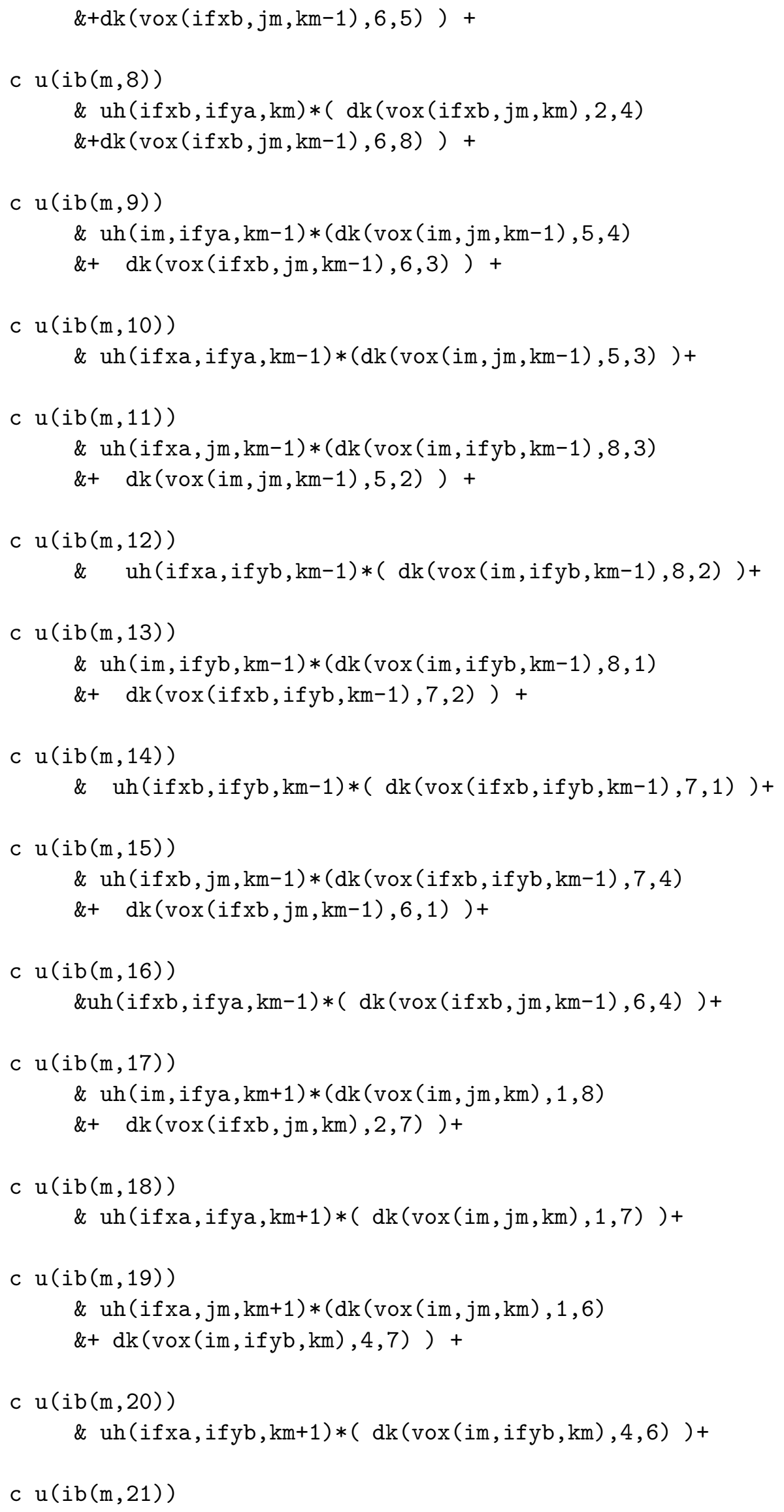




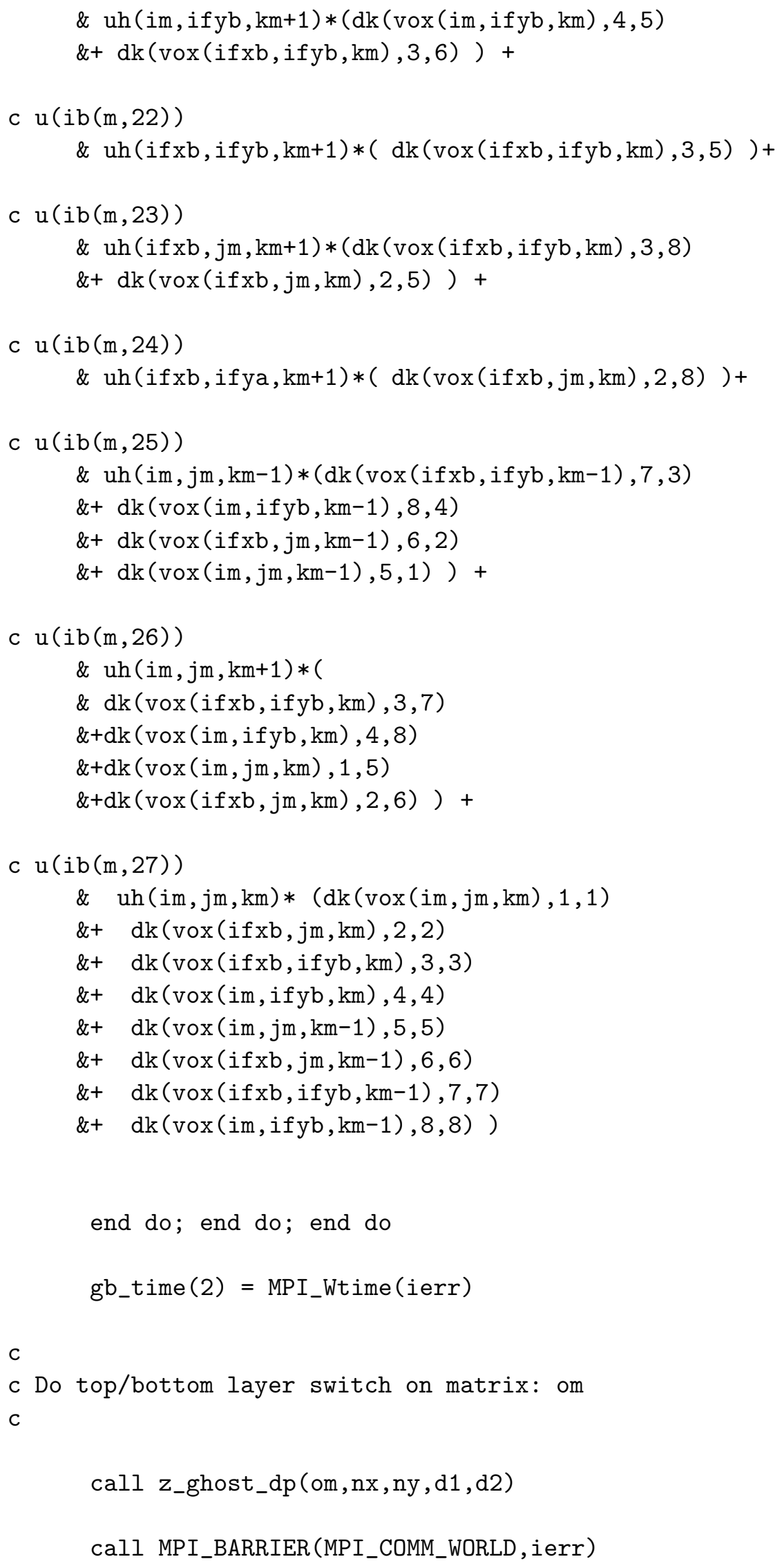




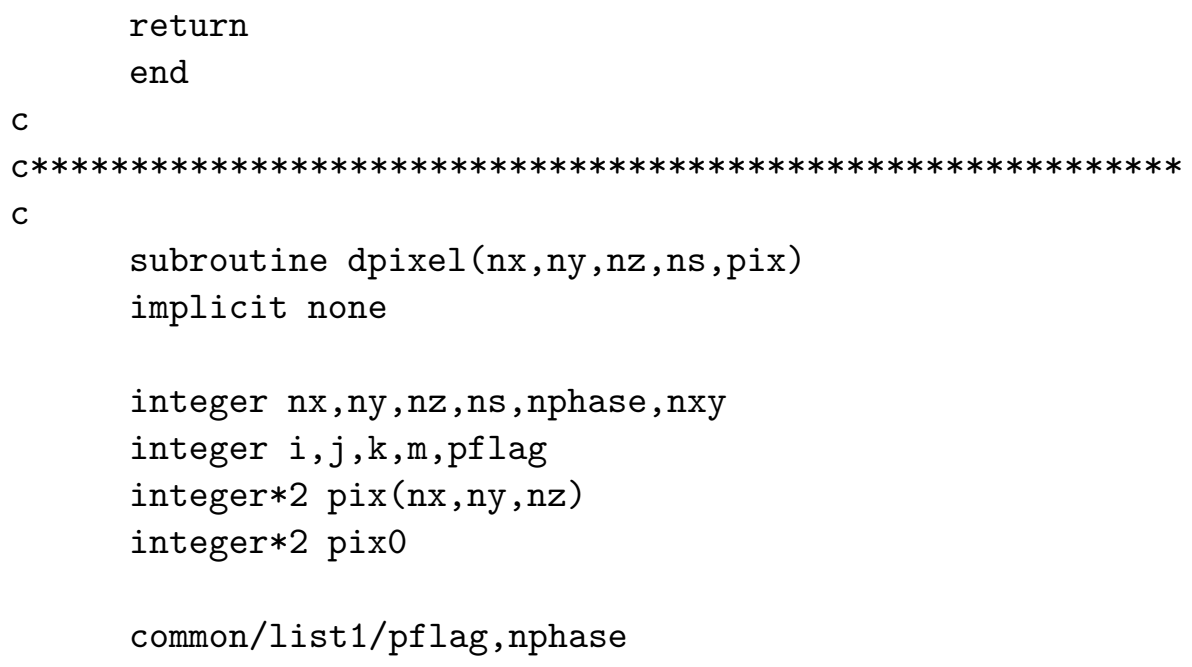

c (USER) If you want to set up a test image inside the program, instead of $c$ reading it in from a file, this should be done inside this subroutine.

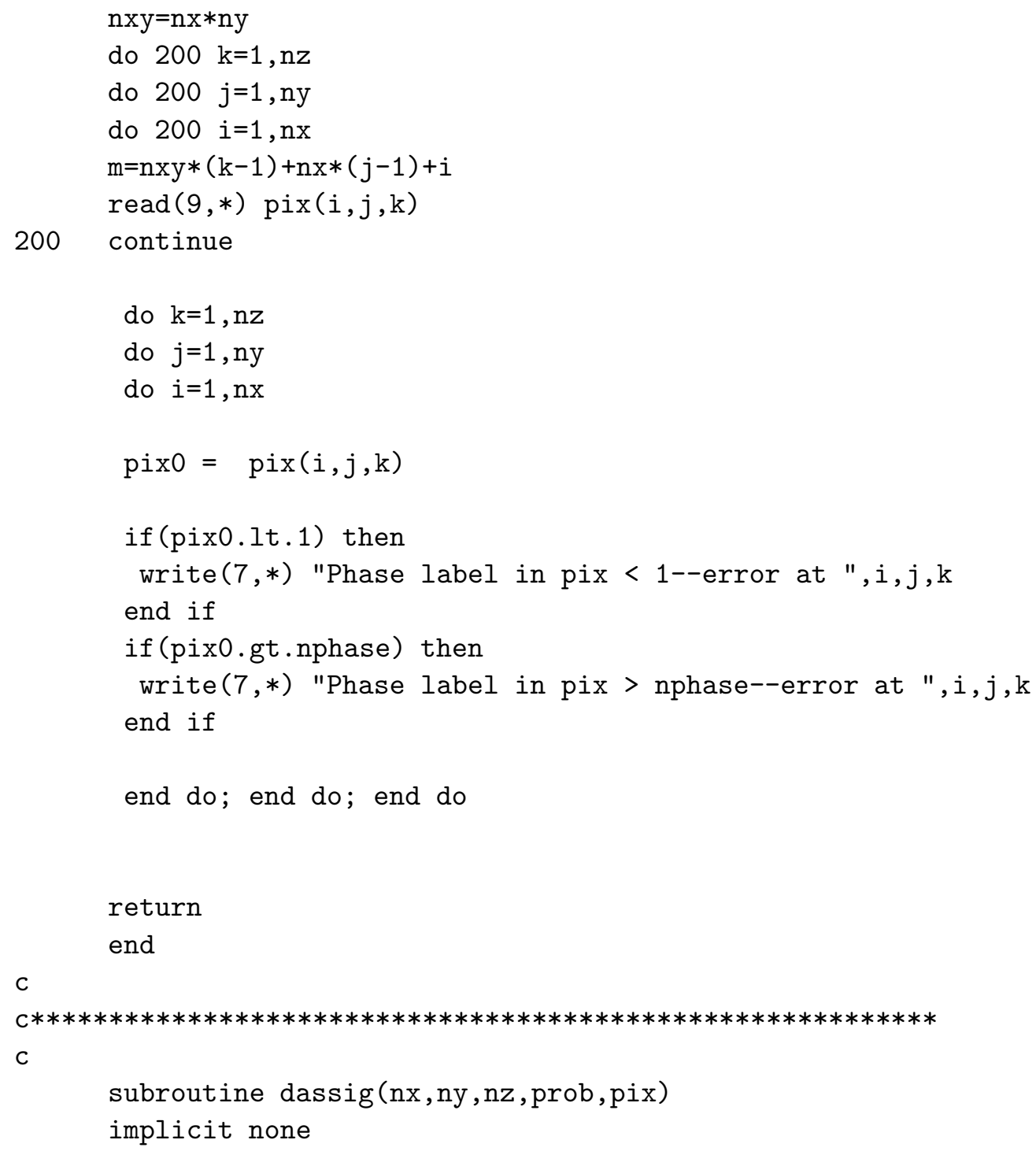




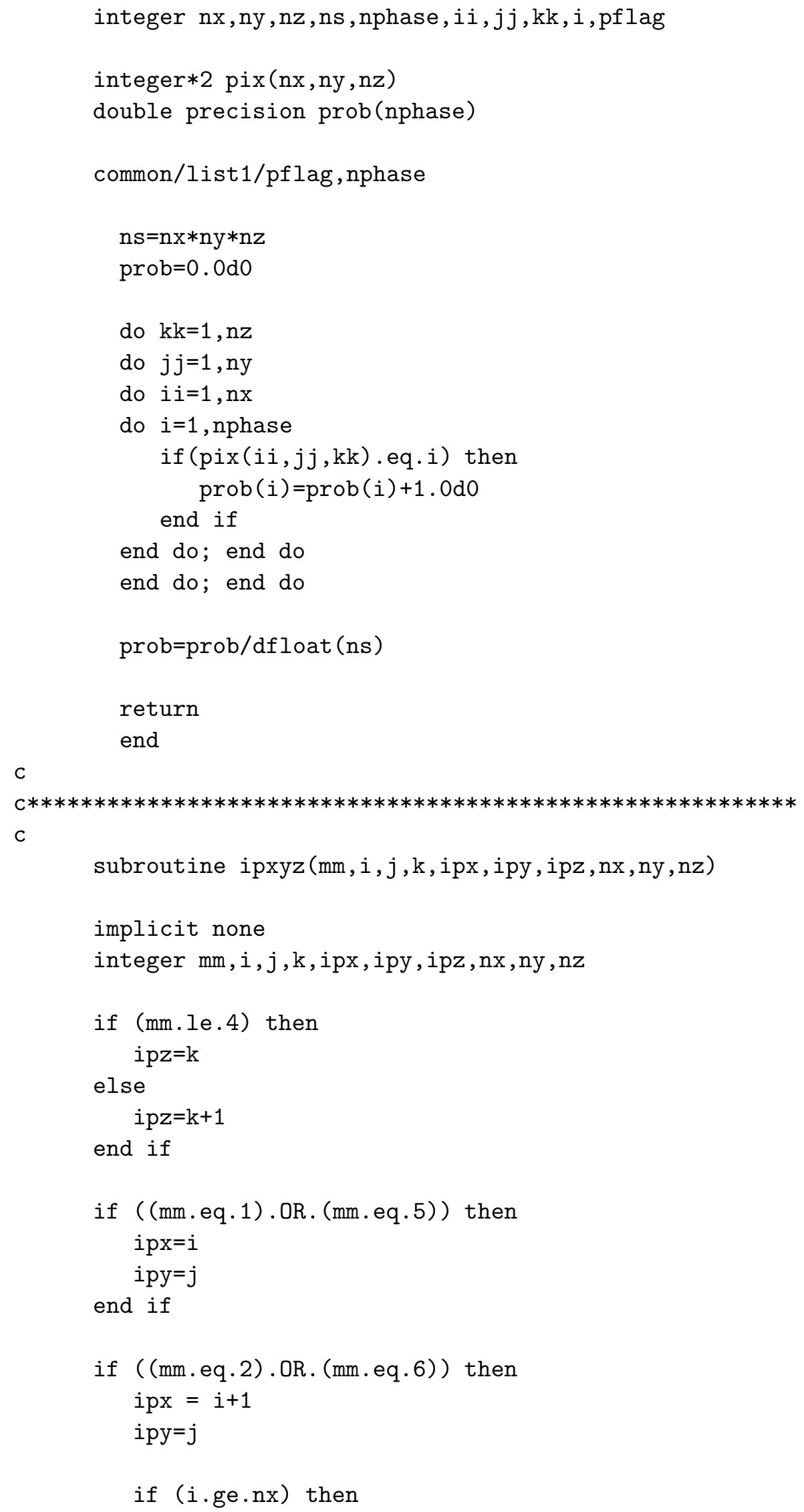




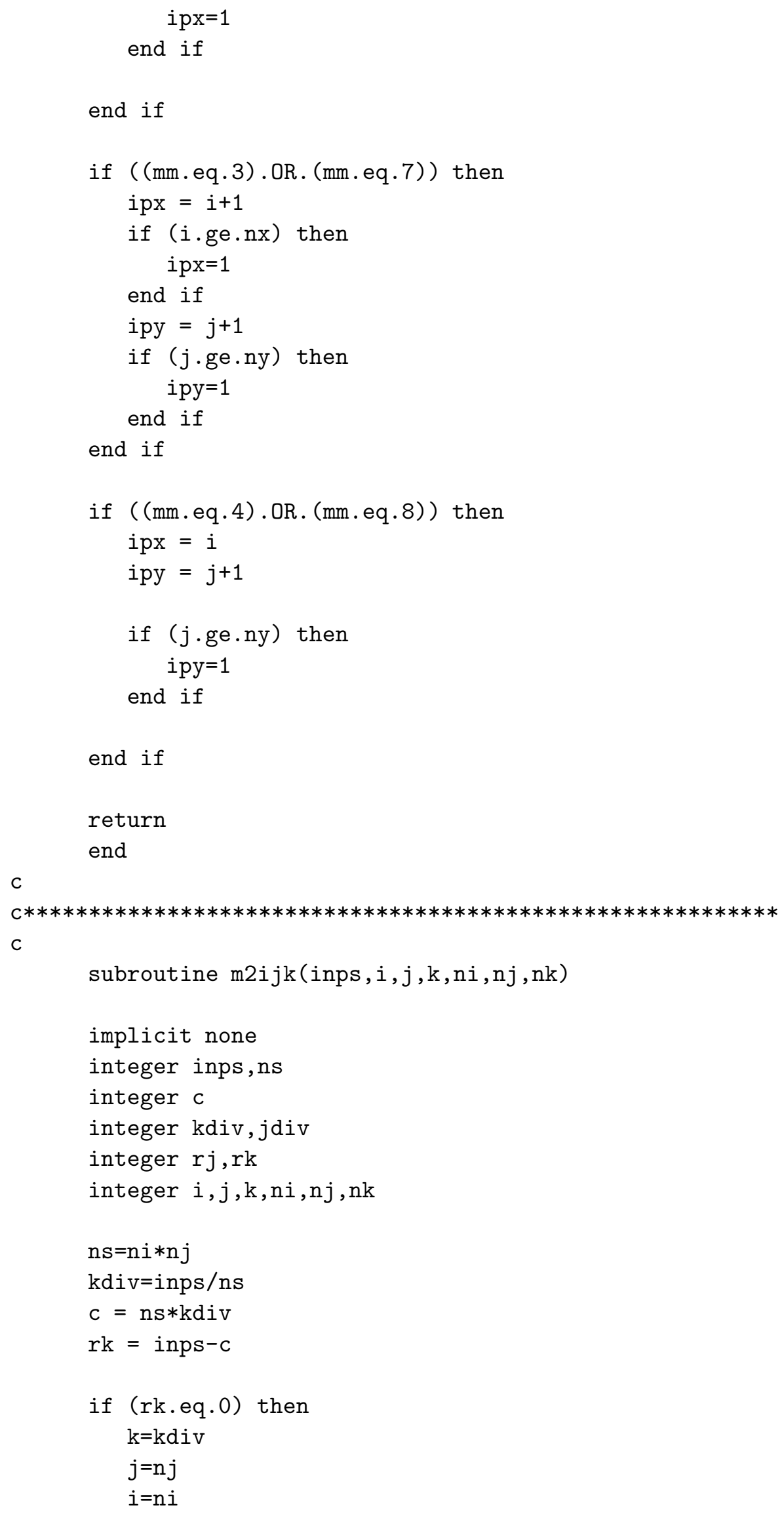




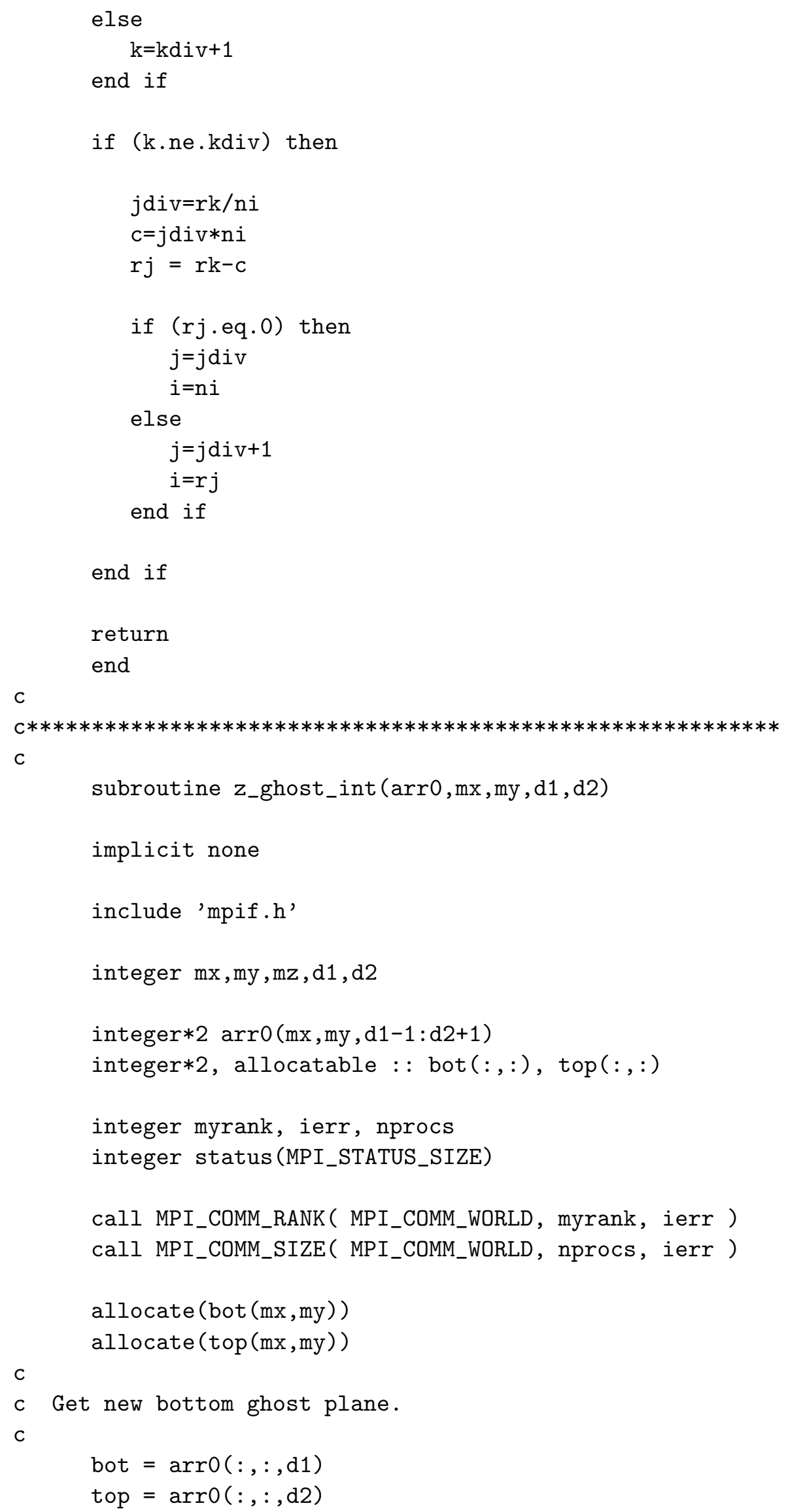




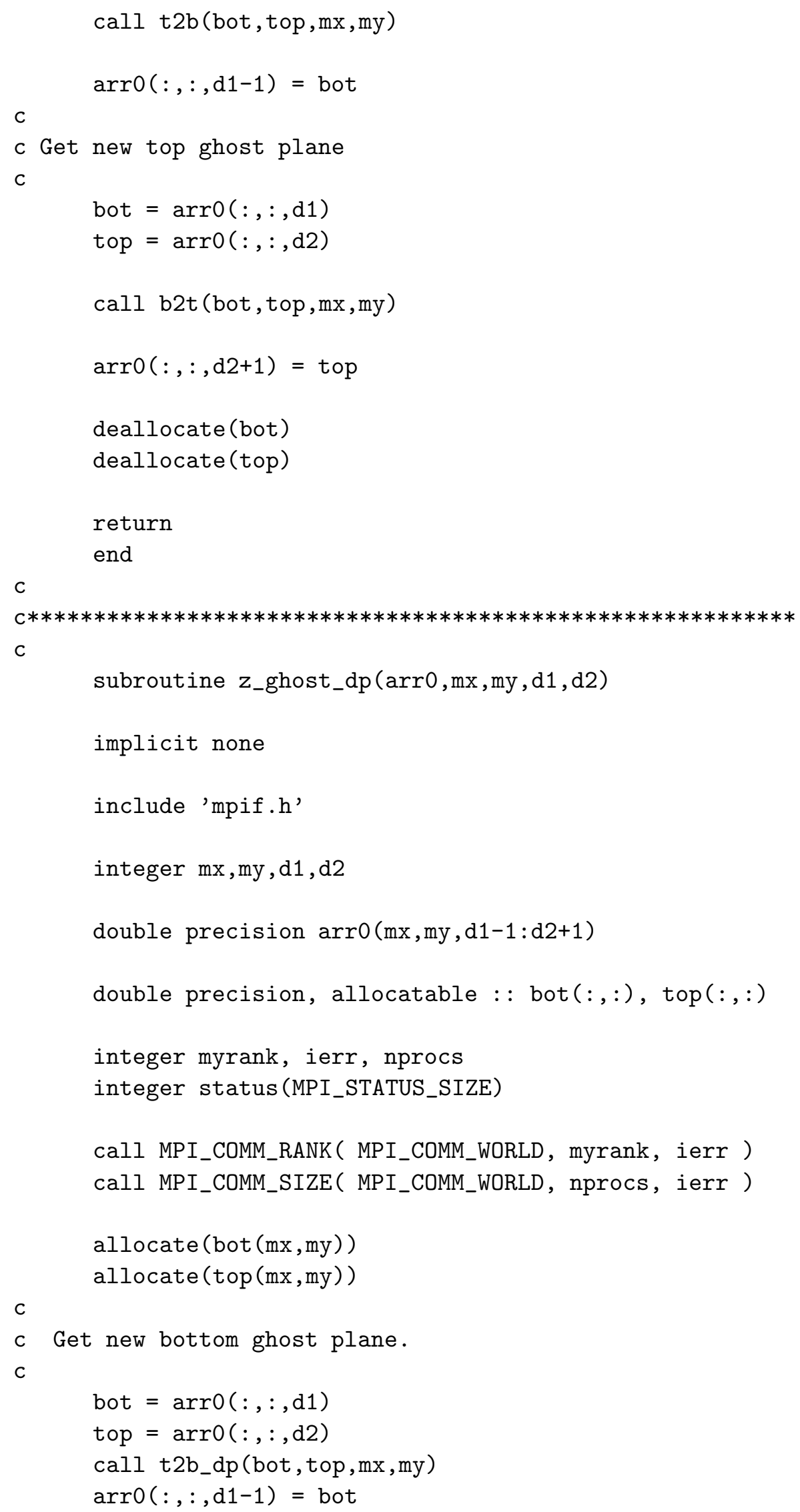


C

c Get new top ghost plane

c

bot $=\operatorname{arr} 0(:,:, \mathrm{d} 1)$

top $=\operatorname{arr} 0(:,:, d 2)$

call b2t_dp (bot, top, mx,my)

$\operatorname{arr} 0(:,:, \mathrm{d} 2+1)=$ top

deallocate(bot)

deallocate(top)

return

end

$\mathrm{C}$

c $* * * * * * * * * * * * * * * * * * * * * * * * * * * * * * * * * * * * * * * * * * * * * * * * * * * * * * * * * * * *$

$\mathrm{C}$

subroutine t2b(b_layer,t_layer, nx, ny)

c This is an INTEGER*2 subroutine.

C

c Used for transferring: pix bottom2top layers

C

c RECV a new t_layer (TOP layer) per node.

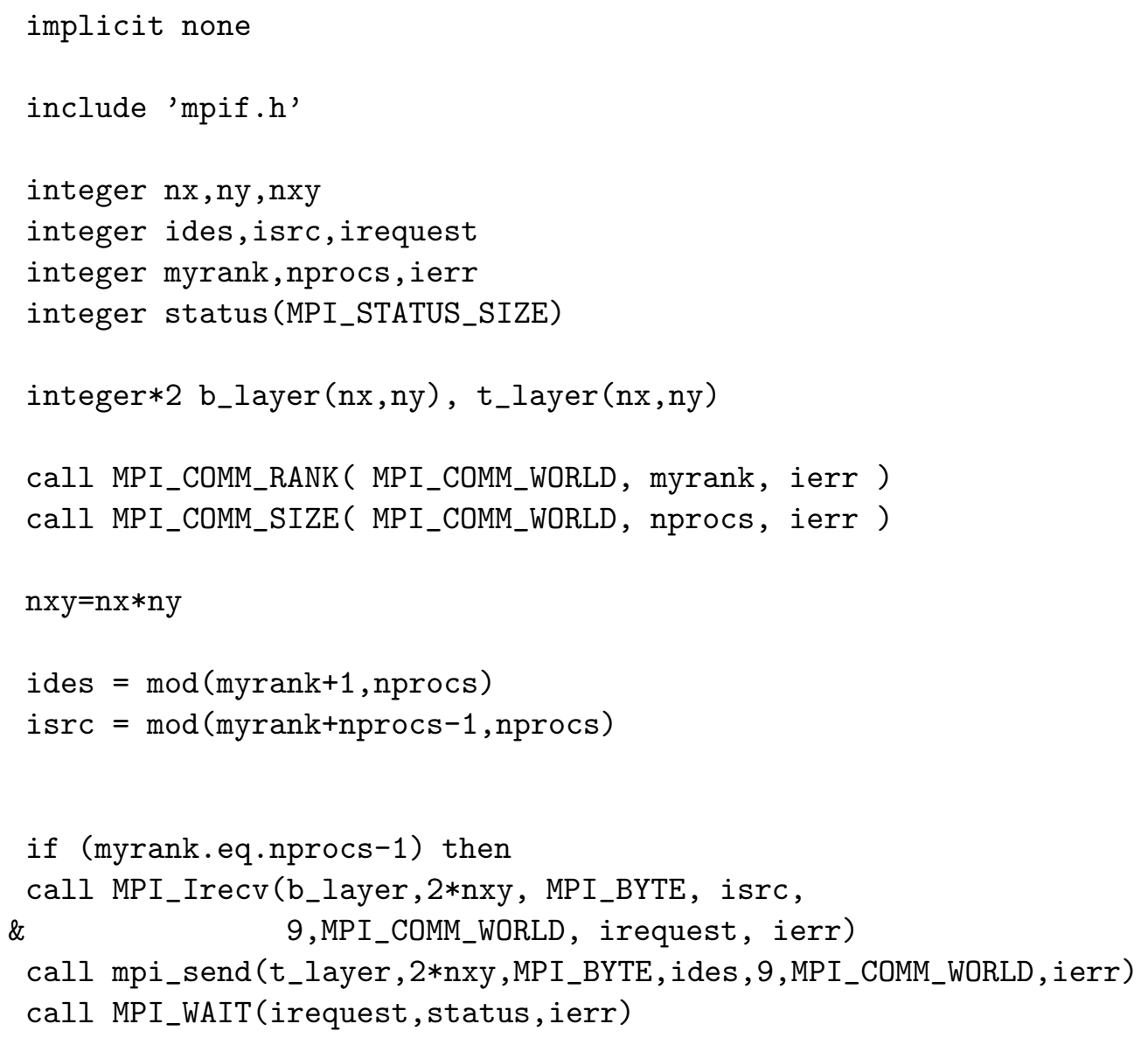




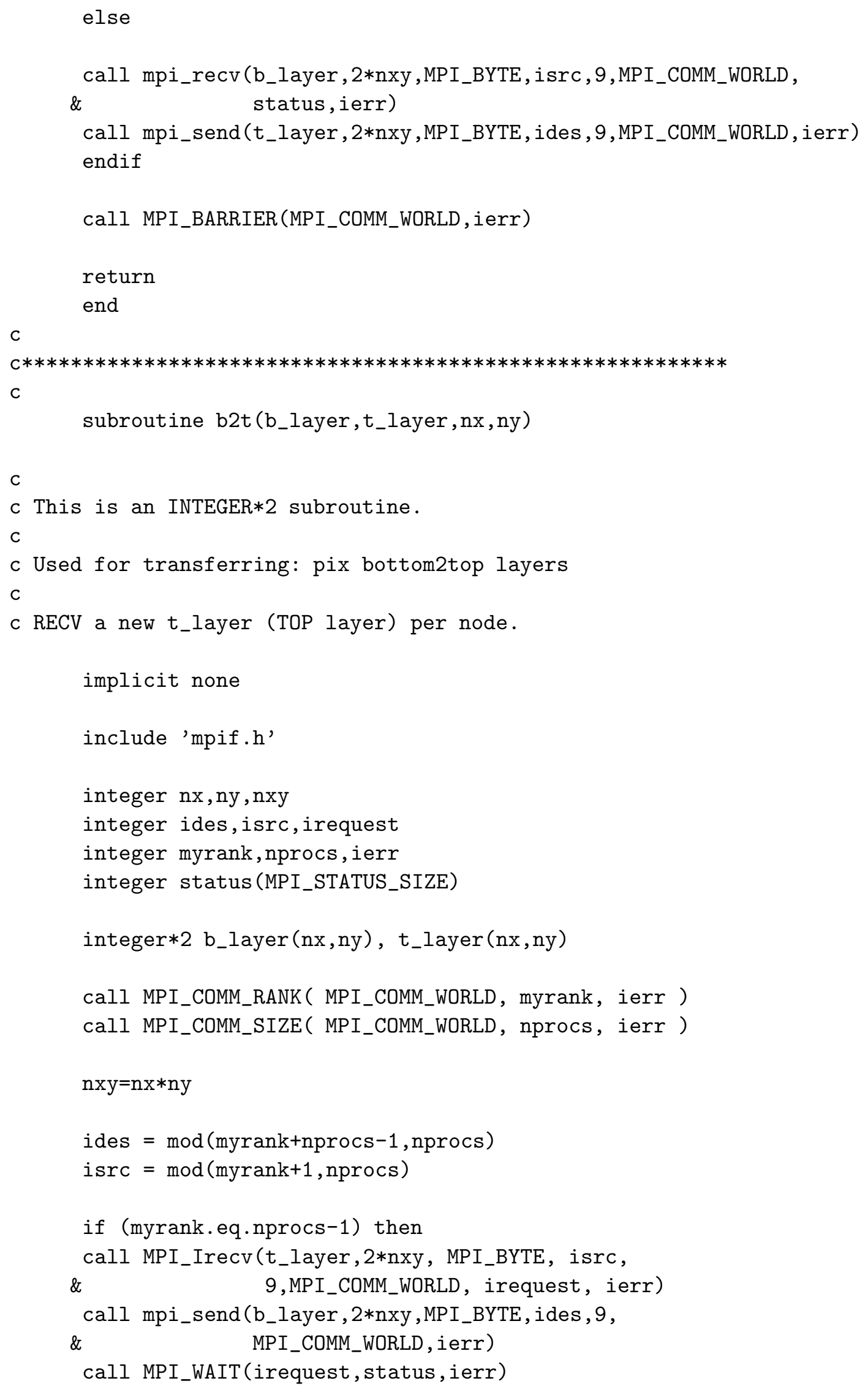




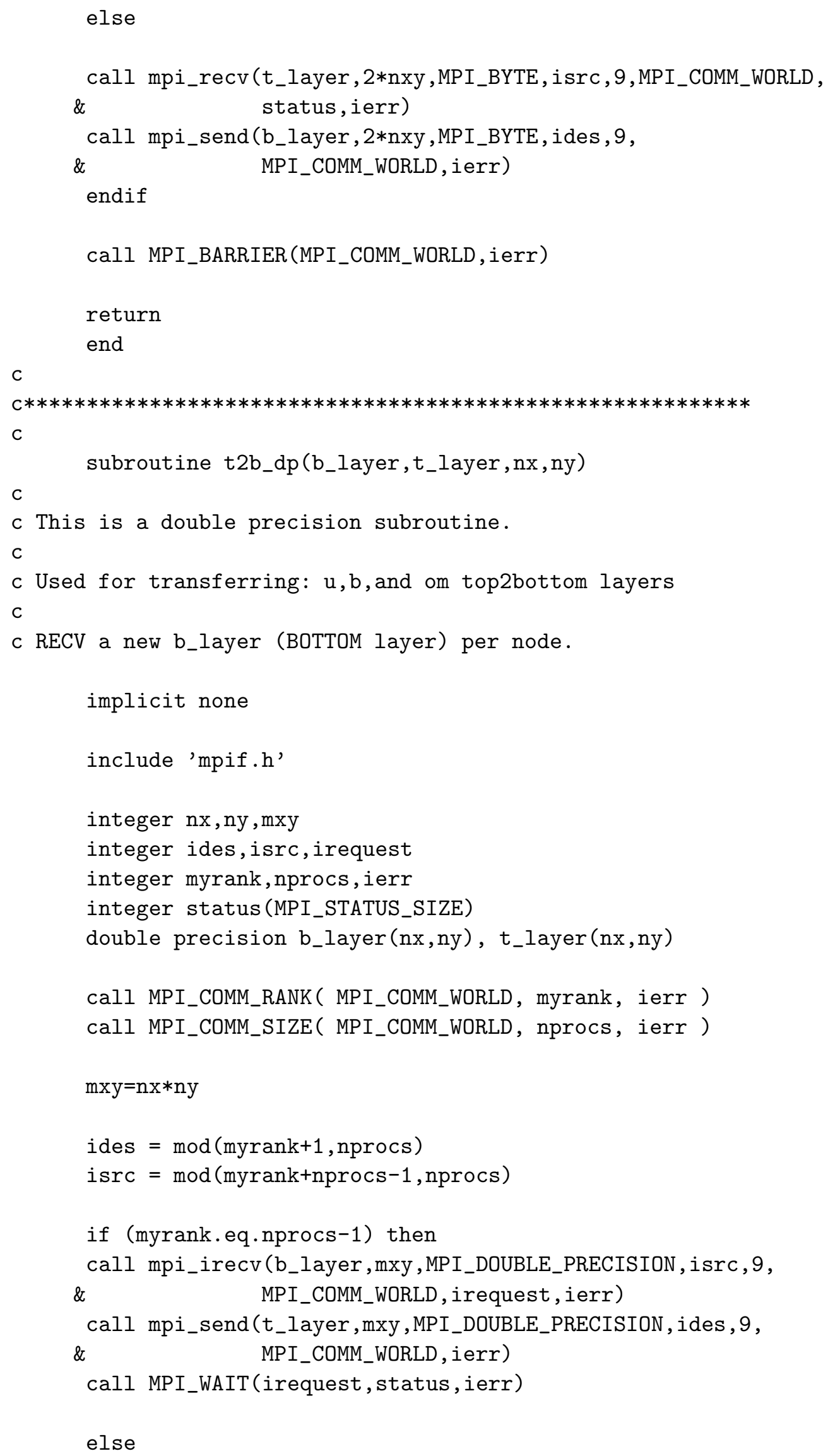




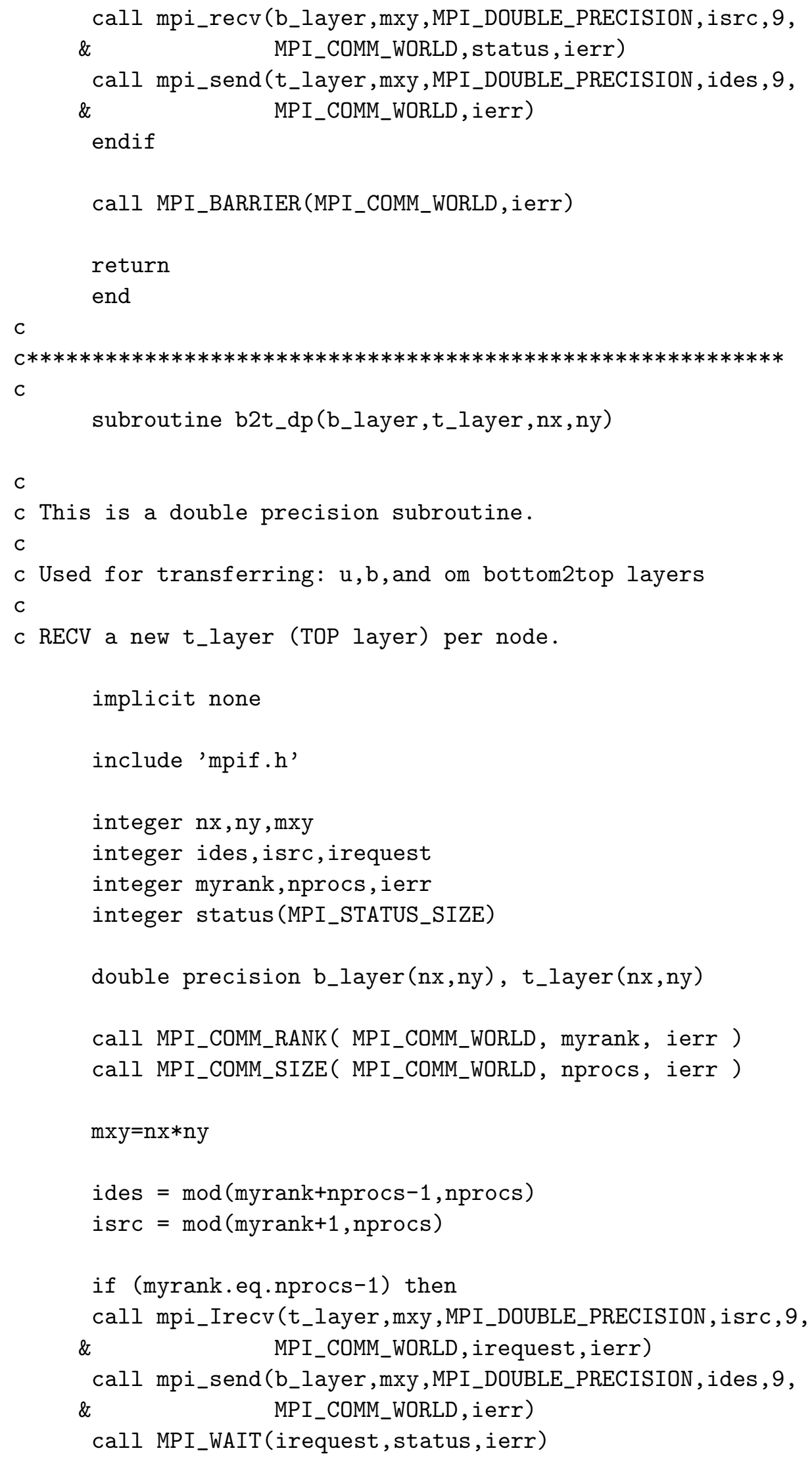




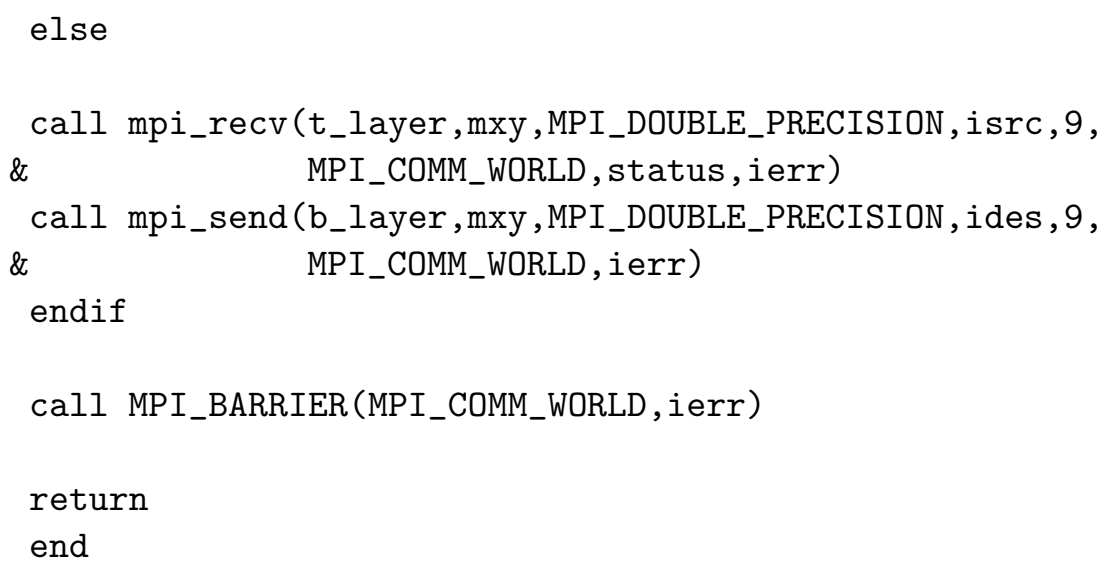




\subsubsection{ELAS3D_MPI.f}

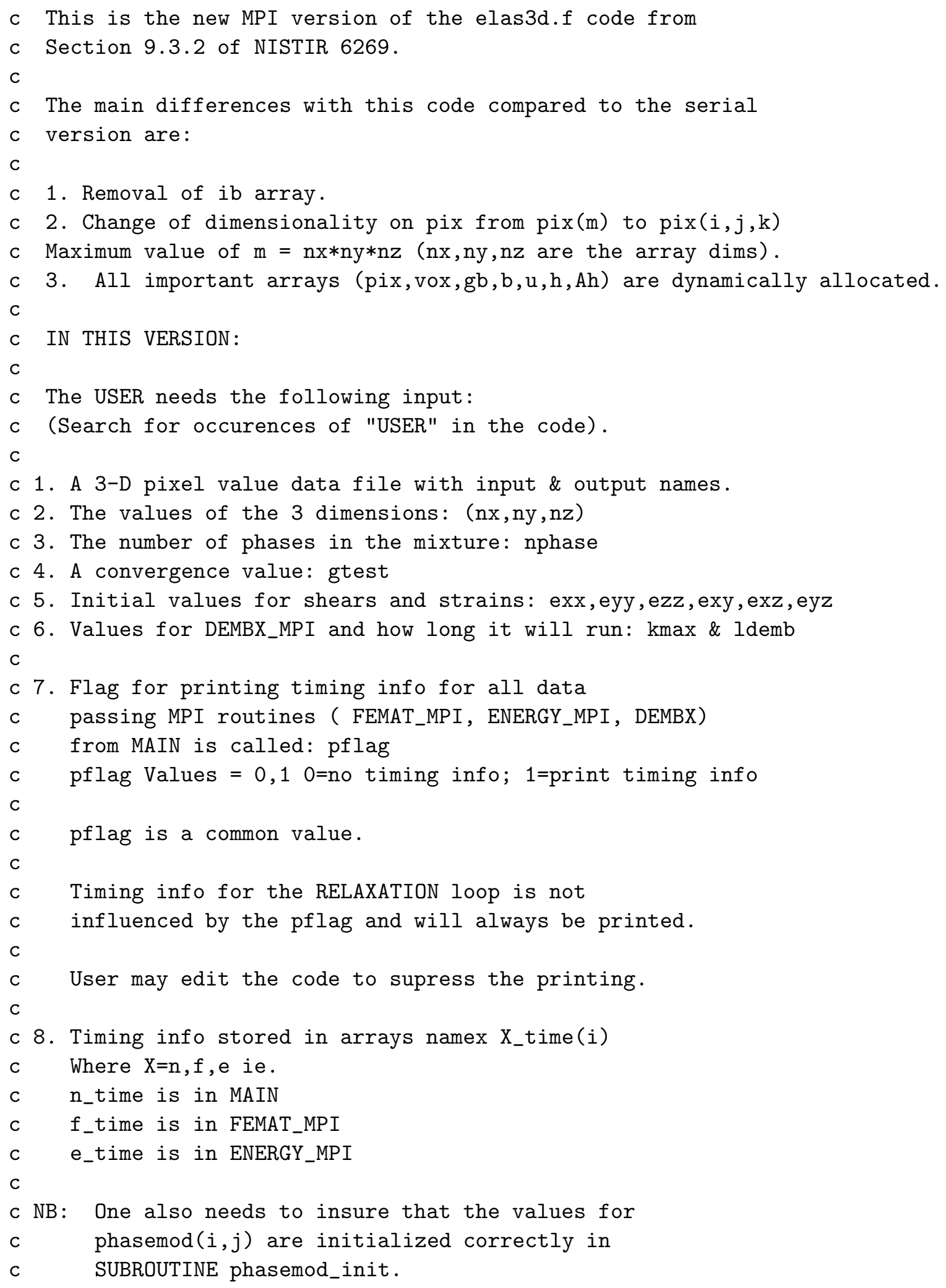


c END of NEW comments.

$C$

BEGIN ORIGINAL comments.

BACKGROUND

c This program solves the linear elastic equations in a

c random linear elastic material, subject to an applied macroscopic strain,

$c$ using the finite element method. Each pixel in the 3-D digital

$c$ image is a cubic tri-linear finite element, having its own

clastic moduli tensor. Periodic boundary conditions are maintained.

c In the comments below, (USER) means that this is a section of code that

$c$ the user might have to change for his particular problem. Therefore the

$c$ user is encouraged to search for this string.

C PROBLEM AND VARIABLE DEFINITION

$c$ The problem being solved is the minimization of the energy

c $1 / 2 \mathrm{uAu}+\mathrm{bu}+\mathrm{C}$, where $\mathrm{A}$ is the Hessian matrix composed of the

c stiffness matrices (dk) for each pixel/element, b is a constant vector

$C$ and $\mathrm{C}$ is a constant that are determined by the applied strain and

$c$ the periodic boundary conditions, and $u$ is a vector of

c all the displacements. The solution

c method used is the conjugate gradient relaxation algorithm.

$c$ Other variables are: $\mathrm{gb}$ is the gradient $=\mathrm{Au}+\mathrm{b}, \mathrm{h}$ and $\mathrm{Ah}$ are

c auxiliary variables used in the conjugate gradient algorithm (in dembx),

c $\operatorname{dk}(n, i, j)$ is the stiffness matrix of the $n^{\prime}$ th phase, $\operatorname{cmod}(n, i, j)$ is

$c$ the elastic moduli tensor of the $n^{\prime}$ th phase, pix is a vector that gives

c the phase label of each pixel, ib is a matrix that gives the labels of

c the 27 (counting itself) neighbors of a given node, prob is the volume

c fractions of the various phases,

c strxx, stryy, strzz, strxz, stryz, and strxy are the six Voigt

c volume averaged total stresses, and

c sxx, syy, szz, sxz, syz, and sxy are the six Voigt

c volume averaged total strains.

\section{c DIMENSIONS}

c The vectors $\mathrm{u}, \mathrm{gb}, \mathrm{b}, \mathrm{h}$, and $\mathrm{Ah}$ are dimensioned to be the system size, $n s=n x * n y * n z$, with three components, where the digital image of the

microstructure considered is a rectangular paralleliped, nx $\mathrm{x}$ ny $\mathrm{x}$ n

$c$ in size. The arrays pix and ib are are also dimensioned to the system size.

c The array ib has 27 components, for the 27 neighbors of a node.

c Note that the program is set up at present to have at most 100

c different phases. This can easily be changed, simply by changing

$c$ the dimensions of $\mathrm{dk}$, prob, and cmod. The parameter nphase gives the

C number of phases being considered in the problem.

c All arrays are passed between subroutines using simple common statements. 
c STRONGLY SUGGESTED: READ THE MANUAL BEFORE USING PROGRAM!!

implicit none

include 'mpif.h'

C

c (USER) Change the nx,ny,nz dimensions at the beginning.

c All important arrays are dynamically allocated.

C

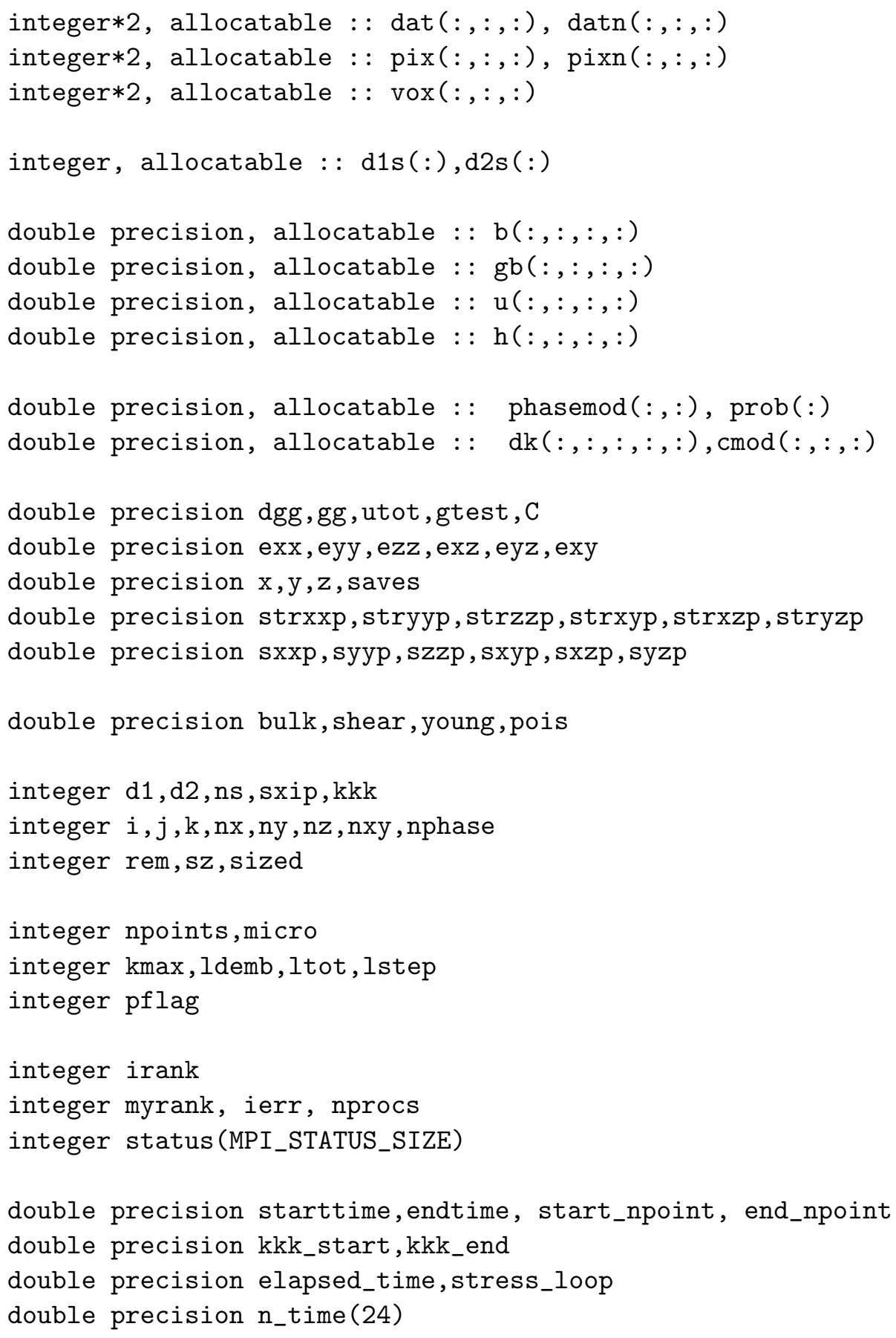




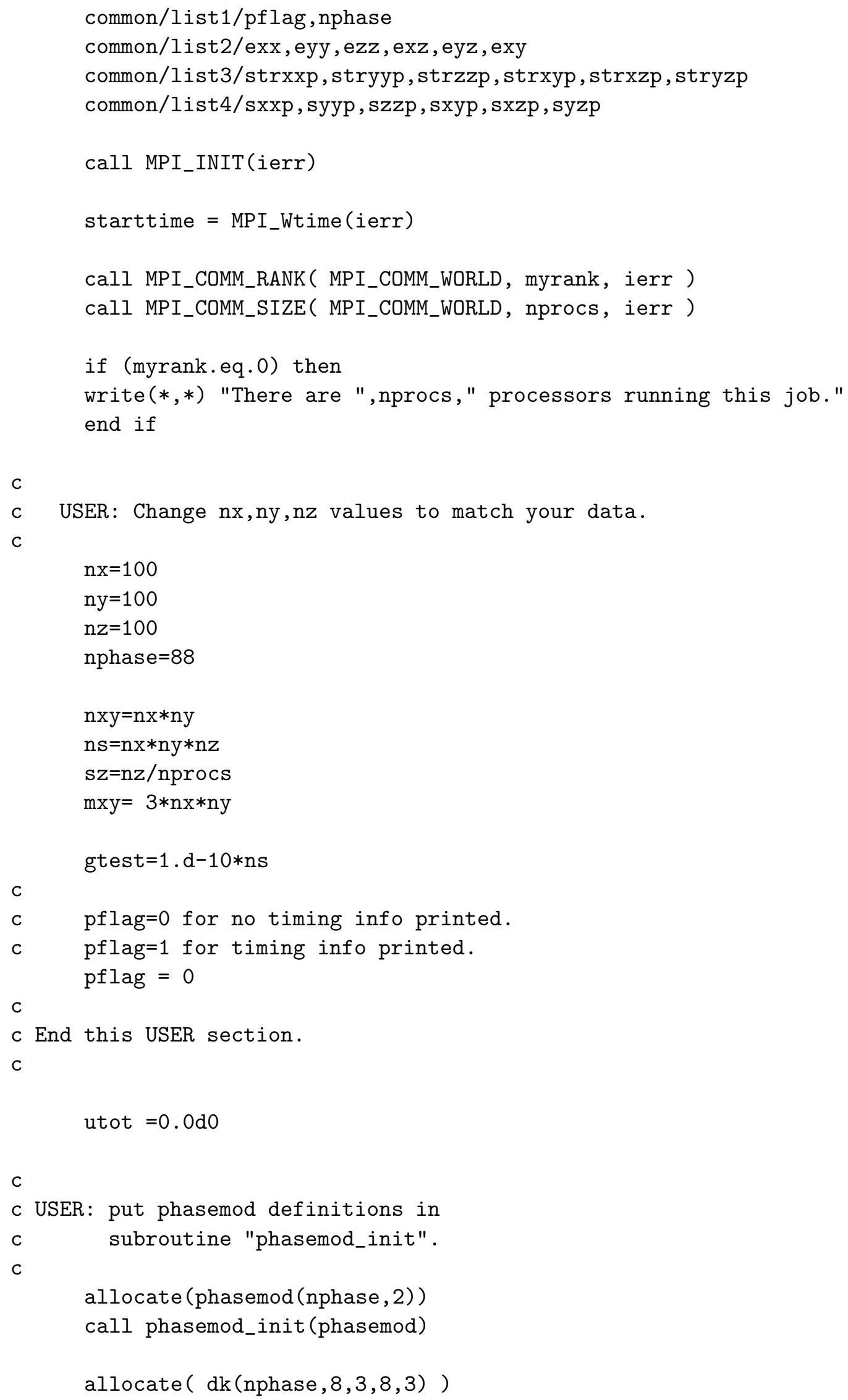




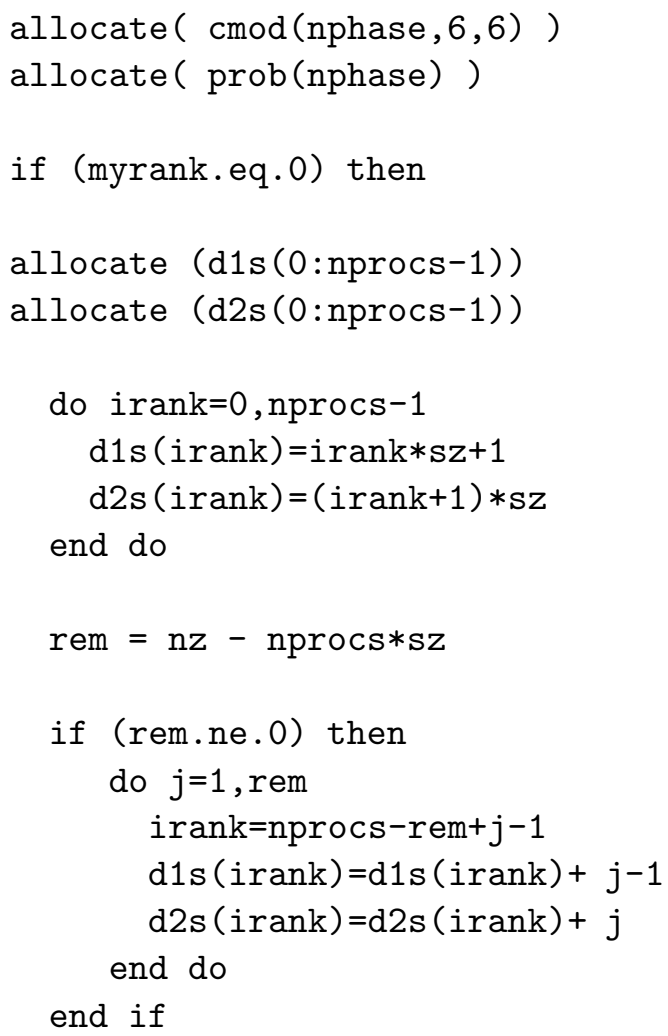

c Send all d1s(i) and d2s(i) from R00T

$c$ to NODE $i$ \& store into d1 \& d2

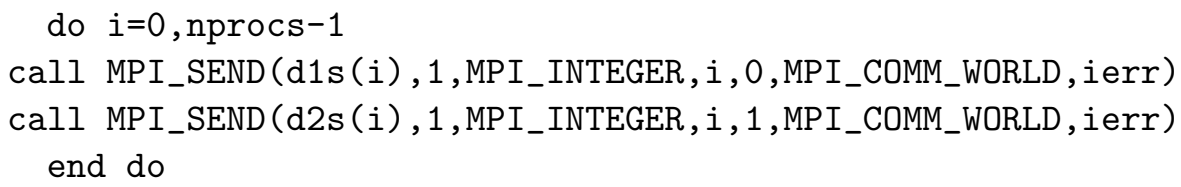


C

c Want the ability to calculate on a series

$c$ of input files based on a value \& some if statements.

c

c Compute the average stress and strain in each microstructure.

c (USER) npoints is the number of microstructures to use.

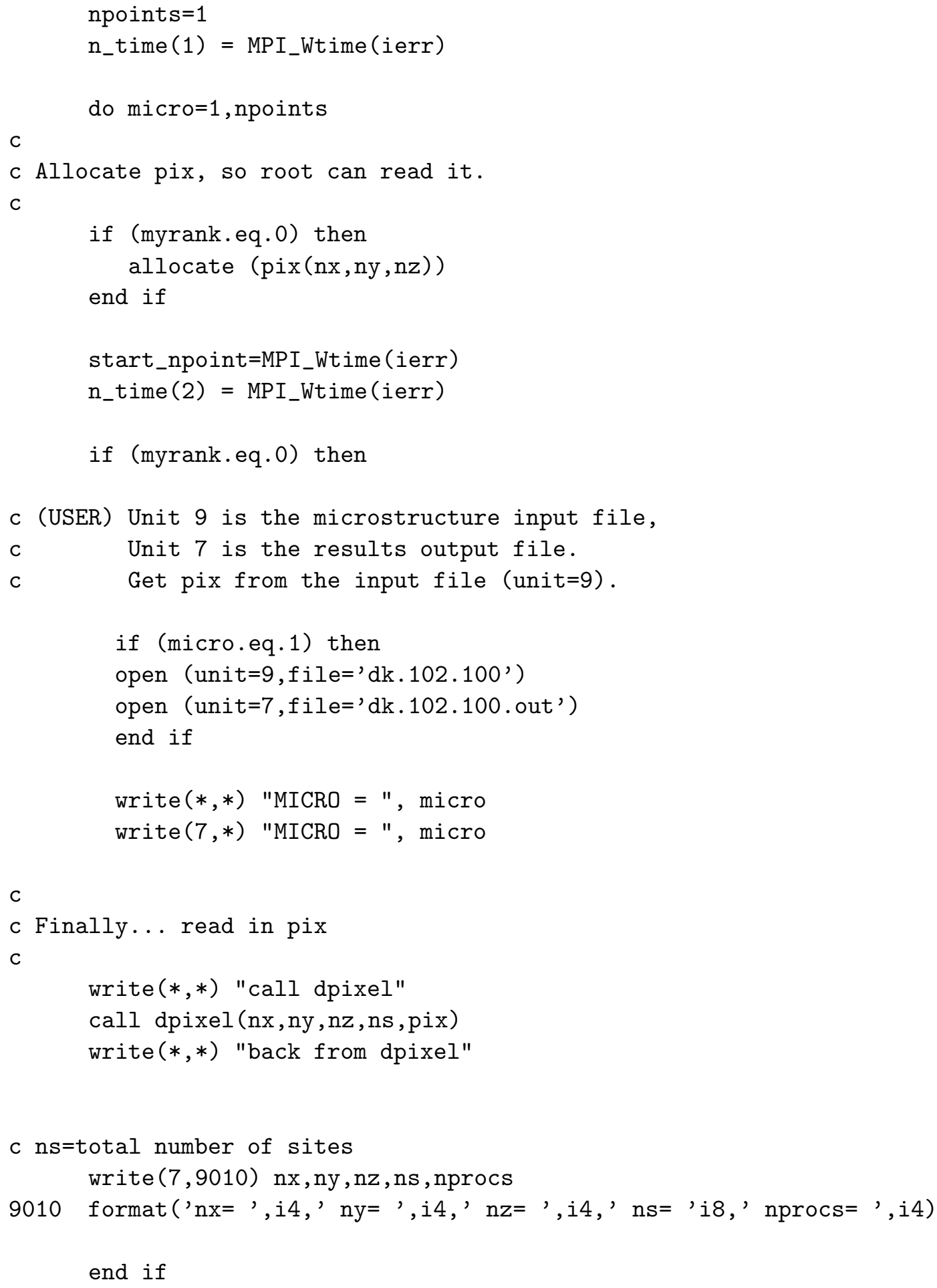


call MPI_BARRIER (MPI_COMM_WORLD, ierr)

c Now that the nodes are set up correctly,

c one can pass the data from the root node (myrank=0)

$c$ to all the rest.

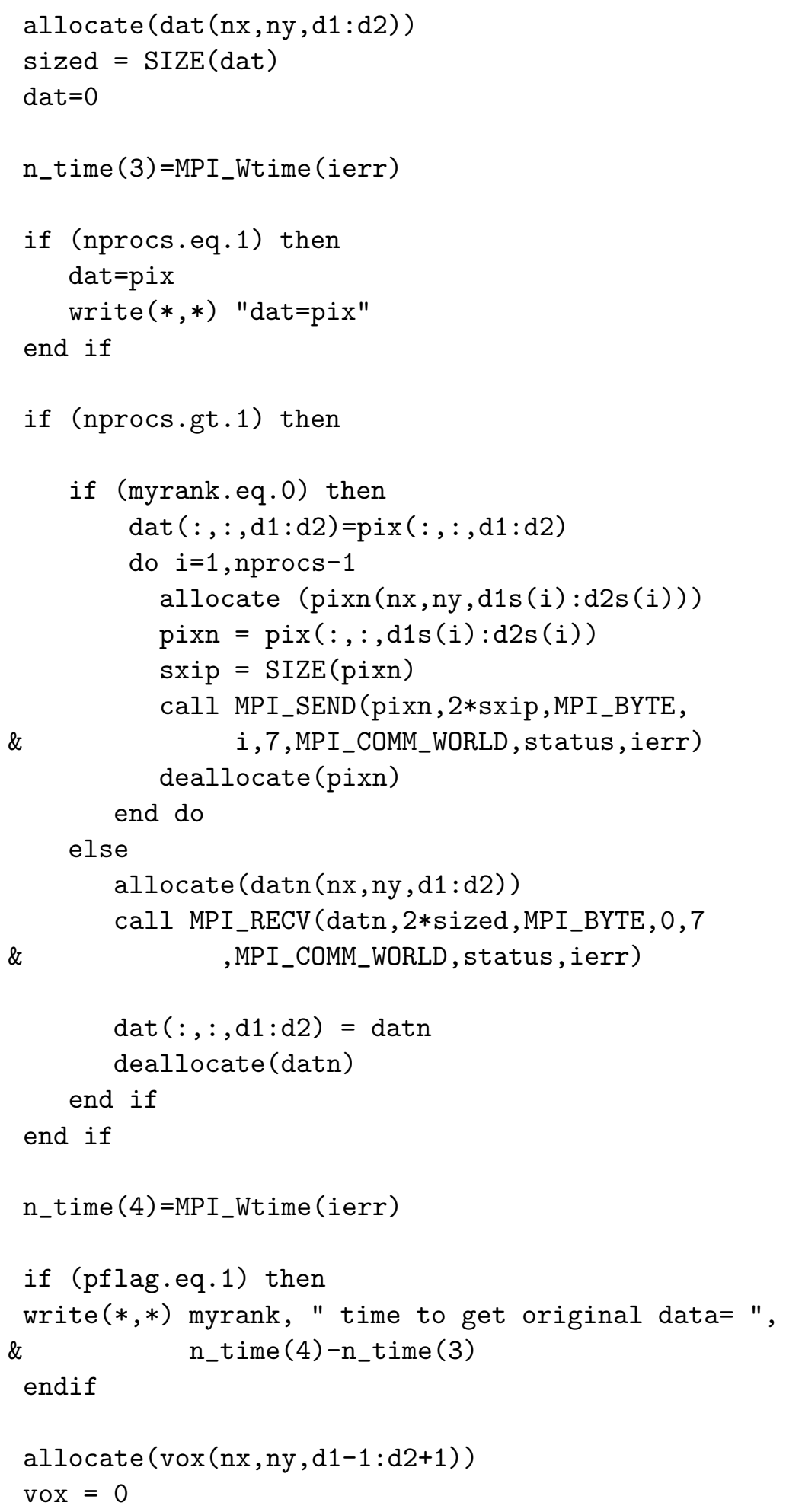




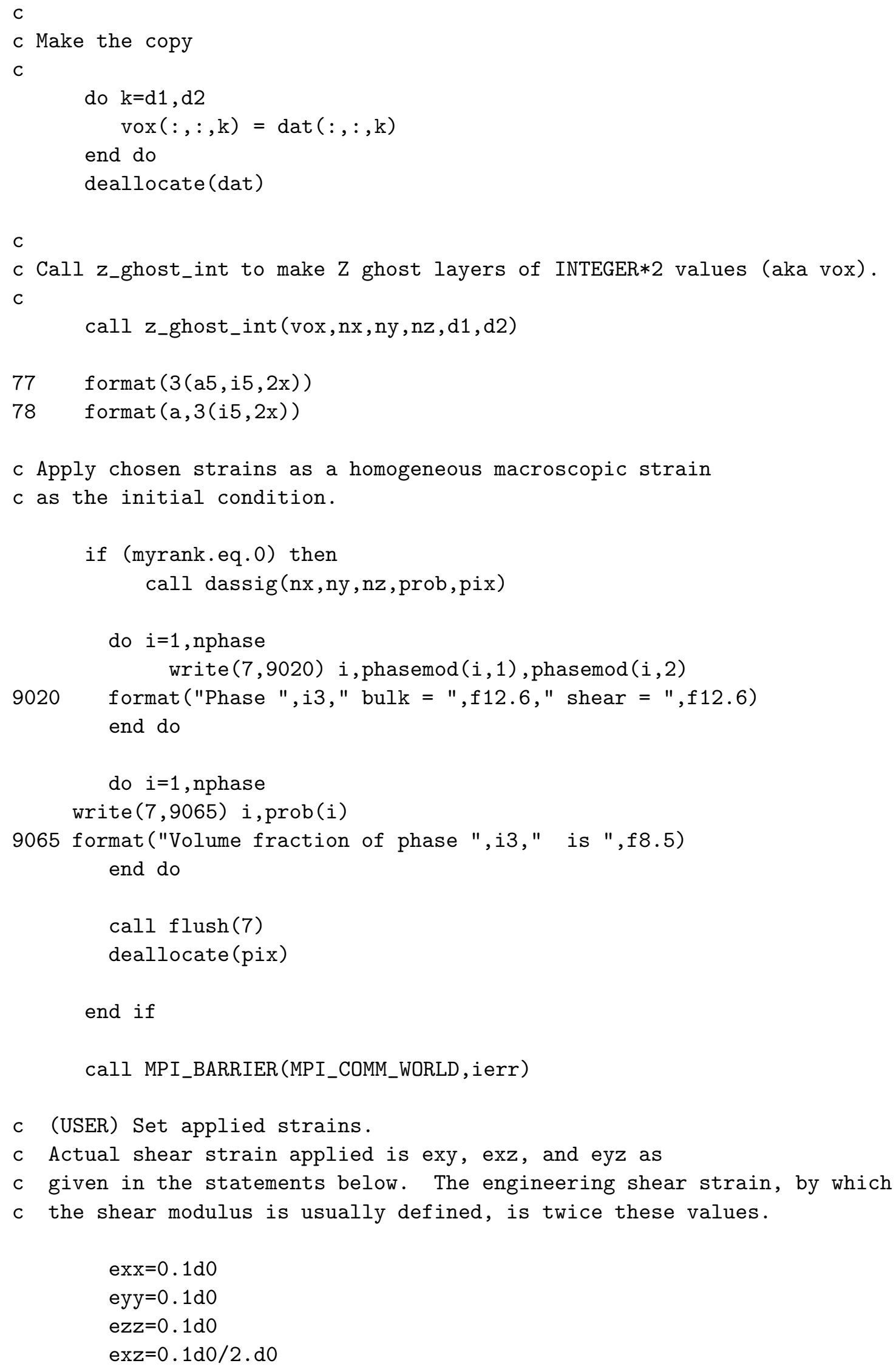




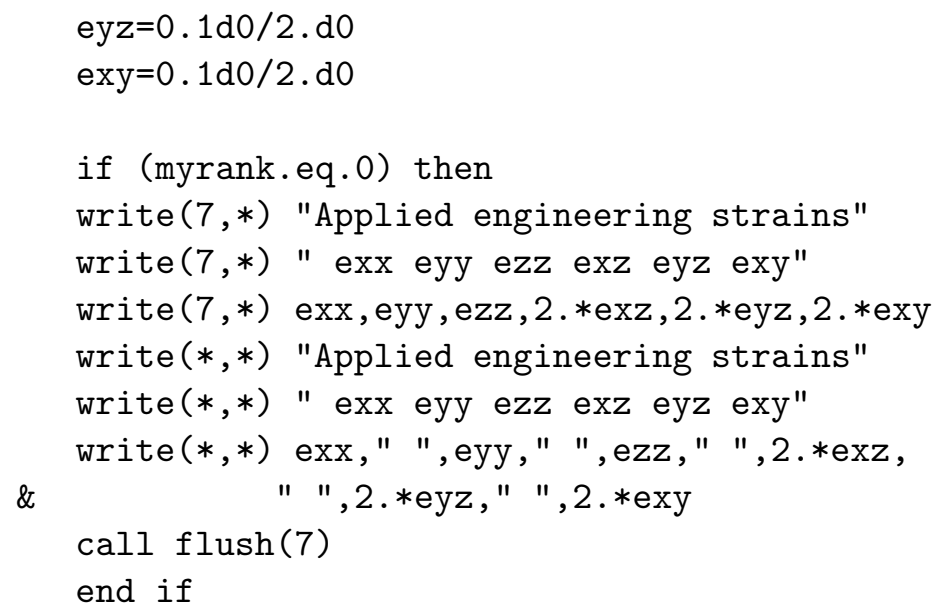

c Set up the elastic modulus variables, finite element stiffness matrices, $c$ the constant, $\mathrm{C}$, and vector, b, required for computing the energy.

c (USER) If anisotropic elastic moduli tensors are used, these need to be c input in subroutine femat.

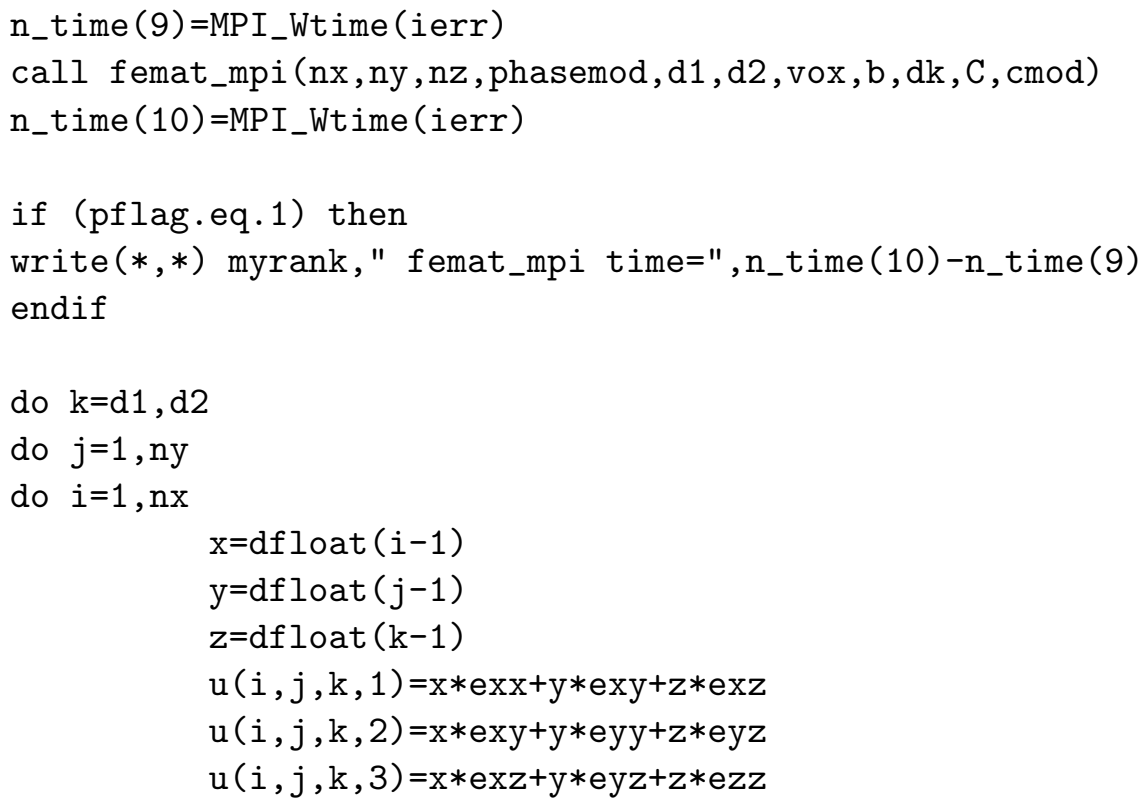

c RELAXATION LOOP

c (USER) kmax is the maximum number of times dembx will be called, with

c ldemb conjugate gradient steps performed during each call. The total

c number of conjugate gradient steps allowed for a given elastic

c computation is $k m a x * l d e m b$.

$\operatorname{kmax}=40$

1 demb $=100$

ltot $=0$ 
c Call energy to get initial energy and initial gradient

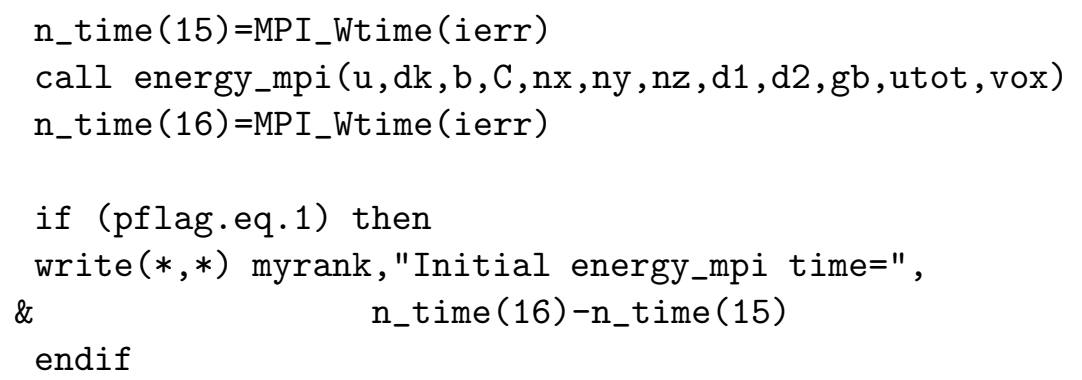

c $g g$ is the norm squared of the gradient ( $g g=g b * g b$ ) $\mathrm{dgg}=0.0 \mathrm{~d} 0$

c call dembx_mpi to go into the conjugate gradient solver

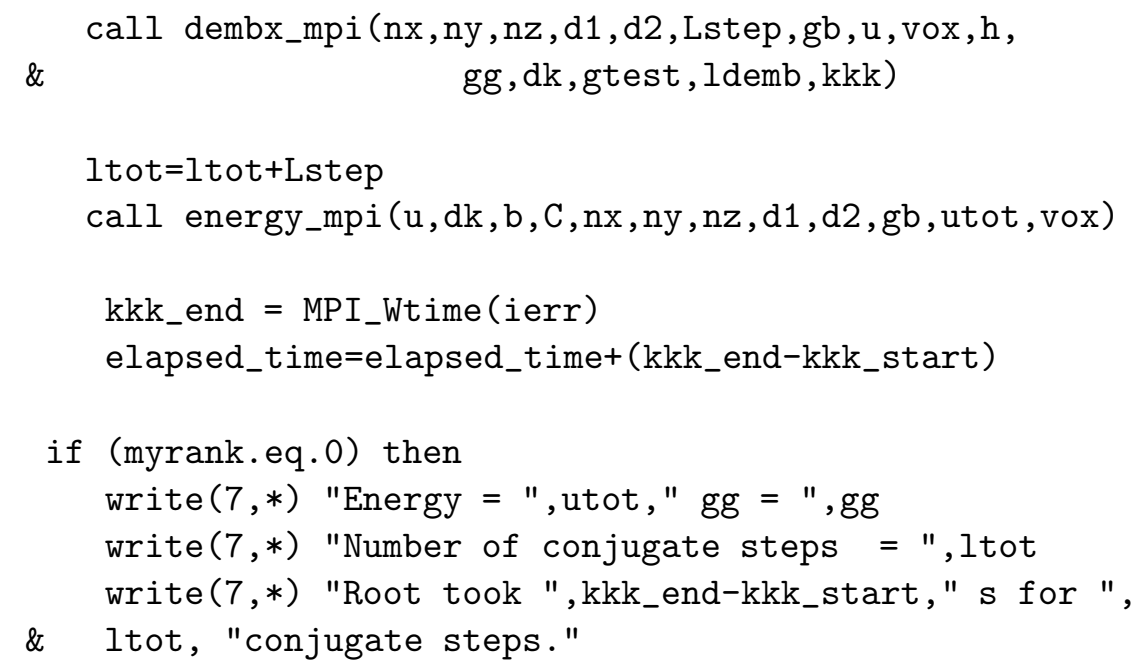




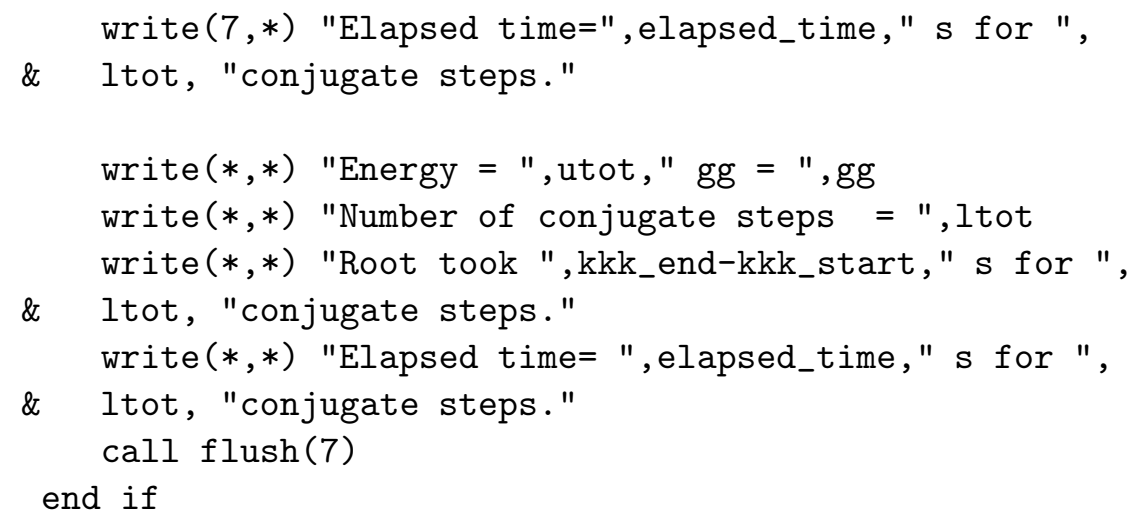

c Call energy_mpi to compute energy after dembx_mpi call. If gg < gtest, $c$ this will be the final energy. If gg is still larger than gtest,

$c$ then this will give an intermediate energy with which to check how the

c relaxation process is coming along.

c If relaxation process is finished, jump out of loop if (gg.le.gtest) goto 444

c If relaxation process will continue, compute and output stresses

$c$ and strains as an additional aid to judge how the

c relaxation procedure is progressing.

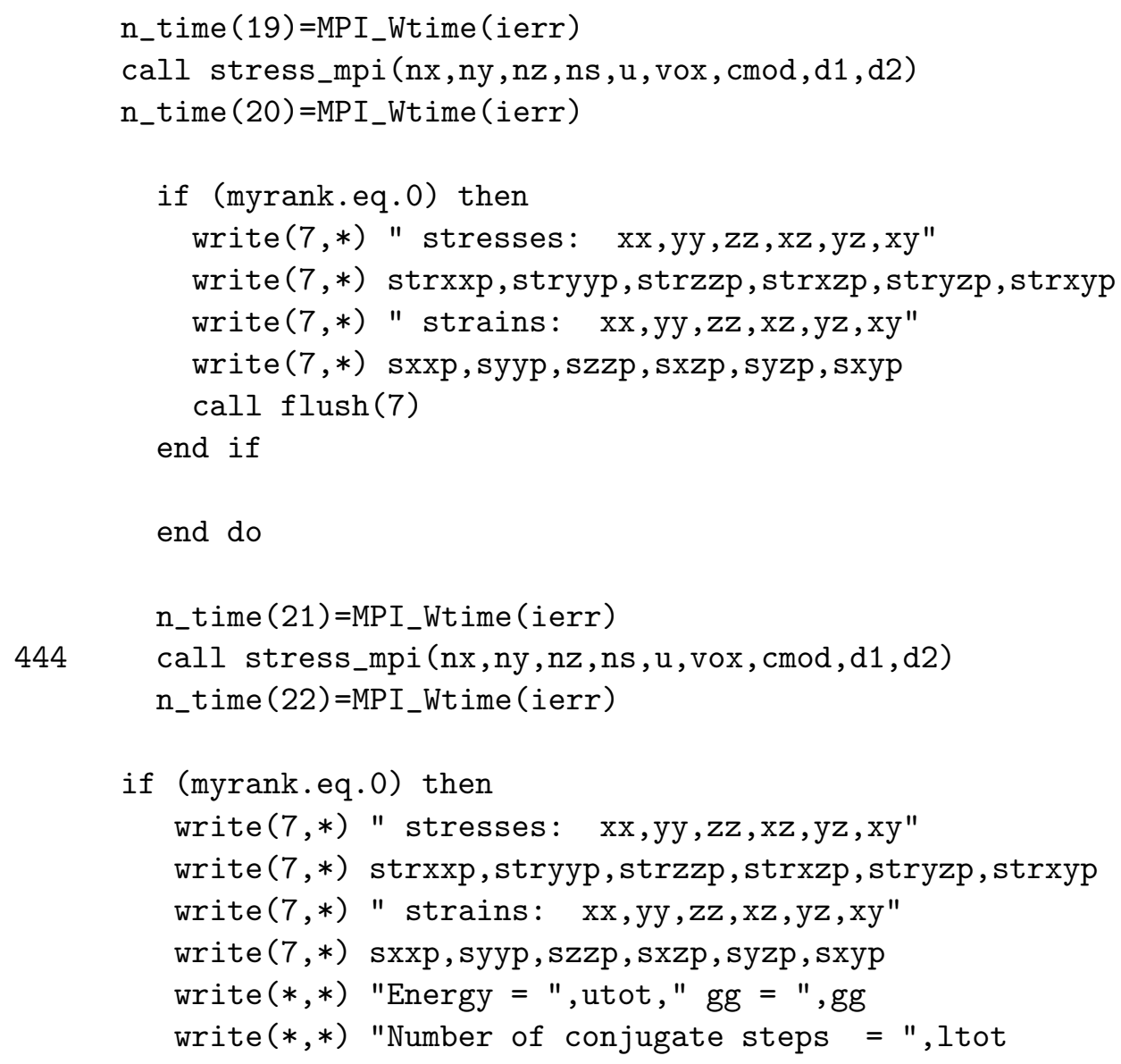


end if

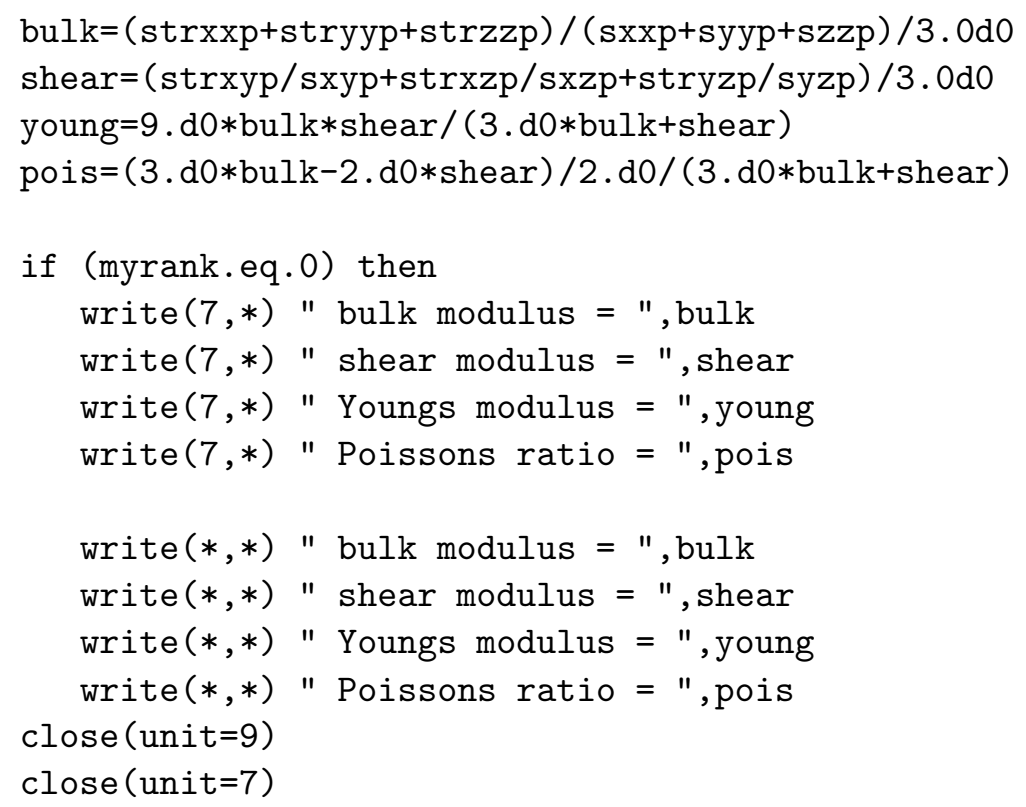




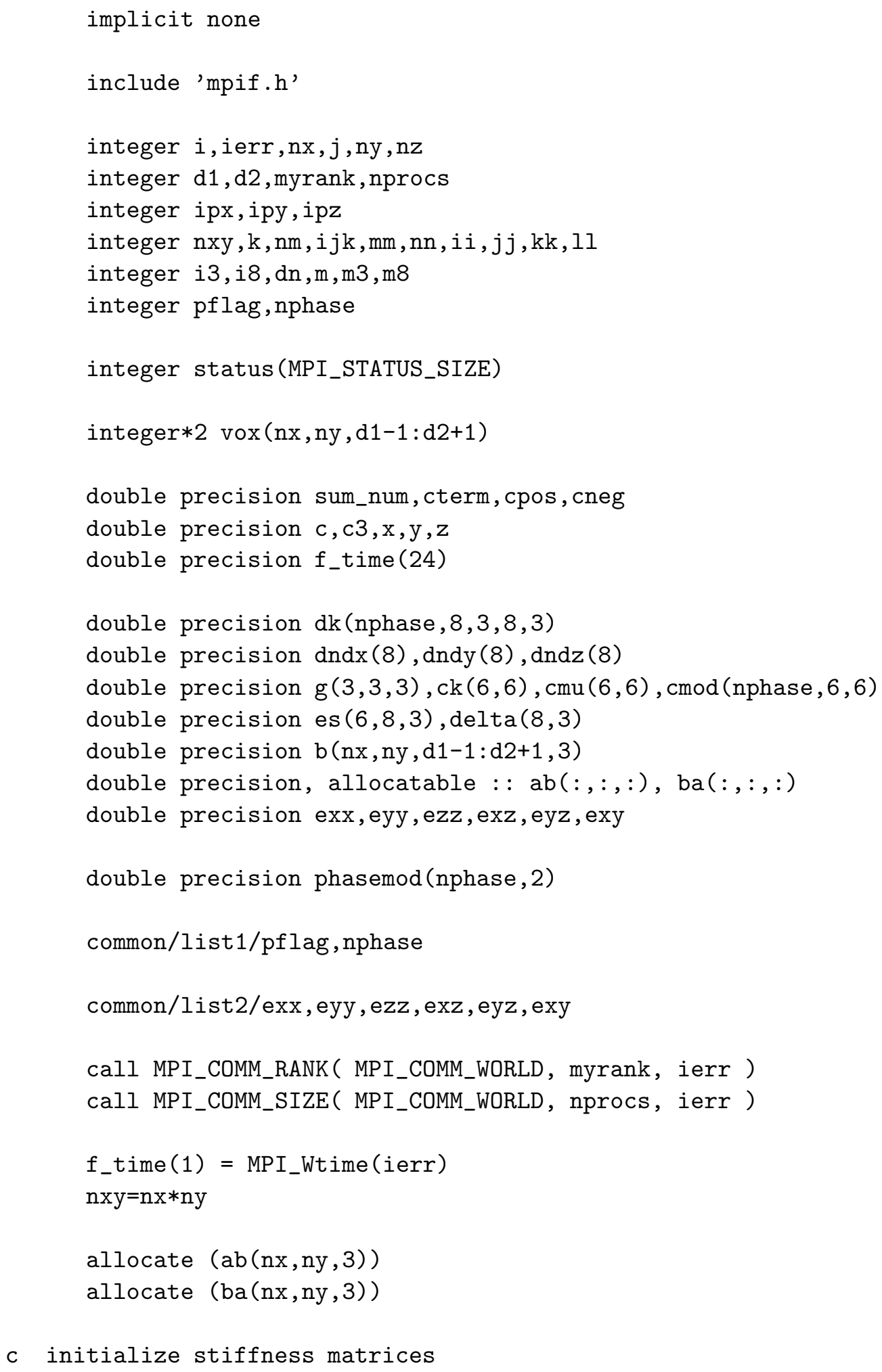




$$
\begin{aligned}
& \operatorname{ck}(1,1)=1.0 \mathrm{~d} 0 \\
& \operatorname{ck}(1,2)=1.0 \mathrm{~d} 0 \\
& \operatorname{ck}(1,3)=1.0 \mathrm{~d} 0 \\
& \operatorname{ck}(1,4)=0.0 \mathrm{~d} 0 \\
& \operatorname{ck}(1,5)=0.0 \mathrm{~d} 0 \\
& \operatorname{ck}(1,6)=0.0 \mathrm{~d} 0 \\
& \operatorname{ck}(2,1)=1.0 \mathrm{~d} 0 \\
& \operatorname{ck}(2,2)=1.0 \mathrm{~d} 0 \\
& \operatorname{ck}(2,3)=1.0 \mathrm{~d} 0 \\
& \operatorname{ck}(2,4)=0.0 \mathrm{~d} 0 \\
& \operatorname{ck}(2,5)=0.0 \mathrm{~d} 0 \\
& \mathrm{ck}(2,6)=0.0 \mathrm{~d} 0 \\
& \operatorname{ck}(3,1)=1.0 \mathrm{~d} 0 \\
& \operatorname{ck}(3,2)=1.0 \mathrm{~d} 0 \\
& \operatorname{ck}(3,3)=1.0 \mathrm{~d} 0 \\
& \operatorname{ck}(3,4)=0.0 \mathrm{~d} 0 \\
& \mathrm{ck}(3,5)=0.0 \mathrm{~d} 0 \\
& \mathrm{ck}(3,6)=0.0 \mathrm{~d} 0 \\
& \operatorname{ck}(4,1)=0.0 \mathrm{~d} 0 \\
& \operatorname{ck}(4,2)=0.0 \mathrm{~d} 0 \\
& \operatorname{ck}(4,3)=0.0 \mathrm{~d} 0 \\
& \operatorname{ck}(4,4)=0.0 \mathrm{~d} 0 \\
& \operatorname{ck}(4,5)=0.0 \mathrm{~d} 0 \\
& \mathrm{ck}(4,6)=0.0 \mathrm{~d} 0 \\
& \mathrm{ck}(5,1)=0.0 \mathrm{~d} 0 \\
& \operatorname{ck}(5,2)=0.0 \mathrm{~d} 0 \\
& \operatorname{ck}(5,3)=0.0 \mathrm{~d} 0 \\
& \operatorname{ck}(5,4)=0.0 \mathrm{~d} 0 \\
& \operatorname{ck}(5,5)=0.0 \mathrm{~d} 0 \\
& \operatorname{ck}(5,6)=0.0 \mathrm{~d} 0 \\
& \mathrm{ck}(6,1)=0.0 \mathrm{~d} 0 \\
& \operatorname{ck}(6,2)=0.0 \mathrm{~d} 0 \\
& \operatorname{ck}(6,3)=0.0 \mathrm{~d} 0 \\
& \mathrm{ck}(6,4)=0.0 \mathrm{~d} 0 \\
& \operatorname{ck}(6,5)=0.0 \mathrm{~d} 0 \\
& \mathrm{ck}(6,6)=0.0 \mathrm{~d} 0 \\
& \mathrm{cmu}(1,1)=4.0 \mathrm{~d} 0 / 3.0 \mathrm{~d} 0 \\
& \mathrm{cmu}(1,2)=-2 \cdot 0 \mathrm{~d} 0 / 3.0 \mathrm{~d} 0 \\
& \mathrm{cmu}(1,3)=-2.0 \mathrm{~d} 0 / 3.0 \mathrm{~d} 0 \\
& \mathrm{cmu}(1,4)=0.0 \mathrm{~d} 0 \\
& \mathrm{cmu}(1,5)=0.0 \mathrm{~d} 0 \\
& \mathrm{cmu}(1,6)=0.0 \mathrm{~d} 0 \\
& \mathrm{cmu}(2,1)=-2 \cdot 0 \mathrm{~d} 0 / 3 \cdot 0 \mathrm{~d} 0 \\
& \mathrm{cmu}(2,2)=4 \cdot 0 \mathrm{~d} 0 / 3 \cdot 0 \mathrm{~d} 0 \\
& \mathrm{cmu}(2,3)=-2 \cdot 0 \mathrm{~d} 0 / 3 \cdot 0 \mathrm{~d} 0 \\
& \mathrm{cmu}(2,4)=0.0 \mathrm{~d} 0 \\
& \mathrm{cmu}(2,5)=0.0 \mathrm{~d} 0
\end{aligned}
$$




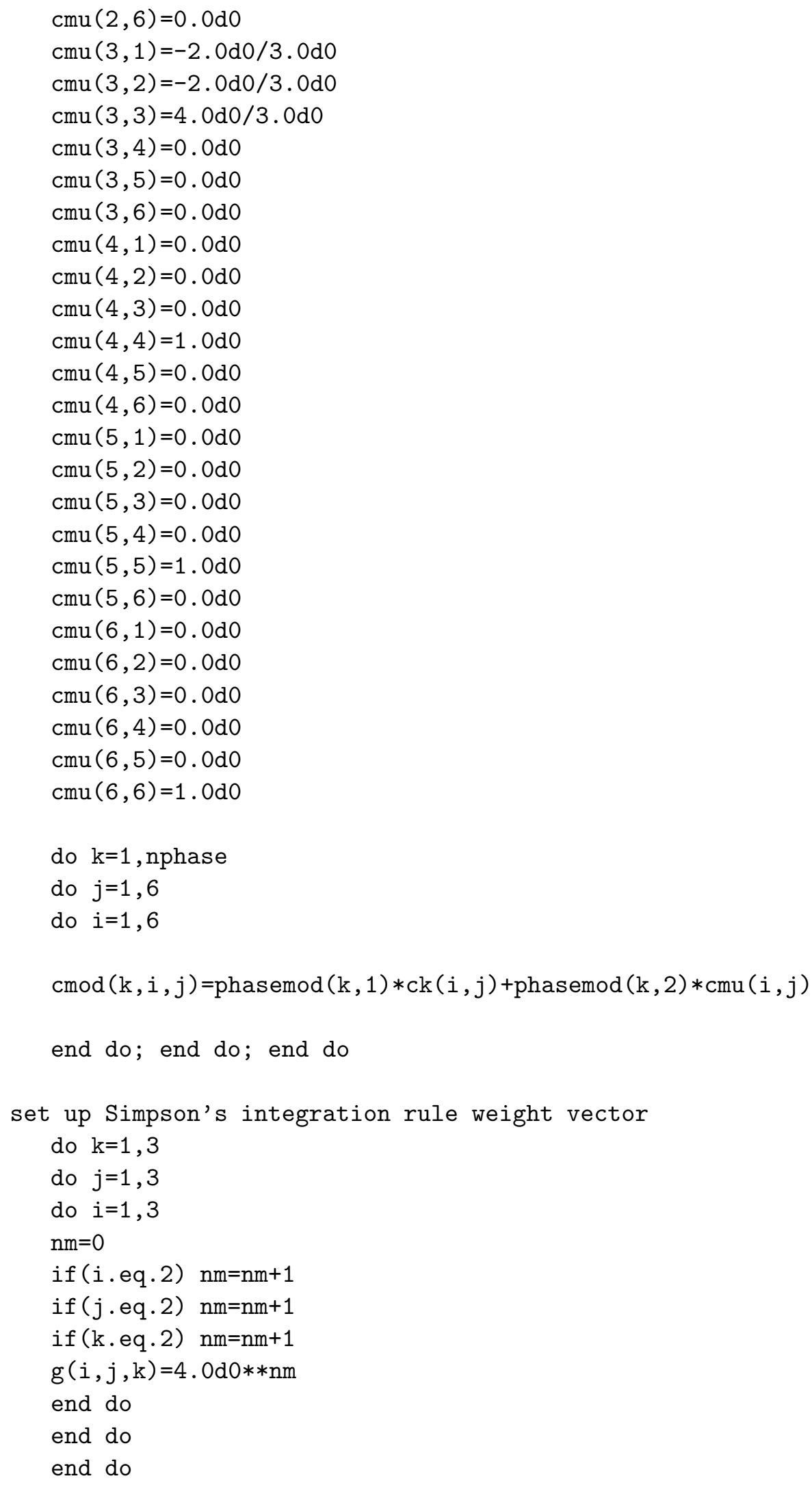

c loop over the nphase kinds of pixels and Simpson's rule quadrature c points in order to compute the stiffness matrices. Stiffness matrices 
c of trilinear finite elements are quadratic in $\mathrm{x}, \mathrm{y}$, and $\mathrm{z}$, so that

c Simpson's rule quadrature gives exact results.

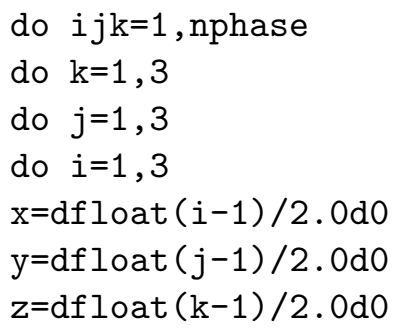

$c$ dndx means the negative derivative, with respect to $\mathrm{x}$, of the shape

c matrix $N$ (see manual, Sec. 2.2), dndy, and dndz are similar.

$$
\begin{aligned}
& \operatorname{dndx}(1)=-(1.0 \mathrm{~d} 0-\mathrm{y}) *(1.0 \mathrm{~d} 0-\mathrm{z}) \\
& \operatorname{dndx}(2)=(1.0 \mathrm{~d} 0-y) *(1.0 \mathrm{~d} 0-z) \\
& \operatorname{dndx}(3)=\mathrm{y} *(1.0 \mathrm{~d} 0-\mathrm{z}) \\
& \operatorname{dndx}(4)=-y *(1.0 \mathrm{~d} 0-z) \\
& \operatorname{dndx}(5)=-(1.0 \mathrm{~d} 0-\mathrm{y}) * z \\
& \operatorname{dndx}(6)=(1.0 \mathrm{~d} 0-\mathrm{y}) * z \\
& \operatorname{dndx}(7)=y * z \\
& \operatorname{dndx}(8)=-y * z \\
& \operatorname{dndy}(1)=-(1.0 \mathrm{~d} 0-\mathrm{x}) *(1.0 \mathrm{~d} 0-\mathrm{z}) \\
& \operatorname{dndy}(2)=-x *(1.0 \mathrm{~d} 0-z) \\
& \operatorname{dndy}(3)=\mathrm{x} *(1.0 \mathrm{~d} 0-\mathrm{z}) \\
& \operatorname{dndy}(4)=(1.0 \mathrm{~d} 0-x) *(1.0 \mathrm{~d} 0-z) \\
& \operatorname{dndy}(5)=-(1.0 \mathrm{~d} 0-\mathrm{x}) * \mathrm{z} \\
& \operatorname{dndy}(6)=-x * z \\
& \operatorname{dndy}(7)=x * z \\
& \operatorname{dndy}(8)=(1.0 \mathrm{~d} 0-x) * z \\
& \operatorname{dnd} z(1)=-(1.0 \mathrm{~d} 0-x) *(1.0 \mathrm{~d} 0-\mathrm{y}) \\
& \operatorname{dnd} z(2)=-x *(1.0 \mathrm{~d} 0-y) \\
& \operatorname{dnd} z(3)=-x * y \\
& \operatorname{dnd} z(4)=-(1.0 \mathrm{~d} 0-\mathrm{x}) * \mathrm{y} \\
& \operatorname{dnd} z(5)=(1.0 \mathrm{~d} 0-x) *(1.0 \mathrm{~d} 0-\mathrm{y}) \\
& \operatorname{dnd} z(6)=x *(1.0 d 0-y) \\
& \operatorname{dnd} z(7)=x * y \\
& \operatorname{dnd} z(8)=(1.0 \mathrm{~d} 0-\mathrm{x}) * \mathrm{y}
\end{aligned}
$$

c now build strain matrix

$$
\begin{aligned}
& \text { es }=0.0 \mathrm{~d} 0 \\
& \text { es }(1,:, 1)=d n d x \\
& \text { es }(2,:, 2)=d n d y \\
& \text { es }(3,:, 3)=d n d z \\
& \text { es }(4,:, 1)=d n d z \\
& \text { es }(4,:, 3)=d n d x \\
& \text { es }(5,:, 2)=d n d z \\
& \text { es }(5,:, 3)=d n d y \\
& \text { es }(6,:, 1)=d n d y
\end{aligned}
$$


es $(6,:, 2)=\operatorname{dnd} x$

c Matrix multiply to determine value at $(\mathrm{x}, \mathrm{y}, \mathrm{z})$, multiply by

$c$ proper weight, and sum_num into $d k$, the stiffness matrix

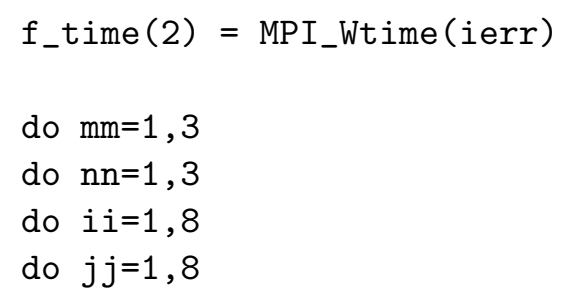

c Define sum over strain matrices and elastic moduli matrix for

c stiffness matrix

sum_num $=0.0 \mathrm{~d} 0$

do $\mathrm{kk}=1,6$

do $11=1,6$

sum_num $=$ sum_num+es $(k k, i i, m m) * \operatorname{cmod}(i j k, k k, l l) * e s(l l, j j, n n)$

end do; end do

$\mathrm{dk}(i j k, i i, m m, j j, n n)=d k(i j k, i i, m m, j j, n n)+g(i, j, k) * s u m \_n u m / 216$.

end do; end do; end do; end do

end do; end do; end do; end do

f_time(3) = MPI_Wtime(ierr)

if (pflag.eq.1) then

write $(*, *)$ myrank, "time to calculate $d k=$,f_time(3)-f_time(2)

endif

c Initialize $\mathrm{b}$ and $\mathrm{C}$

if (myrank.eq.0) then write $(*, *)$ "Initializing b \& C."

end if

$\mathrm{b}=0.0 \mathrm{~d} 0$

$\mathrm{C}=0.0 \mathrm{~d} 0$

$\mathrm{c} 3=0.0 \mathrm{~d} 0$

999 format $(4(i 4,1 x),, 3(f 9.6,1 x))$

C

c $\mathrm{x}=\mathrm{nx}$ face

c

do $i 3=1,3$

do $i 8=1,8$

$\operatorname{delta}(i 8, i 3)=0.0 \mathrm{~d} 0$

if (i8.eq.2.or.i8.eq.3.or.i8.eq.6.or.i8.eq.7) then 


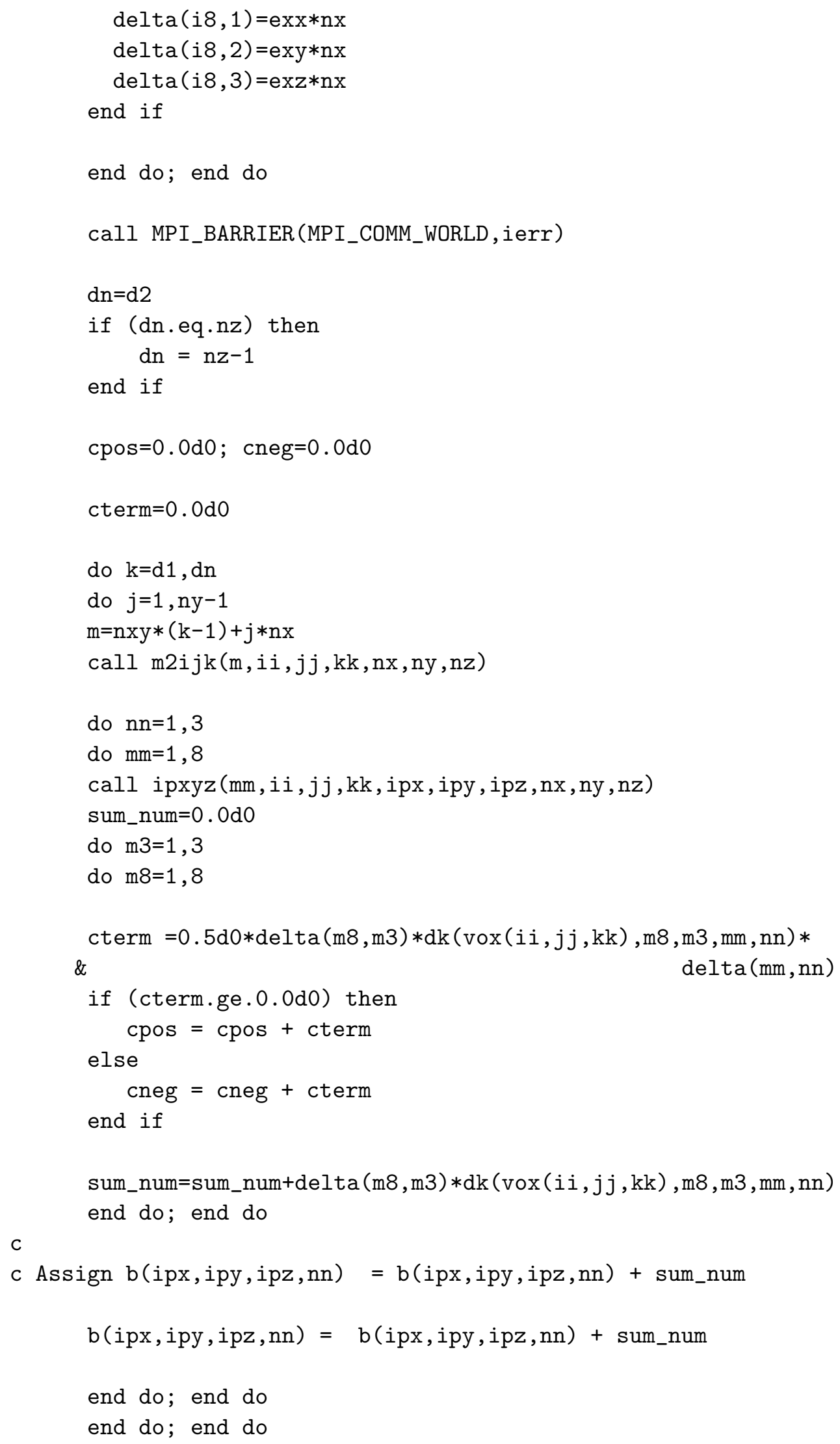


$\mathrm{C}$

$\mathrm{C}$

do $i 8=1,8$

$\operatorname{delta}(i 8, i 3)=0.0 \mathrm{~d} 0$

if (i8.eq.3.or.i8.eq.4.or.i8.eq.7.or.i8.eq.8) then

$\operatorname{delta}(i 8,1)=\operatorname{exy} *$ ny

$\operatorname{delta}(i 8,2)=$ eyy $*$ ny

$\operatorname{delta}(i 8,3)=e y z * n y$

end if

end do; end do

do $i=1, n x-1$

do $\mathrm{k}=\mathrm{d} 1, \mathrm{dn}$

$m=n x y *(k-1)+n x *(n y-1)+i$

call m2ijk(m,ii,jj,kk,nx,ny,nz)

do $\mathrm{nn}=1,3$

do $\mathrm{mm}=1,8$

call ipxyz (mm,ii, jj,kk, ipx, ipy, ipz, nx, ny, nz)

sum_num $=0.0 \mathrm{~d} 0$

do $m 3=1,3$

do $m 8=1,8$

sum_num=sum_num+delta $(m 8, m 3) * d k(\operatorname{vox}(i i, j j, k k), m 8, m 3, m m, n n)$

cterm $=0.5 \mathrm{~d} 0 * \operatorname{delta}(\mathrm{m} 8, \mathrm{~m} 3) * \operatorname{dk}(\operatorname{vox}(\mathrm{i} i, j \mathrm{j}, \mathrm{kk}), \mathrm{m} 8, \mathrm{~m} 3, \mathrm{~mm}, \mathrm{nn}) *$

\&

$\operatorname{delta}(m m, n n)$

if (cterm.ge.0.0d0) then

cpos $=$ cpos + cterm

else

cneg $=$ cneg + cterm

end if

end do; end do

$b(i p x, i p y, i p z, n n)=b(i p x, i p y, i p z, n n)+$ sum_num

end do; end do

end do; end do

c Zface calcs

C

c Only the last node does these series of calculations since

c it contains all the necessary data therefore no data transfer

c occurs.

C

if (myrank.eq.nprocs-1) then 


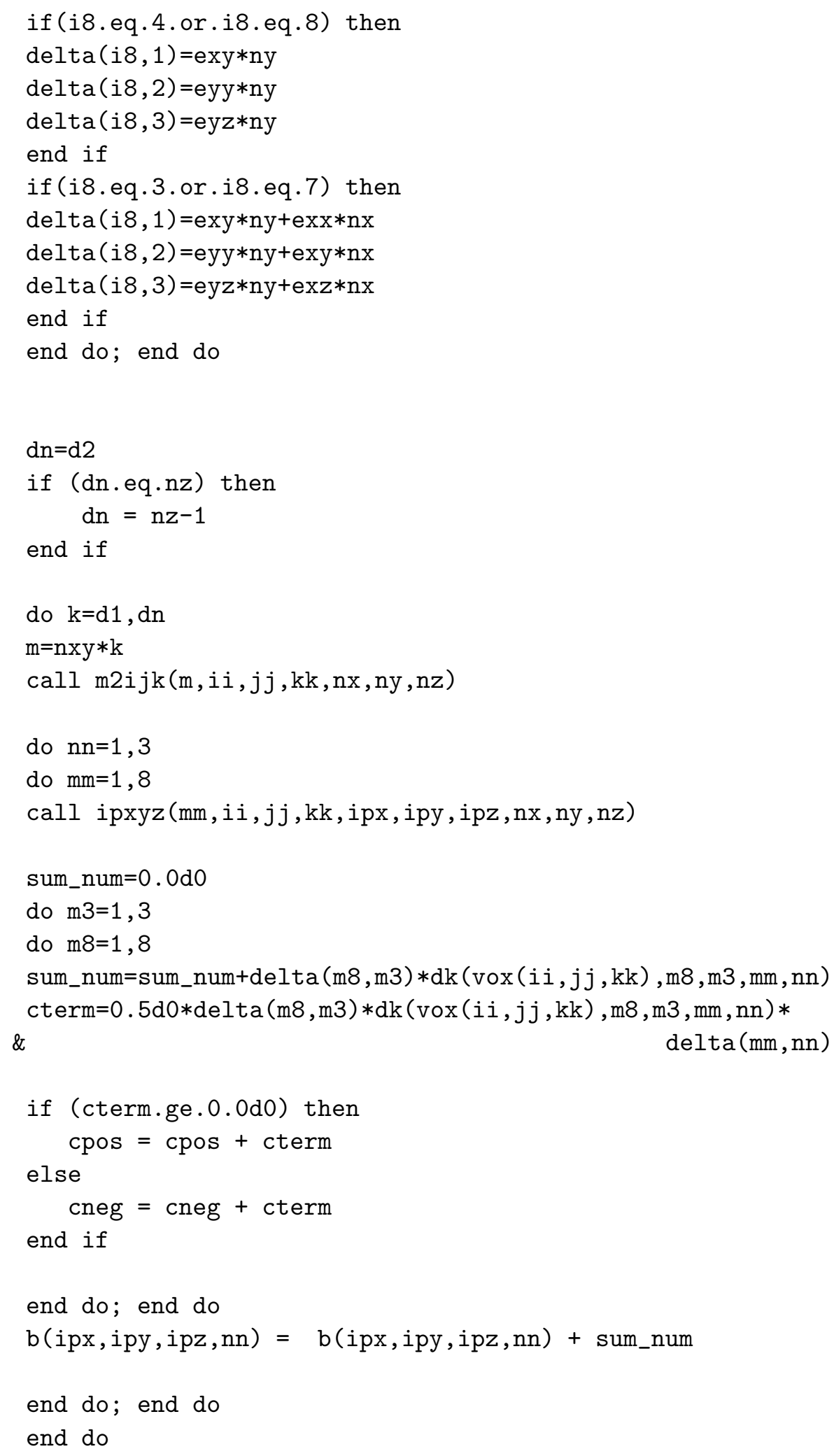




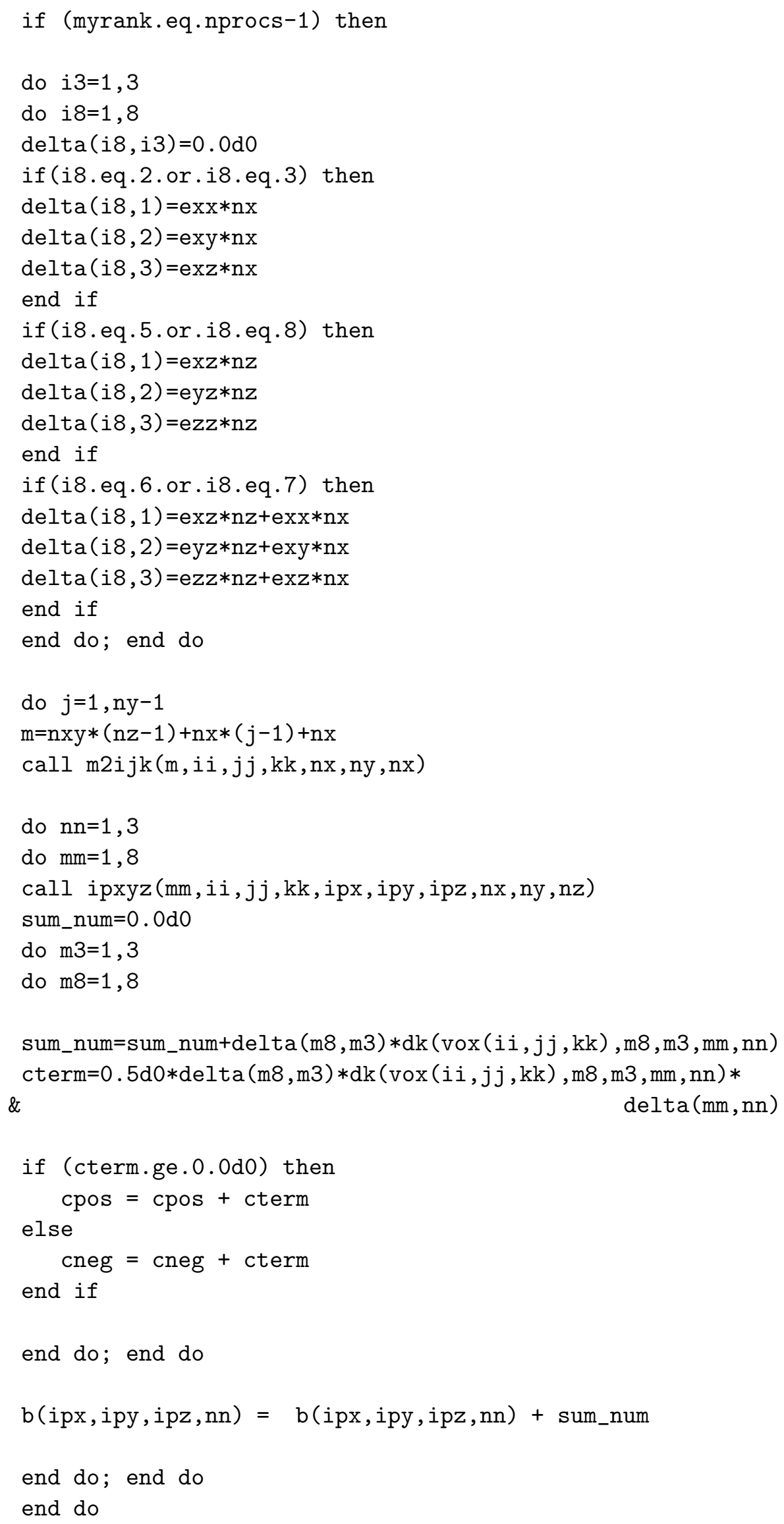


C

c $y=n y ~ z=n z$ edge

C

do $i 3=1,3$

do $i 8=1,8$

$\operatorname{delta}(i 8, i 3)=0.0 \mathrm{~d} 0$

if (i8.eq.5.or.i8.eq.6) then

$\operatorname{delta}(i 8,1)=\operatorname{exz} * \mathrm{nz}$

$\operatorname{delta}(i 8,2)=e y z * n z$

$\operatorname{delta}(i 8,3)=e z z * n z$

end if

if (i8.eq.3.or.i8.eq.4) then

$\operatorname{delta}(i 8,1)=$ exy $*$ ny

$\operatorname{delta}(i 8,2)=$ eyy $*$ ny

$\operatorname{delta}(i 8,3)=e y z * n y$

end if

if (i8.eq.7.or.i8.eq.8) then

$\operatorname{delta}(i 8,1)=e x y * n y+e x z * n z$

$\operatorname{delta}(i 8,2)=$ eyy $*$ ny + eyz $* n z$

$\operatorname{delta}(i 8,3)=e y z * n y+e z z * n z$

end if

end do; end do

do $i=1, n x-1$

$\mathrm{m}=\mathrm{nxy} *(\mathrm{nz}-1)+\mathrm{nx} *(\mathrm{ny}-1)+\mathrm{i}$

call m2ijk (m,ii,jj,kk, nx, ny, nx)

do $\mathrm{nn}=1,3$

do $\mathrm{mm}=1,8$

call ipxyz(mm,ii,jj,kk, ipx,ipy,ipz,nx,ny,nz)

sum_num $=0.0 \mathrm{~d} 0$

do $\mathrm{m} 3=1,3$

do $\mathrm{m} 8=1,8$

sum_num=sum_num+delta $(\mathrm{m} 8, \mathrm{~m} 3) * \mathrm{dk}(\operatorname{vox}(i \mathrm{i}, \mathrm{j} j, \mathrm{kk}), \mathrm{m} 8, \mathrm{~m} 3, \mathrm{~mm}, \mathrm{nn})$

cterm $=0.5 \mathrm{~d} 0 * \operatorname{delta}(\mathrm{m} 8, \mathrm{~m} 3) * \mathrm{dk}(\operatorname{vox}(\mathrm{ii}, \mathrm{jj}, \mathrm{kk}), \mathrm{m} 8, \mathrm{~m} 3, \mathrm{~mm}, \mathrm{nn}) *$

\&

$\operatorname{delta}(\mathrm{mm}, \mathrm{nn})$

if (cterm.ge.0.0d0) then

cpos $=$ cpos + cterm

else

cneg $=$ cneg + cterm

end if

end do; end do

$b(i p x, i p y, i p z, n n)=b(i p x, i p y, i p z, n n)+$ sum_num end do; end do

end do 
C

C $\mathrm{x}=\mathrm{nx}$ y=ny $\mathrm{z}=\mathrm{nz}$ corner

C

do $i 3=1,3$

do $i 8=1,8$

$\operatorname{delta}(i 8, i 3)=0.0 \mathrm{~d} 0$

if (i8.eq.2) then

$\operatorname{delta}(i 8,1)=\operatorname{exx} * \mathrm{nx}$

$\operatorname{delta}(i 8,2)=\operatorname{exy} * \mathrm{nx}$

$\operatorname{delta}(i 8,3)=\operatorname{exz} * \mathrm{nx}$

end if

if (i8.eq.4) then

$\operatorname{delta}(i 8,1)=$ exy $*$ ny

$\operatorname{delta}(i 8,2)=$ eyy $*$ ny

$\operatorname{delta}(i 8,3)=e y z * n y$

end if

if (i8.eq.5) then

$\operatorname{delta}(i 8,1)=\operatorname{exz} * \mathrm{nz}$

$\operatorname{delta}(i 8,2)=e y z * n z$

$\operatorname{delta}(i 8,3)=e z z * n z$

end if

if (i8.eq.8) then

$\operatorname{delta}(i 8,1)=e x y * n y+e x z * n z$

$\operatorname{delta}(i 8,2)=$ eyy $*$ ny + eyz $* n z$

$\operatorname{delta}(i 8,3)=e y z * n y+e z z * n z$

end if

if (i8.eq.6) then

$\operatorname{delta}(i 8,1)=\operatorname{exx} * \mathrm{nx}+\mathrm{exz} * \mathrm{nz}$

$\operatorname{delta}(i 8,2)=e x y * n x+e y z * n z$

$\operatorname{delta}(i 8,3)=e x z * n x+e z z * n z$

end if

if (i8.eq.3) then

$\operatorname{delta}(i 8,1)=\operatorname{exx} * n x+e x y * n y$

$\operatorname{delta}(i 8,2)=$ exy $* n x+$ eyy $*$ ny

$\operatorname{delta}(i 8,3)=e x z * n x+e y z * n y$

end if

if (i8.eq.7) then

$\operatorname{delta}(i 8,1)=e x x * n x+e x y * n y+e x z * n z$

$\operatorname{delta}(i 8,2)=e x y * n x+e y y * n y+e y z * n z$

$\operatorname{delta}(i 8,3)=e x z * n x+e y z * n y+e z z * n z$

end if

end do; end do

$\mathrm{m}=\mathrm{nx} * \mathrm{ny} * \mathrm{nz}$

call m2ijk(m,ii,jj,kk, nx, ny, nx)

do $\mathrm{nn}=1,3$

do $m m=1,8$

call ipxyz(mm,ii,jj,kk, ipx,ipy,ipz,nx,ny,nz) 


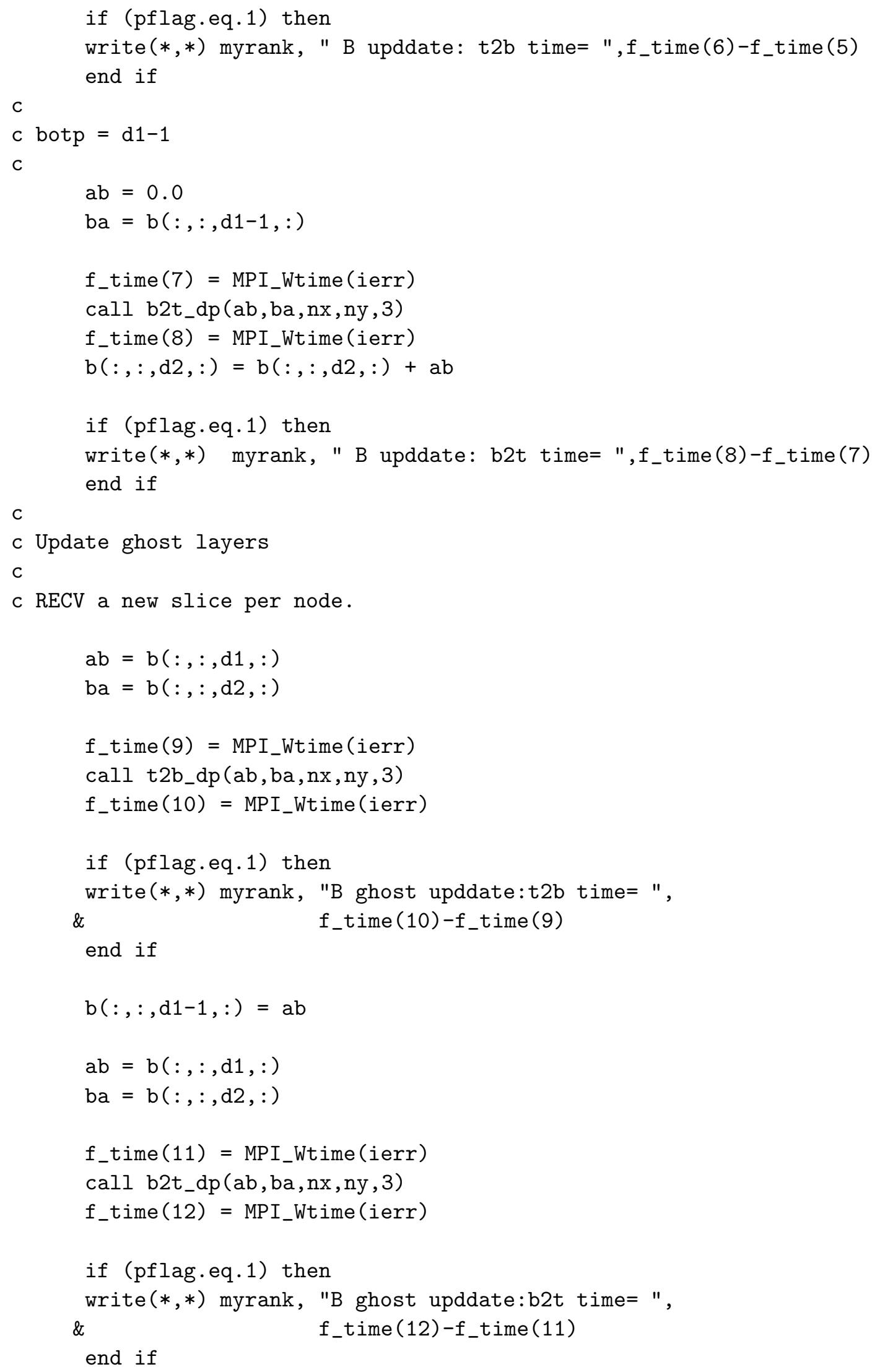




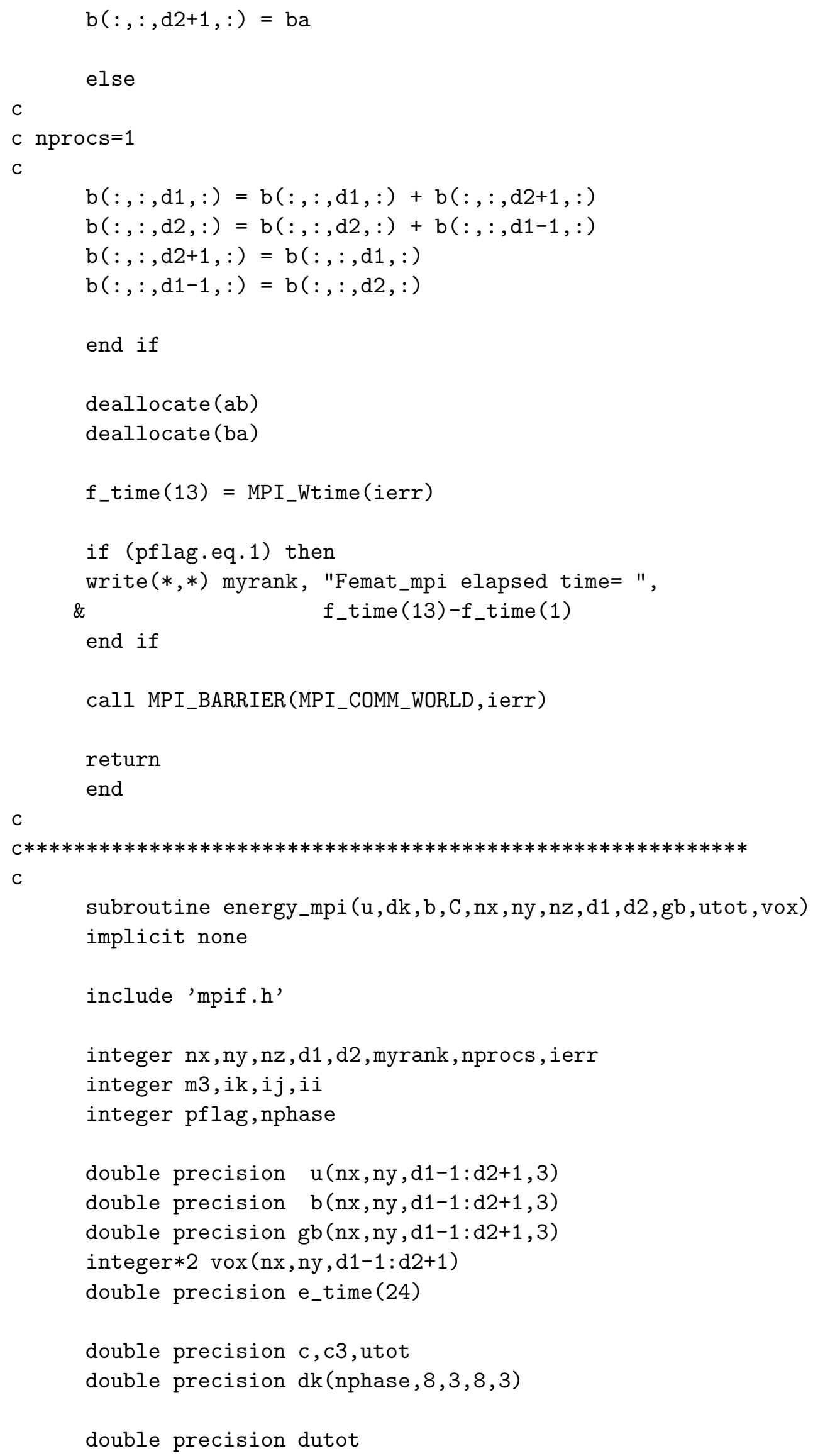


double precision exx, eyy, ezz, exz, eyz, exy

common/list1/pflag, nphase

common/list2/exx, eyy, ezz, exz, eyz, exy

call MPI_COMM_RANK ( MPI_COMM_WORLD, myrank, ierr )

call MPI_COMM_SIZE( MPI_COMM_WORLD, nprocs, ierr )

e_time (1) = MPI_Wtime (ierr)

dutot $=0.0 \mathrm{~d} 0$

c After this call, $\mathrm{gb}$ is calculated and data slabs

c are updated and passed.

call gbah (gb,u, dk, vox, nx, ny, nz, d1, d2)

c Now do the rest of the gb calculations that appear

c in original "energy" subroutine.

C

c utot will be a per processor value.

c Do an MPI_ALLREDUCE on dutot

c so each node will have the current updated version.

$$
\begin{aligned}
& \text { dutot }=0.0 \mathrm{~d} 0 \\
& \text { do } m 3=1,3 \\
& \text { do } i k=d 1, \text { d2 } \\
& \text { do } i j=1, n y \\
& \text { do } i i=1, n x
\end{aligned}
$$$$
\text { dutot=dutot+0.5d0*u(ii, ij, ik,m3)*gb(ii, ij, ik, m3)+ }
$$$$
\text { \& } \quad b(i i, i j, i k, m 3) * u(i i, i j, i k, m 3)
$$

end do; end do; end do; end do

call MPI_ALLREDUCE (dutot, utot, 1,MPI_DOUBLE_PRECISION,

$\&$

MPI_SUM, MPI_COMM_WORLD, ierr)

utot $=$ utot $+\mathrm{C}$

c easier to add $\mathrm{C}$ here than before the above MPI call.

$g b=g b+b$

return

end

C

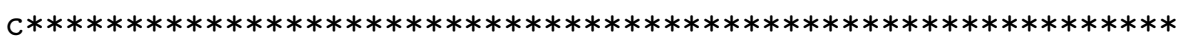

C

subroutine dembx_mpi(nx, ny, nz, d1, d2, Lstep, gb, u, vox, h, 


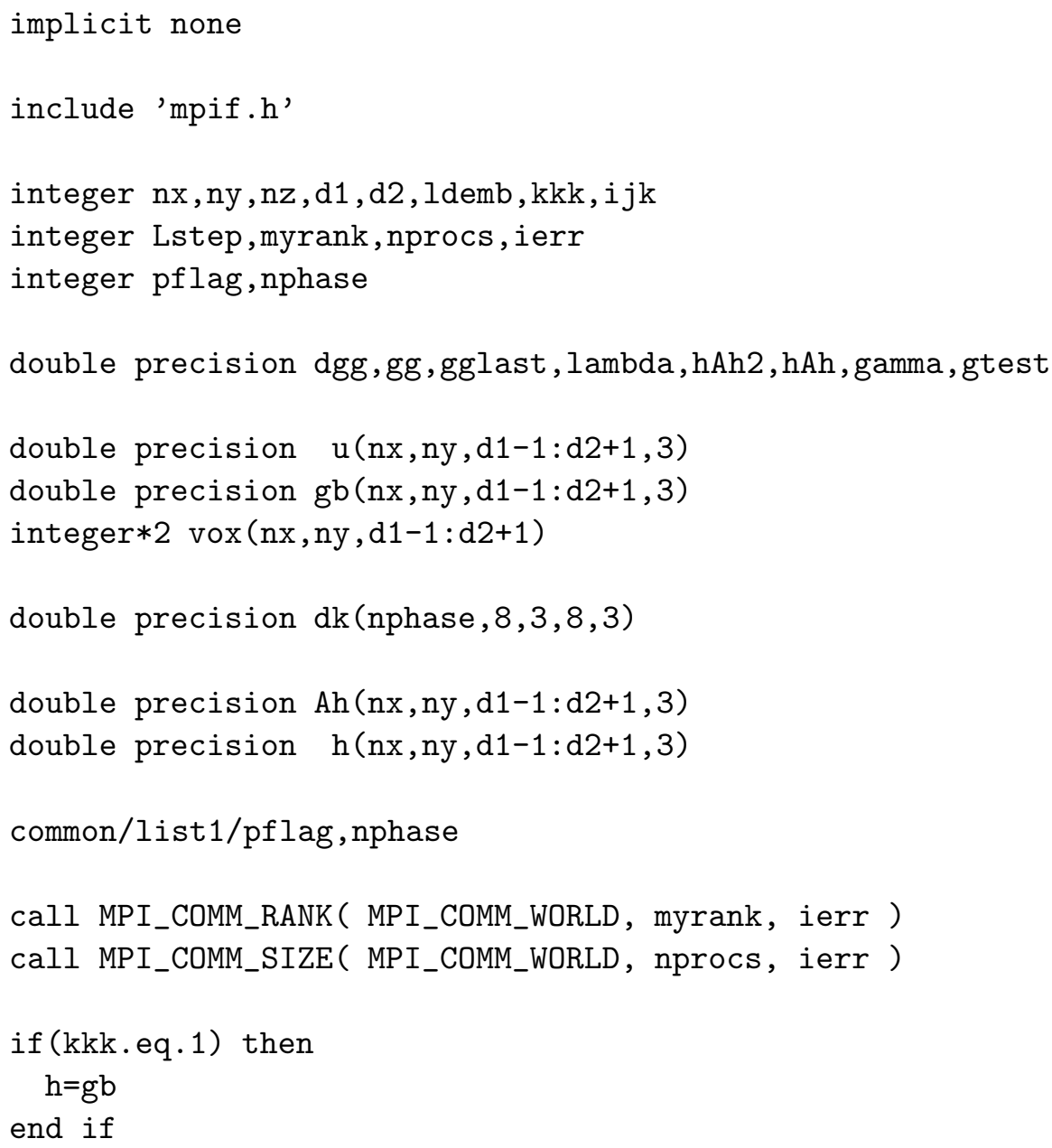




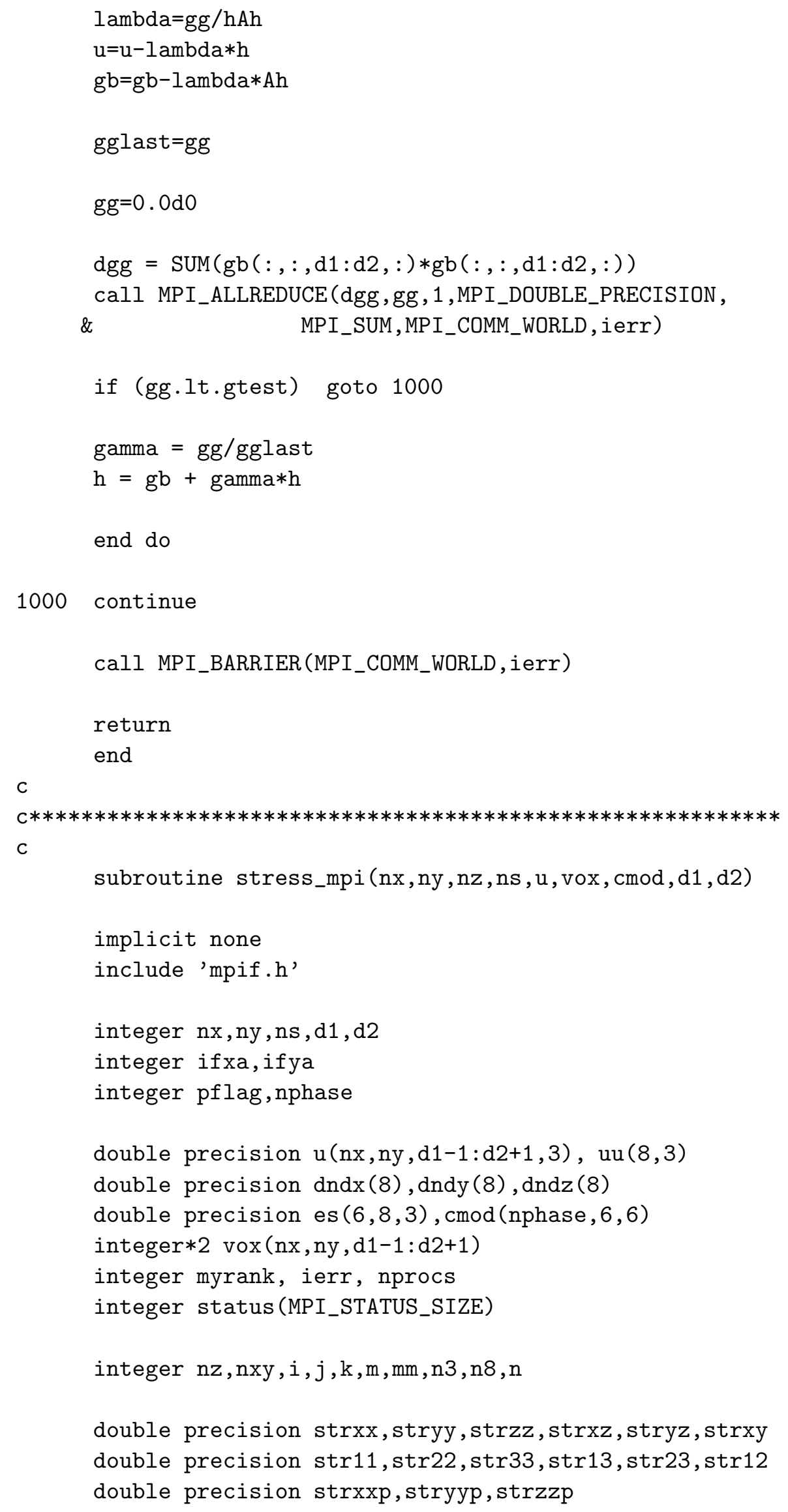




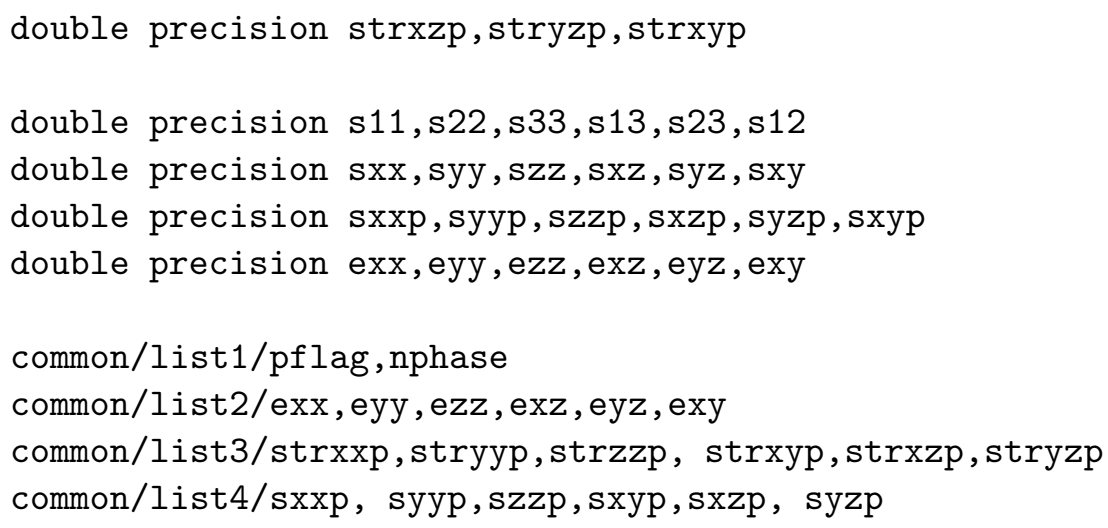

$$
\begin{aligned}
& \operatorname{dndx}(1)=-0.25 \mathrm{~d} 0 \\
& \operatorname{dndx}(2)=0.25 \mathrm{~d} 0 \\
& \operatorname{dndx}(3)=0.25 \mathrm{~d} 0 \\
& \operatorname{dndx}(4)=-0.25 \mathrm{~d} 0 \\
& \operatorname{dndx}(5)=-0.25 \mathrm{~d} 0 \\
& \operatorname{dndx}(6)=0.25 \mathrm{~d} 0 \\
& \operatorname{dndx}(7)=0.25 \mathrm{~d} 0 \\
& \operatorname{dndx}(8)=-0.25 \mathrm{~d} 0 \\
& \operatorname{dndy}(1)=-0.25 \mathrm{~d} 0 \\
& \operatorname{dndy}(2)=-0.25 \mathrm{~d} 0 \\
& \operatorname{dndy}(3)=0.25 \mathrm{~d} 0 \\
& \operatorname{dndy}(4)=0.25 \mathrm{~d} 0 \\
& \operatorname{dndy}(5)=-0.25 \mathrm{~d} 0 \\
& \operatorname{dndy}(6)=-0.25 \mathrm{~d} 0 \\
& \operatorname{dndy}(7)=0.25 \mathrm{~d} 0 \\
& \operatorname{dndy}(8)=0.25 \mathrm{~d} 0 \\
& \operatorname{dndz}(1)=-0.25 \mathrm{~d} 0 \\
& \operatorname{dndz}(2)=-0.25 \mathrm{~d} 0 \\
& \operatorname{dndz}(3)=-0.25 \mathrm{~d} 0 \\
& \operatorname{dndz}(4)=-0.25 \mathrm{~d} 0 \\
& \operatorname{dndz}(5)=0.25 \mathrm{~d} 0 \\
& \operatorname{dndz}(6)=0.25 \mathrm{~d} 0 \\
& \operatorname{dndz}(7)=0.25 \mathrm{~d} 0 \\
& \operatorname{dndz}(8)=0.25 \mathrm{~d} 0
\end{aligned}
$$

c Build averaged strain matrix, follows code in femat, but for average

c strain over the pixel, not the strain at a point.

$$
\text { es }=0.0 \mathrm{~d} 0
$$




$$
\begin{aligned}
& \text { es }(1,:, 1)=d n d x \\
& \text { es }(2,:, 2)=d n d y \\
& \text { es }(3,:, 3)=d n d z \\
& \text { es }(4,:, 1)=d n d z \\
& \text { es }(4,:, 3)=d n d x \\
& \text { es }(5,:, 2)=d n d z \\
& \text { es }(5,:, 3)=d n d y \\
& \text { es }(6,:, 1)=d n d y \\
& \text { es }(6,:, 2)=d n d x
\end{aligned}
$$

c Compute components of the average stress and strain tensors in each pixel

$$
\text { str } \mathrm{xx}=0.0 \mathrm{~d} 0
$$

stryy $=0.0 \mathrm{~d} 0$

strzz $=0.0 \mathrm{~d} 0$

$\operatorname{str} x z=0.0 \mathrm{~d} 0$

stryz $=0.0 \mathrm{~d} 0$

strxy $=0.0 \mathrm{~d} 0$

$\mathrm{sxx}=0.0 \mathrm{~d} 0$

syy $=0.0 \mathrm{~d} 0$

$\mathrm{szz}=0.0 \mathrm{~d} 0$

$\mathrm{sxz}=0.0 \mathrm{~d} 0$

syz $=0.0 \mathrm{~d} 0$

$\mathrm{sxy}=0.0 \mathrm{~d} 0$

strxxp $=0.0 \mathrm{~d} 0$

stryyp $=0.0 \mathrm{~d} 0$

strzzp $=0.0 \mathrm{~d} 0$

strxzp $=0.0 \mathrm{~d} 0$

stryzp $=0.0 \mathrm{~d} 0$

strxyp $=0.0 \mathrm{~d} 0$

$\mathrm{sxxp}=0.0 \mathrm{~d} 0$

syyp $=0.0 \mathrm{~d} 0$

$\mathrm{szzp}=0.0 \mathrm{~d} 0$

$\mathrm{sxzp}=0.0 \mathrm{~d} 0$

syzp $=0.0 \mathrm{~d} 0$

$\operatorname{sxyp}=0.0 \mathrm{~d} 0$

do $470 \mathrm{k}=\mathrm{d} 1$, d2

do $470 \mathrm{j}=1$, ny

do $470 i=1, n x$

$\mathrm{m}=(\mathrm{k}-1) * \mathrm{nxy}+(\mathrm{j}-1) * \mathrm{nx}+\mathrm{i}$

if $((i+1) . G T . n x)$ then

if $x a=1$

else

ifxa $=i+1$

end if 


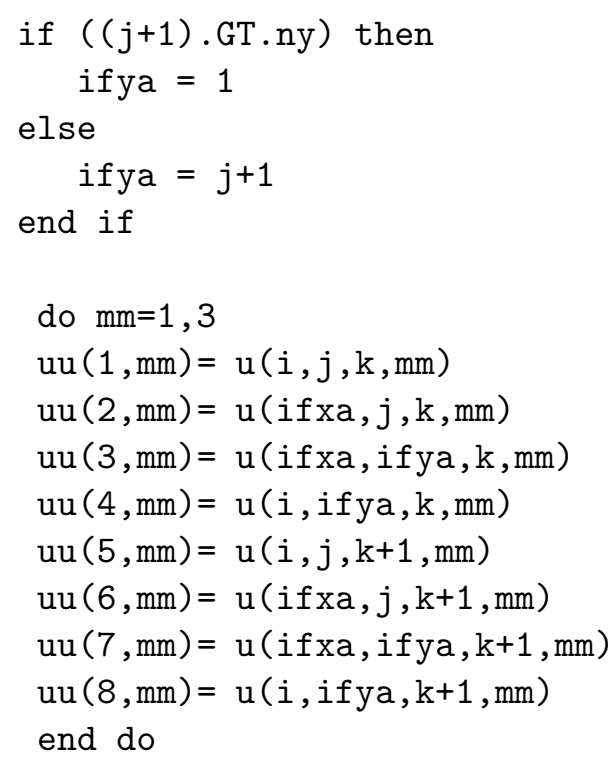

c Correct for periodic boundary conditions, some displacements are wrong c for a pixel on a periodic boundary. Since they come from an opposite c face, need to put in applied strain to correct them.
if (i.eq. nx) then uu $(2,1)=u u(2,1)+e x x * n x$ $\mathrm{uu}(2,2)=\mathrm{uu}(2,2)+\mathrm{exy} * \mathrm{nx}$ uu $(2,3)=\mathrm{uu}(2,3)+\mathrm{exz} * \mathrm{nx}$ uu $(3,1)=u u(3,1)+$ exx $*$ nx uu $(3,2)=u u(3,2)+$ exy $*$ nx $\mathrm{uu}(3,3)=\mathrm{uu}(3,3)+\mathrm{exz} * \mathrm{nx}$ $\mathrm{uu}(6,1)=\mathrm{uu}(6,1)+\mathrm{exx} * \mathrm{nx}$ $\mathrm{uu}(6,2)=\mathrm{uu}(6,2)+\mathrm{exy} * \mathrm{nx}$ $\mathrm{uu}(6,3)=\mathrm{uu}(6,3)+\mathrm{exz} * \mathrm{nx}$ $\mathrm{uu}(7,1)=\mathrm{uu}(7,1)+\mathrm{exx} * \mathrm{nx}$ $\mathrm{uu}(7,2)=\mathrm{uu}(7,2)+\mathrm{exy} * \mathrm{nx}$ uu $(7,3)=u u(7,3)+e x z * n x$ end if
if ( $j$.eq.ny) then
$\mathrm{uu}(3,1)=\mathrm{uu}(3,1)+$ exy $*$ ny uu $(3,2)=u u(3,2)+$ eyy $*$ ny uu $(3,3)=u u(3,3)+$ eyz $*$ ny $\mathrm{uu}(4,1)=\mathrm{uu}(4,1)+$ exy $*$ ny uu $(4,2)=u u(4,2)+$ eyy $*$ ny $\mathrm{uu}(4,3)=\mathrm{uu}(4,3)+$ eyz $*$ ny $\mathrm{uu}(7,1)=\mathrm{uu}(7,1)+$ exy $*$ ny uu $(7,2)=u u(7,2)+$ eyy $*$ ny uu $(7,3)=u u(7,3)+$ eyz $*$ ny uu $(8,1)=u u(8,1)+$ exy $*$ ny uu $(8,2)=u u(8,2)+$ eyy $*$ ny $\mathrm{uu}(8,3)=\mathrm{uu}(8,3)+$ eyz $*$ ny end if if (k.eq.nz) then 


$$
\begin{aligned}
& \mathrm{uu}(5,1)=\mathrm{uu}(5,1)+\mathrm{exz} * \mathrm{nz} \\
& \text { uu }(5,2)=u u(5,2)+\text { eyz } * n z \\
& \mathrm{uu}(5,3)=\mathrm{uu}(5,3)+\mathrm{ezz} * \mathrm{nz} \\
& \text { uu }(6,1)=u u(6,1)+\text { exz } * \text { nz } \\
& \mathrm{uu}(6,2)=\mathrm{uu}(6,2)+\text { eyz } * \mathrm{nz} \\
& \mathrm{uu}(6,3)=\mathrm{uu}(6,3)+\mathrm{ezz} * \mathrm{nz} \\
& \mathrm{uu}(7,1)=\mathrm{uu}(7,1)+\mathrm{exz} * \mathrm{nz} \\
& \mathrm{uu}(7,2)=\mathrm{uu}(7,2)+\text { eyz } * \mathrm{nz} \\
& \mathrm{uu}(7,3)=\mathrm{uu}(7,3)+\mathrm{ezz} * \mathrm{nz} \\
& \text { uu }(8,1)=u u(8,1)+\text { exz } * \text { nz } \\
& \mathrm{uu}(8,2)=\mathrm{uu}(8,2)+\text { eyz } * \mathrm{nz} \\
& \mathrm{uu}(8,3)=\mathrm{uu}(8,3)+\mathrm{ezz} * \mathrm{nz} \\
& \text { end if }
\end{aligned}
$$

c local stresses and strains in a pixel

$$
\begin{aligned}
& \text { str } 11=0.0 \mathrm{~d} 0 \\
& \text { str22 }=0.0 \mathrm{~d} 0 \\
& \text { str } 33=0.0 \mathrm{~d} 0 \\
& \text { str } 13=0.0 \mathrm{~d} 0 \\
& \text { str23=0.0d0 } \\
& \text { str } 12=0.0 \mathrm{~d} 0 \\
& \mathrm{~s} 11=0.0 \mathrm{~d} 0 \\
& \mathrm{~s} 22=0.0 \mathrm{~d} 0 \\
& \mathrm{~s} 33=0.0 \mathrm{~d} 0 \\
& \mathrm{~s} 13=0.0 \mathrm{~d} 0 \\
& \mathrm{~s} 23=0.0 \mathrm{~d} 0 \\
& \mathrm{~s} 12=0.0 \mathrm{~d} 0
\end{aligned}
$$

do $n 3=1,3$

do $\mathrm{n} 8=1,8$

$\mathrm{s} 11=\mathrm{s} 11+\mathrm{es}(1, \mathrm{n} 8, \mathrm{n} 3) * \mathrm{uu}(\mathrm{n} 8, \mathrm{n} 3)$

$\mathrm{s} 22=\mathrm{s} 22+\mathrm{es}(2, \mathrm{n} 8, \mathrm{n} 3) * \mathrm{uu}(\mathrm{n} 8, \mathrm{n} 3)$

$\mathrm{s} 33=\mathrm{s} 33+\mathrm{es}(3, \mathrm{n} 8, \mathrm{n} 3) * \mathrm{uu}(\mathrm{n} 8, \mathrm{n} 3)$

$\mathrm{s} 13=\mathrm{s} 13+\mathrm{es}(4, \mathrm{n} 8, \mathrm{n} 3) * \mathrm{uu}(\mathrm{n} 8, \mathrm{n} 3)$

$\mathrm{s} 23=\mathrm{s} 23+\mathrm{es}(5, \mathrm{n} 8, \mathrm{n} 3) * \mathrm{uu}(\mathrm{n} 8, \mathrm{n} 3)$

$\mathrm{s} 12=\mathrm{s} 12+\mathrm{es}(6, \mathrm{n} 8, \mathrm{n} 3) * \mathrm{uu}(\mathrm{n} 8, \mathrm{n} 3)$

do $\mathrm{n}=1,6$

$\operatorname{str} 11=\operatorname{str} 11+\operatorname{cmod}(\operatorname{vox}(i, j, k), 1, n) * e s(n, n 8, n 3) * u u(n 8, n 3)$

$\operatorname{str} 22=\operatorname{str} 22+\operatorname{cmod}(\operatorname{vox}(i, j, k), 2, n) * e s(n, n 8, n 3) * u u(n 8, n 3)$

str33=str33+cmod $(\operatorname{vox}(i, j, k), 3, n) * e s(n, n 8, n 3) * u u(n 8, n 3)$

$\operatorname{str} 13=\operatorname{str} 13+\operatorname{cmod}(\operatorname{vox}(i, j, k), 4, n) * e s(n, n 8, n 3) * u u(n 8, n 3)$

str23=str23+cmod $(\operatorname{vox}(i, j, k), 5, n) * e s(n, n 8, n 3) * u u(n 8, n 3)$

$\operatorname{str} 12=\operatorname{str} 12+\operatorname{cmod}(\operatorname{vox}(i, j, k), 6, n) * e s(n, n 8, n 3) * u u(n 8, n 3)$

end do

end do; end do

c sum local strains and stresses into global values 
call MPI_ALLREDUCE (sxy, sxyp, 1, MPI_DOUBLE_PRECISION, $\&$

call MPI_ALLREDUCE (syz, syzp , 1, MPI_DOUBLE_PRECISION, $\&$

MPI_SUM, MPI_COMM_WORLD, ierr)

C

c Now root has strxx, stryy, ... sxy

c Let him write out data to disk after this Volume averaging

c

c Volume average of global stresses and strains strxxp=strxxp/dfloat (ns)

stryyp=stryyp/dfloat (ns)

strzzp=strzzp/dfloat (ns)

strxzp=strxzp/dfloat (ns)

stryzp=stryzp/dfloat (ns)

strxyp=strxyp/dfloat (ns)

$\operatorname{sxxp}=\operatorname{sxxp} / d f l o a t(n s)$

syyp=syyp/dfloat (ns)

$\mathrm{szzp}=\mathrm{szzp} / \mathrm{dfl}$ loat (ns)

$\operatorname{sxzp}=s x z p / d f l o a t$ (ns)

syzp $=$ syzp/dfloat (ns)

sxyp $=$ sxyp/dfloat (ns)

if (myrank.eq.0) then

write $(*, *)$ "strxxp = ", $\operatorname{strxxp}$

write $(*, *)$ "stryyp $=$ ", stryyp

write $(*, *)$ "strzzp = ", strzzp

write $(*, *)$ "strxyp = ", strxyp

write $(*, *)$ "strxzp = ", strxzp

write $(*, *)$ "stryzp = ", stryzp

write $(*, *)$ "sxxp $=$ ", $\operatorname{sxxp}$

write $(*, *)$ "syyp $=$ ", syyp

write $(*, *)$ "szzp $=$ ", szzp

write $(*, *)$ "sxyp $=$ ", sxyp

write $(*, *)$ "sxzp = ", $\operatorname{sxzp}$

write $(*, *)$ "syzp = ", syzp

end if

return

end

C

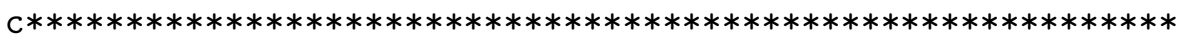

C 


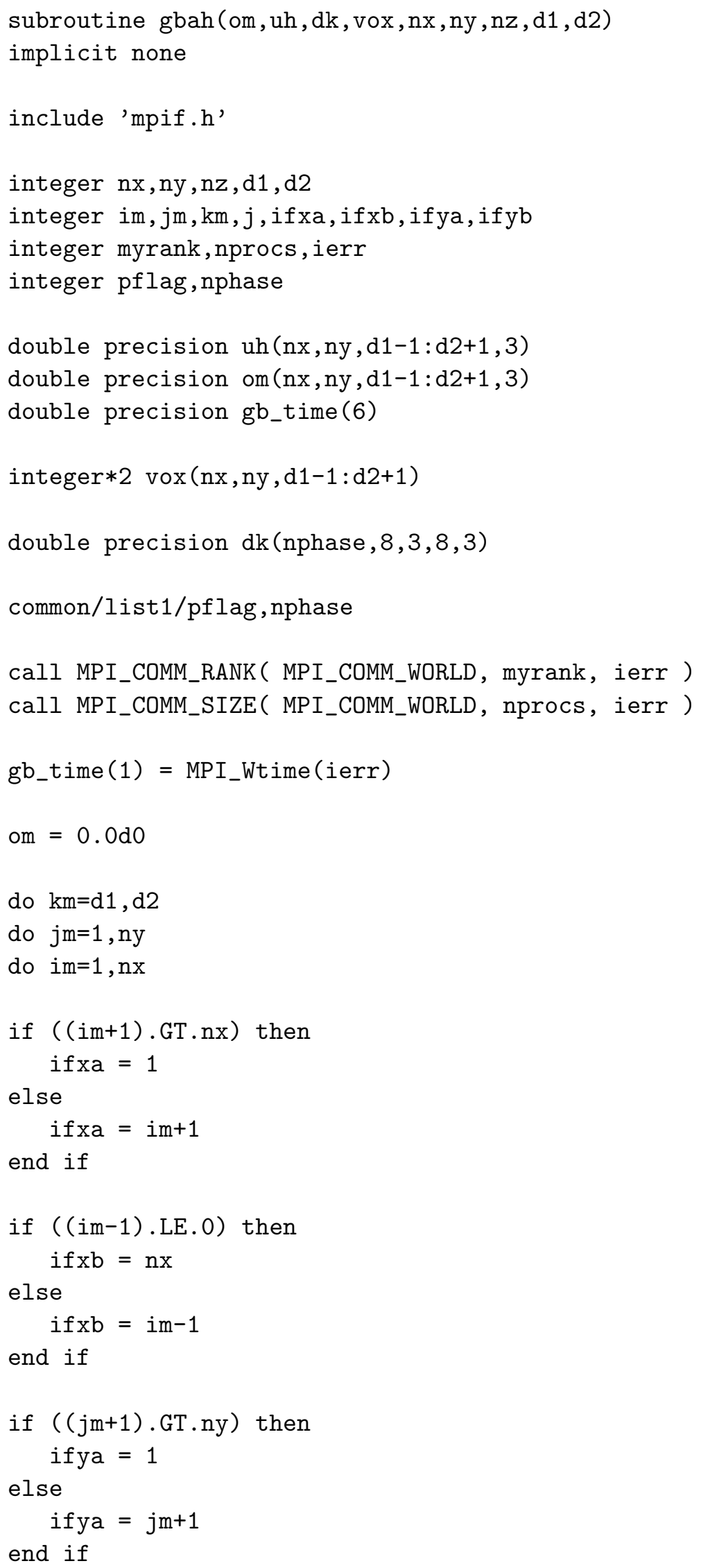


if $((j m-1) . L E .0)$ then

ifyb $=$ ny

else

ifyb $=j m-1$

end if

do $j=1,3$

c SELF TERM

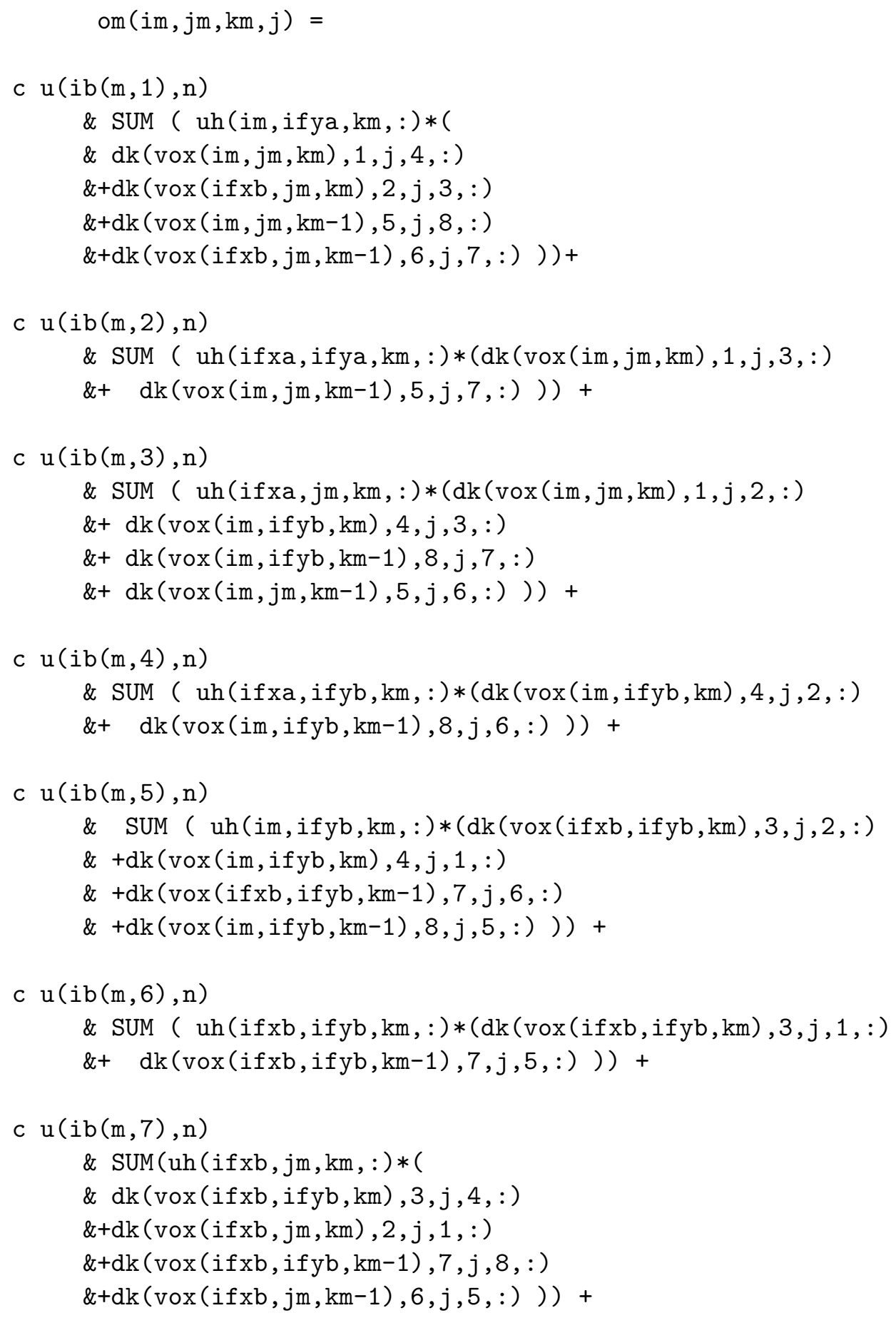




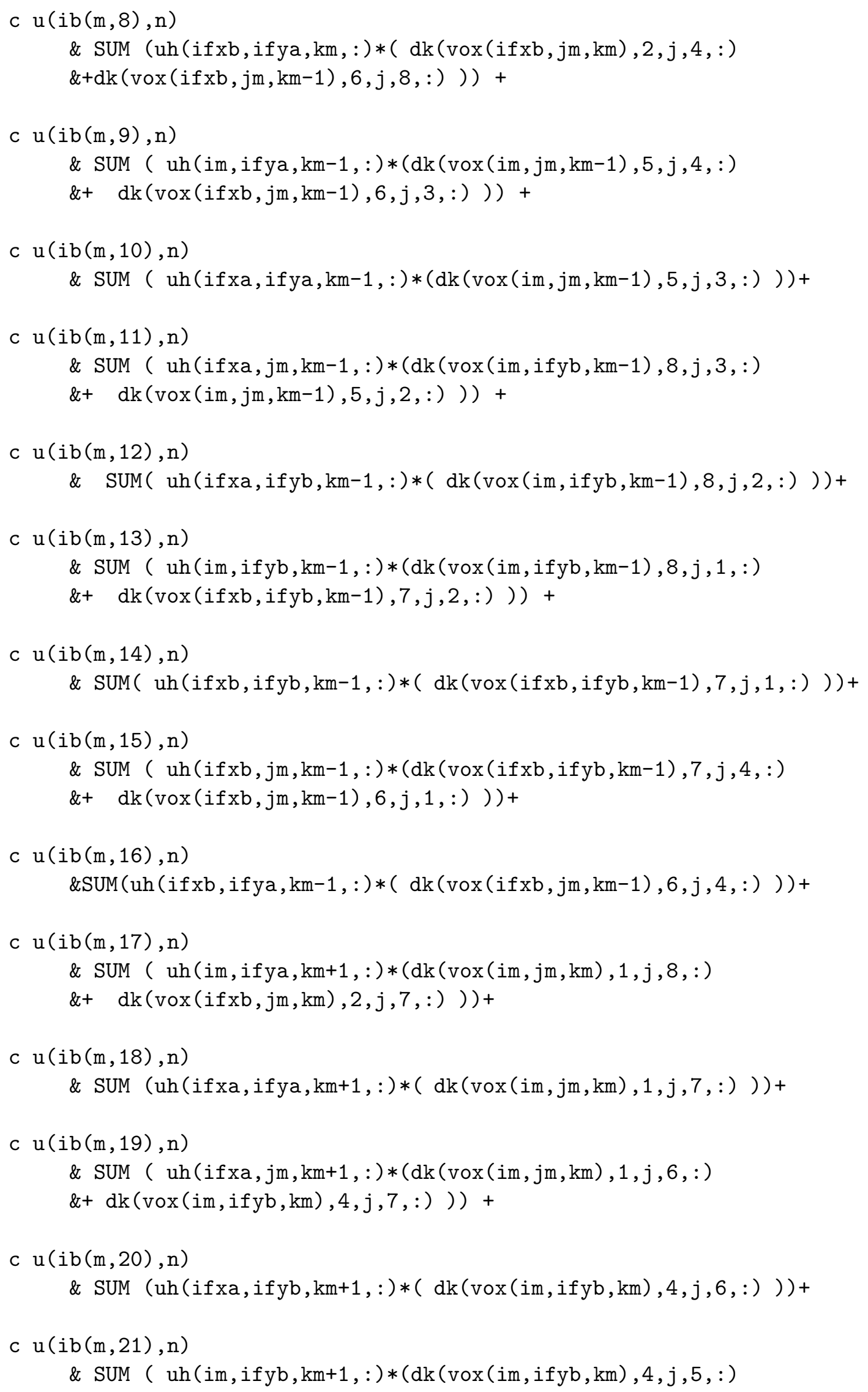




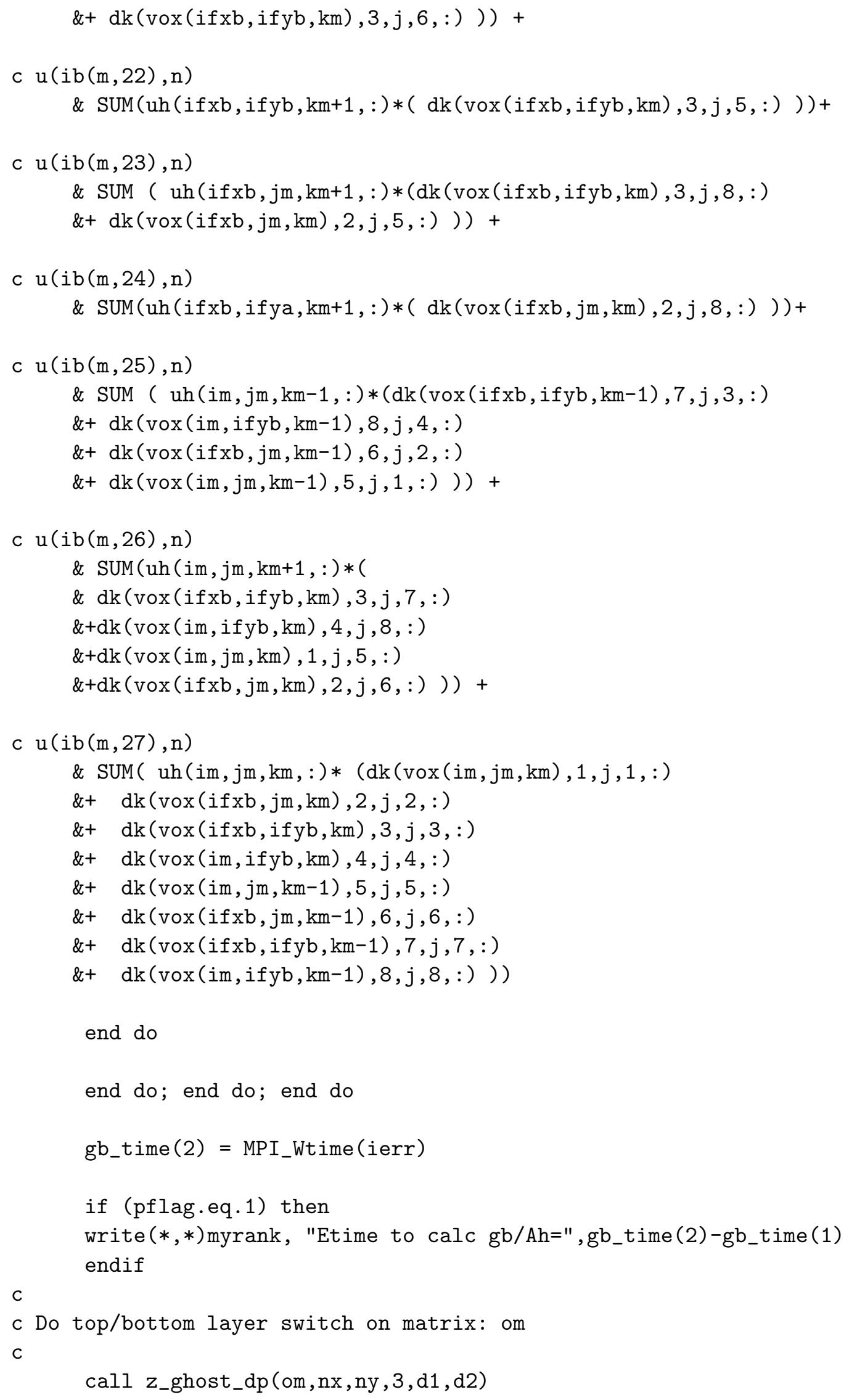




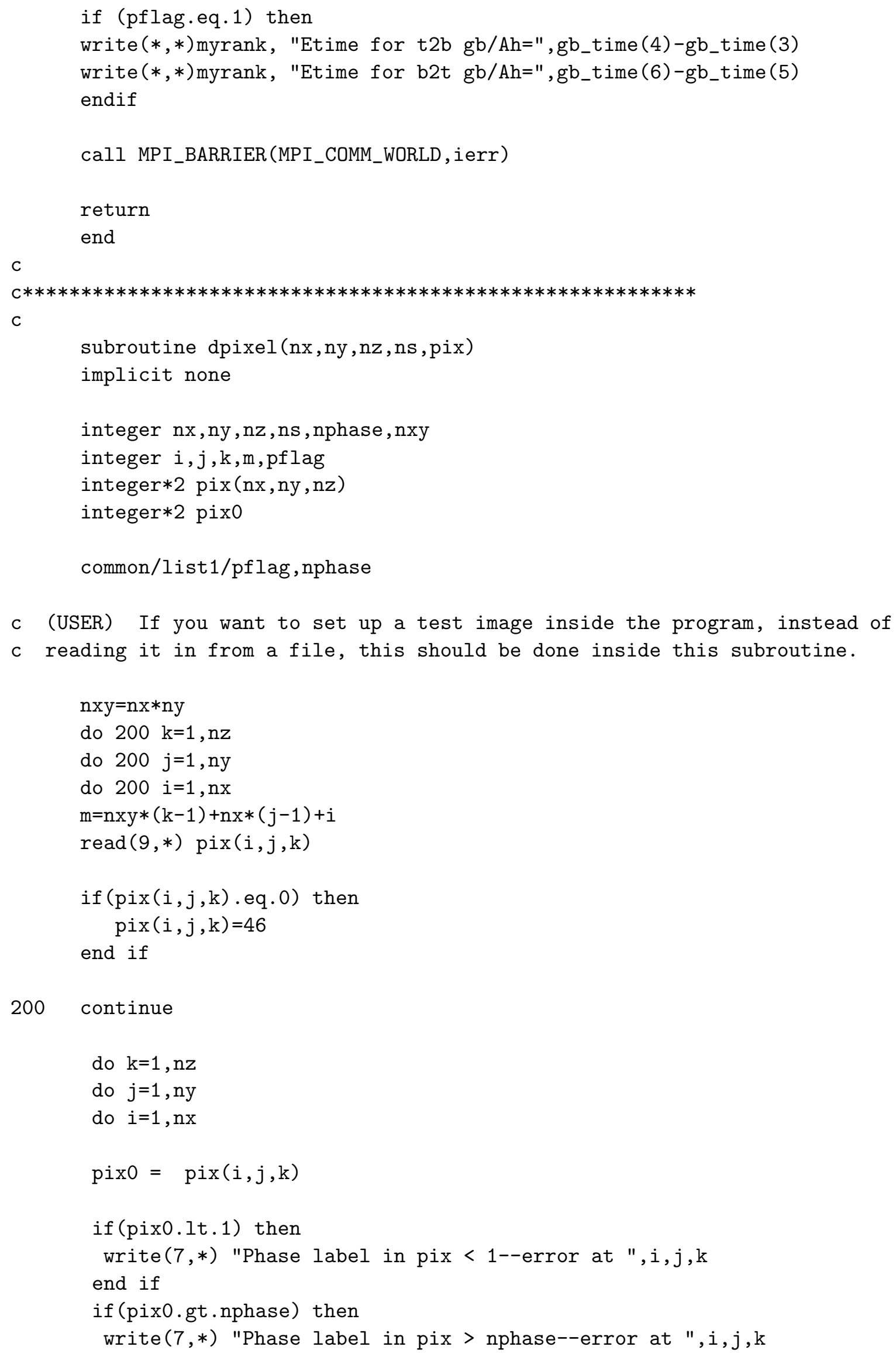

c (USER) If you want to set up a test image inside the program, instead of $c$ reading it in from a file, this should be done inside this subroutine. 
end if

end do; end do; end do

return

end

$\mathrm{C}$

C*************************************************************

$c$

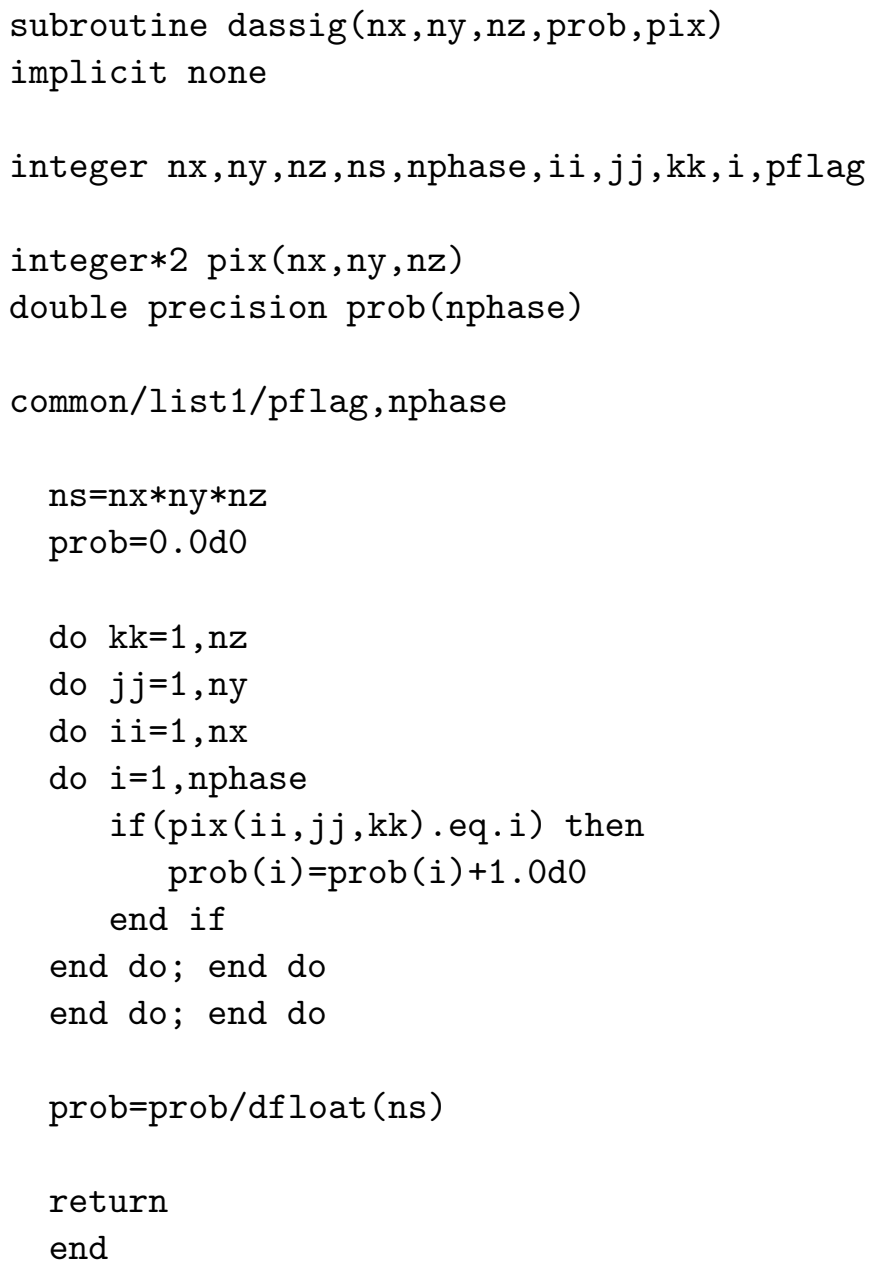




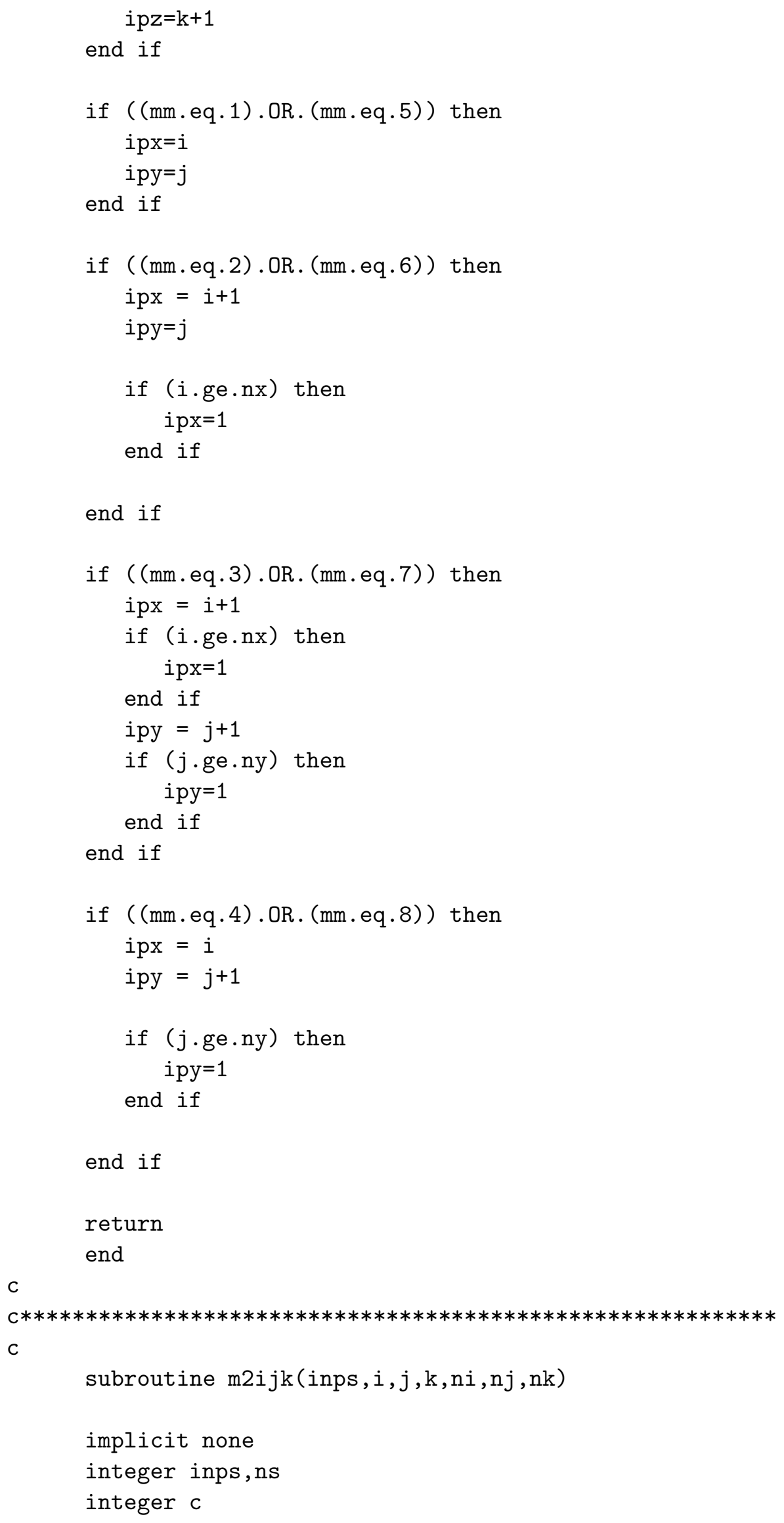




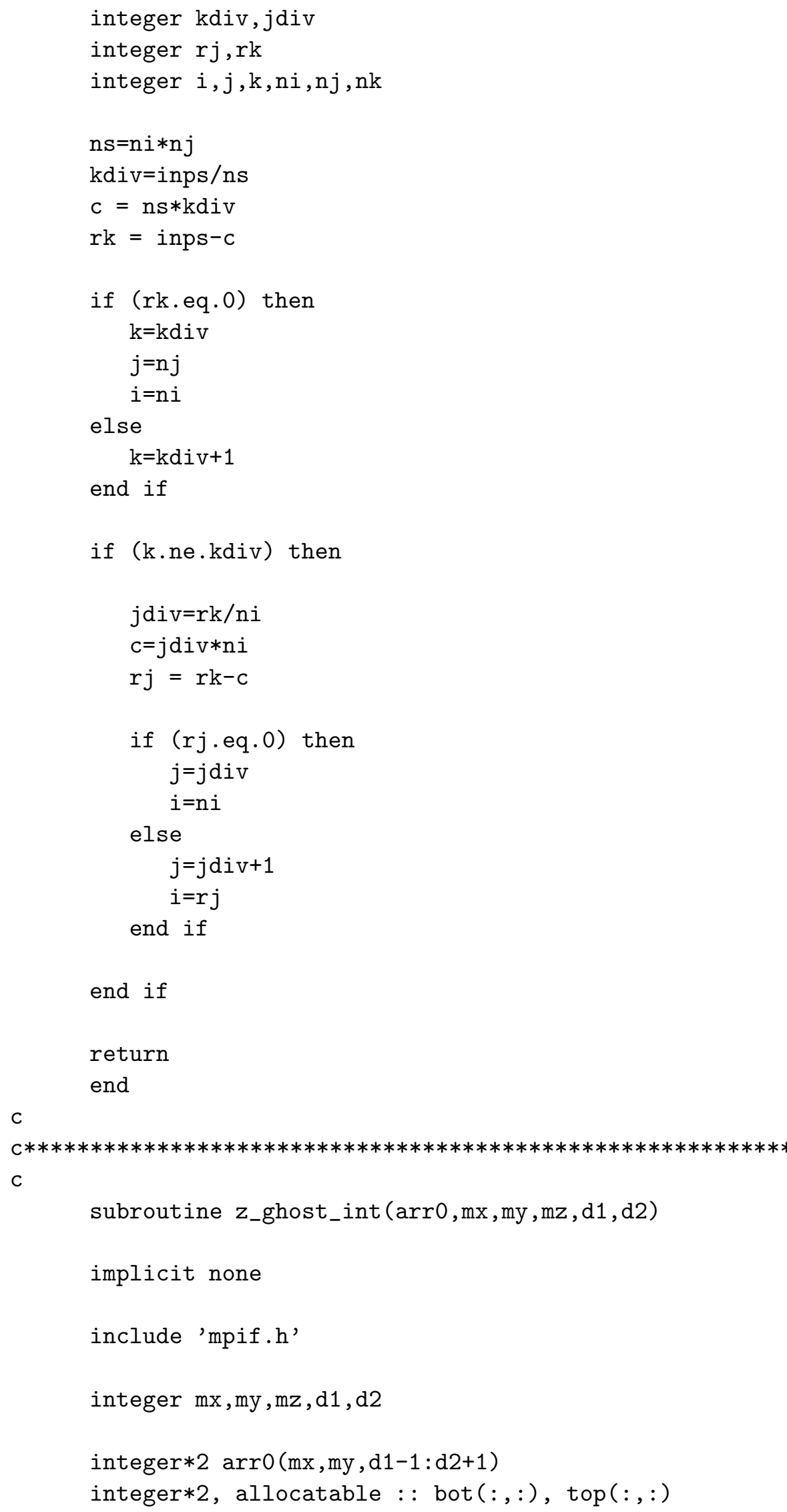


integer myrank, ierr, nprocs

integer status(MPI_STATUS_SIZE)

call MPI_COMM_RANK ( MPI_COMM_WORLD, myrank, ierr)

call MPI_COMM_SIZE( MPI_COMM_WORLD, nprocs, ierr)

C

c Make the Z Ghost

C

allocate (bot ( $m x, m y))$

allocate (top (mx,my))

C

c Get new bottom ghost plane.

C

bot $=\operatorname{arr} 0(:,:, \mathrm{d} 1)$

top $=\operatorname{arr} 0(:,:, d 2)$

call t2b(bot, top, mx,my)

$\operatorname{arr} 0(:,:, d 1-1)=\operatorname{bot}$

C

c Get new top ghost plane

c

bot $=\operatorname{arr} 0(:,:, \mathrm{d} 1)$

top $=\operatorname{arr} 0(:,:, d 2)$

call b2t (bot, top, mx,my)

$\operatorname{arr} 0(:,:, d 2+1)=$ top

deallocate (bot)

deallocate (top)

return

end

$\mathrm{C}$

c

C

subroutine z_ghost_dp (arr0,mx,my,mz,d1,d2)

implicit none

include 'mpif.h'

integer $\mathrm{mx}, \mathrm{my}, \mathrm{mz}, \mathrm{d} 1, \mathrm{~d} 2$

double precision $\operatorname{arr} 0(\mathrm{mx}, \mathrm{my}, \mathrm{d} 1-1: \mathrm{d} 2+1, \mathrm{mz})$

double precision, allocatable : $\operatorname{bot}(:,:,:)$, top $(:,:,:)$ 


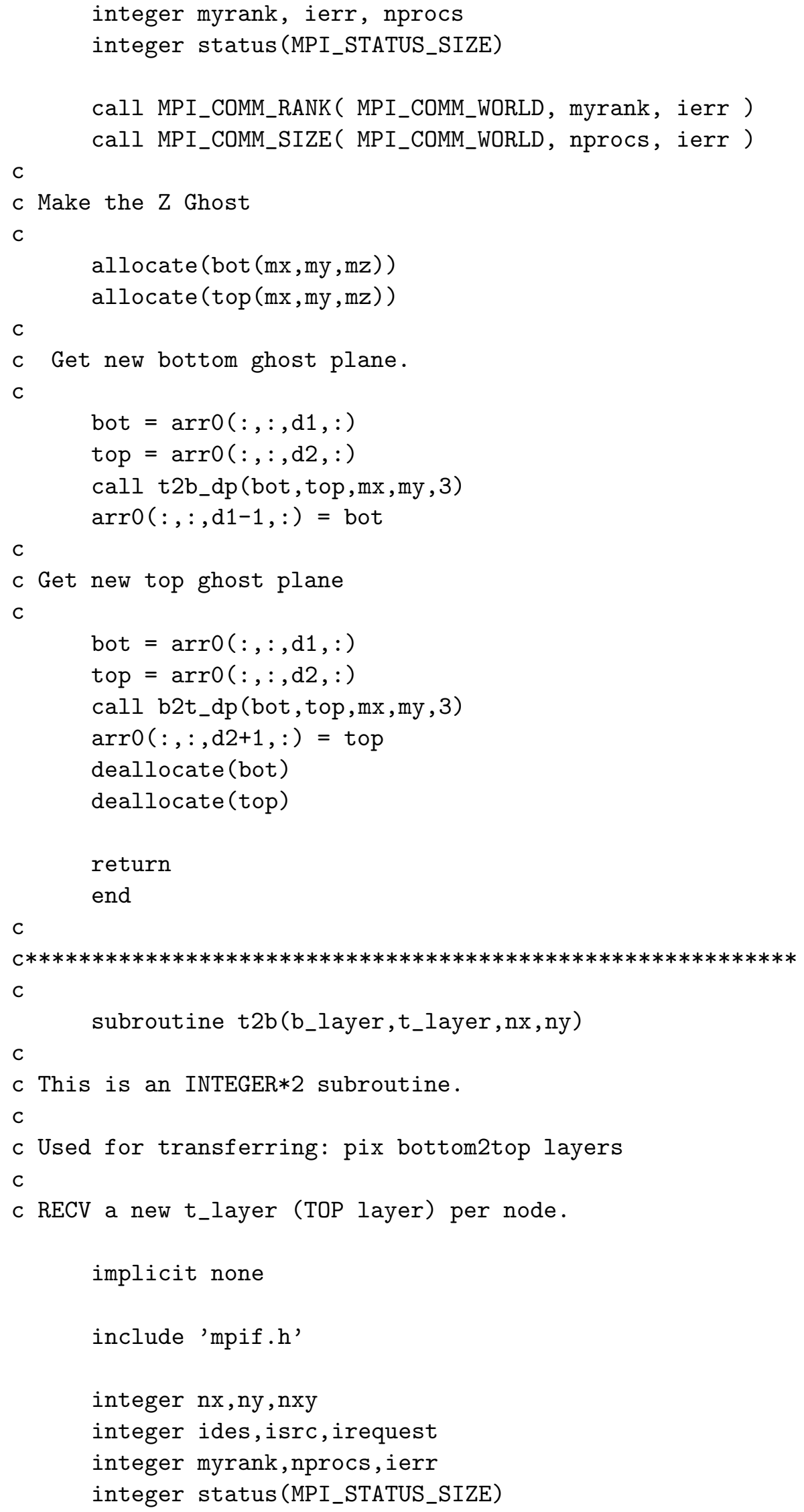




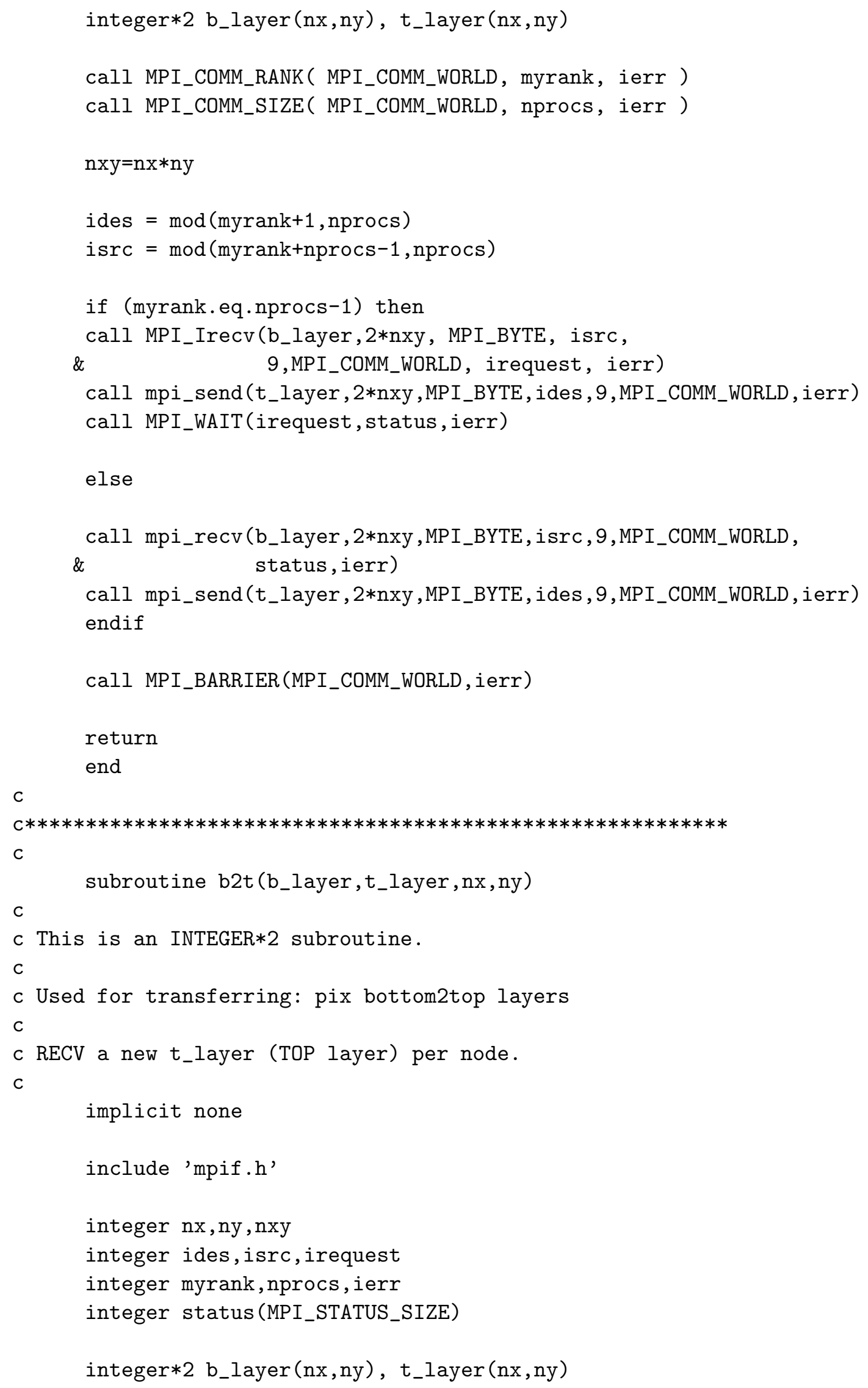




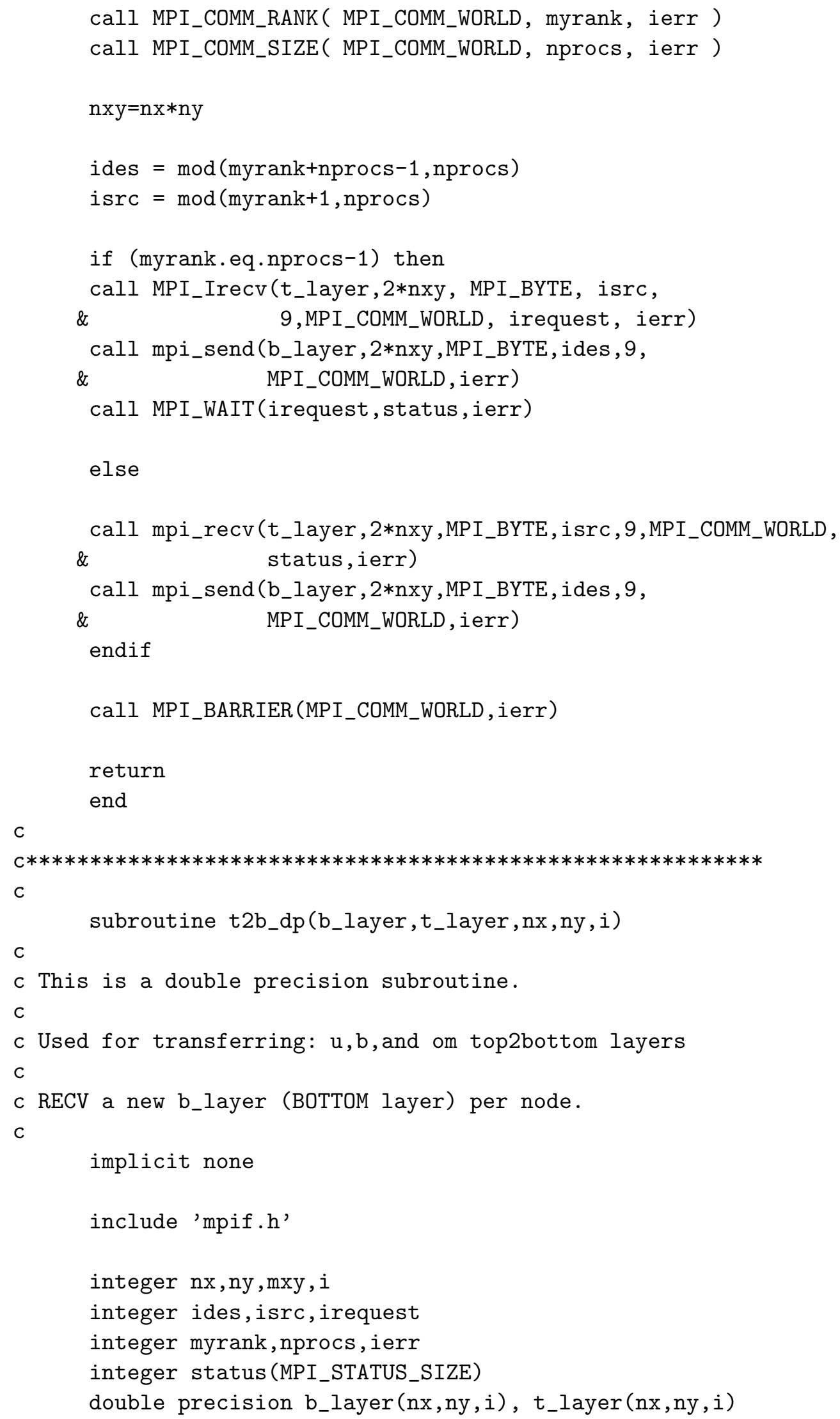




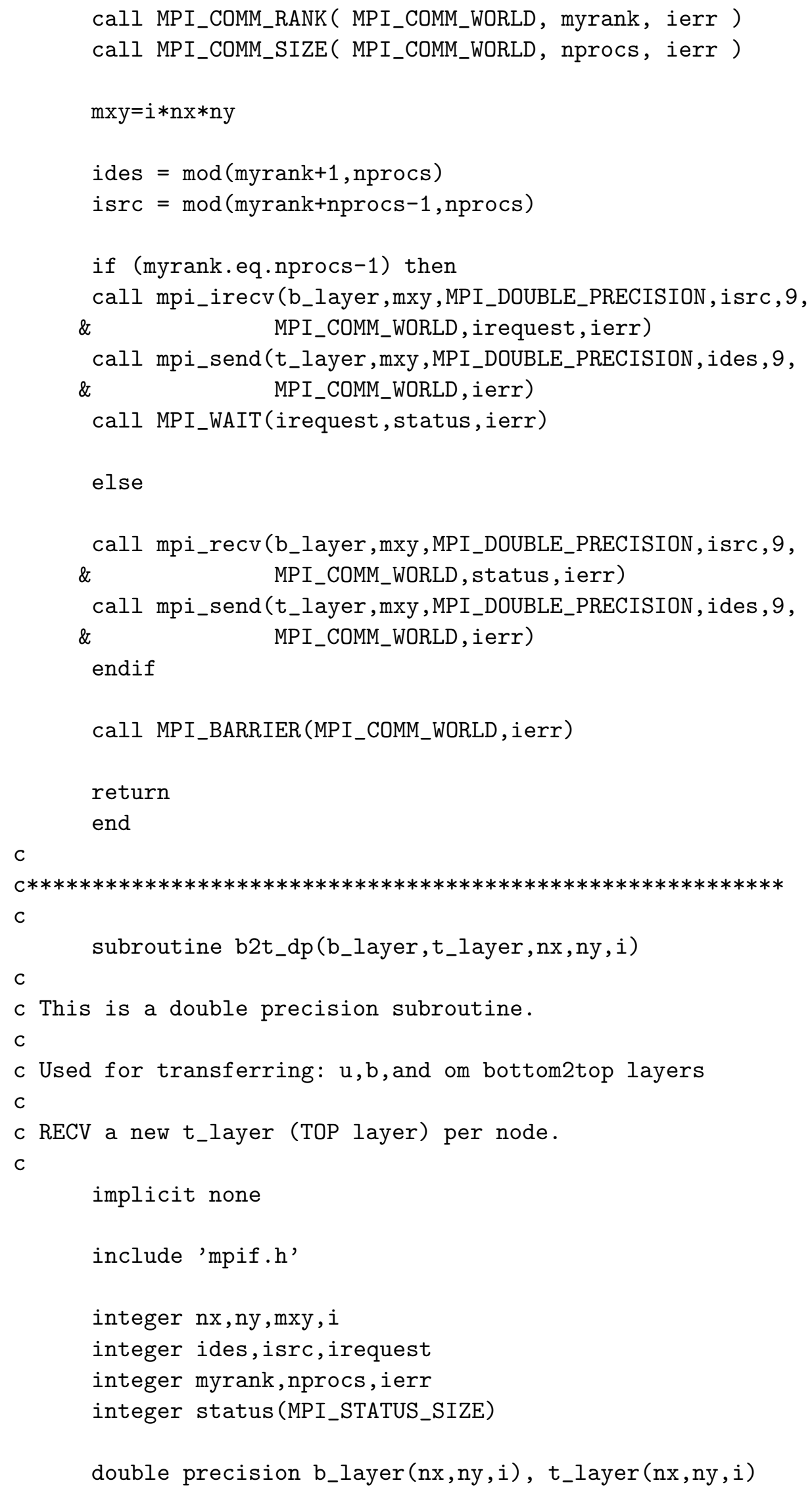




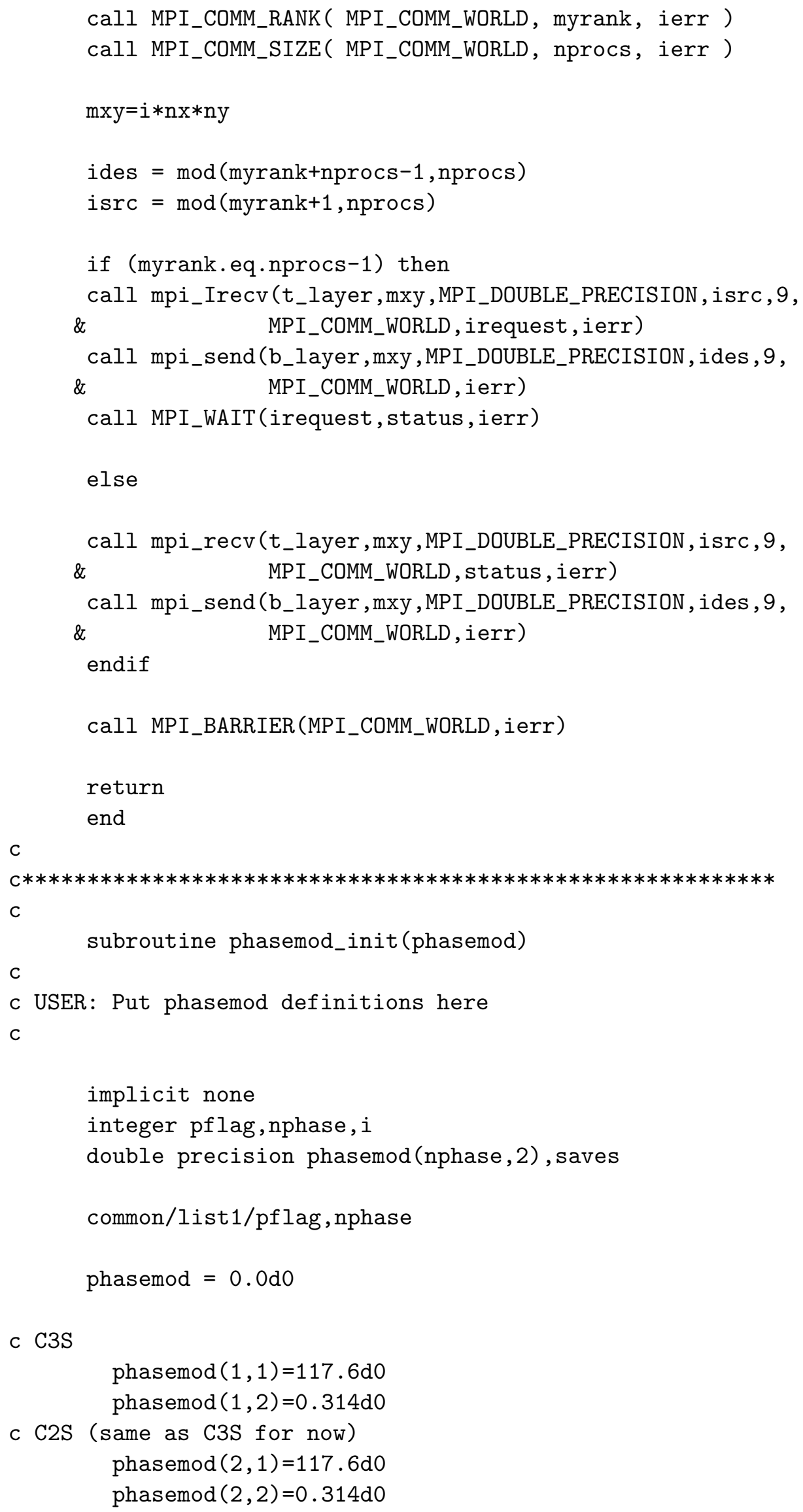


c C3A (same as C3S for now)

phasemod $(3,1)=117.6 \mathrm{~d} 0$

phasemod $(3,2)=0.314 \mathrm{~d} 0$

c C4AF (same as C3S for now)

phasemod $(4,1)=117.6 \mathrm{~d} 0$

phasemod $(4,2)=0.314 \mathrm{~d} 0$

c gypsum (use from paper with Sylvain)

phasemod $(5,1)=45.7 \mathrm{~d} 0$

phasemod $(5,2)=0.33 \mathrm{~d} 0$

c hemihydrate (same as gypsum for now)

phasemod $(6,1)=0.5 *(45.7 \mathrm{~d} 0+80.0 \mathrm{~d} 0)$

phasemod $(6,2)=0.5 *(0.33 \mathrm{~d} 0+0.275 \mathrm{~d} 0)$

$c$ anhydrite (same as gypsum for now)

phasemod $(7,1)=80.0 \mathrm{~d} 0$

phasemod $(7,2)=0.275 \mathrm{~d} 0$

c pozzolan (no pozzolan)

phasemod $(8,1)=0.0 \mathrm{~d} 0$

phasemod $(8,2)=0.0 \mathrm{~d} 0$

$c$ inert

phasemod $(9,1)=0.0 \mathrm{~d} 0$

phasemod $(9,2)=0.0 \mathrm{~d} 0$

c slag

phasemod $(10,1)=0.0 \mathrm{~d} 0$

phasemod $(10,2)=0.0 \mathrm{~d} 0$

c ASG flyash

phasemod $(11,1)=0.0 \mathrm{~d} 0$

phasemod $(11,2)=0.0 \mathrm{~d} 0$

c CAS2 fly ash

phasemod $(12,1)=0.0 \mathrm{~d} 0$

phasemod $(12,2)=0.0 \mathrm{~d} 0$

c $\mathrm{CH}$

phasemod $(13,1)=42.3 \mathrm{~d} 0$

phasemod $(13,2)=0.324 \mathrm{~d} 0$

c $\mathrm{C}-\mathrm{S}-\mathrm{H}$

phasemod $(14,1)=22.4 \mathrm{~d} 0$

phasemod $(14,2)=0.25 \mathrm{~d} 0$

c C3AH6 (same as C-S-H for now)

phasemod $(15,1)=\operatorname{phasemod}(14,1)$

phasemod $(15,2)=$ phasemod $(14,2)$

c ettringite (from C3A) (1/3 gypsum for now)

$\operatorname{phasemod}(16,1)=\operatorname{phasemod}(14,1)$

phasemod $(16,2)=\operatorname{phasemod}(14,2)$

$c$ ettringite (from $\mathrm{C} 4 \mathrm{AF}$ )

phasemod $(17,1)=\operatorname{phasemod}(16,1)$

phasemod $(17,2)=$ phasemod $(16,2)$

C Afm

phasemod $(18,1)=\operatorname{phasemod}(13,1)$

phasemod $(18,2)=$ phasemod $(13,2)$

c FH3 (same as C-S-H for now) 


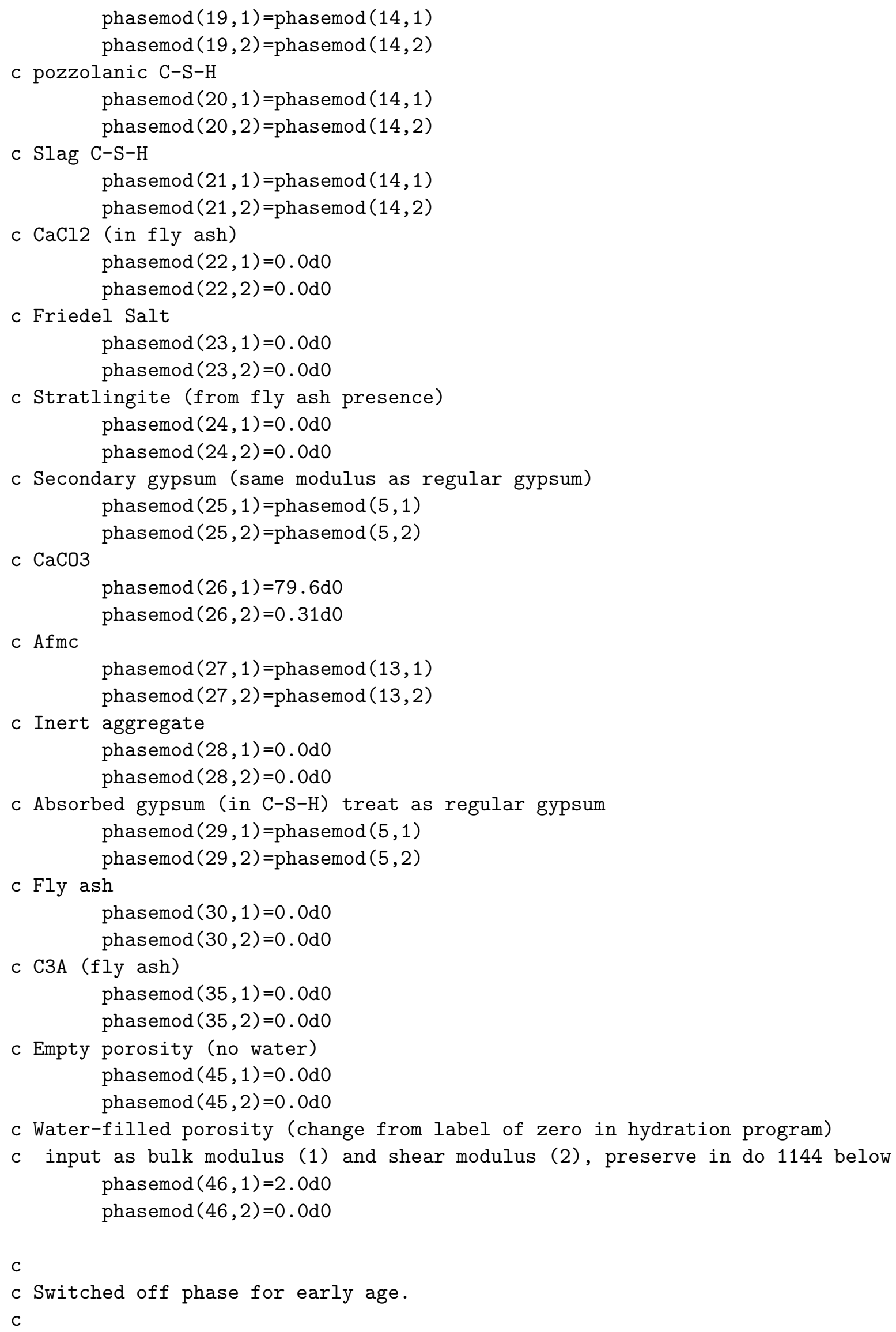




$$
\begin{aligned}
& \operatorname{phasemod}(88,1)=0.0 \mathrm{~d} 0 \\
& \operatorname{phasemod}(88,2)=0.0 \mathrm{~d} 0
\end{aligned}
$$

C

c USER: end of phasemod defs

C

c (USER) Program uses bulk modulus (1) and shear modulus (2), so transform

c Young's modulis (1) and Poisson's ratio (2).

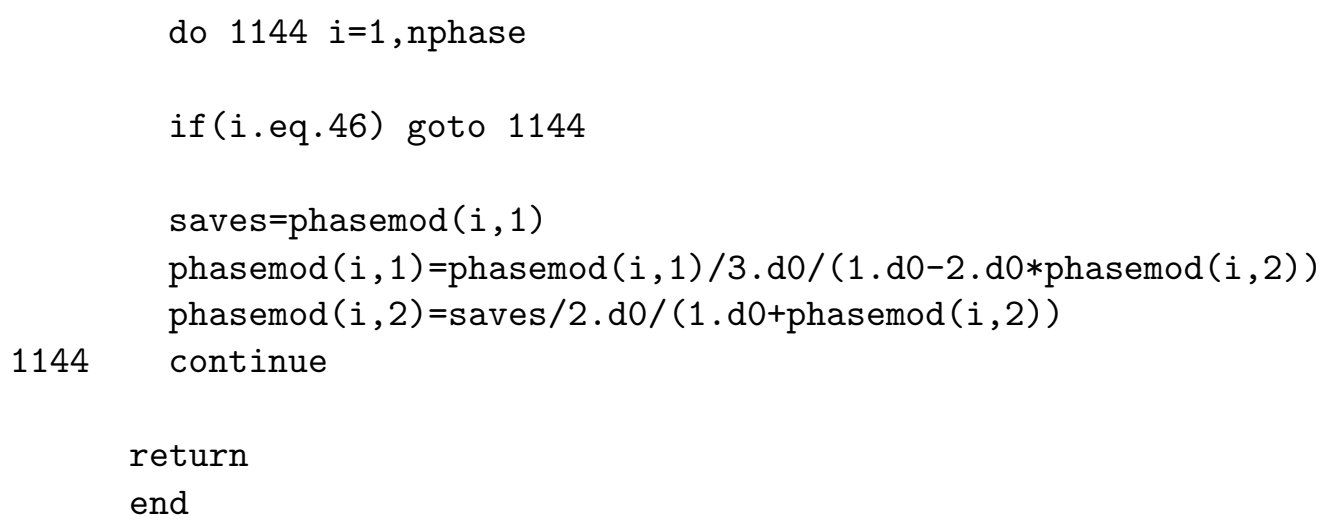




\subsubsection{THERMAL3D_MPI.f}

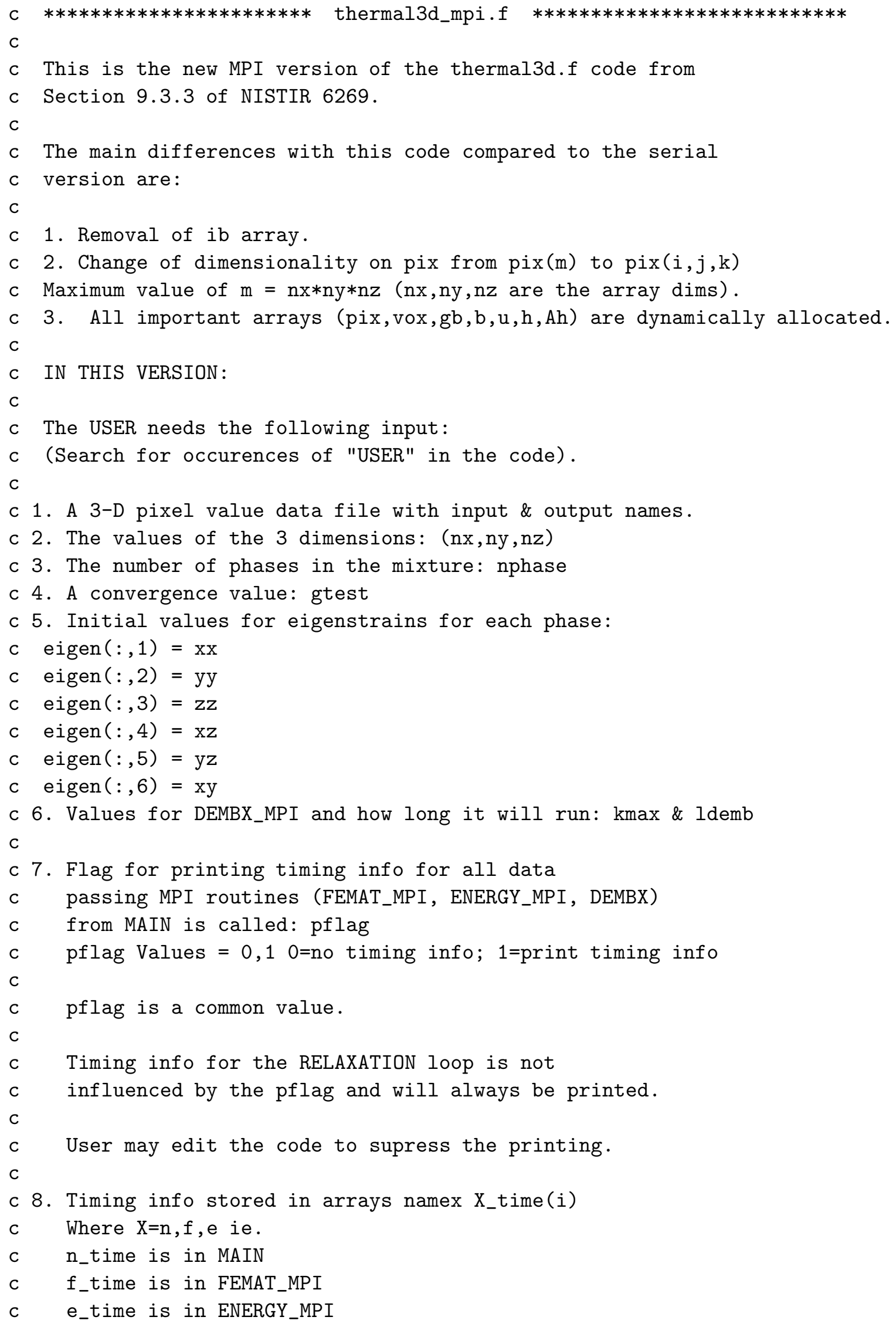


C

c NB: One also needs to insure that the values for

C phasemod $(i, j)$ are initialized correctly in

c SUBROUTINE phasemod_init.

c

c END of NEW comments.

$C$

BEGIN ORIGINAL comments.

BACKGROUND

$C$

c Program adjusts dimensions of unit cell,

c [(1 + macrostrain) times dimension],

c in response to phases that have a non-zero eigenstrain and arbitrary

c elastic moduli tensors.

c All six macrostrains can adjust their values (3-d program), and are

$c$ stored in the last two positions in the displacement vector $u$,

$c$ as listed below. Periodic boundaries are maintained.

c In the comments below, (USER) means that this is a section of code

c that the user might have to change for his particular problem.

c Therefore the user is encouraged to search for this string.

c PROBLEM AND VARIABLE DEFINITION

c The problem being solved is the minimization of the elastic energy

c $1 / 2 u A u+b u+C+T u+Y$, where $b$ and $C$ are also functions of the

c macrostrains.

c The small array zcon computes the thermal strain energy associated

$c$ with macrostrains ( $C$ term), $T$ is the thermal energy term linear in the

c displacements (built from ss), b is the regular energy term linear in the

$c \quad b$ is the regular energy term linear in the

c displacements, $u$ is the displacements including the macrostrains,

$c \mathrm{gb}$ is the energy gradient vector, $\mathrm{h}, \mathrm{Ah}$ are auxiliary vectors,

$c \mathrm{dk}$ is the single pixel stiffness matrix, pix is the phase

$c$ identification vector, and $i b$ is the

c integer matrix for mapping labels from the 1-27 nearest neighbor

c labelling to the 1-d system labelling.

c The array prob(i) contains the volume fractions of the $i$ 'th phase,

c strxx, etc. are the six independent (Voigt notation) volume

c averaged stresses, sxx, etc. are the six independent (Voigt notation)

c volume averaged strains (not counting the thermal strains).

c The variable cmod(i,6,6) gives the elastic moduli tensor

$c$ of the $i$ 'th phase, eigen( $i, 6)$ gives the six independent elements

$c$ of the eigenstrain tensor for the $i$ 'th phase (Voigt notation)

$c$ and $\operatorname{dk}(i, 8,3,8,3)$ is the stiffness matrix of the $i$ 'th

$c$ phase. The parameter nphase gives the number of phases being considered

$c$ in the problem, and is set by the user. 


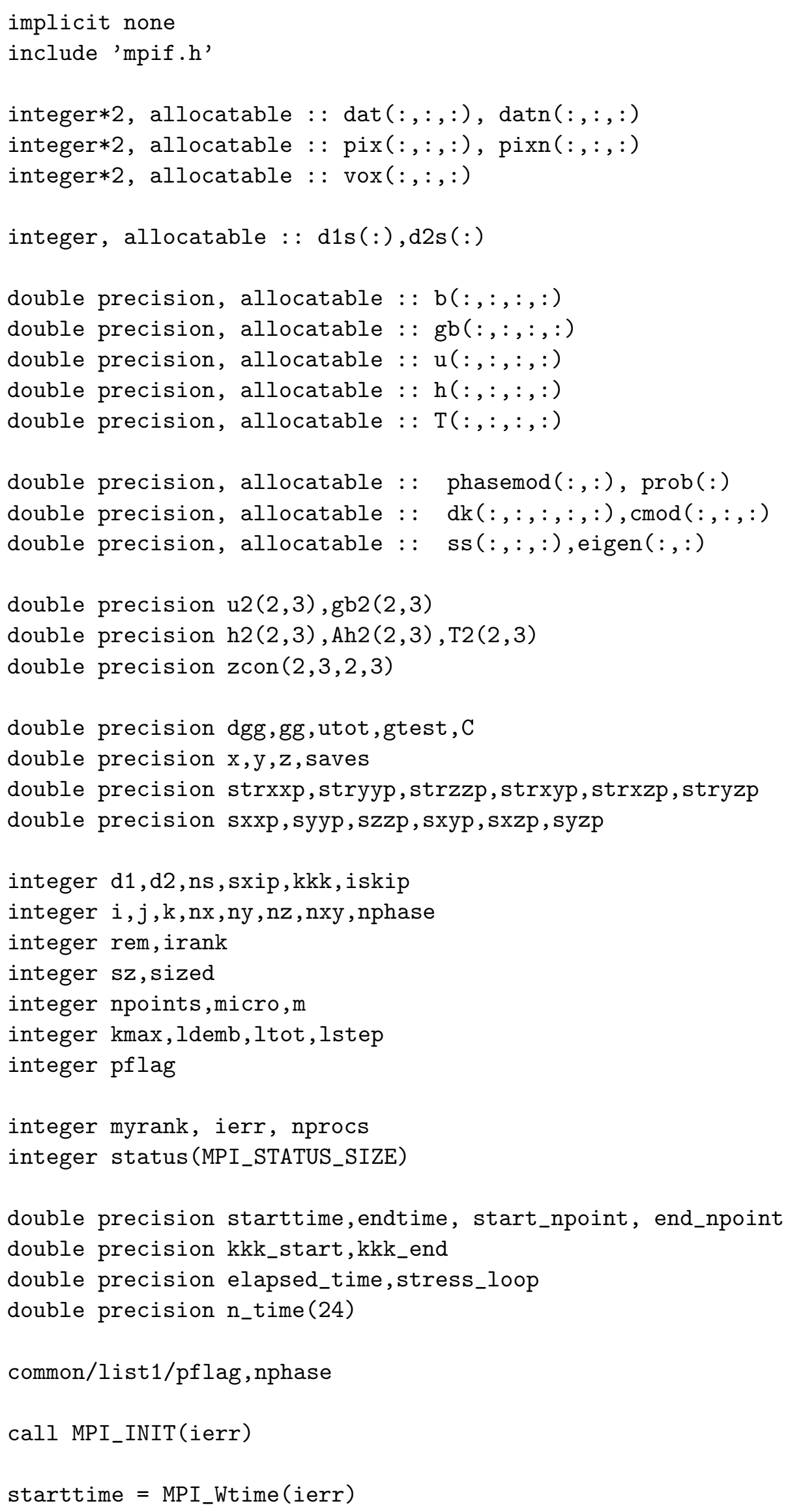


call MPI_COMM_RANK ( MPI_COMM_WORLD, myrank, ierr )

call MPI_COMM_SIZE( MPI_COMM_WORLD, nprocs, ierr)

if (myrank.eq.0) then

write $(*, *)$ "There are ",nprocs," processors running this job." end if

pflag $=0$

c (USER) $\mathrm{nx}, \mathrm{ny}, \mathrm{nz}$ are the size of the lattice

$\mathrm{nx}=200$

ny $=200$

$\mathrm{nz}=200$

c ns=total number of sites

$\mathrm{ns}=\mathrm{nx} * \mathrm{ny} * \mathrm{nz}$

$\mathrm{nxy}=\mathrm{nx} * \mathrm{ny}$

$\mathrm{sz}=\mathrm{nz} / \mathrm{nprocs}$

c (USER) nphase is the number of phases being considered in the problem.

c The values of pix(m) will run from 1 to nphase.

nphase $=3$

c (USER) gtest is the stopping criterion, compared to gg=gb*gb.

c If gtest=abc*ns, when gg < gtest, the rms value per pixel

$c$ of $\mathrm{gb}$ is less than sqrt(abc)

gtest $=1 . d-8 *$ ns

C

c Calculate d1 \& d2 limits for each node.

c Then ROOT passes these values to workers.

c

if (myrank.eq.0) then

allocate (d1s (0:nprocs-1))

allocate (d2s (0:nprocs-1))

do irank $=0$, nprocs -1

d1s $($ irank $)=i r a n k * s z+1$

d2s $($ irank $)=($ irank +1$) *$ sz

end do

rem $=\mathrm{nz}-$ nprocs*sz

if (rem.ne.0) then

do $j=1$,rem

irank $=$ nprocs $-r e m+j-1$ 
1144 continue

c (USER) input eigenstrains for each phase

c $\quad(1=x x, 2=y y, 3=z z, 4=x z, 5=y z, 6=x y)$.

allocate (eigen (nphase, 6))

eigen $(1,1)=0.1 \mathrm{~d} 0$

eigen $(1,2)=0.1 \mathrm{~d} 0$

eigen $(1,3)=0.1 \mathrm{~d} 0$

eigen $(1,4)=0 \cdot d 0$

eigen $(1,5)=0 \cdot d 0$

eigen $(1,6)=0 \cdot d 0$

eigen $(2,1)=0.2 \mathrm{~d} 0$

eigen $(2,2)=0.2 \mathrm{~d} 0$

eigen $(2,3)=0.2 \mathrm{~d} 0$

eigen $(2,4)=0 . d 0$

eigen $(2,5)=0 \cdot d 0$

eigen $(2,6)=0 \cdot d 0$

eigen $(3,1)=0 \cdot d 0$

eigen $(3,2)=0 \cdot d 0$

eigen $(3,3)=0 \cdot d 0$

eigen $(3,4)=0 \cdot d 0$

eigen $(3,5)=0 \cdot d 0$

eigen $(3,6)=0 \cdot d 0$

C

c Allocate other arrays which need d1\&d2 values.

C

allocate (gb $(n x, n y, d 1-1: d 2+1,3))$

$\mathrm{gb}=0.0 \mathrm{~d} 0$

allocate (b (nx, ny , d1 $-1: d 2+1,3)$ )

$\mathrm{b}=0.0 \mathrm{~d} 0$

allocate ( $(\mathrm{nx}, \mathrm{ny}, \mathrm{d} 1-1: \mathrm{d} 2+1,3))$

$\mathrm{T}=0.0 \mathrm{~d} 0$

$\mathrm{gb} 2=0.0 \mathrm{~d} 0$

$\mathrm{T} 2=0.0 \mathrm{~d} 0$

allocate ( $\mathrm{u}(\mathrm{nx}, \mathrm{ny}, \mathrm{d} 1-1: \mathrm{d} 2+1,3))$

allocate $(h(n x, n y, d 1-1: d 2+1,3))$

c Compute the average stress and strain, as well as the macrostrains (overall

c system size and shape) in each microstructure.

c (USER) npoints is the number of microstructures to use.

npoints $=1$

n_time (1) $=$ MPI_Wtime (ierr)

do 8000 micro=1, npoints 
C

c Allocate pix, so root can read it.

C

if (myrank.eq.0) then

allocate $(\operatorname{pix}(n x, n y, n z))$

end if

$$
\begin{aligned}
& \text { start_npoint=MPI_Wtime(ierr) } \\
& \text { n_time(2) = MPI_Wtime(ierr) }
\end{aligned}
$$

if (myrank.eq.0) then

C

c Get pix from the input file (unit=9).

C

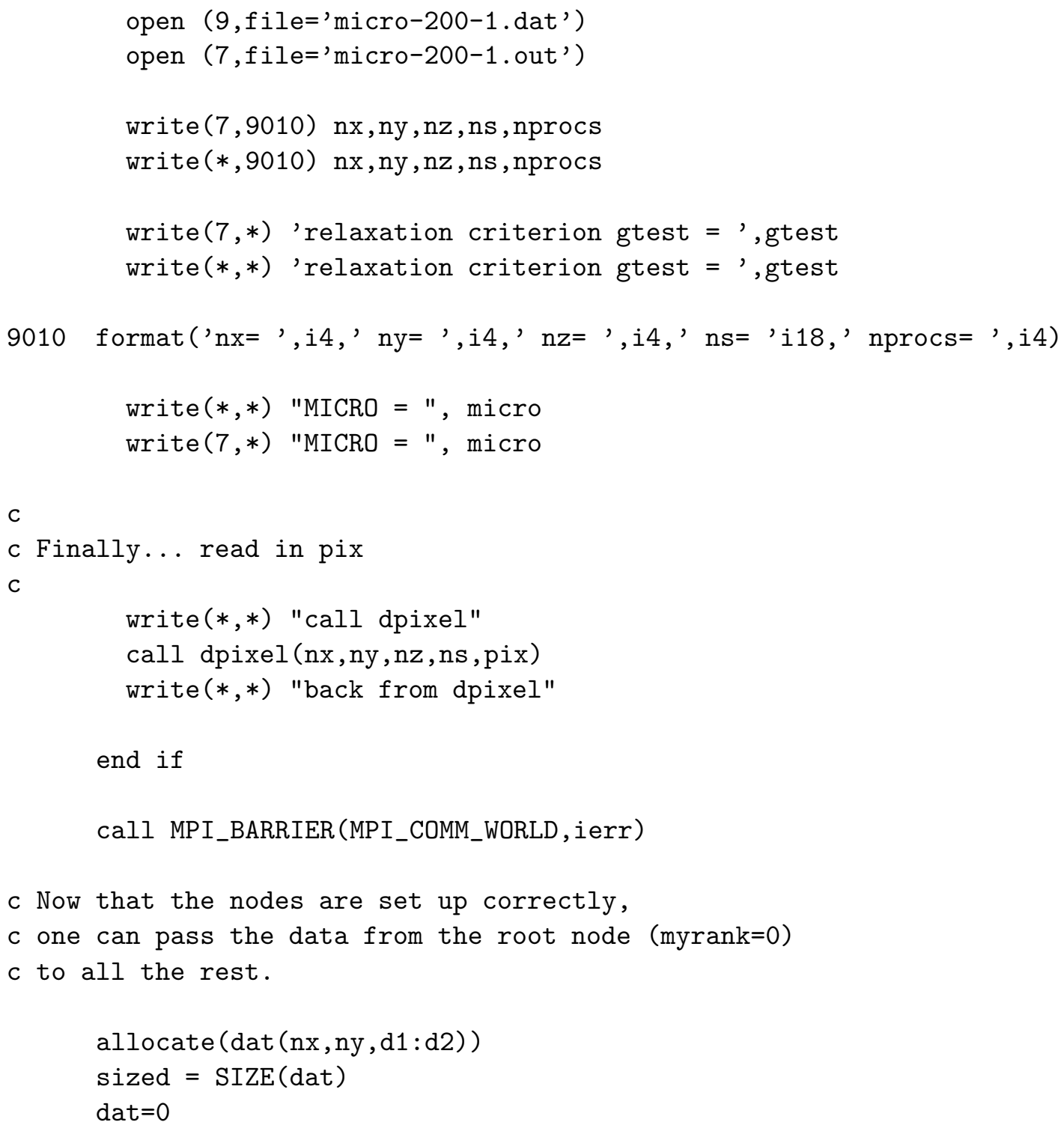




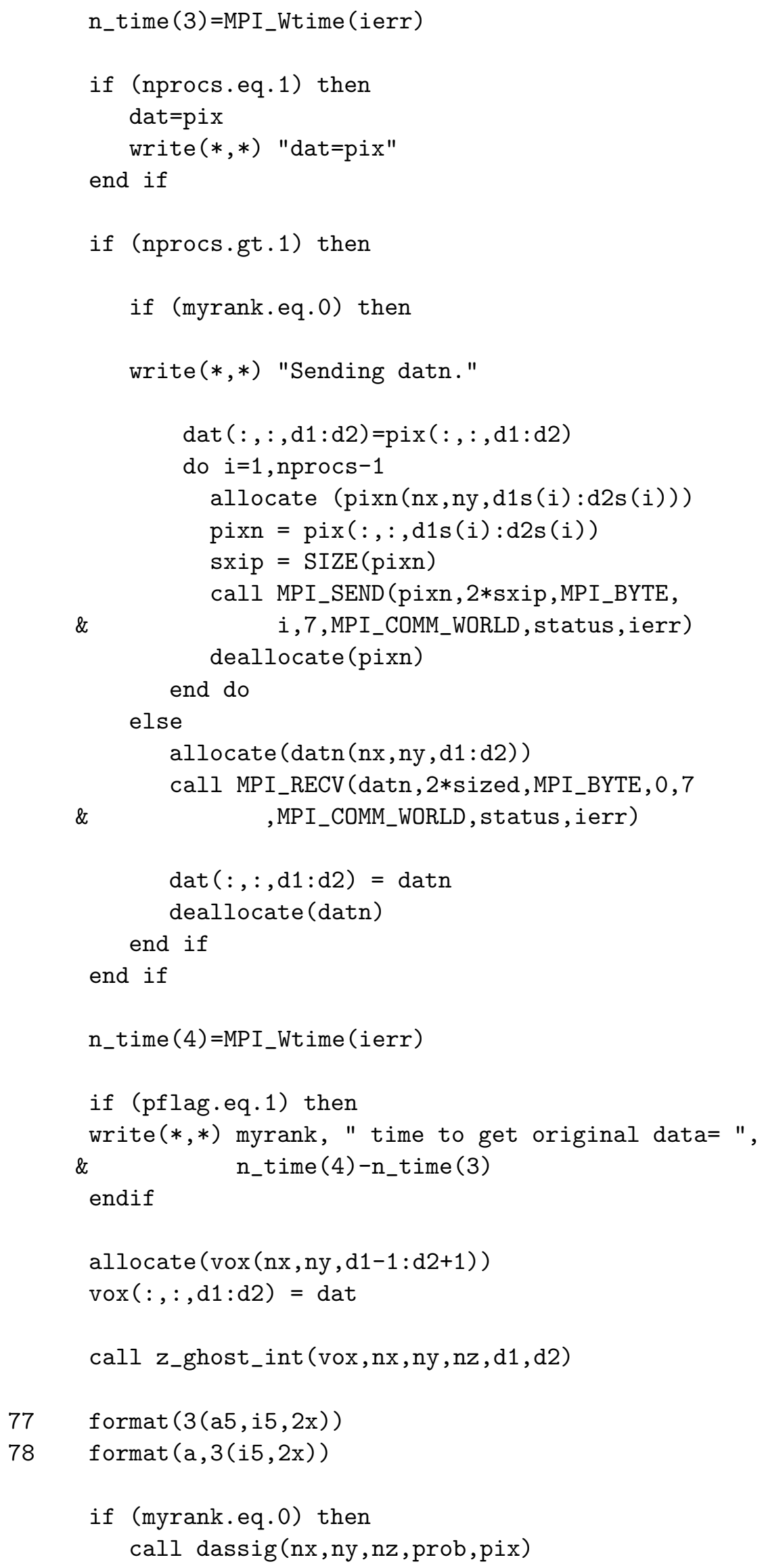




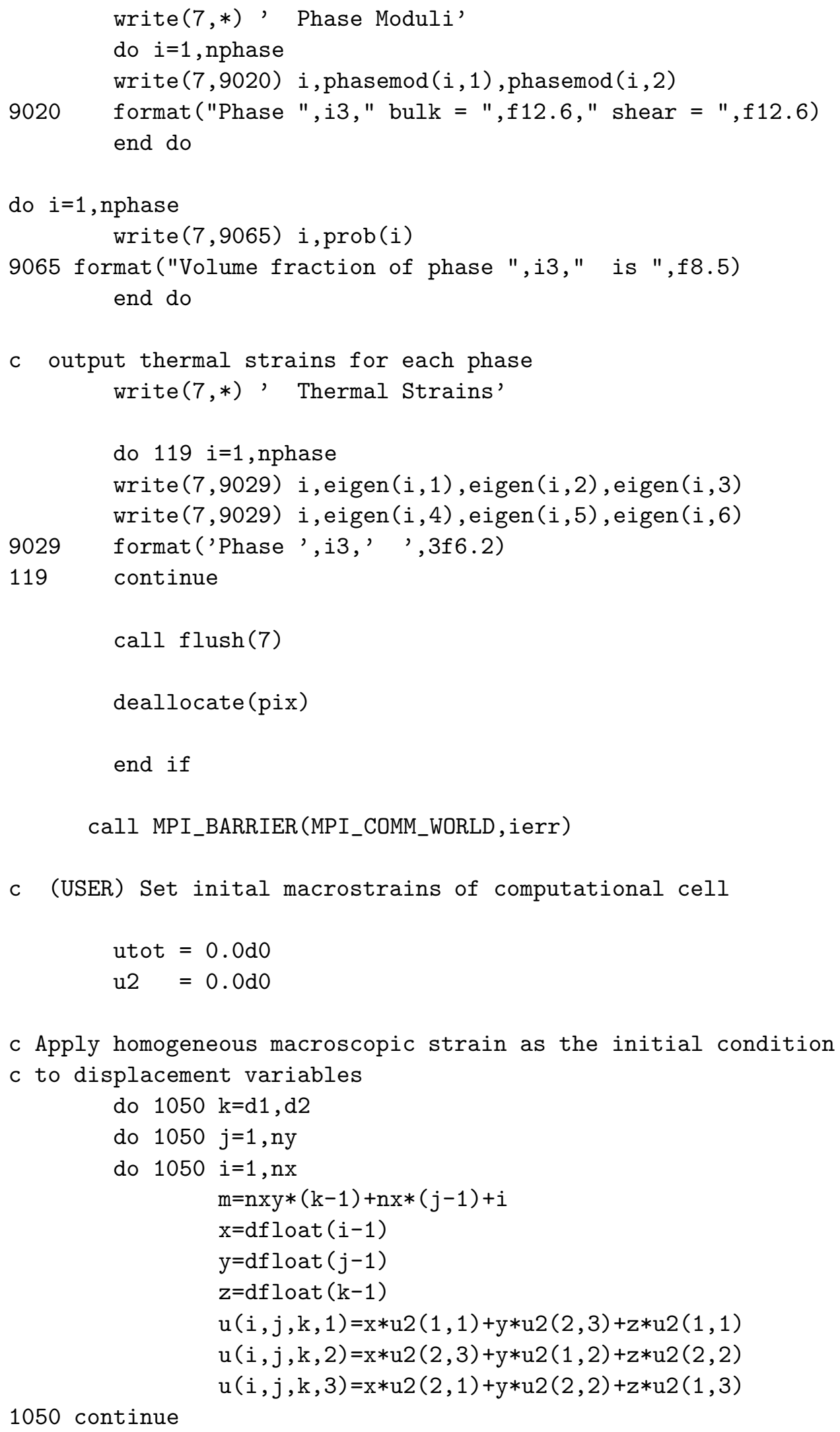

c Apply homogeneous macroscopic strain as the initial condition c to displacement variables

do $1050 \mathrm{k}=\mathrm{d} 1, \mathrm{~d} 2$

do $1050 j=1$, ny

do $1050 i=1, n x$

$m=n x y *(k-1)+n x *(j-1)+i$

$\mathrm{x}=\mathrm{dfloat}(\mathrm{i}-1)$

$y=\operatorname{dfloat}(j-1)$

$\mathrm{z}=\operatorname{dfloat}(\mathrm{k}-1)$

$\mathrm{u}(\mathrm{i}, \mathrm{j}, \mathrm{k}, 1)=\mathrm{x} * \mathrm{u} 2(1,1)+\mathrm{y} * \mathrm{u} 2(2,3)+\mathrm{z} * \mathrm{u} 2(1,1)$

$\mathrm{u}(\mathrm{i}, \mathrm{j}, \mathrm{k}, 2)=\mathrm{x} * \mathrm{u} 2(2,3)+\mathrm{y} * \mathrm{u} 2(1,2)+\mathrm{z} * \mathrm{u} 2(2,2)$

$\mathrm{u}(\mathrm{i}, \mathrm{j}, \mathrm{k}, 3)=\mathrm{x} * \mathrm{u} 2(2,1)+\mathrm{y} * \mathrm{u} 2(2,2)+\mathrm{z} * \mathrm{u} 2(1,3)$

1050 continue

call z_ghost_dp (u, nx, ny , 3, d1, d2) 
c Set up the finite element stiffness matrices, the constant, C,

$c$ the vector, $b$, required for the energy. $b$ and $C$ depend on the macrostrains.

$c$ When they are updated, the values of $\mathrm{b}$ and $\mathrm{C}$ are updated too via

c calling subroutine femat.

c Only compute the thermal strain terms the first time femat is called,

c (iskip=0) as they are unaffected by later changes (iskip=1) in

c displacements and macrostrains.

c Compute initial value of gradient $g b$ and $g g=g b * g b$.

iskip $=0$

$\mathrm{Y}=0.0 \mathrm{~d} 0$

$\mathrm{gg}=0.0 \mathrm{~d} 0$

call femat_mpi2 (nx, ny, nz, phasemod, d1, d2, vox,b,

\& dk, C, cmod, zcon, u, u2, T, T2, eigen, ss, iskip, Y)

call energy_mpi2 ( $n x, n y, n z, d 1, d 2, C$, utot, $Y$, vox ,

$\&$

$\mathrm{dk}, \mathrm{u}, \mathrm{b}, \mathrm{gb}, \mathrm{T}, \mathrm{zcon}, \mathrm{T} 2, \mathrm{u} 2, \mathrm{gb} 2$ )

$\mathrm{dgg}=0.0 \mathrm{~d} 0$

$\mathrm{gg}=0.0 \mathrm{~d} 0$

$\operatorname{dgg}=\operatorname{SUM}(g b(:,:, \mathrm{d} 1: \mathrm{d} 2,:) * g b(:,:, \mathrm{d} 1: \mathrm{d} 2,:))$

call MPI_ALLREDUCE (dgg, gg, 1,MPI_DOUBLE_PRECISION,

\&

MPI_SUM, MPI_COMM_WORLD, ierr)

$$
g g=g g+\operatorname{SUM}(g b 2 * g b 2)
$$

if (myrank.eq.0) then

write $(7,9042)$ utot,gg

write $(*, 9042)$ utot,gg

call flush (7)

9042 format (' energy $=$ ',d15.8,' $g g=$ ',d15.8)

end if

$\mathrm{kmax}=40$

1 demb $=100$

ltot $=0$

iskip $=1$

do $5000 \mathrm{kkk}=1, \mathrm{kmax}$

c Call dembx to implement conjugate gradient routine

call dembx_mpi2 (nx, ny, nz, ns, Lstep, gg, gtest, ldemb, kkk,

$\&$

zcon, u, h, gb , u2 , h2 , gb2, b , d1 , d2, vox, dk)

ltot=ltot+Lstep 
c Call energy to compute energy after dembx call. If gg < gtest, this

c will be the final energy. If $g g$ is still larger than gtest, then this

c will give an intermediate energy with which to check how the

c relaxation process is coming along. The call to energy does not

$c$ change the gradient or the value of $\mathrm{gg}$.

c Need to first call femat to update the vector $b$, as the value of the

$c$ components of $\mathrm{b}$ depend on the macrostrains.

call femat_mpi2 (nx, ny, nz, phasemod, d1, d2, vox, b,

\& $\mathrm{dk}, \mathrm{C}, \mathrm{cmod}, \mathrm{zcon}, \mathrm{u}, \mathrm{u} 2, \mathrm{~T}, \mathrm{~T} 2$, eigen, ss, iskip, $\mathrm{Y}$ )

$$
\begin{aligned}
& \text { call energy_mpi2(nx, ny, nz, d1, d2, C, utot, Y, vox, } \\
& \begin{array}{l}
\text { \& } \\
\mathrm{dk}, \mathrm{u}, \mathrm{b}, \mathrm{gb}, \mathrm{T}, \mathrm{zcon}, \mathrm{T} 2, \mathrm{u} 2, \mathrm{gb} 2)
\end{array}
\end{aligned}
$$

\&

if (myrank.eq.0) then

write $(7,9043)$ utot,gg, ltot

write $(*, 9043)$ utot,gg,ltot

9043 format (' energy $=$ ', d15.8,' $\mathrm{gg}=$ ', $\mathrm{d} 15.8,{ }^{\prime}$ ltot $=$ ', i6)

call flush(7)

end if

c If relaxation process is finished, jump out of loop

if (gg.lt.gtest) goto 444

c Output stresses, strains, and macrostrains as an additional aid in judging

c how well the relaxation process is proceeding.

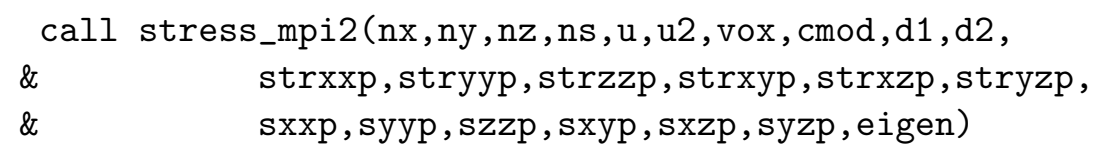

if (myrank.eq.0) then

write $(7, *)$ ' stresses: $\mathrm{xx}, \mathrm{yy}, \mathrm{zz}, \mathrm{xz}, \mathrm{yz}, \mathrm{xy}$ '

write $(7, *)$ strxxp, stryyp, strzzp, strxzp, stryzp, strxyp

write $(7, *)$ ' strains: $\mathrm{xx}, \mathrm{yy}, \mathrm{zz}, \mathrm{xz}, \mathrm{yz}, \mathrm{xy}$ '

write $(7, *)$ sxxp, syyp, szzp, sxzp, syzp, sxyp

write $(7, *)$ ' macrostrains in same order'

write $(7, *)$ u2 $(1,1)$, u2 $(1,2)$, u2 $(1,3)$

write $(7, *)$ u2 $(2,1), \mathrm{u} 2(2,2), \mathrm{u} 2(2,3)$

write $(7, *)$ 'avg $=$ ', $(u 2(1,1)+u 2(1,2)+u 2(1,3)) / 3.0 d 0$

end if

5000 continue

444 call stress_mpi2 (nx, ny, nz, ns, u, u2, vox, cmod, d1, d2,

\& $\quad$ strxxp, stryyp, strzzp, strxyp, strxzp, stryzp, 
if (myrank.eq.0) then

write $(7, *)$ ' stresses: $\mathrm{xx}, \mathrm{yy}, \mathrm{zz}, \mathrm{xz}, \mathrm{yz}, \mathrm{xy}$ '

write $(7, *)$ strxxp, stryyp, strzzp, strxzp, stryzp, strxyp

write $(7, *)$ ' strains: $x x, y y, z z, x z, y z, x y '$

write $(7, *)$ sxxp, syyp, szzp, sxzp, syzp, sxyp

write $(7, *)$ ' macrostrains in same order'

write $(7, *)$ u2 $(1,1)$, u2 $(1,2)$, u2 $(1,3)$

write $(7, *)$ u2 $(2,1)$, u2 $(2,2), \mathrm{u} 2(2,3)$

write $(7, *)$ ' avg $=$ ', $(\mathrm{u} 2(1,1)+\mathrm{u} 2(1,2)+\mathrm{u} 2(1,3)) / 3.0 \mathrm{~d} 0$

end if

deallocate (vox)

8000

continue

C

c Do another calculation using loop var: npoints

c

deallocate (u)

deallocate (b)

deallocate $(\mathrm{gb})$

deallocate $(\mathrm{h})$

deallocate $(\mathrm{T})$

CALL MPI_FINALIZE (ierr)

end

$\mathrm{C}$

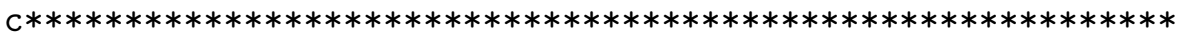

$\mathrm{C}$

subroutine femat_mpi2( $\mathrm{nx}, \mathrm{ny}, \mathrm{nz}$, phasemod,d1, d2, vox, b,

\& dk, C, cmod, zcon, u, u2, T, T2, eigen, ss, iskip, Y)

c

c Subroutine sets up the stiffness matrices, the linear term in the

c regular displacements, b, and the constant term, C, which come from

c the periodic boundary conditions, the term linear in the displacments,

c $T$, that comes from the thermal strains, and the constant term $Y$.

c

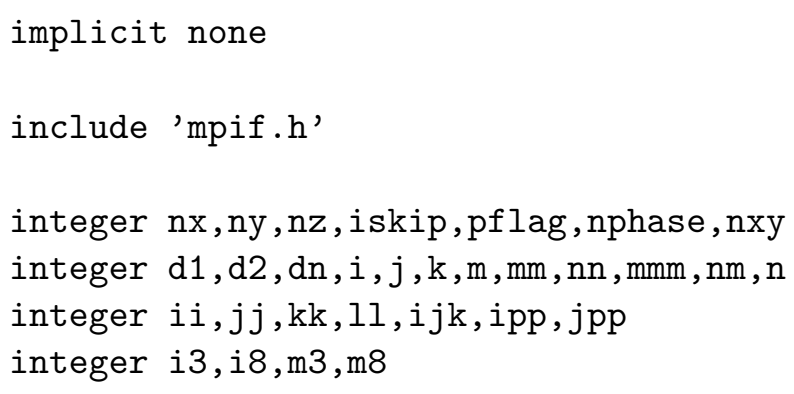


integer ipx,ipy,ipz

double precision sum_num, $\mathrm{x}, \mathrm{y}, \mathrm{z}, \mathrm{C}$

double precision yt,yterm, yneg, ypos, t2temp

double precision exx, eyy, ezz, exz, eyz, exy

integer*2 $\operatorname{vox}(\mathrm{nx}, \mathrm{ny}, \mathrm{d} 1-1: \mathrm{d} 2+1)$

double precision $u(n x, n y, d 1-1: d 2+1,3)$, u2 $(2,3)$

double precision $b(n x, n y, d 1-1: d 2+1,3)$

double precision $\mathrm{T}(\mathrm{nx}, \mathrm{ny}, \mathrm{d} 1-1: \mathrm{d} 2+1,3), \mathrm{T} 2(2,3)$

double precision dk(nphase, $8,3,8,3$ ), phasemod (nphase, 2)

double precision cmod(nphase,6,6), eigen(nphase,6)

double precision ss (nphase, 8,3)

double precision $\operatorname{dndx}(8), \operatorname{dndy}(8), \operatorname{dndz}(8)$

double precision $\mathrm{g}(3,3,3), \mathrm{ck}(6,6), \mathrm{cmu}(6,6)$

double precision es $(6,8,3), z \operatorname{zcon}(2,3,2,3)$, delta $(8,3)$

double precision, allocatable $:: a b(:,:,:)$, ba $(:,:,:)$

double precision contr

C

c MPI VARIABLES

C

integer myrank,nprocs,ierr, status (MPI_STATUS_SIZE)

common/list1/pflag, nphase

call MPI_COMM_RANK ( MPI_COMM_WORLD, myrank, ierr)

call MPI_COMM_SIZE( MPI_COMM_WORLD, nprocs, ierr )

$\mathrm{nxy}=\mathrm{nx} * \mathrm{ny}$

allocate (ab (nx, ny, 3))

allocate (ba $(n x, n y, 3))$

C Generate dk, zcon, $T$, and $Y$ on first pass. After that they are

$c$ constant, since they are independent of the macrostrains. Only b gets

c upgraded as the macrostrains change.

c Line number 1221 is the routine for b.

if (iskip.eq.1) goto 1221

c initialize stiffness matrices 


\section{$\mathrm{dk}=0.0 \mathrm{~d} 0$}

c initialize zcon matrix (gives $\mathrm{C}$ term for arbitrary macrostrains)

$\mathrm{zcon}=0.0 \mathrm{~d} 0$

c (USER) An anisotropic elastic moduli tensor could be input at this point, c bypassing this part, which assumes isotropic elasticity, so that there $c$ are only two independent numbers making up the elastic moduli tensor, $c$ the bulk modulus $\mathrm{K}$ and the shear modulus $\mathrm{G}$.

c Set up elastic moduli matrices for each kind of element

c ck and cmu are the bulk modulus and shear modulus matrices, which

c need to multiplied by the actual bulk and shear moduli in each phase.

$$
\begin{aligned}
& \operatorname{ck}(1,1)=1.0 \mathrm{~d} 0 \\
& \operatorname{ck}(1,2)=1.0 \mathrm{~d} 0 \\
& \operatorname{ck}(1,3)=1.0 \mathrm{~d} 0 \\
& \operatorname{ck}(1,4)=0.0 \mathrm{~d} 0 \\
& \operatorname{ck}(1,5)=0.0 \mathrm{~d} 0 \\
& \operatorname{ck}(1,6)=0.0 \mathrm{~d} 0 \\
& \operatorname{ck}(2,1)=1.0 \mathrm{~d} 0 \\
& \operatorname{ck}(2,2)=1.0 \mathrm{~d} 0 \\
& \operatorname{ck}(2,3)=1.0 \mathrm{~d} 0 \\
& \operatorname{ck}(2,4)=0.0 \mathrm{~d} 0 \\
& \operatorname{ck}(2,5)=0.0 \mathrm{~d} 0 \\
& \operatorname{ck}(2,6)=0.0 \mathrm{~d} 0 \\
& \operatorname{ck}(3,1)=1.0 \mathrm{~d} 0 \\
& \operatorname{ck}(3,2)=1.0 \mathrm{~d} 0 \\
& \operatorname{ck}(3,3)=1.0 \mathrm{~d} 0 \\
& \operatorname{ck}(3,4)=0.0 \mathrm{~d} 0 \\
& \operatorname{ck}(3,5)=0.0 \mathrm{~d} 0 \\
& \operatorname{ck}(3,6)=0.0 \mathrm{~d} 0 \\
& \operatorname{ck}(4,1)=0.0 \mathrm{~d} 0 \\
& \operatorname{ck}(4,2)=0.0 \mathrm{~d} 0 \\
& \operatorname{ck}(4,3)=0.0 \mathrm{~d} 0 \\
& \operatorname{ck}(4,4)=0.0 \mathrm{~d} 0 \\
& \operatorname{ck}(4,5)=0.0 \mathrm{~d} 0 \\
& \operatorname{ck}(4,6)=0.0 \mathrm{~d} 0 \\
& \operatorname{ck}(5,1)=0.0 \mathrm{~d} 0 \\
& \operatorname{ck}(5,2)=0.0 \mathrm{~d} 0 \\
& \operatorname{ck}(5,3)=0.0 \mathrm{~d} 0 \\
& \operatorname{ck}(5,4)=0.0 \mathrm{~d} 0 \\
& \operatorname{ck}(5,5)=0.0 \mathrm{~d} 0 \\
& \operatorname{ck}(5,6)=0.0 \mathrm{~d} 0 \\
& \operatorname{ck}(6,1)=0.0 \mathrm{~d} 0 \\
& \operatorname{ck}(6,2)=0.0 \mathrm{~d} 0 \\
& \operatorname{ck}(6,3)=0.0 \mathrm{~d} 0 \\
& \operatorname{ck}(6,4)=0.0 \mathrm{~d} 0
\end{aligned}
$$




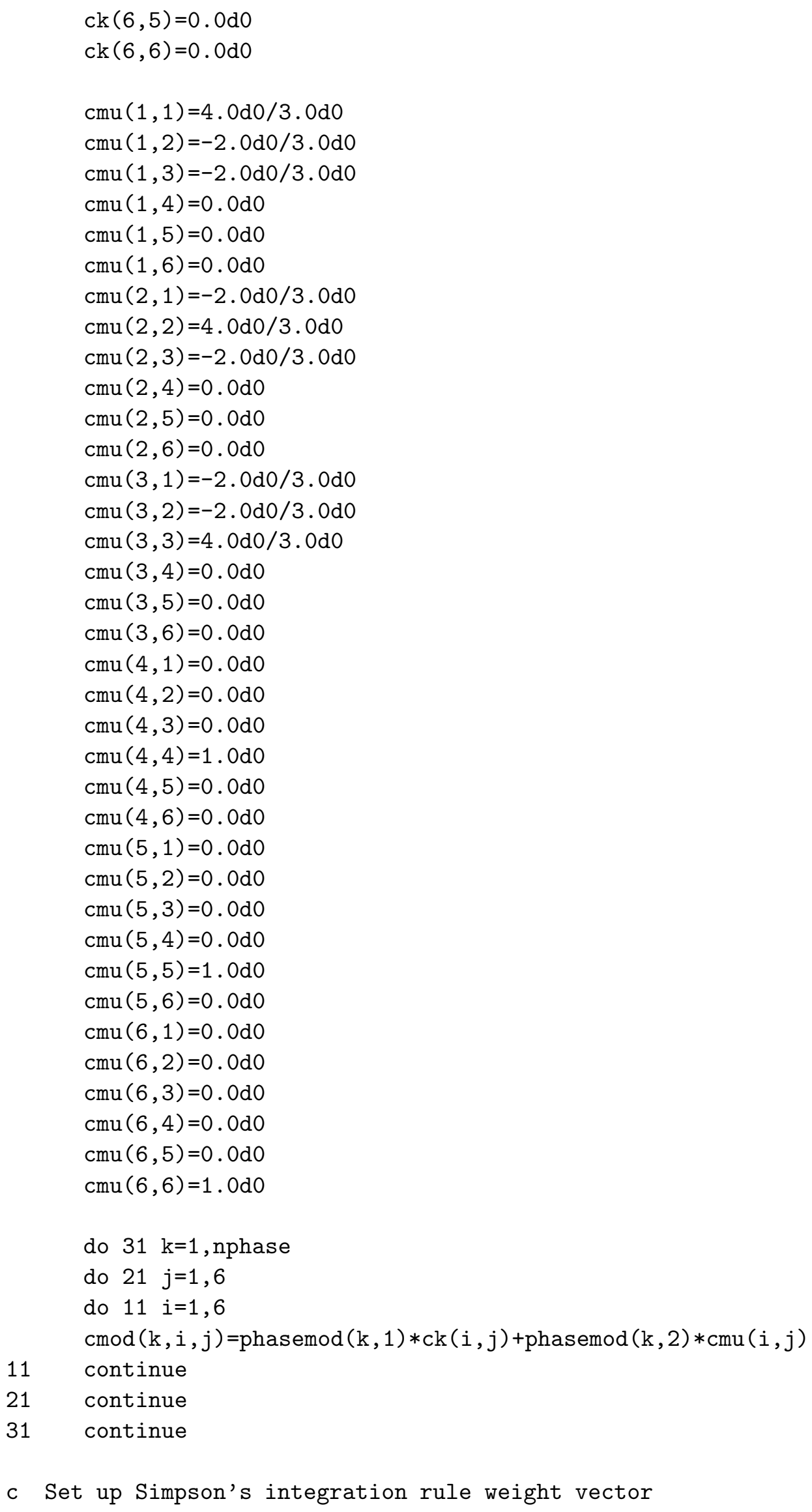

c Set up Simpson's integration rule weight vector 


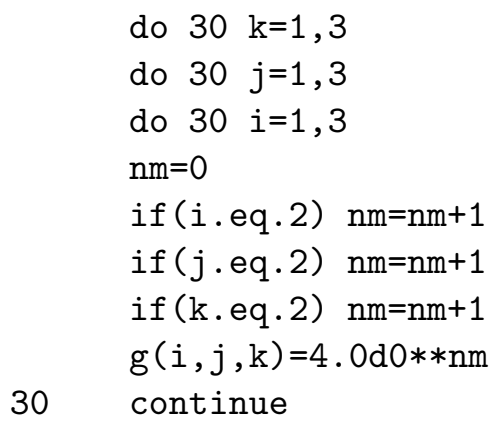
continue

c Loop over the nphase kinds of pixels and

c Simpson's rule quadrature points in order to compute the stiffness

c matrices. Stiffness matrices of trilinear finite elements are quadratic

$c$ in $\mathrm{x}, \mathrm{y}$, and $\mathrm{z}$, so that Simpson's rule quadrature gives exact results.

do 4000 ijk=1, nphase

do $3000 \mathrm{k}=1,3$

do $3000 j=1,3$

do $3000 i=1,3$

$\mathrm{x}=\mathrm{dfloat}(\mathrm{i}-1) / 2.0 \mathrm{~d} 0$

$y=\operatorname{dfloat}(j-1) / 2.0 \mathrm{~d} 0$

$\mathrm{z}=\mathrm{dfloat}(\mathrm{k}-1) / 2.0 \mathrm{~d} 0$

$c$ dndx means the negative derivative with respect to $\mathrm{x}$, of the shape

c matrix N (see manual, Sec. 2.2), dndy and dndz are similar.

$$
\begin{aligned}
& \operatorname{dndx}(1)=-(1.0 \mathrm{~d} 0-\mathrm{y}) *(1.0 \mathrm{~d} 0-\mathrm{z}) \\
& \operatorname{dndx}(2)=(1.0 \mathrm{~d} 0-y) *(1.0 \mathrm{~d} 0-z) \\
& \operatorname{dndx}(3)=y *(1.0 \mathrm{~d} 0-\mathrm{z}) \\
& \operatorname{dndx}(4)=-y *(1.0 \mathrm{~d} 0-z) \\
& \operatorname{dndx}(5)=-(1.0 d 0-y) * z \\
& \operatorname{dndx}(6)=(1.0 \mathrm{~d} 0-\mathrm{y}) * z \\
& \operatorname{dndx}(7)=y * z \\
& \operatorname{dndx}(8)=-y * z \\
& \operatorname{dndy}(1)=-(1.0 \mathrm{~d} 0-\mathrm{x}) *(1.0 \mathrm{~d} 0-\mathrm{z}) \\
& \operatorname{dndy}(2)=-x *(1.0 \mathrm{~d} 0-z) \\
& \operatorname{dndy}(3)=\mathrm{x} *(1.0 \mathrm{~d} 0-\mathrm{z}) \\
& \operatorname{dndy}(4)=(1.0 \mathrm{~d} 0-x) *(1.0 \mathrm{~d} 0-z) \\
& \operatorname{dndy}(5)=-(1.0 \mathrm{~d} 0-\mathrm{x}) * \mathrm{z} \\
& \operatorname{dndy}(6)=-x * z \\
& \operatorname{dndy}(7)=x * z \\
& \operatorname{dndy}(8)=(1.0 \mathrm{~d} 0-x) * z \\
& \operatorname{dnd} z(1)=-(1.0 \mathrm{~d} 0-\mathrm{x}) *(1.0 \mathrm{~d} 0-\mathrm{y}) \\
& \operatorname{dnd} z(2)=-x *(1.0 \mathrm{~d} 0-y) \\
& \operatorname{dnd} z(3)=-x * y \\
& \operatorname{dnd} z(4)=-(1.0 \mathrm{~d} 0-\mathrm{x}) * \mathrm{y} \\
& \operatorname{dnd} z(5)=(1.0 \mathrm{~d} 0-x) *(1.0 \mathrm{~d} 0-\mathrm{y}) \\
& \operatorname{dnd} z(6)=x *(1.0 \mathrm{~d} 0-\mathrm{y}) \\
& \operatorname{dnd} z(7)=x * y \\
& \operatorname{dnd} z(8)=(1.0 \mathrm{~d} 0-x) * y
\end{aligned}
$$

c now build strain matrix 


$$
\begin{aligned}
& \text { es }=0.0 \mathrm{~d} 0 \\
& \text { es }(1,:, 1)=\operatorname{dndx} \\
& \text { es }(2,:, 2)=\operatorname{dndy} \\
& \text { es }(3,:, 3)=\operatorname{dnd} z \\
& \text { es }(4,:, 1)=\operatorname{dnd} z \\
& \text { es }(4,:, 3)=\operatorname{dndx} \\
& \text { es }(5,:, 2)=\operatorname{dnd} z \\
& \text { es }(5,:, 3)=\operatorname{dndy} \\
& \text { es }(6,:, 1)=\operatorname{dndy} \\
& \text { es }(6,:, 2)=\operatorname{dndx}
\end{aligned}
$$

c now do matrix multiply to determine value at $(\mathrm{x}, \mathrm{y}, \mathrm{z})$, multiply by

c proper weight, and sum into $d k$, the stiffness matrix

do $900 \mathrm{~mm}=1,3$

do $900 \mathrm{nn}=1,3$

do $900 \mathrm{i} i=1,8$

do $900 j j=1,8$

c define sum over strain matrices and elastic moduli matrix for

c stiffness matrix

sum_num $=0.0 \mathrm{~d} 0$

do $890 \mathrm{kk}=1,6$

do $890 \quad l l=1,6$

sum_num=sum_num+es $(\mathrm{kk}, i i, \mathrm{~mm}) * \operatorname{cmod}(i j k, k \mathrm{k}, 1 l) * e s(1 l, j j, \mathrm{nn})$

890 continue

$d k(i j k, i i, m m, j j, n n)=d k(i j k, i i, m m, j j, n n)+g(i, j, k) * s u m \_n u m / 216$.

900 continue

3000 continue

4000 continue

c Now compute the ss matrices, which give the thermal strain terms

c for the i'th phase, single pixel.

$$
\begin{aligned}
& \operatorname{dndx}(1)=-0.25 \mathrm{~d} 0 \\
& \operatorname{dndx}(2)=0.25 \mathrm{~d} 0 \\
& \operatorname{dndx}(3)=0.25 \mathrm{~d} 0 \\
& \operatorname{dndx}(4)=-0.25 \mathrm{~d} 0 \\
& \operatorname{dndx}(5)=-0.25 \mathrm{~d} 0 \\
& \operatorname{dndx}(6)=0.25 \mathrm{~d} 0 \\
& \operatorname{dndx}(7)=0.25 \mathrm{~d} 0 \\
& \operatorname{dndx}(8)=-0.25 \mathrm{~d} 0 \\
& \operatorname{dndy}(1)=-0.25 \mathrm{~d} 0 \\
& \operatorname{dndy}(2)=-0.25 \mathrm{~d} 0 \\
& \operatorname{dndy}(3)=0.25 \mathrm{~d} 0 \\
& \operatorname{dndy}(4)=0.25 \mathrm{~d} 0 \\
& \operatorname{dndy}(5)=-0.25 \mathrm{~d} 0 \\
& \operatorname{dndy}(6)=-0.25 \mathrm{~d} 0 \\
& \operatorname{dndy}(7)=0.25 \mathrm{~d} 0
\end{aligned}
$$




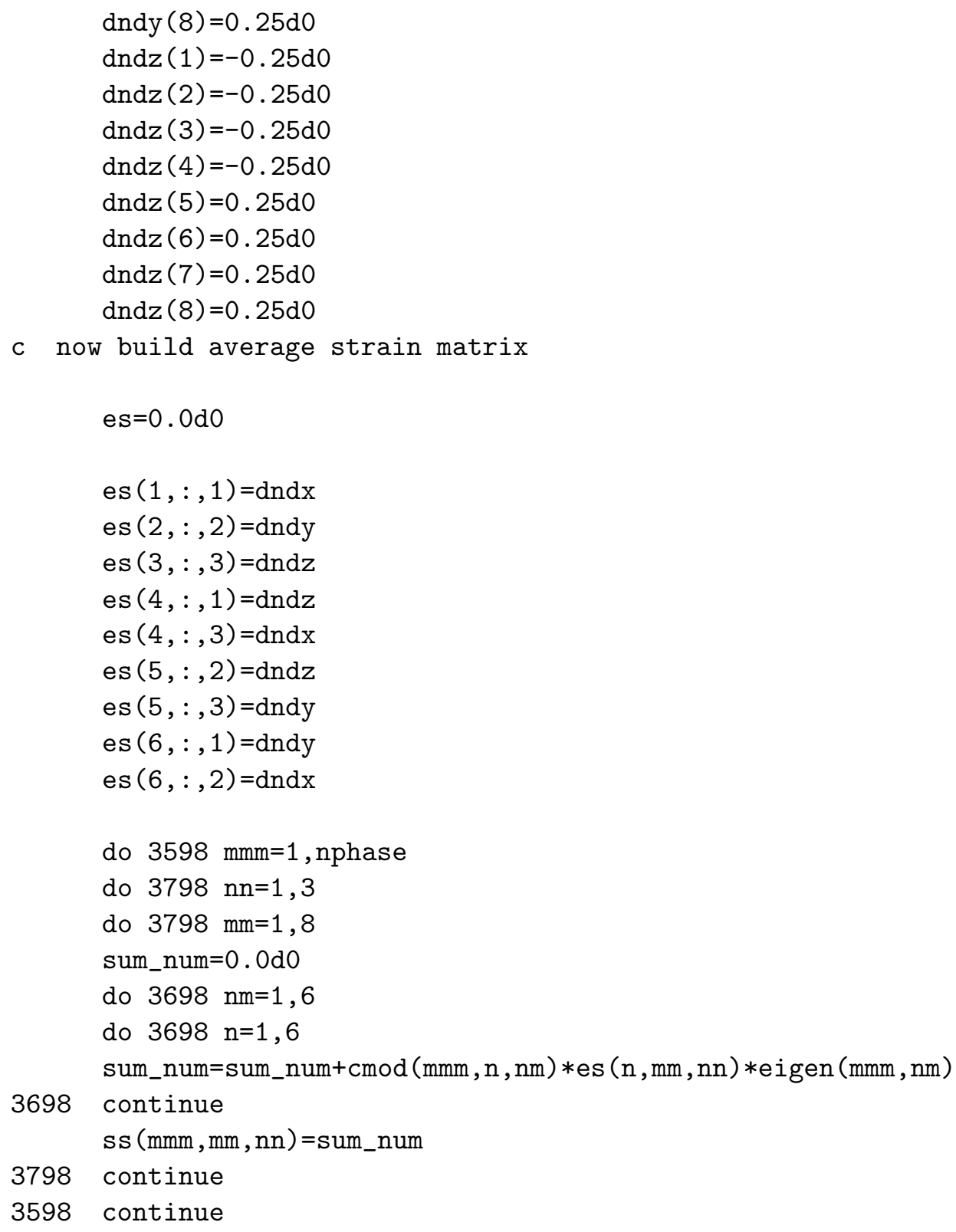

c Now set up linear term, $T$, for thermal energy. It does not depend

c on the macrostrains or displacements, so there is no need to update it $c$ as the macrostrains change. $T$ is built up out of the ss matrices.

$$
\begin{aligned}
& \mathrm{T}=0.0 \mathrm{~d} 0 \\
& \mathrm{~T} 2=0.0 \mathrm{~d} 0
\end{aligned}
$$

c Do all points, but no macrostrain terms

c note: factor of 2 on linear thermal term is cancelled 


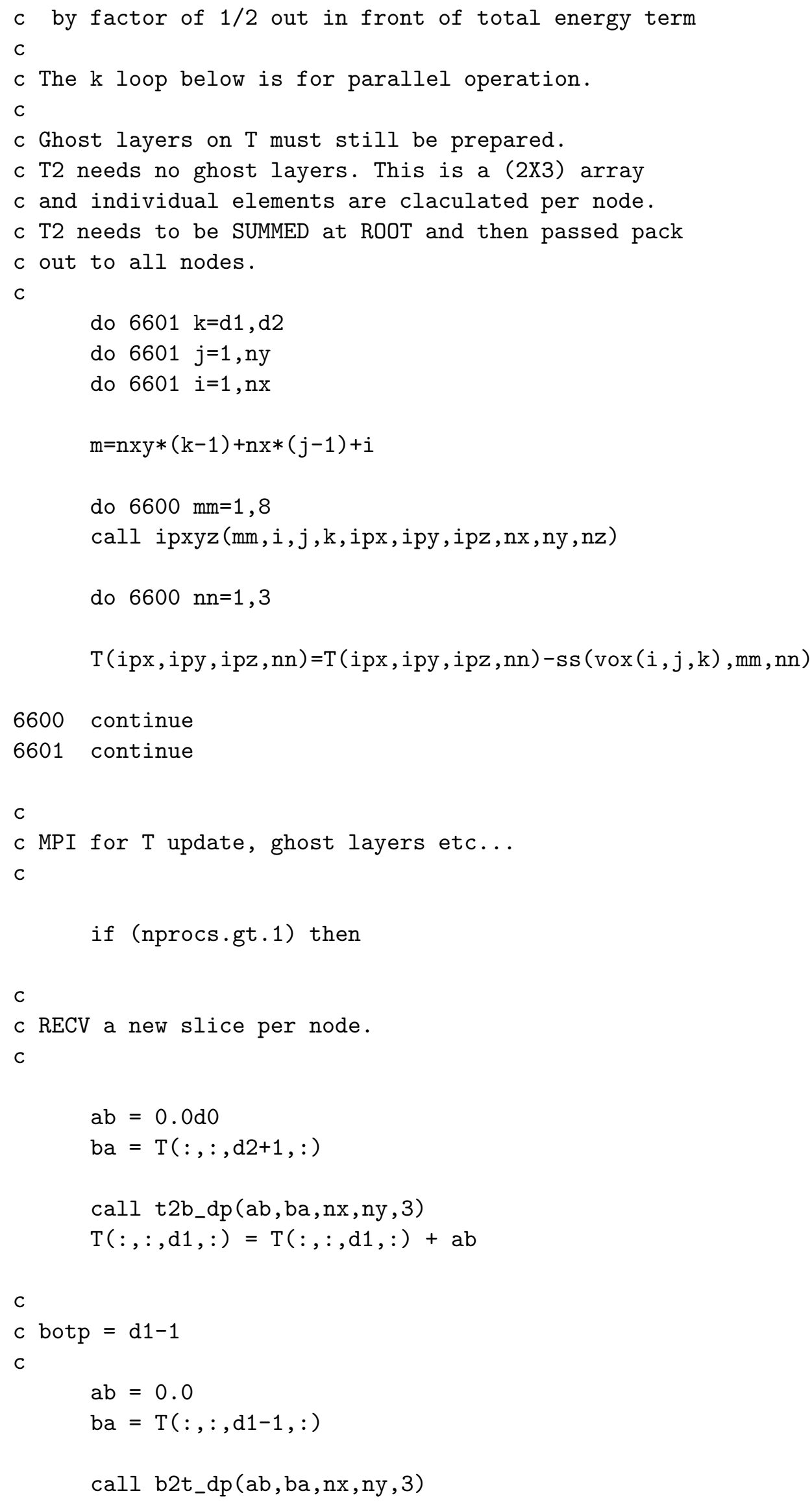




$$
\mathrm{T}(:,:, \mathrm{d} 2,:)=\mathrm{T}(:,:, \mathrm{d} 2,:)+\mathrm{ab}
$$

c Update ghost layers

C

c RECV a new slice per node.

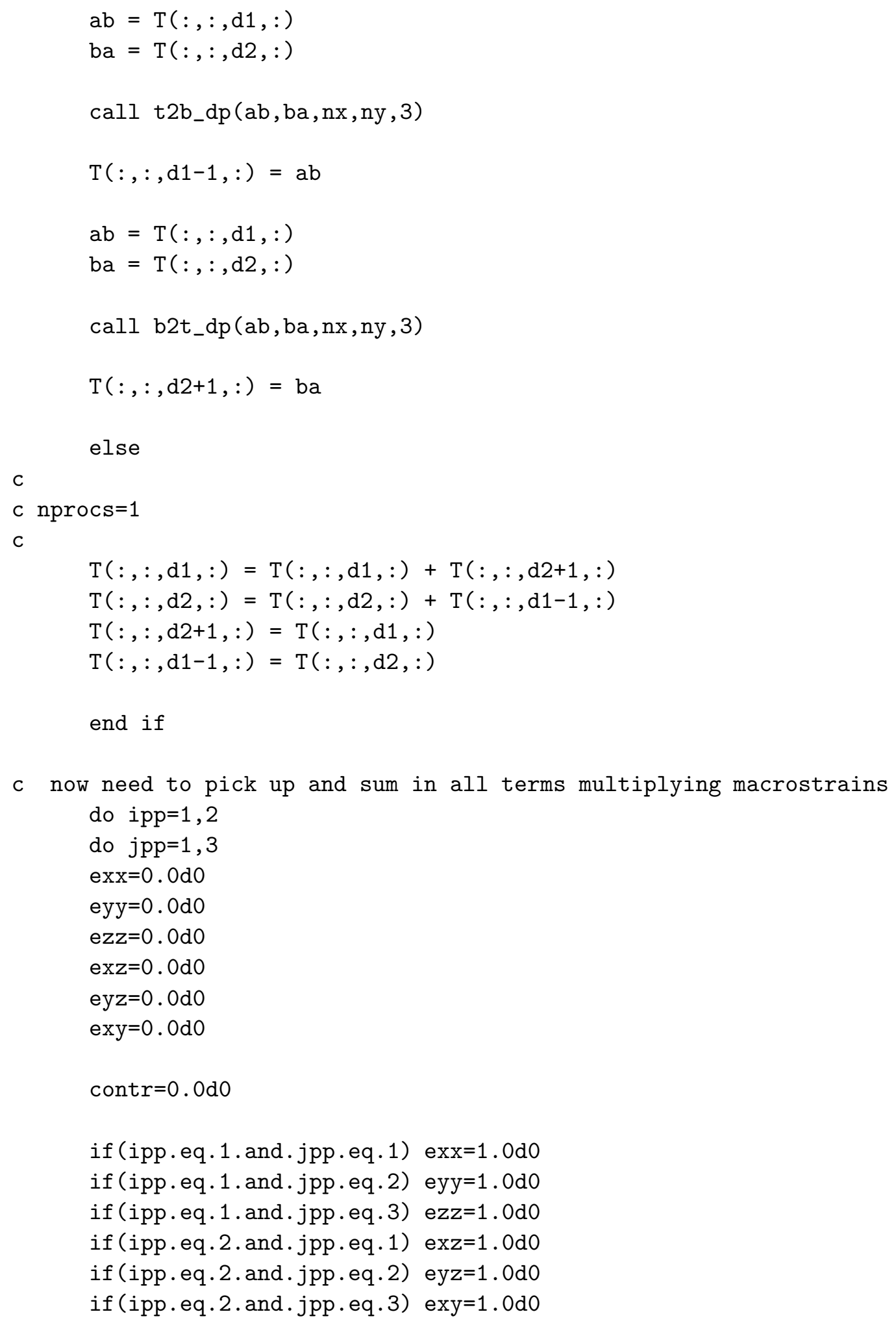




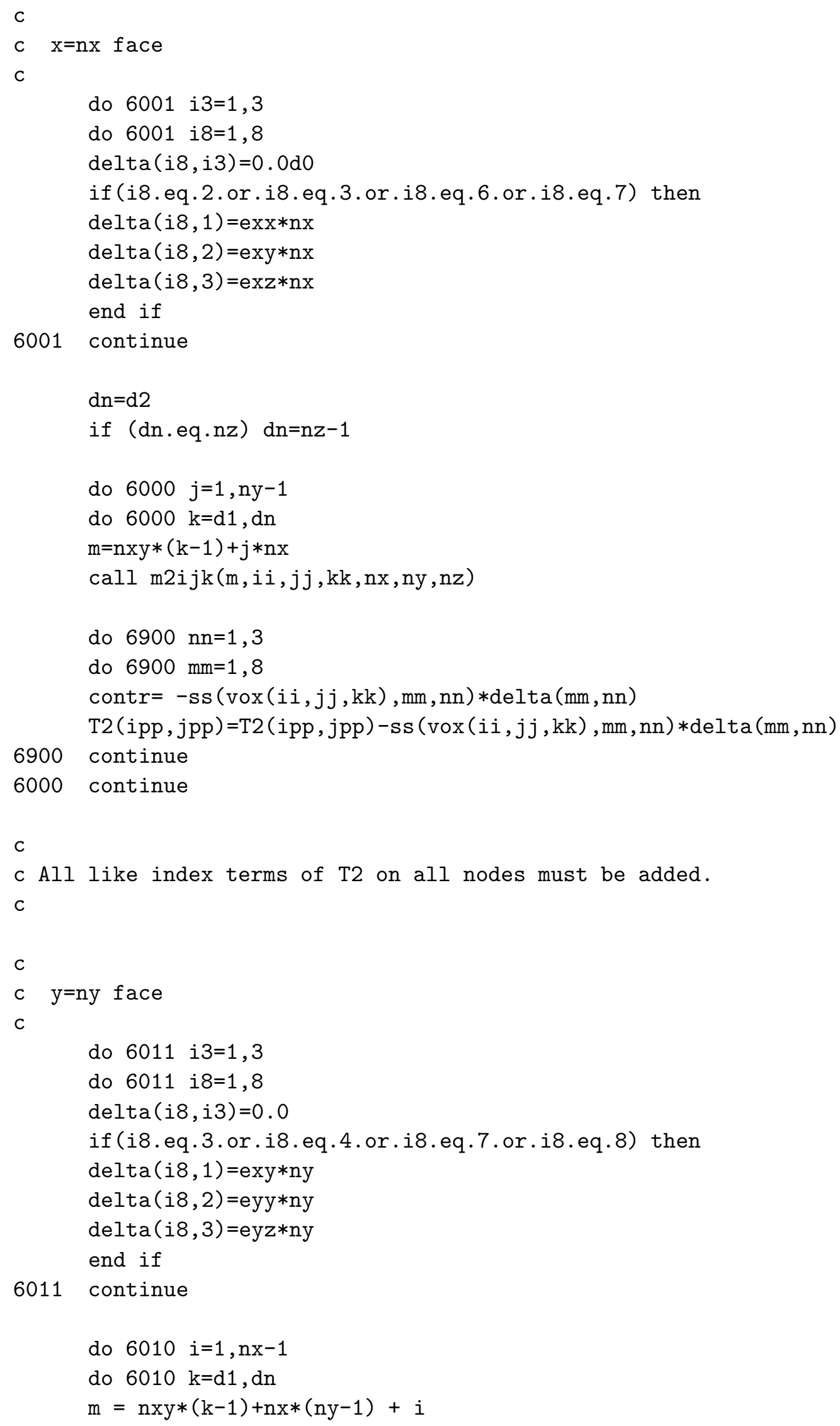




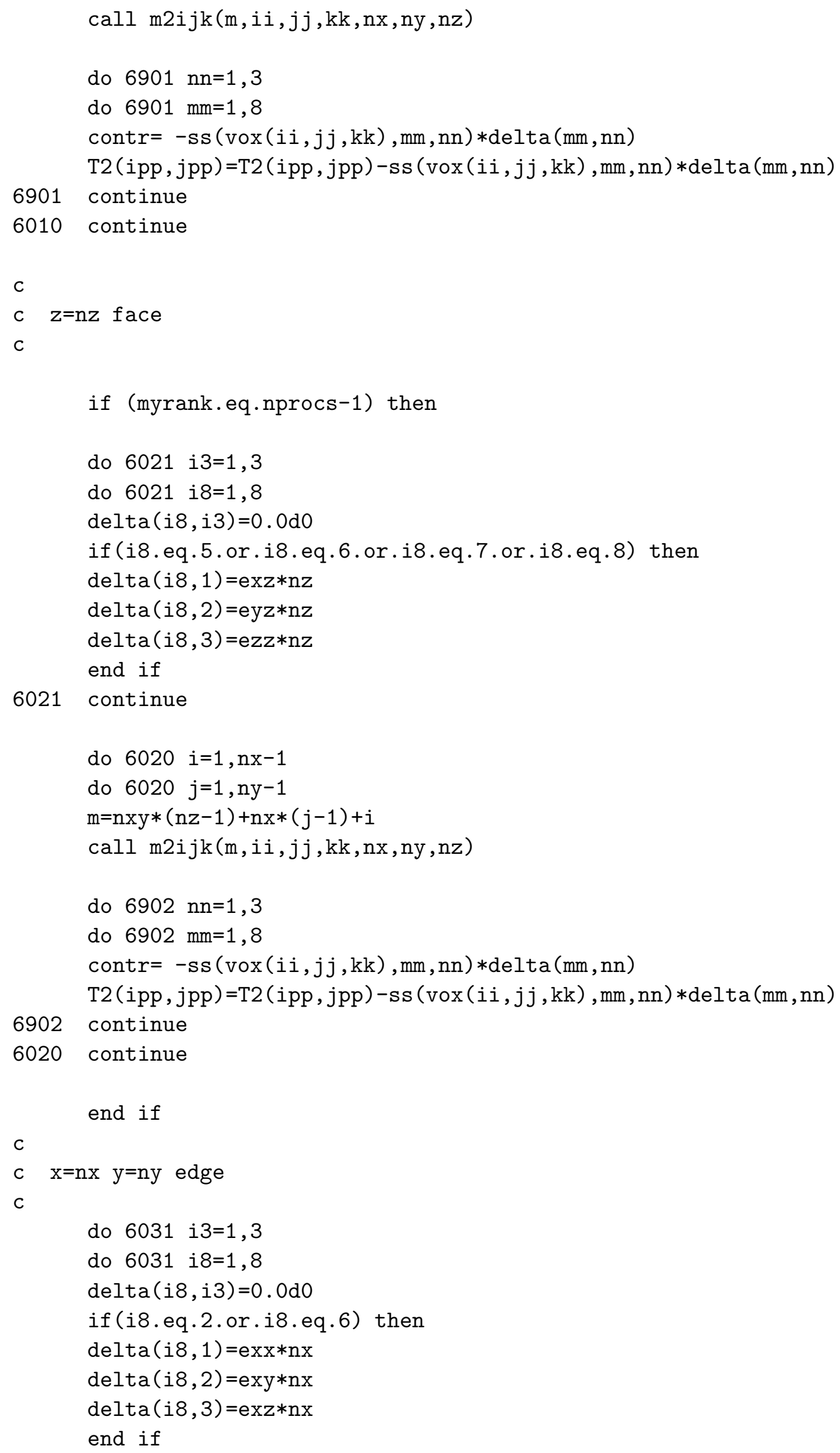




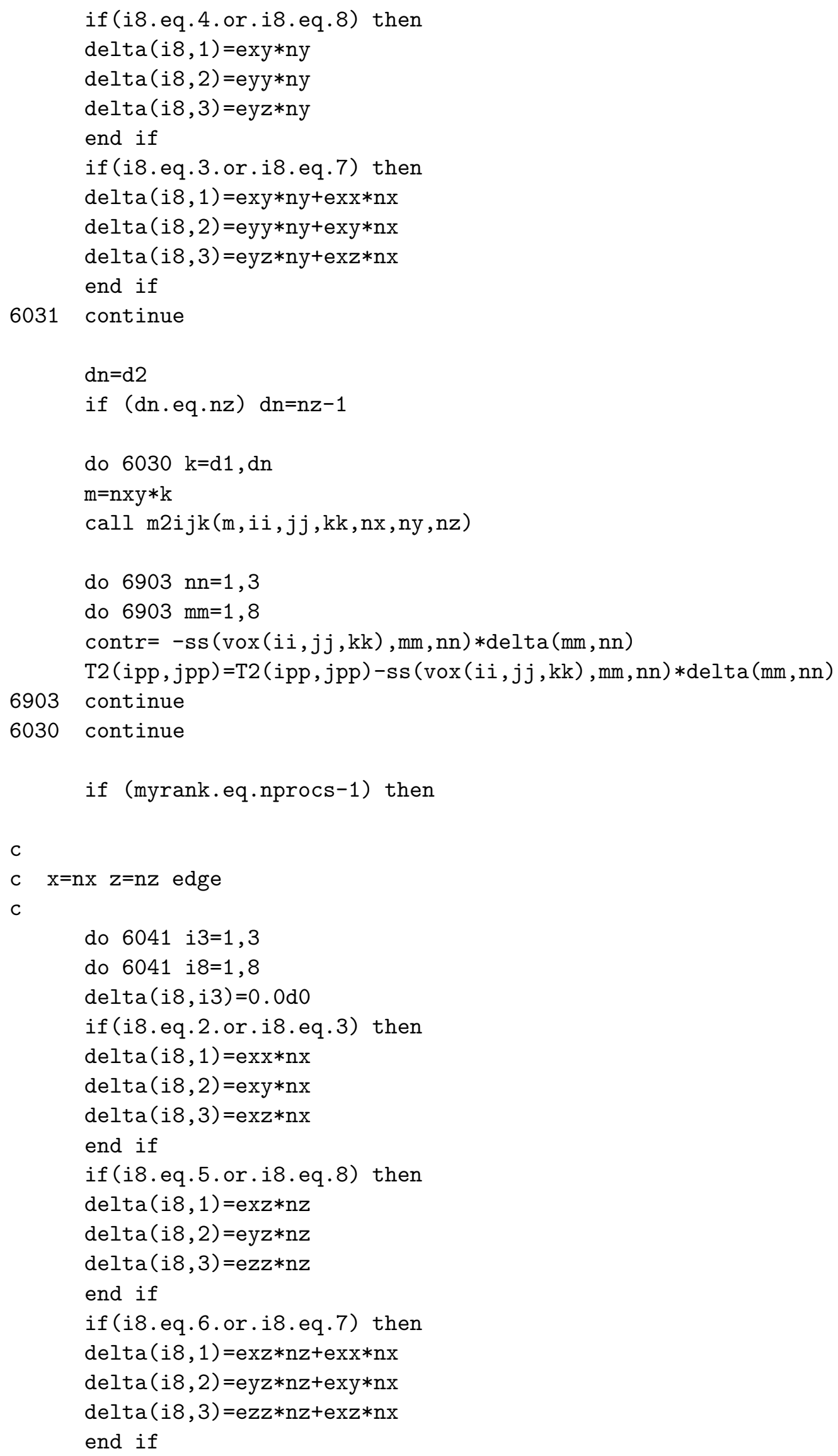




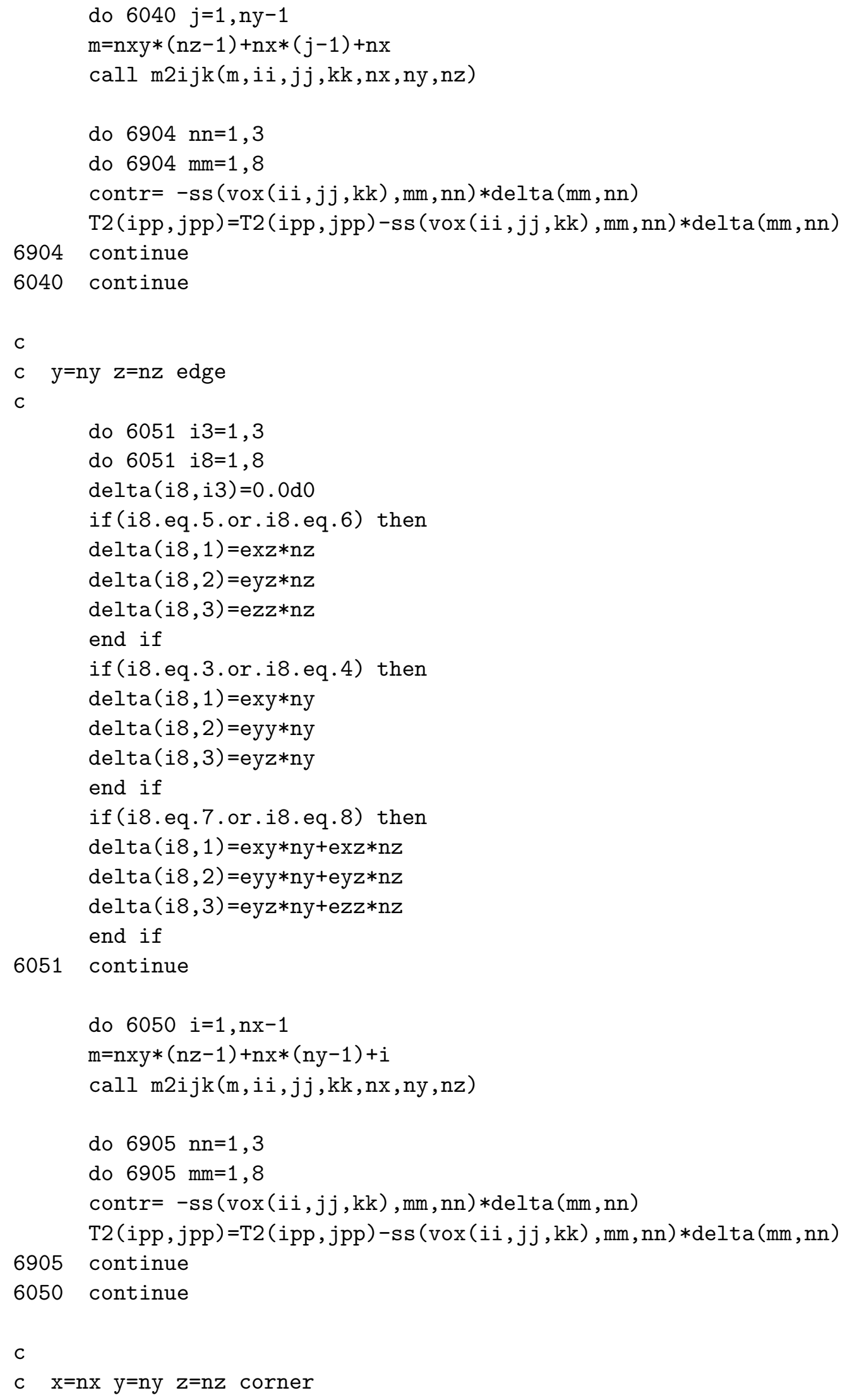


C

do 6061 i3=1,3
do 6061 i8=1,8
delta $(i 8, i 3)=0.0 d 0$
if $(i 8$. eq. 2$)$ then
delta $(i 8,1)=$ exx $* n x$
delta $(i 8,2)=$ exy $* n x$
delta $(i 8,3)=$ exz $* n x$
end if
if $(i 8 . e q .4)$ then
delta $(i 8,1)=$ exy*ny
delta $(i 8,2)=$ eyy $* n y$
delta $(i 8,3)=$ eyz $* n y$

end if

if (i8.eq.5) then

$\operatorname{delta}(i 8,1)=\operatorname{exz} * n z$

$\operatorname{delta}(i 8,2)=e y z * n z$

$\operatorname{delta}(i 8,3)=e z z * n z$

end if

if (i8.eq.8) then

$\operatorname{delta}(i 8,1)=e x y * n y+e x z * n z$

$\operatorname{delta}(i 8,2)=e y y * n y+e y z * n z$

$\operatorname{delta}(i 8,3)=e y z * n y+e z z * n z$

end if

if (i8.eq.6) then

$\operatorname{delta}(i 8,1)=\operatorname{exx} * n x+e x z * n z$

$\operatorname{delta}(i 8,2)=e x y * n x+e y z * n z$

$\operatorname{delta}(i 8,3)=\operatorname{exz} * n x+e z z * n z$

end if

if (i8.eq.3) then

$\operatorname{delta}(i 8,1)=\operatorname{exx} * n x+e x y * n y$

$\operatorname{delta}(i 8,2)=$ exy $* n x+$ eyy $* n y$

$\operatorname{delta}(i 8,3)=e x z * n x+e y z * n y$

end if

if (i8.eq.7) then

$\operatorname{delta}(i 8,1)=\operatorname{exx} * n x+e x y * n y+e x z * n z$

$\operatorname{delta}(i 8,2)=e x y * n x+e y y * n y+e y z * n z$

$\operatorname{delta}(i 8,3)=e x z * n x+e y z * n y+e z z * n z$

end if

6061

continue

$\mathrm{m}=\mathrm{nx} * \mathrm{ny} * \mathrm{nz}$

call m2ijk(m,ii,jj,kk,nx,ny,nz)

do $6906 \mathrm{~mm}=1,8$

do $6906 \mathrm{nn}=1,3$

contr $=-s s(\operatorname{vox}(i \mathrm{i}, \mathrm{j} j, \mathrm{kk}), \mathrm{mm}, \mathrm{nn}) * \operatorname{delta}(\mathrm{mm}, \mathrm{nn})$

$\mathrm{T} 2(\mathrm{ipp}, \mathrm{jpp})=\mathrm{T} 2(\mathrm{ipp}, \mathrm{jpp})-\mathrm{ss}(\operatorname{vox}(\mathrm{i} i, j \mathrm{j}, \mathrm{kk}), \mathrm{mm}, \mathrm{nn}) * \operatorname{delta}(\mathrm{mm}, \mathrm{nn})$

continue 
CALL MPI_ALLREDUCE (yt, $\mathrm{Y}, 1$, MPI_DOUBLE_PRECISION , MPI_SUM, $\&$ MPI_COMM_WORLD, ierr)

else

$\mathrm{Y}=\mathrm{yt}$

end if

c Following needs to be run after every change in macrostrain

c when energy is recomputed.

1221 continue

c Use auxiliary variables (exx, etc.) instead of $u($ ) variable, for

$c$ convenience, and to make the following code easier to read.

$\operatorname{exx}=\mathrm{u} 2(1,1)$

eyy $=u 2(1,2)$

$\mathrm{ezz}=\mathrm{u} 2(1,3)$

exz=u2 $(2,1)$

eyz=u2 $(2,2)$

exy=u2 $(2,3)$

c Now set up vector for linear term that comes from periodic boundary

$c$ conditions. Notation and conventions same as for $\mathrm{T}$ term.

c This is done using the stiffness matrices, and the periodic terms

$c$ in the macrostrains. It is easier to set up b this way than to

$c$ analytically write out all the terms involved.

$\mathrm{b}=0.0 \mathrm{~d} 0$

$\mathrm{C}=0.0 \mathrm{~d} 0$

C

c $x=n x$ face

c

do 2001 i3=1,3

do 2001 i $8=1,8$

$\operatorname{delta}(i 8, i 3)=0.0 \mathrm{~d} 0$

if (i8.eq.2.or.i8.eq.3.or.i8.eq.6.or.i8.eq.7) then

$\operatorname{delta}(i 8,1)=\operatorname{exx} * n x$

$\operatorname{delta}(i 8,2)=\operatorname{exy} * n x$

$\operatorname{delta}(i 8,3)=\operatorname{exz} * n x$

end if

2001 continue

$\mathrm{dn}=\mathrm{d} 2$

if (dn.eq.nz) $d n=n z-1$ 


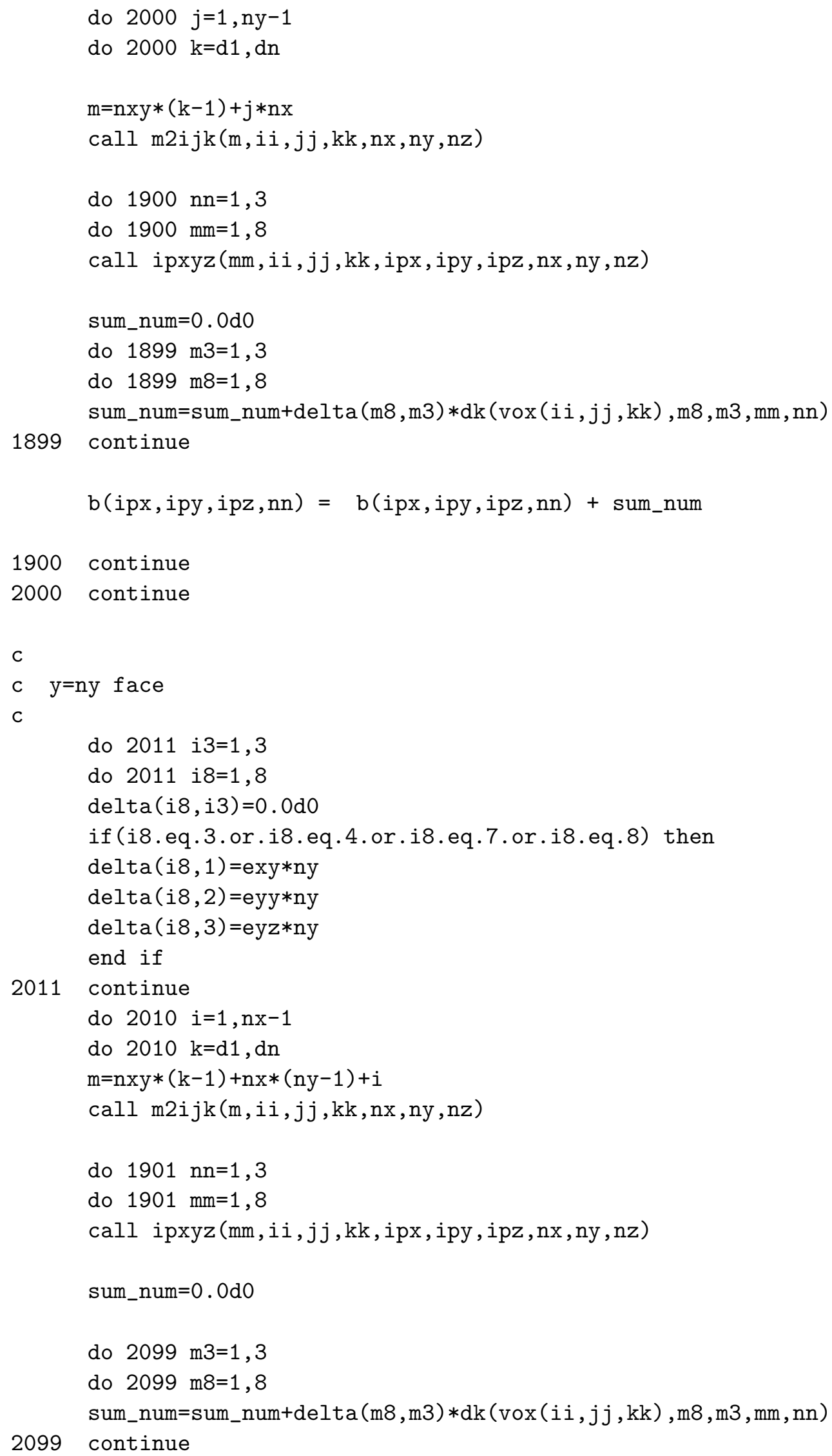




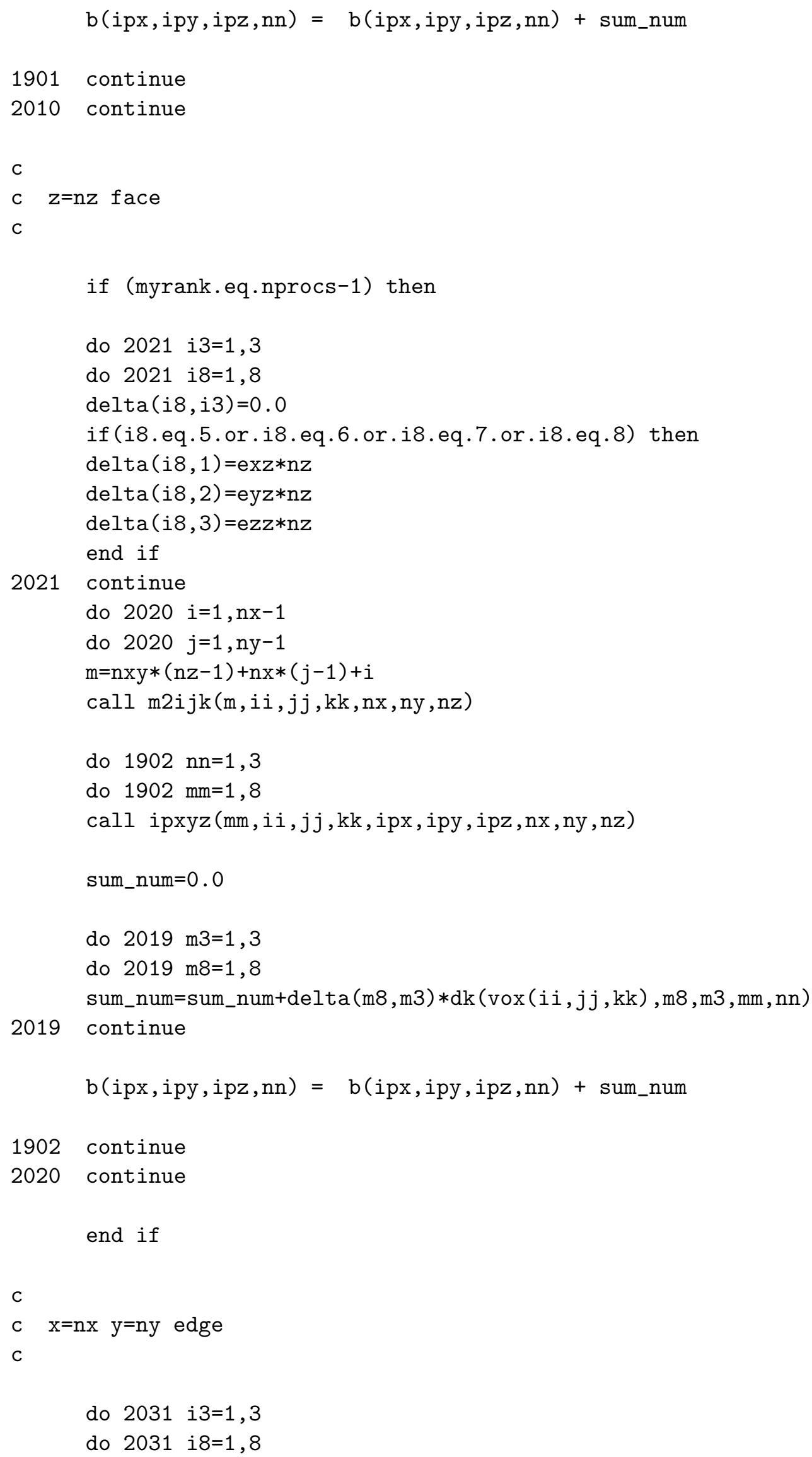




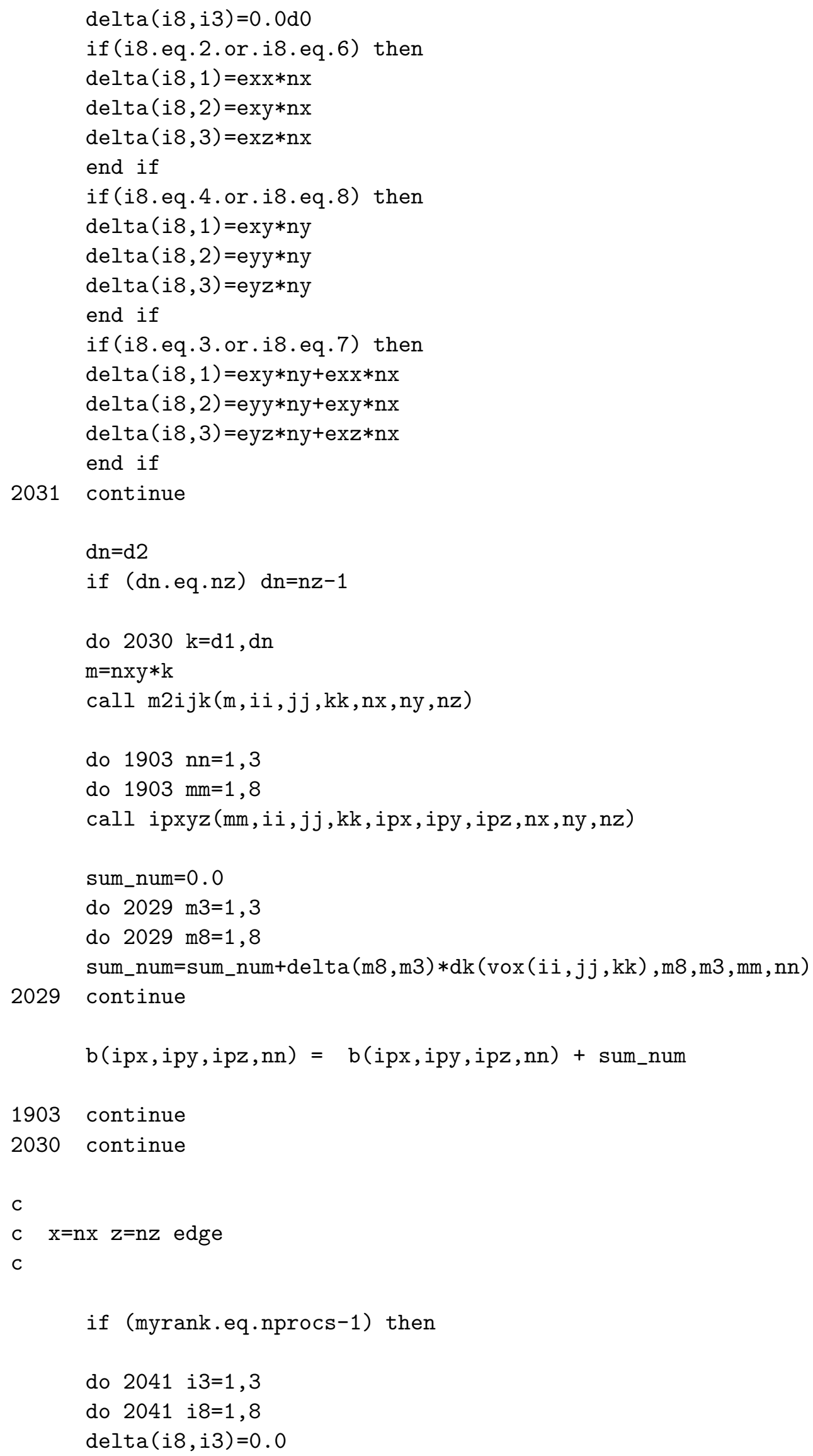




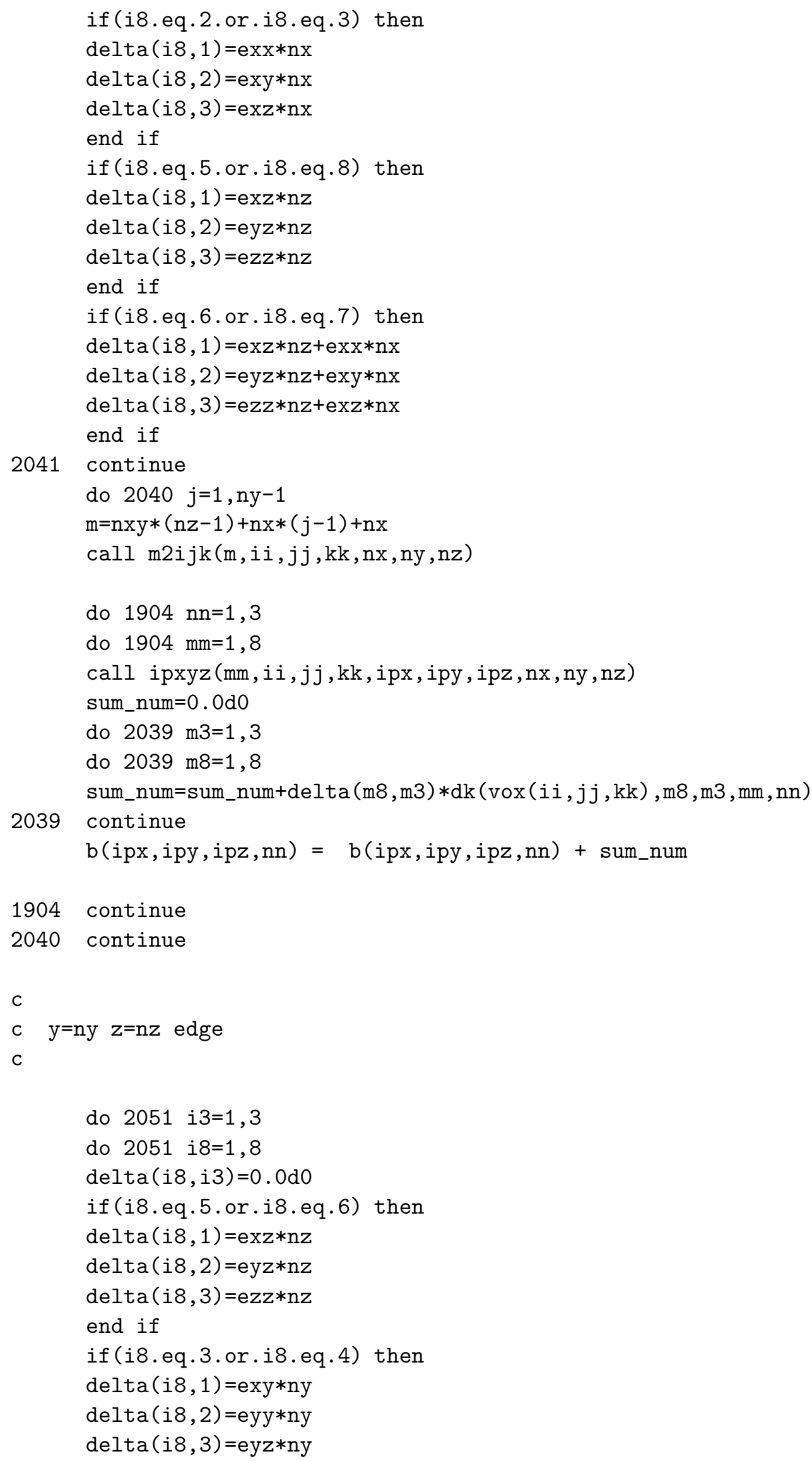




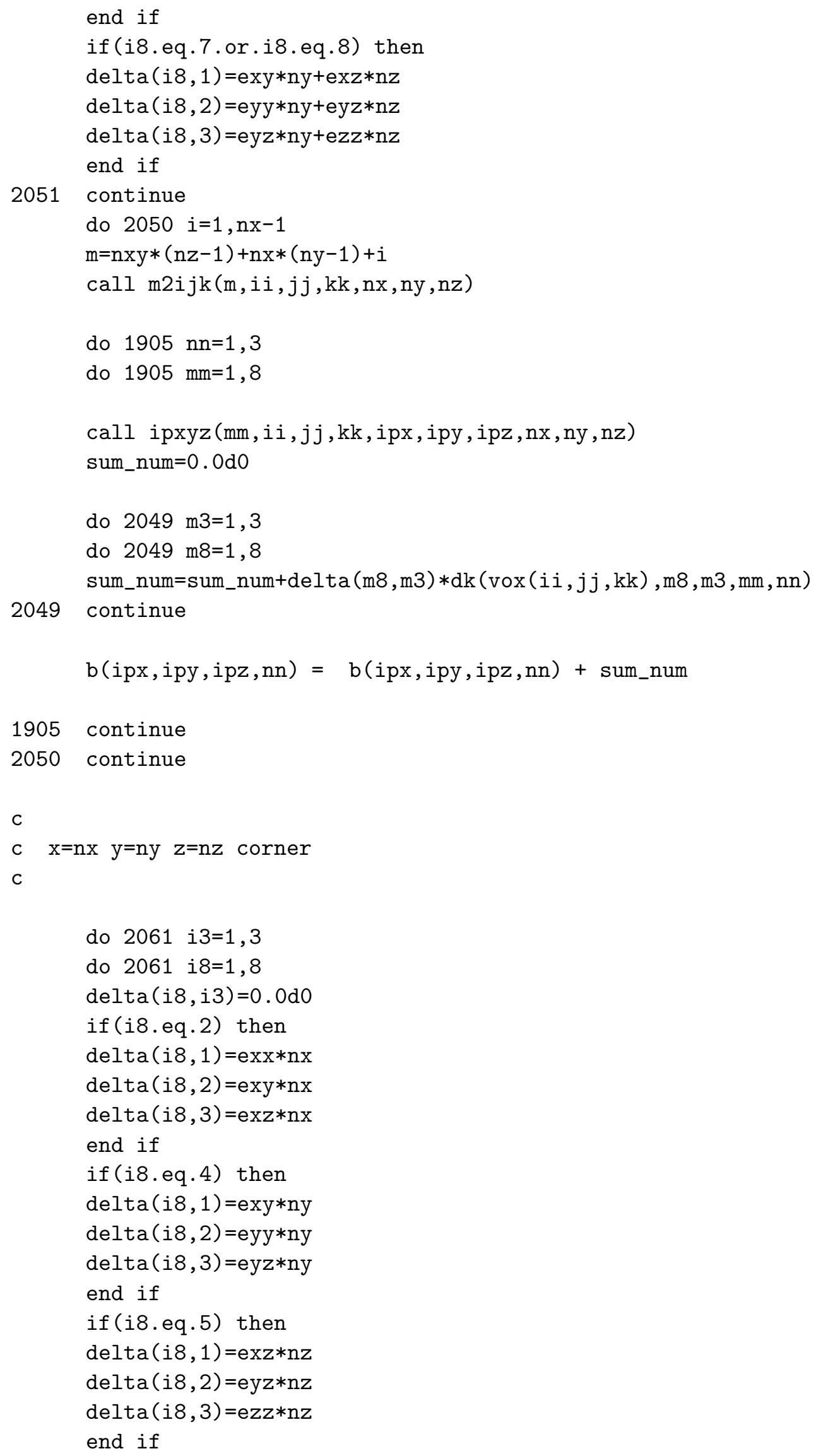




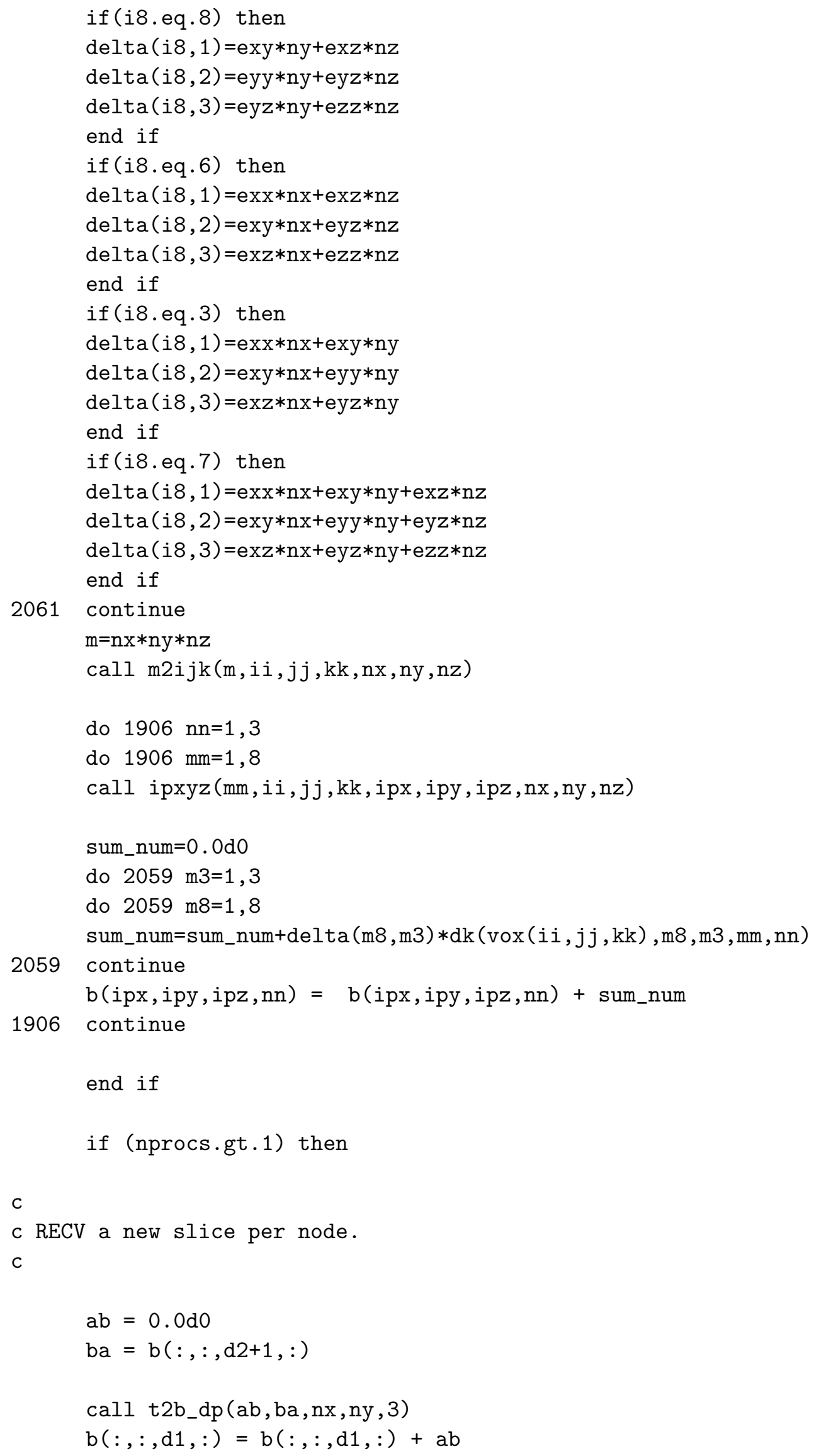


c

c botp $=\mathrm{d} 1-1$

$c$

$$
\begin{aligned}
& \mathrm{ab}=0.0 \\
& \mathrm{ba}=\mathrm{b}(:,:, \mathrm{d} 1-1,:) \\
& \mathrm{call} b 2 t_{-} \mathrm{dp}(\mathrm{ab}, \mathrm{ba}, \mathrm{nx}, \mathrm{ny}, 3) \\
& \mathrm{b}(:,:, \mathrm{d} 2,:)=\mathrm{b}(:,:, \mathrm{d} 2,:)+\mathrm{ab}
\end{aligned}
$$

C

c Update ghost layers

C

c RECV a new slice per node.

$c$

$\mathrm{ab}=\mathrm{b}(:,:, \mathrm{d} 1,:)$

$\mathrm{ba}=\mathrm{b}(:,:, \mathrm{d} 2,:)$

call t2b_dp (ab, ba, nx, ny, 3)

$\mathrm{b}(:,:, \mathrm{d} 1-1,:)=\mathrm{ab}$

$\mathrm{ab}=\mathrm{b}(:,:, \mathrm{d} 1,:)$

$\mathrm{ba}=\mathrm{b}(:,:, \mathrm{d} 2,:)$

call b2t_dp (ab, ba, nx, ny, 3)

$b(:,:, d 2+1,:)=b a$

else

$c$

C nprocs $=1$

C

$\mathrm{b}(:,:, \mathrm{d} 1,:)=\mathrm{b}(:,:, \mathrm{d} 1,:)+\mathrm{b}(:,:, \mathrm{d} 2+1,:)$

$\mathrm{b}(:,:, \mathrm{d} 2,:)=\mathrm{b}(:,:, \mathrm{d} 2,:)+\mathrm{b}(:,:, \mathrm{d} 1-1,:)$

$\mathrm{b}(:,:, \mathrm{d} 2+1,:)=\mathrm{b}(:,:, \mathrm{d} 1,:)$

$\mathrm{b}(:,:, \mathrm{d} 1-1,:)=\mathrm{b}(:,:, \mathrm{d} 2,:)$

end if

deallocate (ab)

deallocate (ba)

return

end

C

c $* * * * * * * * * * * * * * * * * * * * * * * * * * * * * * * * * * * * * * * * * * * * * * * * * * * * * * * * * *$

$C$

subroutine const_mpi(dk, zcon, nx, ny, nz, vox, d1, d2) 
c Subroutine computes the quadratic term in the macrostrains, that comes

c from the periodic boundary conditions, and sets it up as a

c $(2,3) \times(2,3)$ matrix that couples to the six macrostrains

implicit none

include 'mpif.h'

integer nx, ny, nz, nphase, d1,d2, dn,pflag

integer $\mathrm{nxy}, \mathrm{mmm}$

integer $i i, j j, i 3, i 8$

integer $i, j, k, m, m i, m j$

integer ia, ja,ka

integer mm, nn, m3, m8, ipx, ipy, ipz, irank

double precision $\mathrm{dk}$ (nphase, $8,3,8,3), \operatorname{zcon}(2,3,2,3)$, delta $(8,3)$

double precision $\mathrm{pp}(6,6), \mathrm{s}(6,6)$

double precision econ, eterm, epos, eneg, epart

double precision exx, eyy, ezz, exz, eyz, exy

integer $* 2 \operatorname{vox}(\mathrm{nx}, \mathrm{ny}, \mathrm{d} 1-1: \mathrm{d} 2+1)$

C

C MPI vars

C

integer myrank,nprocs,ierr, status (MPI_STATUS_SIZE)

common/list1/pflag, nphase

call MPI_COMM_RANK ( MPI_COMM_WORLD, myrank, ierr )

call MPI_COMM_SIZE( MPI_COMM_WORLD, nprocs, ierr )

c routine to set up 6 × 6 matrix for energy term involving macro-strains

c only, pulled out of femat

c Idea is to evaluate the quadratic term in the macrostrains repeatedly

c for choices of strain like

c $\operatorname{exx}=1$, exy=1, all others $=0$, build up 21 choices, then recombine

$c$ to get matrix elements by themselves

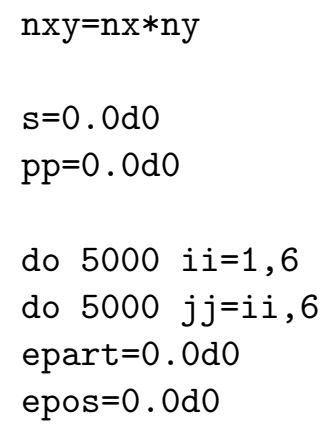




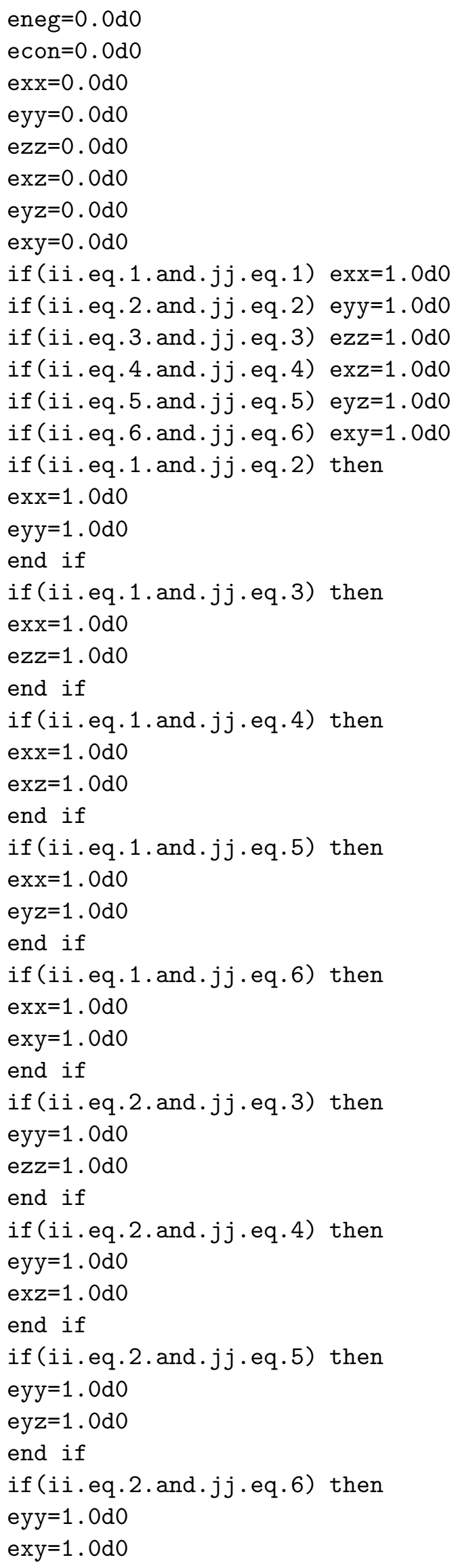




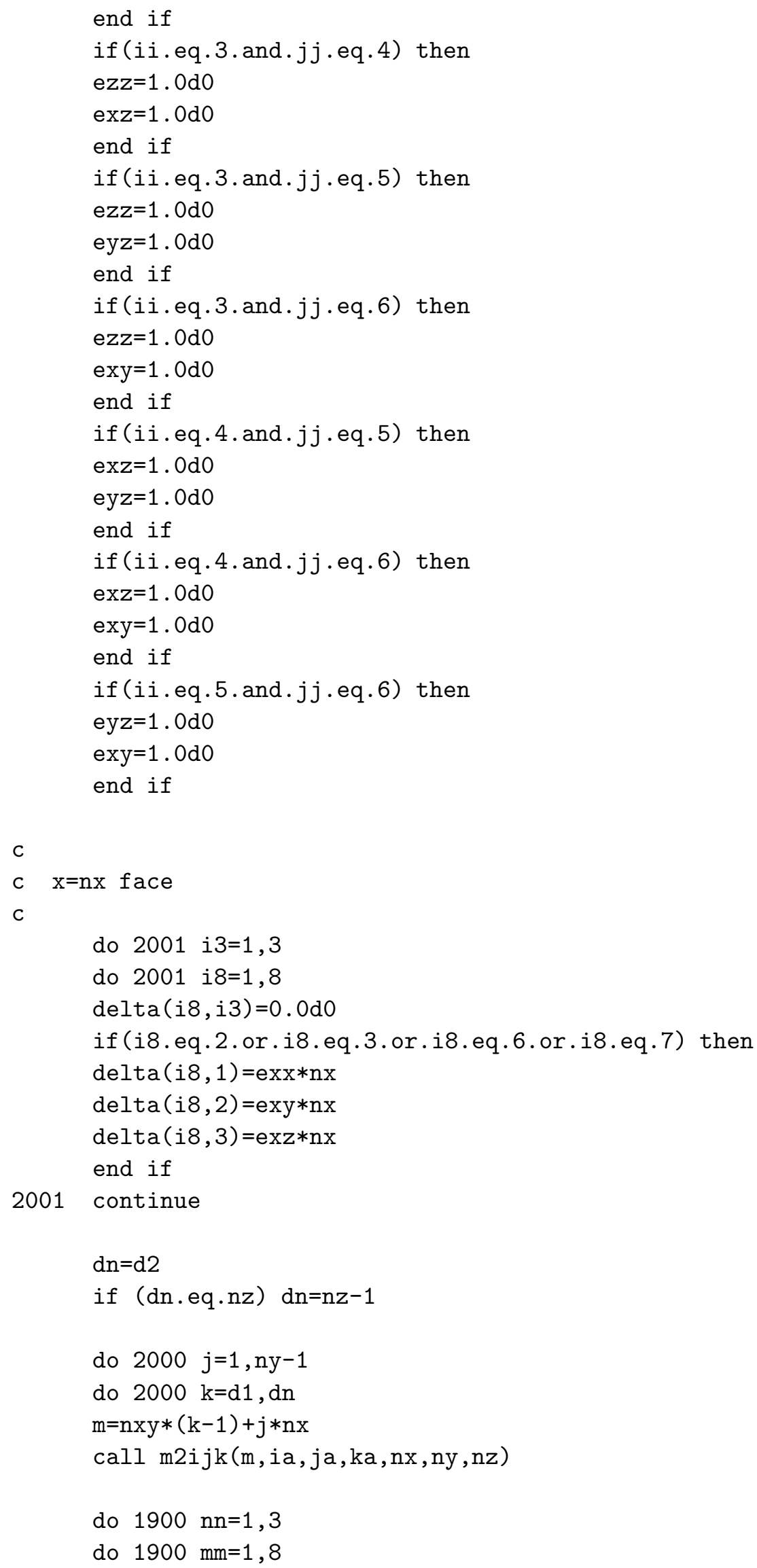




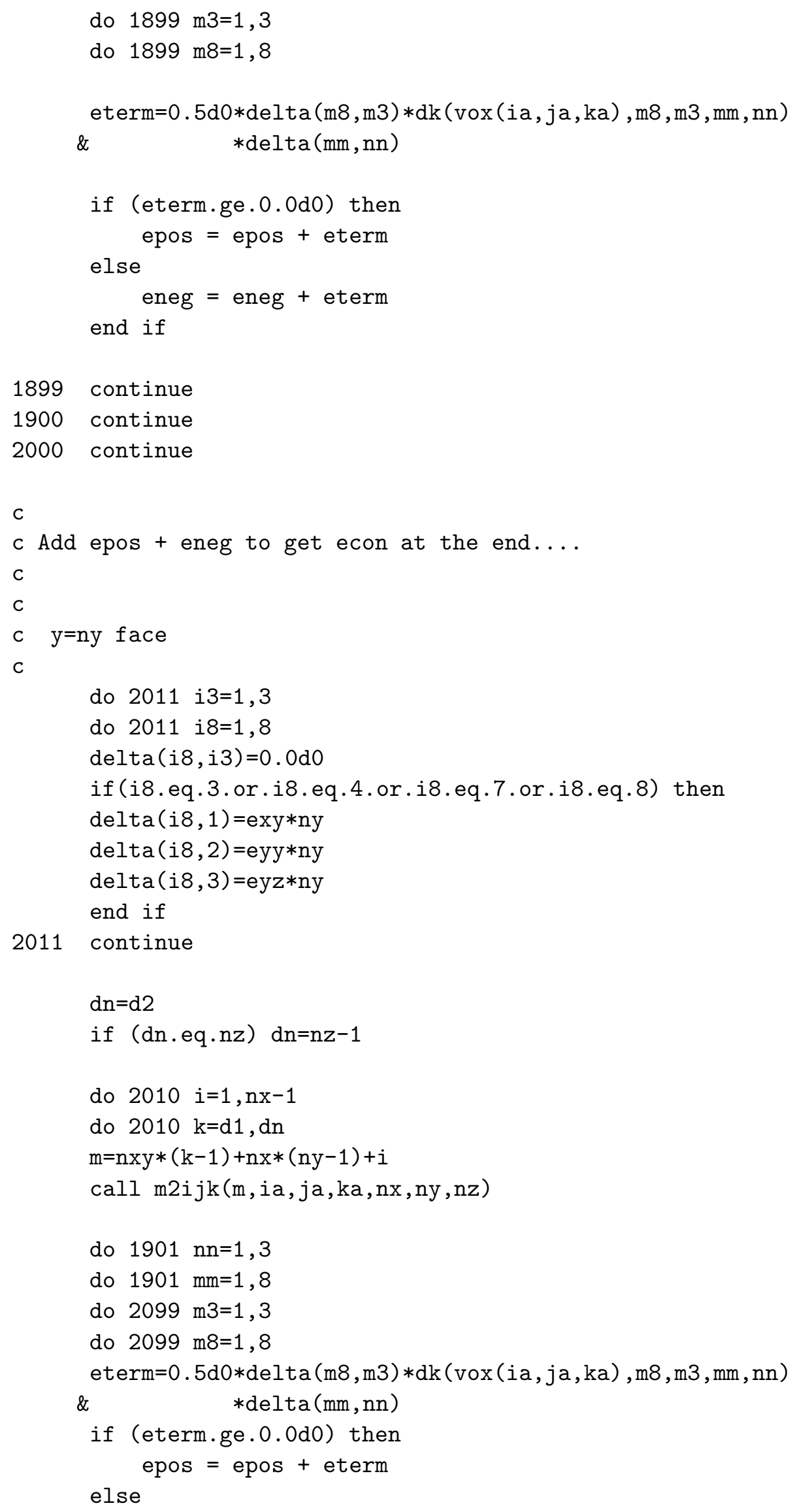




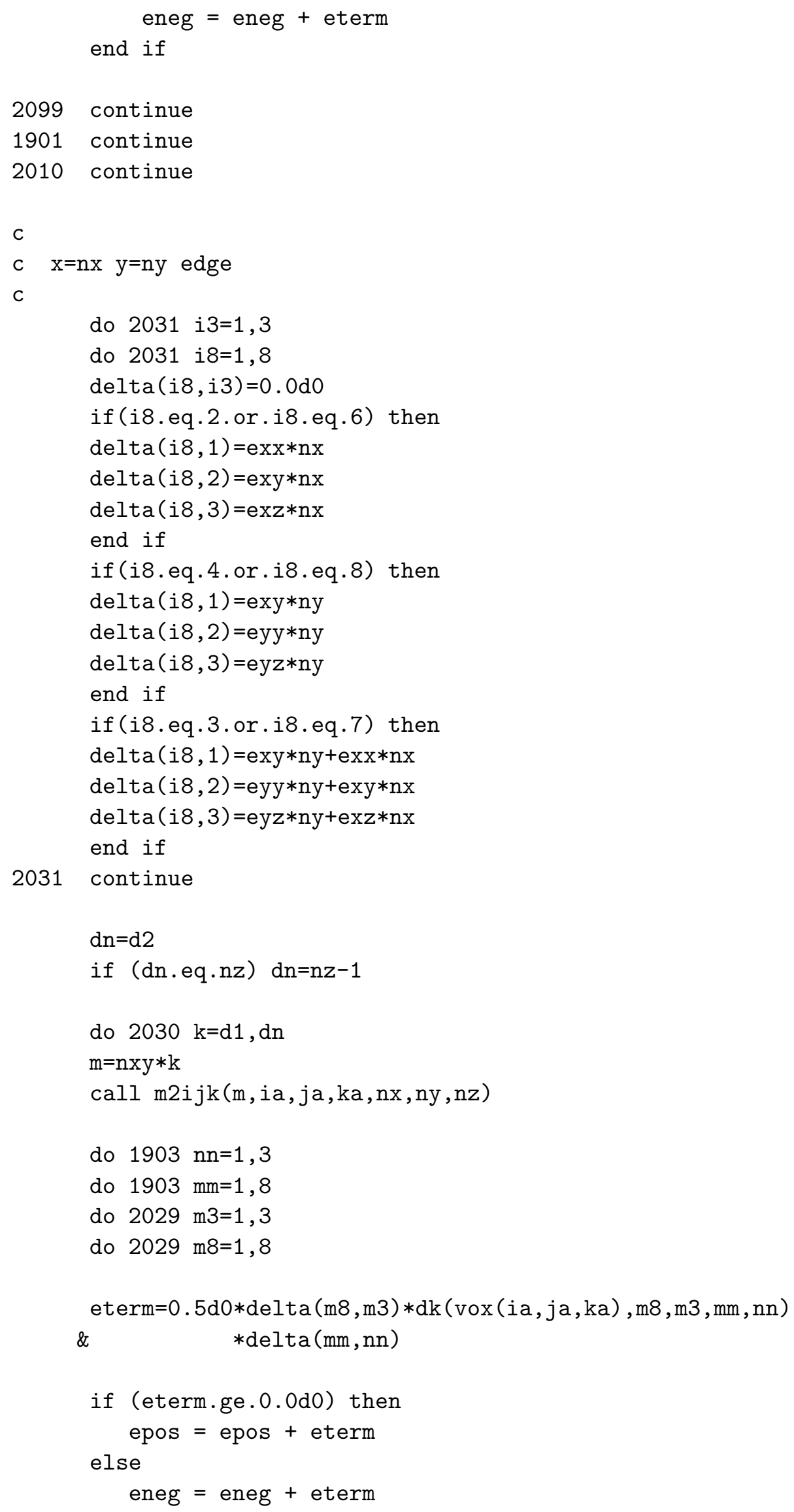




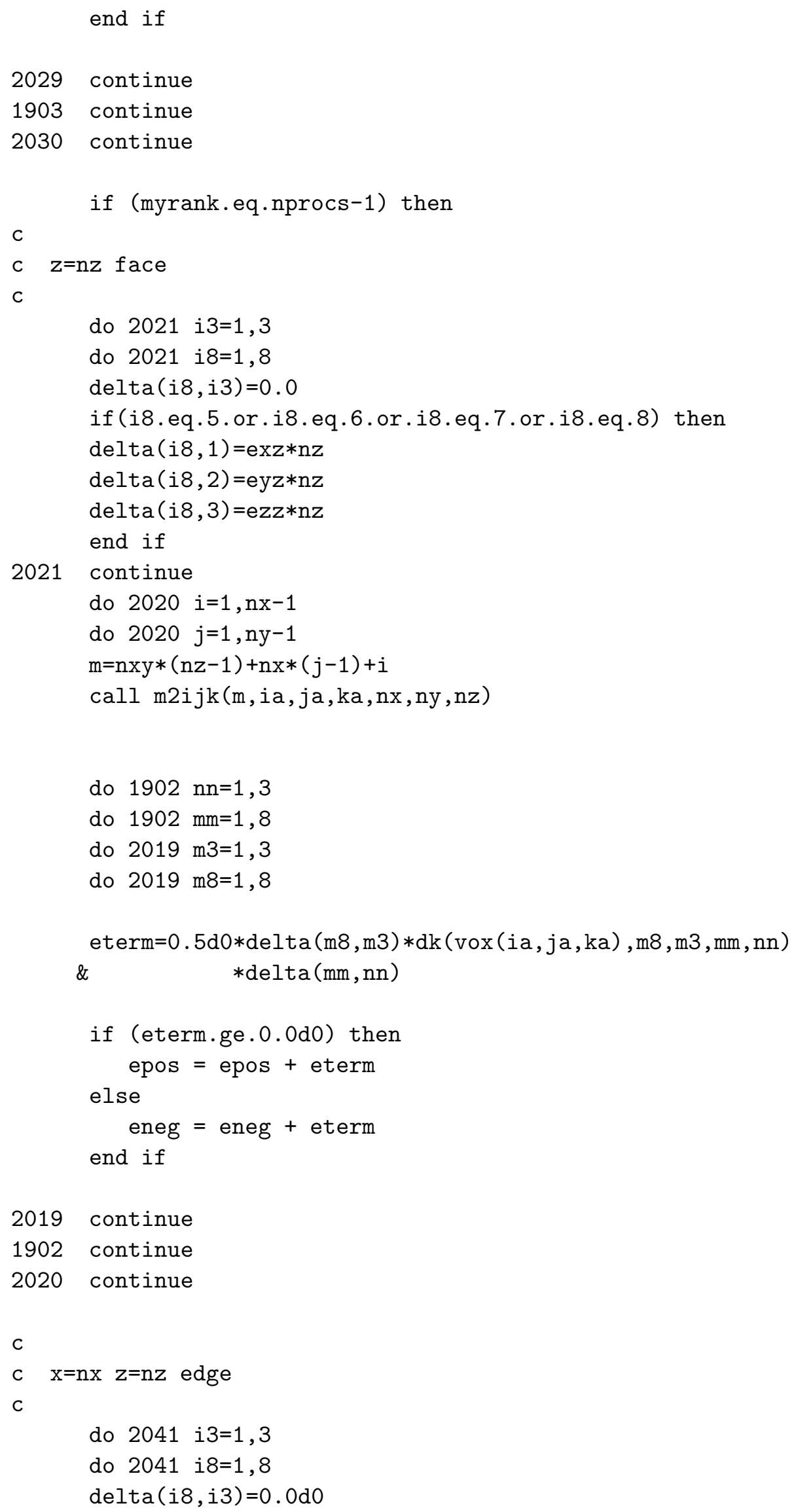




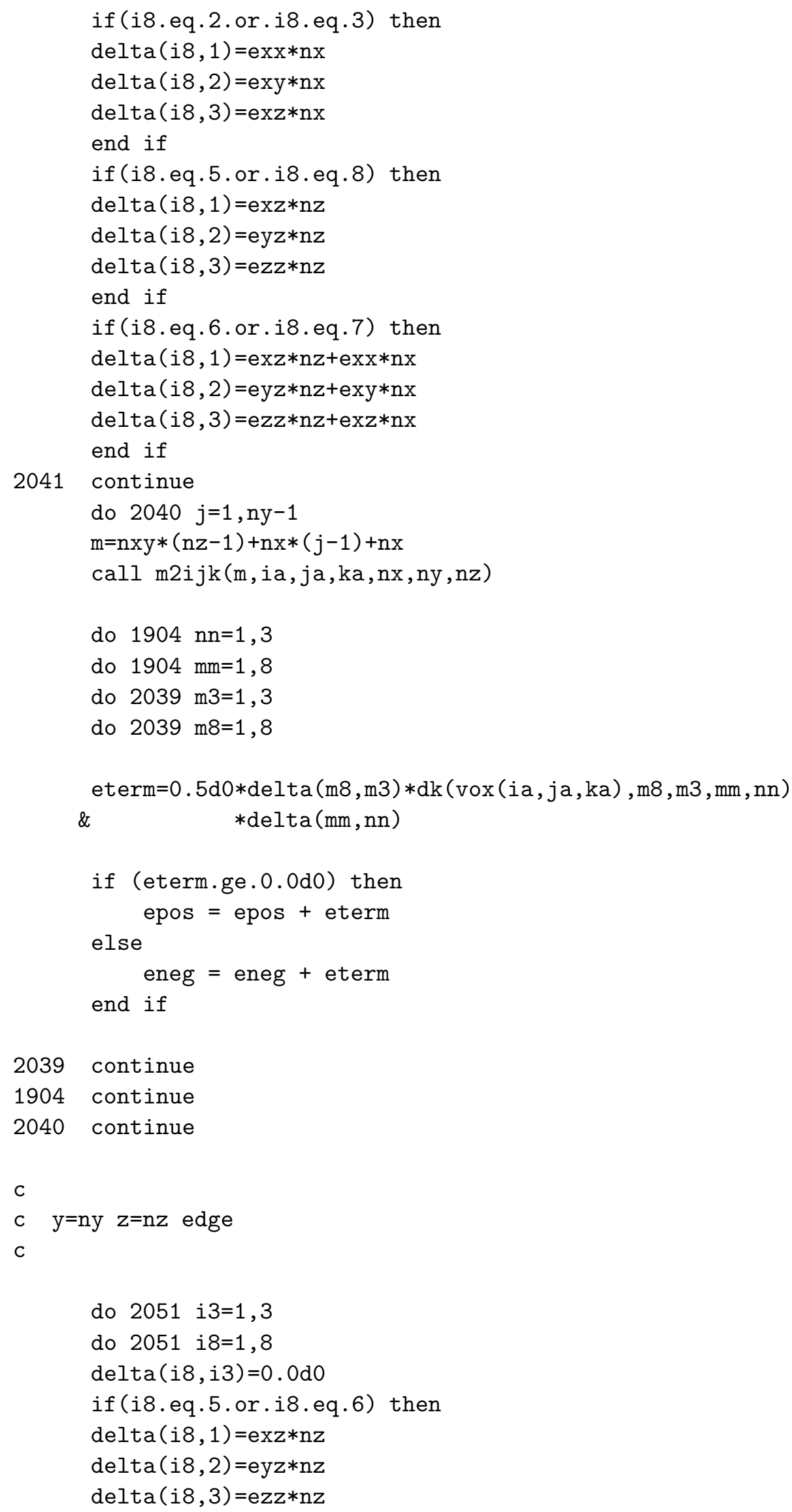




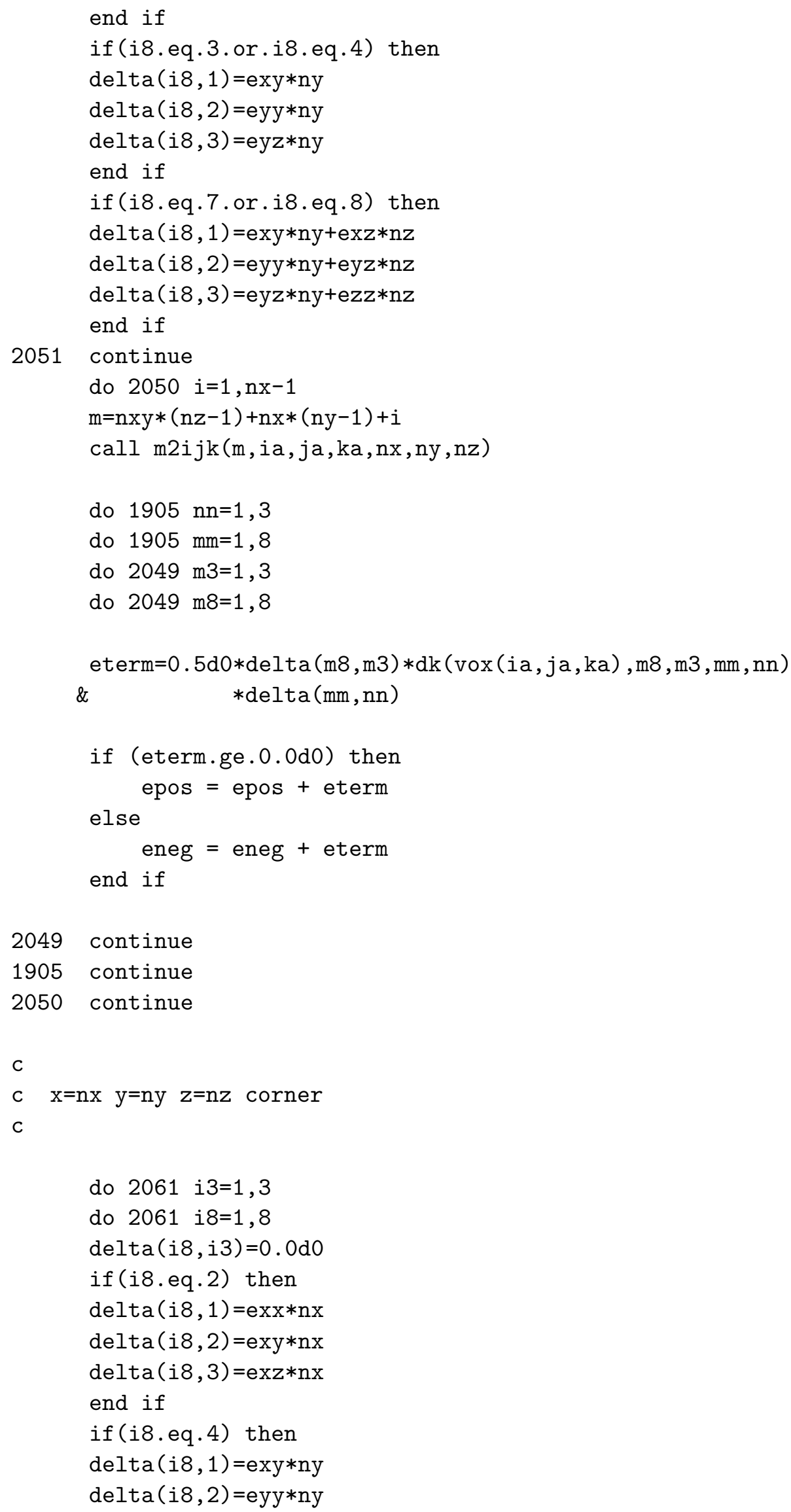




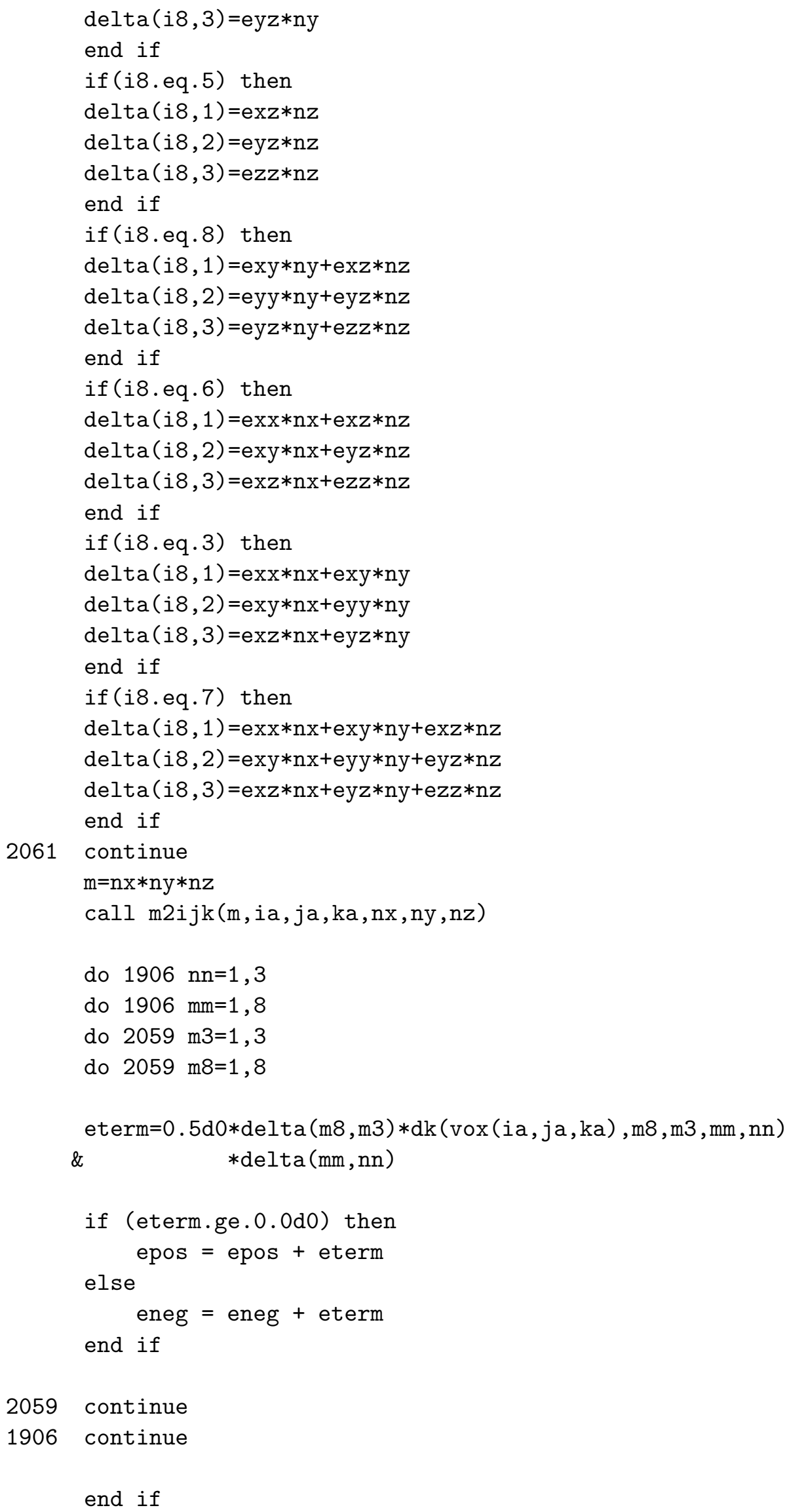

end if 
c Now the nprocs-1 processor is done...

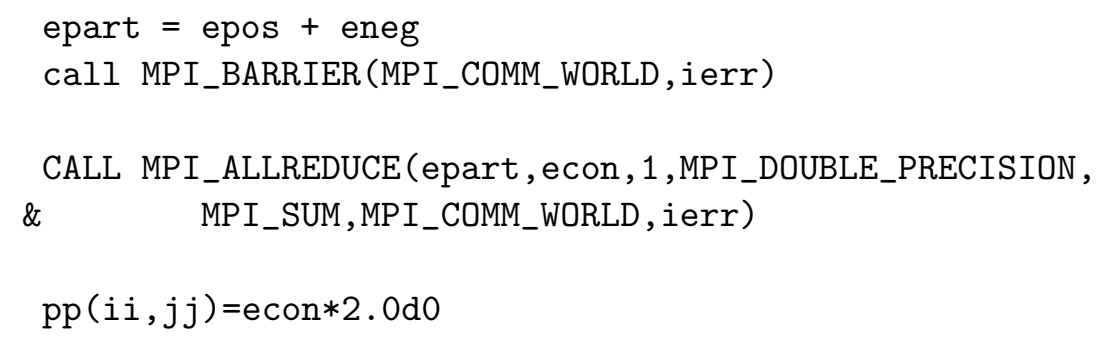

c Subroutine computes the total energy, utot, and the gradient, gb, 
implicit none

include 'mpif.h'

integer nx, ny, nz,ns, nxy, d1, d2, nphase,pflag

integer ii,i2i,i,j,k,m,ij,ik,m3

integer $\mathrm{mi}, \mathrm{mj}$

double precision $u(n x, n y, d 1-1: d 2+1,3), g b(n x, n y, d 1-1: d 2+1,3)$

double precision $b(n x, n y, d 1-1: d 2+1,3), T(n x, n y, d 1-1: d 2+1,3)$

double precision dk(nphase, $8,3,8,3), \operatorname{zcon}(2,3,2,3)$

double precision $u 2(2,3), g b 2(2,3), \mathrm{T} 2(2,3)$

double precision utot, tu, tutot, dutot, C, sum_num, Y double precision dusum

double precision exx, eyy, ezz, exz, eyz, exy

integer*2 $\operatorname{vox}(\mathrm{nx}, \mathrm{ny}, \mathrm{d} 1-1: \mathrm{d} 2+1)$

C

c MPI VARIABLES

C

integer myrank, nprocs, ierr, status (MPI_STATUS_SIZE)

common/list1/pflag, nphase

call MPI_COMM_RANK( MPI_COMM_WORLD, myrank, ierr )

call MPI_COMM_SIZE( MPI_COMM_WORLD, nprocs, ierr )

$\mathrm{ns}=\mathrm{nx} * \mathrm{ny} * \mathrm{nz}$

$\mathrm{nxy}=\mathrm{nx} * \mathrm{ny}$

$\mathrm{gb}=0.0 \mathrm{~d} 0$

call gbah (gb,u, dk, vox, nx, ny, nz, d1, d2)

C

c Same gbah subroutine as in ELAS3D_MPI.F

C

utot $=0.0 \mathrm{~d} 0$

dutot $=0.0 \mathrm{~d} 0$

do $m 3=1,3$ 


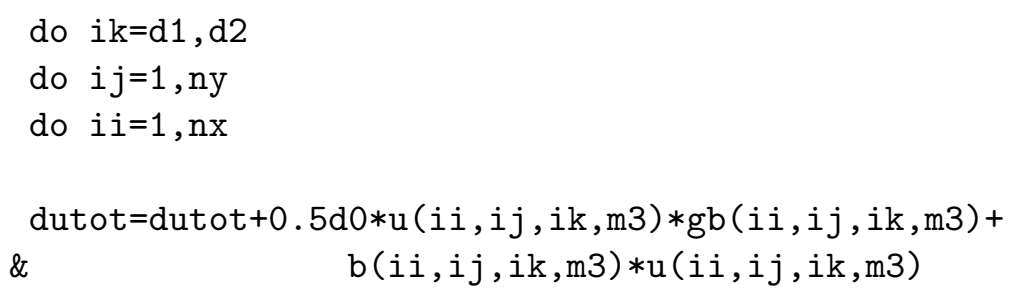




$$
\text { utot }=\text { utot }+ \text { tu }
$$

c Compute gradient with respect to macrostrains.

c put in piece from first derivative of zcon quadratic term

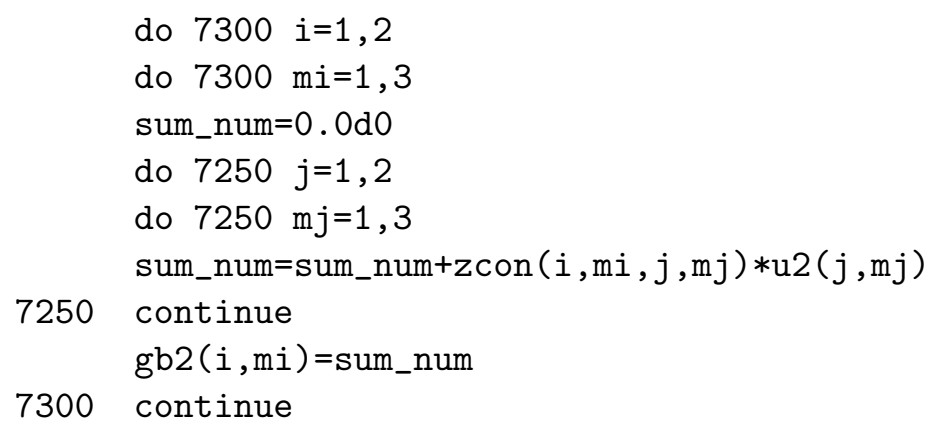

c Add in piece of gradient, for displacements as well as macrostrains, $c$ that come from linear term in thermal energy

$$
\begin{aligned}
& \mathrm{gb}=\mathrm{gb}+\mathrm{T} \\
& \mathrm{gb} 2=\mathrm{gb} 2+\mathrm{T} 2
\end{aligned}
$$

c Now generate part that comes from b. u term

$c$ do by calling $b$ generation with appropriate macrostrain set to 1 to

c get that partial derivative, just use bgrad (taken from femat),

c skip dk and zcon part

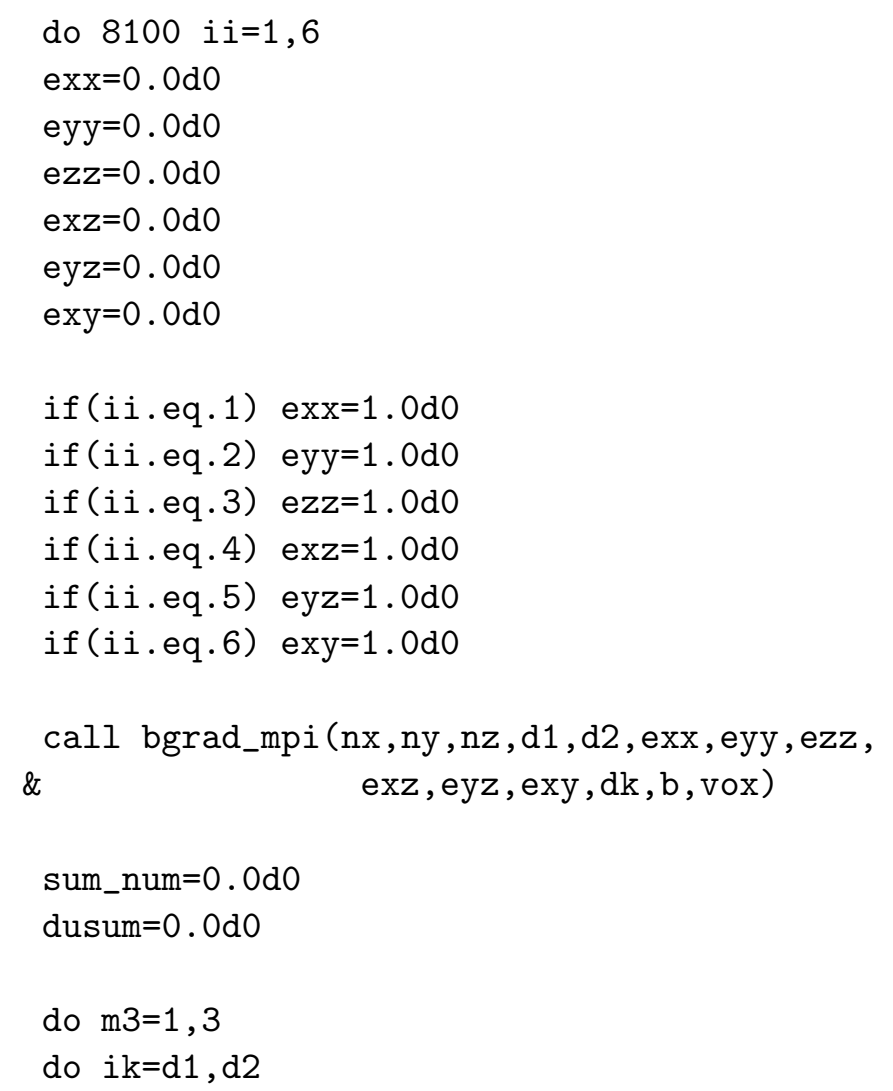




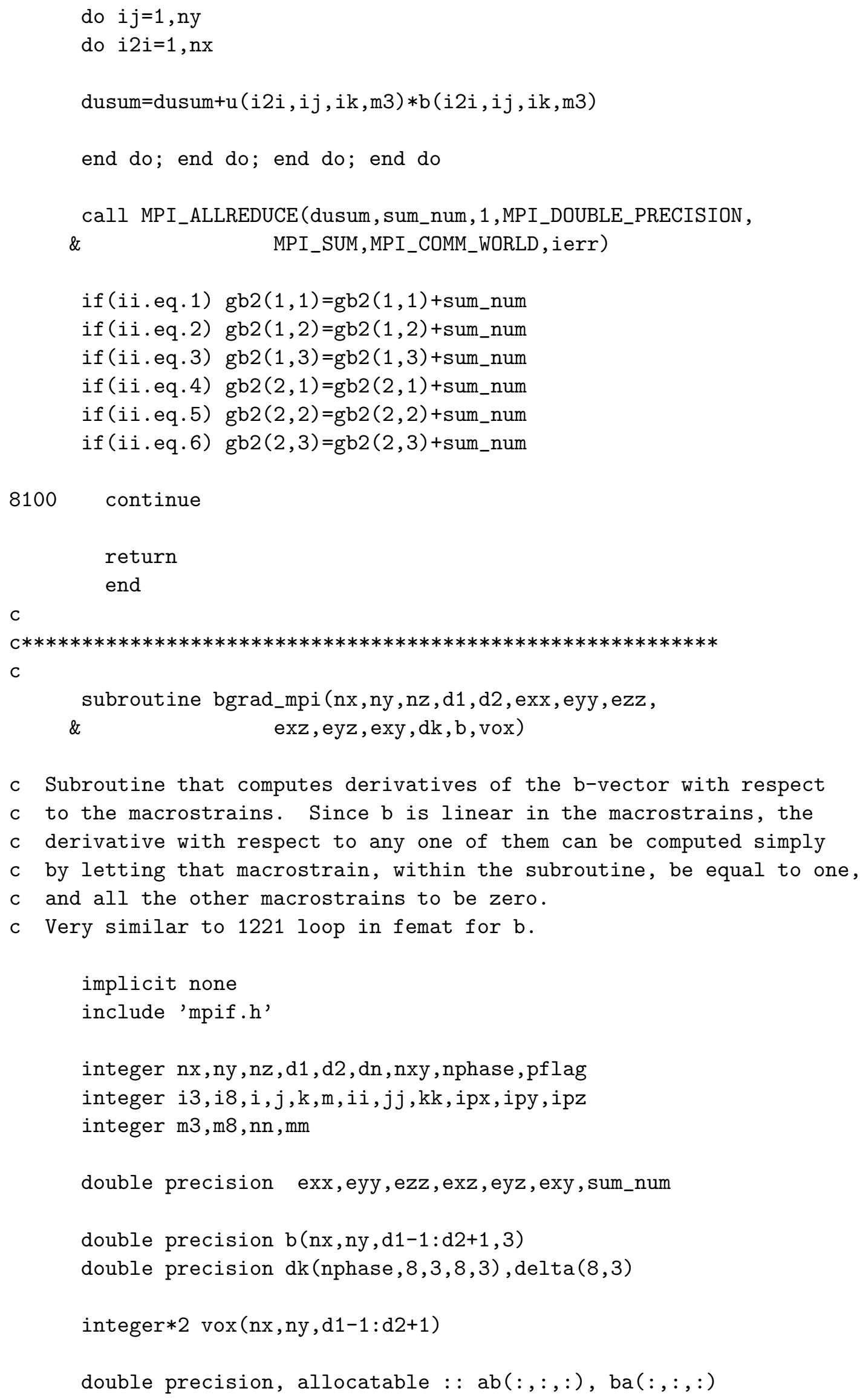

c Subroutine that computes derivatives of the b-vector with respect $c$ to the macrostrains. Since b is linear in the macrostrains, the c derivative with respect to any one of them can be computed simply $c$ by letting that macrostrain, within the subroutine, be equal to one, c and all the other macrostrains to be zero.

c Very similar to 1221 loop in femat for b. 
C

c MPI vars...

C

integer myrank, nprocs, ierr, status (MPI_STATUS_SIZE)

common/list1/pflag, nphase

call MPI_COMM_RANK ( MPI_COMM_WORLD, myrank, ierr )

call MPI_COMM_SIZE( MPI_COMM_WORLD, nprocs, ierr )

$\mathrm{nxy}=\mathrm{nx} * \mathrm{ny}$

c exx, eyy, ezz, exz, eyz, exy are the artificial macrostrains used

c to get the gradient terms (appropriate combinations of 1's and 0's).

allocate $(a b(n x, n y, 3))$

allocate (ba $(n x, n y, 3))$

c Set up vector for linear term

$b=0.0 d 0$

C

c $\mathrm{x}=\mathrm{nx}$ face

C

do 2001 i3=1,3

do 2001 i $8=1,8$

$\operatorname{delta}(i 8, i 3)=0.0 \mathrm{~d} 0$

if (i8.eq.2.or.i8.eq.3.or.i8.eq.6.or.i8.eq.7) then

$\operatorname{delta}(i 8,1)=\operatorname{exx} * n x$

$\operatorname{delta}(i 8,2)=\operatorname{exy} * n x$

$\operatorname{delta}(i 8,3)=\operatorname{exz} * n x$

end if

2001 continue

$\mathrm{dn}=\mathrm{d} 2$

if $(\mathrm{dn} \cdot \mathrm{eq} \cdot \mathrm{nz}) \mathrm{dn}=\mathrm{nz}-1$

do $2000 \mathrm{j}=1, \mathrm{ny}-1$

do $2000 \mathrm{k}=\mathrm{d} 1, \mathrm{dn}$

$\mathrm{m}=\mathrm{nxy} *(\mathrm{k}-1)+\mathrm{j} * \mathrm{nx}$

call m2ijk(m,ii,jj,kk,nx,ny,nz)

do $1900 \mathrm{nn}=1,3$

do $1900 \mathrm{~mm}=1,8$

sum_num $=0.0 \mathrm{~d} 0$

call ipxyz (mm,ii,jj,kk, ipx, ipy, ipz,nx,ny,nz) 


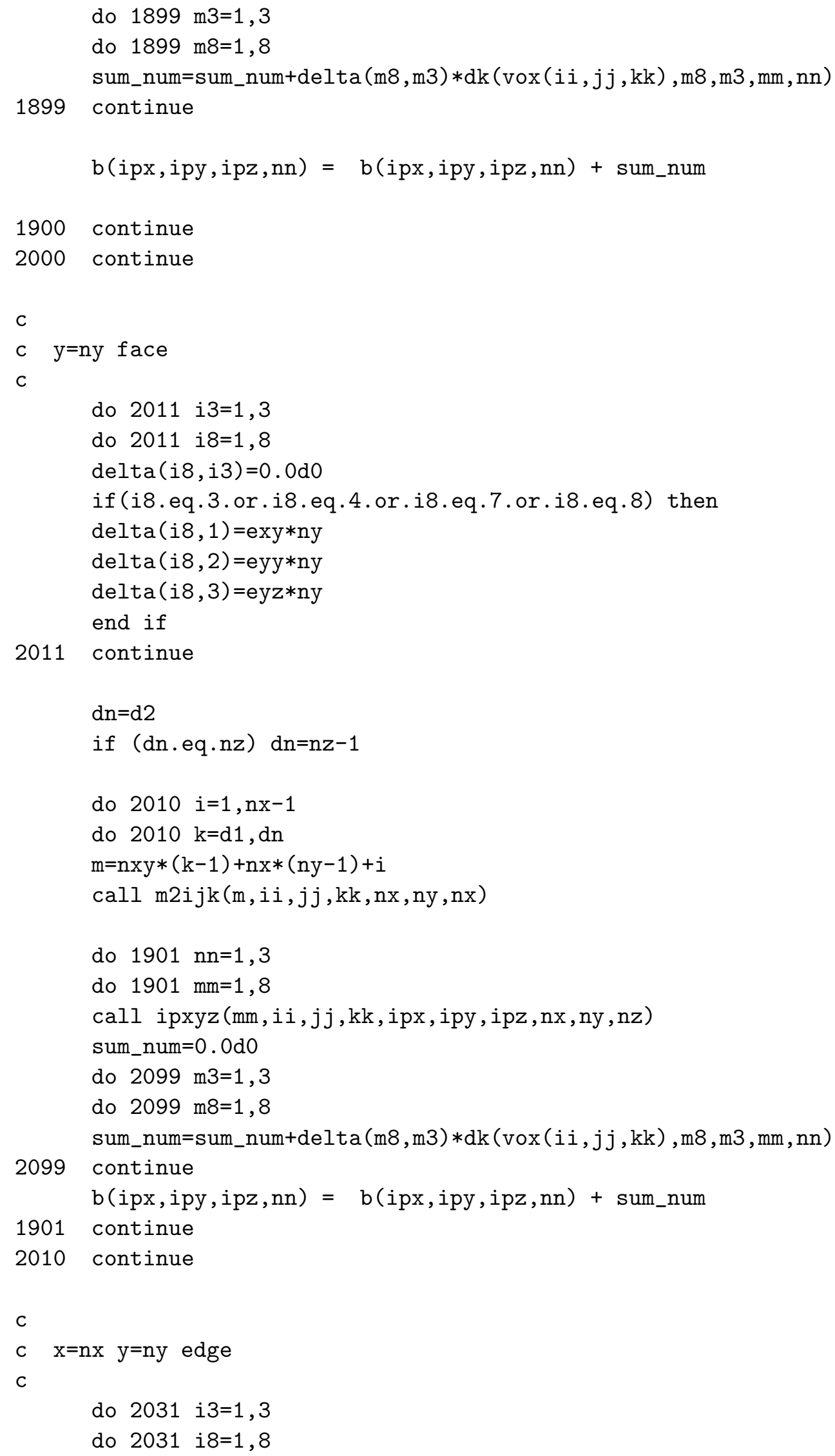




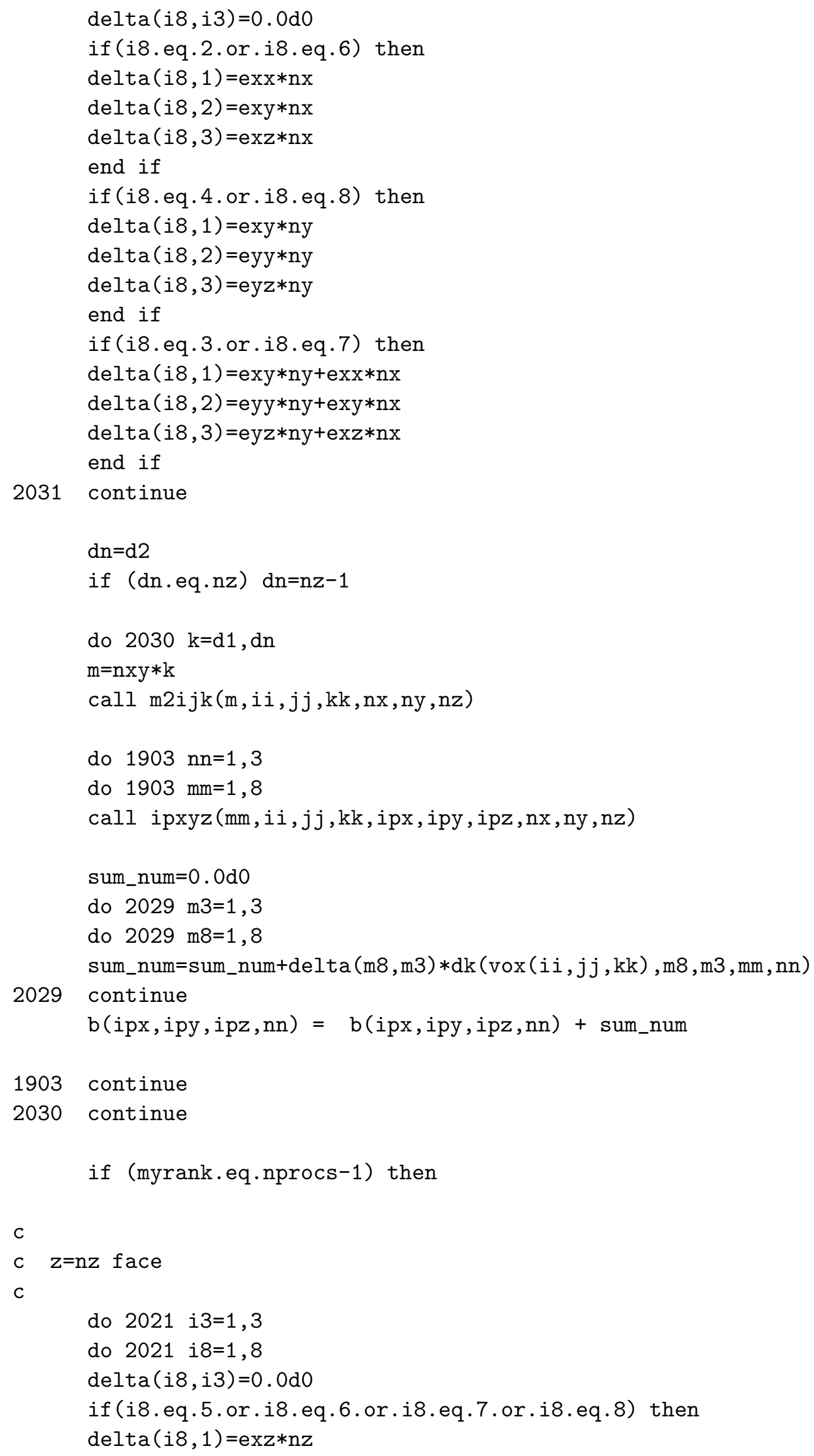




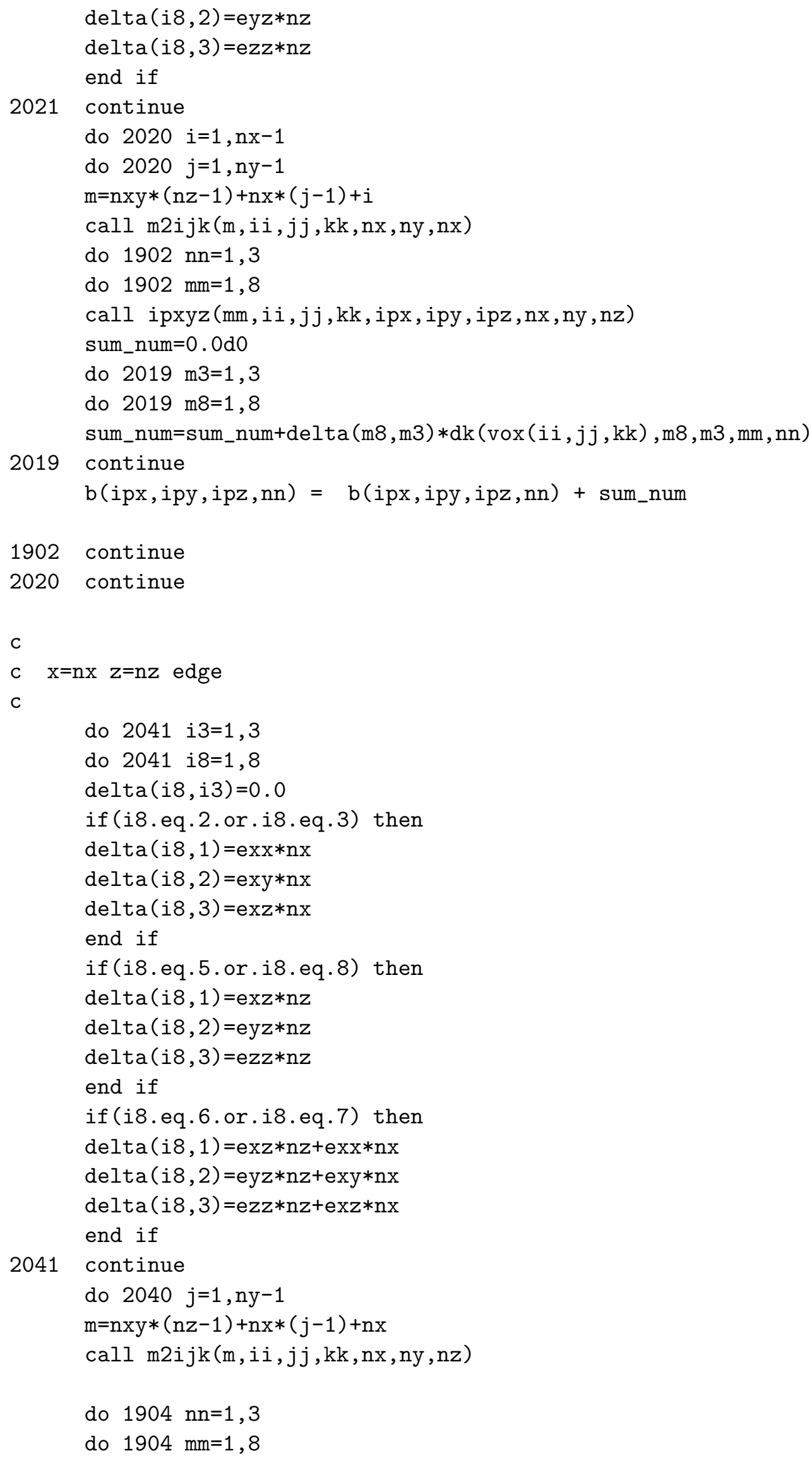




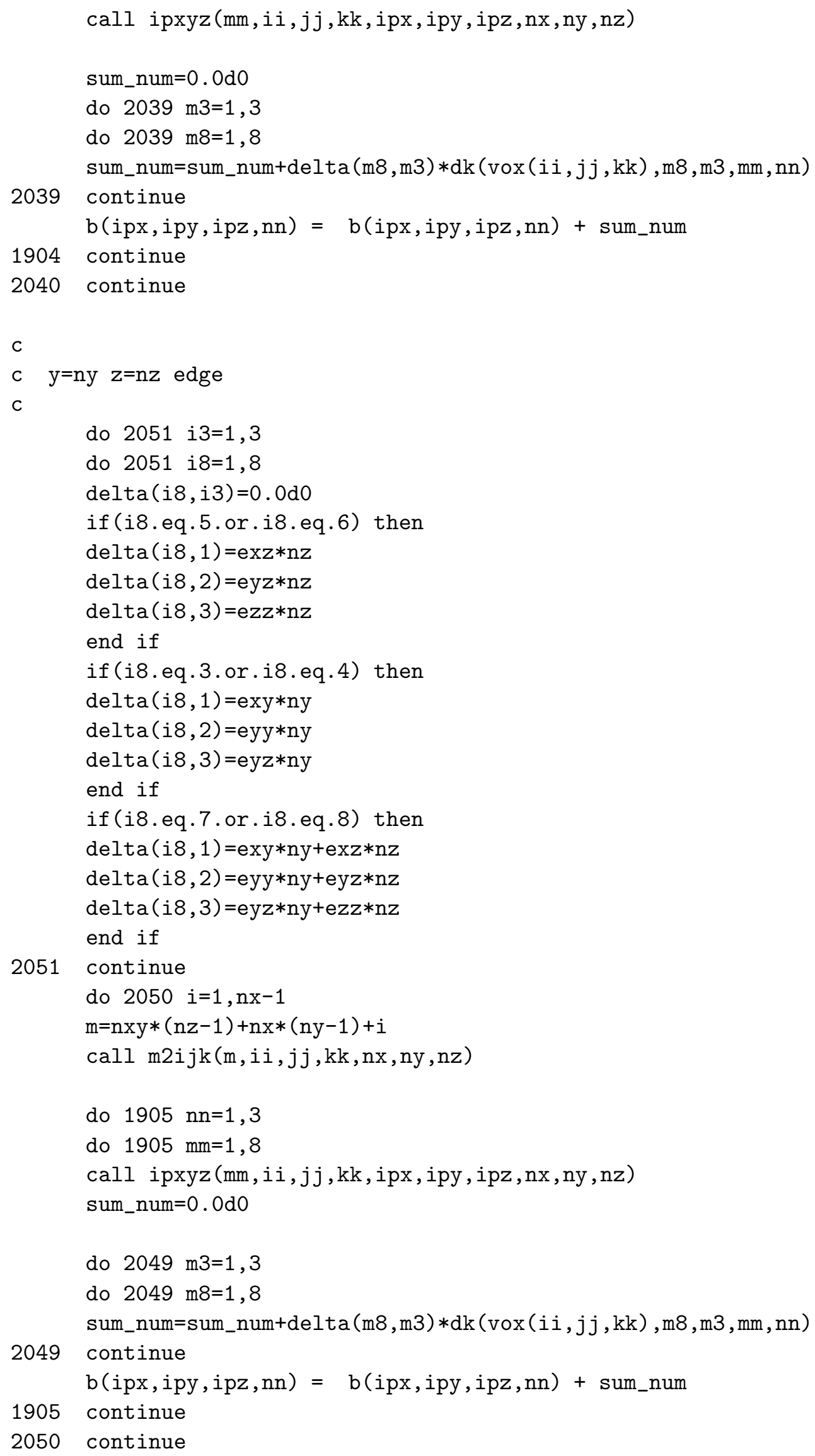


C

C $\mathrm{x}=\mathrm{nx}$ y=ny $\mathrm{z}=\mathrm{nz}$ corner

C

$$
\begin{aligned}
& \text { do } 2061 \text { i3 }=1,3 \\
& \text { do } 2061 \text { i8=1,8 } \\
& \operatorname{delta}(i 8, i 3)=0.0 \mathrm{~d} 0 \\
& \text { if (i8.eq.2) then } \\
& \operatorname{delta}(i 8,1)=\operatorname{exx} * \mathrm{nx} \\
& \operatorname{delta}(i 8,2)=\operatorname{exy} * n x \\
& \operatorname{delta}(i 8,3)=\operatorname{exz} * n x \\
& \text { end if } \\
& \text { if (i8.eq.4) then } \\
& \operatorname{delta}(i 8,1)=\text { exy } * \text { ny } \\
& \operatorname{delta}(i 8,2)=\text { eyy } * \text { ny } \\
& \operatorname{delta}(i 8,3)=e y z * n y
\end{aligned}
$$$$
\text { end if }
$$$$
\text { if (i8.eq.5) then }
$$$$
\operatorname{delta}(i 8,1)=\operatorname{exz} * \mathrm{nz}
$$$$
\operatorname{delta}(i 8,2)=e y z * n z
$$$$
\operatorname{delta}(i 8,3)=e z z * n z
$$$$
\text { end if }
$$$$
\text { if (i8.eq.8) then }
$$$$
\operatorname{delta}(i 8,1)=e x y * n y+e x z * n z
$$$$
\operatorname{delta}(i 8,2)=e y y * n y+e y z * n z
$$$$
\operatorname{delta}(i 8,3)=e y z * n y+e z z * n z
$$$$
\text { end if }
$$$$
\text { if (i8.eq.6) then }
$$$$
\operatorname{delta}(i 8,1)=e x x * n x+e x z * n z
$$$$
\operatorname{delta}(i 8,2)=e x y * n x+e y z * n z
$$$$
\operatorname{delta}(i 8,3)=e x z * n x+e z z * n z
$$$$
\text { end if }
$$$$
\text { if (i8.eq.3) then }
$$$$
\operatorname{delta}(i 8,1)=\operatorname{exx} * n x+\text { exy } * n y
$$$$
\operatorname{delta}(i 8,2)=e x y * n x+\text { eyy } * \text { ny }
$$$$
\operatorname{delta}(i 8,3)=e x z * n x+e y z * n y
$$$$
\text { end if }
$$$$
\text { if (i8.eq.7) then }
$$$$
\operatorname{delta}(i 8,1)=e x x * n x+e x y * n y+e x z * n z
$$$$
\operatorname{delta}(i 8,2)=e x y * n x+e y y * n y+e y z * n z
$$$$
\operatorname{delta}(i 8,3)=e x z * n x+e y z * n y+e z z * n z
$$$$
\text { end if }
$$

$\mathrm{m}=\mathrm{nx} * \mathrm{ny} * \mathrm{nz}$

call m2ijk(m,ii,jj,kk, nx, ny,nz)

do $1906 \mathrm{nn}=1,3$

do $1906 \mathrm{~mm}=1,8$

call ipxyz(mm,ii,jj,kk, ipx, ipy, ipz,nx,ny,nz)

sum_num $=0.0 \mathrm{~d} 0$ 


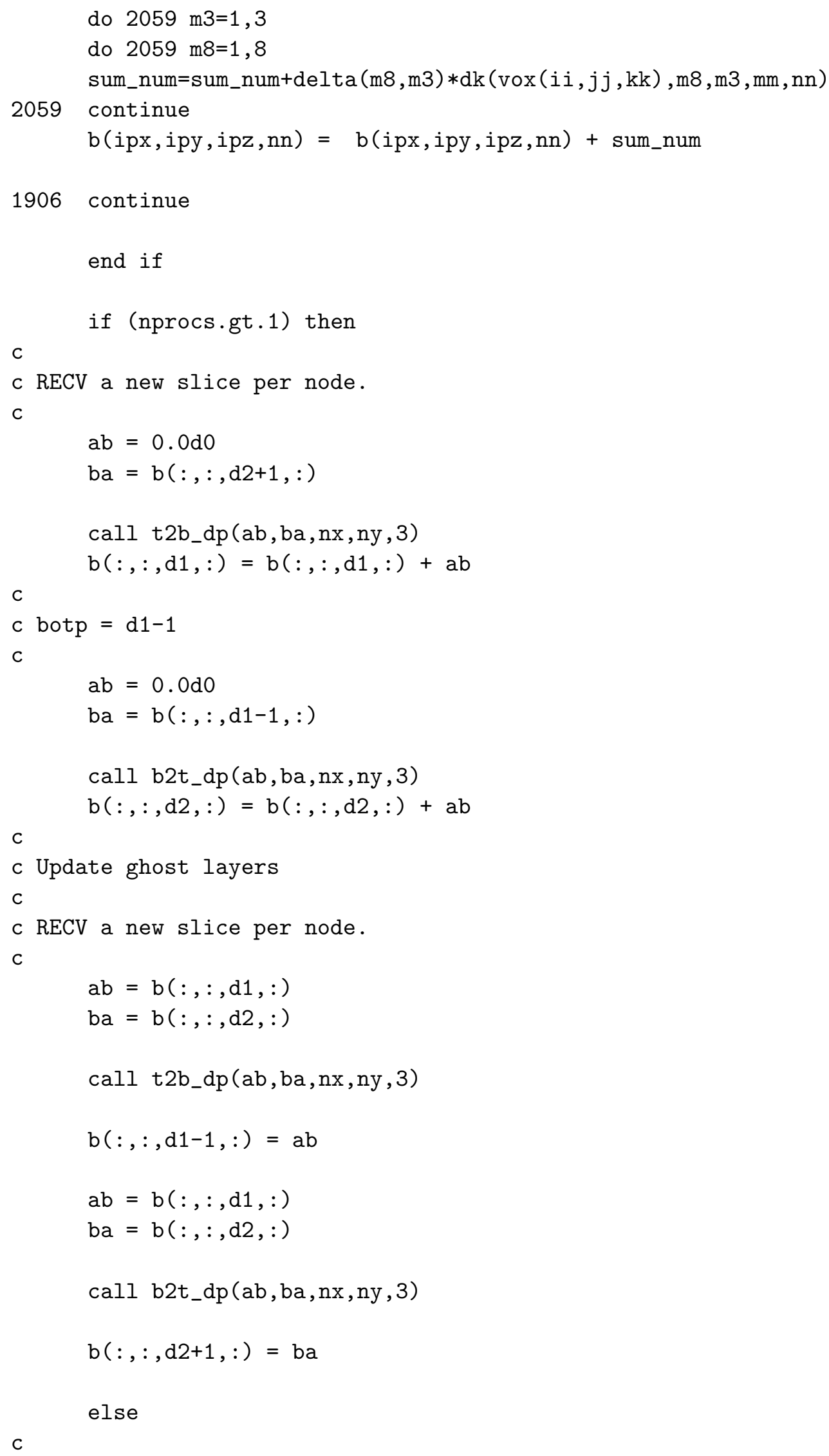




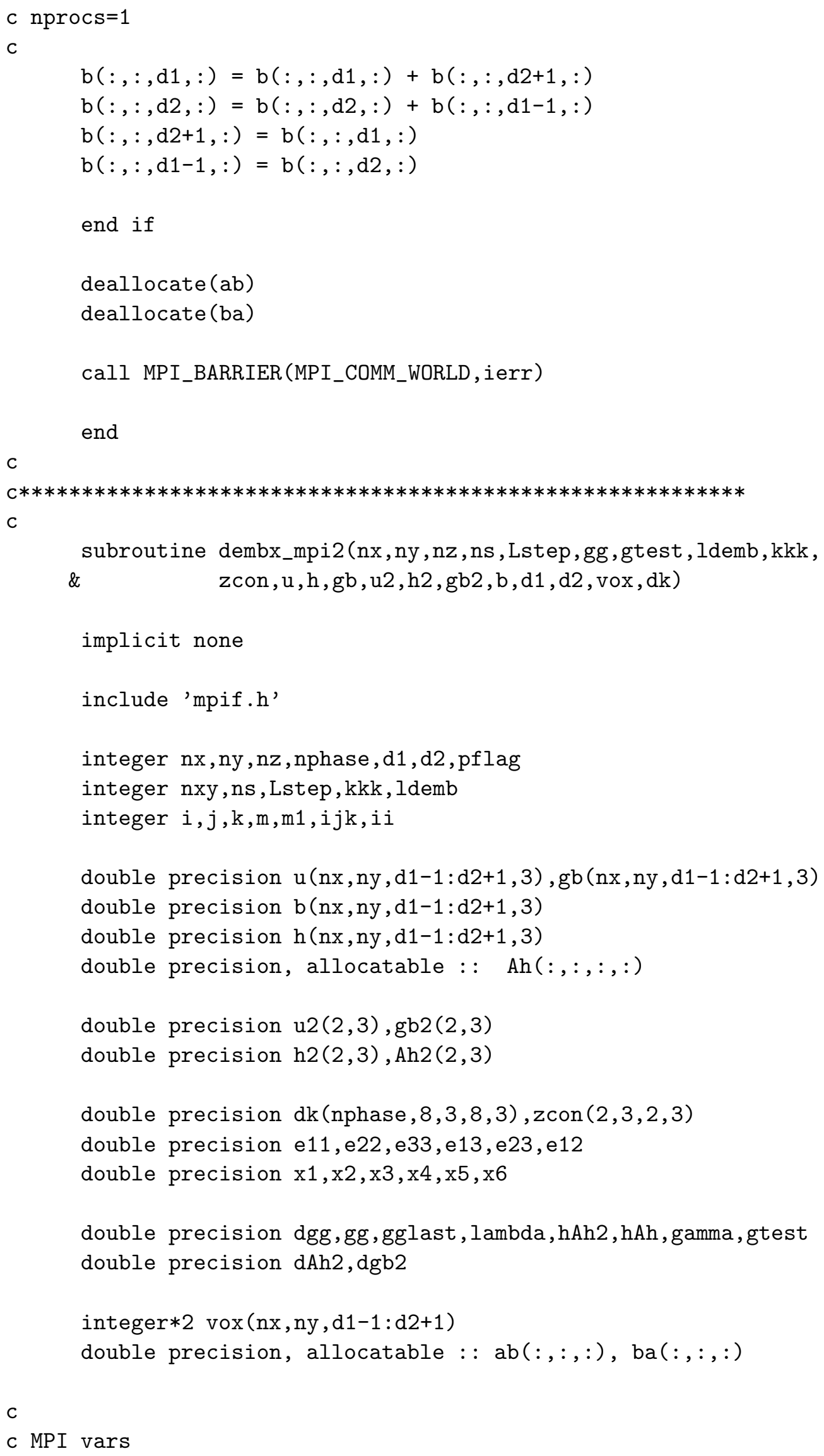


integer myrank,nprocs,ierr, status (MPI_STATUS_SIZE)

common/list1/pflag, nphase

call MPI_COMM_RANK( MPI_COMM_WORLD, myrank, ierr )

call MPI_COMM_SIZE( MPI_COMM_WORLD, nprocs, ierr )

allocate $(a b(n x, n y, 3))$

allocate (ba $(n x, n y, 3))$

allocate (Ah $(n x, n y, d 1-1: d 2+1,3))$

$\mathrm{ns}=\mathrm{nx} * \mathrm{ny} * \mathrm{nz}$

c Initialize the conjugate direction vector on first call to dembx only.

c For calls to dembx after the first, we want to continue using the value

$c$ of $\mathrm{h}$ determined in the previous call. Of course, if npoints

$c$ is greater than 1, then this initialization step will be run each time

c a new microstructure is used, as kkk will be reset to 1 every time

$c$ the counter micro is increased.

if (kkk.eq.1) then

$\mathrm{h}=\mathrm{gb}$

$\mathrm{h} 2=\mathrm{gb} 2$

end if

c Lstep counts the number of conjugate gradient steps taken

c in each call to dembx

Lstep $=0$

do $800 i j k=1,1 d e m b$

Lstep $=$ Lstep +1

$\mathrm{Ah}=0.0 \mathrm{~d} 0$

Ah2 $=0.0 \mathrm{~d} 0$

c Do global matrix multiply via small stiffness matrices, $\mathrm{Ah}=\mathrm{A} * \mathrm{~h}$

$c$ The long statement below correctly brings in all the terms from

$c$ the global matrix A using only the small stiffness matrices.

call gbah (Ah, h, dk, vox, nx, ny, nz, d1, d2)

c The above accurately gives the second derivative matrix with respect

c to nodal displacements, but fails to give the 2nd derivative terms that

$c$ include the macrostrains [ du d(strain) and d(strain)d(strain)].

c Use repeated calls to bgrad to generate mixed 2nd derivatives terms, c plus use zcon in order to correct the matrix multiply and correctly bring

c in macrostrain terms (see manual, Sec. 2.4).

do $8100 i i=1,6$ 


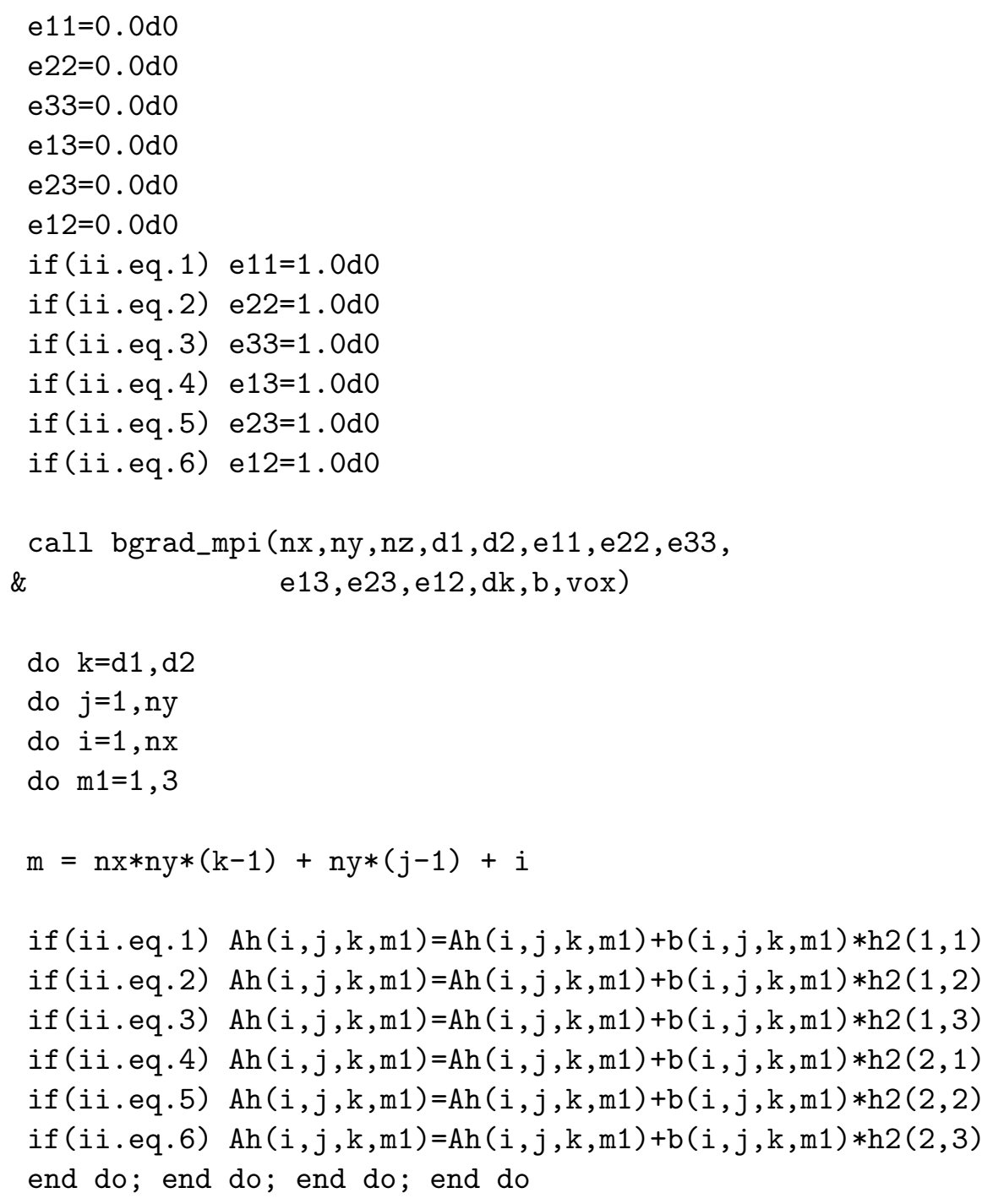




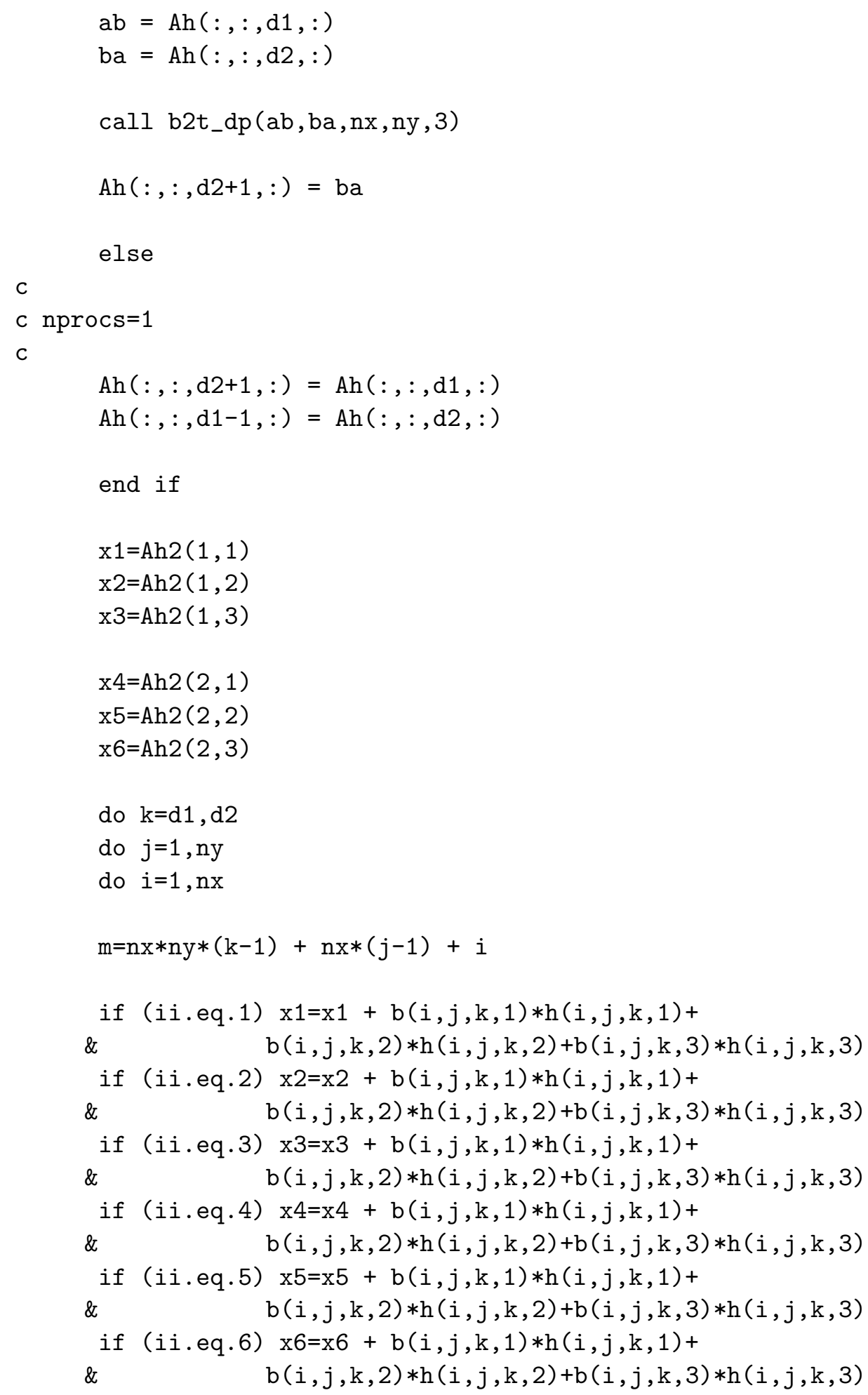




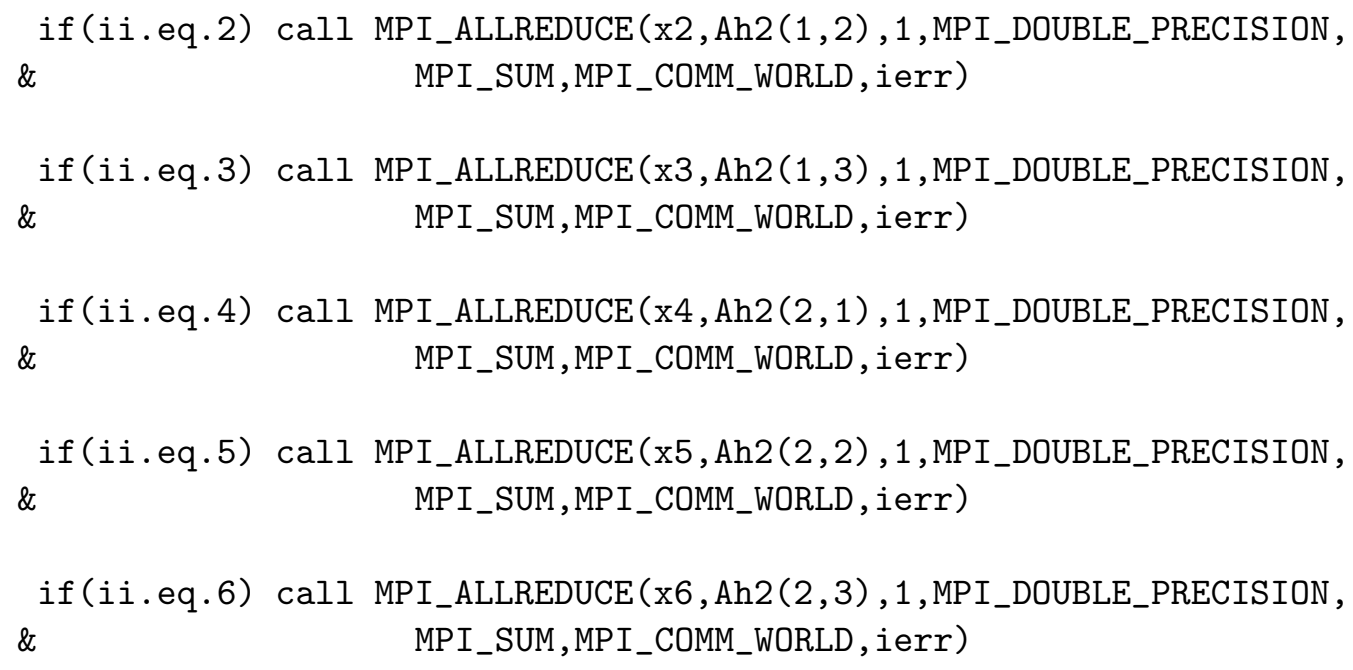

c now do righthand corner terms, ns+1 to ns+2

do $3335 \mathrm{~m}=1,2$

do $3335 \mathrm{~m} 1=1,3$

if (ii.eq.1) $\operatorname{Ah} 2(1,1)=\operatorname{Ah} 2(1,1)+z \operatorname{con}(1,1, \mathrm{~m}, \mathrm{~m} 1) * \mathrm{~h} 2(\mathrm{~m}, \mathrm{~m} 1)$

if (ii.eq. 2) $\operatorname{Ah} 2(1,2)=\operatorname{Ah} 2(1,2)+\mathrm{zcon}(1,2, \mathrm{~m}, \mathrm{~m} 1) * \mathrm{~h} 2(\mathrm{~m}, \mathrm{~m} 1)$

if (ii.eq.3) $\operatorname{Ah} 2(1,3)=\operatorname{Ah} 2(1,3)+\mathrm{zcon}(1,3, \mathrm{~m}, \mathrm{~m} 1) * \mathrm{~h} 2(\mathrm{~m}, \mathrm{~m} 1)$

if (ii.eq.4) $\operatorname{Ah} 2(2,1)=\operatorname{Ah} 2(2,1)+\mathrm{zcon}(2,1, \mathrm{~m}, \mathrm{~m} 1) * \mathrm{~h} 2(\mathrm{~m}, \mathrm{~m} 1)$

if (ii.eq.5) $\operatorname{Ah} 2(2,2)=\operatorname{Ah} 2(2,2)+\mathrm{zcon}(2,2, \mathrm{~m}, \mathrm{~m} 1) * \mathrm{~h} 2(\mathrm{~m}, \mathrm{~m} 1)$

if (ii.eq.6) $\operatorname{Ah} 2(2,3)=\operatorname{Ah} 2(2,3)+\mathrm{zcon}(2,3, \mathrm{~m}, \mathrm{~m} 1) * \mathrm{~h} 2(\mathrm{~m}, \mathrm{~m} 1)$

3335 continue

8100 continue

$\mathrm{hAh}=0.0 \mathrm{~d} 0$

hAh2 $=0.0 \mathrm{~d} 0$

hAh2 $=\operatorname{SUM}(h(:,:, \mathrm{d} 1: \mathrm{d} 2,:) * \operatorname{Ah}(:,:, \mathrm{d} 1: \mathrm{d} 2,:))$

call MPI_ALLREDUCE (hAh2, hAh, 1, MPI_DOUBLE_PRECISION, $\&$ MPI_SUM , MPI_COMM_WORLD, ierr)

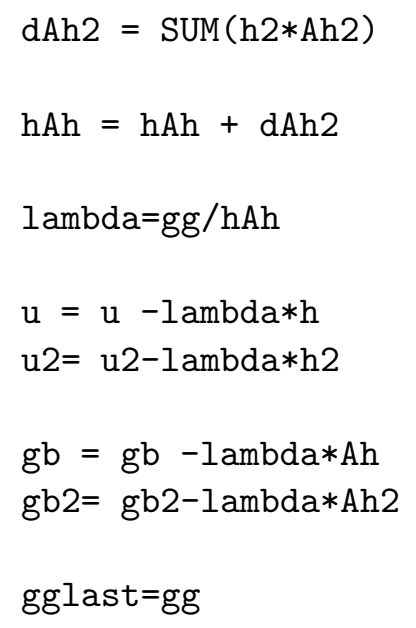




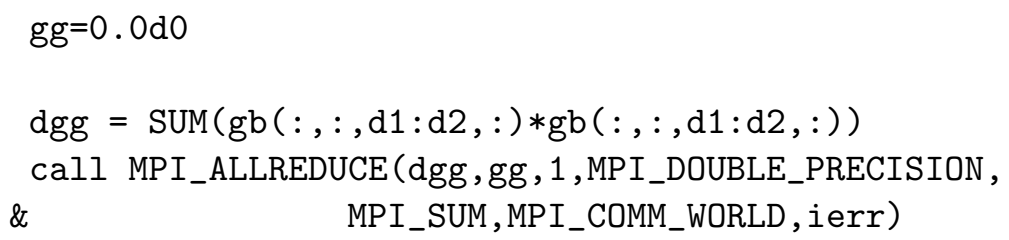

C

c Now add contributions from gb2 to gg.

C

$\mathrm{dgb} 2=\operatorname{SUM}(\mathrm{gb} 2 * \mathrm{gb} 2)$

$g g=g g+d g b 2$

if (gg.lt.gtest) goto 1000

gamma $=$ gg/gglast

$\mathrm{h}=\mathrm{gb}+$ gamma $* \mathrm{~h}$

$\mathrm{h} 2=\mathrm{gb} 2+$ gamma $* \mathrm{~h} 2$

800 continue

1000 continue

C

c u2, h2, gb2, lambda

C

deallocate $(\mathrm{ab})$

deallocate (ba)

deallocate $(\mathrm{Ah})$

return

end

C

c************************************************************

C

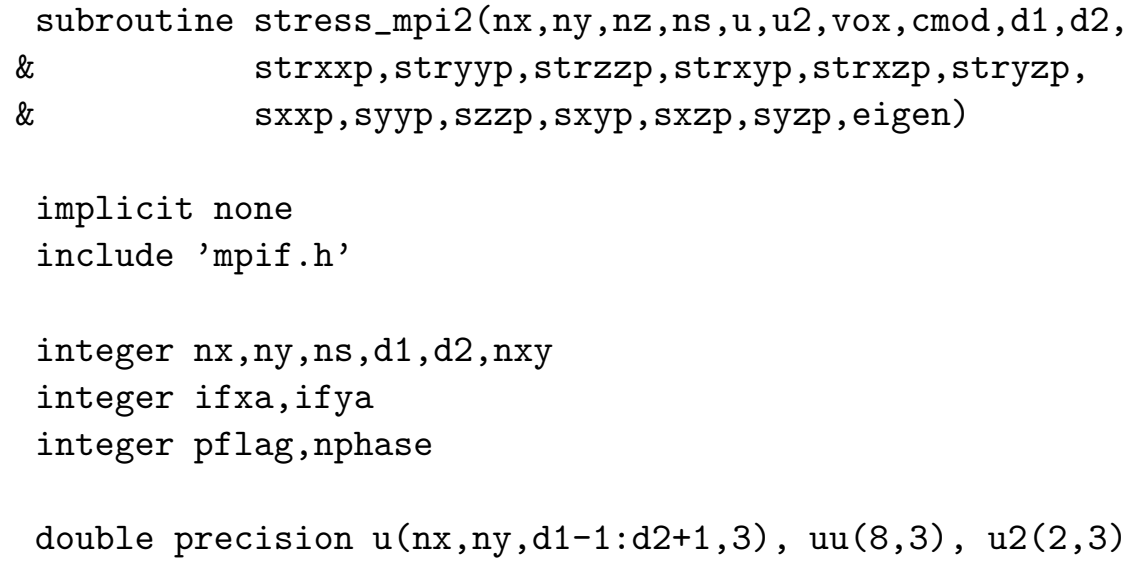




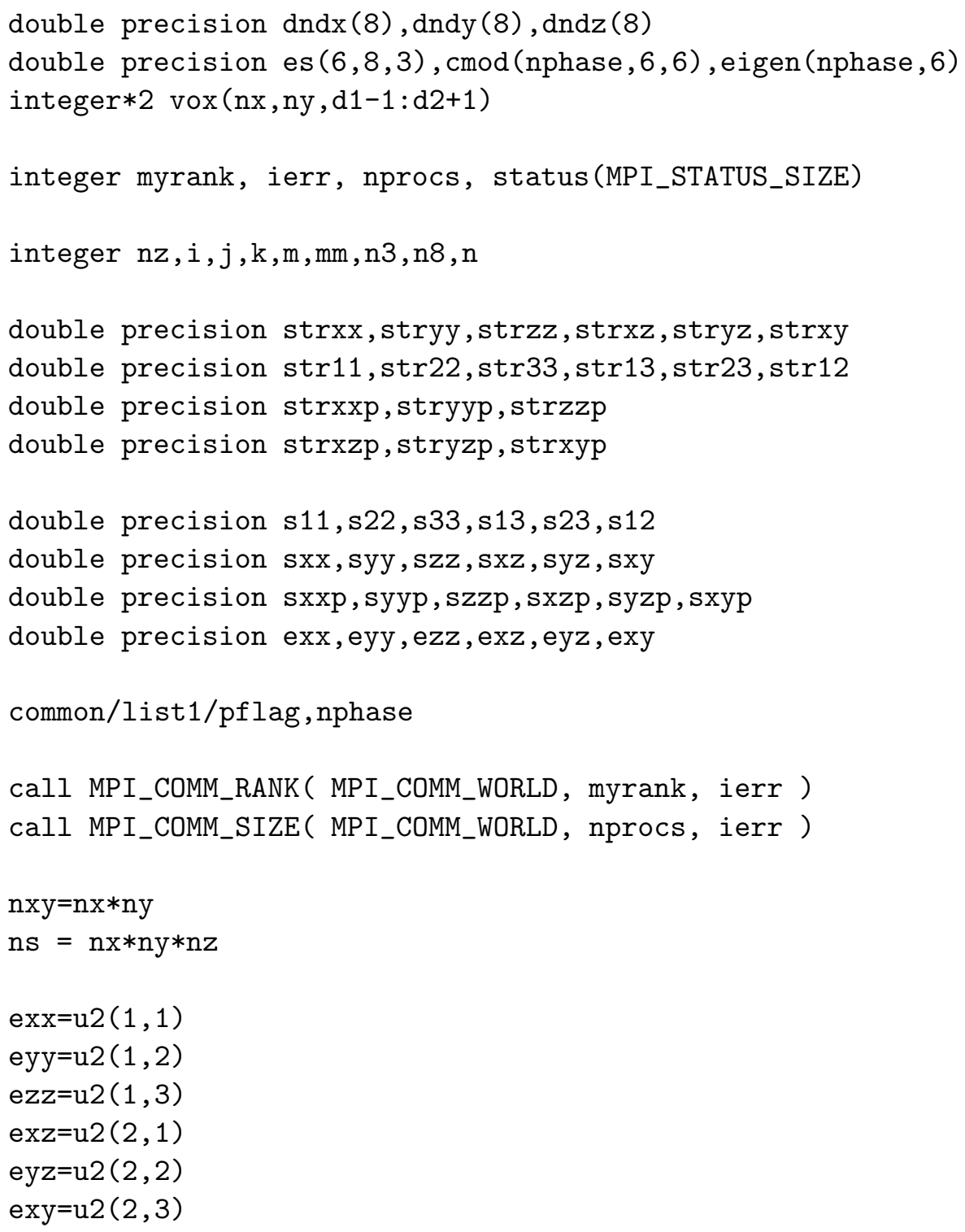

$c$ set up single element strain matrix

c dndx, dndy, and $d n d z$ are the components of the average strain

c matrix in a pixel

$$
\begin{aligned}
& \operatorname{dndx}(1)=-0.25 \mathrm{~d} 0 \\
& \operatorname{dndx}(2)=0.25 \mathrm{~d} 0 \\
& \operatorname{dndx}(3)=0.25 \mathrm{~d} 0 \\
& \operatorname{dndx}(4)=-0.25 \mathrm{~d} 0 \\
& \operatorname{dndx}(5)=-0.25 \mathrm{~d} 0 \\
& \operatorname{dndx}(6)=0.25 \mathrm{~d} 0 \\
& \operatorname{dndx}(7)=0.25 \mathrm{~d} 0 \\
& \operatorname{dndx}(8)=-0.25 \mathrm{~d} 0 \\
& \operatorname{dndy}(1)=-0.25 \mathrm{~d} 0 \\
& \operatorname{dndy}(2)=-0.25 \mathrm{~d} 0 \\
& \operatorname{dndy}(3)=0.25 \mathrm{~d} 0 \\
& \operatorname{dndy}(4)=0.25 \mathrm{~d} 0
\end{aligned}
$$




$$
\begin{aligned}
& \operatorname{dndy}(5)=-0.25 \mathrm{~d} 0 \\
& \operatorname{dndy}(6)=-0.25 \mathrm{~d} 0 \\
& \operatorname{dndy}(7)=0.25 \mathrm{~d} 0 \\
& \operatorname{dndy}(8)=0.25 \mathrm{~d} 0 \\
& \operatorname{dndz}(1)=-0.25 \mathrm{~d} 0 \\
& \operatorname{dndz}(2)=-0.25 \mathrm{~d} 0 \\
& \operatorname{dndz}(3)=-0.25 \mathrm{~d} 0 \\
& \operatorname{dndz}(4)=-0.25 \mathrm{~d} 0 \\
& \operatorname{dndz}(5)=0.25 \mathrm{~d} 0 \\
& \operatorname{dndz}(6)=0.25 \mathrm{~d} 0 \\
& \operatorname{dndz}(7)=0.25 \mathrm{~d} 0 \\
& \operatorname{dndz}(8)=0.25 \mathrm{~d} 0
\end{aligned}
$$

c Build averaged strain matrix, follows code in femat, but for average

c strain over the pixel, not the strain at a point.

$$
\begin{aligned}
& \text { es }=0.0 \mathrm{~d} 0 \\
& \text { es }(1,:, 1)=\operatorname{dndx} \\
& \text { es }(2,:, 2)=\operatorname{dndy} \\
& \text { es }(3,:, 3)=\operatorname{dnd} z \\
& \text { es }(4,:, 1)=\operatorname{dnd} z \\
& \text { es }(4,:, 3)=\operatorname{dndx} \\
& \text { es }(5,:, 2)=\operatorname{dnd} z \\
& \text { es }(5,:, 3)=\operatorname{dndy} \\
& \text { es }(6,:, 1)=\operatorname{dndy} \\
& \text { es }(6,:, 2)=\operatorname{dndx}
\end{aligned}
$$

c Compute components of the average stress and strain tensors in each pixel

$$
\begin{aligned}
& \text { sxx }=0.0 d 0 \\
& \text { syy }=0.0 d 0 \\
& \text { szz }=0.0 d 0 \\
& \text { sxz }=0.0 d 0 \\
& \text { syz }=0.0 d 0 \\
& \text { sxy }=0.0 d 0 \\
& \text { strxx }=0.0 d 0 \\
& \text { stryy }=0.0 d 0 \\
& \text { strzz }=0.0 d 0 \\
& \text { strxz }=0.0 d 0 \\
& \text { stryz }=0.0 d 0 \\
& \text { strxy }=0.0 d 0 \\
& \text { sxxp }=0.0 d 0 \\
& \text { syyp }=0.0 d 0 \\
& \text { szzp }=0.0 d 0 \\
& \text { sxzp }=0.0 d 0 \\
& \text { syzp }=0.0 d 0 \\
& \text { sxyp }=0.0 d 0 \\
& \text { strxxp }=0.0 d 0 \\
& \text { stryyp }=0.0 d 0
\end{aligned}
$$




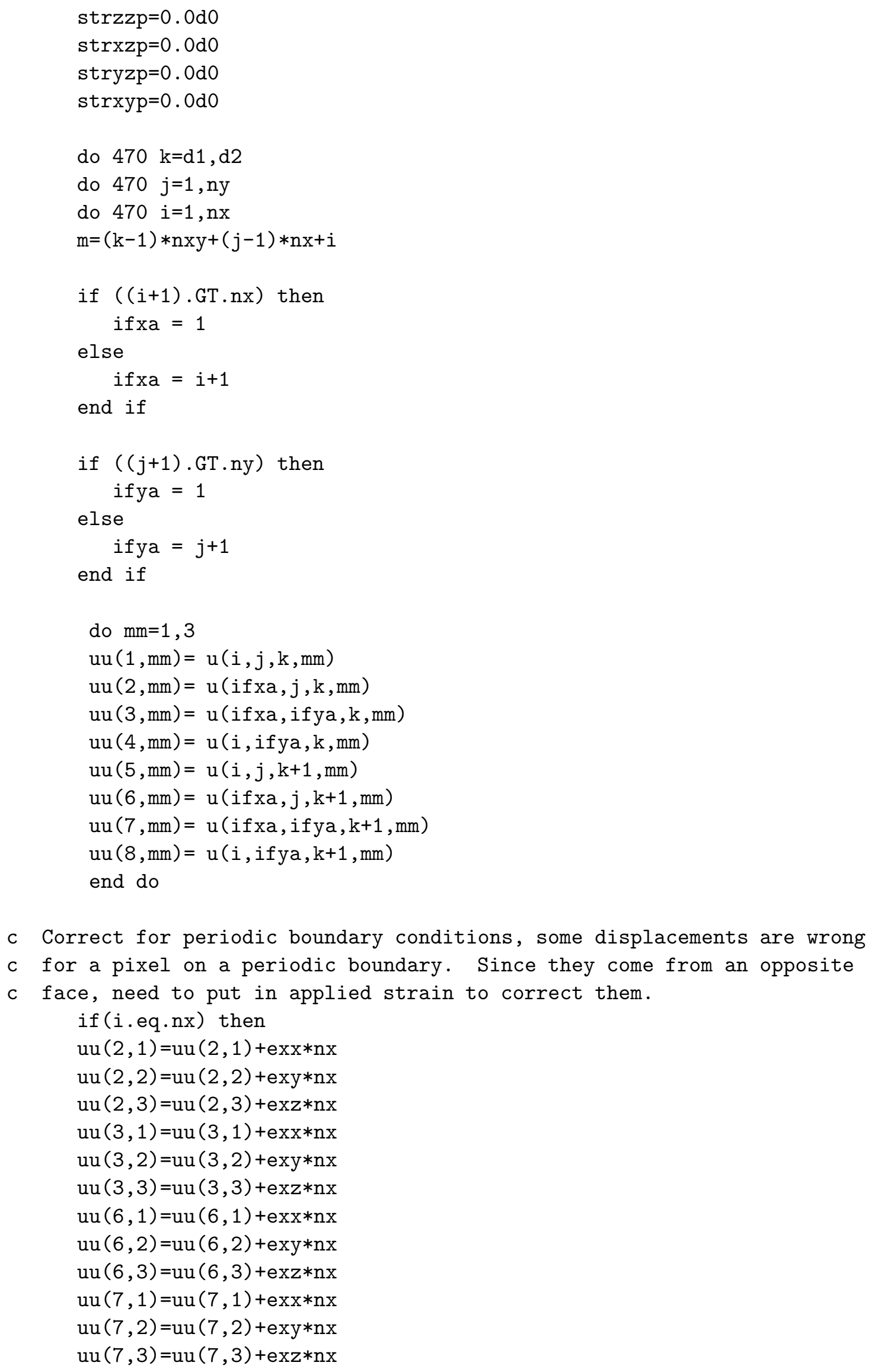

c Correct for periodic boundary conditions, some displacements are wrong

c for a pixel on a periodic boundary. Since they come from an opposite

c face, need to put in applied strain to correct them.

if (i.eq.nx) then

$\mathrm{uu}(2,1)=\mathrm{uu}(2,1)+\mathrm{exx} * \mathrm{nx}$

$\mathrm{uu}(2,2)=\mathrm{uu}(2,2)+$ exy $* \mathrm{nx}$

$\mathrm{uu}(2,3)=\mathrm{uu}(2,3)+\mathrm{exz} * \mathrm{nx}$

$\mathrm{uu}(3,1)=\mathrm{uu}(3,1)+\mathrm{exx} * \mathrm{nx}$

$\mathrm{uu}(3,2)=\mathrm{uu}(3,2)+$ exy $*$ nx

$\mathrm{uu}(3,3)=\mathrm{uu}(3,3)+\mathrm{exz} * \mathrm{nx}$

$\mathrm{uu}(6,1)=\mathrm{uu}(6,1)+\mathrm{exx} * \mathrm{nx}$

$\mathrm{uu}(6,2)=\mathrm{uu}(6,2)+\mathrm{exy} * \mathrm{nx}$

$\mathrm{uu}(6,3)=\mathrm{uu}(6,3)+\mathrm{exz} * \mathrm{nx}$

$\mathrm{uu}(7,1)=\mathrm{uu}(7,1)+\mathrm{exx} * \mathrm{nx}$

$\mathrm{uu}(7,2)=\mathrm{uu}(7,2)+\mathrm{exy} * \mathrm{nx}$

$\mathrm{uu}(7,3)=\mathrm{uu}(7,3)+\mathrm{exz} * \mathrm{nx}$ 
end if

if ( $j . e q . n y)$ then

uu $(3,1)=u u(3,1)+$ exy $*$ ny

uu $(3,2)=u u(3,2)+$ eyy $*$ ny

$\mathrm{uu}(3,3)=\mathrm{uu}(3,3)+$ eyz $*$ ny

uu $(4,1)=u u(4,1)+$ exy $*$ ny

$\mathrm{uu}(4,2)=\mathrm{uu}(4,2)+$ eyy $*$ ny

$\mathrm{uu}(4,3)=\mathrm{uu}(4,3)+$ eyz $*$ ny

$\mathrm{uu}(7,1)=\mathrm{uu}(7,1)+$ exy $*$ ny

$\mathrm{uu}(7,2)=\mathrm{uu}(7,2)+$ eyy $*$ ny

uu $(7,3)=u u(7,3)+$ eyz $*$ ny

$\mathrm{uu}(8,1)=\mathrm{uu}(8,1)+$ exy $*$ ny

uu $(8,2)=u u(8,2)+$ eyy $*$ ny

uu $(8,3)=u u(8,3)+$ eyz $*$ ny

end if

if (k.eq.nz) then

uu $(5,1)=u u(5,1)+e x z * n z$

$\mathrm{uu}(5,2)=\mathrm{uu}(5,2)+\mathrm{eyz} * \mathrm{nz}$

uu $(5,3)=u u(5,3)+$ ezz $* n z$

$\mathrm{uu}(6,1)=\mathrm{uu}(6,1)+$ exz $* \mathrm{nz}$

$\mathrm{uu}(6,2)=\mathrm{uu}(6,2)+$ eyz $* n z$

$\mathrm{uu}(6,3)=\mathrm{uu}(6,3)+$ ezz $* \mathrm{nz}$

$\mathrm{uu}(7,1)=\mathrm{uu}(7,1)+\mathrm{exz} * \mathrm{nz}$

$\mathrm{uu}(7,2)=\mathrm{uu}(7,2)+\mathrm{eyz} * \mathrm{nz}$

$\mathrm{uu}(7,3)=\mathrm{uu}(7,3)+\mathrm{ezz} * \mathrm{nz}$

$\mathrm{uu}(8,1)=\mathrm{uu}(8,1)+$ exz $* \mathrm{nz}$

$\mathrm{uu}(8,2)=\mathrm{uu}(8,2)+\mathrm{eyz} * \mathrm{nz}$

uu $(8,3)=u u(8,3)+e z z * n z$

end if

c local stresses and strains in a pixel

str $11=0.0 \mathrm{~d} 0$

str22 $=0.0 \mathrm{~d} 0$

str33 $=0.0 \mathrm{~d} 0$

str13 $=0.0 \mathrm{~d} 0$

str23 $=0.0 \mathrm{~d} 0$

str12 $=0.0 \mathrm{~d} 0$

$\mathrm{s} 11=0.0 \mathrm{~d} 0$

$\mathrm{s} 22=0.0 \mathrm{~d} 0$

$\mathrm{s} 33=0.0 \mathrm{~d} 0$

$\mathrm{s} 13=0.0 \mathrm{~d} 0$

$\mathrm{s} 23=0.0 \mathrm{~d} 0$

$\mathrm{s} 12=0.0 \mathrm{~d} 0$

c********compute average stress and strain tensor in each pixel**************

C First put thermal strain-induced stresses into stress tensor

do $465 \mathrm{n}=1,6$

$\operatorname{str} 11=\operatorname{str} 11-\operatorname{cmod}(\operatorname{vox}(i, j, k), 1, n) * \operatorname{eigen}(\operatorname{vox}(i, j, k), n)$

$\operatorname{str} 22=\operatorname{str} 22-\operatorname{cmod}(\operatorname{vox}(i, j, k), 2, n) * \operatorname{eigen}(\operatorname{vox}(i, j, k), n)$

$\operatorname{str} 33=\operatorname{str} 33-\operatorname{cmod}(\operatorname{vox}(i, j, k), 3, n) * \operatorname{eigen}(\operatorname{vox}(i, j, k), n)$ 


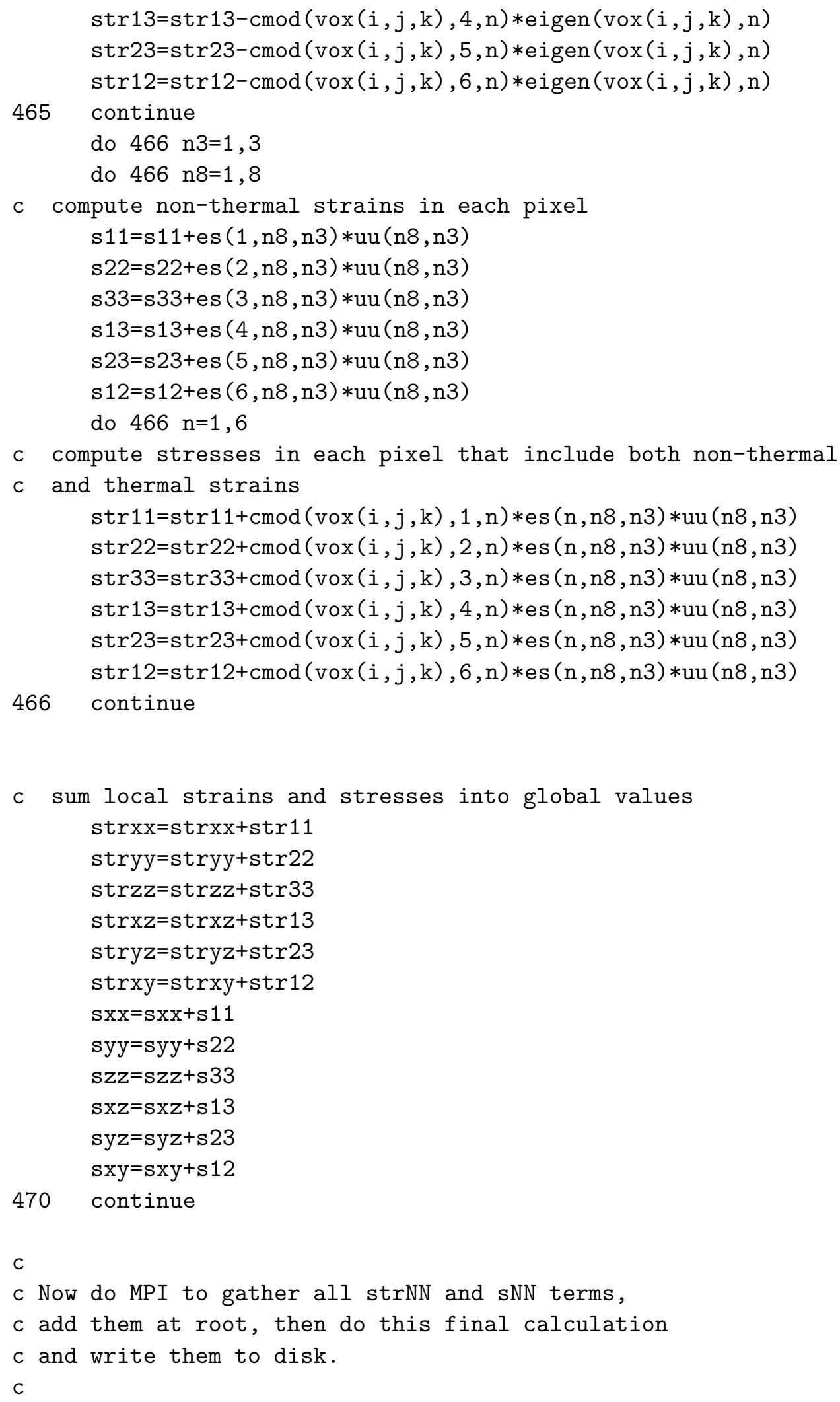

call MPI_ALLREDUCE (strxx, strxxp,1,MPI_DOUBLE_PRECISION,

MPI_SUM, MPI_COMM_WORLD, i err) 


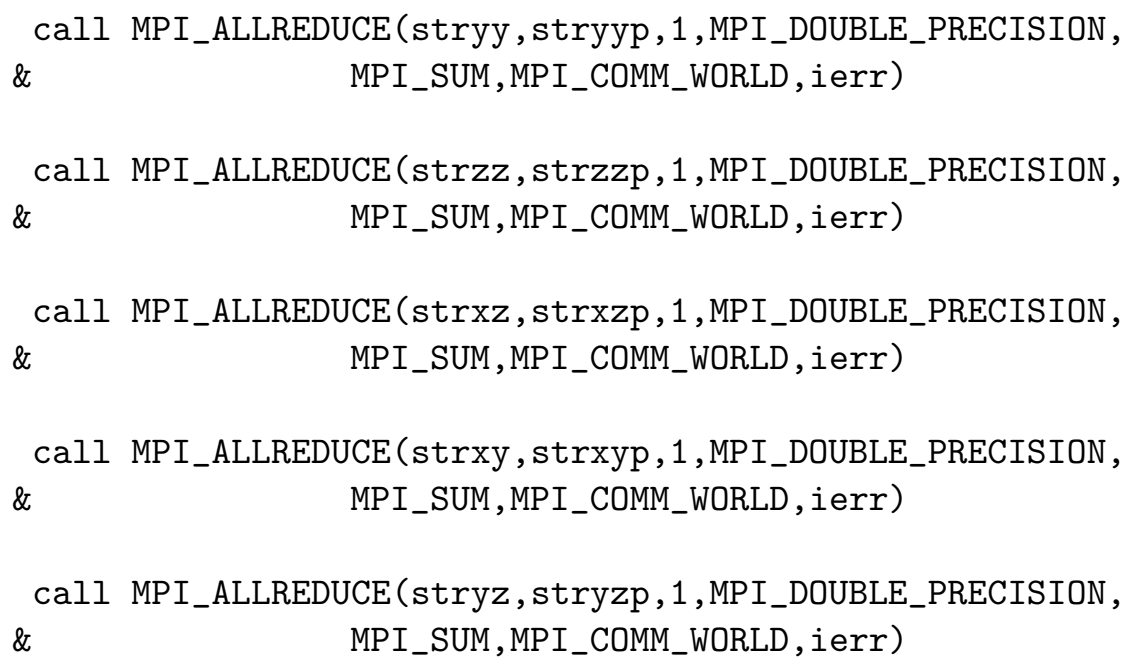




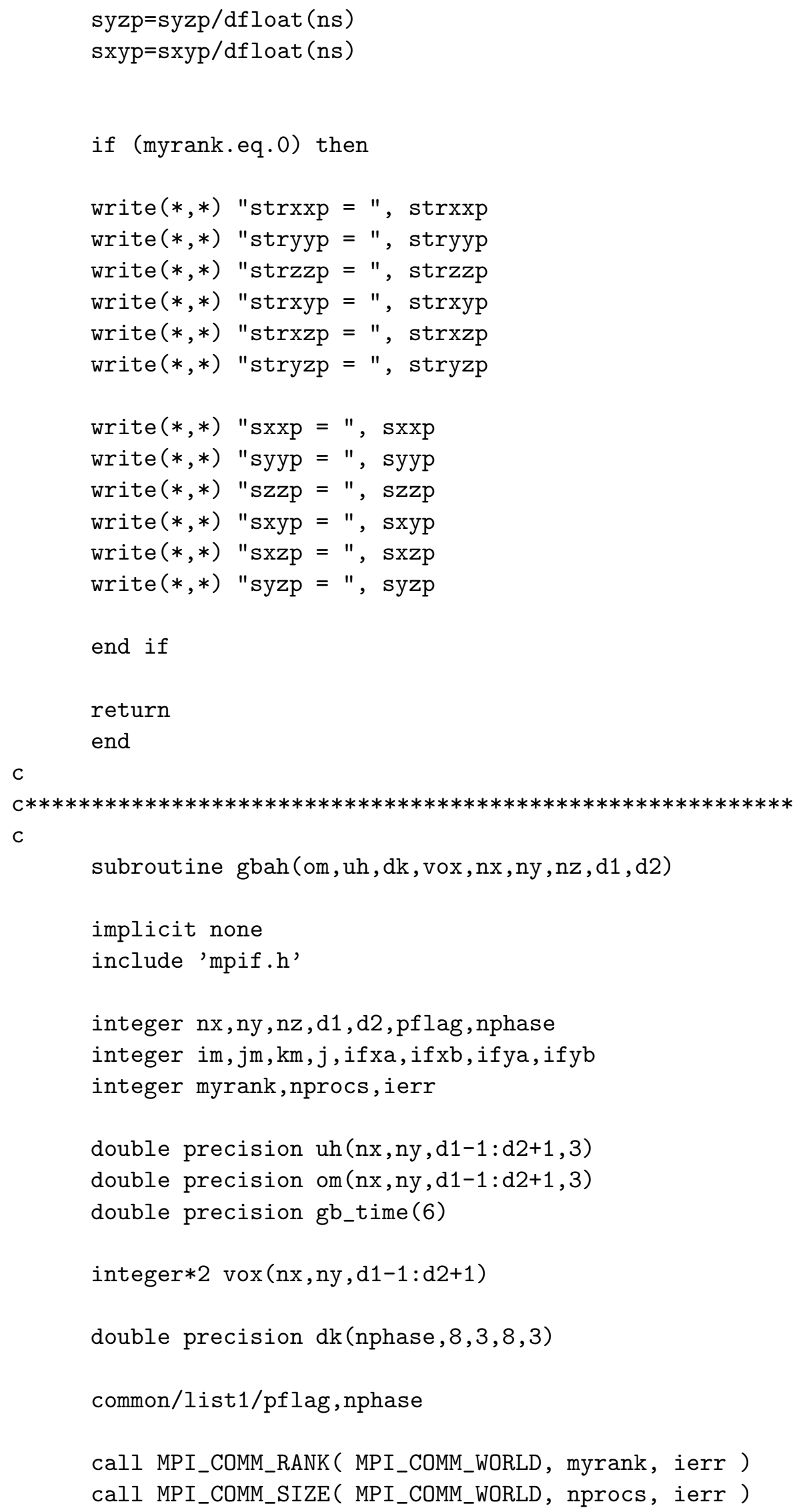




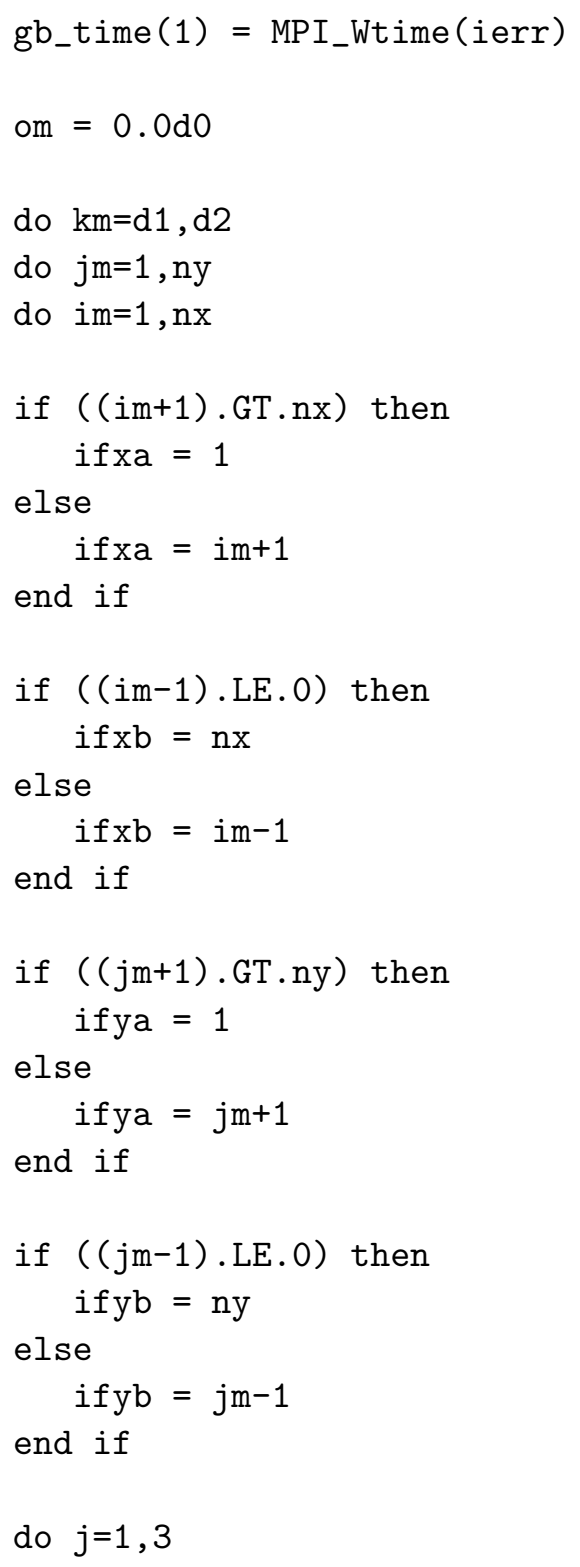

c SELF TERM

$\mathrm{om}(\mathrm{im}, \mathrm{jm}, \mathrm{km}, \mathrm{j})=$

c $u(i b(m, 1), n)$

\& SUM ( uh (im, ifya, $\mathrm{km},:$ ) *(

$\& d k(\operatorname{vox}(i m, j m, k m), 1, j, 4,:)$

$\&+d k(\operatorname{vox}($ ifxb $, j m, k m), 2, j, 3,:)$

$\&+d k(\operatorname{vox}(i m, j m, k m-1), 5, j, 8,:)$

$\&+d k(\operatorname{vox}($ if $x b, j m, k m-1), 6, j, 7,:)))+$

c $u(i b(m, 2), n)$

\& SUM ( uh(ifxa, ifya, $\mathrm{km},:$ ) $*(\mathrm{dk}(\operatorname{vox}(\mathrm{im}, \mathrm{jm}, \mathrm{km}), 1, \mathrm{j}, 3,:$ )

$\&+\operatorname{dk}(\operatorname{vox}(\mathrm{im}, \mathrm{jm}, \mathrm{km}-1), 5, \mathrm{j}, 7,:)))+$ 


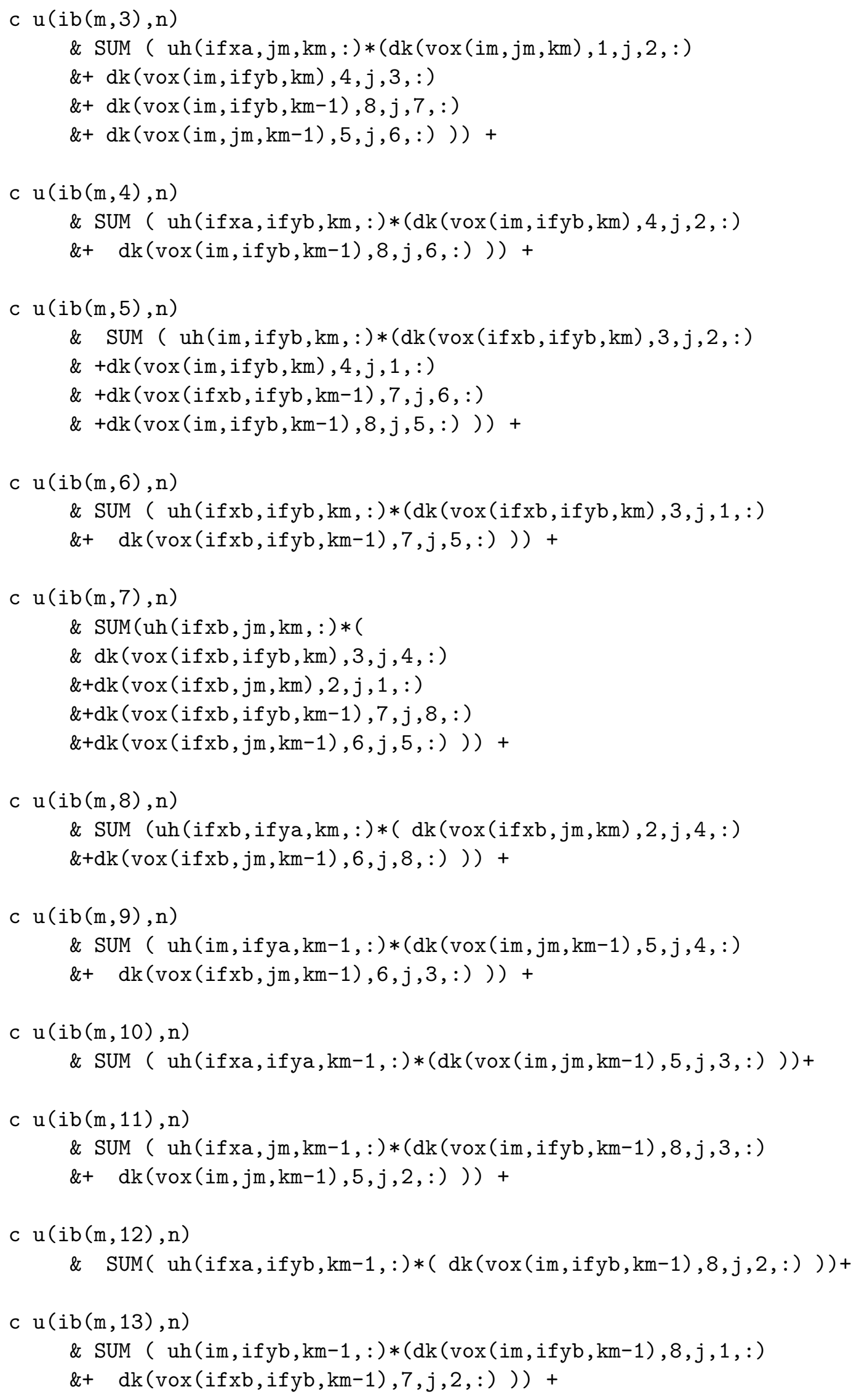




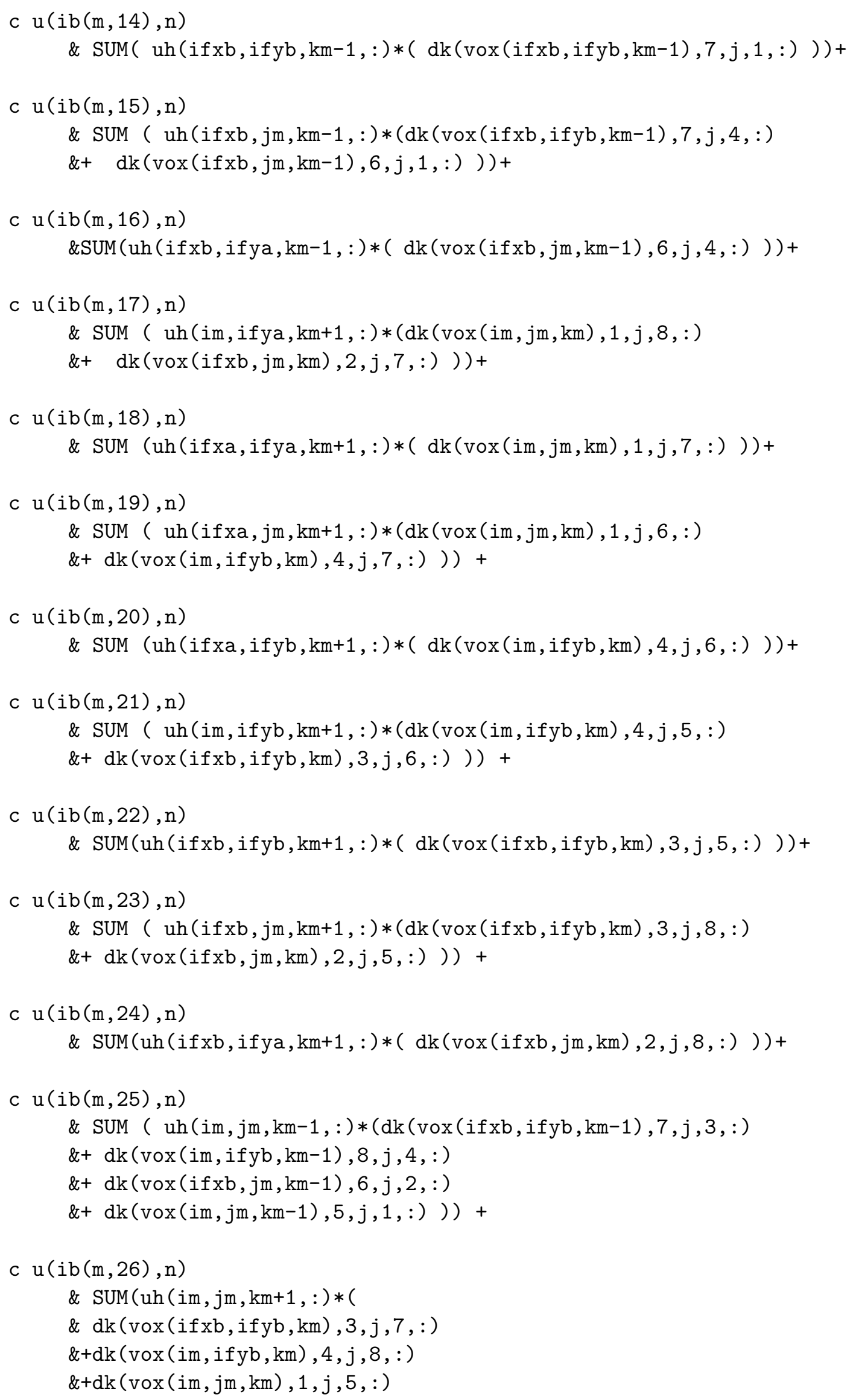




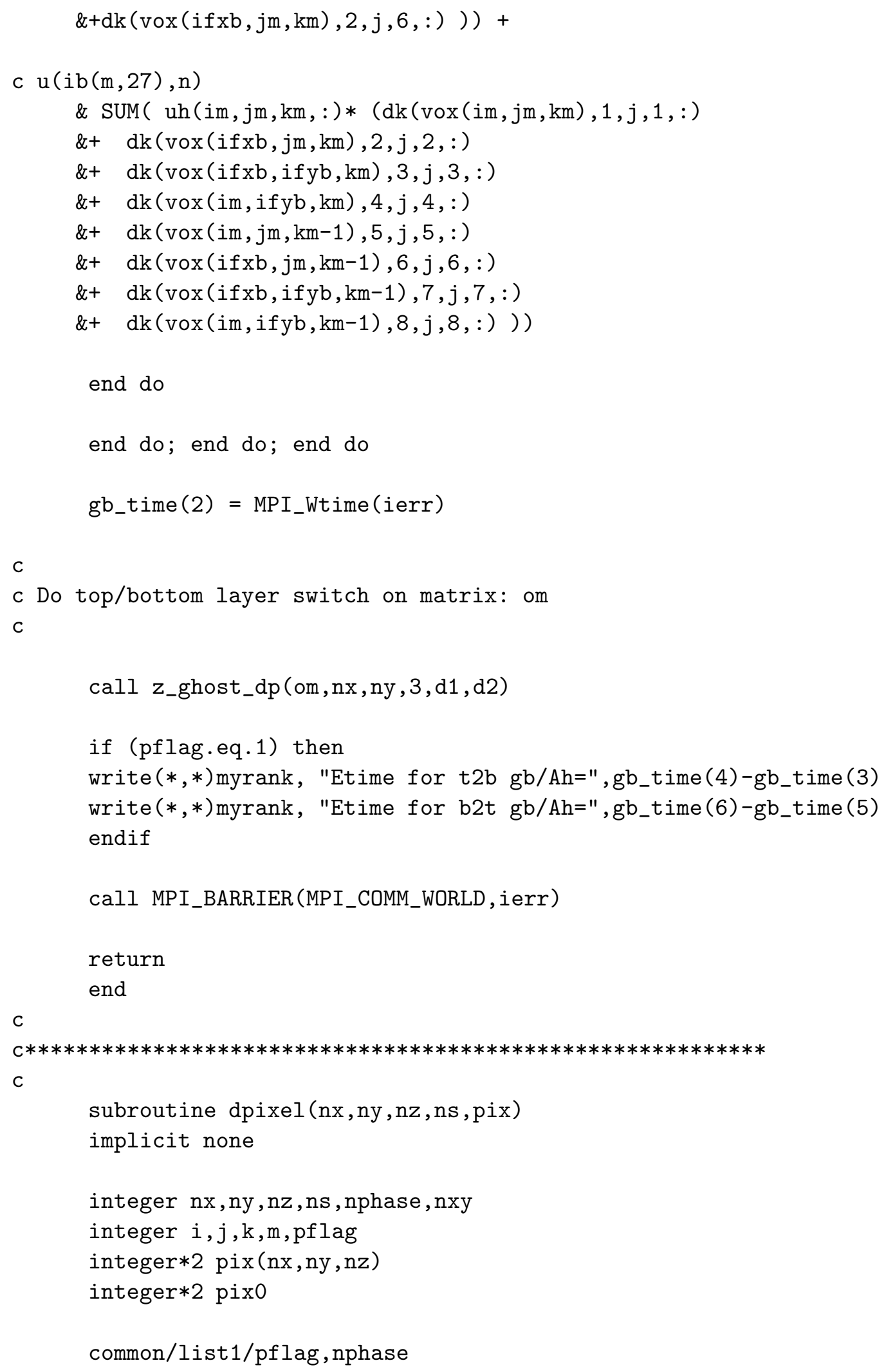

c (USER) If you want to set up a test image inside the program, instead of $c$ reading it in from a file, this should be done inside this subroutine. 


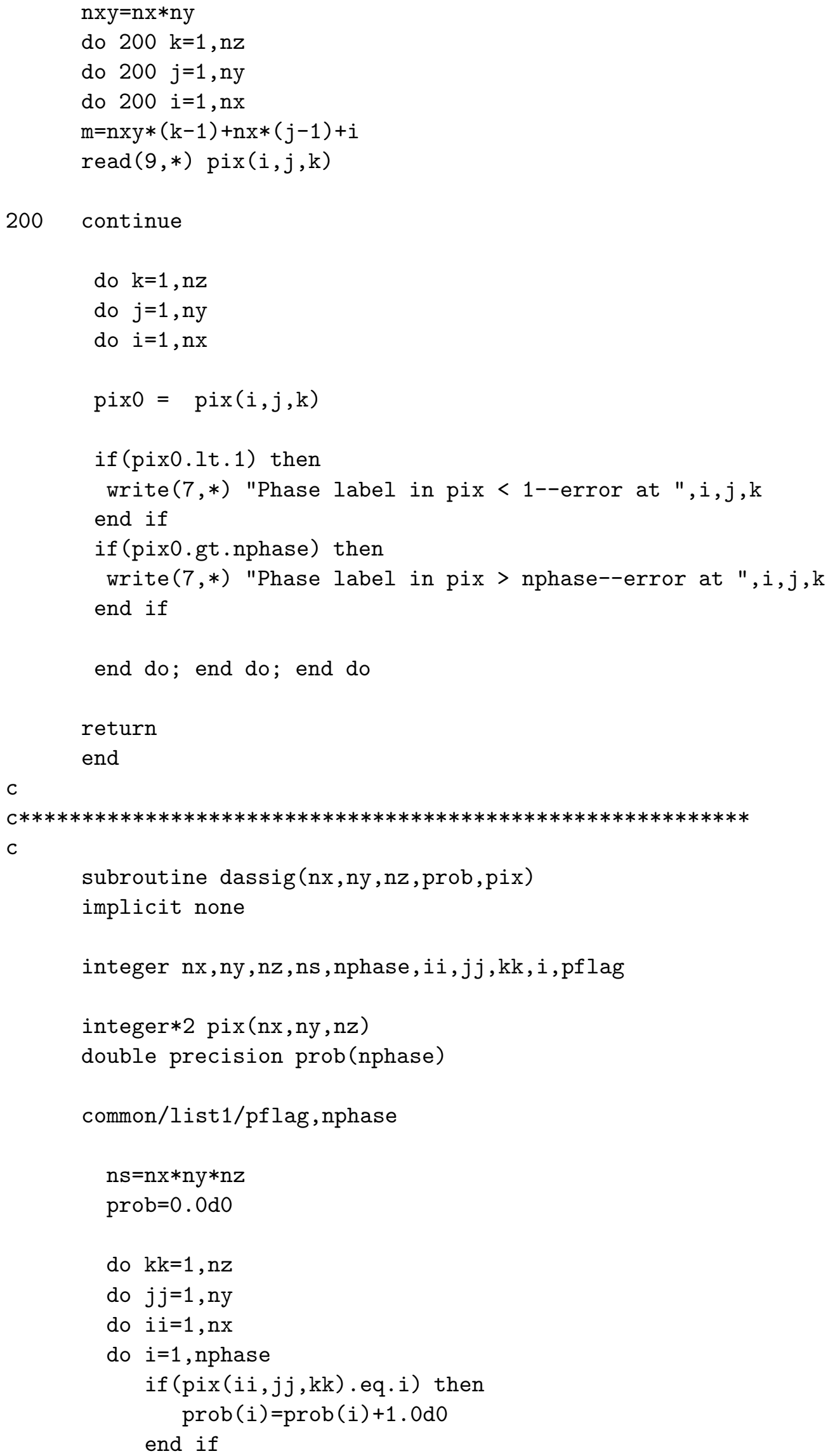

continue

do $\mathrm{k}=1, \mathrm{nz}$

do $j=1$, ny

do $i=1, n x$

$\operatorname{pix} 0=\operatorname{pix}(i, j, k)$

if (pix0.1t.1) then write $(7, *)$ "Phase label in pix < 1--error at ",i,j,k end if 


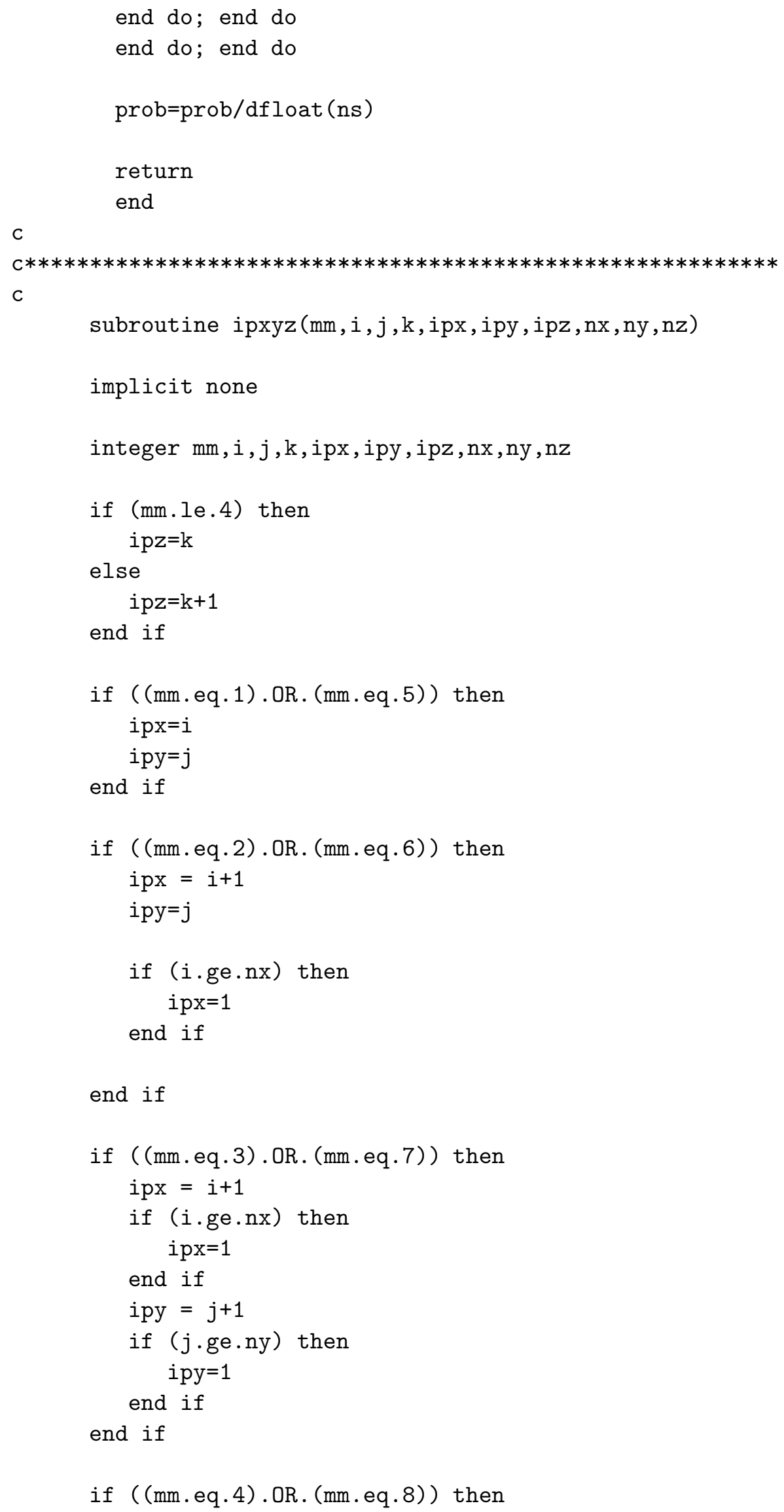




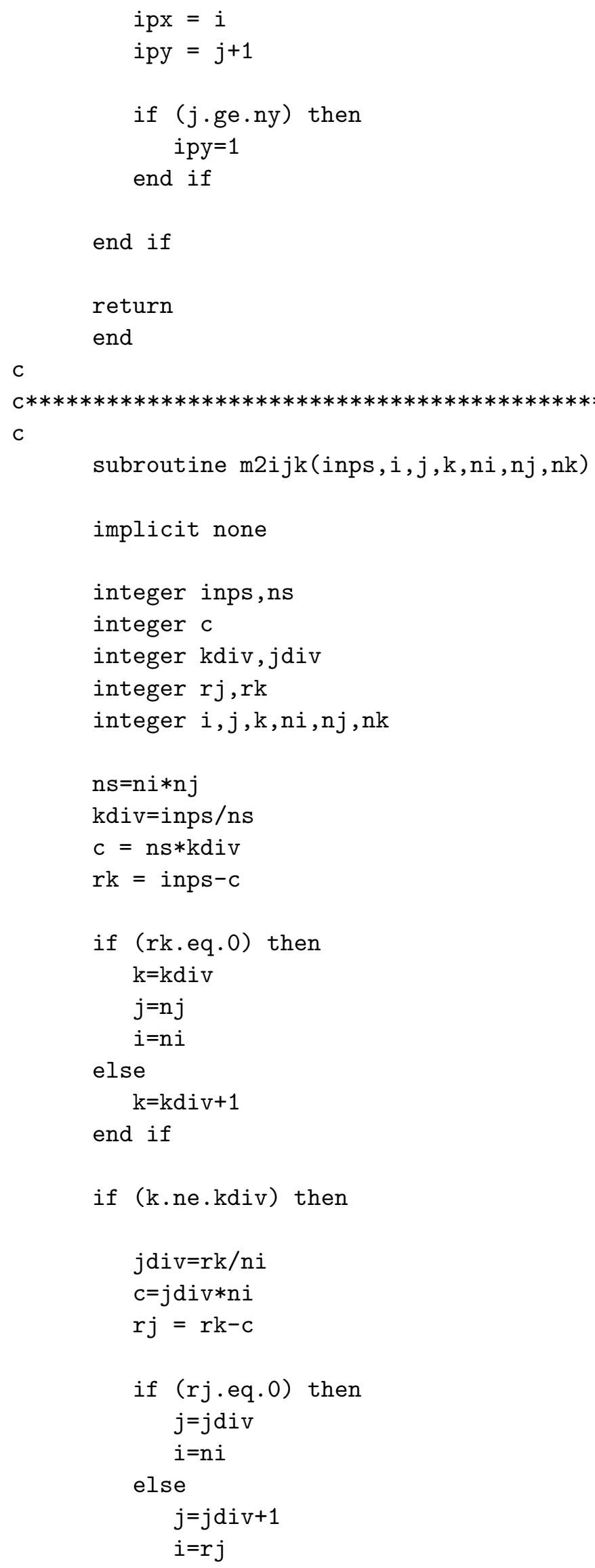


end if

end if

return

end

C

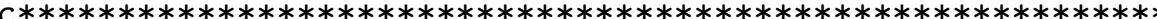

$C$

subroutine z_ghost_int (arr0,mx,my,mz,d1,d2)

implicit none

include 'mpif.h'

integer $\mathrm{mx}, \mathrm{my}, \mathrm{mz}, \mathrm{d} 1, \mathrm{~d} 2$

integer $* 2 \operatorname{arr0}(\mathrm{mx}, \mathrm{my}, \mathrm{d} 1-1: \mathrm{d} 2+1)$

integer*2, allocatable $:: \operatorname{bot}(:,:), \operatorname{top}(:,:)$

integer myrank, ierr, nprocs

integer status(MPI_STATUS_SIZE)

call MPI_COMM_RANK ( MPI_COMM_WORLD, myrank, ierr )

call MPI_COMM_SIZE( MPI_COMM_WORLD, nprocs, ierr )

allocate (bot (mx,my))

allocate (top (mx,my))

C

c Get new bottom ghost plane.

C

bot $=\operatorname{arr0}(:,:, \mathrm{d} 1)$

top $=\operatorname{arr} 0(:,:, d 2)$

call t2b(bot, top, mx,my)

$\operatorname{arr0}(:,:, d 1-1)=\operatorname{bot}$

c Get new top ghost plane

C

bot $=\operatorname{arr} 0(:,:, \mathrm{d} 1)$

top $=\operatorname{arr} 0(:,:, d 2)$

call b2t (bot, top, mx,my)

$\operatorname{arr} 0(:,:, d 2+1)=$ top

deallocate(bot)

deallocate(top) 


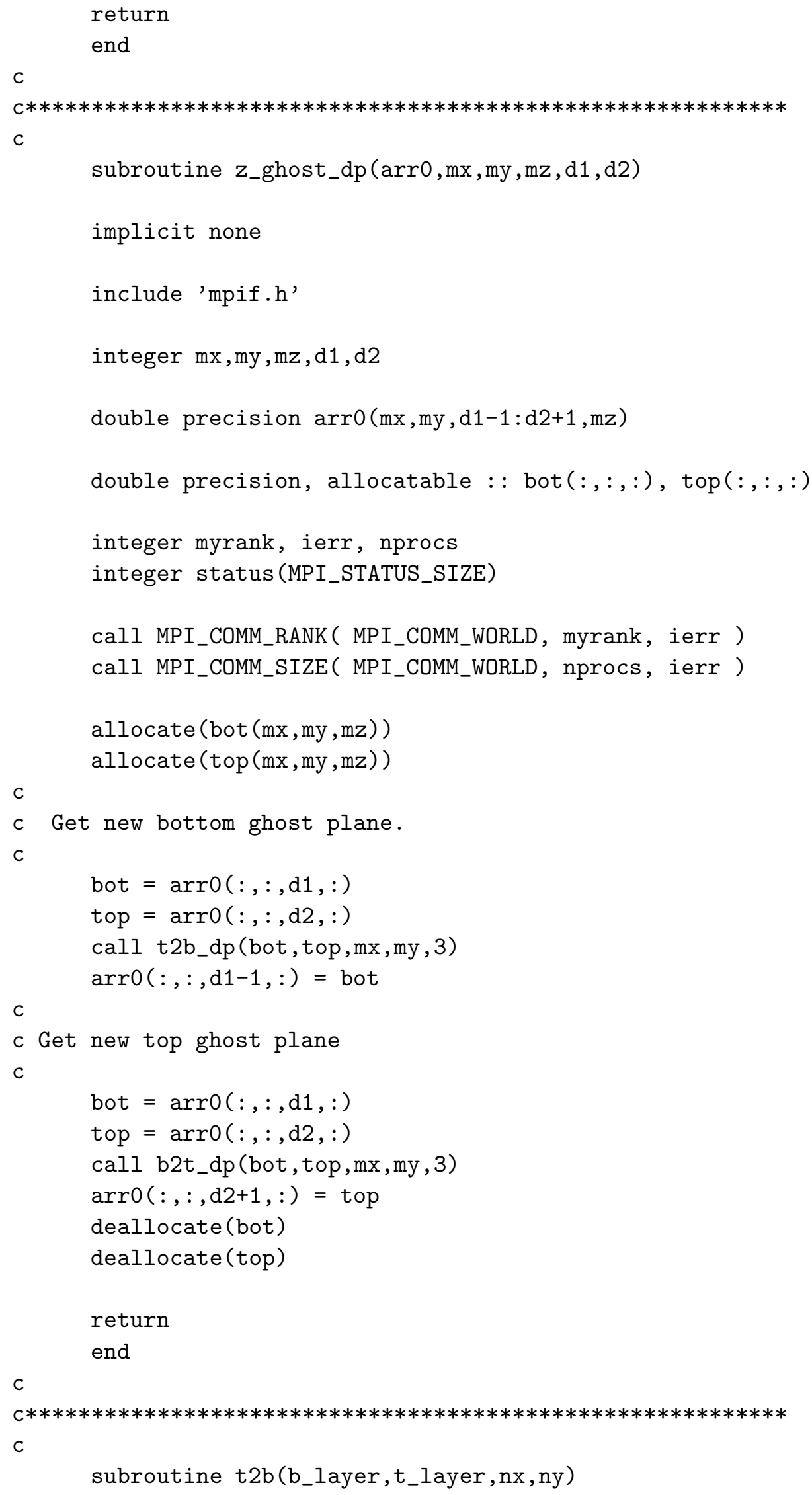


C

c This is an INTEGER*2 subroutine.

C

c Used for transferring: pix bottom2top layers

C

c RECV a new t_layer (TOP layer) per node.

C

C

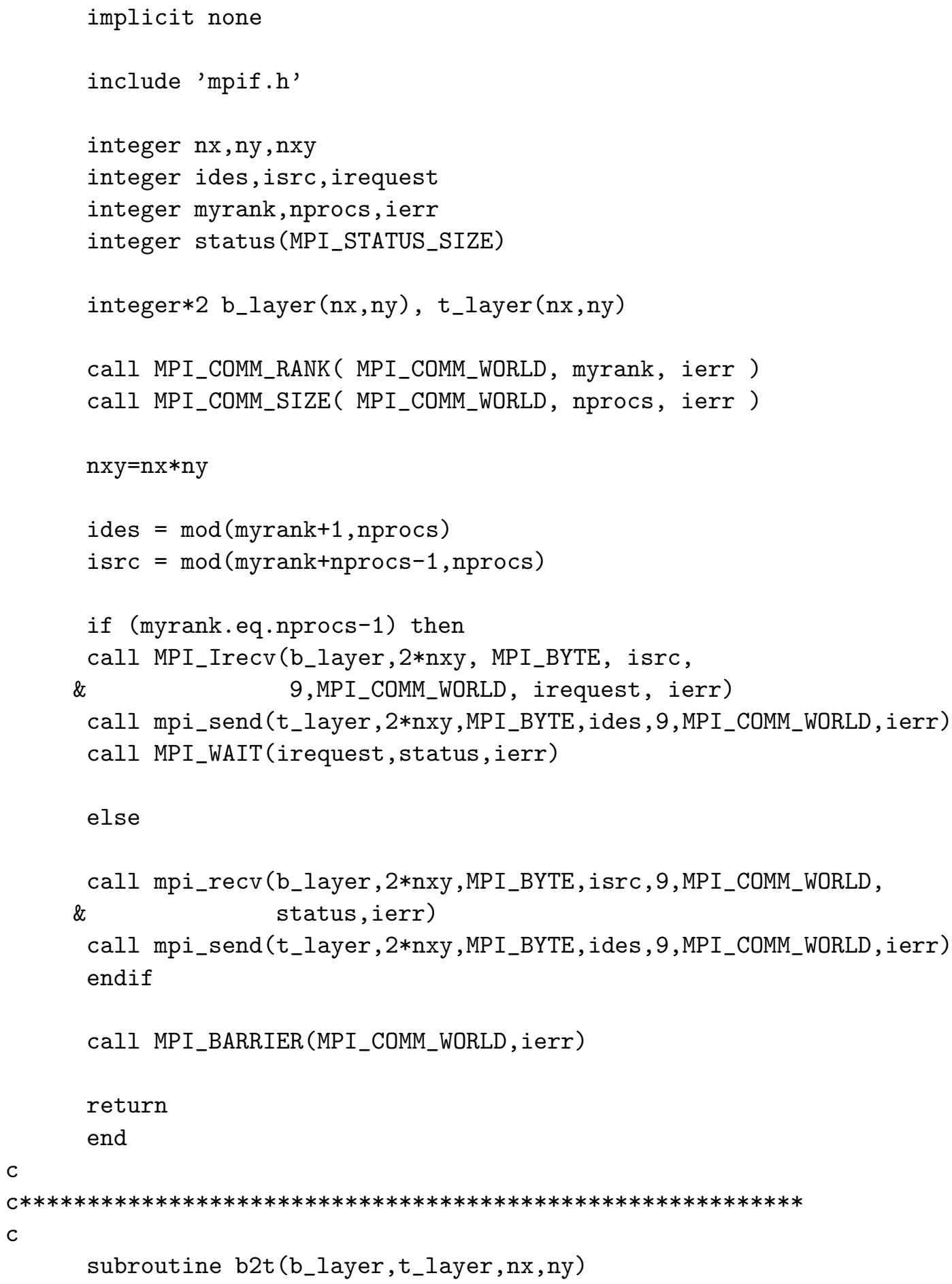


c This is an INTEGER*2 subroutine.

$\mathrm{C}$

c Used for transferring: pix bottom2top layers

$\mathrm{C}$

c RECV a new t_layer (TOP layer) per node.

C

C

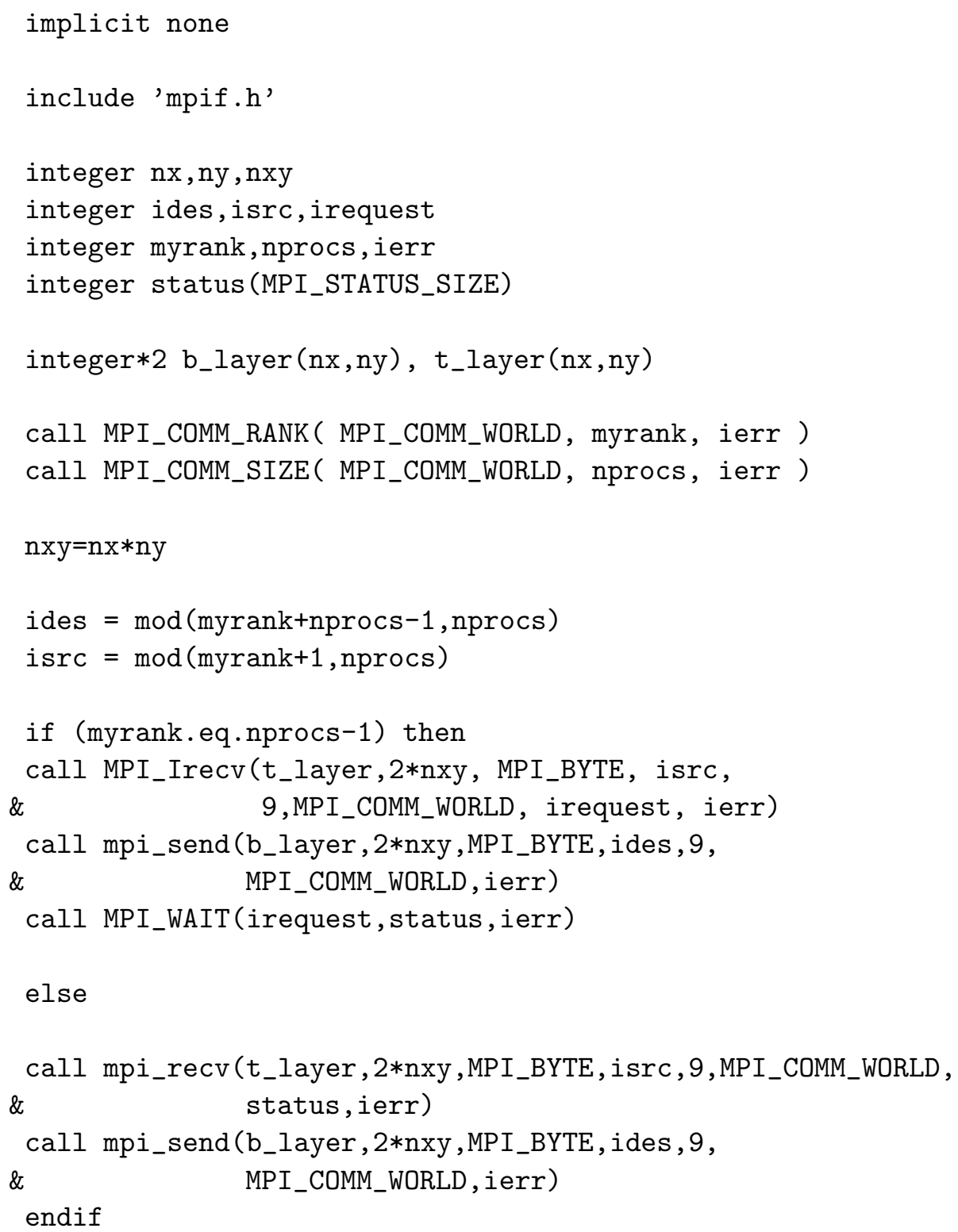


c This is a double precision subroutine.

C

c Used for transferring: $u, b$, and om top2bottom layers

C

c RECV a new b_layer (BOTTOM layer) per node.

C

C

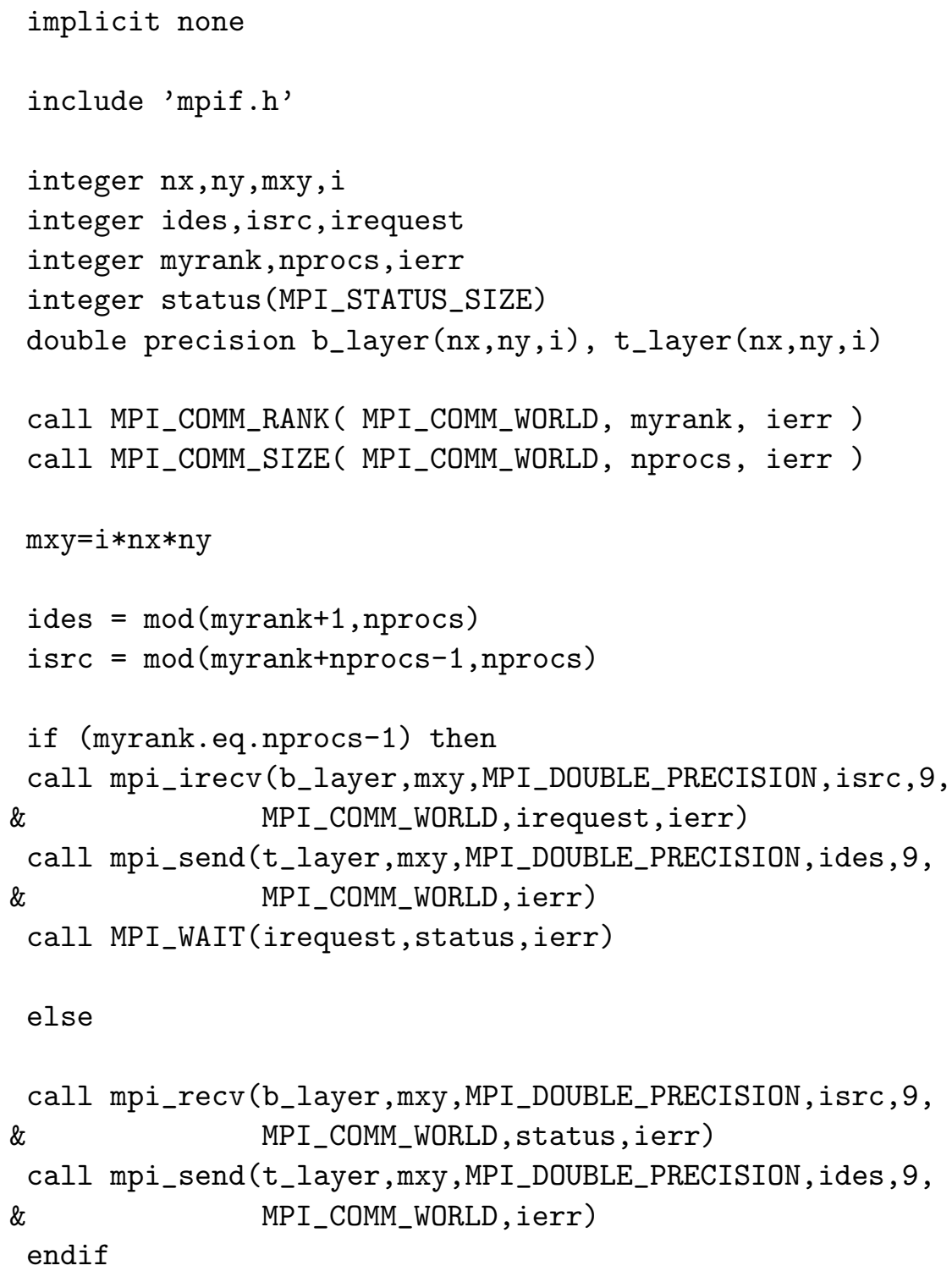


c This is a double precision subroutine.

C

c Used for transferring: $u, b$, and om bottom2top layers

C

c RECV a new t_layer (TOP layer) per node.

C

C

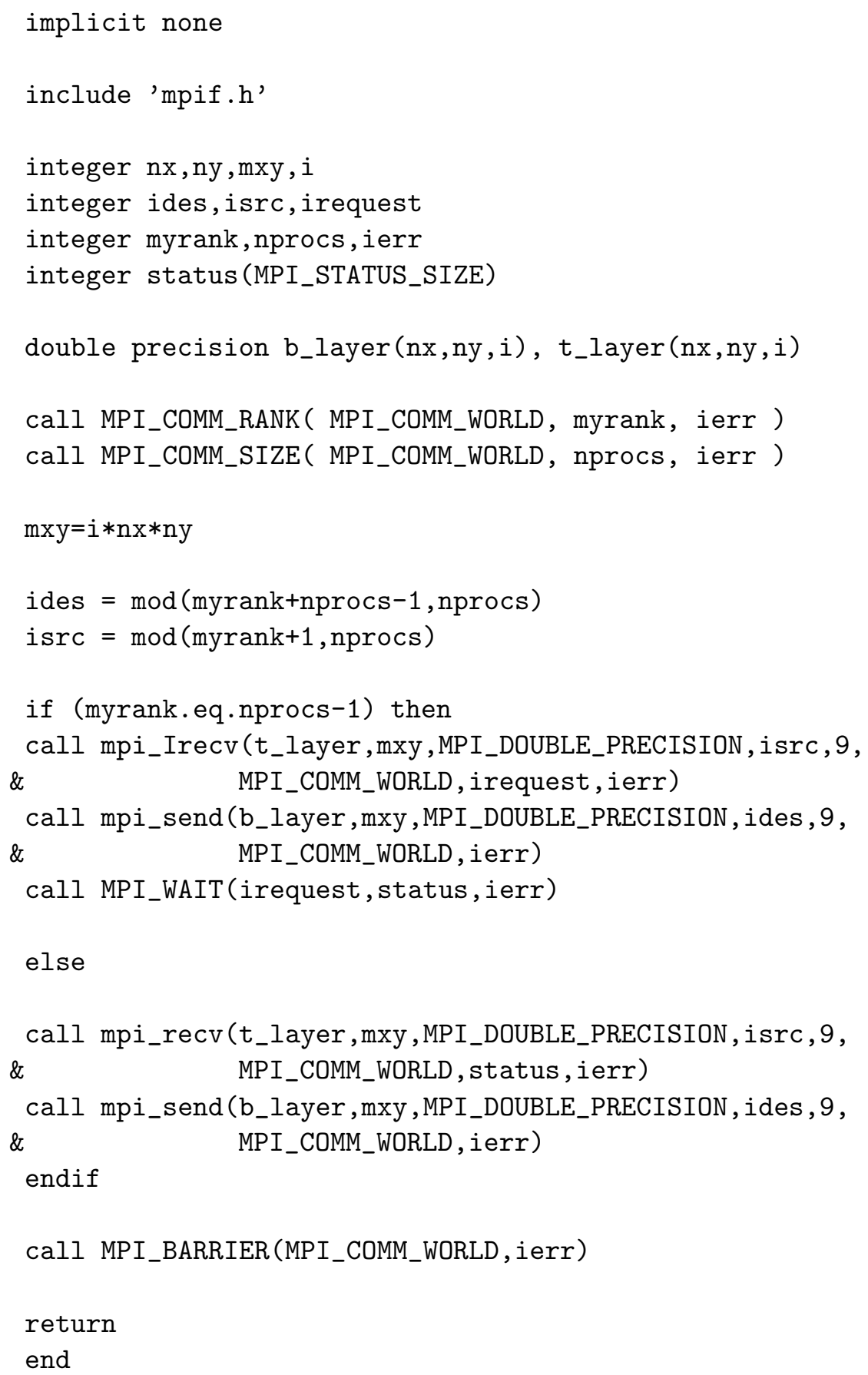




\subsection{Finite Difference}

\subsubsection{DC3D_MPI.f}

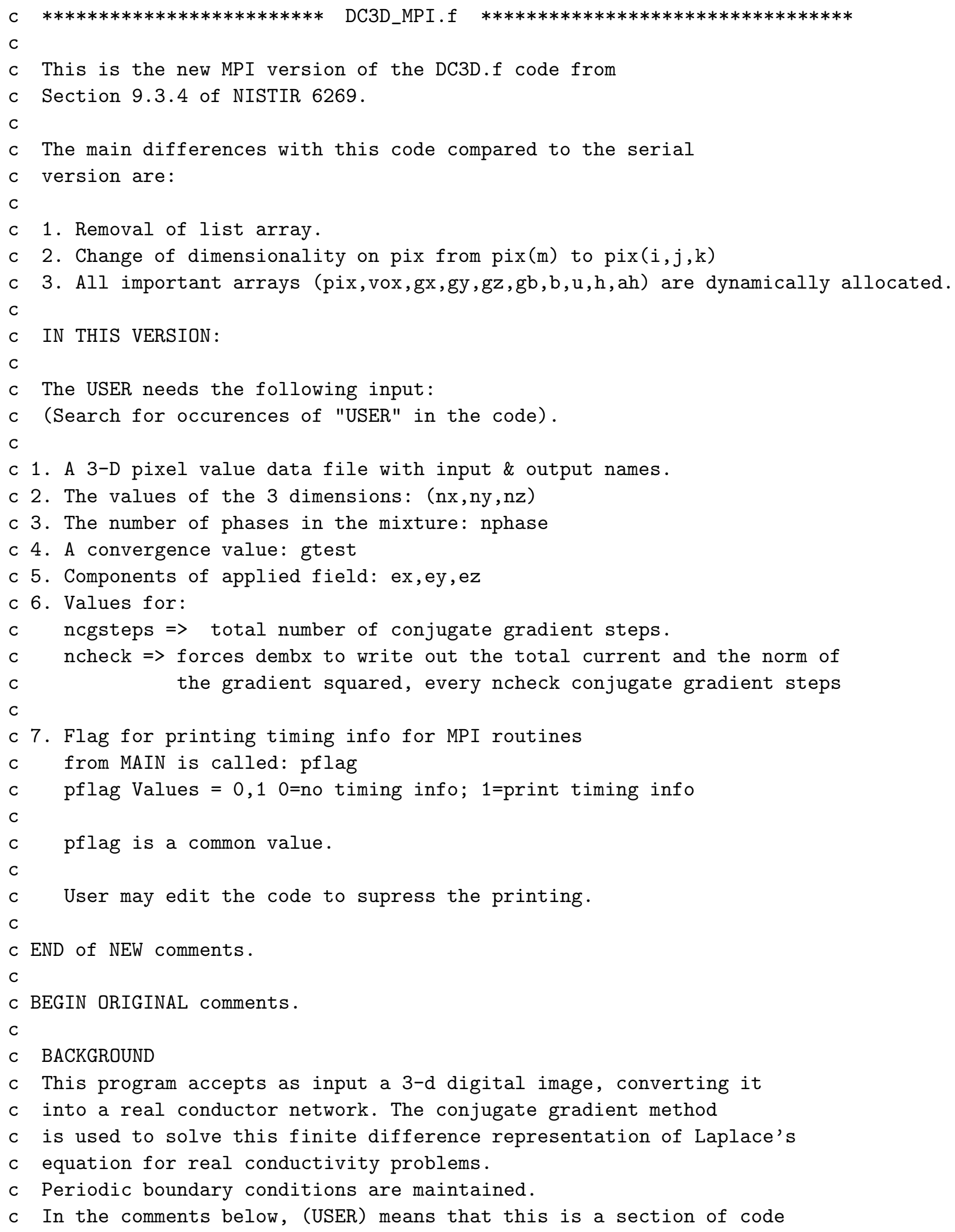




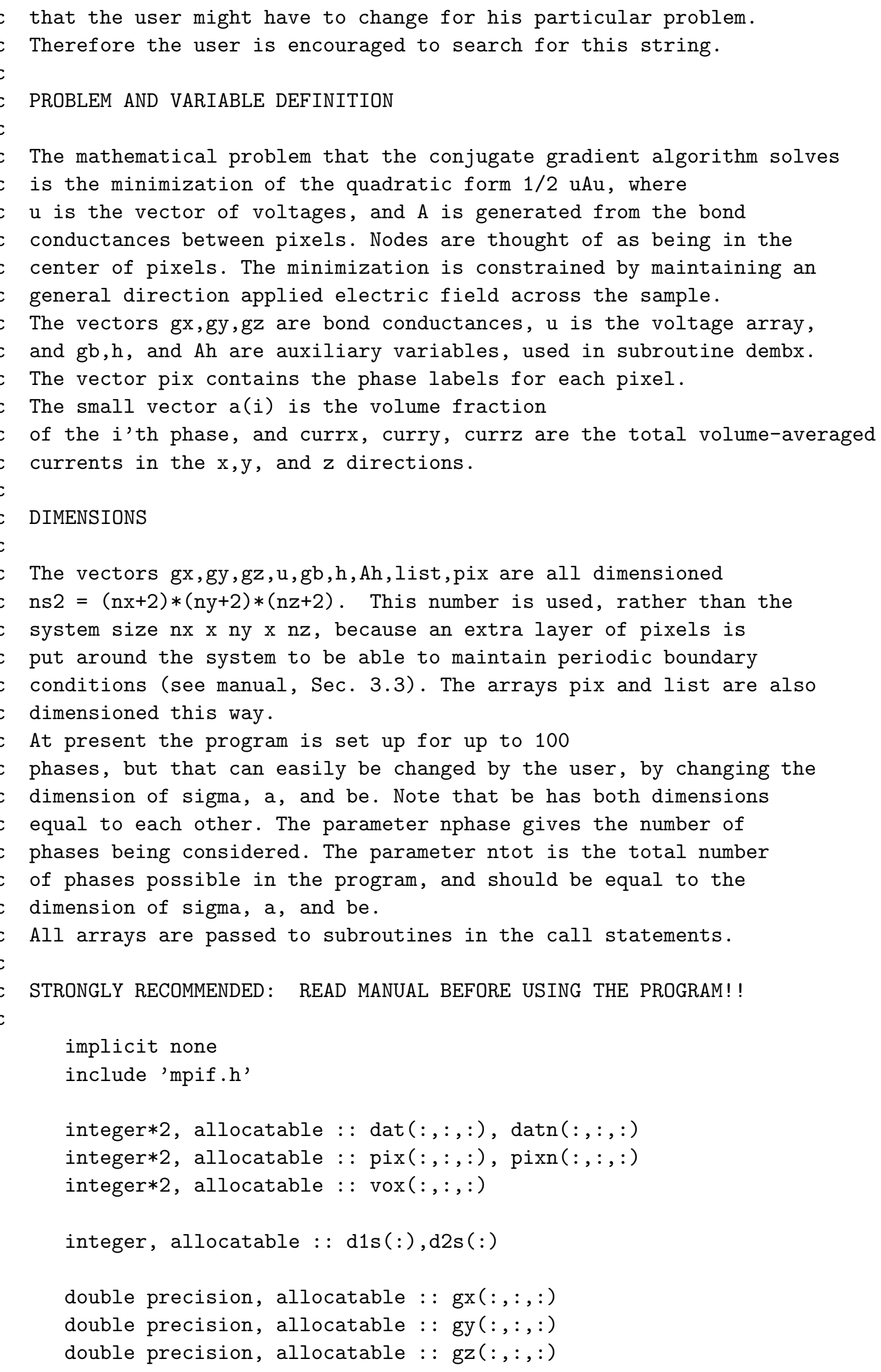




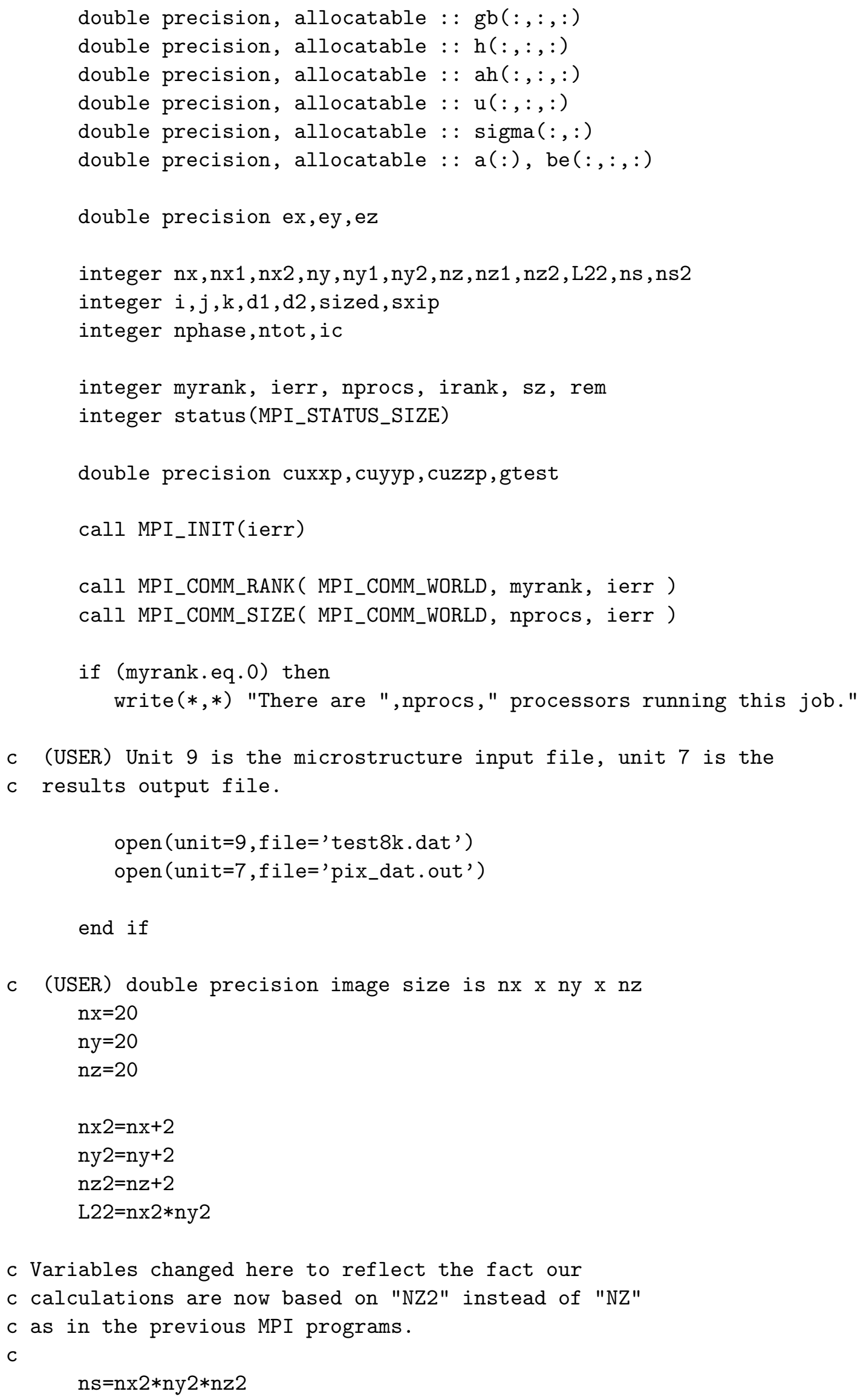




\section{$\mathrm{sz}=\mathrm{nz} 2 / \mathrm{nprocs}$}

if (myrank.eq.0) then

allocate (pix $(\mathrm{nx} 2, \mathrm{ny} 2, \mathrm{nz} 2))$

write $(7,1111) \mathrm{nx}, \mathrm{ny}, \mathrm{nz}, \mathrm{nx} * \mathrm{ny} * \mathrm{nz}$

end if

1111 format(' Image is ',3i6,' No. of double precision sites = ',i8)

c auxiliary variables involving the system size

$\mathrm{nx} 1=\mathrm{nx}+1$

ny1 $1=n y+1$

$\mathrm{nz} 1=\mathrm{nz}+1$

c computational image size ns2 is $\mathrm{nx} 2 \times \mathrm{xy} 2 \mathrm{x} \mathrm{nz} 2$

$\mathrm{ns} 2=\mathrm{nx} 2 * \mathrm{ny} 2 * \mathrm{nz} 2$

c (USER) set cutoff for norm squared of gradient, gtest. gtest is

$c$ the stopping criterion, compared to gb*gb. When gb*gb is less

c than gtest=abc*ns2, then the rms value of the gradient at a pixel

$c$ is less than sqrt (abc).

gtest $=1.0 \mathrm{~d}-16 * \mathrm{~ns} 2$

c (USER) nphase is the number of phases being considered in the problem.

c The values of pix(m) will run from 1 to nphase. ntot is the total

C number of phases possible in the program, and is the dimension of

$c$ sigma, $a$, and be.

nphase $=2$

ntot $=100$

allocate (a (ntot))

allocate (sigma (nphase, 3$)$ )

c Program calculates the d1 \& d2 limits for the principle arrays.

C

C NB: In this program, d1 \& d2 are not based on "nz".

c They are calculated on the basis of "nz2".

$\mathrm{C}$

if (myrank.eq.0) then

allocate (d1s (0:nprocs-1))

allocate (d2s (0:nprocs-1))

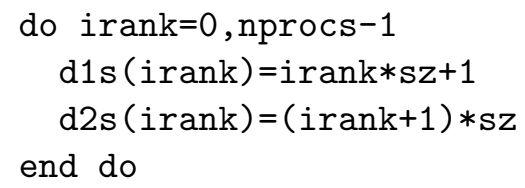




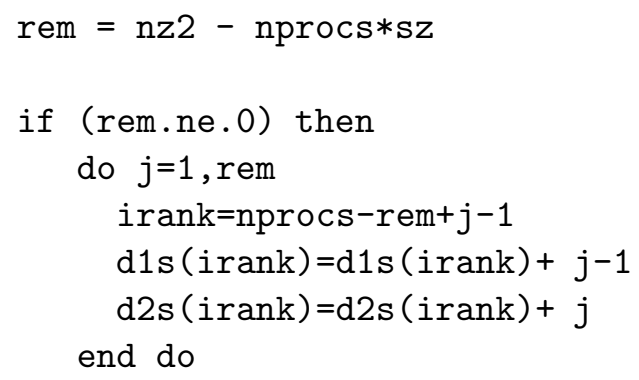




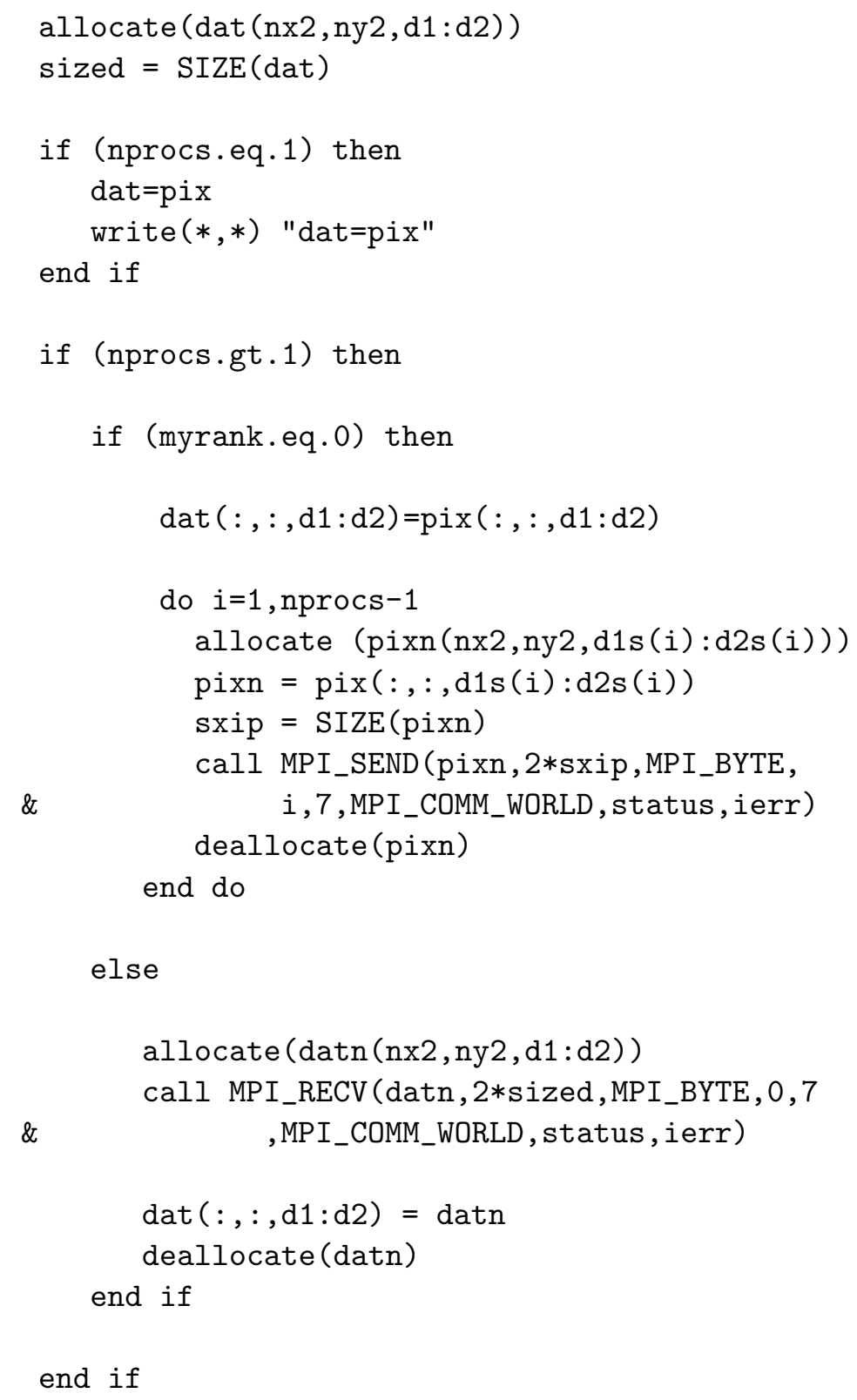


do $\mathrm{k}=\mathrm{d} 1, \mathrm{~d} 2$

$\operatorname{vox}(:,:, \mathrm{k})=\operatorname{dat}(:,:, \mathrm{k})$

end do

deallocate(dat)

C

c Call z_ghost_int to make $Z$ ghost layers of INTEGER*2 values (aka vox).

C

call z_ghost_int (vox, nx2, ny2,nz2, d1, d2)

c (USER) Set components of applied field, E = (ex,ey,ez)

$\mathrm{ex}=1.0 \mathrm{~d} 0$

ey=1.0d0

$\mathrm{ez}=1.0 \mathrm{~d} 0$

if (myrank.eq.0) then

write $(7, *)$ 'Applied field components:'

write $(7, *)$ 'ex $=$ ', ex, ' ey $=$ ', ey, ' ez $=$ ', ez

end if

c Initialize the voltage distribution by putting on uniform field.

allocate $(u(n x 2, n y 2, d 1-1: d 2+1))$

$\mathrm{u}=0 . \operatorname{Od} 0$

do $30 \mathrm{k}=\mathrm{d} 1, \mathrm{~d} 2$

do $30 \mathrm{j}=1, \mathrm{ny} 2$

do $30 i=1, n \times 2$

$u(i, j, k)=-e x * i-e y * j-e z * k$

30

continue

C

c Call z_ghost_dp to make $Z$ ghost layers

$c$ of DOUBLE PRECISION values (aka u).

C

call z_ghost_dp (u, nx2, ny2, nz2, d1, d2)

c (USER) input value of double precision conductivity tensor for

c each phase (diagonal only). 1,2,3 $=\mathrm{x}, \mathrm{y}, \mathrm{z}$, respectively.

$$
\begin{aligned}
& \operatorname{sigma}(1,1)=1.0 \mathrm{~d} 0 \\
& \operatorname{sigma}(1,2)=1.0 \mathrm{~d} 0 \\
& \operatorname{sigma}(1,3)=1.0 \mathrm{~d} 0 \\
& \operatorname{sigma}(2,1)=0.5 \mathrm{~d} 0 \\
& \operatorname{sigma}(2,2)=0.5 \mathrm{~d} 0 \\
& \operatorname{sigma}(2,3)=0.5 \mathrm{~d} 0
\end{aligned}
$$




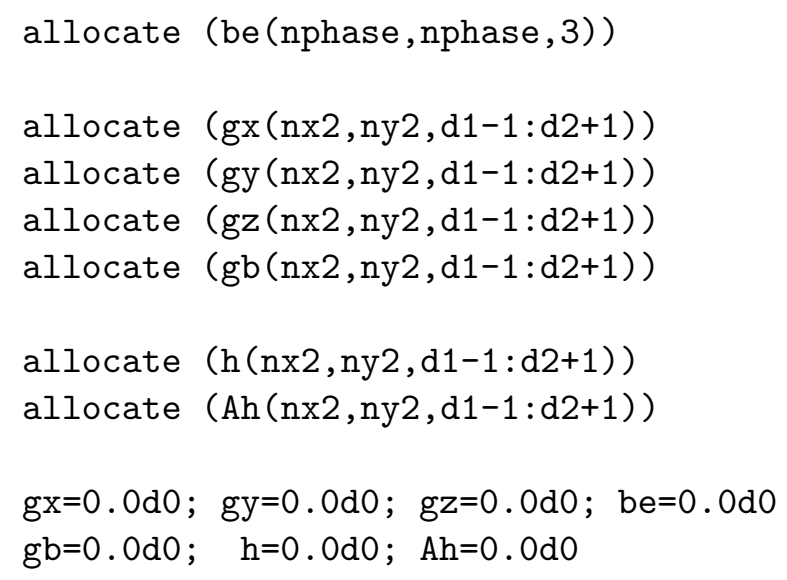




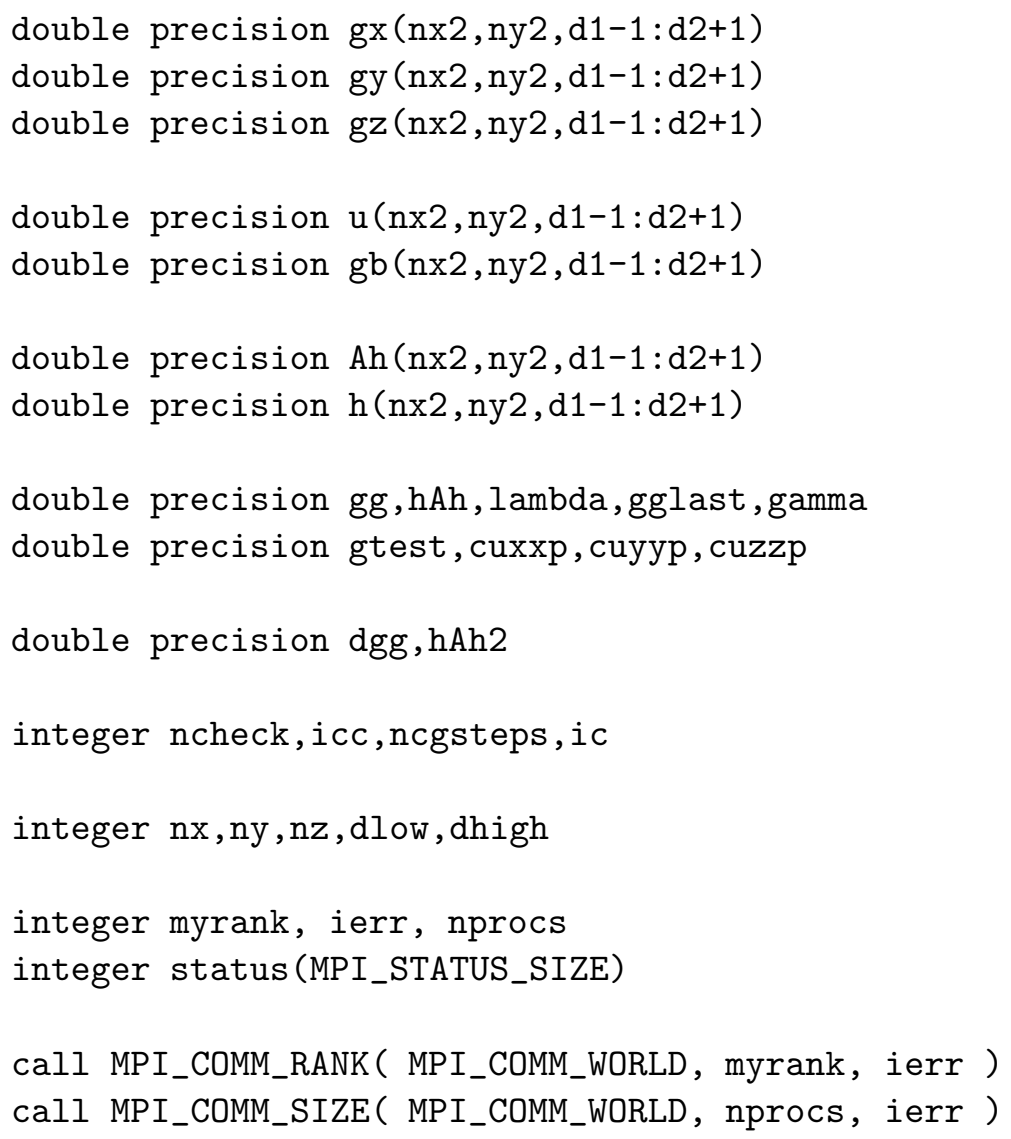

c Note: voltage gradients are maintained because in the conjugate gradient

c relaxation algorithm, the voltage vector is only modified by adding a

c periodic vector to it.

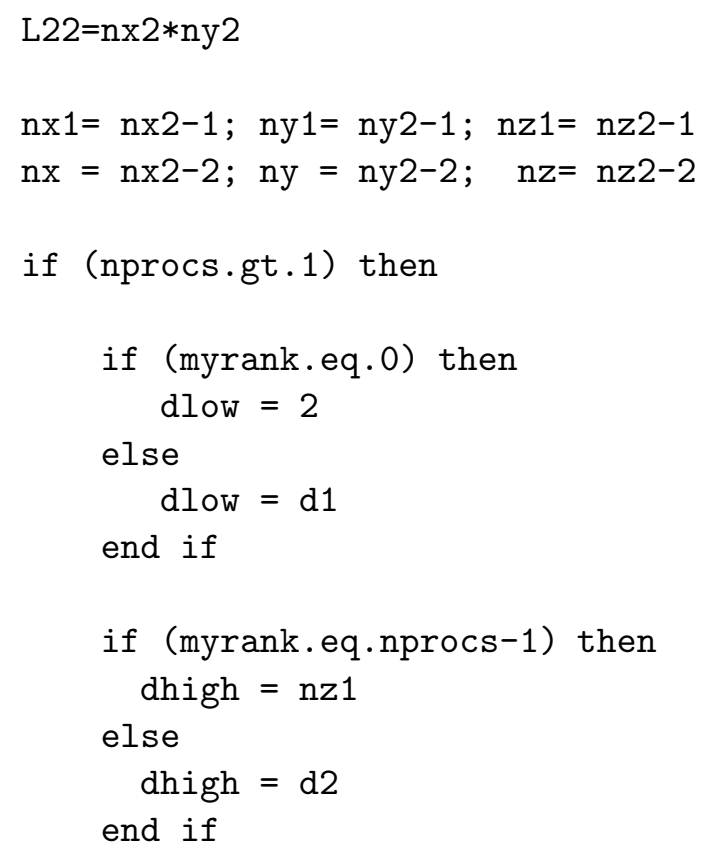


if (nprocs.eq.1) then

dlow $=2$

dhigh=nz1

end if

c First stage, compute initial value of gradient (gb), initialize $h$, the

c conjugate gradient direction, and compute norm squared of gradient vector.

call prod_dc (nx2, ny2, nz2, ns2, gx,gy, gz, u, gb, d1 ,d2)

$\mathrm{h}=\mathrm{gb}$

c Variable gg is the norm squared of the gradient vector $\mathrm{gg}=0.0 \mathrm{~d} 0$

$\mathrm{dgg}=0.0 \mathrm{~d} 0$

gg $=0.0 \mathrm{~d} 0$

$\operatorname{dgg}=\operatorname{SUM}(g b(2: n x 1,2:$ ny 1, dlow $:$ dhigh $) * g b(2: n x 1,2:$ ny 1, dlow $:$ dhigh $))$

call MPI_ALLREDUCE (dgg,gg, 1,MPI_DOUBLE_PRECISION, \& $\quad$ MPI_SUM, MPI_COMM_WORLD, ierr)

c Second stage, initialize Ah variable, compute parameter lamdba, c make first change in voltage array, update gradient (gb) vector

if (gg.lt.gtest) goto 44

call prod_dc (nx2,ny2,nz2,ns2,gx,gy,gz,h, Ah, d1 ,d2)

$\mathrm{hAh}=0.0 \mathrm{~d} 0$

$\mathrm{hAh} 2=0.0 \mathrm{~d} 0$

hAh2 $=\operatorname{SUM}(h(2: n x 1,2:$ ny1, dlow $:$ dhigh $) * \operatorname{Ah}(2: \mathrm{nx} 1,2: \mathrm{ny} 1$, dlow $:$ dhigh $))$

call MPI_ALLREDUCE (hAh2, hAh, 1, MPI_DOUBLE_PRECISION, MPI_SUM, \& MPI_COMM_WORLD, ierr)

lambda $=g g / h A h$

$\mathrm{u}=\mathrm{u}-\mathrm{l}$ ambda $* \mathrm{~h}$

$\mathrm{gb}=\mathrm{gb}-\mathrm{lambda} * \mathrm{Ah}$

c third stage: iterate conjugate gradient solution process until

c gg < gtest criterion is satisfied.

c (USER) The parameter ncgsteps is the total number of conjugate gradient steps

$c$ to go through. Only in very unusual problems, like when the conductivity

c of one phase is much higher than all the rest, will this many steps be 
c used.

ncgsteps $=30000$

do 33 icc $=1$, ncgsteps

gglast $=\mathrm{gg}$

$\mathrm{gg}=0.0 \mathrm{~d} 0$

$\mathrm{dgg}=0.0 \mathrm{~d} 0$

$\operatorname{dgg}=\operatorname{SUM}(g b(2: n x 1,2:$ ny 1, dlow $:$ dhigh $) * g b(2: n x 1,2:$ ny1, dlow:dhigh $))$

call MPI_ALLREDUCE (dgg, gg, 1,MPI_DOUBLE_PRECISION, $\&$

MPI_SUM, MPI_COMM_WORLD, ierr)

if (myrank.eq.0) then call flush(7)

end if

if (gg.lt.gtest) goto 44

gamma $=$ gg/gglast

c update conjugate gradient direction

$\mathrm{h}=\mathrm{gb}+$ gamma $* \mathrm{~h}$

call prod_dc (nx2, ny2, nz2, ns2, gx,gy,gz, h, Ah, d1 ,d2)

hAh $=0.0 \mathrm{~d} 0$

$\mathrm{hAh} 2=0.0 \mathrm{~d} 0$

hAh2 $=\operatorname{SUM}(h(2: n x 1,2: n y 1$, dlow $:$ dhigh $) * \operatorname{Ah}(2: n x 1,2:$ ny 1, dlow $:$ dhigh $))$

call MPI_ALLREDUCE (hAh2, hAh, 1, MPI_DOUBLE_PRECISION, MPI_SUM, \& MPI_COMM_WORLD, ierr)

lambda $=g g / h A h$

c update voltage, gradient vectors

$\mathrm{u}=\mathrm{u}-\mathrm{lambda} * \mathrm{~h}$

$\mathrm{gb}=\mathrm{gb}-\mathrm{lambda} * \mathrm{Ah}$

c (USER) This piece of code forces dembx to write out the total current and

$c$ the norm of the gradient squared, every ncheck conjugate gradient steps,

$c$ in order to see how the relaxation is proceeding. If the currents become

c unchanging before the relaxation is done, then gtest was picked to be

c smaller than was necessary.

ncheck $=30$

if (ncheck*(icc/ncheck).eq.icc) then

if (myrank.eq.0) then 


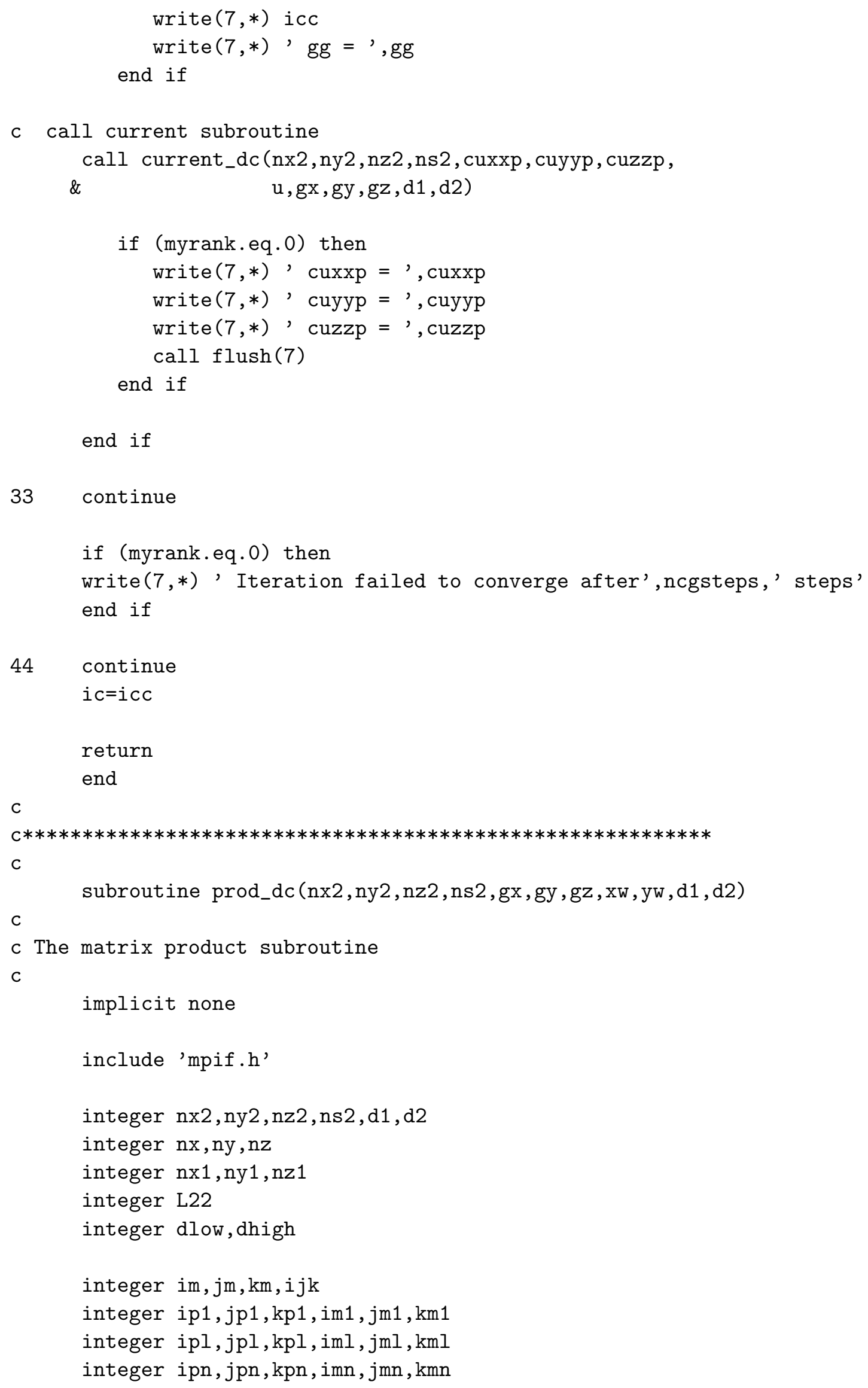


double precision $\mathrm{gx}(\mathrm{nx} 2, \mathrm{ny} 2, \mathrm{~d} 1-1: \mathrm{d} 2+1)$

double precision gy $(\mathrm{nx} 2, \mathrm{ny} 2, \mathrm{~d} 1-1: \mathrm{d} 2+1)$

double precision $\mathrm{gz}(\mathrm{nx} 2, \mathrm{ny} 2, \mathrm{~d} 1-1: \mathrm{d} 2+1)$

double precision $\mathrm{xw}(\mathrm{nx} 2, \mathrm{ny} 2, \mathrm{~d} 1-1: \mathrm{d} 2+1)$

double precision $\mathrm{yw}(\mathrm{nx} 2, \mathrm{ny} 2, \mathrm{~d} 1-1: \mathrm{d} 2+1)$

integer myrank, ierr, nprocs

integer status(MPI_STATUS_SIZE)

integer lowrank, highrank

call MPI_COMM_RANK ( MPI_COMM_WORLD, myrank, ierr )

call MPI_COMM_SIZE( MPI_COMM_WORLD, nprocs, ierr )

lowrank=0; highrank=0

c $\mathrm{xw}$ is the input vector, $\mathrm{yw}=(\mathrm{A})(\mathrm{xw})$ is the output vector

c auxiliary variables involving the system size

$\mathrm{nx} 1=\mathrm{nx} 2-1$

ny $1=$ ny $2-1$

$\mathrm{nz} 1=\mathrm{nz} 2-1$

$\mathrm{nx}=\mathrm{nx} 2-2$

ny $=$ ny $2-2$

$\mathrm{nz}=\mathrm{nz} 2-2$

L22 $=$ nx $2 *$ ny 2

if (nprocs.gt.1) then

if (myrank.eq.0) then

dlow $=2$

else

$\mathrm{dlow}=\mathrm{d} 1$

end if

if (myrank.eq.nprocs-1) then dhigh $=n z 1$

else

dhigh $=\mathrm{d} 2$

end if

end if

if (nprocs.eq.1) then

dlow $=2$

dhigh=nz1

end if 
C

$$
\begin{array}{ll}
\text { C } \mathrm{x} \text { faces } & \\
\mathrm{yw}(\mathrm{nx} 2,:,:) & =\mathrm{yw}(2,:,:) \\
\mathrm{yw}(1,:,:) & =\mathrm{yw}(\mathrm{nx} 1,:,:)
\end{array}
$$

C y faces

$$
\begin{aligned}
& \mathrm{yw}(:, 1,:)=\mathrm{yw}(:, \mathrm{ny} 1,:) \\
& \mathrm{yw}(:, \operatorname{ny} 2,:) \quad=\operatorname{yw}(:, 2,:)
\end{aligned}
$$

c $z$ faces

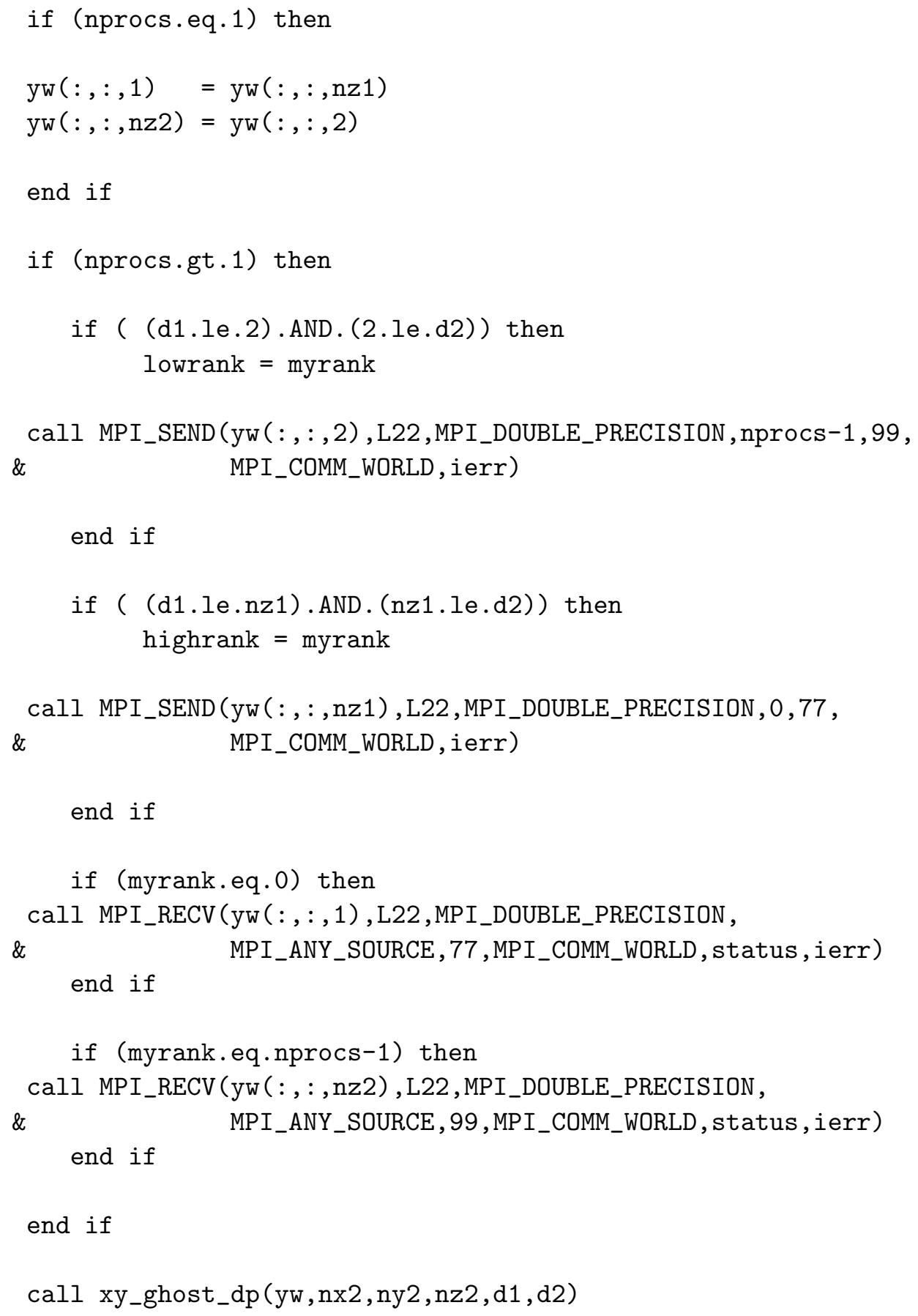


call z_ghost_dp (yw, nx2, ny2, nz2, d1, d2)

return

end

C

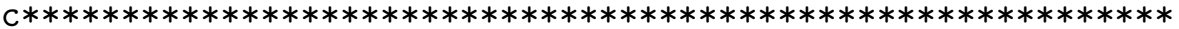

C

subroutine bond_dc(arr0, gx, gy, gz, nx2, ny2, nz2, ns2,

$\&$

sigma, be, nphase, ntot, d1, d2)

implicit none

include 'mpif.h'

integer nx2, ny2,nz2, ns2, nphase,ntot, d1, d2

integer $\mathrm{nx}, \mathrm{ny}, \mathrm{nz}, \mathrm{nx} 1, \mathrm{ny} 1, \mathrm{nz} 1, \mathrm{~L} 22$

integer $i, j, k, m, d l o w$, dhigh, $k 1, j 1, i 1$

double precision $\mathrm{gx}(\mathrm{nx} 2, \mathrm{ny} 2, \mathrm{~d} 1-1: \mathrm{d} 2+1)$

double precision gy $(\mathrm{nx} 2, \mathrm{ny} 2, \mathrm{~d} 1-1: \mathrm{d} 2+1)$

double precision $\mathrm{gz}(\mathrm{nx} 2, \mathrm{ny} 2, \mathrm{~d} 1-1: \mathrm{d} 2+1)$

double precision sigma(nphase,3), be (nphase,nphase,3)

integer*2 $\operatorname{arr} 0(\mathrm{nx} 2, \mathrm{ny} 2, \mathrm{~d} 1-1: \mathrm{d} 2+1)$

integer myrank, ierr, nprocs

integer status(MPI_STATUS_SIZE)

call MPI_COMM_RANK( MPI_COMM_WORLD, myrank, ierr )

call MPI_COMM_SIZE( MPI_COMM_WORLD, nprocs, ierr)

c auxiliary variables involving the system size

$\mathrm{nx}=\mathrm{n} \times 2-2$

ny $=$ ny $2-2$

$\mathrm{nz}=\mathrm{nz} 2-2$

$\mathrm{nx} 1=\mathrm{n} \times 2-1$

ny1=ny2-1

$\mathrm{nz} 1=\mathrm{nz} 2-1$

$\mathrm{L} 22=\mathrm{n} \times 2 * \mathrm{ny} 2$

c Set values of conductor for phase $(i, m)--p h a s e(j, m)$ interface,

c store in array be $(i, j, m), m=1,2,3$. If either phase $i$ or $j$

$c$ has zero conductivity in the m'th direction, then $b e(i, j, m)=0.0$.

$\mathrm{be}=0.0 \mathrm{~d} 0$

do $10 \mathrm{~m}=1,3$

do $10 i=1$, nphase 


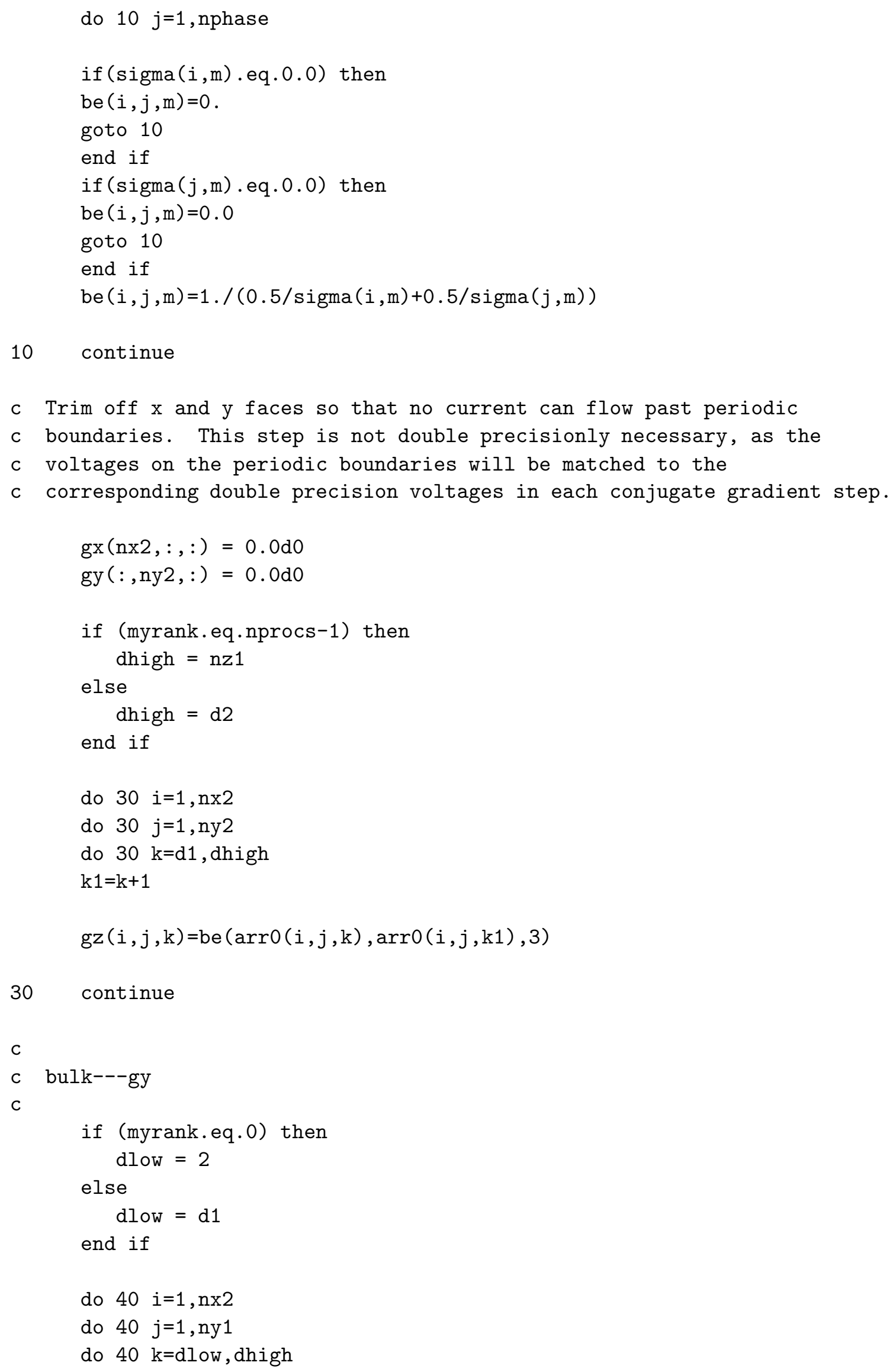




$$
\begin{aligned}
& j 1=j+1 \\
& \operatorname{gy}(i, j, k)=\operatorname{be}(\operatorname{arr} 0(i, j, k), \operatorname{arr} 0(i, j 1, k), 2)
\end{aligned}
$$

40

$$
\text { continue }
$$

c bulk--gx

do $50 i=1, n \times 1$

do $50 j=1$, ny2

do $50 \mathrm{k}=$ dlow, dhigh

$\mathrm{m}=(\mathrm{k}-1) * \mathrm{~L} 22+(\mathrm{j}-1) * \mathrm{n} \times 2+\mathrm{i}$

$i 1=i+1$

$j 1=j$

$\mathrm{k} 1=\mathrm{k}$

$\operatorname{gx}(i, j, k)=\operatorname{be}(\operatorname{arr} 0(i, j, k), \operatorname{arr} 0(i 1, j, k), 1)$

50 continue

$\operatorname{gx}(\mathrm{nx} 2,:,:)=0.0 \mathrm{~d} 0$

gy $(:$, ny $2,:)=0.0 \mathrm{~d} 0$

call xy_ghost_dp (gx, nx2, ny2, nz2, d1, d2)

call z_ghost_dp (gx, nx2, ny2, nz2, d1, d2)

call xy_ghost_dp (gy, nx2, ny2, nz2, d1, d2)

call z_ghost_dp (gy, nx2, ny2, nz2, d1, d2)

call xy_ghost_dp (gz, nx2, ny2, nz2, d1 ,d2)

call z_ghost_dp (gz,nx2, ny2, nz2, d1, d2)

$\operatorname{gx}(\mathrm{nx} 2,:,:)=0.0 \mathrm{~d} 0$

gy $(:$, ny $2,:)=0.0 \mathrm{~d} 0$

return

end

$\mathrm{C}$

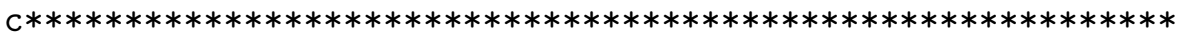

$\mathrm{C}$

subroutine dpixel_dc(pix,nx2,ny2,nz2, a, nphase,ntot)

implicit none

integer $\mathrm{nx} 2, \mathrm{ny} 2, \mathrm{nz2}$, nphase, ntot

integer $\mathrm{nx}, \mathrm{ny}, \mathrm{nz}$

integer $i, j, k, m$

double precision a(ntot) 
integer*2 pix (nx2,ny2,nz2)

c (USER) If you want to set up a test image inside the program, instead

$c$ of reading it in from a file, this should be done inside this subroutine.

$\mathrm{nx}=\mathrm{nx} 2-2$

ny=ny $2-2$

$\mathrm{nz}=\mathrm{nz} 2-2$

c Initialize phase fraction array.

$\mathrm{a}=0.0 \mathrm{~d} 0$

c Use 3-d labelling scheme as shown in manual.

do $100 \mathrm{k}=2, \mathrm{nz} 2-1$

do $100 \mathrm{j}=2, \mathrm{ny} 2-1$

do $100 i=2, n \times 2-1$

$\operatorname{read}(9, *) \operatorname{pix}(i, j, k)$

$\mathrm{a}(\operatorname{pix}(i, j, k))=a(\operatorname{pix}(i, j, k))+1.0 d 0$

100 continue

$a=a / d f l o a t(n x * n y * n z)$

C now map periodic boundaries of pix

c F90 syntax

$$
\begin{aligned}
& \operatorname{pix}(n \times 2,:,:)=\operatorname{pix}(2,:,:) \\
& \operatorname{pix}(1,:,:) \quad=\operatorname{pix}(n \times 2-1,:,:) \\
& \operatorname{pix}(:, n y 2,:)=\operatorname{pix}(:, 2,:) \\
& \operatorname{pix}(:, 1,:)=\operatorname{pix}(:, \text { ny2-1,: }) \\
& \operatorname{pix}(:,:, 1)=\operatorname{pix}(:,:, \mathrm{nz} 2-1) \\
& \operatorname{pix}(:,:, n z 2)=\operatorname{pix}(:,:, 2)
\end{aligned}
$$

c Check for wrong phase labels--less than 1 or greater than nphase

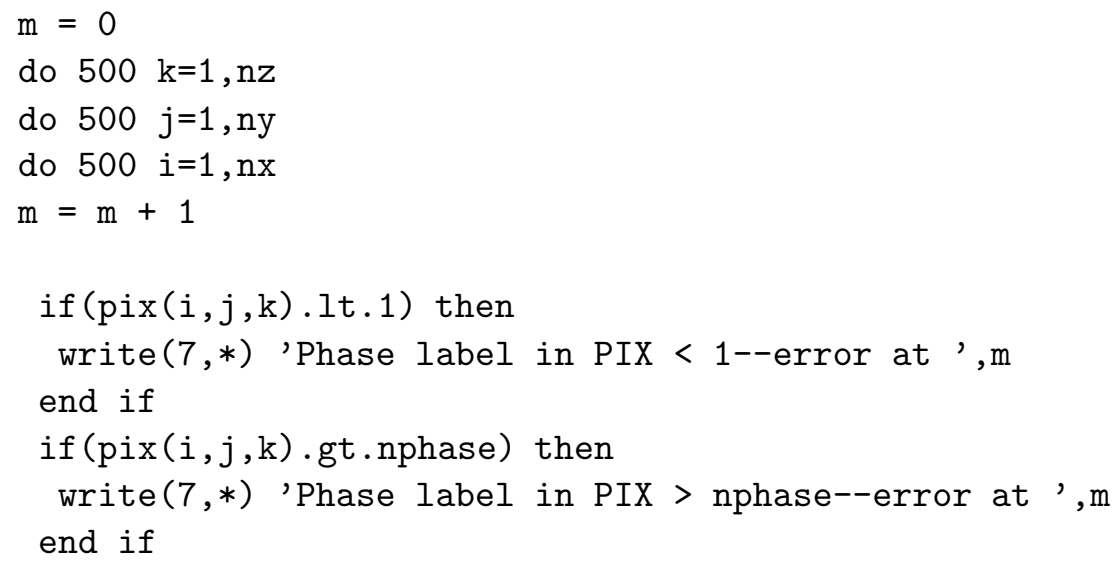




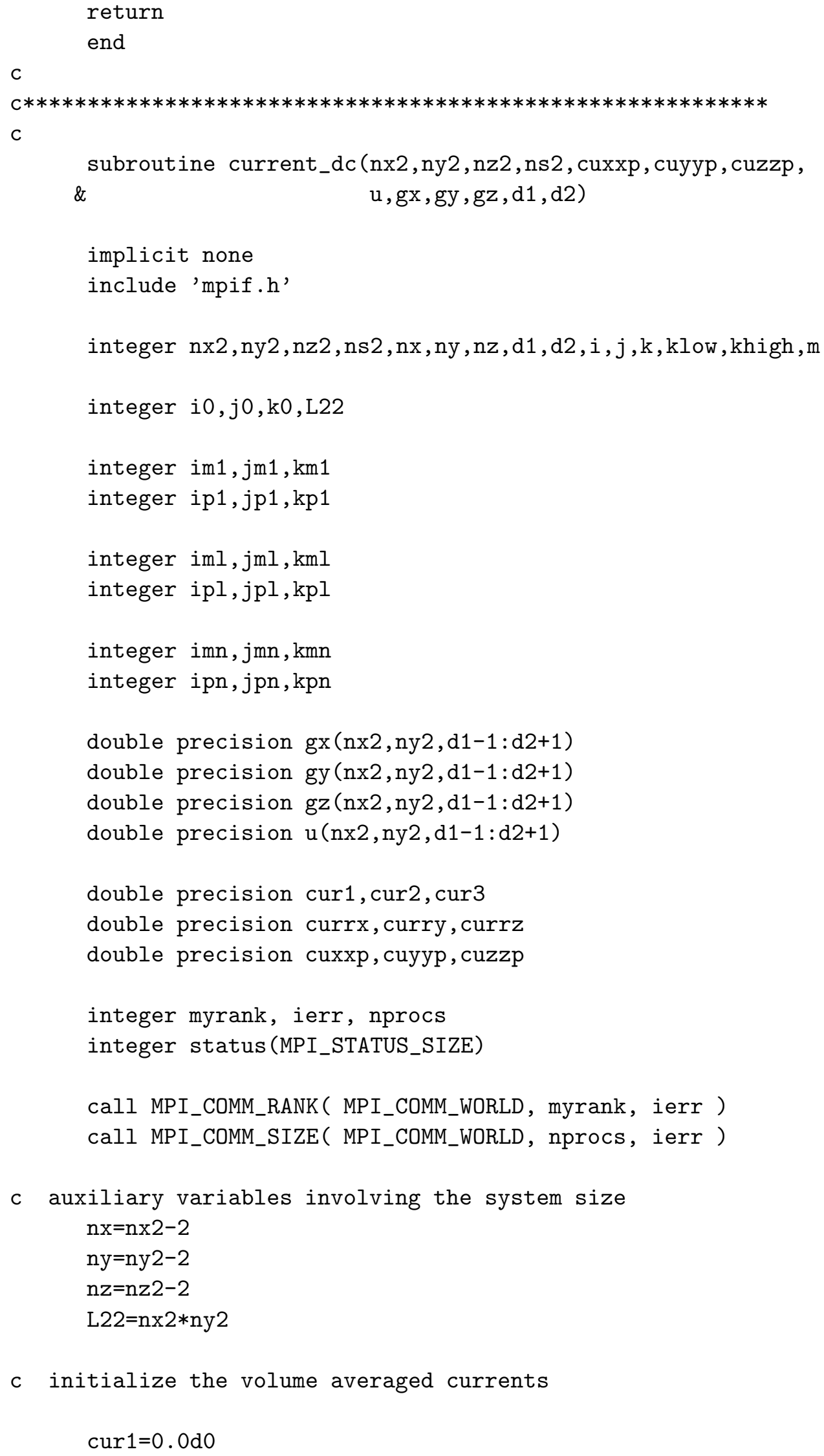




$$
\begin{aligned}
& \operatorname{cur} 2=0.0 \mathrm{~d} 0 \\
& \operatorname{cur} 3=0.0 \mathrm{~d} 0 \\
& \operatorname{currx}=0.0 \mathrm{~d} 0 \\
& \operatorname{curry}=0.0 \mathrm{~d} 0 \\
& \operatorname{currz}=0.0 \mathrm{~d} 0 \\
& \operatorname{cuxxp}=0.0 \mathrm{~d} 0 \\
& \operatorname{cuyyp}=0.0 \mathrm{~d} 0 \\
& \text { cuzzp }=0.0 \mathrm{~d} 0
\end{aligned}
$$

c Only loop over real sites and bonds in order to get true total current

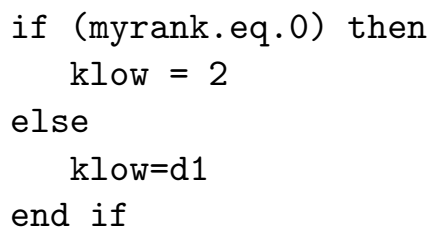

c cur1, cur2, cur3 are the currents in one pixel $\operatorname{cur} 1=0.5 \mathrm{~d} 0 *((\mathrm{u}(\mathrm{im} 1, \mathrm{jm} 1, \mathrm{~km} 1)-\mathrm{u}(\mathrm{i}, \mathrm{j}, \mathrm{k})) * \mathrm{gx}(\mathrm{im} 1, \mathrm{jm} 1, \mathrm{~km} 1)+$ \& $\quad(u(i, j, k)-u(i p 1, j p 1, k p 1)) * g x(i, j, k))$

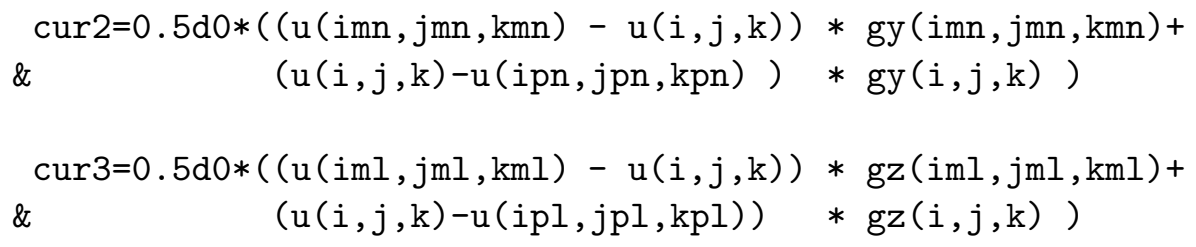

c sum pixel currents into volume averages 


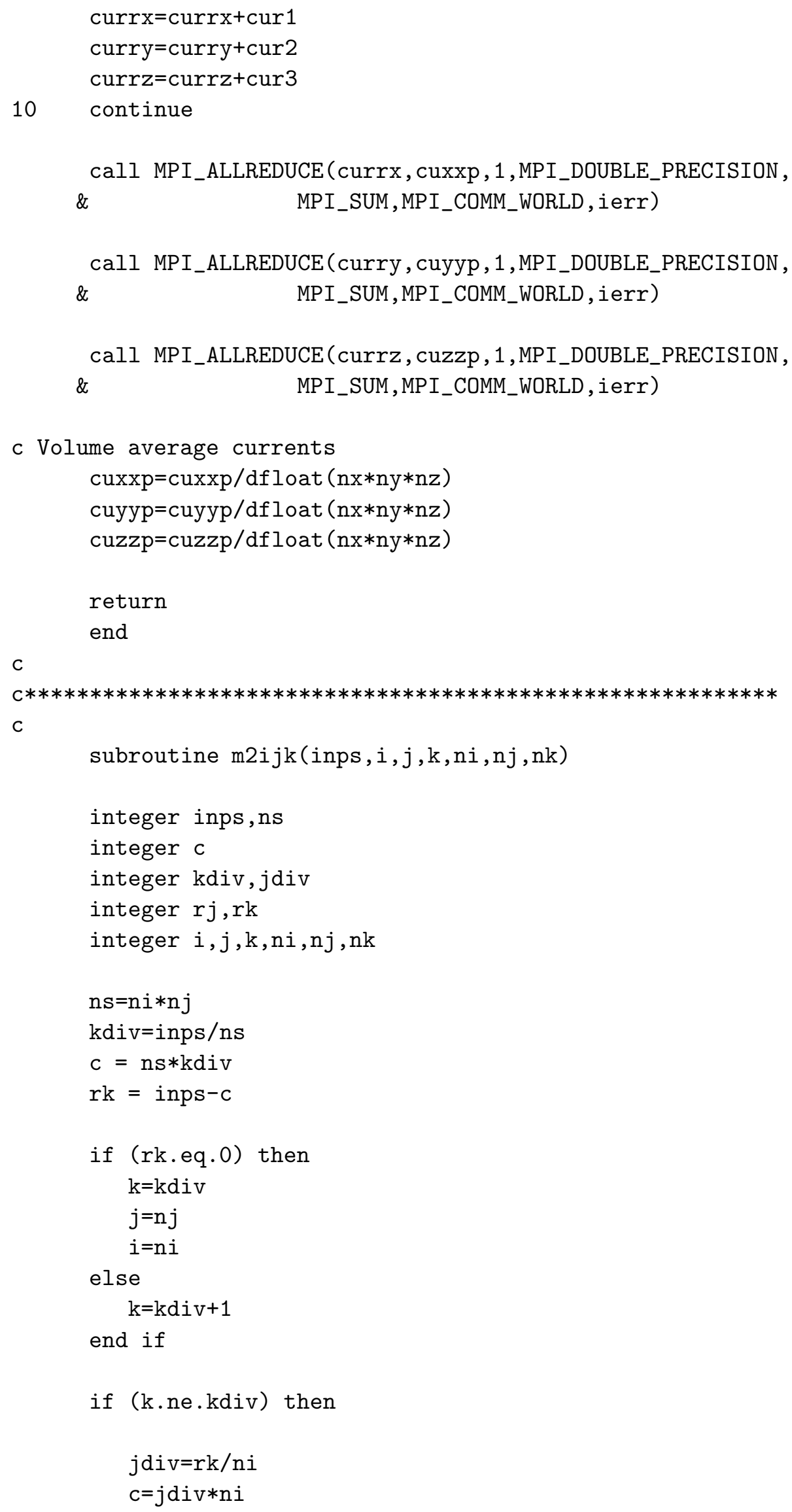




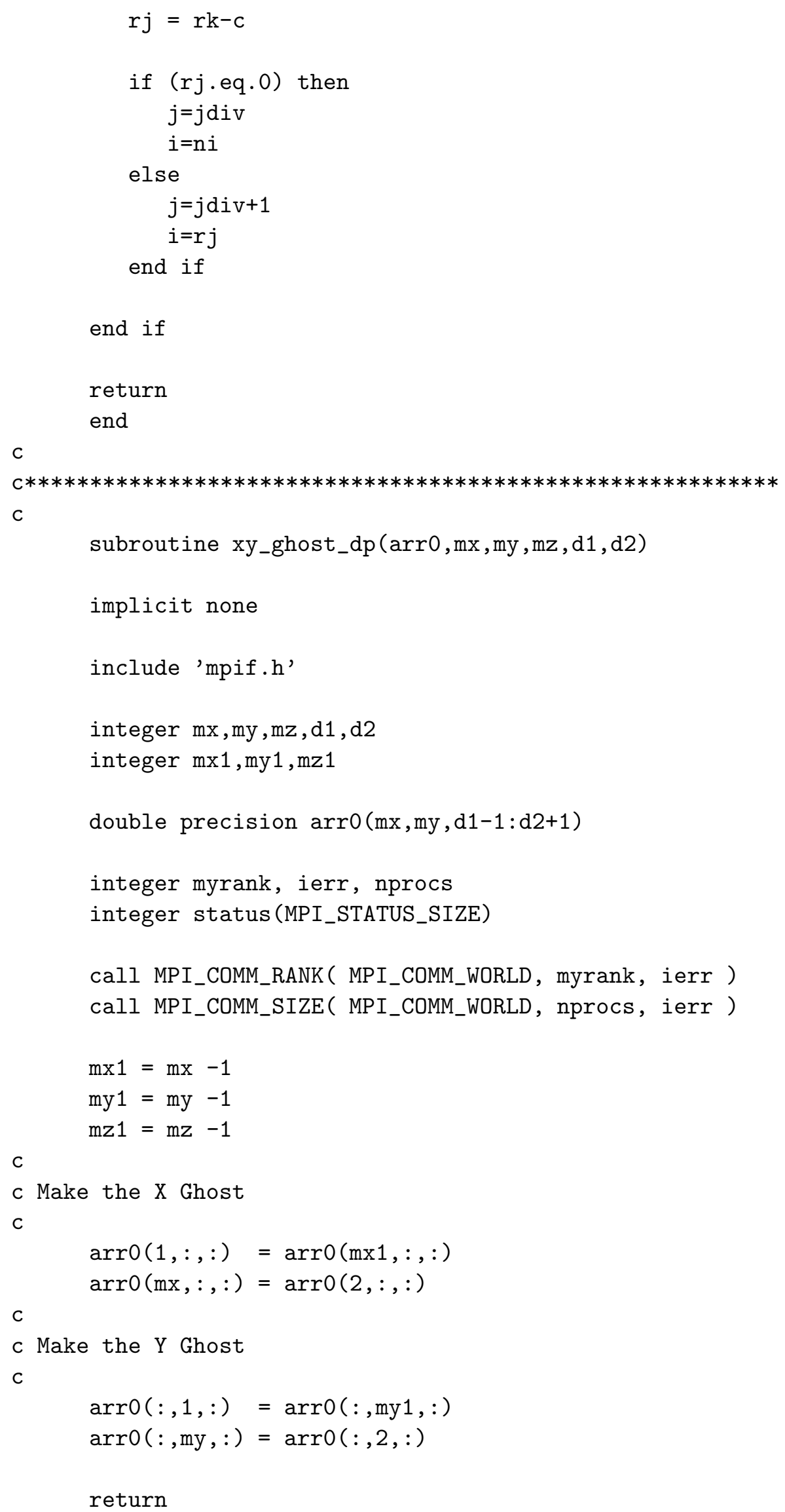


end

C

c $* * * * * * * * * * * * * * * * * * * * * * * * * * * * * * * * * * * * * * * * * * * * * * * * * * * * * * * * * *$

$c$

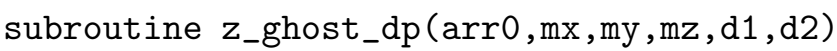

implicit none

include 'mpif.h'

integer $\mathrm{mx}, \mathrm{my}, \mathrm{mz}, \mathrm{d} 1, \mathrm{~d} 2$

double precision $\operatorname{arr} 0(\mathrm{mx}, \mathrm{my}, \mathrm{d} 1-1: \mathrm{d} 2+1)$

double precision, allocatable :: $\operatorname{bot}(:,:), \operatorname{top}(:,:)$

integer myrank, ierr, nprocs

integer status(MPI_STATUS_SIZE)

call MPI_COMM_RANK ( MPI_COMM_WORLD, myrank, ierr )

call MPI_COMM_SIZE( MPI_COMM_WORLD, nprocs, ierr )

C

c Make the $\mathrm{Z}$ Ghost

c

allocate (bot (mx,my))

allocate (top (mx,my))

C

c Get new bottom ghost plane.

$c$

bot $=\operatorname{arr} 0(:,:, \mathrm{d} 1)$

top $=\operatorname{arr} 0(:,:, d 2)$

call t2b_dp (bot, top, mx,my)

$\operatorname{arr} 0(:,:, \mathrm{d} 1-1)=\operatorname{bot}$

C

c Get new top ghost plane

c

bot $=\operatorname{arr} 0(:,:, \mathrm{d} 1)$

top $=\operatorname{arr} 0(:,:, d 2)$

call b2t_dp (bot, top, mx, my)

$\operatorname{arr} 0(:,:, d 2+1)=$ top

deallocate (bot)

deallocate (top)

return

end

C

c*************************************************************

C

subroutine $z_{-}$ghost_int ( $\left.\operatorname{arr} 0, m x, m y, m z, d 1, d 2\right)$ 


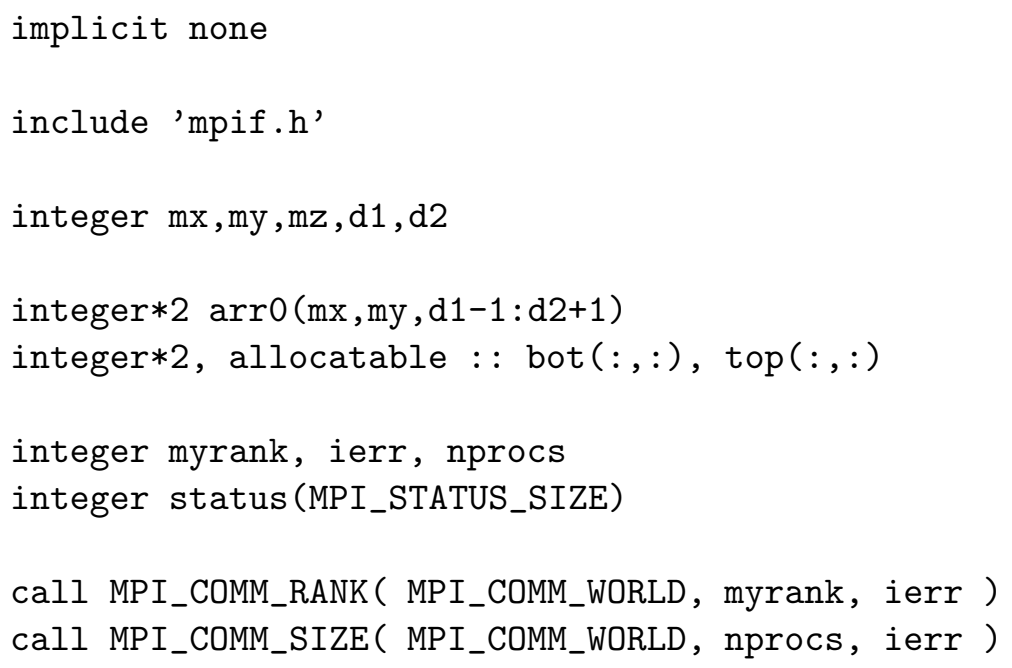


C

c This is an INTEGER*2 subroutine.

C

c Used for transferring: pix bottom2top layers

C

c RECV a new t_layer (TOP layer) per node.

C

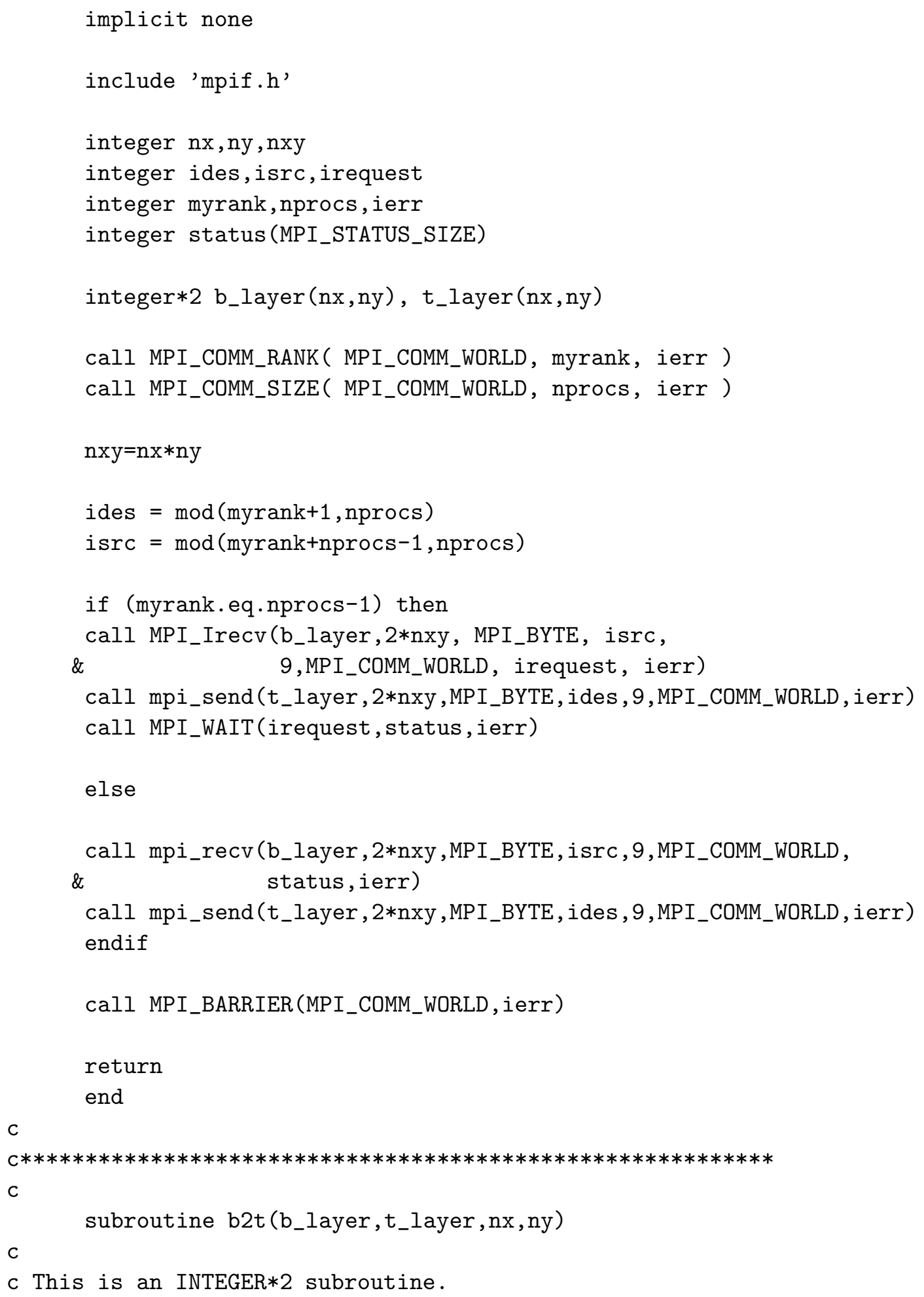


C

c Used for transferring: pix bottom2top layers

C

c RECV a new t_layer (TOP layer) per node.

C

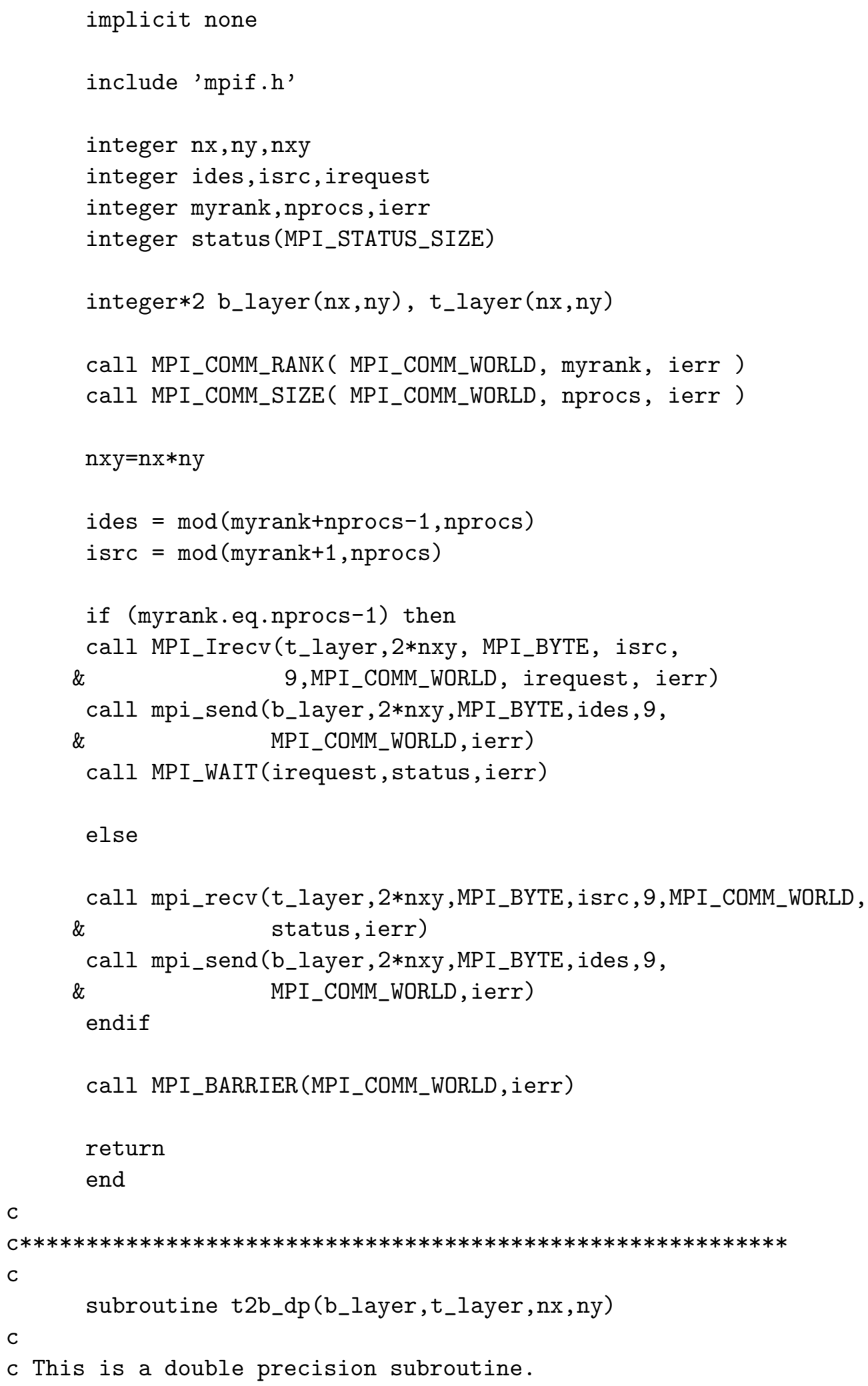


C

c Used for transferring: $u, b$, and om top2bottom layers

C

c RECV a new b_layer (BOTTOM layer) per node.

C

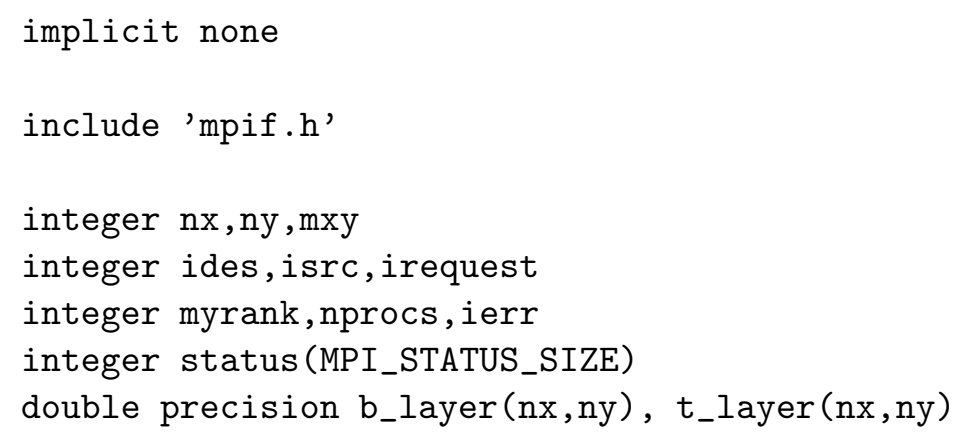


c Used for transferring: $u, b$, and om bottom2top layers

$\mathrm{C}$

c RECV a new t_layer (TOP layer) per node.

C

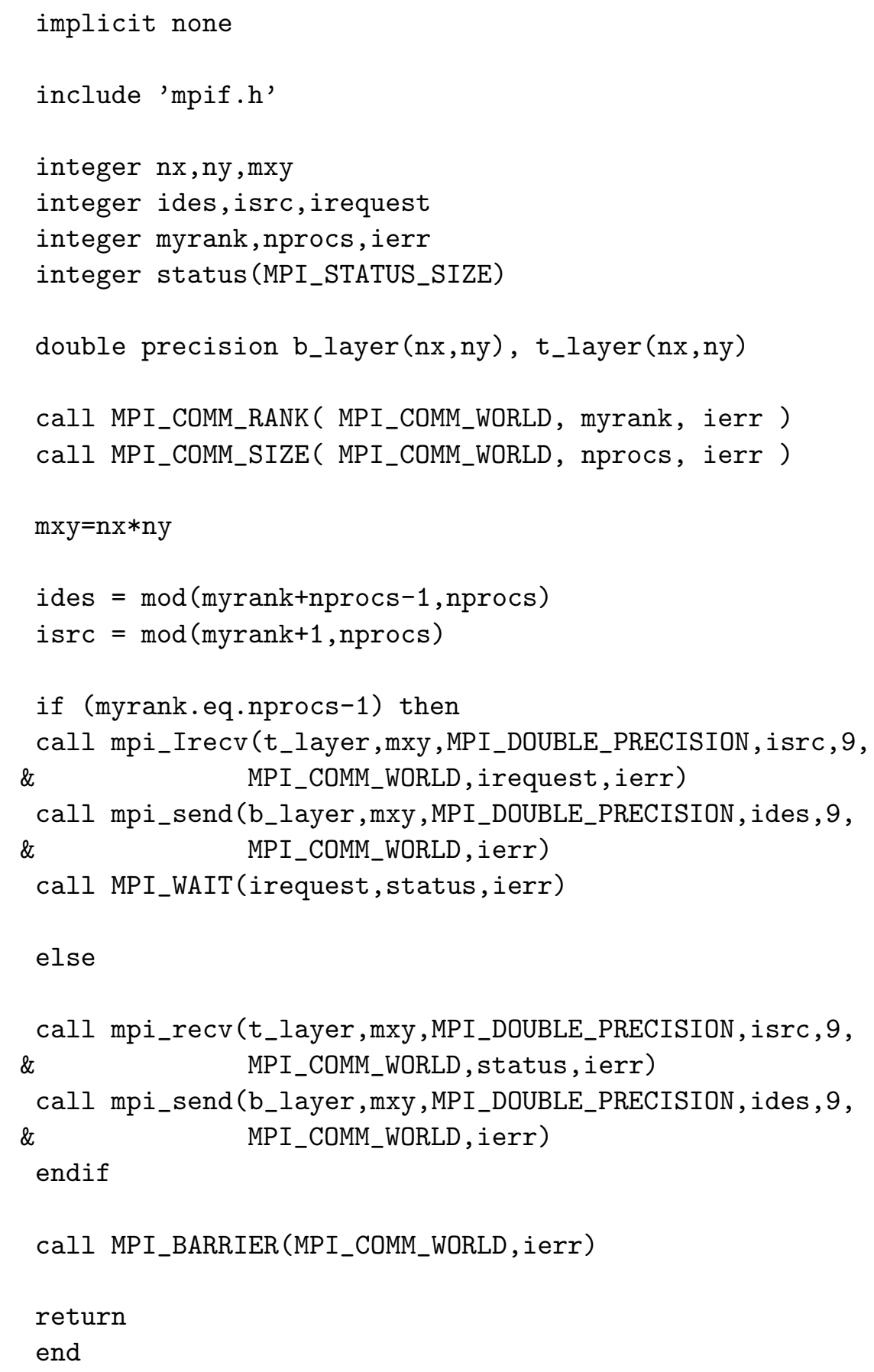




\subsubsection{AC3D_MPI.f}

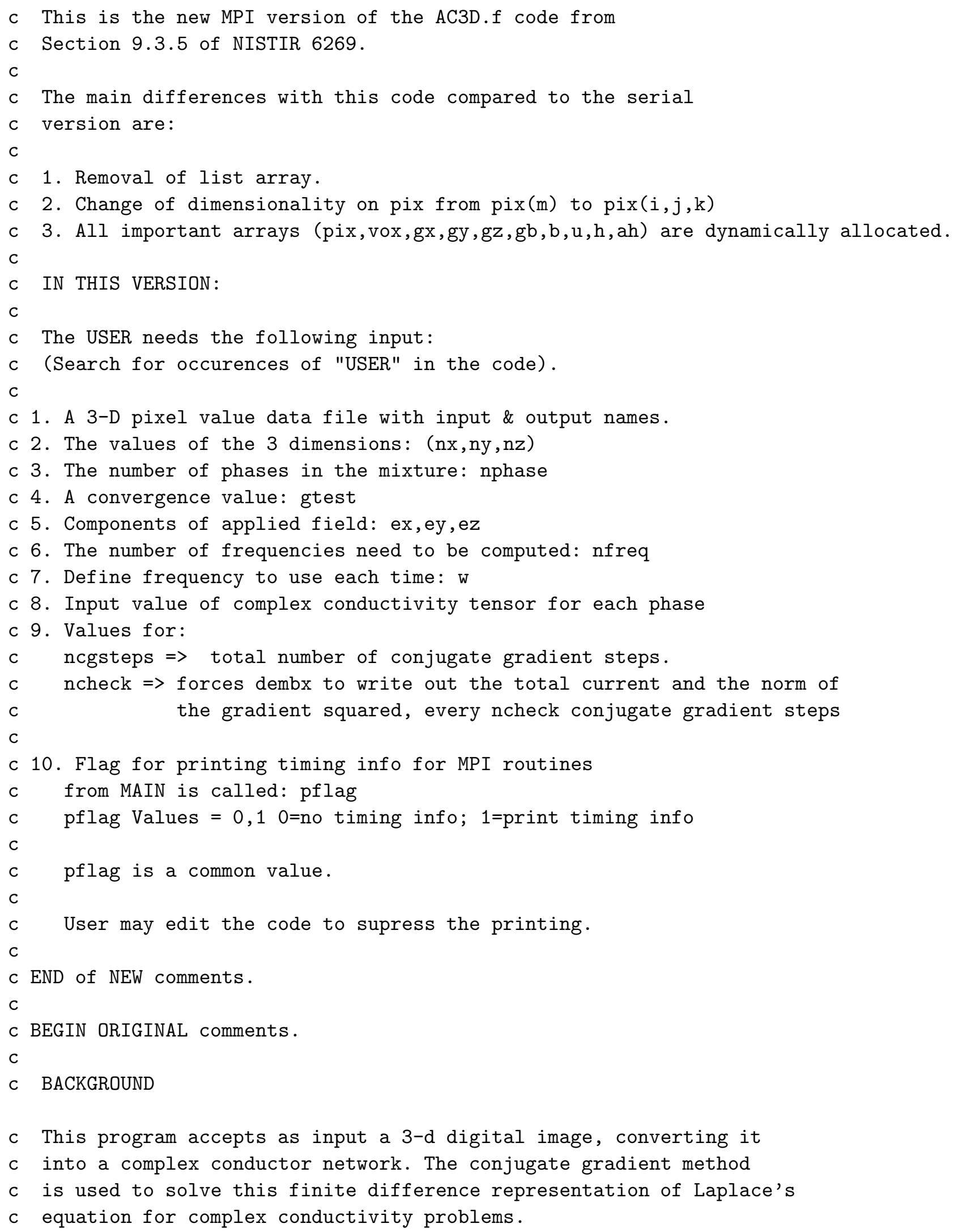

c This program accepts as input a 3-d digital image, converting it

$c$ into a complex conductor network. The conjugate gradient method

c is used to solve this finite difference representation of Laplace's

c equation for complex conductivity problems. 
c Periodic boundary conditions are maintained.

c In the comments below, (USER) means that this is a section of code

c that the user might have to change for his particular problem.

c Therefore the user is encouraged to search for this string.

C PROBLEM AND VARIABLE DEFINITION

c The mathematical problem that the conjugate gradient algorithm solves

$c$ is the minimization of the quadratic form $1 / 2 \mathrm{uAu}$, where

$c \quad u$ is the vector of voltages, and $A$ is generated from the bond

c conductances between pixels. Nodes are thought of as being in the

$c$ center of pixels. The minimization is constrained by maintaining an

c general direction applied electric field across the sample.

c The vectors gx,gy,gz are bond conductances, $u$ is the voltage array,

$c$ and $g b, h$, and Ah are auxiliary variables, used in subroutine dembx.

c The vector pix contains the phase labels for each pixel.

c The small vector $a(i)$ is the volume fraction

$c$ of the ith phase, and currx, curry, currz are the total volume-averaged

c complex currents in the $\mathrm{x}, \mathrm{y}$, and $\mathrm{z}$ directions.

C DIMENSIONS

c The vectors $\mathrm{gx}, \mathrm{gy}, \mathrm{gz}, \mathrm{u}, \mathrm{gb}, \mathrm{h}, \mathrm{Ah}, \mathrm{list}, \mathrm{pix}$ are all dimensioned

c $\mathrm{ns} 2=(\mathrm{nx}+2) *(\mathrm{ny}+2) *(\mathrm{nz}+2)$. This number is used, rather than the

c system size $\mathrm{nx} \times \mathrm{ny} \mathrm{xz}$, because an extra layer of pixels is

c put around the system to be able to maintain periodic boundary

c conditions (see manual, Sec. 3.3). The arrays pix and list are also

c dimensioned this way. At present the program is set up for 100 phases,

$c$ but that can easily be changed by the user, by changing the dimensions

c of sigma, a, and be. Note that be has both dimensions equal to

$c$ each other. The parameter nphase gives the number of phases

$c$ being considered. The parameter ntot is the total number of phases

c possible in the program, and should be equal to the dimension

$c$ of sigma, $a$, and be. All arrays are passed to subroutines in

$c$ the call statements.

C STRONGLY RECOMMENDED: READ MANUAL BEFORE USING THE PROGRAM!!

c (USER) Change these dimensions for different system sizes. All

C dimensions in the subroutines are passed, so do not need to be

c changed. The dimensions of sigma, a, and be should be equal to

c the value of ntot.

implicit none

include 'mpif.h'

integer*2, allocatable $:: \operatorname{dat}(:,:,:)$, datn $(:,:,:)$

integer*2, allocatable $:: \operatorname{pix}(:,:,:), \operatorname{pixn}(:,:,:)$

integer*2, allocatable :: $\operatorname{vox}(:,:,:)$ 


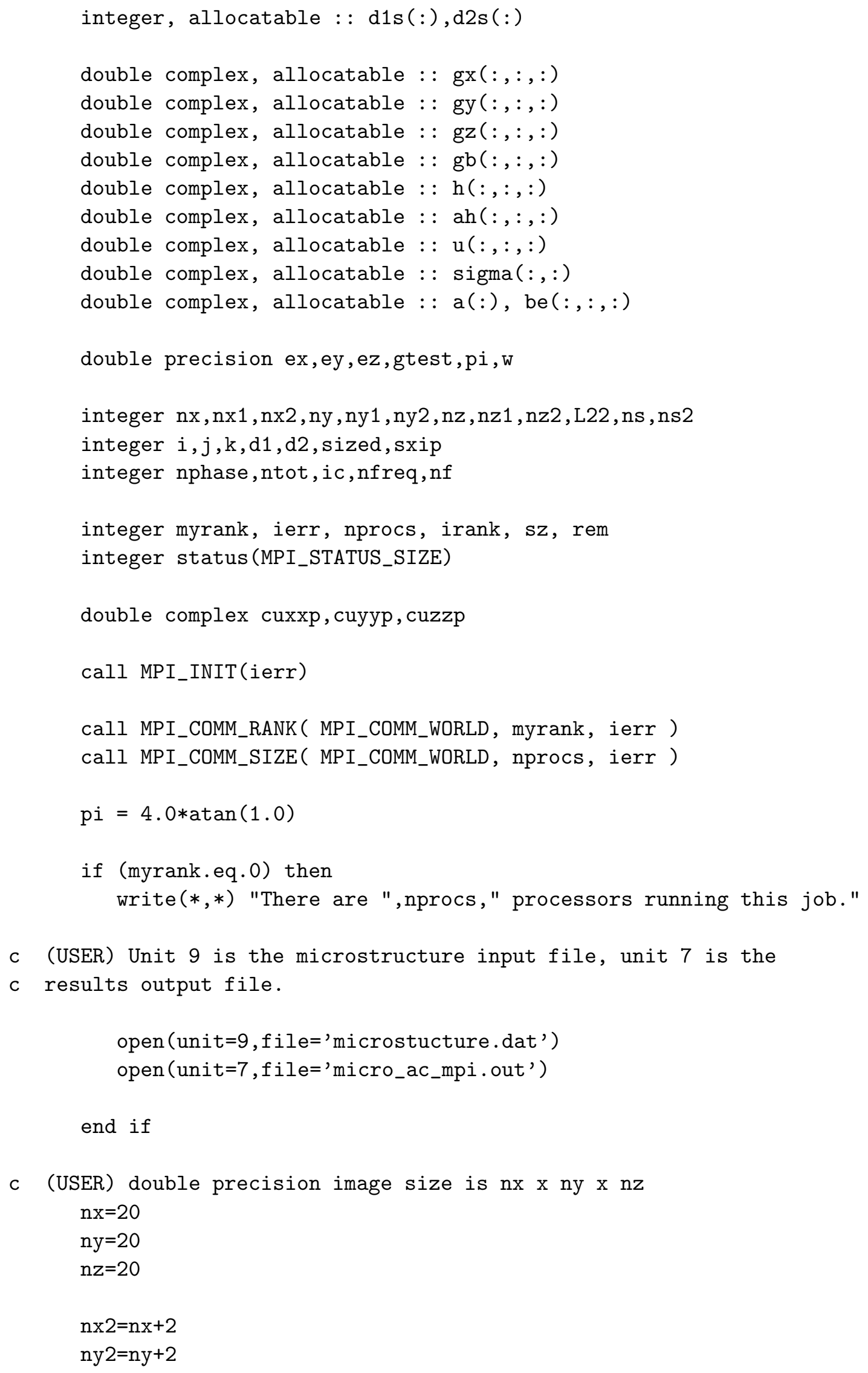


$\mathrm{nz} 2=\mathrm{nz}+2$

L22 $=\mathrm{n} \times 2 *$ ny 2

c Variables changed here to reflect the fact our

c calculations are now based on "NZ2" instead of "NZ"

$c$ as in the previous MPI programs.

C

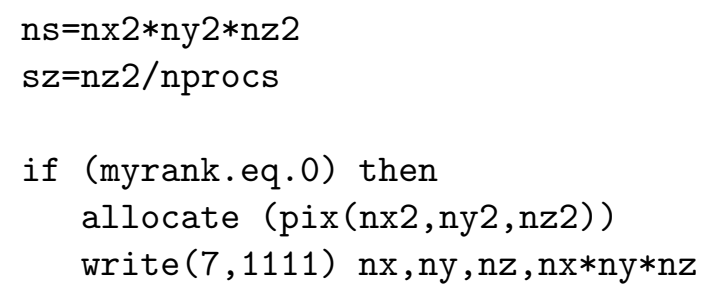

1111 format(' Image is ',3i6,' No. of double precision sites = ',i8)

c auxiliary variables involving the system size $\mathrm{nx} 1=\mathrm{nx}+1$

ny $1=n y+1$

$\mathrm{nz} 1=\mathrm{nz}+1$

c computational image size ns 2 is $n \times 2 \times n y 2 \times n z 2$ ns $2=$ nx $2 *$ ny $2 * n z 2$

c (USER) set cutoff for norm squared of gradient, gtest. gtest is

$c$ the stopping criterion, compared to gb*gb. When gb*gb is less

$c$ than gtest=abc*ns2, then the rms value of the gradient at a pixel

$c$ is less than sqrt (abc). gtest $=1.0 d-16 * n s 2$

c (USER) nphase is the number of phases being considered in the problem.

c The values of pix(m) will run from 1 to nphase. ntot is the total

$c$ number of phases possible in the program, and is the dimension of

c sigma, a, and be.

nphase $=2$

ntot $=100$

allocate(a(ntot))

allocate (sigma (nphase, 3 ))

$\mathrm{C}$

c Program calculates the d1 \& d2 limits for the principle arrays.

C

c In this program, d1 \& d2 are not based on "nz".

c They are calculated on the basis of "nz2".

c

if (myrank.eq.0) then 
c (myrank=0) to all the rest.

c

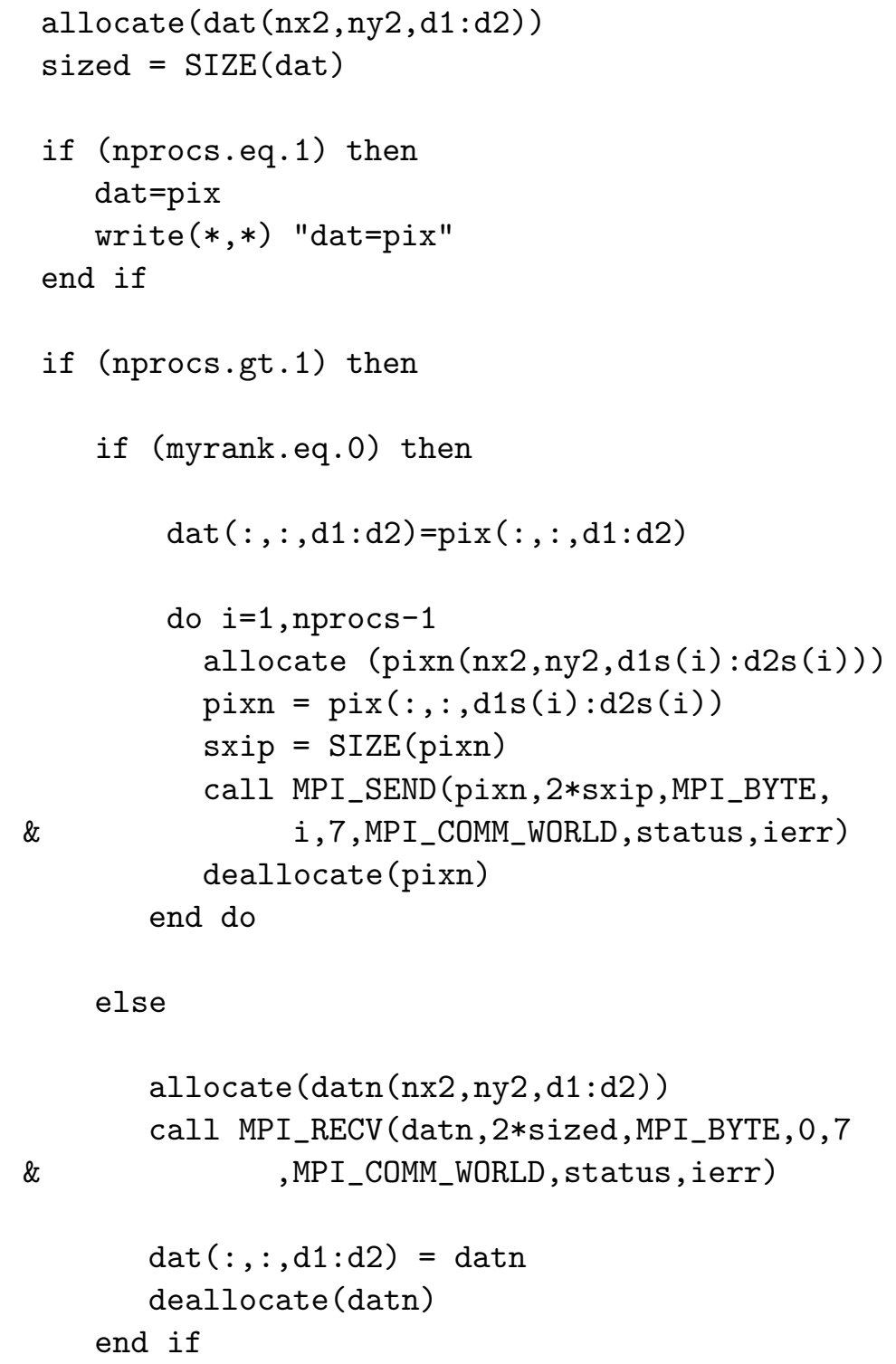




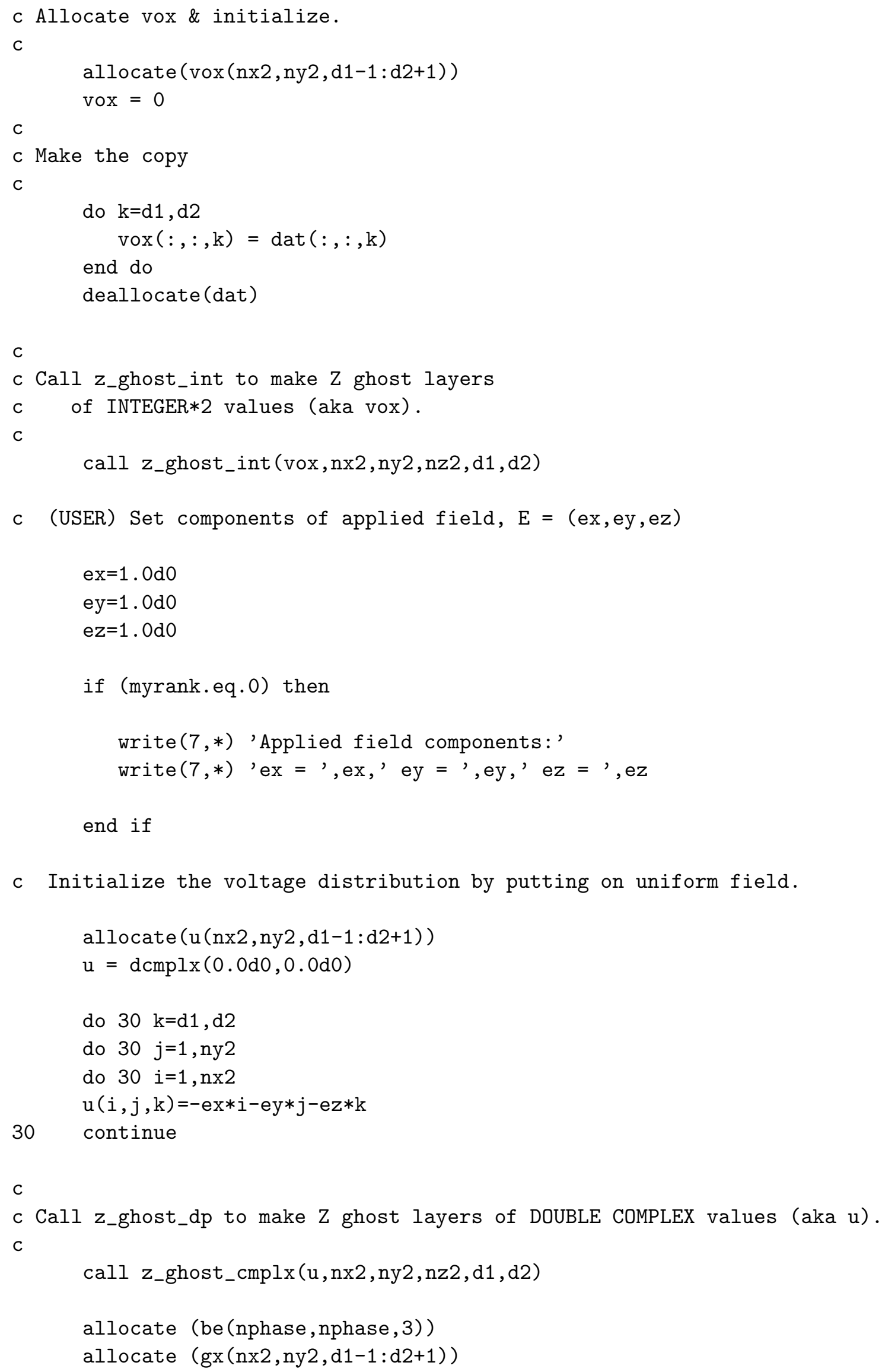




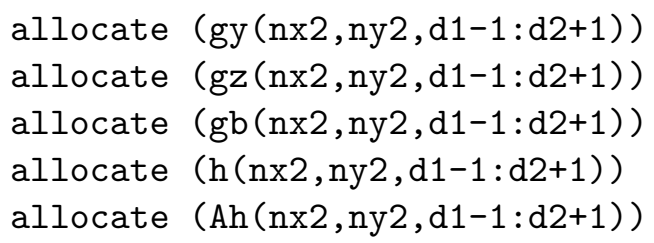

c (USER) Set how many frequencies need to be computed. nfreq $=50$

c Loop over desired frequencies. do $300 \mathrm{nf}=1$, nfreq

c (USER) Define frequency to use each time. Alter this statement to get

c different frequencies. The frequencies are in $\mathrm{Hz}$, according to

$c$ the units used for sigma. $\mathrm{w}=10 . * *((\mathrm{nf}-1) * 11.4 / 49 .-3$.

$c$ convert to angular frequency $\mathrm{w}=\mathrm{w} * 2 . * \mathrm{pi}$

write $(7, *)$ 'No.', nf, ' angular frequency = ',w, ' radians'

c (USER) input value of complex conductivity tensor for each phase

c (diagonal only). $1,2,3=\mathrm{x}, \mathrm{y}, \mathrm{z}$, respectively. $\operatorname{sigma}(1,1)=d \operatorname{cmplx}\left(1.0 \mathrm{~d} 0,10 \cdot d 0 *_{\mathrm{w}}\right)$ $\operatorname{sigma}(1,2)=d \operatorname{cmplx}(1.0 \mathrm{~d} 0,10 . \mathrm{d} 0 * \mathrm{w})$ $\operatorname{sigma}(1,3)=d \operatorname{cmplx}(1.0 \mathrm{~d} 0,10 . \mathrm{d} 0 * \mathrm{w})$ $\operatorname{sigma}(2,1)=\operatorname{dcmplx}(0.5 \mathrm{~d} 0,1 . \mathrm{d} 0 * \mathrm{w})$ $\operatorname{sigma}(2,2)=d \operatorname{cmplx}(0.5 \mathrm{~d} 0,1 . \mathrm{d} 0 * \mathrm{w})$ $\operatorname{sigma}(2,3)=d \operatorname{cmplx}(0.5 \mathrm{~d} 0,1 . \mathrm{d} 0 * \mathrm{w})$

$g x=0.0 \mathrm{~d} 0 ; g y=0.0 \mathrm{~d} 0 ; g z=0.0 \mathrm{~d} 0 ; \mathrm{be}=0.0 \mathrm{~d} 0$ $\mathrm{gb}=0.0 \mathrm{~d} 0 ; \mathrm{h}=0.0 \mathrm{~d} 0 ; \mathrm{Ah}=0.0 \mathrm{~d} 0$

c Subroutine bond sets up conductor network in gx, gy, gz 1-d arrays

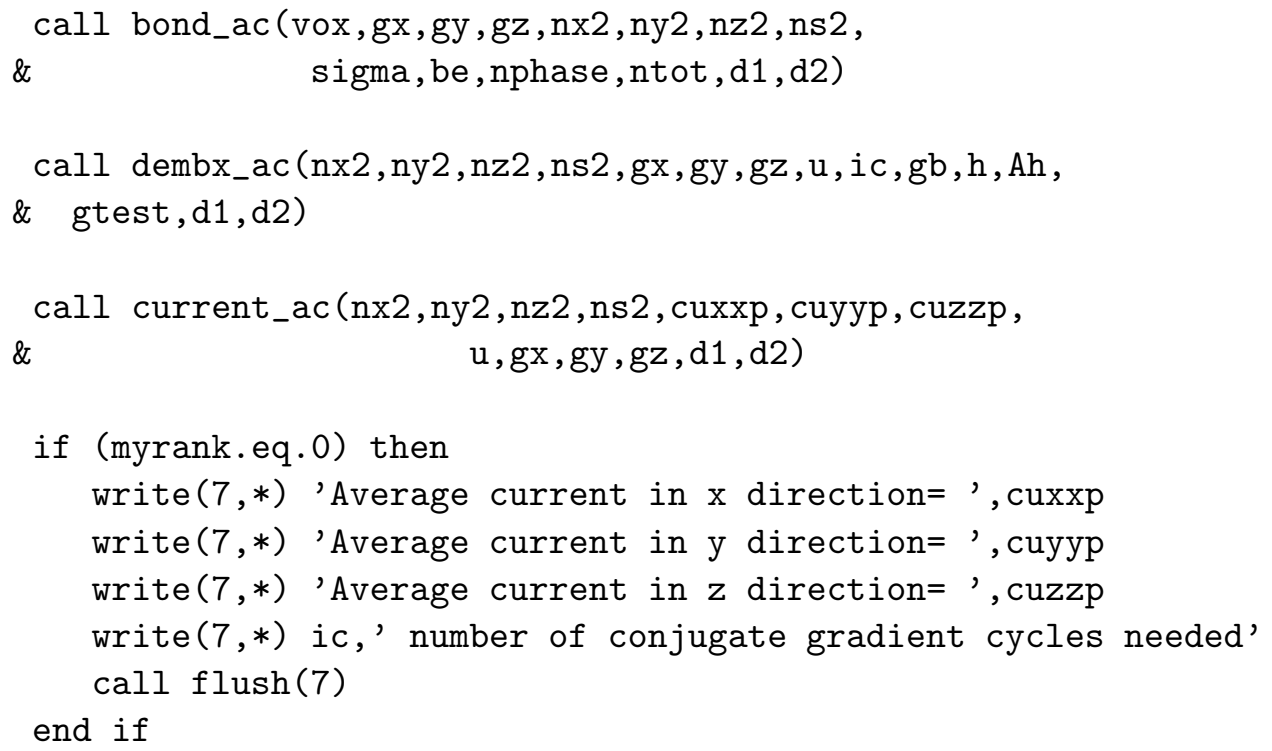


CALL MPI_FINALIZE(ierr)

end

$\mathrm{C}$

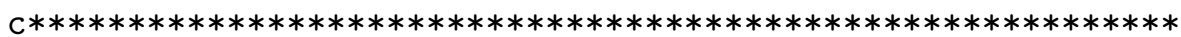

$\mathrm{c}$

subroutine dembx_ac (nx $2, n y 2, n z 2, n s 2, g x, g y, g z, u, i c, g b, h, A h$,

\& gtest, $\mathrm{d} 1, \mathrm{~d} 2$ )

implicit none

include 'mpif.h'

integer $\mathrm{nx} 2, \mathrm{ny} 2, \mathrm{nz} 2, \mathrm{~ns} 2, \mathrm{~d} 1, \mathrm{~d} 2, \mathrm{~L} 22$

integer $\mathrm{nx} 1, \mathrm{ny} 1, \mathrm{nz} 1$

double complex $\mathrm{gx}(\mathrm{nx} 2, \mathrm{ny} 2, \mathrm{~d} 1-1: \mathrm{d} 2+1)$

double complex gy $(\mathrm{nx} 2, \mathrm{ny} 2, \mathrm{~d} 1-1: \mathrm{d} 2+1)$

double complex $g z(n x 2, n y 2, d 1-1: d 2+1)$

double complex $u(n \times 2$, ny2, d1-1:d2+1)

double complex gb(nx2, ny2,d1-1:d2+1)

double complex Ah(nx2,ny2,d1-1:d2+1)

double complex $h(n x 2$, ny2, d1-1:d2+1)

double complex gg,hAh,hAh2, lambda,gglast, gamma

double complex cuxxp, cuyyp, cuzzp

double complex dgg

double precision gtest

integer ncheck, icc,ncgsteps, ic

integer $\mathrm{nx}, \mathrm{ny}, \mathrm{nz}, \mathrm{dlow}$, dhigh

integer myrank, ierr, nprocs

integer status(MPI_STATUS_SIZE)

call MPI_COMM_RANK ( MPI_COMM_WORLD, myrank, ierr )

call MPI_COMM_SIZE( MPI_COMM_WORLD, nprocs, ierr )

c Note: voltage gradients are maintained because in the conjugate gradient

c relaxation algorithm, the voltage vector is only modified by adding a

c periodic vector to it.

$\mathrm{L} 22=\mathrm{n} \times 2 * \mathrm{ny} 2$ 


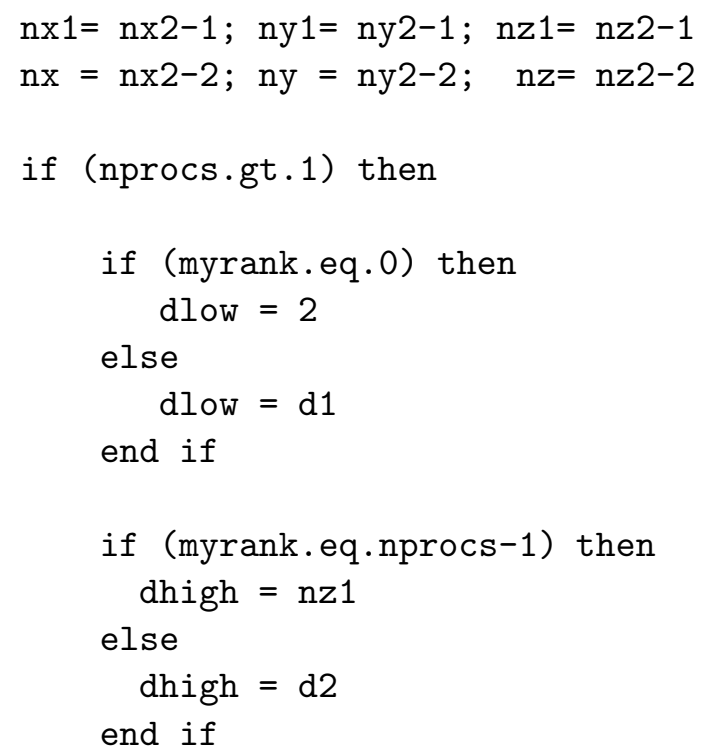

c First stage, compute initial value of gradient (gb), initialize $h$, the

c conjugate gradient direction, and compute norm squared of gradient vector.

call prod_ac (nx2, ny2, nz2, ns2, gx, gy, gz, u, gb, d1, d2)

$\mathrm{h}=\mathrm{gb}$

c Variable gg is the norm squared of the gradient vector

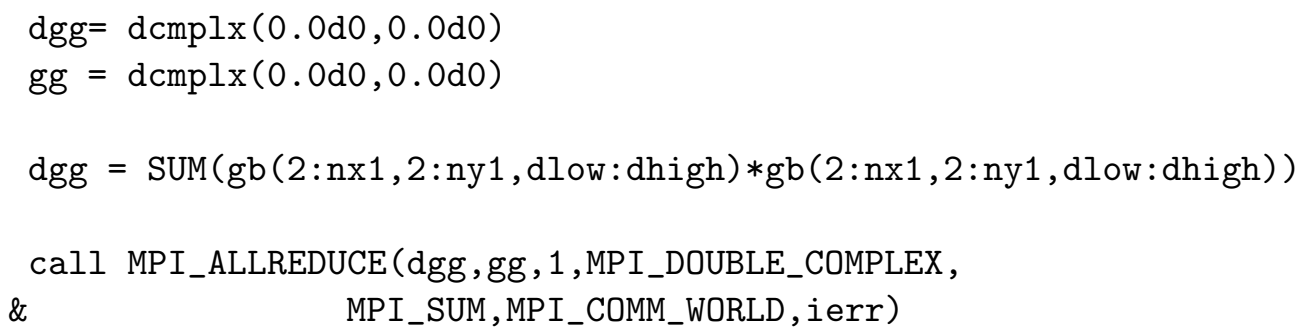

c Second stage, initialize Ah variable, compute parameter lamdba, c make first change in voltage array, update gradient (gb) vector

if (abs (real (gg)).lt.gtest) goto 44

call prod_ac (nx2, ny2, nz2, ns2, gx, gy, gz, h, Ah, d1 , d2) 


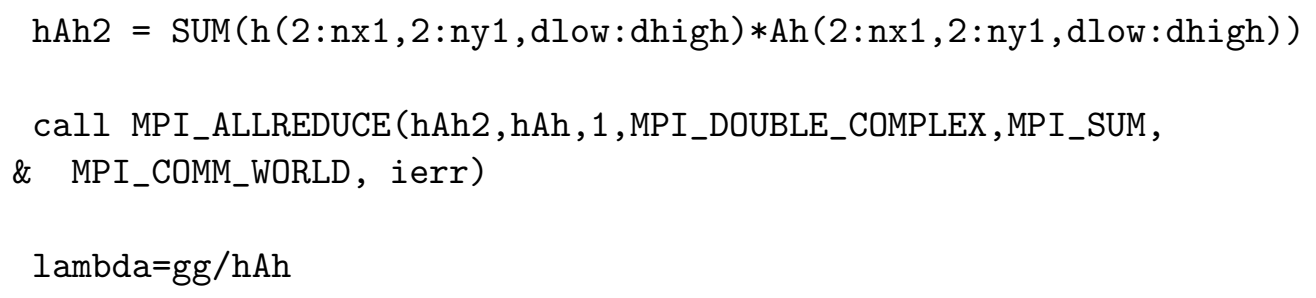

c (USER) This piece of code forces dembx to write out the total current and $c$ the norm of the gradient squared, every ncheck conjugate gradient steps, $c$ in order to see how the relaxation is proceeding. If the currents become c unchanging before the relaxation is done, then gtest was picked to be c smaller than was necessary.

ncheck $=30$

if (ncheck*(icc/ncheck).eq.icc) then

if (myrank.eq.0) then write $(7, *)$ icc write $(7, *)$ ' $g g=$ ',gg end if

c call current subroutine call current_ac (nx2, ny $2, \mathrm{nz} 2, \mathrm{~ns} 2$, cuxxp, cuyyp, cuzzp, \& $\mathrm{u}, \mathrm{gx}, \mathrm{gy}, \mathrm{gz}, \mathrm{d} 1, \mathrm{~d} 2$ )

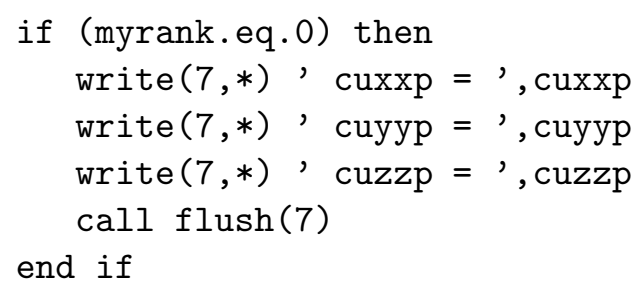


end

$\mathrm{C}$

c $* * * * * * * * * * * * * * * * * * * * * * * * * * * * * * * * * * * * * * * * * * * * * * * * * * * * * * * * * *$

C

subroutine prod_ac (nx2, ny2,nz2, ns2,gx,gy,gz, xw , yw , d1 , d2)

C

c The matrix product subroutine

c

implicit none

include 'mpif.h'

integer $\mathrm{nx} 2, \mathrm{ny} 2, \mathrm{nz} 2, \mathrm{~ns} 2, \mathrm{~d} 1, \mathrm{~d} 2$

integer $\mathrm{nx}, \mathrm{ny}, \mathrm{nz}$

integer $\mathrm{nx} 1, \mathrm{ny} 1, \mathrm{nz} 1$

integer L22

integer dlow, dhigh

integer $\mathrm{im}, \mathrm{jm}, \mathrm{km}, \mathrm{ijk}$

integer ip1,jp1,kp1,im1,jm1,km1

integer ipl,jpl,kpl,iml,jml,kml

integer ipn,jpn,kpn, imn, jmn, kmn

double complex $\mathrm{gx}(\mathrm{nx} 2, \mathrm{ny} 2, \mathrm{~d} 1-1: \mathrm{d} 2+1)$

double complex gy $(\mathrm{nx} 2, \mathrm{ny} 2, \mathrm{~d} 1-1: \mathrm{d} 2+1)$

double complex $g z(n x 2, n y 2, d 1-1: d 2+1)$

double complex $\mathrm{xw}(\mathrm{nx} 2, \mathrm{ny} 2, \mathrm{~d} 1-1: \mathrm{d} 2+1)$

double complex yw $(\mathrm{nx} 2, \mathrm{ny} 2, \mathrm{~d} 1-1: \mathrm{d} 2+1)$

integer myrank, ierr, nprocs

integer status(MPI_STATUS_SIZE)

integer lowrank, highrank

call MPI_COMM_RANK ( MPI_COMM_WORLD, myrank, ierr )

call MPI_COMM_SIZE ( MPI_COMM_WORLD, nprocs, ierr )

lowrank=0; highrank=0

c $\mathrm{xw}$ is the input vector, $\mathrm{yw}=(\mathrm{A})(\mathrm{xw})$ is the output vector

c auxiliary variables involving the system size

$\mathrm{nx} 1=\mathrm{nx} 2-1$

ny1=ny2-1

$\mathrm{nz} 1=\mathrm{nz} 2-1$

$\mathrm{nx}=\mathrm{nx} 2-2$

ny $=$ ny $2-2$

$\mathrm{nz}=\mathrm{nz} 2-2$

$\mathrm{L} 22=\mathrm{n} \times 2 * \mathrm{ny} 2$ 
if (nprocs.gt.1) then

if (myrank.eq.0) then

dlow $=2$

else

dlow $=d 1$

end if

if (myrank.eq.nprocs-1) then

dhigh $=n z 1$

else

dhigh $=\mathrm{d} 2$

end if

end if

if (nprocs.eq.1) then

dlow $=2$

dhigh=nz1

end if

c Perform basic matrix multiplication, results in incorrect information at c periodic boundaries.

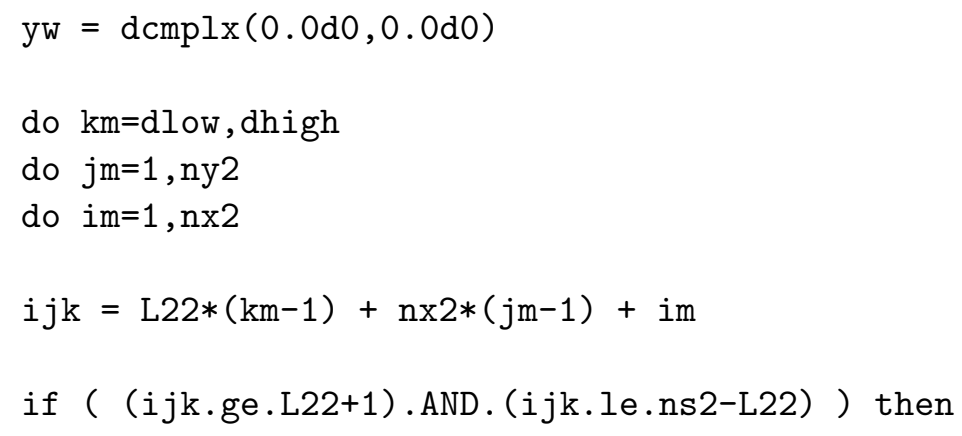

$\mathrm{C}$

c Calculation 1

$\mathrm{C}$

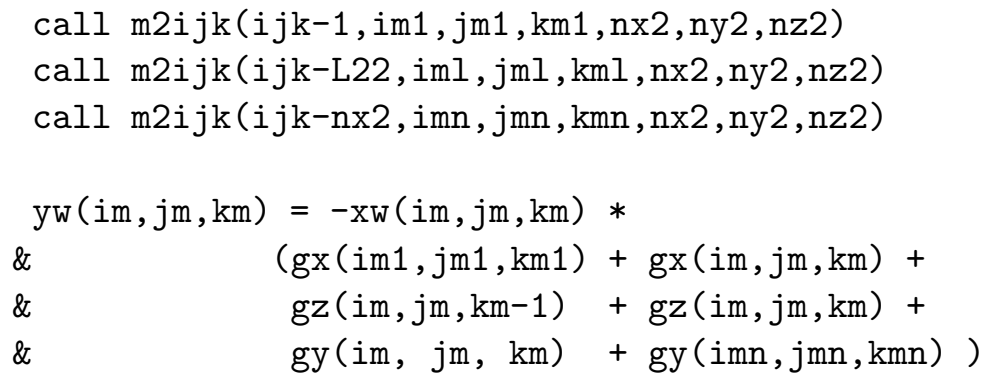

C

c Calculation 2 


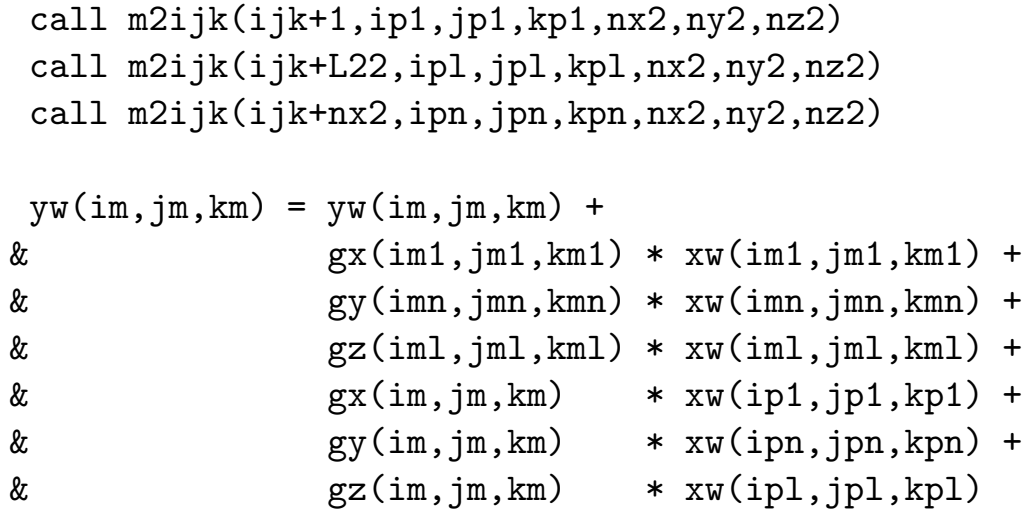

end if

end do; end do; end do

C

c Calculation 3

$\mathrm{C}$

C Correct terms at periodic boundaries

C

C $\mathrm{x}$ faces

$\mathrm{yw}(\mathrm{nx} 2,:,:)=\mathrm{yw}(2,:,:)$

$\mathrm{yw}(1,:,:) \quad=\mathrm{yw}(\mathrm{nx} 1,:,:)$

c $\mathrm{y}$ faces

$\mathrm{yw}(:, 1,:)=\mathrm{yw}(:, \mathrm{ny} 1,:)$

$\mathrm{yw}(:, \mathrm{ny} 2,:) \quad=\mathrm{yw}(:, 2,:)$

c $\quad$ f faces

if (nprocs.eq.1) then

$\mathrm{yw}(:,:, 1) \quad=\mathrm{yw}(:,:, \mathrm{nz} 1)$

$\mathrm{yw}(:,:, \mathrm{nz} 2)=\mathrm{yw}(:,:, 2)$

end if

if (nprocs.gt.1) then

if ( (d1.le.2).AND. (2.le.d2)) then lowrank $=$ myrank

cal1 MPI_SEND (yw ( : , : , 2), L22,MPI_DOUBLE_COMPLEX, nprocs-1, 99, $\&$ MPI_COMM_WORLD, ierr)

end if 


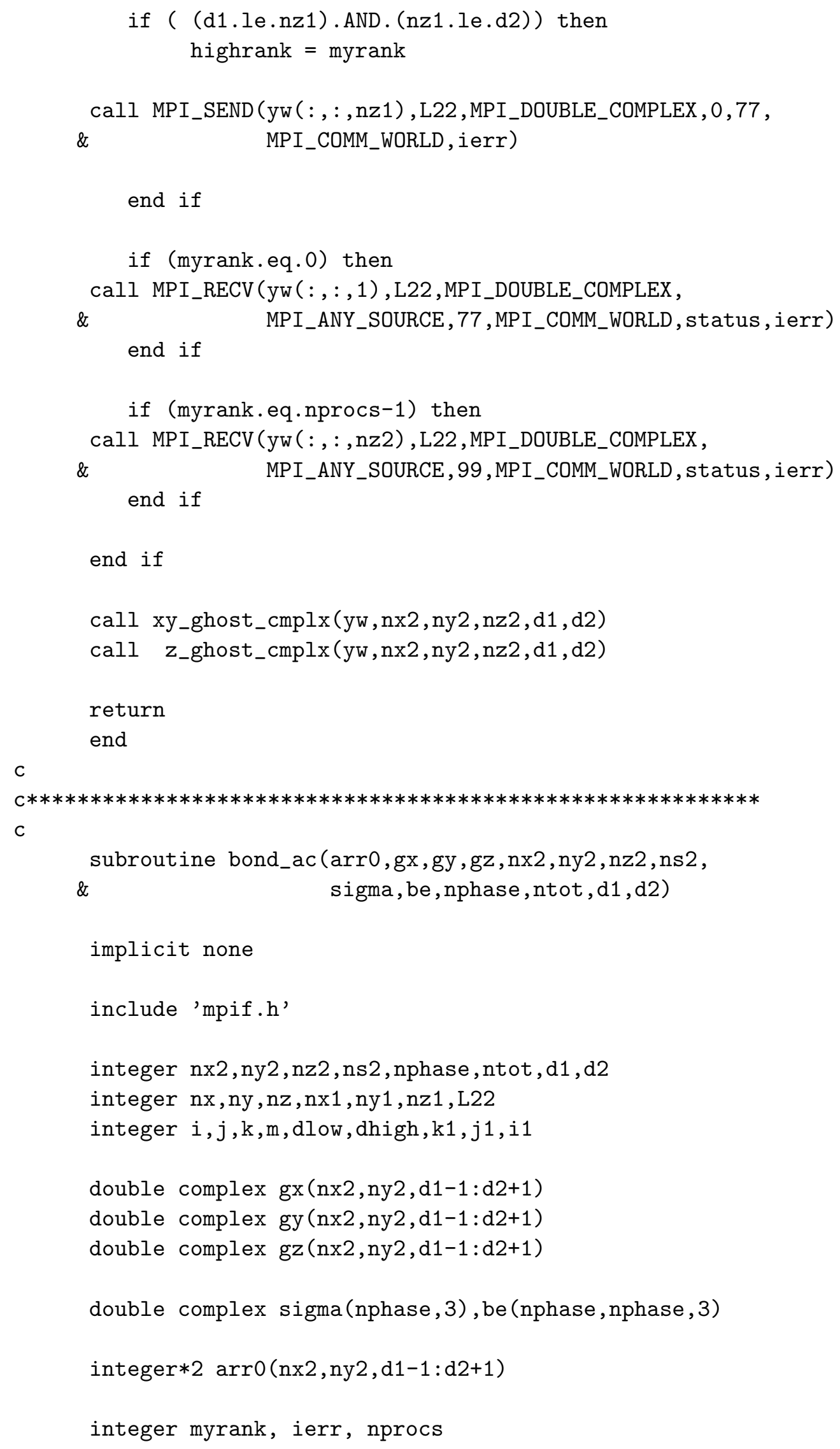


integer status(MPI_STATUS_SIZE)

call MPI_COMM_RANK ( MPI_COMM_WORLD, myrank, ierr )

call MPI_COMM_SIZE( MPI_COMM_WORLD, nprocs, ierr )

c auxiliary variables involving the system size

$$
\begin{aligned}
& \mathrm{nx}=\mathrm{nx} 2-2 \\
& \mathrm{ny}=\mathrm{ny} 2-2 \\
& \mathrm{nz}=\mathrm{nz} 2-2 \\
& \mathrm{nx} 1=\mathrm{nx} 2-1 \\
& \mathrm{ny} 1=\mathrm{ny} 2-1 \\
& \mathrm{nz} 1=\mathrm{nz} 2-1 \\
& \mathrm{~L} 22=\mathrm{nx} 2 * \mathrm{ny} 2
\end{aligned}
$$

c Set values of conductor for phase $(i, m)--p h a s e(j, m)$ interface,

$c$ store in array be( $i, j, m), m=1,2,3$. If either phase $i$ or $j$

$c$ has zero conductivity in the $m^{\prime}$ th direction, then be $(i, j, m)=0.0$.

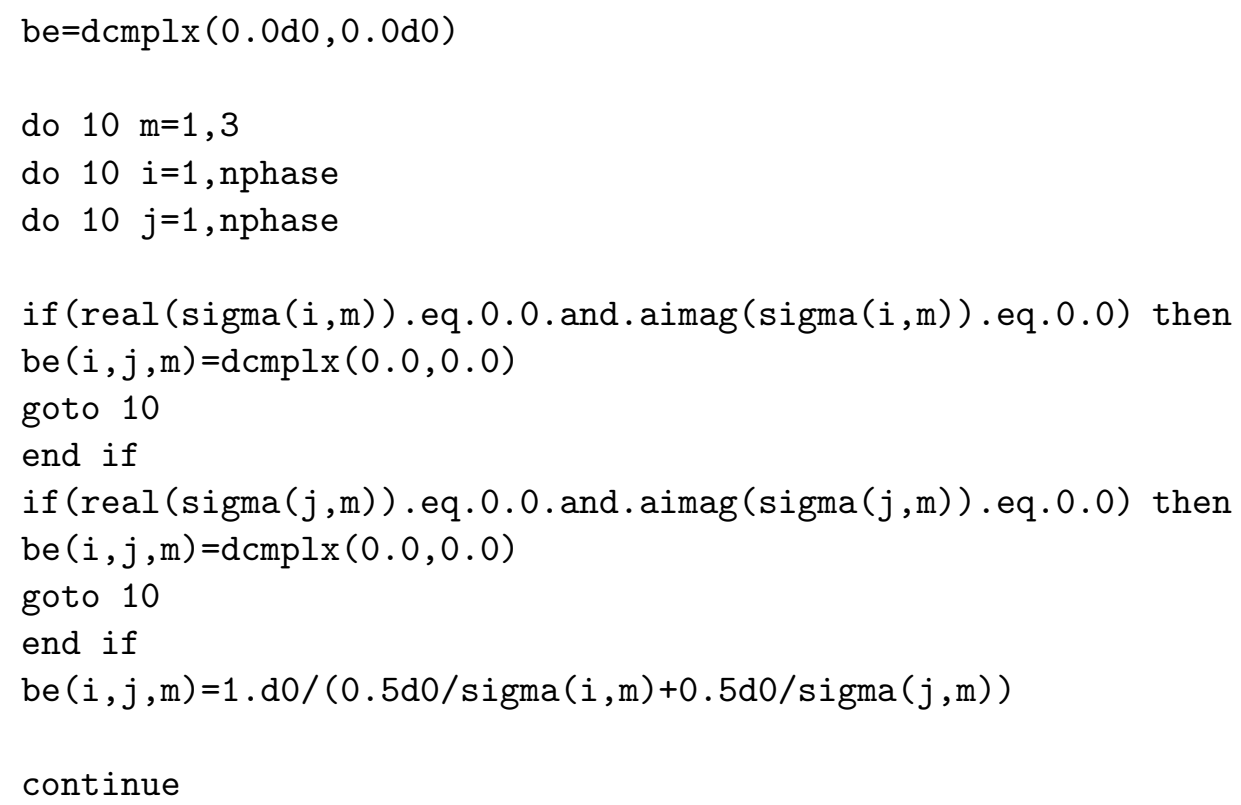

c Trim off $x$ and $y$ faces so that no current can flow past periodic

c boundaries. This step is not double precisionly necessary, as the

c voltages on the periodic boundaries will be matched to the

c corresponding double precision voltages in each conjugate gradient step.

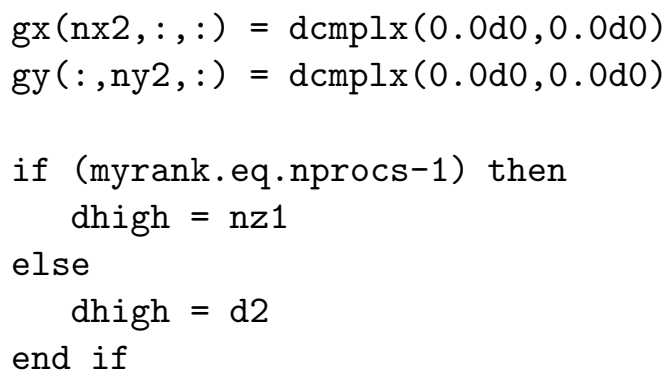




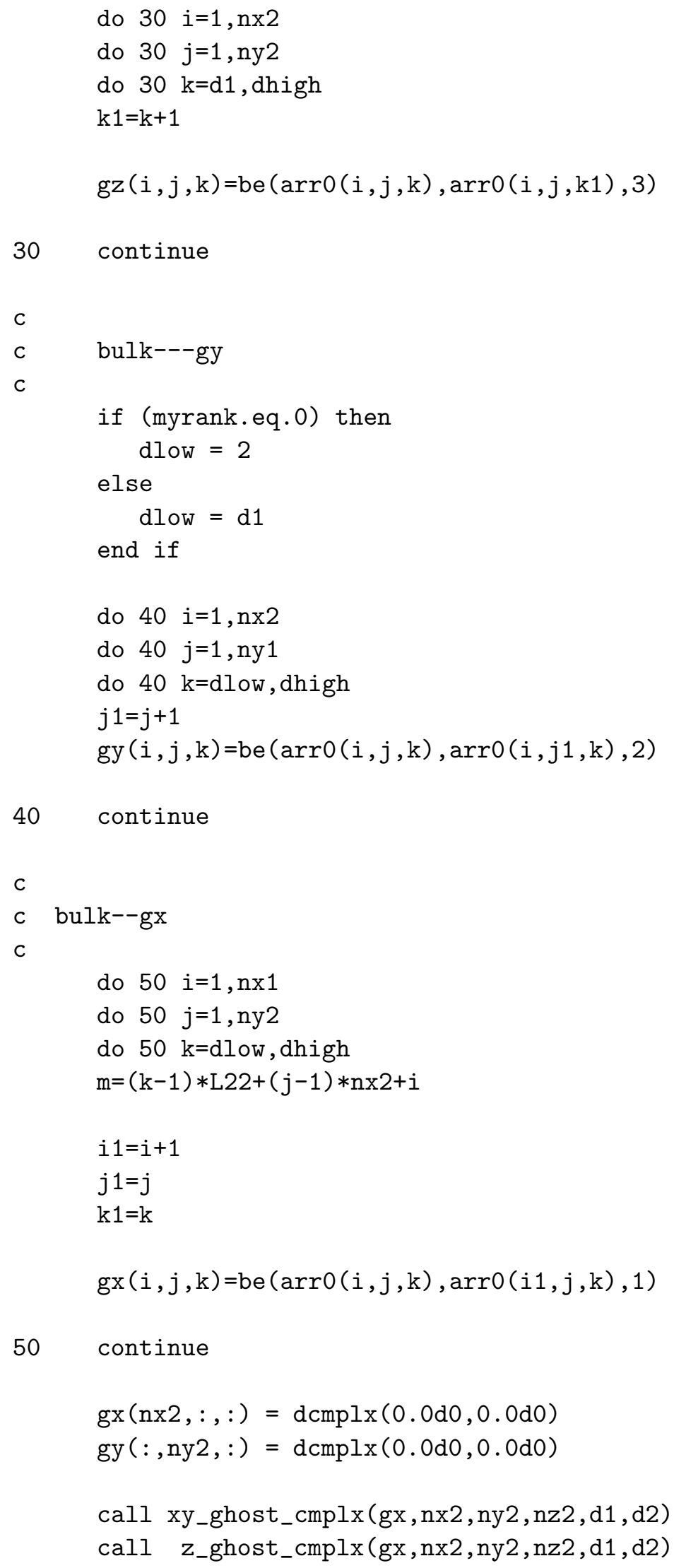




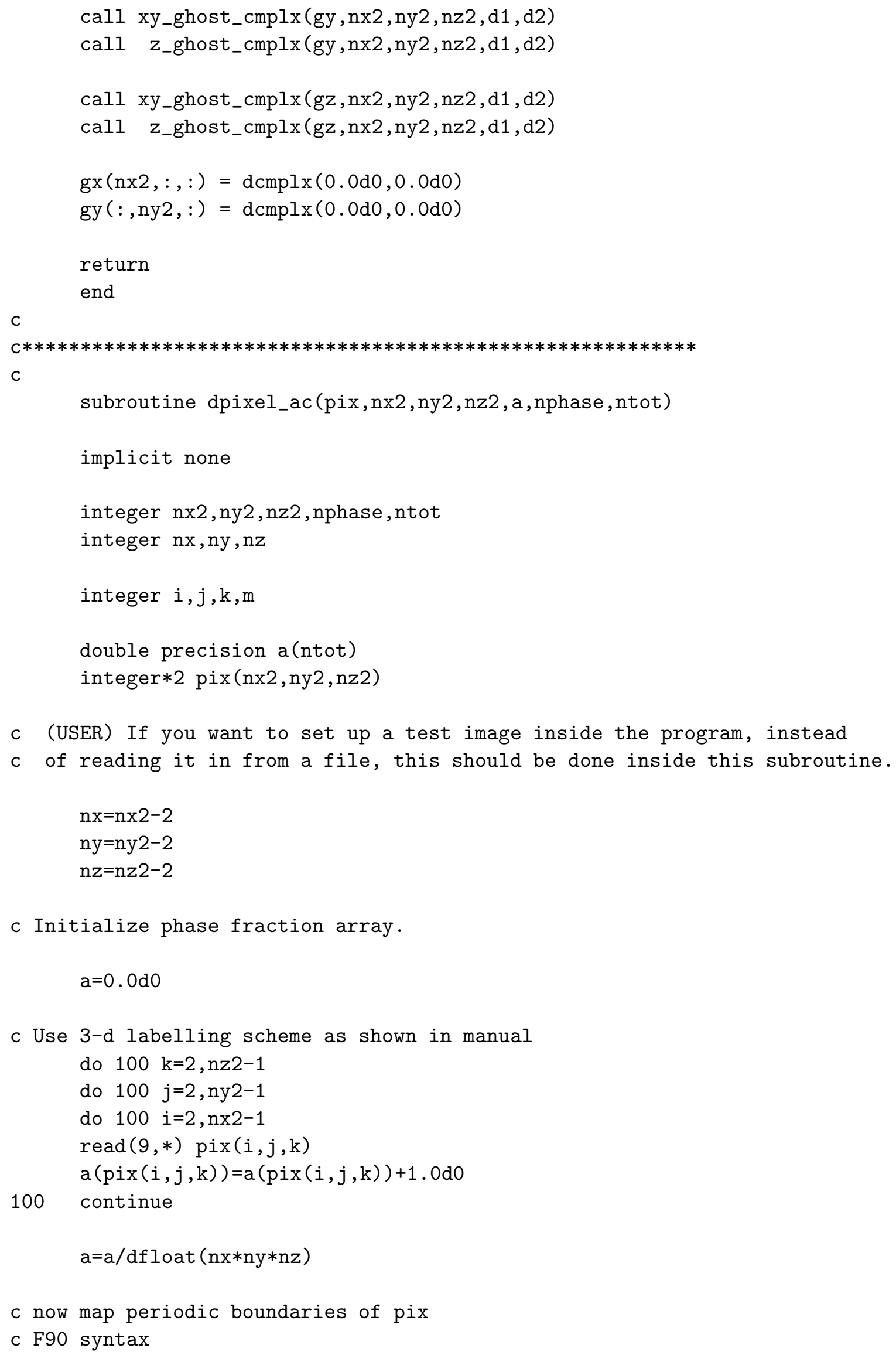

c Initialize phase fraction array.

$\mathrm{a}=0.0 \mathrm{~d} 0$

c Use 3-d labelling scheme as shown in manual

do $100 \mathrm{k}=2, \mathrm{nz2}-1$

do $100 \mathrm{j}=2$, ny2-1

do $100 i=2, n \times 2-1$

$\operatorname{read}(9, *) \operatorname{pix}(\mathrm{i}, \mathrm{j}, \mathrm{k})$

$\mathrm{a}(\operatorname{pix}(\mathrm{i}, \mathrm{j}, \mathrm{k}))=\mathrm{a}(\operatorname{pix}(\mathrm{i}, \mathrm{j}, \mathrm{k}))+1.0 \mathrm{~d} 0$

100 continue

$\mathrm{a}=\mathrm{a} / \mathrm{dfl}$ loat $(\mathrm{nx} * n y * n z)$

c now map periodic boundaries of pix

c F90 syntax 


$$
\begin{aligned}
& \operatorname{pix}(n \times 2,:,:)=\operatorname{pix}(2,:,:) \\
& \operatorname{pix}(1,:,:) \quad=\operatorname{pix}(n \times 2-1,:,:) \\
& \operatorname{pix}(:, \operatorname{ny} 2,:)=\operatorname{pix}(:, 2,:) \\
& \operatorname{pix}(:, 1,:) \quad=\operatorname{pix}(:, \text { ny2-1,: }) \\
& \operatorname{pix}(:,:, 1)=\operatorname{pix}(:,:, n z 2-1) \\
& \operatorname{pix}(:,:, \mathrm{nz} 2)=\operatorname{pix}(:,:, 2)
\end{aligned}
$$

c Check for wrong phase labels--less than 1 or greater than nphase

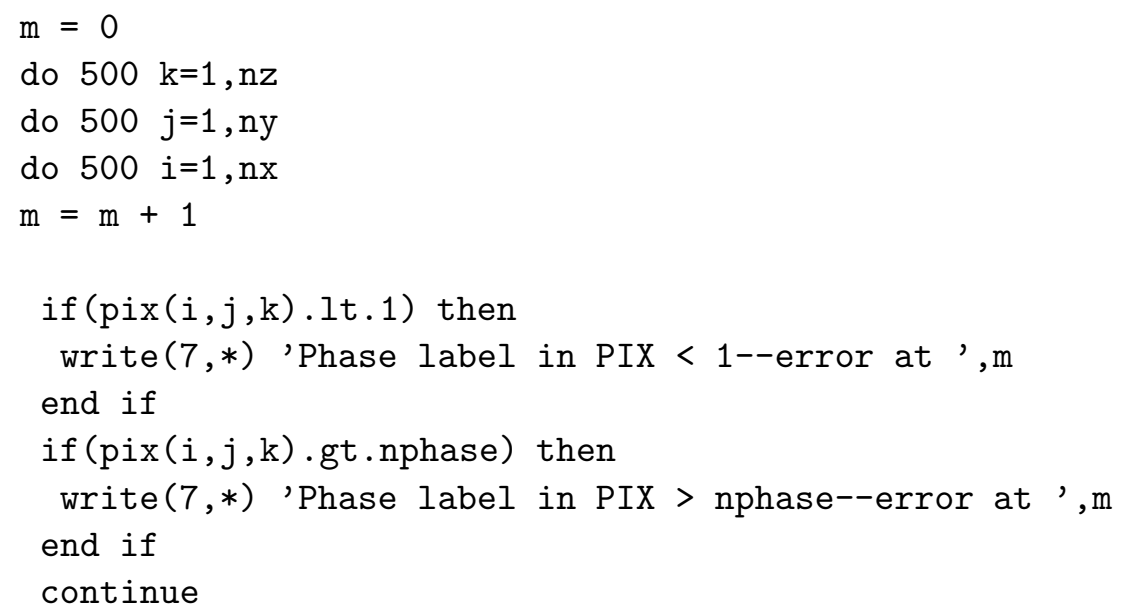

500 continue

return

end

C

C*************************************************************

C

subroutine current_ac(nx2, ny2,nz2, ns2, cuxxp, cuyyp, cuzzp, \&

$\mathrm{u}, \mathrm{gx}, \mathrm{gy}, \mathrm{gz}, \mathrm{d} 1, \mathrm{~d} 2$ )

implicit none

include 'mpif.h'

integer nx2,ny2,nz2,ns2,nx,ny,nz,d1,d2,i,j,k,klow,khigh,m

integer $\mathrm{i} 0, \mathrm{j} 0, \mathrm{k} 0, \mathrm{~L} 22$

integer im1,jm1, km1

integer ip1,jp1,kp1

integer iml,jml, $\mathrm{kml}$

integer ipl,jpl,kpl

integer imn, jmn, $\mathrm{kmn}$

integer ipn,jpn,kpn 


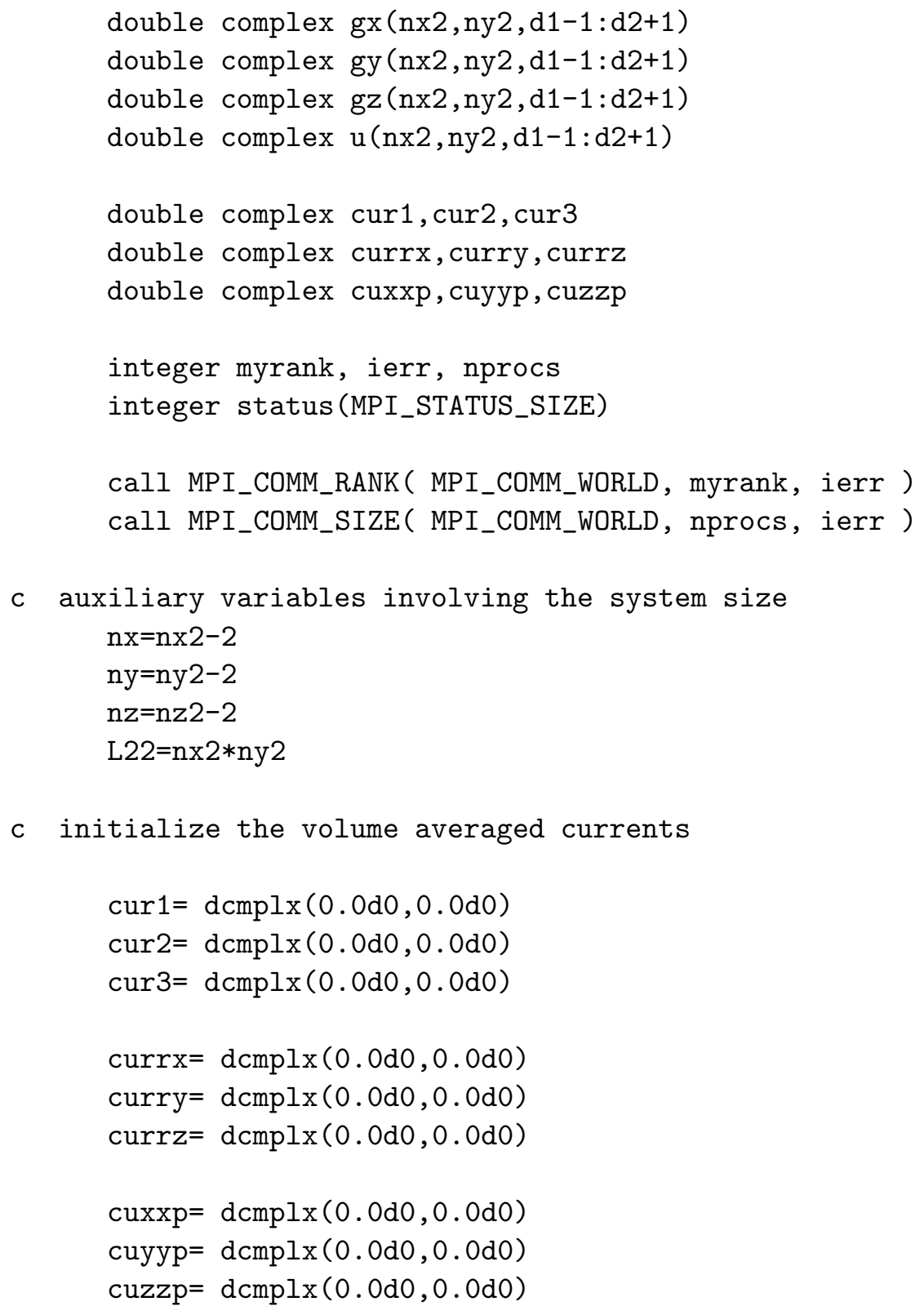

c initialize the volume averaged currents

$$
\begin{aligned}
& \operatorname{cur} 1=\operatorname{dcmplx}(0.0 \mathrm{~d} 0,0.0 \mathrm{~d} 0) \\
& \operatorname{cur} 2=\operatorname{dcmplx}(0.0 \mathrm{~d} 0,0.0 \mathrm{~d} 0) \\
& \operatorname{cur} 3=\operatorname{dcmplx}(0.0 \mathrm{~d} 0,0.0 \mathrm{~d} 0) \\
& \text { currx }=\operatorname{dcmplx}(0.0 \mathrm{~d} 0,0.0 \mathrm{~d} 0) \\
& \text { curry }=\operatorname{dcmplx}(0.0 \mathrm{~d} 0,0.0 \mathrm{~d} 0) \\
& \text { currz }=\operatorname{dcmplx}(0.0 \mathrm{~d} 0,0.0 \mathrm{~d} 0) \\
& \operatorname{cuxxp}=\operatorname{dcmplx}(0.0 \mathrm{~d} 0,0.0 \mathrm{~d} 0) \\
& \operatorname{cuyyp}=\operatorname{dcmplx}(0.0 \mathrm{~d} 0,0.0 \mathrm{~d} 0) \\
& \operatorname{cuzzp}=\operatorname{dcmplx}(0.0 \mathrm{~d} 0,0.0 \mathrm{~d} 0)
\end{aligned}
$$

c Only loop over real sites and bonds in order to get true total current

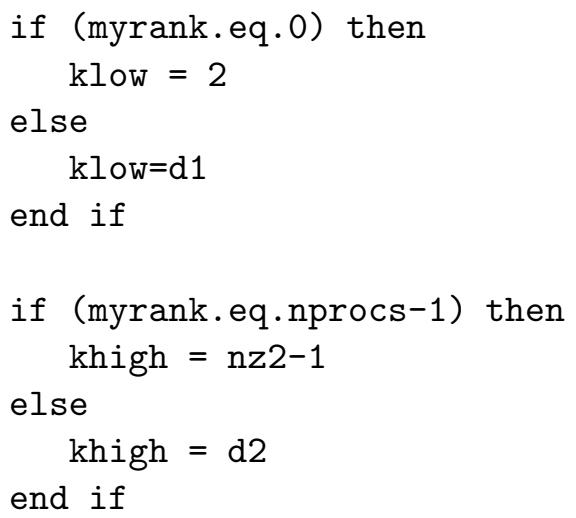




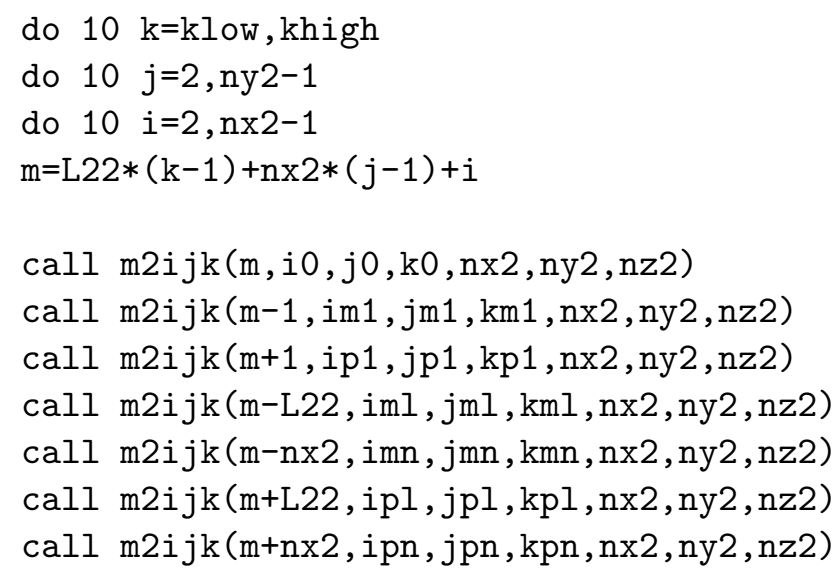

c cur1, cur2, cur3 are the currents in one pixel

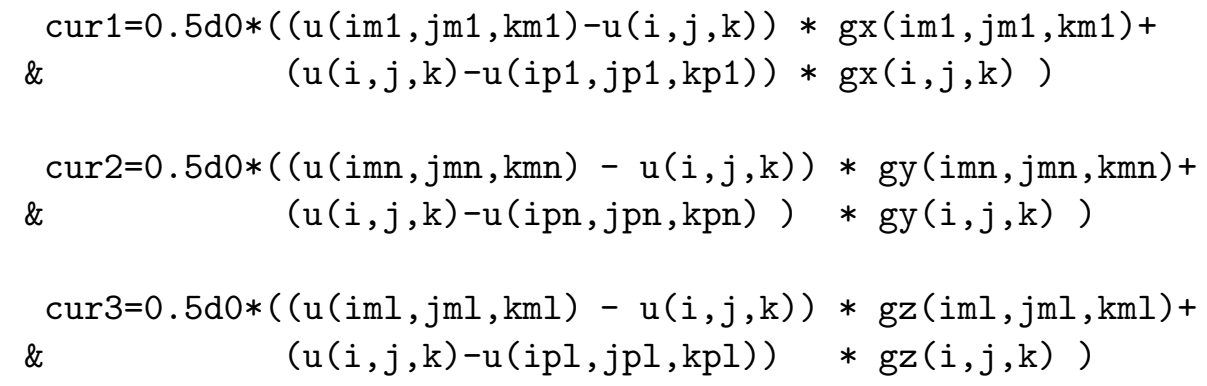

c Volume average currents $\operatorname{cuxxp}=\operatorname{cuxxp} /$ df loat $(\mathrm{nx} * \mathrm{ny} * \mathrm{nz})$ cuyyp $=$ cuyyp $/$ dfloat $(n x * n y * n z)$ cuzzp $=$ cuzzp $/$ dfloat $(n x * n y * n z)$

return

end

$\mathrm{C}$

c $* * * * * * * * * * * * * * * * * * * * * * * * * * * * * * * * * * * * * * * * * * * * * * * * * * * * * * * * * *$

C 


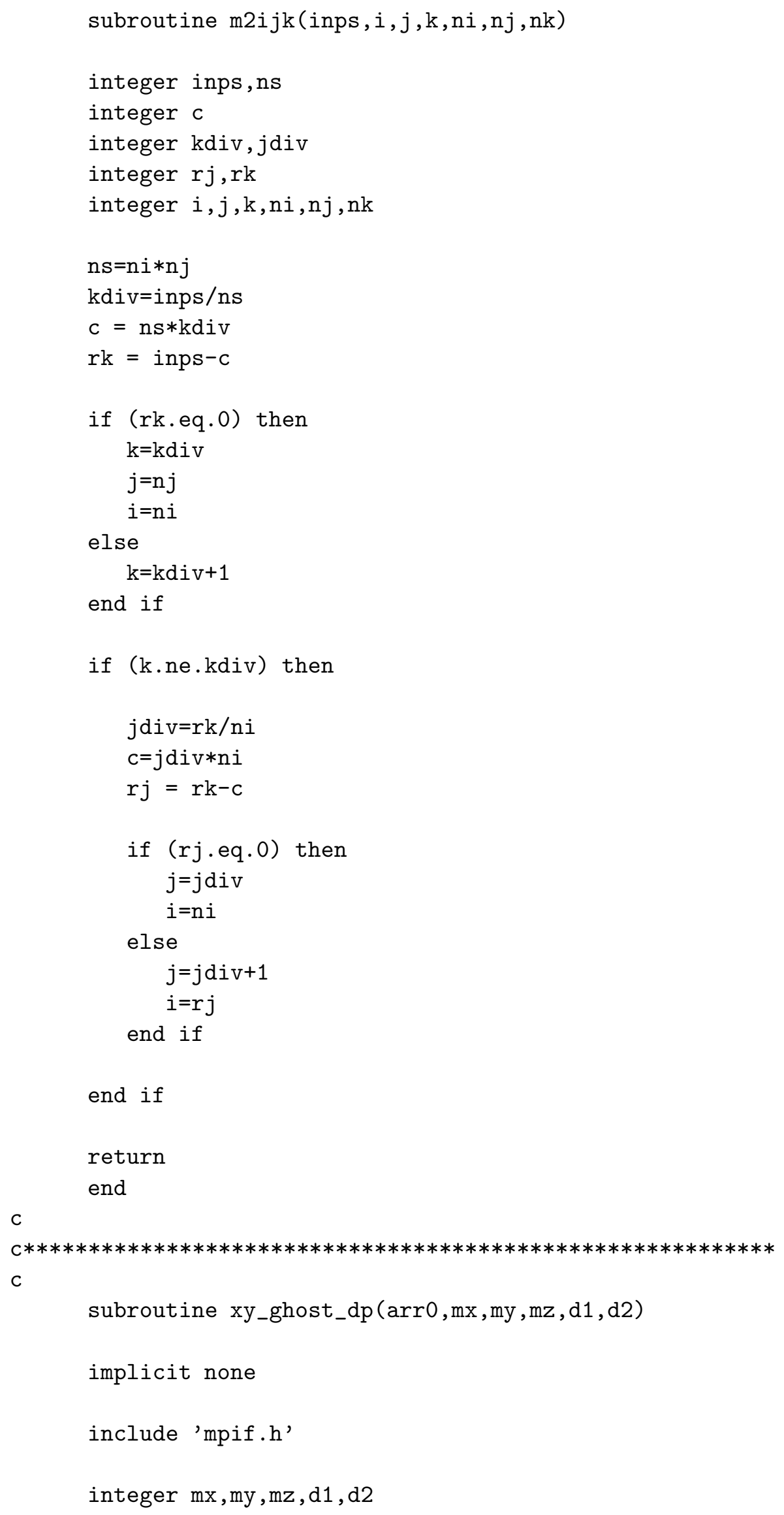




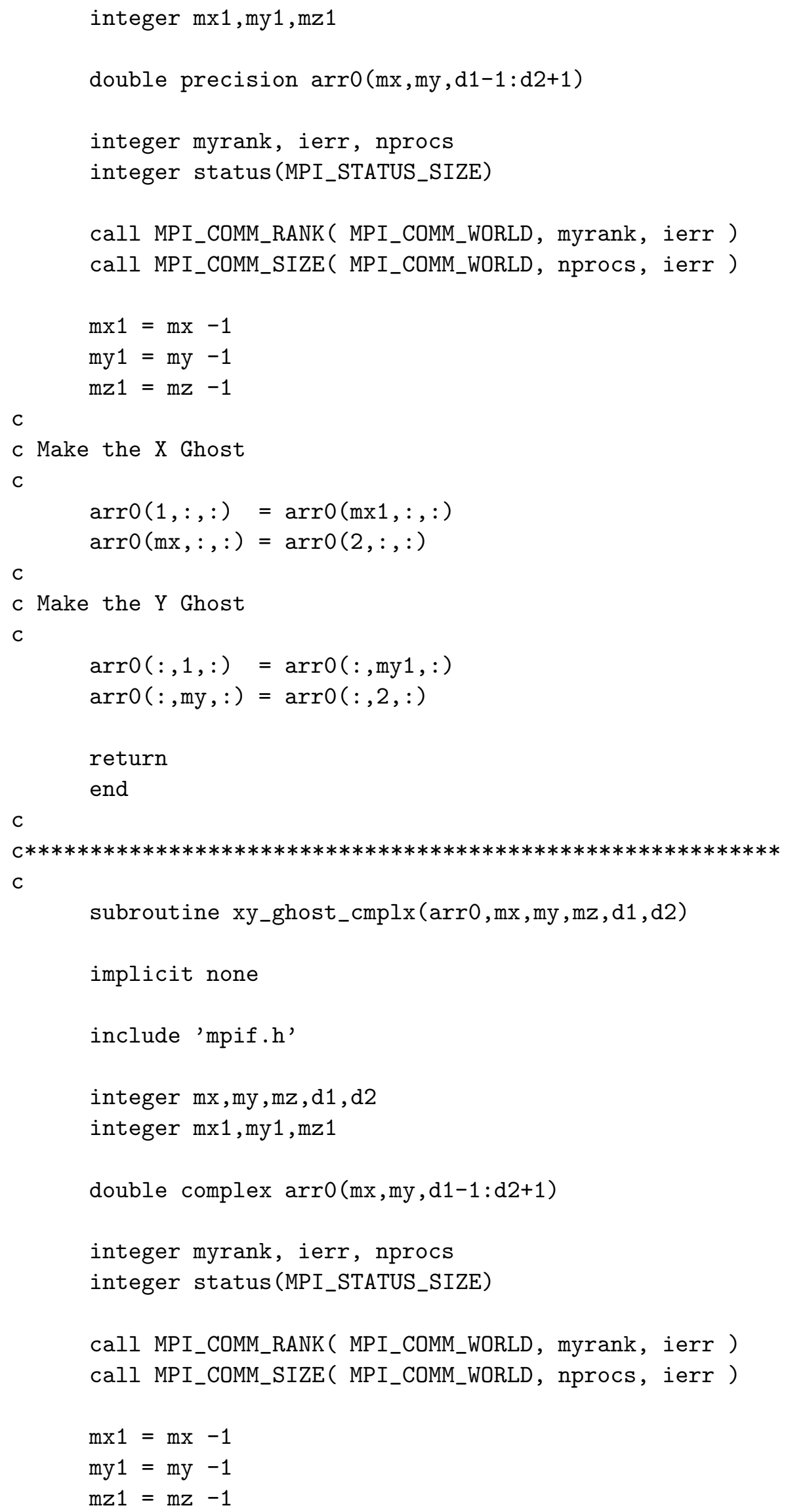


C

c Make the X Ghost

$c$ $\operatorname{arr} 0(1,:,:)=\operatorname{arr0}(m x 1,:,:)$

$\operatorname{arr} 0(m x,:,:)=\operatorname{arr} 0(2,:,:)$

C

c Make the Y Ghost

C

$\operatorname{arr0}(:, 1,:)=\operatorname{arr0}(:, m y 1,:)$

$\operatorname{arr} 0(:, m y,:)=\operatorname{arr} 0(:, 2,:)$

return

end

$\mathrm{C}$

C $* * * * * * * * * * * * * * * * * * * * * * * * * * * * * * * * * * * * * * * * * * * * * * * * * * * * * * * * * * * * *$

$\mathrm{C}$

subroutine z_ghost_dp (arr0,mx,my,mz,d1,d2)

implicit none

include 'mpif.h'

integer $\mathrm{mx}, \mathrm{my}, \mathrm{mz}, \mathrm{d} 1, \mathrm{~d} 2$

double precision $\operatorname{arr} 0(\mathrm{mx}, \mathrm{my}, \mathrm{d} 1-1: \mathrm{d} 2+1)$

double precision, allocatable : $\operatorname{bot}(:,:), \operatorname{top}(:,:)$

integer myrank, ierr, nprocs

integer status(MPI_STATUS_SIZE)

call MPI_COMM_RANK( MPI_COMM_WORLD, myrank, ierr )

call MPI_COMM_SIZE( MPI_COMM_WORLD, nprocs, ierr )

C

c Make the Z Ghost

C

allocate (bot $(m x, m y))$

allocate (top (mx,my))

C

c Get new bottom ghost plane.

c

$$
\begin{aligned}
& \text { bot }=\operatorname{arr} 0(:,:, d 1) \\
& \text { top }=\operatorname{arr} 0(:,:, d 2) \\
& \text { call t2b_dp }(\text { bot, top,mx,my }) \\
& \operatorname{arr} 0(:,:, d 1-1)=\text { bot }
\end{aligned}
$$

C

c Get new top ghost plane

C

$$
\text { bot }=\operatorname{arr} 0(:,:, d 1)
$$




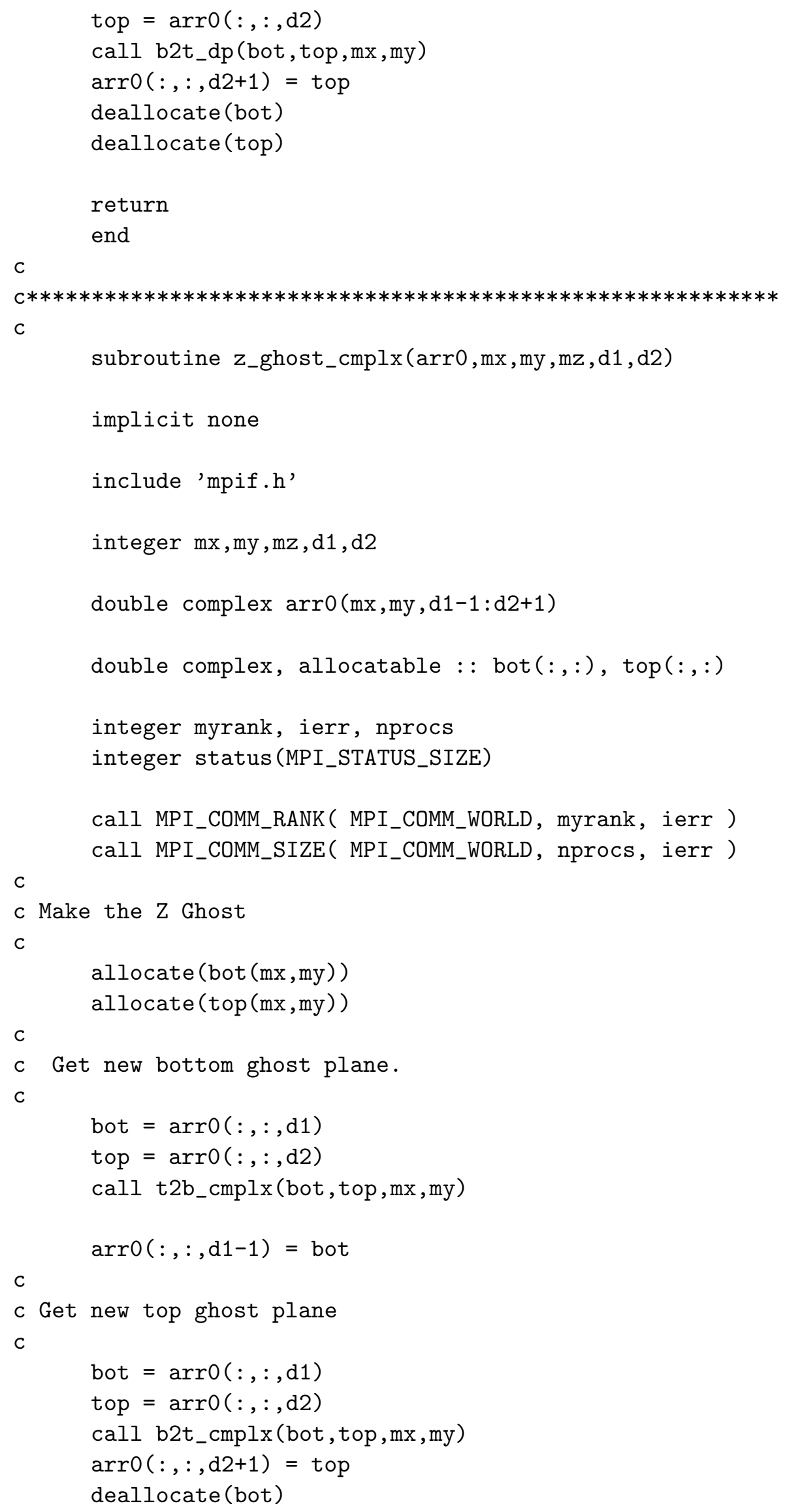




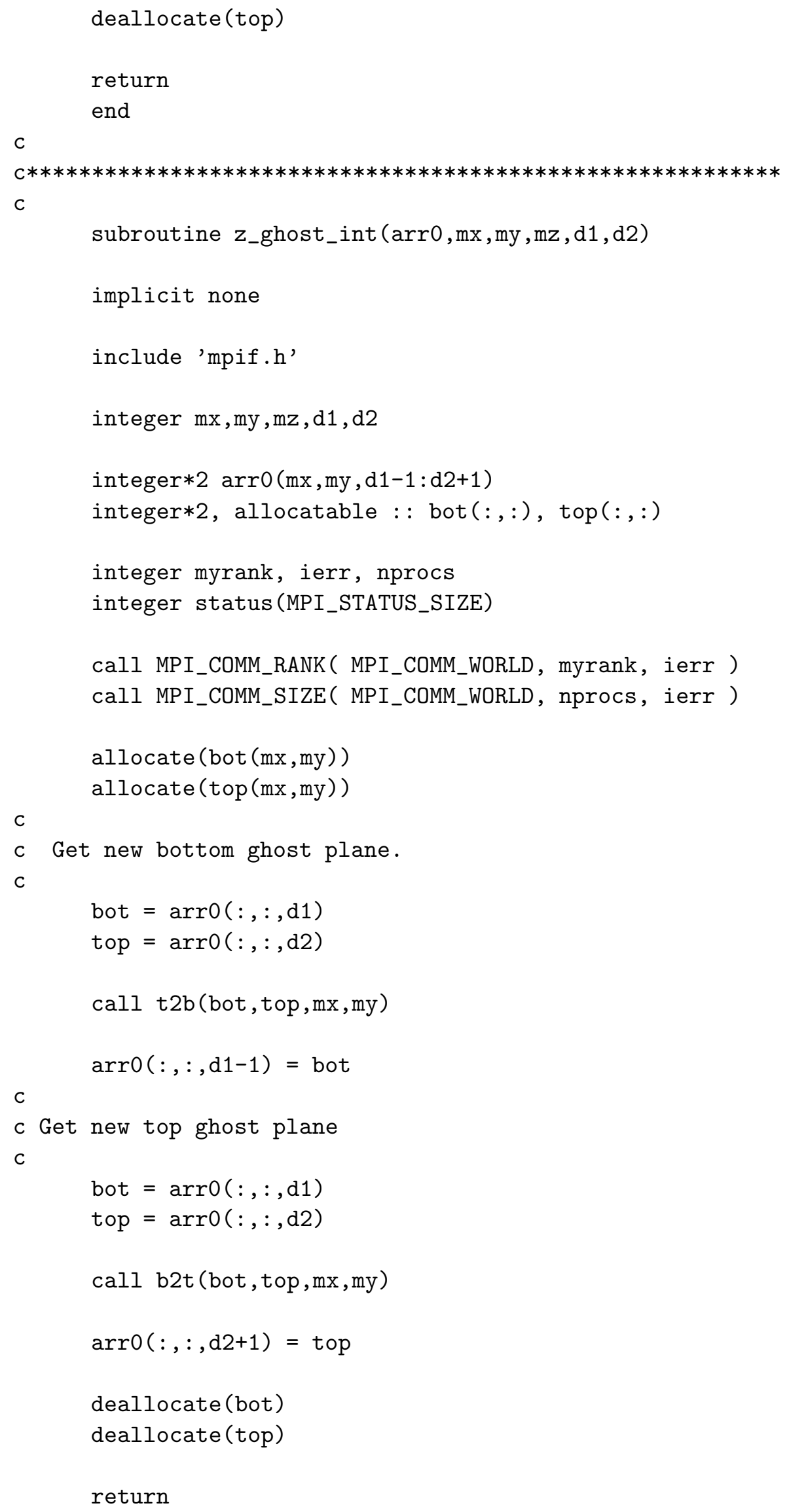


end

C

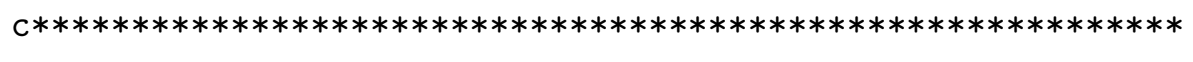

C

subroutine t2b(b_layer,t_layer, nx, ny)

C

c This is an INTEGER $* 2$ subroutine.

C

c Used for transferring: pix bottom2top layers

C

c RECV a new t_layer (TOP layer) per node.

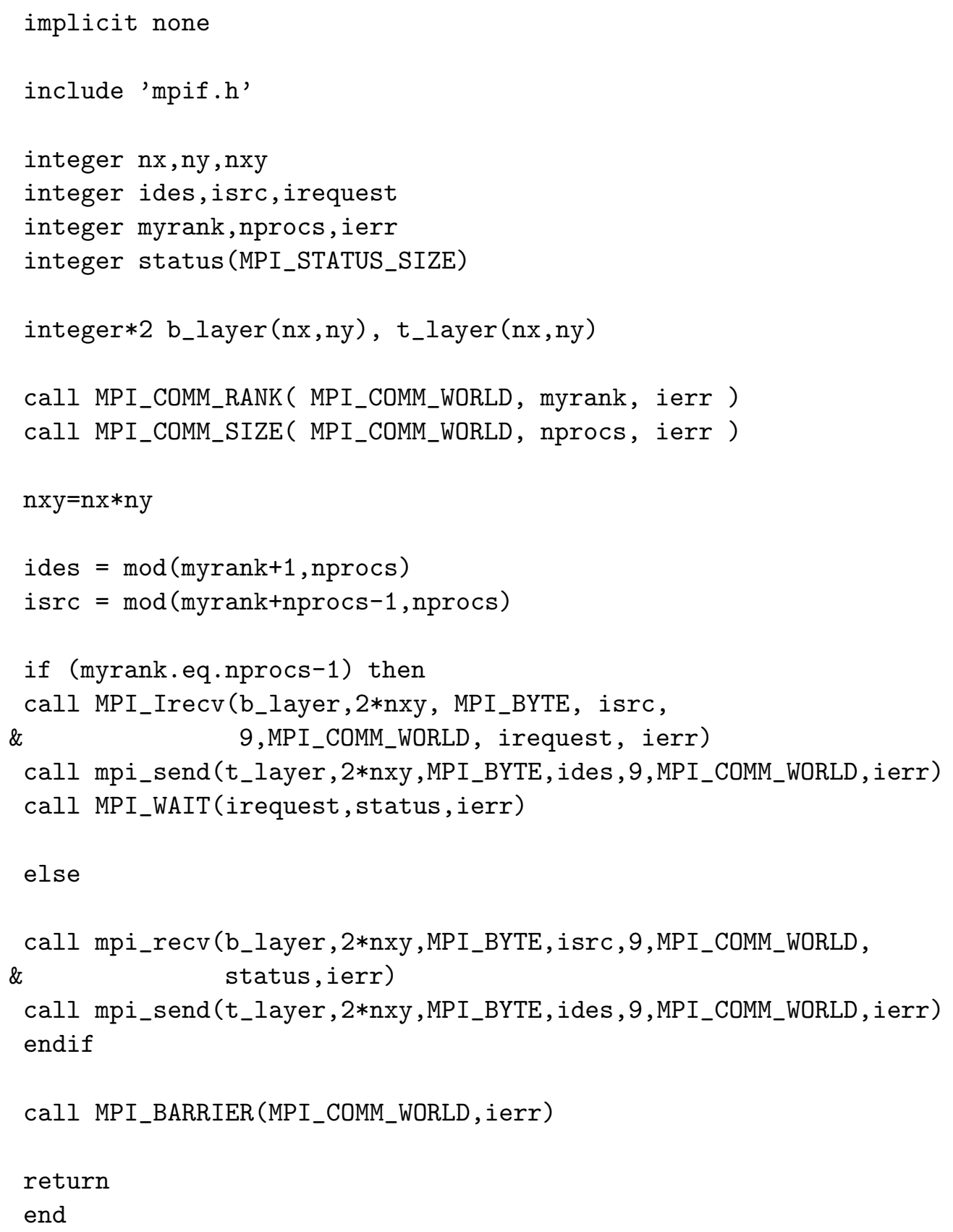




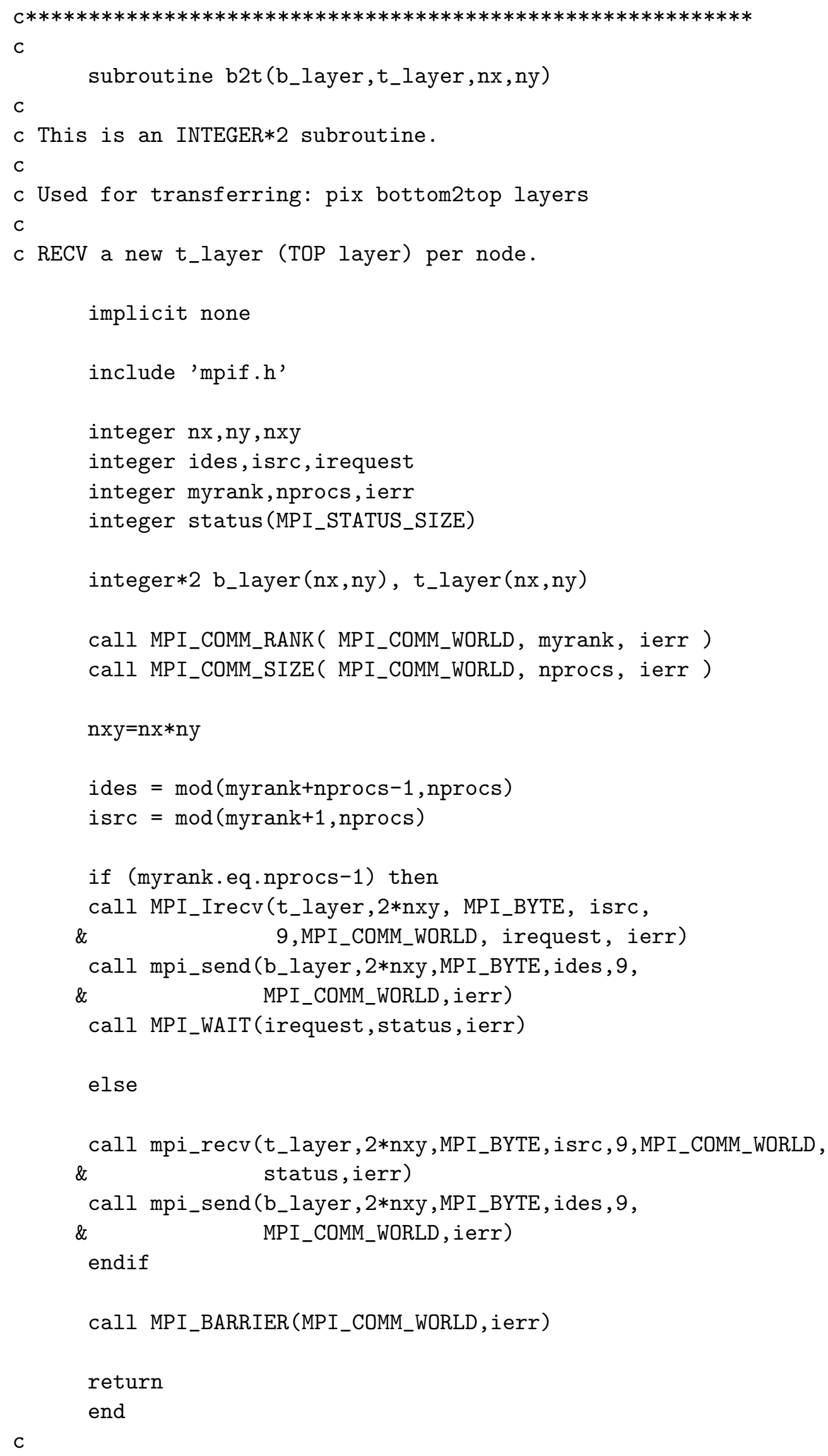




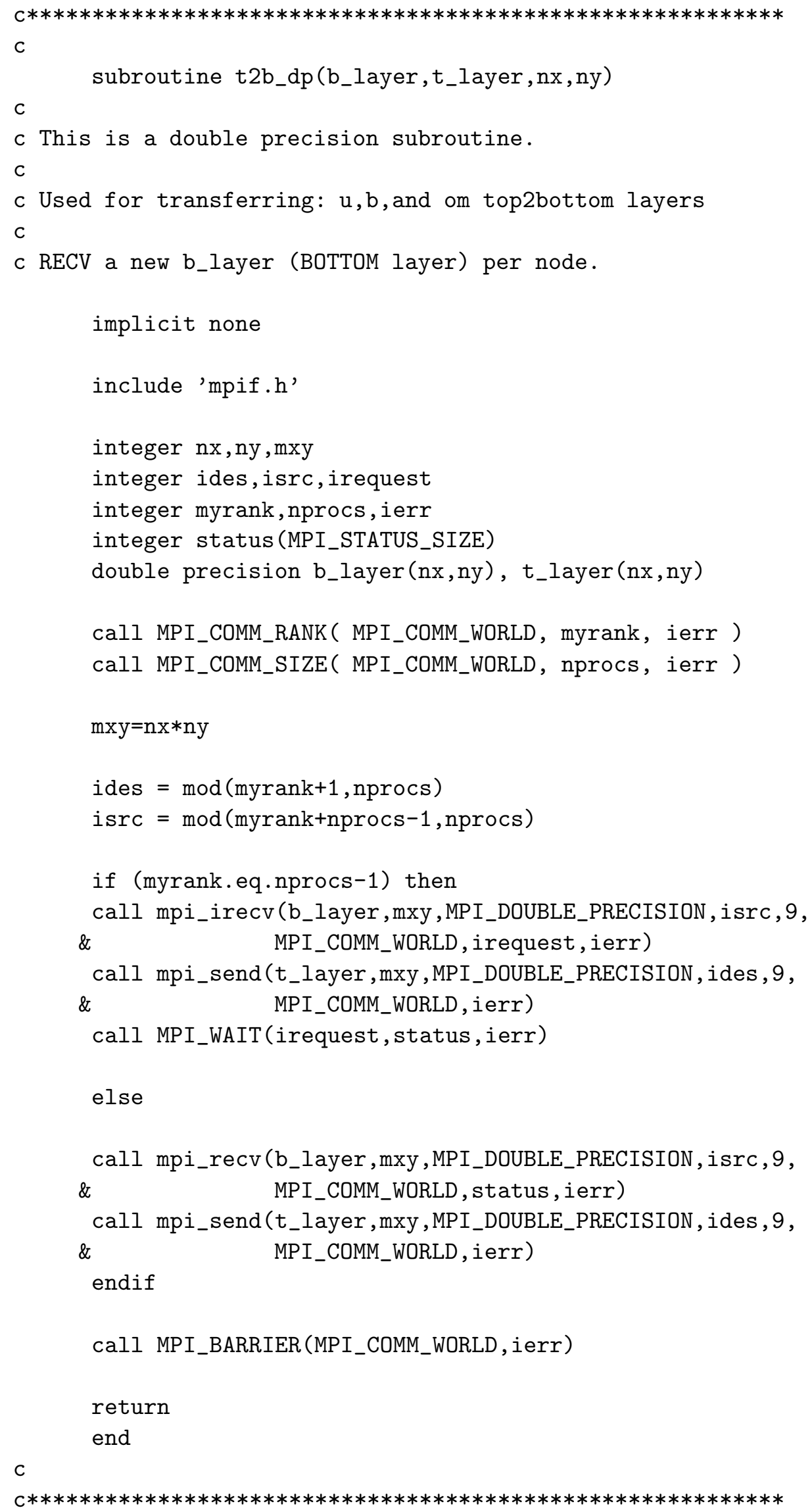


C

subroutine b2t_dp(b_layer,t_layer, nx, ny)

C

c This is a double precision subroutine.

C

c Used for transferring: $u, b$, and om bottom2top layers

$C$

c RECV a new t_layer (TOP layer) per node.

implicit none

include 'mpif.h'

integer $\mathrm{nx}, \mathrm{ny}, \mathrm{mxy}$

integer ides, isrc, irequest

integer myrank, nprocs, ierr

integer status(MPI_STATUS_SIZE)

double precision b_layer(nx,ny), t_layer (nx,ny)

call MPI_COMM_RANK( MPI_COMM_WORLD, myrank, ierr )

call MPI_COMM_SIZE( MPI_COMM_WORLD, nprocs, ierr )

$m x y=n x * n y$

ides $=\bmod ($ myrank + nprocs -1, nprocs $)$

isrc $=\bmod (\operatorname{myrank}+1, \mathrm{nprocs})$

if (myrank.eq.nprocs-1) then

call mpi_Irecv(t_layer, mxy,MPI_DOUBLE_PRECISION, isrc, 9 ,

\& MPI_COMM_WORLD, irequest, ierr)

call mpi_send (b_layer, mxy, MPI_DOUBLE_PRECISION, ides, 9 ,

\& MPI_COMM_WORLD, ierr)

call MPI_WAIT (irequest, status, ierr)

else

call mpi_recv(t_layer,mxy,MPI_DOUBLE_PRECISION, isrc, 9 ,

\& MPI_COMM_WORLD, status, ierr)

call mpi_send (b_layer,mxy,MPI_DOUBLE_PRECISION, ides, 9 ,

\& MPI_COMM_WORLD, ierr)

endif

call MPI_BARRIER(MPI_COMM_WORLD, ierr)

return

end

c 


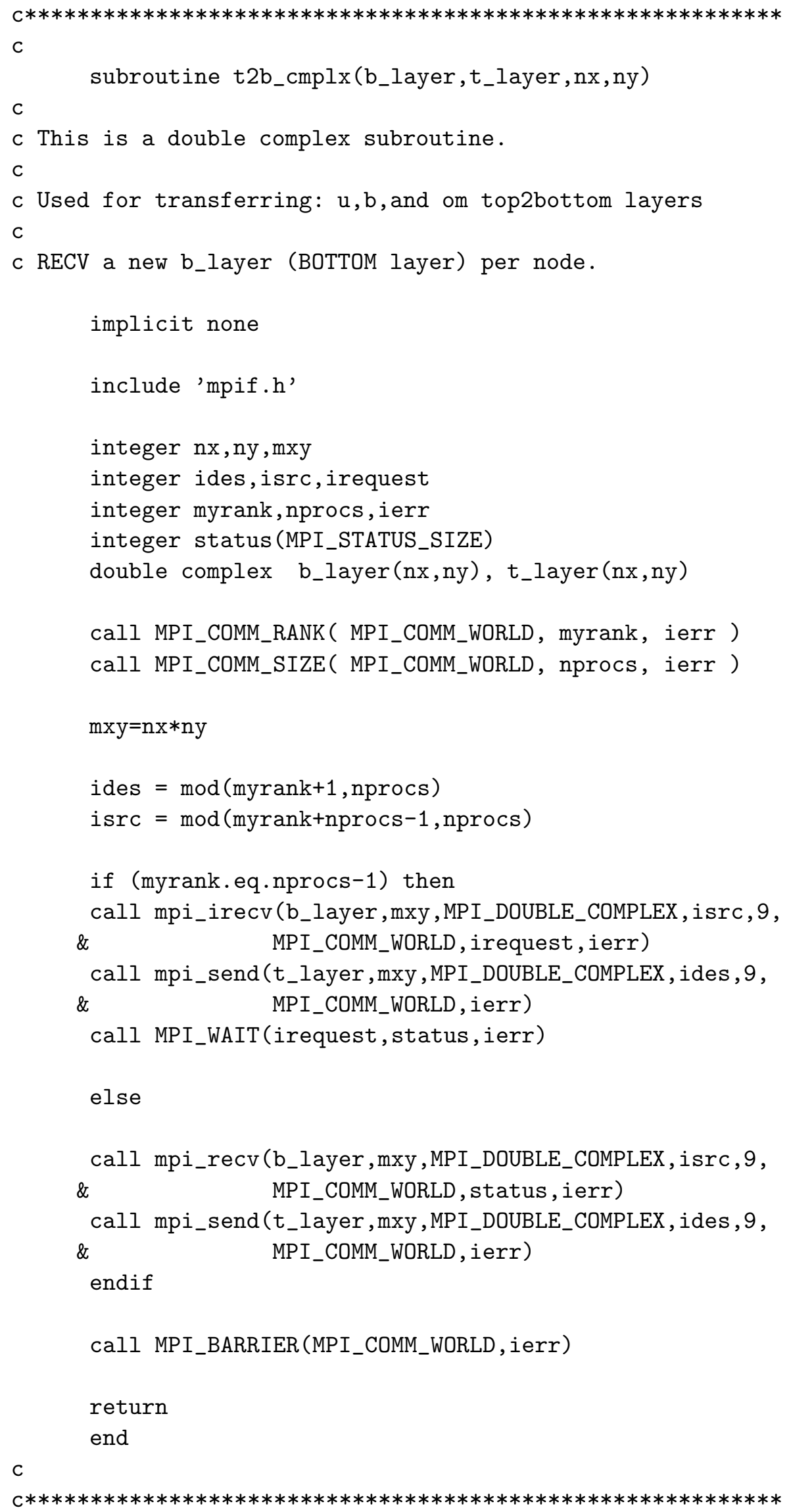


C

subroutine b2t_cmplx(b_layer,t_layer, nx, ny)

C

c This is a double complex subroutine.

C

c Used for transferring: $u, b$, and om bottom2top layers

C

c RECV a new t_layer (TOP layer) per node.

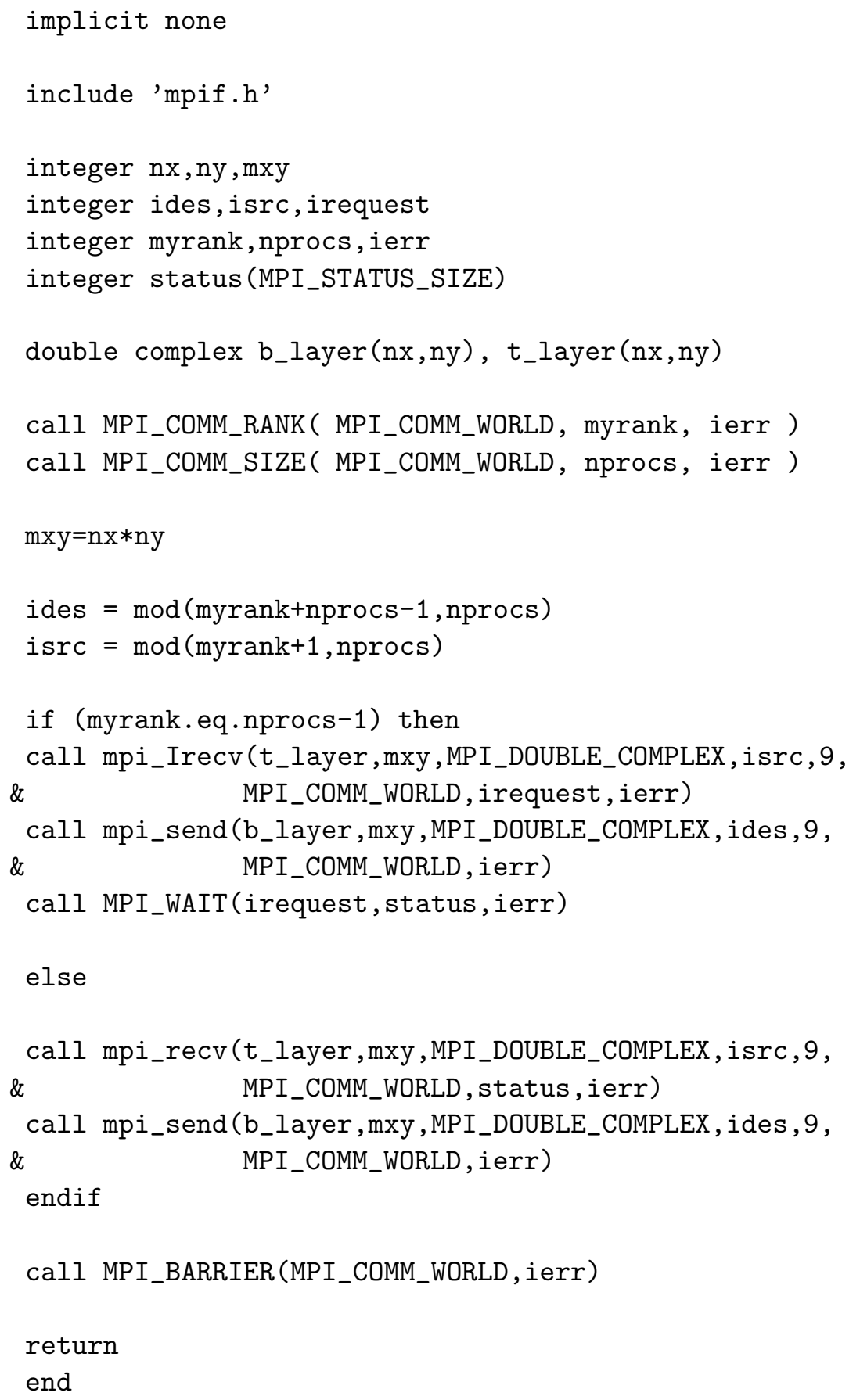




\section{Tools}

\section{$7.1 \quad$ MEMAPP.f}

This FORTRAN90 program makes approximate calculations for the memory requirements need for a given job based on (nx,ny,nz) triplet and number of phases. The user is queried for the $(n x, n y, n z)$ triplet, the number of phases and also the specific program. The resulting output is a list of the approximate memory requirements per processing node.

This is useful information since the user will be able to use these results to decide how much memory will be required per node of their cluster. User should have knowledge of the amount of RAM on each of their cluster nodes.

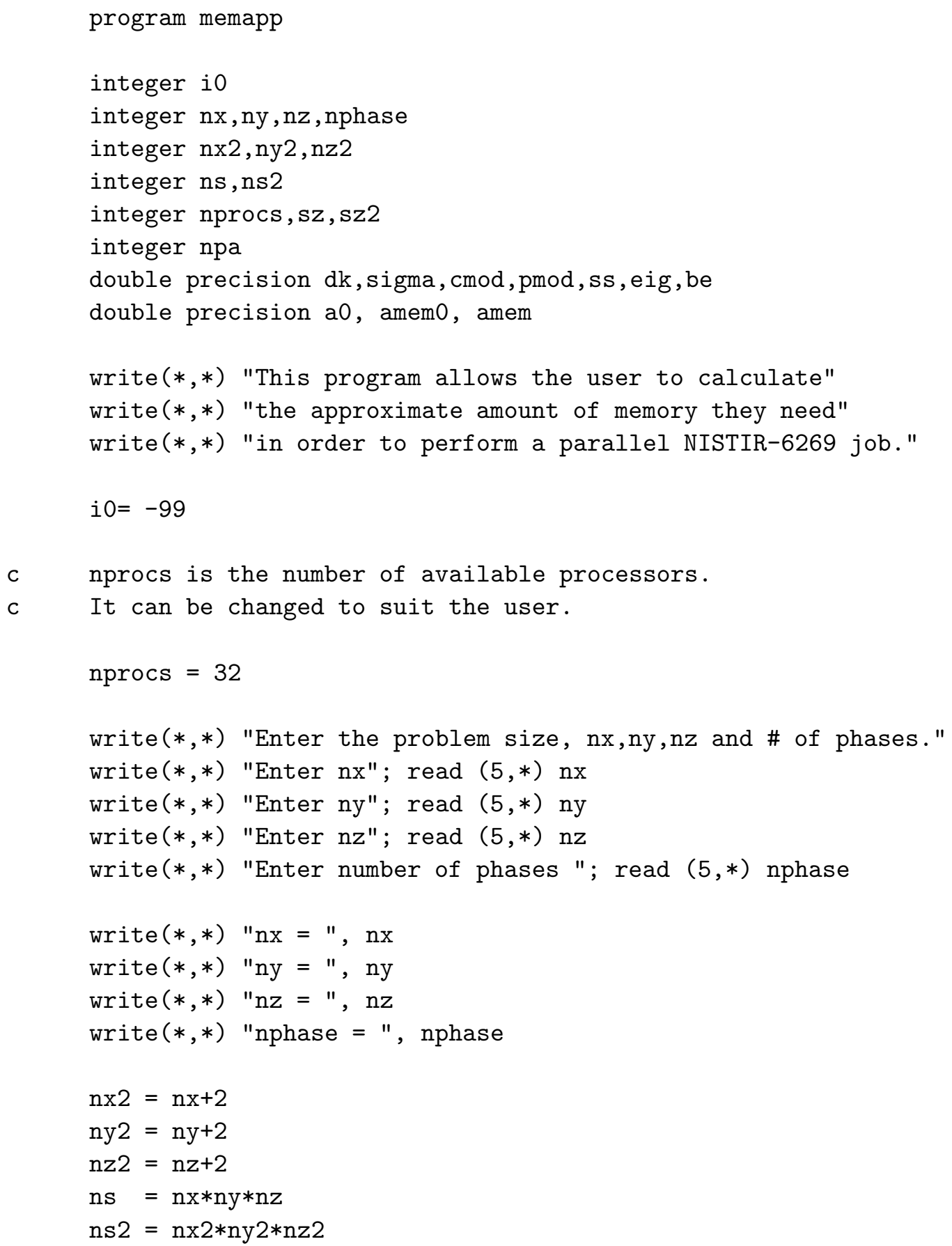




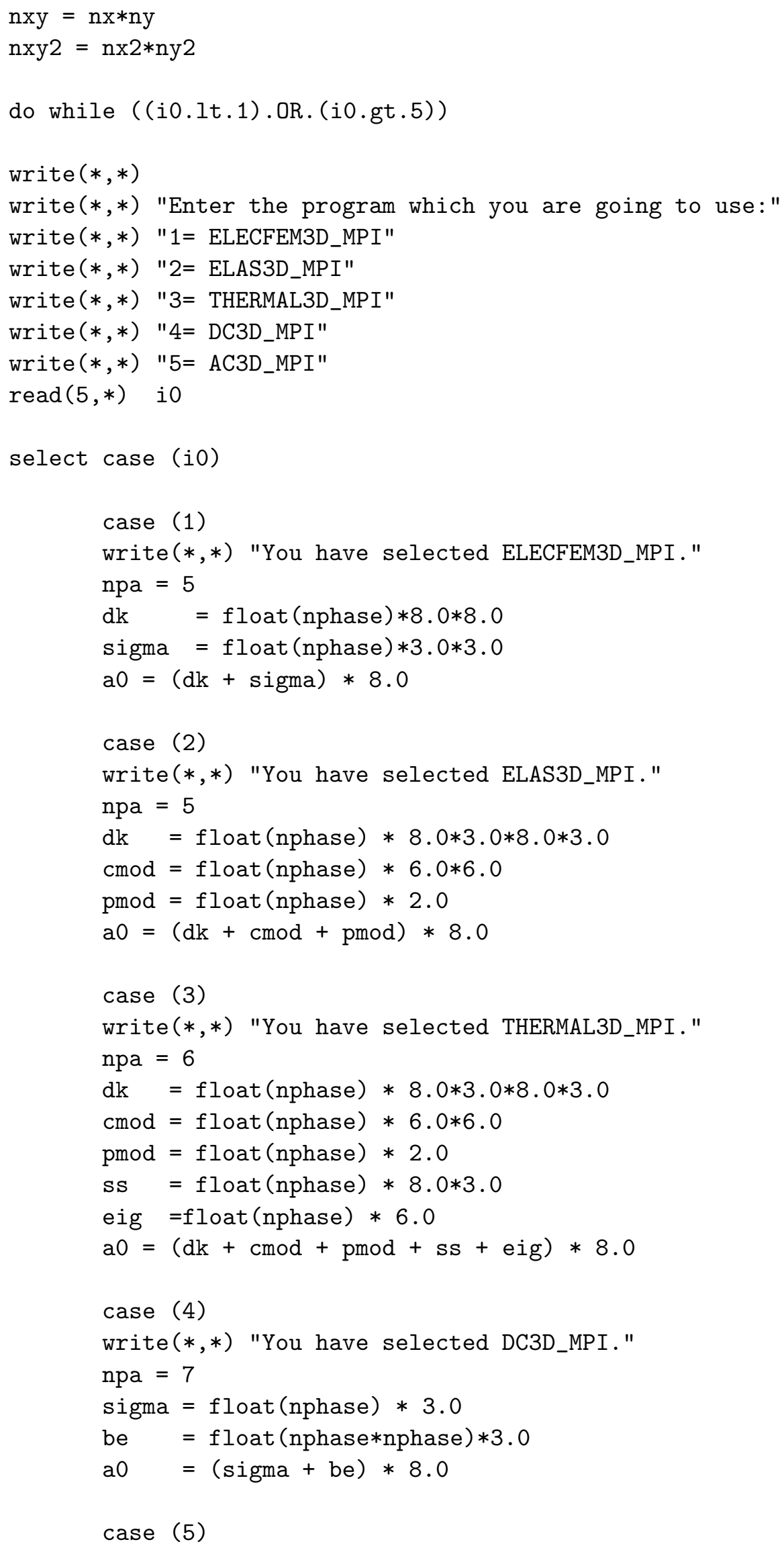




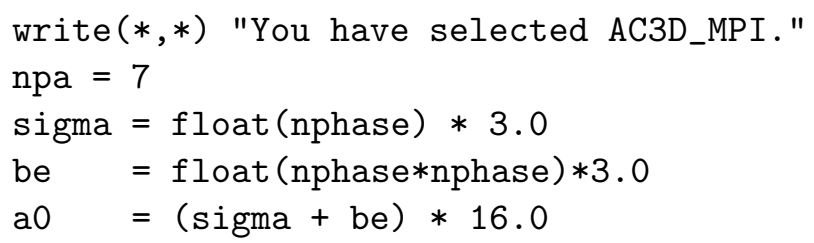

end select

end do

c Output also written to file: memory.dat

open (unit=7,file='memory.dat')

do $n=1$, nprocs

$\mathrm{sz}=\mathrm{nz} / \mathrm{n}$

rem $=\mathrm{nz}-\mathrm{n} * \mathrm{sz}$

if (rem.ne.0) then

$\mathrm{sz}=\mathrm{sz}+1$

end if

$\mathrm{sz} 2=\mathrm{nz} 2 / \mathrm{n}$

rem2 $=\mathrm{nz2}-\mathrm{n} * \mathrm{sz} 2$

if (rem2.ne.0) then $s z 2=s z 2+1$

end if

select case (i0)

C

c Memory for ELECFEM3D

C

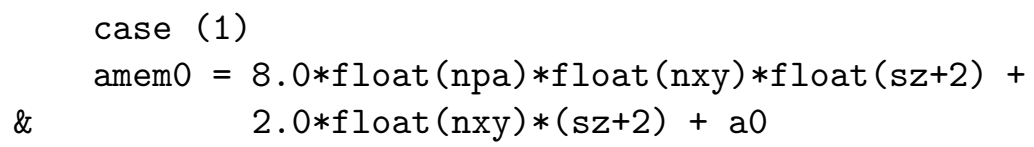

C

c Memory for ELAS3D

C

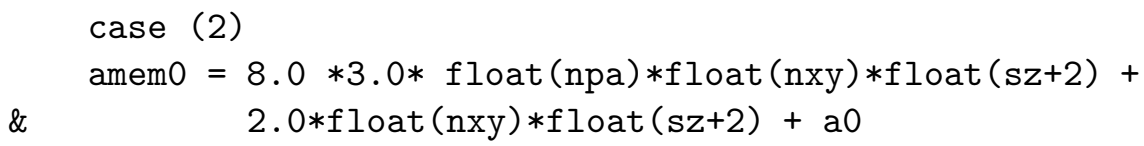


c Memory for DC3D

c

$$
\text { case (4) }
$$

C

c Memory for AC3D

c case (5)

end

\section{References}

[1] Edward J. Garboczi. Finite element and finite difference programs for computing the linear electric and elastic properties of digital images of random materials. NIST Interagency Report NISTIR 6269, Building and Fire Research Laboratory, National Institute of Standards and Technology, 1998.

[2] William H. Press, Brian P. Flannery, Saul A. Teukolsky, and William T. Vetterling. Numerical Recipes. Cambridge University Press, 1986. 